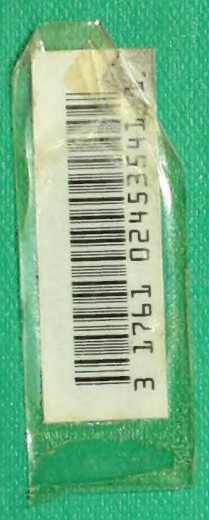




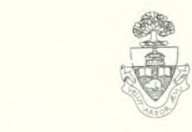

UNIVERSITY OF TORONTO LIBRARI

purchased for the
Geology Collection

from the

VARSITY PALAEONTOLOGY

FUND 
Digitized by the Internet Archive in 2010 with funding from University of Ottawa 





\section{LE TERRAIN}

\section{CARBONIFËRE MARIN}

DE LA France GeNTRALE 
CLepront-Ferrand. - TYPographie et lithographie g. MoNT-Louis 


\title{
LE TERRAIN
}

\section{CARBONIFERE VIARIN}

\section{DE LA FranCE CENTrale}

I. ÉTUde PALÉONTOLOGIQUE ET STRATIGRAPHIQUE DES FAUNES

II. TRAISGRESSION DE LA MER GARBONIFERE

III. ANGIENS GLAGIERS DE LA PÉRIODE HOUILLĖRE SUPÉRIEURE DANS LA FR INGE GENTRALE

AVEC COUPES GEOLOGIQUES ET DIX-SEPT PLANCHES DE FOSSILES

EN HÉLIOGRAVURE DE LA MAISON DUJARDIN

PAR

\section{A. JULIEN}

PROFESSEUR DE GÉLOGIE ET MINÉRALOgIE A L'UNIVERSITÉ DE CLIRMONT-FERRAND

\author{
PARIS \\ MASSON ET Cie, ÉDTTEURS \\ LIBRAIRES DE L'ACADEMIE DE MEDECINE \\ 120, BOULEVARD SAINT-GERMAIN
}




$$
\begin{aligned}
& \text { QE } \\
& 729 \\
& J 85
\end{aligned}
$$

$B R A R Y$

NA.V +1972

atrensity of TOROA 


\section{INTRODUG'TON}

Coxsidérations prélininaires. - Depuis une trentaine d'années, les faunes et les flores fossiles ensevelies dans les terrains tertiaires et houillers de la France centrale ont été l'objet de travaux considérables qui ont porté leur connaissance à un rare degré de perfection. Nous rappellerons, en premier lieu, le grand ouvrage de M. Alph. Milne-Edwards, intitulé : Recherches anatomiques et paléontologiques pour servir it l'histoire des Oiseaux fossiles de la France, qui renferme la description de la célèbre faune ornithologique du calcaire à Phryganes de Saint-Gérand-le-Puy. A cette œuvre magistrale vimrent s'ajouter, quelques années plus tard, les savantes monographies de M. Oustalet et de M. Filhol relatives, l'une aux curieux insectes de Corent, et les autres aux mammifères oligocènes de Saint-Gérand-le-Puy et de Ronzon. Le marquis de Saporta, dont la science déplore la perte récente, et le très distingué botaniste de l'Université catholique de Lille, M. l'abbé Boulay, ont, à leur tour, étudié sans relîche et publié dans de nombreux mémoires nos belles flores tertiaires et quaternaires qui, depuis les empreintes tongriennes des arkoses de Ravel, jusqu'à celles du dusodyle de Saint-Saturnin formé dans la vallée de la Monne au début de la période actuelle, nous offrent, fait unique en Europe, une série végétale presque sans lacunes.

Les Flores et les Faunes des lambeaux de terrain houiller du Pliteau Central ont également, dans le cours de la même période, donné lieu ì des publications du plus haut intérêt. En 1877, parut d'abord le bel onvrage de M. Grand'Eury sur La Flore carbonifere du département de la Loire et du centre de la France, fruit de longues années d'études et de recherehes persévérantes. Dès son apparition, ce travail nous révélait à la fois la riehesse exubérante de la végétation houillère, l'existence de types alor's mal connus, reconstitués" par l'auteur avec une sagacité admirable, et il nous démontrait en même temps, son âge plus récent que celui de la flore des bas- 
sins de la Belgique, d'Angleterre et de Westphalie. Puis, dans ces dernières années, les œurres de MII. Zeiller, B. Renault, Ch. Brongniart et Sauvage qui ont utilisé avee une science consommée les immenses matériaux recueillis dans les tranchées de Commentry, par la sollicitude éclairée de l'honorable et distingué directeur du bassin, M. H. Fayol. Malgré tant d'activité scientifique, malgré tant d'efforts accomplis, il existe encore une lacune regrettable dans la comnaissanes des faunes anciennes de la France centrale. Nous voulons parler de la faune carbonifêre marine qui n'a donné lieu, depuis près d'un demi-siecle, ì aucune recherche spéciale. Cette faune est cependant d'une singulière viriété, et n'était l'extrême mauvais étut de conservation des éléments qui la composent, elle pourrait rivaliser à ce point de vue avec celles de ta Belgique et de l'Irlande, illustrées par les travaux de de Koninck et de Mac-Coy. Elle ofire, par sureroît, un intérêt géologique de premier ordre que révèle bientist son étude à l'observateur attentif. En effet, grâce à son parallélisme complet avec la faune belge, depuis la base du tournaisien jusqu'au sommet du visécn, ainsi qu’à sa répartition géographique spéciale, sa comparaison arce les fauncs carbonitères du reste de la France, toutes exclusivement visécnnes, projette bientôt me lumière éclatante et décisive sur l'ensemble des laits qui se sont déroulés dans le cours de la période carbonifère, particulièrement sur la grande invasion de la mer à eette époque, en France et en Europe.

Ce tranail a prour but de combler eette lacune, non point complètement, il est vai, mais dans les tris larges et tris suffisantes limites que le mauvais état de conservation des fussiles, occasionné par un métamorphisme intense, nous a improsins. Il a aussi pour but de tirer de nos études de paléontologie et de straliqraphlies comparies toutes les conséquenees relatives à la géologie générale 'qu'ellessernterment.

Mais avant de faire connaître le plan auquel nous nous sommes arrêté, nous aproma deverir expreser d'abord, dans une brève notice, l'historique des travaux

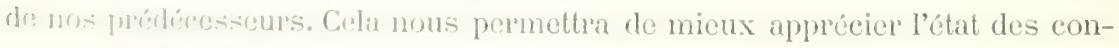
maissunes arequises an début de nos propres études et d'attribuer à ehacun la purt du justica distributive qui lui revient dans eette lente ef pénible álaboration

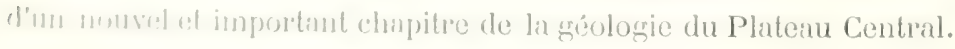

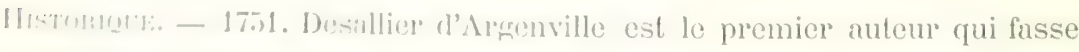

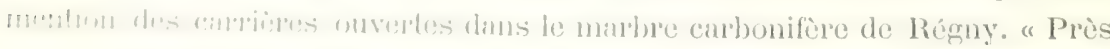

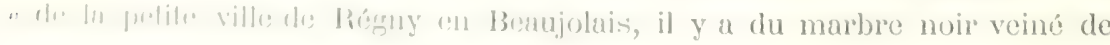


"blane qui se polit très bien; mais il résiste peu au grand air. Les débris de " la carrière servent à faire de la chanx ${ }^{(1)}$. "

1795. Alléon-Dulac mentionne à son tour les carrières de marbre de Lá Forest, près Thizy, Saint-Symphorien-de-Lay et Régny. Il constate dès cette époque une certaine analogie entre ce marbre et celui de Flandre.

"La pierre de Régny, qui est un marbre noir veiné de blanc, fut connue en " cette ville au commencement de ce siècle. L'on en fit, il y a environ trente ans,

" une consommation assez considérable pour des cheminées et des tables; " mais la difficulté et le prix excessif du charroi, et surtout le défaut qu'a ce " marbre de ne pouvoir pas résister au feu pour les cheminées, en fit bientôt " perdre l'usage. Il a été avantageusement remplacé par le marbre de Suisse. "La variété des veines et des coulcur's de ce marbre est infinie, quoique en "général il ait du rapport avec le marbre de la Flandre ou de la Porte") Sainte (2). ")

Et plus loin, nous lisons : “ A La Forest, château et fief dans la paroisse du " bourg de Thizy, on a ouvert une carrière de marbre noir ou bleu trìs foncé, " veiné de blanc, qui est à fleur de terre, par tables et non par blocs, incli"nées de l'orient au couchant sous un angle de $40^{\circ}$ environ ${ }^{(3)}$. ")

1797. Deux ans plus tard, Passinges, professeur d'histoire naturelle ì l'Ecole centrale de Roanne, signale dans le marbre de Régny la présence d'entroques : " Le marbre contient dans une certaine couche, qui peut avoir l'épais» seur d'un pied, une assez grande quantité d'entroques de différentes gros” seurs. ) Le même naturaliste signale ì Thizy le grès anthracifère qui reconvre le marbre: "Dans le bourg même de Thizy, il y a une masse de marbre ") assez grossier qui est de la même nature que celui de Régny et il est courert ") d'un grès granitique (4). »

1825. En 1825, Valuy décrit le calcaire de Régny qui renferme des Polypiers, des Orthocératites et des débris de quelques autres coquilles. Il décourre une empreinte de roseau dans les schistes argileux de Lay superposés an calcarre de Régny, et cite pour la première fois à Propières et à Azolette, dans to

(1) Essai sur l'histoire naturelle des fossiles rqui se trouvent dans toutes les provinces de France. Oryctologic. III ${ }^{e}$ partie, page 479.

(2) Alléon-Dulac. Memoires pour sercir it l'histoire nuturelle des départements du Rhone et de lu Loire. T. II, p. 103, 1795.

(3) Alléon-Dulac. Loc. cit., p. 168.

(4) Passinges. Mém. jour serwis à l'hist. nat. du département de la Loire. Journul des Mines, L. VI ob VII, 1797, et Anrules de l'Aureryne, t. XiII, p. 359 et 378. 1810. 
Beaujolais, les exploitations de calcaire de transition noir, traversé de veines blanches de spath calcaire qui contient quelques débris de Polypiers et d'Orthocératites (1).

Nous avons tenu à donner in extenso les extraits ci-dessus de ces vieux auteurs parce que en quelques lignes nous y voyons apparaître en germe la notion des principaux caractères du terrain carbonifère dans le Roannais, le Lyonnais et le Beaujolais, saroir : la découverte et la mise en exploitation des marbres de Régny, de Thizy, de Propières et d'Azolette, gisements restés classiques; la division en couches de ces marbres; leur dislocation; leur analogie avec le marbre carbonifère de Flandre dont ils ignorent, bien entendu, l'âge relatif; la notion de fossiles marins dont la liste ne fut guère augmentée plus tard par Grüner; le grès anthracifère avec ses roseaux (Bornia), et enfin sa superposition au marbre.

1830. En 1822, Brochant de Villers, nommé directeur de la Carte géologique de France, en confia l'exécution à E. de Beaumont et Dufrénoy. Une ligne séparative tirée de Honfleur sur Alençon, Avallon et Chalon-sur-Saône, puis le long de la Saône et du Rhône jusqu'à la Méditerranée, délimitait le domaine de chacun d'eux. Dufrénoy fut chargé de l'Ouest, Elie de Beaumont de l'Est. Les observations de Dufrénoy relatives aux montagnes du centre de la France furent consignées dans un Mémoire inséré dans les Annales des Mines ${ }^{(2)}$. Cet auteur, qui ne sut pas distinguer le terrain carbonifère du terrain cambrien, assimila vaguement le marbre de Régny au terrain de transition des Anglais.

1835. Le 30 août de l'année 1835, Legrand, chef de l'Administration des Mines, décida l'exécution des cartes géologiques départementales. Le capitaine Rozet fut chargé du massif de montagnes qui sépare la Loire de la Saône et du lihnne, et l'ingénien Grüner, du département de la Loire. Nous verrons polus hin le résultat des travaux de ces deux savants.

18:37. Wans un mémoire important publié à cette date, dans les Bulletins de la

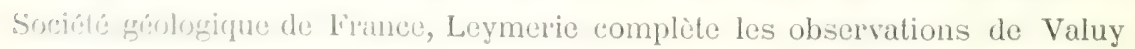
que lis mort, dit-il, a cnlevé trop tôt à la science. Il décrit d'abord le terrain de

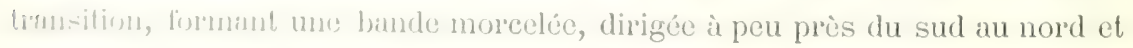
comprant nas pratie des deux versants de la chaîne primordiale qui sépare la

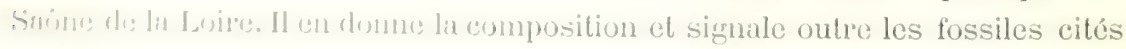

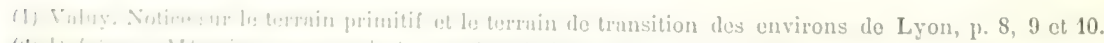

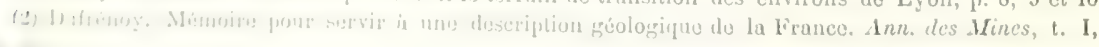


par Valuy, coux qu’il y a trouvés lui-même, savoir deux espèces de Térébratules qui sont d'après M. Michelin :

\section{Terebratula resupinata, Sow.}

- lateralis, Sow.

Les encrines sont assez abondantes et appartiennent pour la plupart au genre Cyathocrinites et peut-être à l'espèce Cyathocrinites rugosus, Miller.

Les Polypiers lui ont paru principalement affectés aux minces couches argileuses; la cassure offre très fréquemment des anneaux et des lames spathiques qui ne sont autre chose que des anneaux de Crinoïdes.

Il s'efforce ensuite de fixer l'âge de ce terrain et le rapporte avec doute au terrain silurien ainsi que le démontrent les citations suivantes:

" J'ai été conduit d'abord par diverses considérations à rejeter l'idée que nos " conglomérats et nos grès pouvaient appartenir au millstone-grit ou au vieux " grès rouge. Je me suis attuché ensuite aux calcaires que j’ai comparés suc" cessivement au calcaire carbonifere (Mountain limestone), systeme calca" reux supérieur de Dumont, et au calcaire de la Grauracke (Dudley, Ent, " Plymouth-Rocks), système calcareux inférieur de Dumont; et c'est à ce " dernier calcaire que le nôtre m’a paru devoir être rapporté. Voici mes princi" paux motifs: $1^{\circ}$ Grande analogie entre los caractères minćralogiques de " notre calcaire et ceux du calcaire de la Grauwacke des Anglais et du calcaire " infériem de M. Dumont, roche que M. Buckland a rapprochée du calcaire de "Dudley; 20 même épaisseur de couches que dans eette dernière localité; " mème emploi; existence dans les deux ealcaires de nombreux débris de Cri"noïdes et de plaques à Polypiers. Il faut avouer cependant que jusqu'i pré"sent on n'a pas reconmu chez nous les Trilobites si ahondants a Dudley, " mais je ferai observer que l'absence de ces fossiles se présente égalument " dans le système calcareux inférieur de la Belgíque, ce qui n’a pais empếché " M. Buckiland de le rapporter au calcaire de Dudley; $3^{\circ}$ enfin la position de "nos calcaires entre deux systemes, ou plutôt au milieu d'un systeme unique " de conglomérats, de grès et de schistes présentant heancoup d'analogie avec " la graumacke des Anglais.

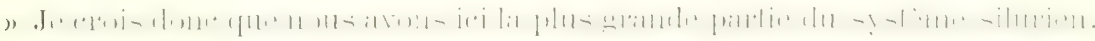

"Toutefois, je n'emmets colte opinion qu'aree doute, car jo sens linen moi") même que pour me pronuncer aree quelque assurance, il me niancque oncore 
"des documents que je ne puis me procurer que par de nouvelles études diringées surtout vers la recherche des fossiles et des superpositions (1). ”

18.10. Rozet, à la suite des recherehes accomplies pour l'exécution de la carte qui lui avait été dévolue, attribua au terrain silurien des Anglais les marbres de Régny, Saint-Victor et Thizy. Nous remarquerons en passant que ce sarant non plus, n'a su distinguer le cambrien du carbonifère (2).

1810. De Terneuil. Dans une note lue dans la séance du 2 mars de la Société géologique de France, note intitulée: Sur l'importance de la limite qui sépare le culcaire carbonifere des formations qui lui sont inférieures, nous trouvons la mention suivunte: " En France, nous n'avons de calcaire de montagne bien ") caractérisé que sur les frontières de Belgique et à Marquise, près de Bou") logne, oì il repose sur le terrain silurien. Cependant j'y rapporte encore, à " cause de leurs fossiles et malgré d'imposantes autorités, les calcaires supé. rieurs de Sablé prés du Mans, ceux de la montagne de Tarare et ceux de "Regny au nord-ouest de Lyon. Bien que ces calcaires soient peu riches en " fos-iles, ils contiennent quelques espèces qui sont très abondantes dans le "calcuire carbonitëre, et qui n’ont jamais été trouvées dans les vrais terrains n siluriens, dins ceux qui recouverts par le calcaire de montagne, sont hors " de trute contestution. Ios untres terrains de la France sont siluriens ou cam" briens."

De Vernenil ne cite dans cette note aucum fossile de Régny et le nom de Jourdan n'est pas une seule fois mentionné dans le volume. Toutefois, d'Archiac: cru rappelent dans son Rapport sur les progrès de la paléontologie en fronce rette déchuration de de Ternenil, a écrit ees lignes que nous reproduisuns: "I)e Vernetuil et Jonrelan ont signalé dans les calcaires qui s'étendraient

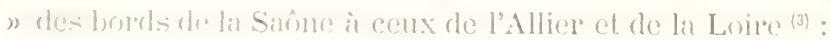

Procluetus corre.

Chonetess pupilionacen.

Spririfirm bismlentus.

()rthis arenistrin.

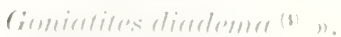

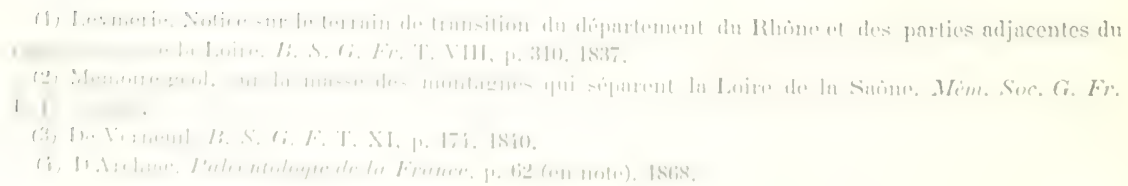


Apres la lecture de cette note, Leymerie fit une observition sur le passage du mémoire de M. de Verneuil où il est question d'un calcaire des environs de Régny que l'auteur eomprend dans la formation earbonifère. Suirant Leymerie, ce lambeau de terrain calcaire se trouve it côté de bassins curbonifères bien développés, mais il paraît en être tout à fait indépendant et se rapporter à la formation silurienne (1).

1840. Grüner chargé, comme nous l'avons vu plus haut, dès 1835, de la carte géologique du département de la Loire, publia après cinq années d'explorations un remarqquable mémoire concernant la stratigraphie de ce département, mémoire dont le manuscrit resta entre les mains de Dufrénoy pour l'útablissement du texte de la carte géologique de Franee et qui ne fut imprimé qu'en 1811 dans les Annales des Mines (2). Il y subdivise en trois étages le terrain de transition. L'étage inférieur' formé de schistes satinés avec banes de lydieme, de quartzites et de conglomérats siliceux est attribué au cambrien; l'étage moyen et l'étage supérieur sont, à l'exemple de Leymerie, classús dans le terrain silurien; l'étage moyen est formé de gris argilo-quartzeux, de calcaire gris bleû́tre très fossilifère et de schistes argilo-talqueux. L'étage supérieur est constitué par le grès anthracifere avee poudingues ì la base. Des éruptions de porphyre granitoïde séparent les étages moyen et supérieur.

C'est dans ce mémoine qu'il donne la liste suivante des fossiles trouvés par lui à Régny et déterminés par Voltz.

Orthis, vois. de Striatella.

Orthis, nov. sp.

Spirifer resupinatus ou esp. rois.

Terebratula. Pen déterminable.

Syringopora?

Etomphalus?

Il justifie l'attribution qu'il fuit au silurien des Anglais dans la phrase sui vante: "Ces fossiles, quoique peu nombreux, semblent cependint bien, par" "l'absence dos Productus et l'abondance des Orthis, caractériser le ter'tain " comme teranin silurien des Anglais. "

Il mentionne également la décourerte de belles empreintes de plantes dans le grès anthracifère do Neulize.

(1) Leymeric, B. S. G. F. I1,, ib., 13, 179.

(2) Gräner. Ménoire sur la mature des terrains de transition et de porphyre du départenent do lat Loire. Annales tes Mines, 3e série. T. XIX, 1. 53. Imprimó en $18+1$. 
1841. Dufrénor. Cette classification fut adoptée par Dufrénoy dans le chapitre relatif au Terrain ancien et au Terrain de transition des montagnes du centre de la France, inséré dans l'Explication de la carte géologique de la France (1).

Toutefois, l'illustre collaborateur d'E. de Beaumont lui fit subir une légère modification en classant le grès anthracifère, division supérieure du silurien de Grïner, dans le déronien à cause des analogies que semblait offrir ce terrain avec le terrain dévonien que Murchison venait de décrire.

1841. E. de Beaumont rapproche les calcaires à Strophomènes de Chénebié et Schirmeck dans les Tosges méridionales, de ceux qu'on observe entre la Saône et la Loire et dans la partie méridionale du Morvan entre Autun et Decize, et qui contiennent des amas stratifiés de calcaire avec encrines et quelques autres fossiles en petit nombre. Pour lui, tous ces terrains font partie d'un nême système que les roches éruptives ont disloqué. Ces terrains qu'Elie fle Benumont rapporte au système de transition supérieur doivent, à son avis, étre rangés dans l'étage dévonien (2).

1812. Viquesnel déerit le terrain de transition de la vallée du Sichon et les porphỵes variés qui le traversent, mais sans en fixer l'âge ${ }^{(3)}$.

181. L'ingúnien I3oulanger eut à décrire pour l'établissement de la carte géologrque du dipartement de l'Allier le terrain de transition de ce département qui affleure en trois régions différentes, savoir: à Diou, à Saint-Léon Ul dans la vallée du Sichon, de Cusset à Ferrières. Il ne sut point distinguer l'âgerelatif des terrains de ces trois luealitis, ni le déronien de Diou, ni le canthicen de Saint-Léon et d'Aromne, ni le carbonifère de l'Ardoisière et de Firriner. Il chvisagua ces lrois lambeaux comme les restes d'une formation unique dépresice a l'époque silurieme, et plus tard disloquée par l'éruption des frandes prophroüdes du Mayet-de-Montagne (t).

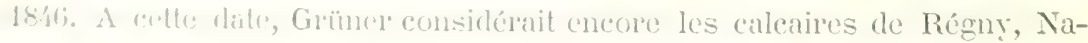
conme, Sinint-Germain-Laval ot Néronde, comme appartenant au terrain silu-

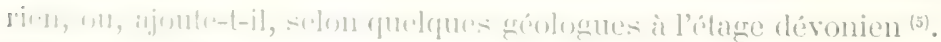

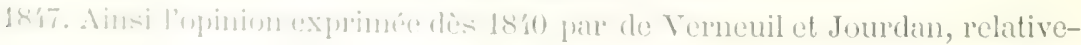

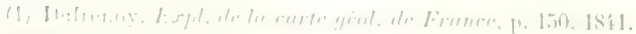

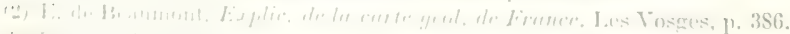

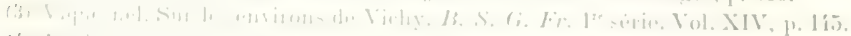

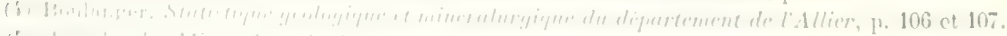

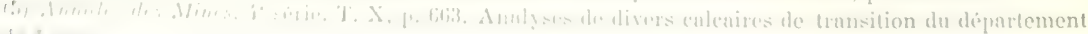
t!. 1,7 
ment à l'âge carbonifère des fossiles enfermés dans les marbres de transition du Lyomnais, du Forez et du Beanjolais, n'avait obtenu l'udhésion d'aucun des ingénieurs qui collaboraient à l'exécution de la carte géologique de France, puisque nous voyons quelques années plus tard Elie de Beaumont, Dufrénoy, Grüner et Boulanger, auxquels venaient s'adjoindre Rozet et Leymerie, considérer les marbres et les schistes de Régny, etc., et les grès qui leur sont superposés comme appartenant à la formation silurienne ou dévonienne. Pendant ce temps, Jourdan avait continué ses recherches, et de 1840 à 1848 il n'avait pas recueilli moins de 2,000 fossiles dans les carrières de Cherbué, Montagny, Thizy, Régny, Vaux, ete., qui tous démontraient victorieusement que les assises qui les renferment devaient être remontées du silurien ou du dévonien dans l'étage carbonifère marin. A la collection de Jourdan, déposée au Musée de la ville de Lyon, venaient s'ajouter encore, d'après une indication de Drian, celle de Fournet à la Faculté des sciences et la collection personnelle de Thiollière, qui toutes les deux renfermaient de beaux échantillons de fossiles des mêmes carrières.

Grüner nous a conservé la liste des fossiles de la collection Jourdan qu'il a reproduite dans son ouvrage sur la géologie du département de la Loire. Nous la donnons ci-dessous:

\section{Cyathophyllum dianthus?} cerentite's.

Syringopora remulosa. relaxa.

Stomatopora serpens.

Cyathocrinites rugosus.

Terebratula lata? pinnatus.

- hastata.

- leevis.

- limertr.

- lincatri planosulcata.

- subglobosa.

- inflata.

Spirifer trigonalis.

- voisin de trigonalis.

- subylobularis.

Orthis resupinata.

- Michelini.

- "1rulumurles. 
Productus scoticus.

- antiquatus.

- pulchellus.

- palliutus.

Chonetes papilionacea.

- curratus.

- Laguessi.

Porcellice influta.

Orthoceratites regularis ${ }^{(1)}$.

D'Archiae, en 1868, a exprimé son opinion sur la valeur de cette liste: " M. Grüner" cite d'après M. Jourdan, dans les calcaires de Régny, 30 espèces " de fossiles dont nous ne pouvons reproduire les noms qui nous sont pour la " plupart inconnus et ne sont suivis d'aucune indication d'auteur (2). ")

1818. De Terneuil, sur l'invitation de Jourdan, vint à Lyon visiter les collectims de ce dernier et il confirma pour la seconde fois l'attribution à la faune can wonifiere des fussiles qui les composaient. En outre, il signala dans une lettre it Elie de Beaumont les recherches du savant lyomais. L’illustre géologue sempressa d'adhérer ì une interprétation qui émanait d'une aussi haute autorité palésntologricque, et l'on trouve l'extrait suivant de la lettre de de Verneuil dans l'article Systime de montagnes du Dictionnaire d'histoire naturelle. "J'ai studić demièrement les différents calcaires des environs de Roanne, et les ai tuns recommus pour des calcaires carbonifères comme ceux de Sablé (Brelingles)

Lin entret, de Verneuil ne se contenta pas de l'oxamen des fossiles. Il visita dans es mime voyagre, en compargnie de Jourdan, les gîtes de Cherbué, Pradines, Condelles, Montaguy et Thizy.

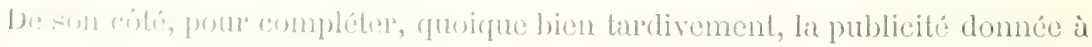

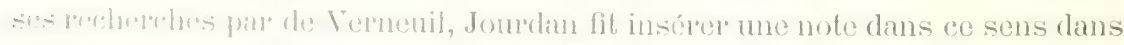

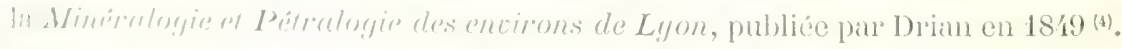

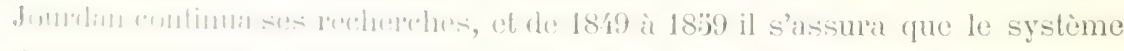

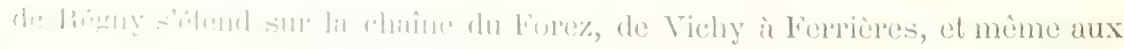

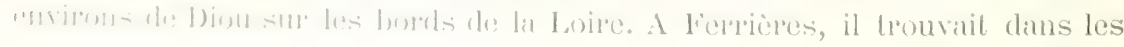

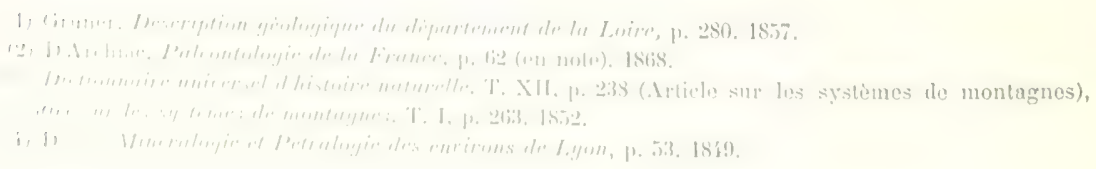


grès de cette dernière localite des fossiles marins, et à Diou l'Atrypa reticularis (qu'il mangeait ì tort dans la même formation (1).

1851. Dans l'été de 1851, Murchison éludiant la vallée du Sichon, découvrit dans le voisinage de l'Ardoisière une petite faune qu'il soumit à l'examen de de Verneuil. Ce dernicr y recommut les especes suivantes:

Productus fimbriatus, Sowerby, ou une espèce à épines sur les côtes transverses qu'on ne peut distinguer de celle-ci.

Ch. papilionacer? Phill.

Orthis crenistria, Phill.

Solenopsis, M'Coy.

Une seconde espèce de Productus, petite et striée Iongitudinalement.

Coquilles univalves en fragments.

Phillipsia, e'est-ì-dire le bouclier pointu de ce trilobite.

2 espèces d'Encrines.

C'était une faune carbonifère qui étendait le système de Régny jusqu'aux enrirons de Vichy. Malheureusement Murchison confondit les schistes trid minces à moitié décomposés et légèrement ferrugineux, qui ronfermaient ce: fossiles, aree le puissant système d'ardoises cambriennes de la vallée du Sichon. Toutefois, il paraissait assez disposé ì mettre au même nivęu le marbre de Ferrières et celui qu'il avait découvert à l'Ardoisière (2).

1855. Fournet, dans un mémoire bien connu, intitulé: Recherches stur l'extension des terrains houllers en France, nous fait connaitre le r'ésultat de ses: études personnelles sur le calcaire carbonifère qu'il avait étudié dès 1835. Sañ doute ces études n'ont eu aucune influence sur la marche de la science, puisqu’il n'a rien publié sur ce sujet avant 1855. Néanmoins, nous croỵons pour' honorer la ménoire de ce vieux géologue qui évoquait plutôt par la tomrnure de son esprit l’idée d'un mineur du xvin siècle égaré dans lo xrxe el dont les travaux visaient surtout l'étude des filons et du métamorphisme, devoir dire un mot des fáts qu'il expose dans le mémoire ci-dessus, si étranger du reste par les conceptions aux vues de la géologie moderne. Dès 1835, appelé par le Gouvernement de la direction des mines de Pontgibaud qu'il administrait depuis six ans, ì l'enseignement de la géologie à la likeulté des sciences de Lyon nouvellement instituée, et chargé simultanement de la restauration des célebres mines de Chessy et Sain-13el, il se mit à explorer rapidement la rérion

(1) Fournet. Extension des terruins houillers, p. 122.

(2) Murchison. Quert. Juurn, of the geol, Soe of London. 'I. V'II. 
Iyonnaise. Le premier résultat de son investigation fut l'établissement de la démarcation des trois zones primitive, primaire ct carbonifère. On en trouve déjà une esquisse dans son discours de réception lu à l'Académie de Lyon en 1835 et dans son mémoire à l'Institut en 1837. Mais il faut arriver à l'année 1859 pour trouver une étude détaillée de cotte ligne de démareation qui existe entre les micaschistes et le cambrien d'une part, et d'autre part entre le cambrien et le carhnnifère (I). Dans ce dernier mémoire, Fournet établit également la dislocation des terrains carbonifères dans le Beaujolais par les porphyres de Tarare et les syćnites, roches éruptives formant d'après lui un double système, dont l'un se prolonge entre la Loire et l'Azergue et jusque dans le Beaujolais et le Mâcommis; ct dont l'autre constitue l'arête beaujolaise qui se confond avec te premier système à partir des Echarmaux (p. 337).

En 18:36, deux puits avaient été creusés pour la recherche de l'anthracite à Valsonne. Fournet put recueillir dans les déblais de magnifiques et nombreuses empreintes de plantes et il les soumit à Brongniart qui constata leur identití aree celles du terrain houiller. Ce fut sans doute cette observation très interressinte qu'il eut tort de passer sous silence jusqu'en 1855, jointe à la découverte qu'il fit de fossiles marins dans les marbres de Régny, Thizy et le bassin de la Vaulxonne, ainsi qu'à la constatation de la liaison intime des grès à plantes aree les schistes à lentilles de marbre bleu fossilifère qu'ils recouvrent pentrut, co fut, dis-je, cette observation qui, sans nul doute, convainquit Fournet di: tris bonne home qu'il arait lix sous les yeux un représentant dans la linnere contrale du Nombain limestone des Anglais.

1)is 1S:37, linurnet avait visite tous les gisements aujourd'hui comus du cal-

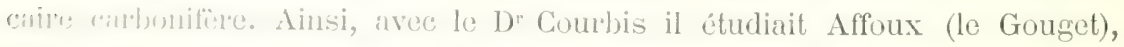
Trün+, linguy, puis Azolette, Propières, signalés jadis par Valuy et dont il

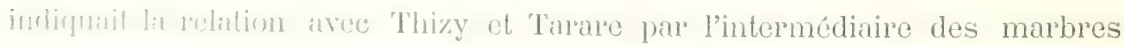
des Sind-13onmet-le-Troney. Il explorait aussi les carrières du bassin de la

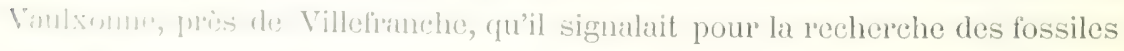
i) In, Initian.

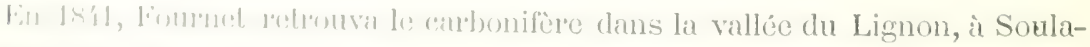

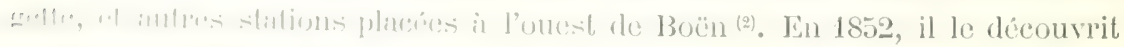

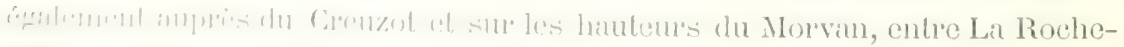

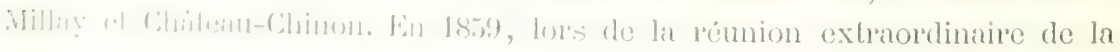


Société géologique de France à Lyon, il dirigea quelques excursions et donna une excellente description du grès anthracifere de Saint-Symphorien-de-Lay (1). Entre temps, en 1846, dans le cour's d'un royage d'exploration dans les Vosges, il découvrit le carbonifère à Planchez-les-Mines et reconnut son identité arec celui du département du Rhône.

Pour bien préciser la nature des recherches de Fournet, il faut rappeler qu'il encourageait son collègue et ami Jourdan à recueillir des fossiles dans les marbres, afin de voir confirmer ses propres vues par la paléontologie, seience, dit-il, à la mode à cette époque et dont il sentait l'importance, non point pour' lui-même, mais pour être mieux compris de ses auditeur's.

La question est done posée, par le récit rétrospectif de Fournet, de savoir si la détermination de l'àge carbonifère de nos marbres et grìs anthraciferres appartient en réalité à Fournet qui en revendiquait la priorité, ou bien à Jourdan et de Verneuil ainsi que cela résulte des pages précédentes. Or, ù notre avis, e'est bien à ces deux derniers savants que revient le mérite de cette découverte, car, dès 1840, puis en 1848, de Verneuil avait déclaré carbonifères les fossiles découverts par Jourdan et mis paléontologiquement hor's de doute l'existence de ce terrain; tandis que de 1835 à 1855 Fournet gardait un silence absolu sur ses travaux, ses découvertes et ses opinions personnelles. Il ne l'a rompu qu'une fois, nous semble-t-il, pour faire connaître à Bronn (2) sa classification des roches de la région lyonnaise dans laquelle il range dans le silurien les schistes, les grès à plantes, toute la formation carbonifère, en un mot, des environs de Tarare. Une telle contradiction, véritablement inexplicable, nous met dans l'impossibilité d'enlever à Jour'dan le mérite de ses recherches paléontologiques, et à Grüner l'établissement de la stratigraphie du même terrain.

Ajoutons cependant que Jourdan mérite bien les mêmes eritiques pour avoir indéfiniment gardé le silence sur ses propres recherehes, d'une si réelle importance, car sans l'heureuse intervention de de Verneuil on se demanderait peutêtre encore à l'heure actuelle quel peut bien être l'âge des terrains carbonifùres de la France centrale?

1857-1859. Grüner publie sa Description géologique et minéralogique du département de la Loire, ouvre d'une importance eapitale pour l'itude stratigraphique du carbonifère.

(1) J. Fournet. Géologie lyonnaise, p. 333. 1861.

(2) Neues Jahrbuch (aout 18'3), P. 707, et Notice historique sur le vir et les tracunx de Fonrnet. E. Chantre, [. 6 tet 65.1870. 
Nous arons vu plus haut que, dix ans avant, il croyait encore à l'âge silurien de ce terrain. Il fut, comme Elie de Beaumont, détrompé par les déclarations si catégoriques de de Verneuil, après la visite en 1848 des collections de Jourdan, et surtout après la lettre mémorable à E. de Beaumont relative à ce sujet.

Aussi n'est-il plus question de terrain silurien dans cet ouvrage, et le terrain corbonifère est-il rétabli officiellement à sa rraie place et mis en pleine lumière. La Description géologique de la Loire fut présentée par l'auteur à la Société géologique de France dans la séance du 21 février 1859. Nous ne donnerons pis l'analyse de cet ouvrage jusqu'à nos jour's demeuré classique. Nous rappellerons seulement la classification générale à laquelle Grüner était arrivé, car nous aurons à la discuter et à la modifier plus loin.

1. Terrain houiller proprement dit :

Porphrye quartzifère (système du Forez N. 15 O.);

$2 \circ$ Grès à anthracite (millstone-grit des Anglais);

Porphyre micacé granitoïde (système des ballons E. 15 S.).

30 Granwacke ou calcaire carbonifère.

1868. Grïncr fait comaître dans la Creuse des lambeaux de dépôts schisteux et de grìs arec rares lentilles de calcaire gris bleuâtre, semi-cristallin, bitumineux, surmonté d'argiles anthraciteuses. Cet ensemble représente, d'après le savant ingénieur, le terrain anthracifure du Roannais. Il signale la découverte d'in article d'enerine dans le marbre du Chat-Cros (1).

1873. De Koninck. Dans la livraison du Bulletin de la Société géologique de France, consacréc à la réunion extruordinaire tenue à Roanne le 30 août 1873 et presidée par Grüner, on a inséré la liste des fossiles de Régny qui se trouvaient ì celte éporque dans les collections de l'Ecole des Mines et qui avaient be dérmines par de Koninck. Nous la reproduisons ici :

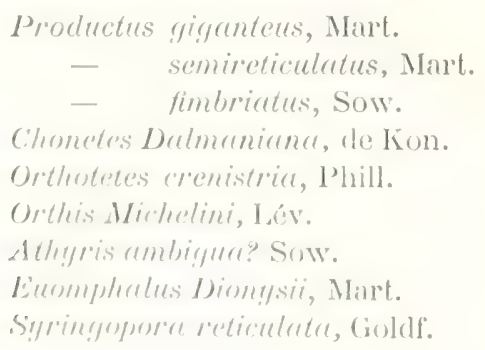


A cette liste en est jointe une autre du même gisement provenant de la collection Jourdan et déterminée par le savant lyounais lui-même :

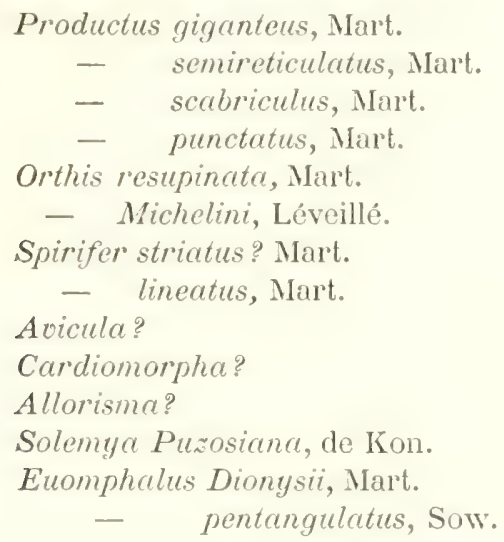

Ensemble 19 espèces formant la totalité des espèces carbonifères de la France centrale, publiées et connues en 1873.

Tel était l'état des connaissances relatives au terrain carbonifère en 1872 dans l'année où nous avons commené nos propres recherches. L'extension de ce terrain dans le Plateau Central était succinctement connue. Il restait à en fixer les nombreux affleurements disséminés sur cette vaste surface sur une carte géologique. Il fallait, par conséquent, les distinguer nettement du terrain cambrien qu'ils recouvrent transgressivement, en le débordant fréquemment, pour reposer sur le terrain primitif. La stratigraphie qui est généralement très simple, formée sur tous les points de deux termes, l'un inférieur et marin, l'autre supérieur et continental n'était guère connue que pour la Loire, aree beaucoup d'incertitude pour les couches de la base, que Griuncr' fait rentrer avec doute dans le cambrien et qui appartiennent en réalité partic au cambrien, partie au waulsortien.

Quant à la paléontologie, seule base d'une commassance certaine et définitive, elle était restée dans l'état où l'avait laisséc Jourdan en 18 17, pan conséquent inutilisable, soit pour fixer lo earactere et l'âge des grisements fossilifères, soit pour établir une comparaison avec la Belgique et l'Angleterre, pays classiques par excellence, voire meime aree les gisements frantenis déconverts dans l'intervalle. 
La lecture du mémoire de Murchison sur la vallée du Sichon fut le point de départ de nos recherches. Grâce aux loisirs des vacances annuelles, je pus recueillir, tant à Régny qu'à l'Ardoisière, quelques millier's de spécimens suffisants pour atteindre le but que je recherchais. J'ajoutais plus tard à ces deux belles faunes celle du Morvan dont la première localité, située entre SavignyPoil-Foil et le champ de la Barette, a été signalée pour la première fois par M. Michel-Lévy dans la légende de la feuille d'Autun, et je recueillis ainsi d'autres milliers de fossiles de l'époque tournaisienne. Ces trois localités permettaient d'établir dès lors un parallélisme complet avec les trois grandes faunes belges de Tournai, de Dinant et de Visé. Appuyé sur cette base solide, jétendis successivement mes recherches à tous les gisements connus ou simplement signalés du Plateau Central, m’efforçant simultanément de décourrir les relations des lambeaux carbonifères avec le substratum cambrien ou cristallophyllicn, et de rechercher des fossiles à la base même des lambeaux carbonifères. L'idée heureuse que j'eus de transporter les marbres cristallins d'un blanc bleun̂tre situés à la base de notre terrain, au Musée royal d'histoire naturelle de Bruxelles, eut pour conséquence la découverte de leur identité arec los narbres waulsortiens belges, particulièrement avec le marbre des Pancquys. Cela me permit de mettre la dernière main à la connaissance du carbonitère marin de la france centrale en faisant ressortir nettement le parallèle complet el définitif de ce terrain avec celui de la Belgique.

Dans l'inter'valle, pour' mener' à bonne fin cette étude, j'avais parfois recours ì lir science consommée de de Koninck, l'illustre paléontologiste belge, qui a Gevé à lá comaissance de la faune carbonifère marine ce monument que le monde entier admire et qu'une mort prématurée l'a seule empêché de réaliser interralement. A plusieurs reprises je me suis plu à transporter moi-même mon riches recoltes ì Liege of ì recevoir directement les enseignements du culdure palórntologiste. Je me plais a recommâtre ici toute la gratitude que je lui drois an ac qu'il m'a facilite dans de grandes proportions et singulièrement

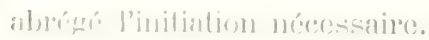

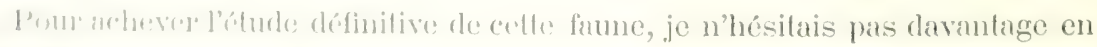

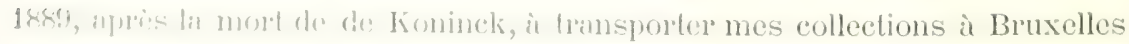

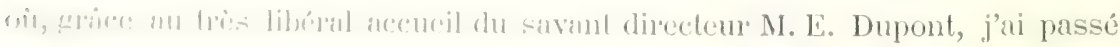

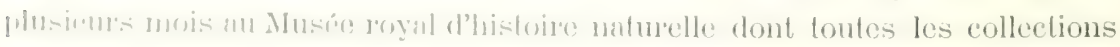

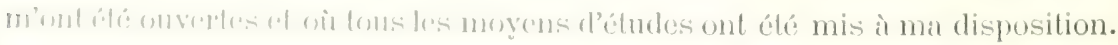

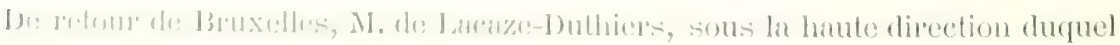


je m’étais familiarisé jadis au Muséum avec l'étude des invertébrés, m’offrit à son tour l'hospitalité dans les pièces de son service à la Sorbonne, et je pus faire procéder à la photogravure de tous les spécimens figurés dans les 17 planches de l'ouvrage, par la maison Dujardin.

On se convainera, en effet, en examinant ces figures qu'il était bien impossible de les représenter pour le plus grand nombre à l'aide du crayon, et la reproduction en photogravure a de plus cet avantage de montrer sous leur aspect récl les roches carbonifères fossilifères de nos divers gisements et de permettre un contrôle facile de la détermination spécifique des empreintes. On se convaincra aussi en les examinant qu'il était impossible de rédiger pour chaque spécimen des diagnoses qui eussent été forcément incomplètes et sans intérêt. - Aussi avons-nous résolu de nous servir des diagnoses déjà publiées, soit par de Koninck et M. J. Fraipont, A. d'Orbigny et de Verneuil; soit en les traduisant des différents auteurs étrangers, Davidson, de Semenow, Phillips et M'Coy, Portlock et Martin, etc. Leur réimpression avait à nos yeux un avantage considérable, c'était celui d'être utile aux amateur's qui ne possèdent pas cette énorme littérature carbonifère qu'on ne peut réunir qu'à grands frais et qui est cependant indispensable à ceux qui voudraient continuer nos études dans la France centrale. Le travail considérable auquel nous nous sommes livré nous a permis de nous familiariser encore plus avec la faune carbonifère, car il n'est rien d'aussi instructif que d'instituer pour soi-même l'historique d'une espèce en la suivant depuis sa première mention par un vieil auteur le plus souvent ignoré du plus grand nombre, jusqu'à sa dernière et plus récente description par un des grands paléontologistes du siècle, tels que Davidson et de Koninck; et e'est le motif qui nous a conduit à donner parfois quelques détails historiques, fruit partiel de notre labeur', principalement sur' un certain nombre de Brachiopodes et d'Echinodermes.

Quant aux espèces nouvelles poul la création desquelles nous avons été d'une sobriété extrêne, et aux espèces rales ou peu comnues, nous en arons décrit les caractères avee le plus grand soin. En voici la liste :

1. Phillipsia Barrundei.

2. Brachymetopus Duponti.

3. Macrochilina Pireti.

4. Lepetopsis Leforti.

5. Solenopsis Bielueshii.

6. Ctpricurdella Julieni, de Koninck.

7. Leiopteria can den Broctir. 


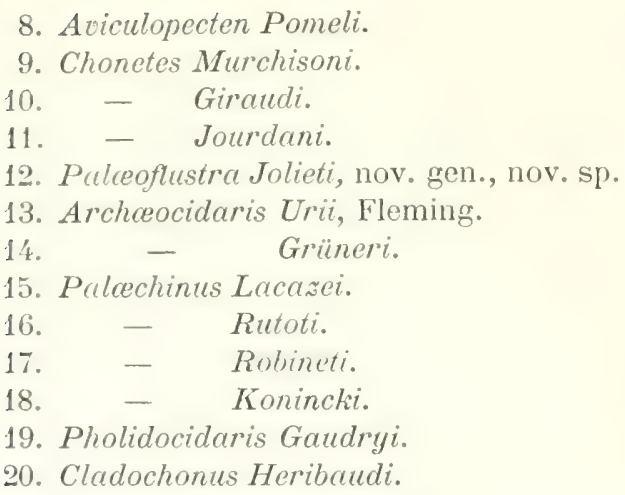

Nous aurions pu en augmenter fucilement le nombre, répétons-nous encore, muis nous avons préféré ne pas utiliser de mauvais échantillons, laissant aux chercheurs futurs l'heureuse chance d'en trouver de meilleurs, et le soin de les faire entrer dans le domaine de la science.

Avant d'exposer le plan de cet ouvrage, nous tenons encore à adresser ici nos remerciements à tous ceux, célèbres ou modestes et obscurs qui nous ont aidé de leurs encouragements ou de leur concours. Un souvenir reconnaissant ¿ Joachim Barrande et à Edouard de Verneuil qui nous ont prodigué dans des lettres que nous sommes heureux de posséder leur's plus vifs eneouragements ¿u début de nos études; ì de Koninck sans les enseignements duquel nous n'aurions peut-être pu les réaliser.

Thus nos sentiments de gratitude la plus profonde à M. Edouard Dupont qui nous a aceopdé l’hospitalité la plus large, la plus libérale au Musée royal f'histrile naturelte de Bruxelles et ì ses savants collaborateurs qui sont devenus fun nuns antant d'amis; ì M. Julien Fraipont tout spécialement qui a birn vouln examiner nu certain nombre de nos plus mauvais spécimens; à motre xestent mui Adolphe Pirot qui nous a ouvert à Tournai son incompa-

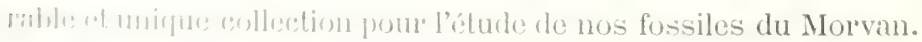

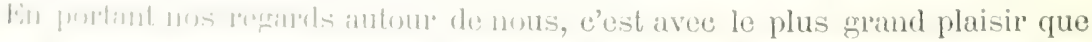

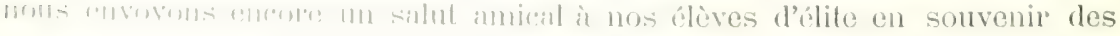

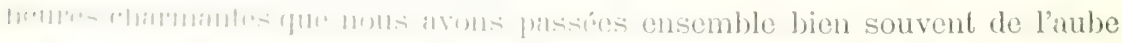

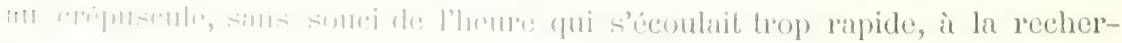

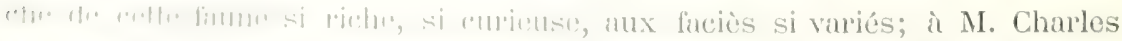

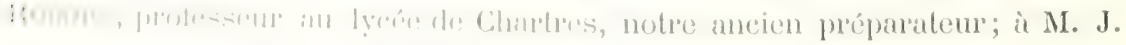


Usclade; à M. Richard, actuellement conservateur des collections du prince de Monaco; ì notre préparateur actuel, M. J. Giraud, qui nous a donné une aide utile dans la préparation de cet ourrage.

Plax. - Ce travail débute par une première partie paléontologique comprenant la description des 192 espèces marines déterminées, et la diagnose, par M. l'abbé Boulay, des plantes anthracifères recueillies au cours de nos recherches;

Viennent ensuite :

$1^{\circ}$ L'étude comparative et stratigraphique des faunules réunies par gisements;

$2^{\circ}$ L'étude comparative de nos faunules avec celles de quelques localités étrangères et celles de tous les gisements français répartis sur la surface du territoire;

$3^{\circ}$ Un chapitre spécial relatif à l'âge de nos grès anthracifères ;

$4^{\circ}$ Le tableau général du synchronisme de nos assises marines et des assises de grès anthracifère du Morvan, du Beaujolais, du Forez, du Bourbonnais et de lin Marche;

5 Enfin un dernier chapitre concernant la transgression carbonifère dans le Morvan, le Plateau Central et le reste de la France ainsi qu'en Europe, suivi d'un Essai de parallélisme entre les deux grandes transgressions des mer's carbonifère et helvétienne, avec leur grandiose cortège de phénomènes éruptifs, orogéniques et glaciaires. 



\title{
LE TERRAIN CARBONIFĖRE MARIN
}

\author{
DE LA France CENTRALE
}

\section{CLAASSE DES POISSONS}

\author{
ORDRE DES SÉLAGIENS
}

FAMILLE DES CESTRATIONTIDÉS

GROUPE DES COCHLIODONTIDÉS

Genre GOGHLIODUS, Agassiz, 1838

Cochliodus acutus, AGAssiz (sins description) 1838

Non figuré.

Caractères spécifiques. - Dent postérieure oblongue, obliquement atténuée en arrière en un angle d'environ $65^{\circ}$; côté antérieur taillé en biais et dirigé en arrière et en dedans sous un angle d'environ 60\% surfice ornée de 3 côtes obliques fortement accusées, séparées par de profondes dépressions; la première côte très proéminente, étroite, aiguä, coïncidant avec le côlé antérieur qui est très abrupt, séparé ordinairement de la dépression adjacente par un étroit sillon obsolète; côte moyenne plus large et placée plutôt en arrière du milieu de la dent, s'inclinant graduellement ver's la base de la côte antérieure, mais d'une façon plus abrupte ver's la dépression posterieure plus profonde et plus étroite qui la sépare de la côte marginale postérieure; celle-ci est saillante, étroite, arrondie, ef forme l'extrémité postérieure de la dent. Surface entière- 
ment couverte de ponctuations dues aux extrémités des canaux vasculaires (au nombre de 8 environ par ligne), elle est recouverte par places d'une très mince couche de ganoïne.

Longueur moyenne de la dent postérieure, 1 pouce 2 lignes; largeur du bord antérieur, 8 lignes. - M'Coy ${ }^{(1)}$.

Gisement. - Rẻgny; une seule dent.

\section{GROUPE DES PÉTALODONTIDÉS}

GENRE PETAlodus, R. OWEN, 1840

Petalodus Hastingsiæ, R. Owen, 1840

Non figuré.

Caractères spécifiques. - Dents à couronne pétaloïde, mince, convexe extérieurement ct de forme subtriangulaire; le côté opposé est faiblement concave et bordé à sa base par un certain nombre de plis de ganoïne imbriqués et parallèles entre eux.

Surfaces extérieure et intérieure minces et polies. Bord supérieur garni de fines stries verticales n'altérant pas le tranchant. Bord inférieur de la surface extéricure de la conronne ayant une forme sigmoüdale, le bord inférieur de la surface intérieure avec une forme subsemicirculaire. Racine occupant presque la moitié de la longueur de la dent, linguiforme, légèrement épaissie inférieurement et irrégulièrement striée en long. - De Koninck ${ }^{(2)}$.

Gisement. - L'Ardoisiere.

\section{GROUPE DES PSAMMODONTIDES}

GLNRE PSAMmodus, Agassiz, 1833

Comprefireses gúnérigues. - Les dentis rénéralement désignées sous ce nom

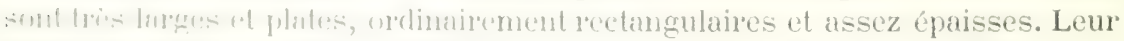

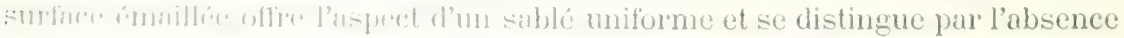

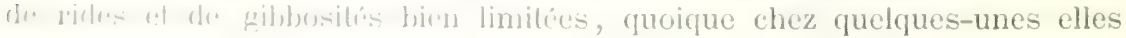


soient plus ou moins ondulées, convexes ou légèrement mammelonnées. La base est à peu près de même étendue que la couronne. Celle-ci est composée d'un grand nombre de petits tubes médullaires verticaux, subparallèles entre eux et correspondant aux pores de la surface. - De Koninck (1).

\section{Gisement. - Régny.}

Observation. - Nous avons trouvé dans ce gisement une dent appartenant avec certitude à ce genre, mais spécifiquement indéterminable.

(1) Loc. cit., p. 41.1878 



\title{
CLASSE DES CRUSTACÉS
}

\author{
ORIDRE DE PHYLLOGAR IDÉS
}

\author{
GROUPE DES MALACOSTRACÉS
}

GENRE DITHYROCARIS, SCOULER

Nous avons trouvé à diverses reprises à l'Ardoisière et à Régny des pièces de la carapace de Dithyrocaris. Nous nous r'éservons de rechercher des pièces plus complètes afin de pouvoir faire une étude spécifique, ce qui nous a été impossible jusqu'ici.

\section{ORDRE DES TRILOBITES}

\author{
FAMILLE DES PROETIDÉS
}

GROUPE DES ENTOMOSTRACES

\section{GENRE GRIFFITHIDES, PORTLOCK, 1843}

\section{Griffithides seminiferus, PhLLıPs, 1836}

Pl. V, fig. 14, 15, 16 .

Historique. - Espèce créée par Phillips sous le nom d'Asaphus seminiferus. Voici la diagnose qu'il en a donnce : "Tête poroso granulée. Lobe médian " offrant 2 sillons latéraux et 2 tubercules a la base; abdomen avec des lobes » enflés; côtes raboteuses pourvues de 8 i 10 ponctuations, subglobuleuses, 
») inégalement saillantes. Limbe non strié. Premier segment du lobe médian » mucroné(1). »

La même espèce a été décrite par de Koninck ${ }^{(2)}$, en 1842, sous le nom de Phillipsia gemmulifera. Il en a été de même pour M'Coy ${ }^{(3)}$.

Caractères spécifiques. - Folme générale ovale-oblongue. Bouclier céphalique arqué; glabelle grande, gibbeuse, surplombant le bord antérieur. Lobe basal pyriforme. Anneau occipital large, séparé au-dessus et au-dessous par un large sillon. Yeux petits, réniformes, lisses. Partie saillante de la joue libre grossièrement et irrégulièrement granulée; limbe des joues lisse; angles latéraux non prolongés en pointes génales.

9 segments tholaciques. Rachis plus large que les plèvres, diminuant seulement très légèrement vers le pygidium.

Chaque segment orné-d'une rangée simple de granules grossiers (environ 8 sur chaque côté et 10 sur l'axe). Sillons délimitant l'axe fortement marqués; segments arqués; terminaisons des plèvres arrondies, partie interne lisse.

Pygidium composé de 12 segments soudés. Axe s'effilant graduellement en cône pour se terminer par une extrémité obtuse. Côtes latérales au nombre de 9 environ, ornées chacune d'une rangée simple de tubercules au nombre de 8 ou 10 sur l'axe et de 8 environ sur chaque plèvre. Limbe du pygidium étroit, bord du limbe coudé. - Woodward (4).

Gisements, - La Varville, assez commun; Siguret.

Explication des figures. - PI. V, fig. 14. Pygidium. La Varville.

Fig. 15. Le même, grossi.

Fig. 16. Autre spécimen, id.

Fig. 10. Glabelle de Griflithides spp.? La Varville.

Fig. 11. I a meme grossie 4 fois.

\section{Griffithides globiceps ? PhILlips, 1836}

Ton fignu'é.

Nous avons trouvé dans un bloc de marbre noir de Saint-Germain-Laval un jygridium que nous mpportons an G. globiceps Phillips. Ce pygidium présonte, "n chetet, lus ciractires habituels de cette espèce. Il est arrondi, il est formé de 11 somitus sutudés. L'axe se termine en une extrémité mousse à une distance comirlanble dn bord. Les plivres sont sillomées et se terminent avant d'atfrindre le brerl du benclier caudal, laissant une marge lisse assez large.

firsment. - Saint-Germain-Laval, un seul specimen.

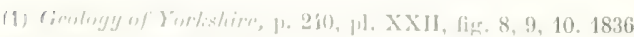

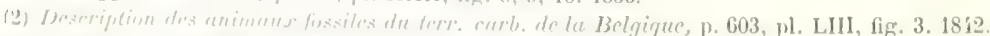

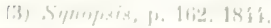

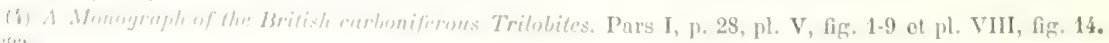
1483 
Genre Phillipsia, Portlock, 1843

I. Phillipsia Barrandei, Nov, sp.

Nous arons trouvé dans nos fouilles de La Varville une très jolie tête de Phillipsia, malheureusement incomplète, et un fragment d'une empreinte de thorax qui se rapporte probablement ì la même espèce car tous les débris de Trilobites que j'ai recueillis en dehors du Brachymetopus Duponti appartiennent au Griffithides seminiferus, et ce fragment de thorax ne saurait provenir de cette espèce. Je le rapproche done, sous toutes réscrves, de la tête mentionnéc ci-dessus.

Caractères spécifiques. - A. Téte. La portion visible comprend seulement la glabelle, le lobe palpébral gauche et l'anneau occipital. La glabelle est ovale allongée, très proéminente. Elle présente 3 pares de sillons laterrax, un sillon antérieur peu visible, un sillon médian un peu plus long, plus profond, et un sillon postérieur très accusé se dirigcant obliquement vers le milieu de l'anneau occipital, qu'il atteint presque, limitant ainsi un lobe posterieur de forme triangulaire.

Le lobe palpébral saillant séparé par un fort sillon de la glabelle, a son bord extérieur semi-circulaire et s’étend depuis l'extrémité du lobe médian jusqu'au milieu du lobe postérieur.

L'anneau occipital, très large dans sa partie médiane, se rétrécit un pen sur les côtés.

Tonte la surface de la glabelle est couverte de gros tubereules serrés disposés en quinconce. Le sillon qui sépare le lobe palpébral de la glabelle est lisse, et le bord externe de ce lobe est orné d'une rangée de tubercules plus fins.

L'anneau oceipital présente quelques granules épar's beancoup plus fins que ceux de la glabelle.

Dimensions. - Longueur de la glabelle, $5 \mathrm{~mm} 5$.

Largeur de la glabelle, prise au niveau dos lobes postórieurs, $5 \mathrm{~mm} 5$.

Longueur du lobe palpébral, 2mm.

Largeur du lobe palpébral, 1/2mm.

B. Thorax. Empreinte externe d'un fragment de thorax comprenant la moitié droite de l'axe et les ploves du côté droit. Le fragment présente 7 annemux de l'axe et autant de plèves. L'axe ost oné de 6 rangées longritudinales de granules; les deux filos médianes formées de granules plus gros. Les plères présentent également 6 langées de gramules, dont les deux médianes sont composées de forts granules, les 4 autres rangées étant formées de granules égaux, mais plus petits que les premiers. 
Dimensions. - Longueur des 7 segments, $5^{\mathrm{mm}}$.

Largeur de l'axe, $7^{\mathrm{mm}}$.

Longueur des plèvres, $4^{\mathrm{mm}}$.

Gisement. - La Varville.

Nous dédions cette jolie espèce de Phillipsia à l'illustre Barrande, en souvenir des encouragements qu'il nous a prodigués au début de nos recherches.

Explication des figures. - Pl. V, fig. 12. Portion de tête.

Fig. 13. La même, grossie 4 fois.

Fig. 17. Empreinte de la portion de thorax décrite ci-dessus.

Fig. 18. La même, grossie 4 fois.

\section{Phillipsia Eichwaldi, Fischer sP., 1825}

Pl. XV, fig. $1 a$.

Pl. XVII, fig. 1, 2, 3.

Curactères spécifiques. - 'Tête semi-circulaire, glabelle légèrement gibbeuse au front, mais ne dépassant pas le bord fixe qui entoure sa partie antérieure et qui forme aussi des lobes palpébraux arrondis. Lobes basilaires nettement delimités, affectant une forme triangulaire, précédés de deux courts sillons latéraux de chaque côté. La tête est marquée de deux pores situés de chaque côté de la glabelle en avant des yeux. Yeux plutôt grands et fortement réticulés, les facettes étant plus grandes que dans le $P h$. gemmulifer $a$; anneau occipital large, bien marqué, offrant un seul tubercule au centre et une rangre de fines granulations ou de granules ténus le long de son bord postérieur. Il en existe de semblables sur l'axe du thorax. Joues libres élevées, petites, mais entourées par un hord large, plat, taillé en biseau du côté de l'extérieur et fincment strié en dessous. Pointes génales allongées atteignant lo go anneau thoracique. Le bouclier céphalique dans son entier, sauf le limbe extérienr est ornó de trés fines gramulations. L'axe du thorax et du pygidium ust tris distinet, chaque segment offrant le long de son bord postérieur une rangúp de très fines granulations semblables à des épines.

Pygidium composé de 16 somites coaleseents. Axe central orné comme cratui dn throrax; plives au nombre de 11 environ. Limbe lisse, contour armondi et stricia fle fines lignes parallèles. - Woodward (1).

fisement. - L'Ardoisiere; commun.

Érplicution des figneres, - I'1. XV, figr. 1 a. Pygidium sur l'arête cardinale d'un Producthes gyejuntens. - L'Ardoisiere.

1'1. XVII, figr 1. Groupe offrant un Phillipsic Eichealdi entior, un pygidium à còté, et it droitu un individu entier mais incomplètement représenté dans la figure. La taille du spérimen rontion est de : esentimetres.

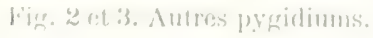

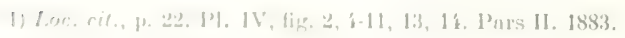


GENRE BRAGHYMETOPUS, M'COY, 1844.

Brachymetopus Duponti, Nov. SP.

Pl. V, fig. 6, 7, 8,9.

Nous avons trouvé dans nos recherches à la Varville, au milieu de plusieurs milliers de fossiles, deux pièces présentant un très grand intérêt : c'est une tête unique de Brachymetopus et un pygidium également unique d'un Trilobite du même genre. Ces deux pièces rarissimes ne se trouvaient pas sur le même échantillon de roche, mais seulement sur deux fragments du même banc. Cela nous engage à les considérer comme deux pièces se rapportant à la même espèce, qui est nouvelle dans la science, et que nous dédions à M. Dupont, le savant directeur du Musée royal d'histoire naturelle de Bruxelles. Nous décrirons donc cette tête et ce pygidium sous le nom de Brachymetopus Duponti.

On sait combien ce genre créé par M'Coy est paurre en espèces, car, jusqu'à notre découverte, on n'en connaissait que 5, savoir:

Brachymetopus M'Coyi, Portlock, 1843.

$\begin{array}{ll}\text { - } & \text { discors, M'Coy, } 1844 . \\ \text { - } & \text { ouralicus, de Verneuil, } 1845 . \\ \text { - } & \text { Straeleckii, M'Coy, 1847. } \\ \text { - } & \text { hibernicus, Woodward, } 1883 .\end{array}$

Caractères spécifiques. - Tête subelliptique allongée, entour'ée d'un limbe de faible dimension, s'élargissant en avant et se terminant en arrière de chaque côté en une pointe génale très courte. Ce limbe, garni de granules plus nombreux à la partie antérieure, est séparé de la tête par un léger sillon qui s'unit en arrière au sillon occipital sous un angle aigu. La glabelle est simple, peu proéminente, allongée; elle occupe à peu près les deux tiers de la longueur de la tête. Les yeux étroits, allongés, subréniformes et peu saillants, sont placés ver's le milieu des joues. Toute la surface de la tête est ornée de granules, dont quelques-uns, plus gros, entourent le sommet de la glabelle.

Dimensions de la tête : longueur totale, $3^{\mathrm{mm}}$; largeur, $\mathbf{4}^{\mathrm{mm}}$; longueur de la glabelle, $2^{\mathrm{mm}}$; largeur, $\mathrm{I}^{\mathrm{mm}}$; largeur maximum du limbe (en avant), $1 / \mathbf{2}^{\mathrm{mm}}$.

Thorax incommu.

Pygidium subsemicirculaire. Lobe axial atteignant presque le bord et ayant à peu pris le tiers de la largeur totale, tròs convexe, composé de 14 segments étroits. Le premier anneau axial offre un large tubereule spinifère médian. Tous les anneaux sont ornés de granules en nombre variable.

Les plèves, sillonnées, sont au nombre de 9, les deux dernières il peine distinetes. La partie située en avant du sillon se termine par une dent qui se continue par une épine; la partie postérieure, plus nettement granulée, se 
recourbe en arrière et vient s'unir à la partie antérieure de la plèvre suivante pour former la dent. En outre des granules, les lobes latéraux présentent chacun 3 gros tubercules spinifères situés, le premier sur la partie médiane de la première plèvre, le second sur la quatrième, et le dernier sur la sepfième, ces deux derniers un peu plus rapprochés du bord extérieur. Le bord du limbe est dentelé. Il présente 21 épines : 10 de chaque côté et 1 terminale située à la partie médiane du pygidium. Ce nombre de 20 épines démontre qu'il y a en réalité 10 segments latéraux, dont le dernier devenu invisible par l'absence de granules et la coalescence avec le segment précédent n’est révélé que par son épine.

Dimensions. - Longueur totale, $4^{\mathrm{mm}}$; largeur', $7^{\mathrm{mm}}$; largeur de l'axe au sommet, $2 \mathrm{~mm}$.

Gisement. - La Varville.

Explication des figures. - Pl. V, fig. 6. Tête grossie 4 fois; la pointe génale est mal venue.

Fig. 7. Pygidium, grandeur naturelle.

Fig. 9. Le même, grossi 4 fois.

Fig. 8. Empreinte externe également grossie 4 fois. Cette empreinte qui est en creux parait en relief.

Nota. - Dans la liste (1) que M. de Koninck a donnée en 1885 des fossiles du Morvan que je lui avais soumis, le Brachymetopus Duponti a été rapporté par lui au $B$. discor's après un examen évidemment trop superficiel. Notre description et les figures suffisent pour montrer l'erreur dans laquelle était tombé l'éminent paléontologiste belge.

\section{ORDRE DES OSTRACODES}

\section{FAMILLE DES CYPRIDINIDÉS}

\section{GRNE ENTOMIS, RUPERT JONES, 1873}

Entomis concentrica, DE KoNINCK, SP., 1844.

I'. V, fig. 19.

Corractiones spécifiques. - Valves ovalaires subreniformes, très bombées, diviseres an deux forties di peu près égnles par un sillon onduleux transversal assoz profond at dont le bord supericur est tranchant. Toute la surface des

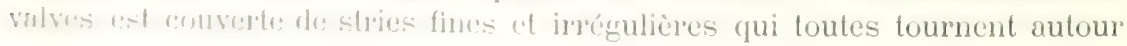


d'un centre commun, situé au milieu de la partie inférieure de chaque valve. Leur's bords sont contournés en dedans, et donnent lieu à la formation d'une forte dépression longitudinale au milieu de laquelle on remarque la suture linéaire.

Dimensions. - Elle a environ $7^{\mathrm{mm}}$ do longueur. - De Koninck (1).

Gisement. - La Varville.

Explication des figures. - Pl. V, fig. 19. Valve grossie 4 fois.

\section{FAMILLE DES CYPRIDÉS}

GenRe BAIRdiA, M'Coy, 1844.

Le genre Bairdia et d'autres genres d'Ostracodes sont abondamment représentés dans le terrain carbonifère marin du Plateau Central. Ils pullulent dans certaines couches; ainsi à l'Ardoisière, et surtout dans le Morvan, à la Varville, à Siguret, etc. Nous avons laissé provisoirement leur étude de côté, nous proposant d'en faire l'objet d'une monographie plus tard; nous nous contentons d'en signaler l'existence.

La pl. V, fig. 20, montre un fragment de la roche de la Varville, présentant à sa surface de nombreux Ostracodes.

(1) Loc. cit, p. 587. Pl. LII, fig. 4 a, b, 5 a, b. 1844. 



\title{
CLASSE DES CEEPHALOPODES
}

\author{
ORDRE DES TÉTRABRANCHIAUX
}

FAMILLE DES NAUTILIDÉS

Genre NAUtilus, J. P. Breynius, 1732.

I. Nautilus sulcatus, J. SOwErBy, 1826.

Pl. XV, fig. $10,11$.

Caractères spécifiques. - Coquille discoïde munie de fines stries transversales. Tours de spire enflés à peu près complètement visibles, ayant de chaque côté deux grands sillons et plusicurs petits. Dos concave.

L'un des sillons caractéristiques qui donnent aux tours de spire une apparence si particulière est limité aux tours intéricurs; le dos est concave et borné par deux carènes saillantes; il y a aussi une arête saillante entre les deux sillons principaux des flanes; le reste des flanes est enflé avec quelques légères impressions sur la partie la plus saillante. L'ouverture est deux fois aussi haute que large; ses bords sont très onduleux; le siphon est placé audessous de la carène principale qui sépare les deux sillons latéraux. Les cloisons sont nombreuses à bords unis. - Sowerby (1).

M. de Koninck a donné une diagnose très détaillée de cette espèce, on peut y recourir ${ }^{(2)}$.

Gisement. - L'Ardoisière; pas très rare.

Explication des figures. - Pl. XV, fig. 10. Empreinte externe d'un spécimen un peu plus grand que ceux de Belgique. - L'Ardoisière.

Fig. 11. Moulage du mẻne.

(1) Mineral Conchology. Traduction Wesor. 1'. 589, pl. 571, 1ig. 1, 2, 3.

(2) Faune du calc. carb. de la Belgique. Poissons et G. Nautile, p. 126, 11. XXVil, tig. 1, 2, 3, 4. 1878. 
II. Nautilus subsulcatus, Phillips, 1836

Non figuré.

Caractères spécifiques. - Discoïde, lisse avec maintes stries sigmoïdales aiguës et quelques-unes spirales. Tours de spire quadrangulaires. Dos concave le long du milieu, coupé de biais sur le côté; les côtés concaves vers le bord extérieur et convexes vers le talus marginal abrupt.

Siphon près du bord extérieur. Ouverture oblongue, analogue à celle du N. sulcatus. - Phillips ${ }^{(1)}$.

Gisement. - L'Ardoisière.

III. Nautilus globatus, J. DE C. Sowerby, 1825.

Non figuré.

Caractères spécifiques. - Coquille globuleuse, lisse, ombiliquée. Tours de spire peu nombreux, plus ou moins aplatis sur le dos et augmentant rapidement de diamètre. Ombilic profond à bords anguleux. Tour's intérieurs cachés. Ouverture large, arquée, largement émarginée sur le dos. - Sowerby (2).

Gisement. - L'Ardoisière.

IV. Nautilus costellatus, M'Coy, 1844 .

Non figuré.

Caractères spéciflques. - Discoïde, tour's intérieur's visibles, tour's de spire très convexes; dos arrondi; côté ombilical abrupt, lisse. Tours ornés d'environ 19 côtes longitudinales, étroites, égales et saillantes, séparés par des intervalles un peu plus grands que deux fois leur propre diamètre; cloisons Irès convexes. Siphon central. - M'Coy ${ }^{(3)}$.

Gisement. - L'Ardoisière.

V. Nautilus sulciferus, Phillips, 1836.

Non firgure.

Caracteres spécifiques. - Coquille discoïde dont les tours de spire s'élarghssent rapidenent, lem section est naviculare, deux fois plus large que haute at terminge latéralomont par des angles aigus. Chaque côté de la spire porte

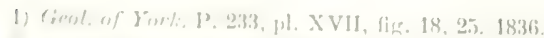

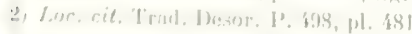

(3) Synespais, 11, 17, 101. 11, fis. ? 
une carène tranchante; le bord ventral auquel cette carene sert de limite est faiblement mais régulièrement bombé; immédiatement à côté de chaque carène, on observe sur ce bord quelques fines côtes longitudinales, qui, à l'origine, sont au moins au nombre de 10, mais qui diminuent rapidement et disparaissent presque complètement au deuxième tour de spire.

La face dorsale de la spire est également ornéc de minces côtes semblables à celles de la face ventrale; clles sont au nombre de 12 de chaque côté et persistent un peu plus longtemps que les premières; toutes ces côtes sont coupées en traver's par de fines stries d'aceroissement, bien distinctes et légèrement courbées.

Ombilic très large, infundibuliforme. Cloisons peu profondes à suture latérale faiblement arquée et non sigmoïdale. - De Koninck (1).

Gisement. - Régny, un seul exemplaire.

\section{FAMILLE DES ORTHOCERATIDESS}

GeNRE ORTHOGERAS, J. P. Breviuts, 1732.

I. Orthoceras Goldfussianum, DE KonINCK, 1844.

Non figuré.

Caractères spécifiques. - Coquille de taille médiocre, régulièrement conique et d'un accroissement assez lent en diamètre.

Cloisons peu profondes, rapprochées dans le jeune êge, et s'écartant progressivement les unes des autres de facon que la distance des dernieres soit de $3 \mathrm{~mm}$. On en compte environ 40 sur un individu complet. La dernière loge occupe le tiers de la longueur totale de la coquille. Les bords de son ouverture sont tranchants et très faiblement contractés et non simués.

Le siphon est sub-central.

Surface du test lisse ou marquée de quelques légères stries d'aceroissement à peine visibles à l'œil nu.

Longueur maximum, $15^{\mathrm{cm}}$; diamètre transverse de l'ouverture, $24^{\mathrm{mm}}$; angle apicial, $8^{\circ}$. - De Koninck ${ }^{(2)}$.

Gisement. - L'Ardoisière.

(1) Loc. cit., p. 142. Pl. XXIX, fig. 5, et pl. XXXI, fig. 7. 1878.

(2) Loc, cit., p. 66. Pl. XXXVII], fig. 8, 9. 1878. 
II. Orthoceras Martinianum, DE KONINCK, 1844.

Pl. IV, fig. 1 .

Caractères spécifiques. - Coquille de taille moyenne, grêle, sensiblement conique, très effilée à son extrémité inférieure. Les cloisons sont faiblement bombées et assez rapprochées les unes des autres.

Siphon étroit et filiforme à l'origine, atteignant un diamètre de $3 / 4$ de millimetre à la dernière cloison; il n'est pas continu; le goulot des cloisons est court et peu saillant; sa situation est un peu excentrique; surface du test entièrement lisse.

Longueur maximum, 20 205 ; diamètre de l'ouverture, 15 $\mathrm{mm} 5$; angle apicial, $5^{\circ}$ - - De Koninck (1).

Gisement. - La Varrille.

Explication des figures. - Pl. IV, fig. 1. Empreinte d'un fragment de O. Martinianum, de $35^{\mathrm{mm}}$ de long. On y observe le fond de la chambre d'habitation et les 13 dernières loges, chacune d'elles mesure une hauteur de $2^{\mathrm{mm}}$; l'angle apicial est de $5^{\circ}$; diamètre de la dernière cloison, $11^{\mathrm{mm}}$. - La Varville.

\section{FAMILLE DES CYRTOCERATIDÉS}

\section{GENRE GYRTOGERAS, GOLdFuss, 1832.}

1. Gyrtoceras unguis, Phillips, 1836.

Non figuré.

Diarnose de Phillips : "Brusquement arqué vers l'extrémité émoussée, sec"tion circularime (3).

Curactères spécifiques. -.. Coquille faiblement arquée vers son extrémité prointue et presque droite dans sa partie supérieure; section transverse presque circulaine d'alond, puis devenant ovale ì une faible distance de la pointe iniliale et conservant celte forne jusqu'ì l'extrémité. Ouverture normale à bords linuchlounls.

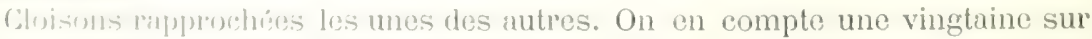

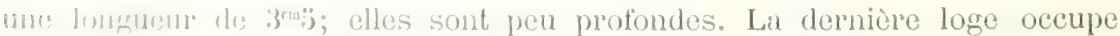
movima la moilis do la longume totale de la eoquille. On la trouve rarement

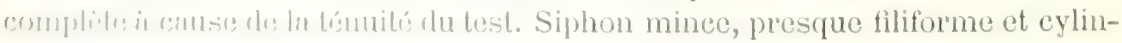


drö̈de; il est subeentral et situé un peu plus près du bord ventral que du bord dorsal. Surface entiùrement lisse.

Longuenr maximum, 10 $1 \mathrm{~cm}^{\mathrm{m}}$; diamètre antéro-postérieur de l'ouverture, $20^{\mathrm{mm}}$; diamitre transverse, $24^{m m}$. - De Koninck ${ }^{(1)}$.

Gisement. - L'Ardoisière.

II. Gyrtoceras Puzosianum, DE KonINCK, 1843.

Non figuré.

Caractères spécifiques. - Coquille d'assez petite taille ayant la forme d'une petite corne aiguë, légèrement courbée, à section transverse circulaire d'abord et faiblement elliptique vers son extrémité antérieure. Cloisons assez profondes. Distance des cloisons terminales, 2mm; dernière loge oceupant environ le tiers de la longueur totale. Siphon mince et situé un peu en dehors du centre, ver's le côté ventral.

Surface ornée de 20 à 22 côtes longitudinales, aiguës, s'étendant de la pointe initiale jusqu'ù une certaine distance de l'ouverture où elles disparaissent presque complètement; clles alternent avec le même nombre de côtes plus minees et moins élevées, ayant leur origine ì quelques millimètres de l'extrémité inféricure et disparaissant en même temps que les premières.

Dimensions. - Longueur totale, $9^{\mathrm{cm}}$; les deux diamètres de l'ouverture sont 15 et $17^{\mathrm{mm}}$; angle apicial, $8^{\circ}$. - De Koninck (2).

Gisement. - Régny; très rare.

(1) Faunc du calc. carbon. de la Belgique. T. V, Cyrtoceras, etc., p. 18. Pl. XXXIV, fig. 2-5. 1880.

(2) Loc. cit., p. 34. Pl. XXXII, fig. 10, 11. 1880. 



\title{
GLASSE DES GLSTEROPODES
}

\author{
ORDRE DES PROSOBRANGHES
}

\author{
FAMILLE DES NERITOPSIDÉS
}

Genre NATIGopsis, M'Coy, 1844.

I. Naticopsis propinqua, DE KonINCK, 1881.

Pl. IV, fig. 19.

Caractères spécifiques. - Coquillo de taille médiocre, globuleuse, à spire déprimée, composée de 4 ou 5 tours de spire enroulés de fuçon que les premiers ne dépassent les dernier's que de $2^{\mathrm{mm}}$. La spire n’est pas régulièrement convexe; sa partie inférieure ust plus bombée que le reste et un peu aplatie du côté de lí suture formée d'un large sillon peu profond. Au bord de la suture se trouvent une quantité considérable de petits pliss obliquement longitudinaux, dont l'extrémité supérieure se confond avec les stries d'accroissement de la coquille, lesquelles, avec les plis, forment les seuls ornements de la surface. L'ouverture est grande, ovale et légèrement oblique; son bord columellaire est chargé, du crité de la suture, d'une forte callosité lissu; celle-ci s’amincit vers le bord supérieur, qui est arrondi. Lo test est issez mince et se brise facilement.

Dimensions. - Longueur, 32mm, épaisseur, 26 $6^{\mathrm{mm}}$; hauteur de l'ouverture, $21^{\mathrm{mm}}$; largeur, $16^{\mathrm{mm}}$; onverture de l'angle spiral, environ 1/45 . De Koninck (1).

Gisement. - La Varville.

Explication des figures. - PI. IV, fig. 19. Empreinte extorne ćcrnsce d'un spécinnen montrant nettement les caractéros do la suture, détorminće d'après les types mèmes do la collection de M. Piret, de Tournai, qui ont servi it lit monographie de M. de Koninck.

(1) Faune du calc. carb. de le Belgique, 1881, 1. VI, 1. 18. 1'l. I, fig. 4, 5, 27, 28. 
II. Naticopsis planispira, Phillips, 1836.

Non figuré.

Diagnose de Phillips : "Columelle calleuse en dessus, sommet des tours plan, couvert de fortes stries d'accroissement (1). »

De Koninck en a dommé une Iongue description (2), que nous ne croyons pas devoir reproduire puisque notre espèce n’est pas figurée.

Gisement. - L'Ardoisière.

Dans la Pl. XI, fig. 25, nous ayons représenté un moule interne de Naticopsis de Montmain, dont le test fait défaut et qui est, par suite, indéterminable. Aussi ne l'avons-nous fiit figurer que pour indiquer la présence du genre dans le gisement et provoquer de nouvelles recherches.

\section{FAMIILE DES PYRAMIDELLIDÉS}

\section{Genie maCRoGhilina, G. BAYLE, 1880.}

Diagrose du genre: "Coquille plus ou moins ventrue, buccinoïde, à spire aiguë; ouverture évasé, subovale, simple; bord externe mince et tranchant, ì jrofil sinueux; bord supérieur arqué; columelle calleuse, faiblement tordue sur (tle-mêne; callosité ne s'étendant que sur la partie supérieure du bord interne. Surfice lisses on ornere de stries d'accroissement. )

\section{Macrochilina Pireti, nov, sp).}

1'. VIII, fig. 18.

Crenceteres sjercifiques. - Corpuile de grande: taille atteignant $63^{\mathrm{mm}}$ de lon-

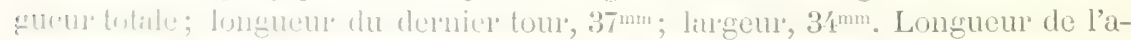

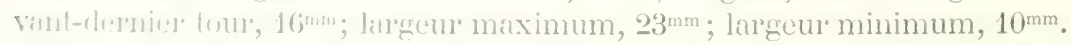

A pratir de aet arant-dernier tour" : longuen des premiers dont le nombre

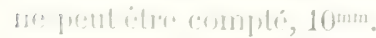

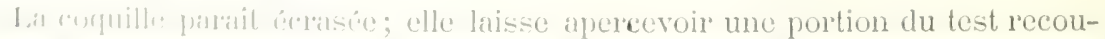
wrant to mumbe interme. Sus dimensions diflèrent de celles de presque toutes

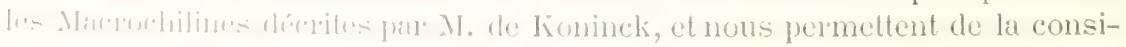

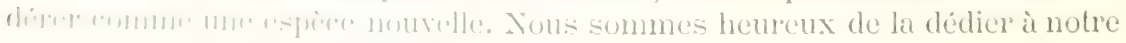

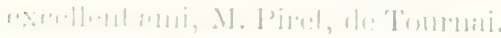

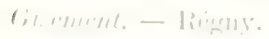

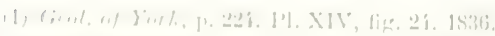

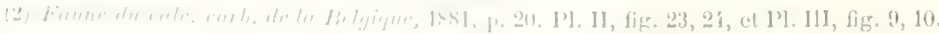




\section{GENRE LOXONEMA, Pitllips, 1811.}

I. Loxonema vittatum, DE KoNINCK, 1881.

Non figure.

Caractères spécifiques. - Coquille d'assez grande taille, de forme conique, composée de 15 ou 16 tours de spire, dont les 5 ou 6 derniers tours sont très convexes et garnis à leur bord inférieur d’une bande étroite, plane, qui borde la suture linéaire des tour's; les premiers tour's sont moins converes, et la bande suturale y est beaucoup moins prononcée. L'ouverture est un peu oblique et subovale. La surface est lisse.

Dimensions. - Longueur maximum, 64nm ; épaisseul, 20mm; hauteur de l'ouverture, $13^{\mathrm{mm}}$; largenr de lis même, $10^{\mathrm{mm}}$; ouverture de l'ungle spiral, $30^{\circ}$. - De Koninck (1).

Gisement. - Siguret; très rare, un seul spécimen.

II. Loxonema acutum, DE KonINCK, 1881.

P. IV, tị... 21 .

Caractères spécifiques. - Coquille composée de 15 oul 16 tours de spine convexes, it suture simple assez profonde. Ouverture subovale, un peu plus longue que large. Surface ordinairement lisse, mais laissant apercevon de fines stries d'accroissement sur quelques rares spécimens.

Dimensions. - Longueur, $30^{\mathrm{mm}}$; ćpaisseur, $8^{\mathrm{mm}}$; ouverture de l'angle apjeial, $18^{\circ}$. - De lioninek (2).

Gisement. - La Varville.

Explicution des figures. - Pl. IV, fig. 21. Moule interne. - La Varville; tress rare.

III. Loxonema priscum, Goldfuss, 1832.

1'l. V'III, lim. 17.

Caractères spécifiques. - Coquille d’assez grande laille, turriculéc, composćc de 12 à 15 tours de spire, dont le profil ust généralement sigmoïdal; chanfue tour de spire est convexe vers sa partic supérienre el déprimé inférieurement;

(1) Loc. cit., p. 45. Pl. VI, fig. 26, 27. 1851.

(2) Loc. cit., p. 40. Pl. IV, fig. 31, 35, 36, 37. 1881. 
son extrémité inférieure s'étale en bande mince sur la partie supérieure du tour' précédent, en formant ainsi un assez large sillon spiral auquel la sutme linéaire sert de limite inférieure; ouverture allongée-ovale; surface à peu près lisse.

Dimensions. - Longueur, environ $75^{\mathrm{mm}}$; épaisseur $18^{\mathrm{mm}}$; hauteur de l'ourerture, $14^{\mathrm{mm}}$; largeur de la même, $9^{\mathrm{mm}}$; ouverture de l'angle spiral, $15^{\circ}$. De Koninck (1).

Gisement. - Régny.

Explication des figures. - Pl. VIII, fig. 17. Moule interne. Cet échantillon, malgré son mauvais état de conserration, ne peut être rapproché que de l'espèce ci-dessus. C'est l'opinion de M. J. Fraipont, auquel je l'ai soumis. - Régny.

IV. Loxonema nanum, DE KONINCK, 1881.

Non figuré.

Caractères spécifiques. - Petite coquille acieulaire, composée de 8 ou 9) tour's de spire régulièrement convexes, à suture bien marquée et à surface lisse.

Dimensions. - Longueur, $4^{\mathrm{mm}}$; épaisseur, $1^{\mathrm{mm}}$; ouverture de l'angle spiral, 9. - De Koninck ${ }^{(2)}$.

trisement. - L'Ardoisière.

V. Loxonema Lefebvrei, LÉveILLÉ, 1835.

Pl. IV, fig. 20.

Coltro-juec classique a été décrite, pour la première fois, par Ch. Léveillé, sous lonom de Rissort? Lefeburei, dans les Mémoires de la Société géologique de Frrence, 1. II, p. 40. Voici la diagnose qu'il en a domée:

"Corpuille turriculée; tours do spire tres nombreux, finement striés dans "Is sens do l'aceroissement. Butuche presque entière; columelle droite, bord

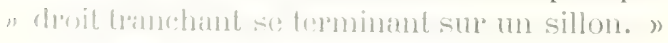

Gisimentis. - Siguret, Lit Varville.

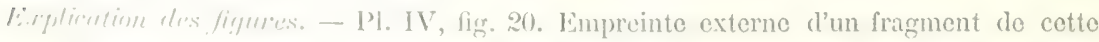

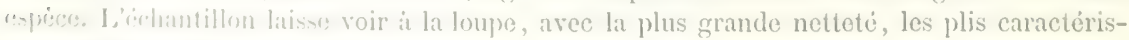
megues de lat rpirie. - Siguret.

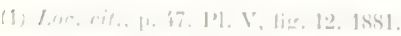

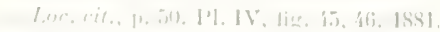


VI. Loxonema propinquum, DE KonINCK, 1881.

Non figuré.

Caractères spécifiques. - Coquille d'assez grande taille, subconique, composée de 9 ou 10 tour's de spine pluśs ou moins renflés dans leur partie supérieure, déprimés inférieurement et séparés l'un de l'autre par une suture linéaire; leur profil est sigmoïdal. Ouverture faiblement ovalaire, un peu plus haute que large. Surface couverte de stries d'aceroissement irrégulières ef assez peu apparentes; leur direction est arouée sur les tours inferiemrs of sigmoïdale sur le dernicr.

Dimensions. - Longueur, environ 6m; épaisseur, 25" $6^{\mathrm{mm}}$; hanteur do l'vu-

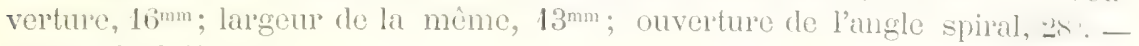
De Koninek (1).

Gisements. - Régny, Néronde.

VII. Loxonema constrictum, IV. MARTiN, 1809.

Non figuré.

Historique. - Cette espèce elissique a été llécrite et figurée par IV. Martin, sous le nom de "Conchyliolithus turbinites? (constrictus) conico-turritus lievis, " anfractibus superno subcoarctatis : cingulo crenato marginali (2). ")

Curacteres spécifiques. - Coquille de taille moyeme, conique, composée de 10 ì 12 tours de spire ì profil sinueux, occasionné par l'existenee d'un léger rentlement dans leur partie supérieure. Son bord sutural est orné d'me? série de petits tubereules allongés plus minees et plus serrés sur les premiers tours, produite par l'aceroissement suceessif de la coquille et correspondint ordinairement à diverses stries très fines dépendant de la mème canse. L'onverture est ovale et un jeu plus longue que large. Le test est asiscz ápais el solide.

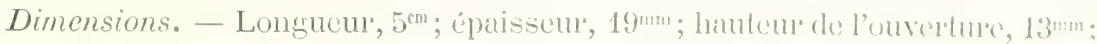
largeur de la même, gnm; onverture de l'angle spiral, 28". - De honinck 3.

Gisement. - L'Ardoisière.

(1) Lac, cit., 1., 53. Pl. VI, lige. 8, 9. 1881 .

(2) P'etr. Derb. Pl. XxxtIII, fig. 3. 1809.

(3) Loc. cit., 1. 56. 1'1. VI, fig., 19, 201, 21. 1881. 
GENRE SGALITES, CONRAD, 1842.

Scalites humilis, DE KoNINCK, 1881 .

Pl. IV, fig. 16.

Caracteres spécifiques. - Coquille de petite taille, conoïde, aussi longue que large, composée de 5 tour's de spire. Spire déprimée, à peine saillante, à suture suljcanalieulée; le dernier tour très développé et très embrassant, occupe à lui soul presque la totalité de la longueur de la coquille; sa partie supérieure est peu convexe, ce qui fait que le profil de la coquille possède une forme conoïdale lorsqu'on la pose sur l'extrérnité de la spire. L'ouverture est légèrement oblique; alle serait semi-lunaire sans l'angle que son bor'd inférieur forme avec le bord externe. Le test est fort mince et très fragile; on ne découvre à sa surface que de faibles stries d'aceroissement un peu sinueuses sur la carène.

Dimensions. - Longueur et épaisseur, $18^{\mathrm{mm}}$; hauteur de l'ouverture, $14^{\mathrm{mm}}$; largeur de li même, $8^{\mathrm{mm}}$; ouverture de l'angle spiral, 135。. - De Koninck (1).

Gisement. - Siguret.

Explicution des figures. - Pl. IV, tig. 16. Un fragment montrant les trois premiers tours de spire.

\section{FAMILLE DES TURBINIDES}

Ciexize TURBonitella, De Koninck, 1881.

Turbonitella biserialis, PInclips, 1836.

Non liqfuré.

Dirrgure de Phillips : "Coquille ovale-conique, tours de spire offrant une double rangere de tubereules allongés ef alternant entre enx ${ }^{(2)}$. 》

Vuici les rimensions de celte jolie petite coquille, extraites de la diagnose de

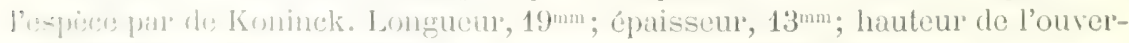

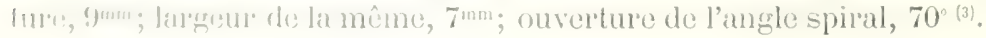

fis ment. - L'Ardrisiente.

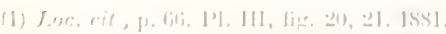

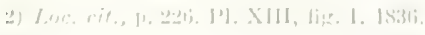

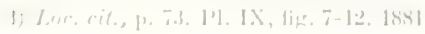


GenRE PORTLOGKIA, DE KONINCK, 1881.

Portlockia pygmæa, DE KONINCK, 1813.

Non figuré.

Caracteres spécifiques. - Petite coquille de forme subconique, composée de 6 tour's de spire convexes, dont le dernier occupe la moitié de la longueur totale de la coquille; la surface est ornée de 7 sillons spiraux, parallèles entre eux et à peu près de mème force, à l'exception du sillon sutural qui est un peu plus large; ces sillons, qui sont canaliculés, donnent naissance à un même nombre de carènes tranchantes, dont les deux ou trois inférieures sont légèrement crénelées. Ouverture presque circulaire.

Dimensions. - Longueur, $6^{\mathrm{mm}}$; épaisseur, $5^{\mathrm{mm}} 5$; ouverture de l’angle spiral, $76^{\circ}$. De Koninck (1).

Gisement. - La Varrille.

\section{FAMILLE DES TROCHIDÉS}

GENRE FLEMINGIA, DE KONINCK, 1881.

Flemingia Hisingeriana, DE KonINCK, 1843.

Pl. XI, fig. 22.

Caractères spécifiques. - Coquille de taille moyenne, régulièrement conique, composée de 6 ou 7 tours de spire à base presque plate, dont le pourtour est formé d'une carène assez tranchante, produite pal' une légère dépression qui en longe le bord. La suture est linéaire; l'ouverture est oblique, subrhomboïdale et plus large que haute; son bol`d columellaire, mince et légèrement incliné, se rattache au bord antérieur, qui est horizontal, par un angle obtus. La fossette ombilicale est largement concave et lisse. La surface est traver'sée obliquement par de fines stries irrégulières d'aceroissement.

Dimensions. - Longueur, environ $30^{\mathrm{mm}}$; largeur transverse, $31^{\mathrm{mm}}$; hauteur de l'ouverture, $11^{\mathrm{mm}}$; largeur de la même, 14m ; ouverture de l'angle spiral, environ $75^{\circ}$. De Koninck (2).

Gisements. - Néronde, l'Ardoisière.

Explication des figures. - Pl. XI, fig. 22. Un spécimen légérement déformé. Nérondo.

(1) Loc, cit., p. 83. Pl. IX, fig. 49, 50, 51 ; f1. X, fig. 11, 12. 1881.

(2) Loc. cit., p. 99. Pl. VII, fig. 38, 39. 1881. 


\section{FAMILLE DES EUOMPHALIDÉS}

GENRE PLATYSCHISMA, M'Coy, 1844.

Platyschisma helicoides, J. DE C. Sowerby, 1828.

Non figuré.

Diagnose de Sowerby : "Coquille presque discoïde, lisse, à spire très courte, massive, à tour's l’enflés, arrondis. Base ombiliquée; ouverture oblongue ${ }^{(1)} \cdot$ »)

De Koninck, à la suite de N'Coy, l'a classée dans le genre Platyschisma.

Gisement. - L'Ardoisière.

\section{GENRE STRAPAROLLUS, D. DE MONTFORT, 1810.}

Straparollus Dionysii, D. DE MoNTFort, 1810.

Pl. VIII, fig. 16.

Caractères spécifiques. - Coquille de taille moyenne, ayant la forme d'un cône dont le sommet serait légèrement tronqué et arrondi, composée de 7 ou 8 tour's de spire convexes, un peu déprimés dans leur partie inférieure et nettement séparés les uns desautres par une suture profonde. Les deux ou trois premier's tour's élant ordinairement enroulés dans un même plan sont cause de la forme tronfucie du sommet de ha spire. L'ouverture est à peu près circulaire; son bor'd externe est niner, oblique, un peu prolongé dans son milieu et muni d'un lange sinus situé ì une petite distance de la suture. La surface des échantillons arlultes ot bien conservés est ordinarement couverte de fortes stries d'aceroissement obliques of irrégulieres; ees stries sont croisées à angle droit

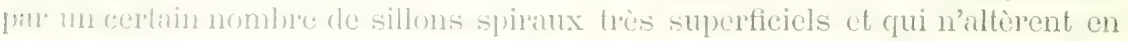
l'én la direction de ces mênes stries. Le lest est généralenent solide et épais. L'ombilic est tres ouvert, infundibuliforme; il laisse facilement apereevoir tous les lours des spire dont la coquille est eomposéc et dont la surface visible est répulimement convexes.

Dimensions d'un irfermtillon de grandeur maximum. - Longueur, $4 \mathbf{1}^{\mathrm{mm}}$;

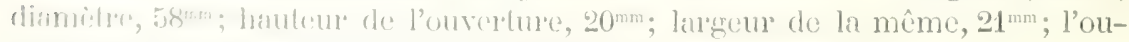
verlure de l'angle spinal varie de $90^{\circ}$ à $100^{\circ}$. - De Koninck (2).

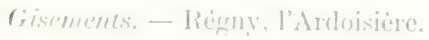

IErplieutern des figures. - P’. VIII, figr. 16. Spécimen aplati, ru du cóté de la spire. Rigny.

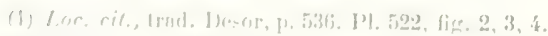

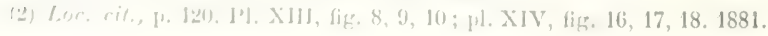


II. Straparollus convolutus, DE KoNinck, 1881.

Non figuré.

Caractères spécifiques. - Coquille de taille moyenne, de forme conique tris surbaissée, composée de 6 ou 7 tour's de spire à section transrerse presque circulaire et s'enveloppant très peu dans leur enroulement. La suture est très profonde; le péristome est entier ef l'ouverture est presque constamment circulaire, parce que chez les individus adultes, l'extrémité antérieure de la spire tend à s'isoler et à se détacher du tour qui l’a précédée. Li sinuosité du bord externe est très faible; l'ombilic est largement ouvert et permet d'y suirre aisément les divers tours de spire dont la eoquille est composée. Test minee; surface garnie de fines stries d'accroissement plus ou moins irrégulières.

Dimensions. - Longueur, 25 $5^{\mathrm{mm}}$; diametre de la base, 42m ; hauteur et largeur de l'ouverture, $12{ }^{\mathrm{mm}}$; ouverture de l'angle spiral, 115\%. - De Koninek (1).

Gisement. - Siguret.

III. Straparollus lævigatus, LÉvellté, 1835.

Pl. IV, fig. 15.

Caractères spécifiques. - Petite coquille discoïde, à spire à peu près plane en dessous et concave en dessus, composée de 5 tour's convexes, se lecourrant très peu dans lemr enroulement et séparés par une suture en forme de sillon spiral bien prononcé. Ouverture très faiblement ovale et transverse; bord externe tranchant, presque droit; une très faible sinuosité de la partie médiane du bord inférieur dome lieu à la formation d'un léger relèvement de la partie de la spire correspondant ì cette sinuosité, sans cependant produire de carène bien prononcée. La surface des premiers tours de spire est lisse; celle du dernier tour est ordinairement garnie de fines stries d'aceroissement dont la direction permet de reconnaître la forme de l'ouverture. Le test est fort mince et très fragile.

Dimensions. - Hauteur du dernier tour de spire, 4m ; diametre transrerse, $10^{\mathrm{mm}}$; hauteur de l'ouverture, $3^{\mathrm{mm}} 3 / 4$; lingeur de lia méme, $4^{\mathrm{mm}}$. - De Koninck (2).

Gisement. - La Tarrille.

Explication desfigures. - PI. IV, fig. 15. Spcieimen va par la partic infericure.

(1) Loc. cit., p. 124. P'. XIX, fig. 7, 8, 1, 18s1.

(2) Loc. cit., p. 127. Pl. XXI, fig. 19, 20, 21, 22. 1881. 
IV. Straparollus pileopsideus, Phillips, 1836.

Pl. XI, fig. 24.

Pl. XV, fig. 13.

Caractères spécifiques. - Petite coquille discoïde, à spire presque plane ou légèrement concave, composée de 5 tour's d'un accroissement lent, ne s'embrassant que partiellement dans leur enroulement. Suture canaliculée, relativement profonde, limitée par un léger renflement de la partie inférieure des tours de spire dont la base est régulièrement convexe. Ombilic large laissant facilement voir tous les tour's de spire. Ouverture ovale un peu plus haute que large. Surface ornéo de stries irrégulières d'aceroissement dont la direction est peu oblique.

Dimensions. - Hauteur du dernier tour de spire, $5^{\mathrm{mm}}$; diamètre de la base, $13^{\mathrm{mm}}$; hauteur de l'ourerture, $4^{\mathrm{mm}}$; largeur de la même, $3^{\mathrm{mm}} 5$. - De Koninck (1).

Gisements. - Néronde; l'Ardoisière, commune dans ce gisement.

Explicution des figures. - Pl. XI, fig. 24. Echantillon déformé. Néronde.

Pl. XV, fig. 13. Petit spécimen de l'Ardoisière.

J'ai donné au Miusce de Bruxelles, sur la prière de M. de Koninck, un très joli échantillon de l'Ardoisière gui figure dans les vitrines sous le $n^{\circ} 4080$.

GENRE EUOMPHALUS, J. SOWERBY, 1814.

I. Euomphalus acutus, J. Sowerby, 1818.

Non figures.

Cerructeres spécifiques. - Coquille de taille moyenne, plus large que longue, irrégulierement conique, composée de 7 tours de spire disposés en gradins. Pantir inferiene des lours de spire plane, perpendiculare aux tours antérieurs et limité prur une carene obtuse, peu saillante. L'ombilie est infundibuliforme, largen profond; l'ouroture est sculiforme, un peu oblique et plus haute que large. Thest assez nince. Sunface gannie d'une infinité de stries d'aceroissement inrpulieres, fris sonsibles quoique ne produisant jamais de lamelles saillantes; leur direction arecuse mu sinus asser profond sur l'angle de la spire.

Jimensions. - Longrueur, 36 $36^{\mathrm{mm}}$; diametre de la base, $47^{\mathrm{mm}}$; hauteur de l'ou-

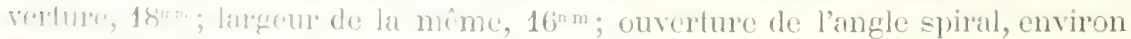
$90 \%$ - I) Koninck (2).

Gisement. - L'Ardoisiere, rare.

(1) Lere rit, 11, 124, 1', XIV, lige, 22, 23, 24, 1881.

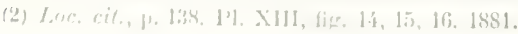




\section{Euomphalus crotalostomus, NI'Coy, 1844.}

Pl. IX, fig. 12, 13.

Caractères spécifiques. - Coquille discoïde, déprimée; spire légèrement saillante, composée de 5 tours; un espace déprimé ou aplati sur la partie supérieme de chaque tour, limité par une carène obsolète. Dos aplati-convexe. Base aplatie. Ombilic très large. Bouche dilatée. Surfuce oftrant des lignes transverses ir'égulières d'accroissement.

Cette espèce atteint quelquefois un pied de diamètre. La spire est très légèrement élevée. La bouche des vieux individus est dilatée en forme de somnette (d'où le nom spécifique). Les restes des bonches successives étalés en collerettes donnent un aspect particulièrement raboteux au der'nier tour. - N'Coy (").

Outre cette diagnose, qui est la plus ancienne, M'Coy en a donné une autre plus complete, en 1855, dans son grand ouvrage en collaboration aree Sedgwick (2).

Gisement. - Néronde.

Explication desfigures. - Pl. 1X, fig. 12. Fragment d'un dernier tour á l'état de moule interne, su de profil, et dont une partie seulement a été figurée. - Néronde.

Fig. 13. Le méme, vu de dos, pour montrer la caréne ( $($ ).

J'ai été heureux de trouver cette belle espèce car elle a servi, comme on le sait, ì M. Dupont de fossile caractéristique de la base de l'étage de Dinant.

III. Euomphalus amœnus, DE Kionnck, 1881.

Pl. VIII, fig. 14.

Caractères spécifiques. - Coquille d'assez petite taille, régulièrement conique, composée de 6 tours de spire anguleux, disposés en gradins; la partie de la surface inférieure comprise entre la carène spirale et la suture est étroite et un peu crense; carène aiguë et proéminente. Ouverture scutiforme, plus haute que largre, peu oblique, à sinus très étroit et peu profond. Ombilic relativement étroit et dont le diamètre n’attuint que lo tiers de celui de la base. Test mince, à surface couverte de fines stries d'uecroissement peu simneuses.

Dimensions. - Longueur, $15^{\mathrm{mm}}$; diamètre de la basc, $23^{\mathrm{mm}}$; hauteur de l'ouverture, $9^{\mathrm{mm}}$; largeur, $8^{\mathrm{mm}}$; angle spiral, $120^{\circ}$. - De Koninck ${ }^{(3)}$.

Gisement. - Régny.

Explicution des figures. - Pl. VIII, fig. 14. - Spécimen aplati, vu du cóté de lin spirc.

(1) Synopsis, p. 36. Pl. VII, fig. 4. 184 .

(2) Syst. descript. of the British I'uleor. Jossils, p. 539.

(3) Loc. cit., p. 143. P1. XII, fig. 20, 21, 2.2. 1881. 


\section{Euomphalus catilliformis, DE KonInCK, 1881.}

Pl. XI, fig. 23.

Caractères spécifiques. -- Coquille discoïde, composée de 6 tours de spire, s'enroulant de façon que leur bord inférieur se trouve à peu près dans un même plan, ou que les premier's tour's soient légèrement concaves. La carène spirale dont la surface inférieure des tours est garnie, se trouve placée dans la partie médiane de cette surface. La surface supérieure de ces mêmes tour's est généralement convexe, rarement un peu anguleuse. L'ouverture est scutiforme, aussi haute que large. L'ombilic est très ouvert et laisse voir tous les tour's de spire. Surface ornée de stries d'aceroissement irrégulières, peu perceptibles sur les premicrs tour's mais très apparentes sur le dernier.

Dimensions. - Diamètre de la base, $51^{\mathrm{mm}}$; hauteur du dernier tour, $18^{\mathrm{mm}}$; hauteur et largeur de l'ouverture, $15^{\mathrm{mm}}$. - De Koninck (1).

Gisements. - Néronde, Ferrières?

Explication des figures. - Pl. XI, fig. 23. Spécimen vu du côté ombilical, montrant nettement $t$ tours, identiques at la fig. 6 de la pl. XIX de l'ouvrage de De Koninck. Nous avons sommis cet échantillon i M. J. Fraipont qui a eu l'obligeance de l'examiner, et nous a donné son aris relativement is cette identification. - Néronde.

Le spécimen de Ferrières est représenté par un moule en plàtre déposé dans les vitrines du Mísée royal d'histoire naturelle de Bruselles sous le $n^{\circ} 3,000$ et sous le nom d'Euomphalus pentangulatus? Nous croyons que ce moulage avait été adressé à de Koninck par Jourdan, de Lyon. Cé dernier, en effet, nons avait déclaré dès 1872 avoir découvert dans les grès de cette localité de grandes Delphinules (lisez Euomphales).

GENRE SCHIZOSTOMA, BRONN, 1835.

Schizostoma cratériforme, DE KonINCK, 1881.

1"1. IV, figr. 1:3, 1\%.

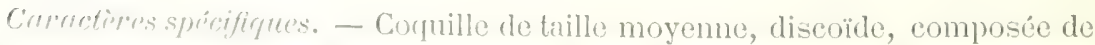

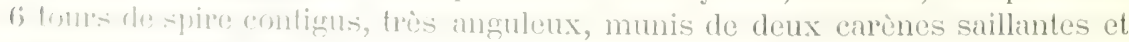

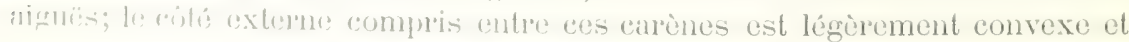

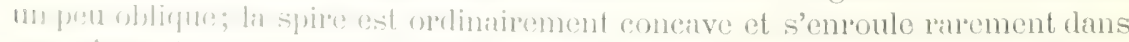
lin minne plin; ses sulures sont assez profondes, surtout aux dernier's tours.

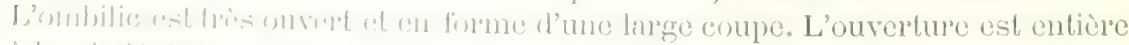

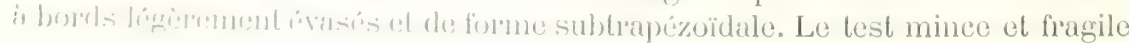


n'est que faiblement épaissi anx angles. La surface est ornée de fines stries d'aceroissement dont la direction est un peu sinueuse sur le côté supérieur, et courbe sur le côté extérieur de la spire.

Dimensions. - Hauteur du dernier tour de spire, 20 20m; dimmètre, 55 $5^{\mathrm{mm}}$; hautemr de l'ouverture, $16^{\mathrm{mm}}$; largeur de la même, $15^{\mathrm{mm}}$. - De Koninck (1).

Gisements. - La Varrille, Siguret.

Explication des figures. - Pl. IV, lig. 13. Fragment d'un spécimen montrant les quatre premiers tours. - Siguret.

Fig. 14. Moulage d'un autre spécimen. - La Varville.

Un beau spécimen de cette espèce provenant de Llook-Point (Irlande) est déposé dans le Musée royal de Bruxelles.

\section{Schizostoma catillus, WV. Martin, 1809.}

\section{Non figuré.}

Curactères spécifiques. - Cocuille de grande taille, discoïde, à spire concave composée de 7 ou 8 tour's contigus, anguleux en dessus et en dessous et séparés par une suture profonde; leur côté externe est faiblement bombé et su dirige un peu obliquement vers le haut. Sa surface est ornée de quelques lignes spirales que l'on l'observe que sur les spécimens bicn conservés. L'ourerture est subtrapézoïdale, un peu plus haute que large of à péristome entier; ses côtés inférieur et supéricur forment chacun un angle aigu avee le côtés externe; l'angle infériemr, un peu plus aigu que le supérieur, correspond ì la carène inférieure qui est plus saillante que la supérieure; l'suverture y est sinuéce, tandis qu'elle ne l'est point du côté opposé. L'ombilie est très large, assez furofond et regulierement concare; les sutures des divers tours de spire qui eontribuent ì le former sont linciares et peu profondes. 'Tunte la surfice est courerte de stries d'aceroissement irrégulières; ees sutures, un peu courbes sur lit partic comprise entre la suture of la carine, produisont un sinus assec étroil sur celle-ei et se dirigent ensuite par une lógère courbe obligue directement jusqu'au fond de l'ombilic; elles ne doment jamais naissance à dos lamelles imbriquées, comme chez certaines espèces d'Eumphalos. - Du Koninck (2).

Gisement. - L'Ardoisière.

(1) Loc. cit., p. 156. P1. XVII, fig. 4, 5, 6. 1881.

(2) Loc. cit, p. 154. P'. XVII, fig. 1, 2, 3, et pl. XXI, fig. 1, 2, 3. 1881. 


\section{Genre Phanerotinus, J. DE C. Sowerby, 1843.}

I. Phanerotinus serpula, DE KoNINCK, 1843.

Pl. IV, fig. 17.

Caractères spécifiques. - Coquille de taille moyenne, à spire à peu près plane, composée de 3 ou 4 tour's disjoints sur toute leur étendue et laissant un espace libre assez considérable entre eux. Le contour des sections transverses des premier's tours de spire est généralement un peu déprimé et subovale, tandis que l'ouvertur'e des spécimens adultes est de forme circulaire. La sur face est ornée de fines stries d'accroissement interrompues sur la partie inférieure de la spire par 2 sillons spiraux, subparallèles entre eux, très superficiels, et limitant la partie correspondant au sinus de l'ouverture; ce dernier est relativement assez large et très peu profond. Le test est mince et très fragile; les exemplaires complets sont très rares.

Dimensions. - Hauteur de la spire, $18^{\mathrm{mm}}$; diamètre de la spire, $45^{\mathrm{mm}}$; diamètre de l'ouverture, $13^{\mathrm{mm}}$. - De Koninck (1).

Gisements. - La Varville, Siguret.

Explication des figures. - Pl. IV, fig. 17. Empreinte d'un fragment montrant les trois premiers tours. - Siguret.

II. Phanerotinus nudus, J. DE C. Sowerby, 1843.

Pl. VIII, fig. 15.

Nons avons rapproché un fragment de Phanerotinus que nous avons recueilli it lispryy, de l'espèce crúce par Sowerby, et dont de Koninck a donné une diagnose et un dessin insuffisants (z).

Gisement. - Régny.

(1) Lu, rit, 1. VIII, p. 4. I1. XXII, fig. 1, 2. 3. 1883.

(2) Lese rit. 15. 7. I1. XXII, fig. 16, 17, 18. 1883. 


\section{FAMILLE IOES HALIOTIDFS}

\section{GENRE PTYGHOMPHALUS, Agassiz, 1845.}

\section{Ptychomphalus, SP.?}

Pl. XY, fig. 12.

Nous avons fait figurer le moulage d'une cmpreinte que nous langeons dans ce genre, bien que le côté ombilical nous soit inconnu. Aucune espèce figurée par les différents auteurs ne peut être identifice avee notre spécimen; le Ptychomphalus coniformis de Koninck, est celui qui paraît s'on rapprocher les plus.

Gisement. - L'Ardoisière.

Nous signalons cette espèce aux cherchenrs futurs.

Genre BAYLEA, DE KONINCK, 1883.

Baylea spirata, DE KioNinck, 1883.

Non figuré.

Garactères spécifiques. - Coquille de taille moyenne, conique, ì peu pris aussi large que longue, composée de 7 ou 8 tours de spire très angulenx et garnis d'une carene tranchante et saillante sur' lo bord mène de la partio anguleuse. La bande du sinus longe cette carene sur la partie horizonlale des teurs de spire et y est accompagnée de trois minees curènes spirales. Au-dessus de l'angle, se trouvent 7 ou 8 carènes spirales assez fortes et súprurés les mus

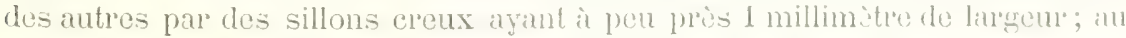
delà de celles-ei, il en existe un plus grand nombre de plus minces dont l'ípaisseur va en diminuant à mesure qu'elles se l'applochent do l'axe de la conuilla. L'ouverture est un peu plus haute gue large.

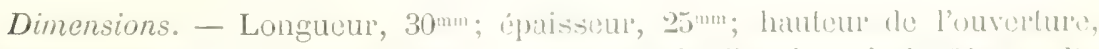
$13^{\mathrm{mm}}$; largeur de la même, 12mm ; onverture de l'angle spiral, 68'. - Do kininckin.

Gisements. - Rérny, Néronde; assez commun.

(1) Loc, cit:, p. 71. 11. XXXII bis, fig. 10. 1883. 


\section{GeNre PORCELLIA, LEveillé, 1835.}

Porcellia Puzo, Léveillé, 1835.

Pl. IV, fig. 11, 12.

Diagnose de Léveillé : " Coquille granulée par le croisement des stries dans "le sens de l'enroulement et dans celui de l'accroissement; ces stries d'acn croissement se dirigent d'avant en arrière; une rangée de tubercules de " chaque côté de la partie ventrale; un sillon dorsal; bouche quadrangu"laire (1). ")

Gisement. - Siguret.

Explication des figures. - Pl. IV, fig. 11. Empreinte externe.

Fig. 12. Nloulage de la même.

\section{FAMILLE DES BELLEROPHONTIDÉS}

Gente BELlerophoN, Denys de Montfort, 1808.

I. Bellerophon sublœvis, Potiez et Michaud, 1838.

P. III, fig. $19($ ( $)$.

P. IV, fig. 10.

Diagnose de Potie a et Micheued : "Testî fossili, parvâ, suborbiculari, globosâ, " longitudinaliter striatâ; striis minutissimis obliquis; aperturâ coarctatâ, " cmarginatî; sinu oblongo."

Dimensions. - Plus grand diamètre transversal de l'ouverture, 10 ì $12 \mathrm{~mm}$; Inognemir d'avant cn arrière, 12 i $15^{\mathrm{mm}}$; hauteur, 15 à $18^{\mathrm{mm}}$.

Corpuille petite, suborbiculairo, converte de très légères stries longitudinales, qui prutent du dos, et se prolongent, en formant inflexion, vers la base des oneillettes, it l'endroit ou serait l'ombilic s'il existait; ouverture étroite, échan-

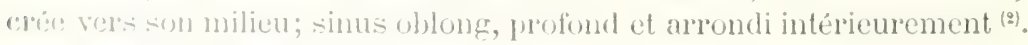

Gisement. - Iat Varville, Siguret.

Ervliention des firgures. - 1’. III, fig. 19) (u). Fragment déformé, vu du cóté du dos. Siment?

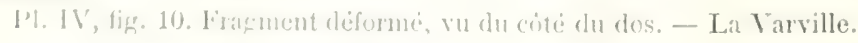

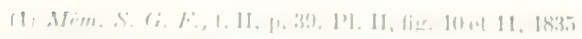

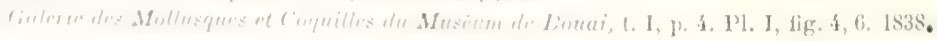


II. Bellerophon scalifer, DE KíNINcK, 1883.

Pl. VIII, fig. 13.

Caractères spécifiques. - Coquille de taille moyenne, globuleuse, aussi haute que large, bande carénale large, quelquefois l'égulièrement creuse, jamais saillante et bordée de chaque côté d'une mince crite filiforme; la partie médiane est couverte de lamelles imbriquées, arquées, moins nombreuses et plus laroges que les lameiles qui ornent le reste de la surface et qui viement rejoindre la bande sous un angle très ouvert. L'ouverture est grande, régulièrement voùtée; ses oreilles latérales ne sont pas fort développées; l'encroûtement de la partic intérieure de l'ouverture est assez étendu, mais il est assez mince quoique oblitérant complètement la bande carénale et les autres ornements de la surface. Les ombilies sont trìs étroits et bien marqués. Cette espèce a été dérouverte par Jourdan, dans le calcaire de Régny. - De Koninck (1).

Gisement. - Régny.

Explication des figures. - Pl. VIII, fig. 13. Spécimen un peu déformé, vu de dos.Régny.

Genre WAAGENIA, DE KoNinck, 1883.

Waagenia Ferussaci, A. D'OrHGNY, 1838.

Pl. VIII, fig. 12.

Caracteres spécifiques. - Coquille de taille moyenne, globuleuse, sonvent un peu plus haute que large. La bancle earemale est un peu sailinte, asser étroite, romposée d'écailles imbriquées phus nombrenses que les lamelles, lankex, reigulières et imbriquées qui couvent lia surfice; celles-ei, en partant de lar

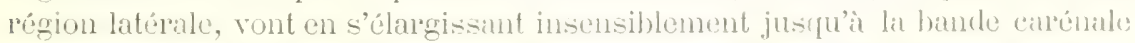
sans presque s'infléchir. L'ouverturr est réniforme; son borel externe est tranchant; une assez forte callosité lisse s'etend sur le bord columellare al conver complètement les ornements de la surface. De charque crits de l'ourerture, il

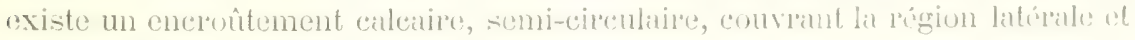
faisant saillie an-dessus de lat sulfiece.

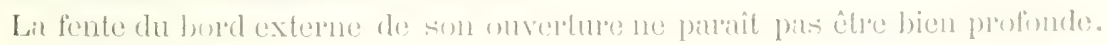

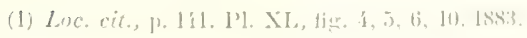


Dimensions. - Hauteur, 37 $7^{\mathrm{mm}}$; largeur, $35^{\mathrm{mm}}$; hauteur de l'ouverture, $15^{\mathrm{mm}}$. - De Koninck (1).

Gisement. - Régny:

Erplication des figures. - Pl. VIII, fig. 12. Fragment de la face dorsale.

\section{FAMILLE DES CALYPTR EIDÉS}

\section{Genre Gapulus, Denys de Montfort, 1810.}

\section{Capulus uncus? DE Kionisck, 1883.}

Pl. IV , fig. 18.

Curactères spécifiques. - Coquille de taille movenne, très comprimée latéralenent, à sommet mince, composé d'un tour de spire et assez proéminent. Le côté droit, légèrement convexe, s'arrondit et forme un large lobe sur son bord; lo côté gauche est, au contraire, un peu concave et son bord arance un peu moins que celui du côté opposé. Les deux côtés se rejoignent par la partie dorsale qui est faiblement carénée et assez régulièrement courbée en avant; à l'extrómité antérieure de cette partie, il existe un sinus assez large et peu profond. L'onverture est irrégulière, sinueuse et beancoup plus longue que large. Le lest est garni de stries et de faibles rides irrégulières d'aceroissement.

Dimensions. - Longueur, 26mm; hauteur du côté droit, 19 $6^{\mathrm{mm}}$; largeur de l'ouverture, $8^{\mathrm{mn}}$; longrueur de la même, 19 $\mathrm{gmm}^{\mathrm{mm}}$ - De Koninek ${ }^{(2)}$.

Gisement. - La Varville.

Erplication des figures. - PI. IV, fig. 18. Empreinte interne, légèrement déformée, gne nous identifions, sous réserve, il l'espèce ci-dessus, à cause du maurais état de conservation.

Il ron cast de méme pour l'espèce suivante.

II. Gapulus fimbriatus? DE KoNinck, 1883.

Sons firmé.

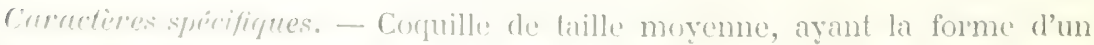

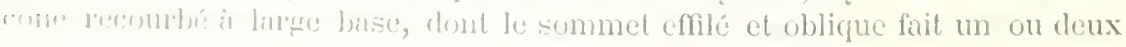


tours sur lui-même. Si partie dorsale est assez régulièrement convexu ver's le sommet, it une certaine distance duquel la coquille subit un plissement longitudinal qui se continue jusqu'aux bords de l'ouverture; le nombre des plis ainsi produits est variable et s'élève quelquefois jusqu'ì 10. L'ouverture est grande et subcirculare, abstraction faite des lobes plus ou moins prononcés qui se produisent sur les bords. Ceux-ci sont minces, tranchants, tres fragiles et rarement bien conservés. I a surface est ornée de stries ondulées d'aceroissement, pen régulières et parallibes au hord.

Dimensions. - Longueur, $35^{\text {mm }}$; dimmitre transverse de l'ourerture, 228m. - De Koninek (1).

Gisement. - Donaine des lioches.

Obsercation. - Ces deux Capulus, de mème que toutes nos espéces de Tournai, ont etrí identifiés arec le plus grand soin, non seulement avec les types du Musée royal d'histoire naturelle de Bruxelles, mais encore avec les types qui composent l'admirable et unique collection de notre excellent ami, M. Piret. Il nous a permis de les étudier is Tournai, en nous accordant l'hospitalité la plus gracieuse et la plus cordiale, ef nous lui en témoignons: de nouveau ici toute notre gratitude.

GENRE LEPETOPSIS, WHITFIELD, 1882.

I. Lepetopsis Leforti, Nov. SP.

Pl. IV, fig. 9.

Diagnose: Corquille de taille moyenne, de forme régulierement alliplique, très déprimée. Sommet sub-central, mammelonné ef situé un peu en arrière? du centre. La partie postérieure est un per plus bombée que la partie antérieure. Surface lisse, n'offrant que quelques stries irregulieres d'aceroinsement.

Dimensions. - Longueur, 27mm; largeur, 17mm; hauterur, $4^{\text {mm }}$; distance? du sommet au bord antérieur', $15^{\mathrm{nm}}$.

Gisement. - La Varville.

Nous dédions cette espèce it notre excellent ami, M. Lefort, fle Novers, at qui nous on devons la communication.

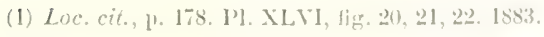


II. Lepetopsis Busscherianus, DE Ryckholt, 1853.

1'. VIII, fig. 11.

Caractères spécifiques. -- Petite coquille de forme ovale, très déprimée, à sommet légèrement excentrique, peu proéminent. Surface ornée de minces côtes longitudinales et un peu confuses. Test très mince et fragile.

Dimensions. - Longueur, 12mm ; largeur, $11^{\mathrm{mm}}$; hauteur, 2mm. - De Koninck (t).

Giscment. - liegny.

Explication des figures. - Pl. VIII, fig. 11. Spécimen un peu déformé.

\title{
ORDRE DES SOLÉNOCONQLES
}

\author{
FAMILLE DES DENTALIDÉS
}

GenRe DENTALium, Linné, 1759.

Nous avons découvert qualques spécimens très mal conselvés el indétermimables te co gemes. Noms nous bornoms à signaler leur présence dans la mer

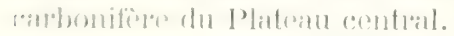

Giserment. - Régny, l'Ardoisière.

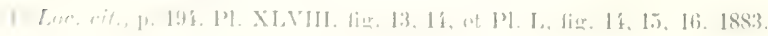




\title{
CLASSE DES LANELLIBRANCHES
}

\author{
ORDRE DES SIPHONÉS
}

FAMILLE DES ANATINIDÉS

GENRE EDMONDIA, DE KONINCK, 1843.

I. Edmondia orbiculata, DE KonINCK, 1885.

Pl. X, fig. 17 .

Caractères spécifiques. - Coquille presque complètement circulaire, renflée dans sa partie médiane et régulièrement bombée, crochets sub-médians; bord cardinal légèrement courbé et se reliant aux bords latéraux par des courbes dont l'antérieure est un peu plus arquée que la postérieure. Surfnce converte de fines stries concentriques d'accroissement. Test mince et fragile. - De Koninck ${ }^{(1)}$.

Gisenent. - Régny.

Eeplication des figures. - Pl. X, fig. 17. Valve droite.

II. Edmondia scalaris, M'Cor, 1844.

Pl. X, fig. 16, 19 .

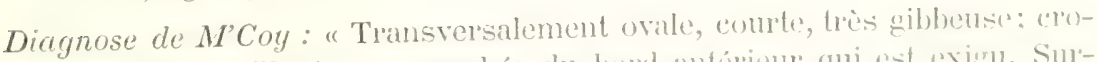
" chets grands, saillants, rapprochés du bord anterieur qui cist exigu. Sur" face couverte de 10 grandes côtes anguleuses. (Venerupis sculuris) (n). p

(1) Faune du calc. carb. de la Belgique. T. XI. 1. 41. I'1. XI, lier. 28, 29), 1885

(2) Synopsis, 1. 67. Pl. X, tig. 6. 
M. de Koninck attribue à cette espèce de M'Coy des spécimens belges qui ont de 12 ì 15 plis.

Gisement. - Régny.

Explicution des figures. - Pl. X, fig. 16. Valve droite. - Régny.

Fig. 19. Spécimen de plus grande taille, engagé dans la roche. - Rẻgny.

Edmondia filigrana, DE KoxINCK, 1855 .

Nun figuréc.

Caracteres spécifiques. - Coquille de petite taille, plus longue que large, subovale, subéquilatérale, peu épaisse; bord antérieur un peu plus convexe que le postírieur; bord ventral un peu plus arqué en avant qu'en arrière; crorhets sub-médians, petits, contigus, presque droits; surface garnie d'uno grande quantité de minees côtes concentriques, peu pereeptibles à la simple rum. - De linninck (1).

Gisement. - Regny.

IT. Edmondia amabilis, DE KonINCK, 1885.

1). Vill, fig. 19.

Caractèress spécifiques. - l’etite eofuille déprimée, subovale, un peu plus longue que large; bord cardinal droit; bords libres tormant une courbe dont colles du bord antérieur est mieux arrondie que celle du bord opposé; côté antérirur un peu plus hant que lo cotó postérieur; eroehets très petits, peu rentlés; -nrfirre grmis du côtes concentriques, minces, saillantes, séparées entre elles

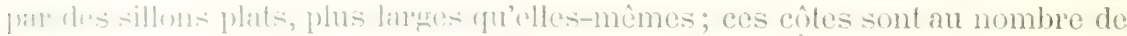
20) iो 2.

Jimmsions. - 1. kinimak ${ }^{1: 1}$.

risirment. - limgly.

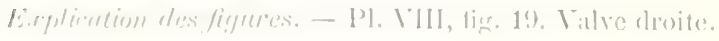


V. Edmondia? selecta, DE LONINCK, 1885.

Pl. X, fig. 18.

Curactères spécifiques. - Coquille de taille moyenne, allongée, sub-elliptique, déprimée vers ses extrémités; crochets légùrement épaissis, situés au quart antérieur de sa longueur totale; bord antérieur régulièrement arrondi; bord postérieur obliquement courbú d'arrière en avant et de haut en bas, se reliant au bord ventral très peu convexe; surface couverte d'un très grand nombre de plis concentriques.

Dimensions. - Longueur, 40 $0^{\mathrm{mm}}$; hauteur, 22mm épaisseul, $15^{\mathrm{mm}}$ - Do Koninck (1).

Gisement. - Régny.

Explication des figures. - Pl. X, fig. 18. Valve droite.

VI. Edmondia? amœena, DE KonINCK, 1885.

Pl. X, fig. 15.

Caractires spécifiques. - Coquille d'assez grande taille, obliquement allongée, subovale, beaucoup plus large en arriere qu'en arant, où le bord est plus fortement et plus régulièrement courbé; crochets petits, presque terminaux. Surface couverte de stries et de sillons concentriques, irrégulièrement distribués et donnant lieu à des côtes inégales, mais n'atteignant jamais une épaisseur qui dépasse un demi-millimètre.

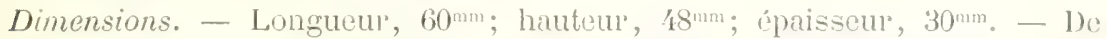
Kioninck (2).

Gisement. - Régny.

Explication des figures. - Nous avons rapporté, avec doute, il cette espéce, lir valve sgruche figurée pl. X, fig. 15 .

VII. Edmondia? angusta, IE KKoNinck, 1885.

Pl. XI, fig. 26.

Caractères spécifiques. - Coquille de taille mérliocre, subovale, derrx fuis

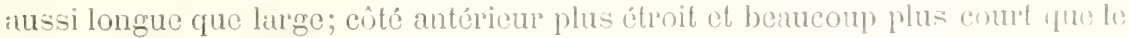

(1) Loc. cit. P1. XI, fig. 47, 48. 185.5.

(2) Loc. cit., 1) 47. P!, X, fig. 1, 2. 
cûté opposé; bord cardinal droit; crochets très petits et fortement recourbés en arant, situés au quart antérieur de la ligne cardinale; surface ornée de fines stries d'accroissement très superficielles et peu régulières.

Dimensions. - Longueur, $31^{\mathrm{mm}}$; hauteur, $15^{\mathrm{mm}}$; épaisscur, $10^{\mathrm{mm}}$. - De Koninck (1)。

Gisement. - Néronde.

Explication des figures. - Pl. XI, fig. 26. Valve droite.

Genre SANGUinolites, M'Coy, 1844.

1. Sanguinolites inconspicuus, DE KoNINCK, 1885.

Non figuré.

Caractères spécifiques. - Coquille subuvale, côté antérieur arrondi, côté posférieur déprimé et obliquement tronqué, bord ventral régulièrement arqué, crochets petits, peu renflés, inclinés en avant. Pli diagonal prineipal anguleux, aceompagné de deux plis rayonnants, écuidistants mais beaucoup moins apparents et ne faisant presque pas saillie; lunule petite et étroite; l'écusson dorsal, au contraire, très long, lanećolé et assez profond; surface garuie de fines stries concentriques d'accroissement, peu profondes et peu régulières.

Dimensions. - Longueur, $33^{\mathrm{mm}}$; hauteur, 15 $\mathrm{mm}$; épaisseur, $11^{\mathrm{mm}}$. - Do Kroninck (2).

Gisement. - La Varville. un seul échantiilon.

11. Sanguinolites tricostatus, Portlock, 1843.

1'. X. 1i_. I'.

Corretères spérifiques. - Coquille de taille moyenne, allongée, deux fois et womir ansi longrue que linge, diagonalement gibbeuse; bords cardinal et ven-

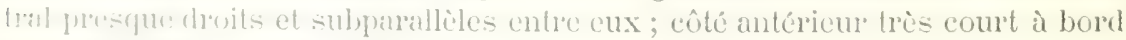

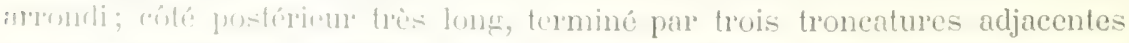
formant antre alles dus angles tres ouverts; crochets petits, contigus et forte-

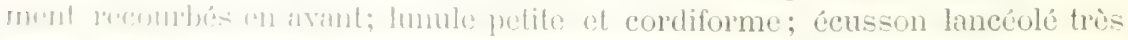

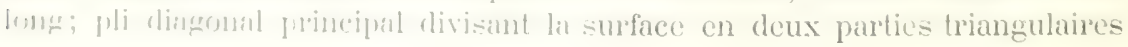


subigales, anguleuses et bien définies; deux autres plis rayonnants, moins saillants, oceupent en outre la moitió postérieure de lir valve et la partageant en trois languetles triangulaires égales; toute la surfice est ornée d'un rísenu formé de minees plis d'aceroissement, quelquefois un peu lamelleux, que coupent en travers de fines stries rayomantes à peine perceptibles à lia simple vue et produisunt ainsi une réticulation qui ne m’a été offerte par aucune autre espice.

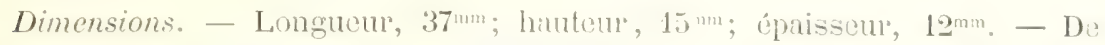
Koninek (1).

Gisement. - Régny.

Explication des flyures. - 1'1. X, fig. 14. Vialve droite.

\section{FAMLLLE DES SOLENIDES}

GenRE SOLENOPSIS, M'Coy, 1811.

I. Solenopsis Bielawskii, Nov. SP.

Pl. X, fig. 13.

Nous arons fait figurer, pl. X, fig. 13, un fragment re valve droite d'un Solenopsis brisé à la partie postérieure. Sus dimensions ne permettent pils de le confondre avec Solenopsis pelagicus Goldtuss.

Voici ses dimensions:

Longueur, $37^{\mathrm{nm}}$.

Hauteur, $10^{\mathrm{mm}}$.

Distance du crochet au bord anterient, $11^{\text {mm }}$; largour du miplat (qui longe les bord cal dinal a $25^{\mathrm{mm}}$ du crochet, $2 \mathrm{~mm}$.

La surface est couverte de stries tris nombrouses, irregulieres; lo bord rontral rectiligne, est parallèle au bord cordinal.

Gisement. - Régny.

Nous n'avons trouvé que eet unique exemplatire que nous dédions it notre excellent ami, 11. Biclawski, bien connu par ses travaux d'érudition.

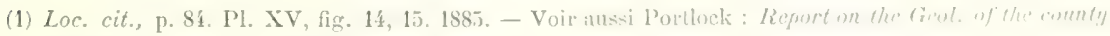
of Londondery, p. 11. I'I. XXXIV, fig. 17. 1811. 


\section{Solenopsis pelagicus, Goldfuss, 1832.}

Non figuré.

Curactères spécifiques. - Coquille étroite, trìs allongée, très inéquilatérale; côté antérieur court et arrondi, côté postérieur très long, allant en se rétrécis. sant, tronqué et faiblement baillant à son extrémité; crochets petits, situés à une certaine distance du bor'd antéricur; une faible dépression oblique et assez large s'étend des crochets au bor'd ventral et l'atteint vers le quart antérieur de sa longueur'; un pli obtus, quoique bien défini, ayant également son origine aux clocheis, se dirige diagonalement vers l'angle formé par la jonction des bords postérieur et ventral; cet angle est à peu près droit; la surface est couverte de fortes stries d'aceroissement un peu inégales, se relevant à angle droit sur la crête du pli diagonal pour atteindre le bord cardinal. - De Koninck (i).

Giscment. - L'Ardoisière. - Un seul échantillon incomplet, mais de dimensions égales à celles de la figrure de de Koninck.

\section{FAMILLE DES CYPRINIDÉS}

\section{GENRE CYPRICARDELLA, HALL, 1856.}

\section{Gypricardella? Julieni, nE Koxinck.}

I'I. VII, tin. 1.

Dirgnose. - Petite coquille suborbiculaire, un peu plus longue que large, convexe; bond antéreur plus court et nieux arrondi que le bord postérieur; rerochets petits, peu sailtants. Chamiere ineonnue. Surface couverte de stries consentriques excessivement fines ot rigulières, aucune autre coguille ne lui cost romparable sons ce l'apport. Ainsi, sur un espace de $5^{\mathrm{mm}}$ mesurés dans la

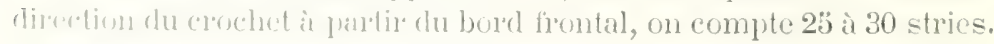

Jimensions. - I.ongurur, 12mm; largeur, $9^{\mathrm{mm}}$. A. Julien.

Gistments. - liegny, Saint-Genmain-Laval, Néronde, Montmain, Propières; excessivement athendantre.

Nims avions rommuniqué un frand nombre de spécimens a M. de Koninck, il y a plu-

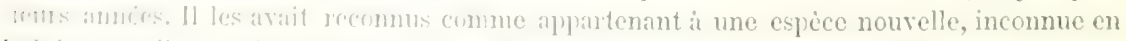

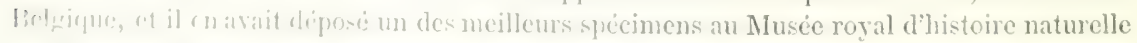
th. limuxelles. It est exposed dans les vitrines qui renferment les fossiles carbonifères étrangers 
sous le $n^{\circ} 4090$ et avec l'étiquette suivante: Lucinella? Juliennii. Nous croyons plutót que c'est une Cypricardelle et nous rectifions le nom spécifique, en donnant une diagnose de cette espèce que nous a dédiće le savant belge qui, du reste, no l’a point décrite.

Explicution des figures. - Pl. VIII, fig. 1. Spécimen de Régny (Voir Chonetes murenisoni).

\section{FAMILIE DES TRIDACNIDÉS}

\section{GENRE GONOGARDIUM, BRONN, 1835.}

I. Conocardium minax, Phillips, 1836.

\section{Pl. XV, fig. 15.}

Caractères spécifques. - Petite coquille allongée, subtrigone, bombée; hir partie antérieure obliquement tronquée, cordiforme, convexe, ornée d’un rostre conique à sa base, et cylindrique sur le reste de son prolongement. La région médiane de la coquille est rentléc et s'avance obliquement sur les côtés et sous la partie antérieure cordiforme; elle se continue en arriere, assez insensiblement, avec la partie postérieure aliforme, qui est béante à son extrémité. Les crochets sont bien accentués, l'ecourbés obliquement en avant. La l'égion copdiforme est ornée de petites stries obliques, partant du bord antériemp; les plus inférieures se perdent au niveau du bourrelet latéral, les autres convergent vers les crochets. Des côtes plus épaisses garnissent les récrions mérlime et postérieure de la coquille; chles rayomnent des crochets vers les bords; colles de l'extrémité aliforme sont plus larges que les autres et séparées par des sillons plus ou moins profonds. De nombreuses petites strics concentriques of ondulées d'aceroissement traversent ces côtes.

Dimensions. - Longueur à la base du rostre, variant de 9 a $17^{\mathrm{mm}}$ suivint lir taille; hauteur, de 7 à $14^{\mathrm{mm}}$; épaisseur, de 6 à $14^{\mathrm{mm}}$. - Do Koninck (1).

Gisement. - L'Ardoisière; assez commun.

Explication des figures. - Pl. XV, fig. 15. Portion de la valve ganche. J'ar fait don au Musee royal d'histoire naturelle de Bruxelles, pur l'intermédiaire de M. de Koninek, d'un très joli spécimen de cette espèce ; il est exposé dans les vitrines de cet établissenent rivervèes aux fossiles carbonifères étrangers sous le $n^{\circ} \cdot 1090$.

(1) Loc, cit, p. 3. 1'. XIX, fig. 22, 23, 22, 25. 1855. 


\title{
ORDRE DES ASIPHONLS
}

\section{FAMILLE DES NUCULIDÉS}

GenRe NUGULA, J.-B. DE LAMARCK, 1799.

\begin{abstract}
Carcutères génériques. - Coquille subtrigone ou ovale, parfaitement close, is sommets tournés du côté postérieur qui est court; surface lisse ou ornée; épiderme olive; face interne nacréc; bords lisses ou crénelés; charnière anguleuse, ayant de chaque côté une rangée de dents tranchantes; dans l'angle de lir charniere ef immédiatement au-dessous des crochets, une fossette saillante drotincée à contenir to cartilage interne; impression palléale simple. - De kininek
\end{abstract}

GEYRE NUGULANA, H.-F. LINK, 1807.

Curcecteres génériques. - Coquille oblongue, arrondie en avant, rostrée et quatguetois un peu bailante en arrière; charnière étroite, composée de deux pangress de petiles dents pointues formant entre elles un angle obtus et séparuss sous les crochets par un petit cuilleron saillant à l'intérieur et destiné au ligmment. Crochets assor proéminents; borls lisses; surface interne nacrée; impression palléale échancrée par un petit sinus qui atteint l'adducteur antérisull. - 1)e Koninck (-).

\section{FAMILLE DES PECTUNCULINES}

GENIR: TELLINOMYA, J. HALL, 1843.

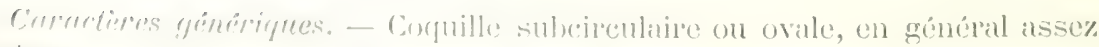

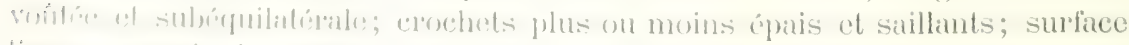

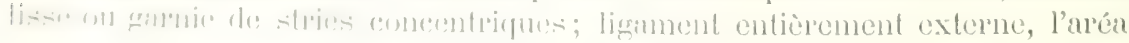


ligamentaire faisant défuut; ligne cardinule courbe avee deux séries de dents transverses dont les plus petites sont au centre; impression palléale simple. - De Koninck (1).

Gisements. - L'Ardoisière, Régny. - Les coquilles appartenant ì ces trois genres ne sont pas rares á Régny ni à l'Ardoisière, mais leur mauvais état de consersation ne m'a pas permis, jusqu'á présent, d'arriver ì des déterminations spécifiques certaines.

\section{FAMILLE DES ARCIDES}

\section{GENRE PARALLELODON, MEEK ET IVORTHEN, 1866.}

I. Parallelodon bistriatus, PortLock, $1813^{(2)}$,

Pl. IV, fig. 7,8 .

Caractères spécifiques. - Petite cocuille oviale, oblongue, un peu gribbeuse. diagonalement renflée à partir des crochets; bord cardinal droit, subanguleux à son extrémité postérieure qui est faiblement tronquée; côté antérieur obliquement arrondi, plus épais que le côté opposé qui est déprimé et s'allonge en se rétrécissant; surface couverte de plis concentriques plus ou moins imbriqués, plus larges en arrière qu'en avant et couverts chez les individus bien conservés d'un dessin plus ou moins réticulé, qui disparaît facilement par' l'usure ou par l'âge; la charnière se compose de trois petites dents antérieures très obliques et courtes, et de deux dents postérieures très minces, parallès au bord cardinal, mais n'oceupant pas tout l'espace compris entre les crochets et l'extrémité postérieure.

Dimensions. - Longueur, 13mm hauteur, $6^{\mathrm{mm}}$; épaisseur, $5^{\mathrm{mm}}$. - 1) Koninck ${ }^{(3)}$.

Gisements. - La Varrille, Siguret; abondant.

Explication des figures. - PI. IV, fig. 7. Valve droite ì demi engagce dans la roche.

La Varrille.

Fig. 8. La mème, grossie 4 fois.

Un magnificute échantillon de La Varville a été domné par nous à MI. de Koninck ponte ètre déposé au Musée royal d’histoire naturelle de Bruxelles, ou on pent le voir dans la vitrine renfermant les fossiles carbonifères étrangers.

(1) Loc. cit, Lamellibranches, 1. 138.

(2) Loc. cit, p. 440. 1'1. XXXvi, fig. 13. 1843.

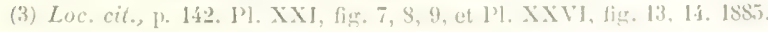


II. Parallelodon argutus, Phillips, 1836.

\section{Non figuré.}

Caractères spécifiques. - Petite coquille allongée, gibbeuse, tronquée obliquement à sa partie postérieure; une carène bien prononcée traverse diagonalement toute sa surface qui en outre est garnie de nombreuses stries concentriques assez r’égulières et assez profondes. Crochets assez renflés et situés au quart antérieur de la ligne cardinale, ils sont très rapprochés et séparés par une fussette liganentaire très étroite et presque linéaire.

Dimensions. - Longueur, 14m $\mathbf{r}^{\mathrm{mm}}$, hauteur, 8mm; épaisseur, $6^{\mathrm{mm}}$ - De Koninck (l).

Gisement. - Rigny.

III. Parallelodon Lacordaireanus, DE KontNck, 1842.

Non figuré.

Caracteres spécifirues. - Coquille de taille moyenne, allongée, subrhombö̈dale, renilée, très inéquilatérale; côté postérieur un peu obliquement Lronqué et plus anguleux ver's le bord ventral; celui-ci est arqué et s'unit au brnd antérieur par une courbe convexe; les parties antérieure et médiane de la rocquile sont rentlées, tandis que le prolongement postérieur en est déprimé; alle est divisée par une carène oblique et obtuse, qui partant des crochets, la haverse diagonalement; fineette ligamentare assez large et plane; surface garnie de côtes lamelleuses, minces et imbriquées, de largeur inégale et traversines pur des stries rayonmantes, assez régulières et assez faiblement marquées sur la majeure partie des régions antérieure et moyenne, mais ordinairement

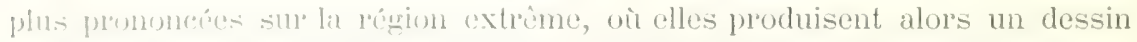
truillisis; charnière composée de 3 ou 4 a dents antérieures, assez courtes ef ohlirpus, ot de 2 ou 3 dents postericures, tres longues, laminaires et subparalli.lus an borted catrlinal.

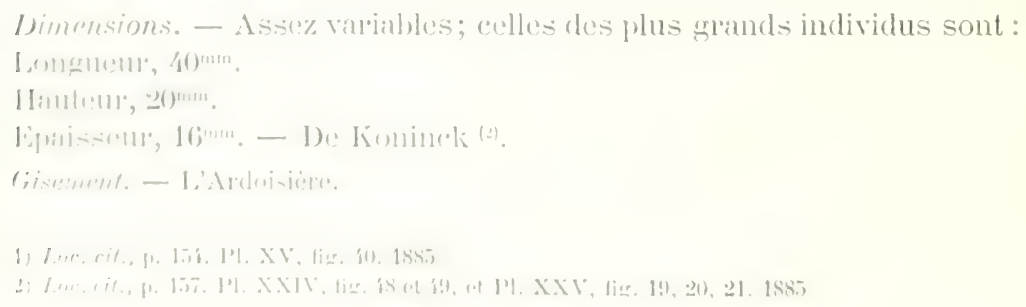


IV. Parallelodon meridionalis, DE KONINCK, 1885.

Non figulé.

Caracteres spécifiques. - Coquille de taille moyeme, subrhomboïdale, bord cardinal droit, bord antérieur court formant un angle droit avec le bord cardinal et s'arrondissant en arant avec le bord ventrul qui est un peu convexe et subparallèle au bord cardinal. Extrémité postéricure tronquée à bord presque droit et formant un angle obtus avec le bor'd cardinal. Crochets renfiés. Facette ligamentaire assez étroite et lancéolée. Surface il carène très obtuse, couverte de nombreuses stries d'aceroissement croisées par des sillons rayomants obsoletes.

Dimensions. - Longucul maximum, 52mm; hauteul, 23m; épaisseur, $21^{m m}$. - De Koninck ${ }^{(1)}$.

Gisement. - La Varville.

V. Parallelodon comoïdes, DE KONINCK, 1885.

Pl. X, fig. 12.

Caractères spécifqques. - Petite coquille allongée, sublectangulaire, un peu obliquement tronquéce en arrière, arondie en avant et à bords cardinal et ventral parallèles; une carène très pronencée, traversant diagonalement los valves des erochets rer's l'angle for'mé par l'inter'section des bords postéricur el ventral, les partuge en deux parties un peu inégales; leur surtiace est ornés d'un grand nombre do minces plis concentriques que traversent des stries rayonnantes plus nombreuses encore et très fines. La facette ligimentaire est bien prononcée et plane. La charnière est incomnue.

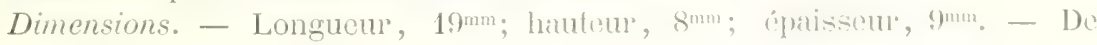
Koninck (2).

Gisements. - Régny, Saint-Germain-Laval, l'Ardoisière.

Explication des figures. - PI. X, fig. 12. Valve rlroite - Régny.

(1) Loc. cit., p. 158. 1'1. XXIX, fig. 7-13, ete. 1855.

(2) Loc. cit., p. 159. Pl. XXIV, fig. 52, 53. 1585. 
VI. Parallelodon mytiloides, DE KonINCK, 1885.

Non figuré.

Caractères spécifiques. - Coquille de taille médiocre allongée, subovale; bord cardinal droit, terminé en arrière par un angle obtus, lequel est formé par le bord postérieur recourbé se joignant au bord ventral ; celui-ci est presque droit et converge faiblement vers le sommet; crochets assez petits, renflés et fort antérieurs; surface presque lisse et uniquement garnie de stries concentriques d'accroissement plus ou moins profondes et régulières; test mince; charnière inconnue.

Dimensions. - Longueur, 19mm; hauteur, 9 $9^{\mathrm{mm}}$; épaisseur, $6^{\mathrm{mm}}$ - De Koninck (1).

Giscment. - Régny.

\section{FAMILLE DES PINNIDES}

\section{GENRE AVIGULOPINNA, MEEK, $186 \%$}

\section{Aviculopinna spathula, M'Coy, 1853.}

Nonfigrulé.

Caractères sprécifiques. - Corpuille allongée, pointue à son extrémité anté-

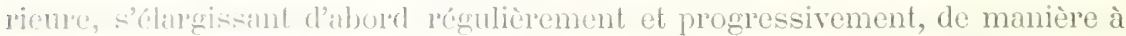
produire une fome trimentaire qui se modifie vers le milieu de la longueur, où les valves cessent de s'élargir at où lo bord ventral prend une direction posegue parallele it colle du bord cardinal; partie antéricure très convexe,

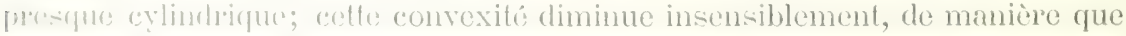

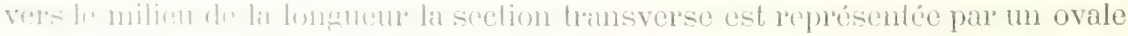

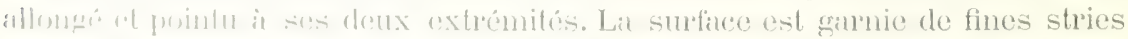

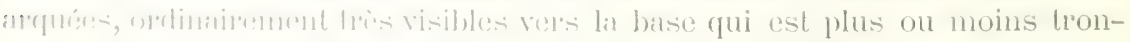

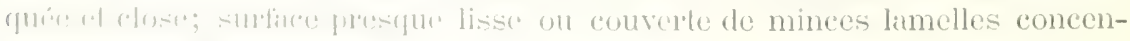

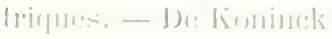

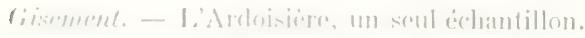

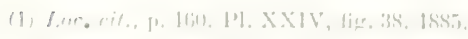

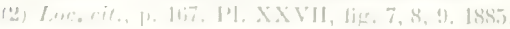




\section{FAMILLE DES MIYTILIDÉS}

GENRE MOdIOLA, LAMARCK, 1801.

1. Modiola fusiformis, DE KoNINCK, 1881.

Pl. X, fig. 10.

Caractères spécifiques. - Coquille de taille moyenne, fusiforme, très allongée, oblique, peu bombée. Bord cardinal légèrement arqqué ou droit, mesurant environ la moitié de la longueur totale. Bord ventral presque droit. Crochets terminaux. Sa surface est garnie d'un grand nombre de fines rides concentriques d'aceroissement.

Dimensions. - Longueur, $70^{\mathrm{mm}}$; hauteur, 22mm ; épaisseur, environ $20^{\mathrm{mm}}$ - De Koninek (1).

Gisement. - Saint-Germain-Laral.

Explication des figures. - Pl. X, fig. 10. Nous arons figuré sous ce nom une modiole très voisine de la fusiformis. Elle en différe toutefois par une longuenr un pen moindre du bord cardinal, et une taille plus faible de la conpuille qui n'a pas plus de $3 y^{\text {mm }}$ de long; mais elle s'en rapproche par sa forme et par ses fines stries d'aceroissment. Nous n'arons pas jugé il propos d'en faire une espèce distincte, car notre spécimen appartient incontestablement au groupe de Modiola fusiformis.

I. Modiola cuneiformis, DE KoNINCK, 1885.

Non figuré.

Caractères spécifiques. - Coquille de taille médiocre, oblongue, oblique, régulièrement bombée, beaucoup plus haute en arrière qu'en avant. Bord cardinal arqué formant un angle obtus avee lo bord antérieur; celui-ci est presque droit et très court. Bords ventral et postirieur arrondis. Cirochets antérieur's et assez bien isolés. Quelques rides d'aecroissement à la surface.

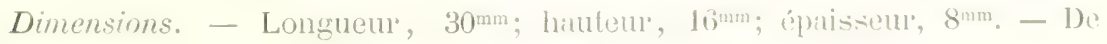
Koninck (2).

Gisement. - Régny.

(1) Loc. cit., p. 174. P1. XXVIII, fig. 2, i, 5, 6, 7, 29, 30. 185\%

(2) Loc. cit., 1). 179. P1. XXYII, fir. 31. 1585. 


\section{FAMILLE DES AVICULIDÉS}

\section{GENRE POSIDONIELLA, DE KONINCK, 1885.}

Posidoniella vetusta, R. Sowerby, 1829.

Pl. XV, fig. 14.

Diagnose de Sowerby : "Coquille ovoïde, convexe, lisse avee des ondula» tions concentriques régulières. Crochets courts, pointus, courbés. Un espace ") concave au bord antérieur ressemblant à une grande lunule. Charnière n) courte n").

Gisement. - L'Ardoisière.

Explication des figur'es. - Pl. XV, fig. 14. Monlage de l'unique spécimen que j’ai découvert dans ce gisement; ce spécimen a été déposé par l'obligeant intermédiaire de M. de Koninck et sur sa demande dans les vitrines du Musée royal d'histoire naturelle de Bruxelles; il y est inscrit sous le $n^{\circ} 4006$.

\section{GENRE LEIOPTERIA, HALL, 1883.}

I. Leiopteria hirundo, DE KonINCK, 1885.

Pl. X, fig. 11.

Curacteres spécifirues. - Coquille d'assez grande taille, allongée, oblique, convexe, équivalve. Les oreillettes antérieures petites, à extrémité arrondie; les postérieures nettement séparées du corps de la coquille, très longues, aliformes, à bords postéricurs tris échanerés, mesurant un peu moins des deux liers de la longneur totale. Crochets contigns, dirigés en avant et presque antérients. Le bord cirdinal un peu moins long que le corps de la coquille est délimité par un rebord étroit au niveau de l’orcillette postéricure. Surface couverte d'un frand nombre de stries d'aceroissement; les stries du corps de la corpuille sont beancoup plus régulieres et plus profondes que celles de la partie aliforme, qui sont nowins marequées et plus confuses.

Dimensions. - Longueur, 50mm; larocur du bord cardinat, 44mm; hauteur,

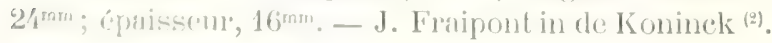

Gisernenl. - Reigny.

Eropliculion des figures. - P1. X, lig. 11. limpreinte d'une valve gauche. M. Fraipont it qui jo l'ai sumise, lat considère commo un jeune individu de cette espèce.

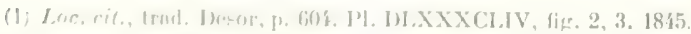

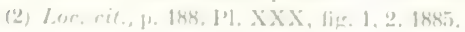


11. Leiopteria lunulata, Phlllips, 1836.

Non figuri.

Curactères spécifiquess.-- Corpuille d'assez glande taille, allongée, très oblique d'arant en arriere et de haut en bas; la valve régulièrement bonbée. L'oreillette antérieure fort petite, à extrémité libre arrondic, l'oreillette pustérieure 6 fois plus longue que la première et nesurant plus de la moitié de la Iongueur totale; elle est effilée à son extrémité libre et très excavée en arrière; elle présente un léger rebord le long de la ligne carlinale. Crochets petits, allongés, arrondis en avant, situés sur le cinquième antérieur de la longueur du bord cardinal, celle-ci mesure environ les 23 de la longueur totale. La surface est recouverte de nombreuses petites côtes, plus regulières près du crochet que vers le bord ventral. C'est ru niveau du pli qui sépare l'oreillette poslérieure du corps de la valve que les stries sont leplus rapprochées et le plus fines.

Dimensions. - Longueur totale, $34^{\mathrm{mm}}$; longueur du bord cardinal, $30^{\mathrm{mm}}$; hanteur totale, $32 \mathrm{~mm}$ - J. Fraipont in de Koninck (1).

Gisement. - Régny.

III. Leiopteria Van den Broecki, Nov. SP.

Pl. IV, fig. 6.

Nous désignons sous ee nom une Leiopteria dont nous arons trouvé 3 ou 4 spécimens à La Varville et qui, par sa forme et ses dimensions tient le milieu entre Leiopteria enaciata de Kon, et Leiopteria columbo de lion. La première n'est pas rare dans le calcaire carbonifère de Furfooz (Et. II), la deuxième est rare dans le calcaire carbonifère de Visć (Et. III). On sait qu'aucume espèce de ce genre n'a encore été trouvée dans l'Etage I, de Tournai.

Diagnose. - Notre petite coquille est allongée, très oblique, légèrement bombée suivant une zone s'étendant des crochets à l'extrémité postéricure du bord inférieur. Sa surface est couverte de fines lamelles d'accroissement très régulières.

Voici les dimensions qu'elle présente:

Diamètre umbono-ventral, $20 \mathrm{~mm}$;

(1) Loc, cit., p. 188. P1. XXX, fig. 4. 1885. 
Longueur de l'oreillette postérieure, $9^{\mathrm{mm}}$; antérieure, $6^{\mathrm{mm}}$.

Gisement. - La Varville.

Explication des figures. - Pl. IV, fig. 6. Valve droite.

Nous dédions cette espèce á notre escellent ami, M. Ernest Van den Broeck, conservateur au Musée royal d'histoire naturelle de Bruxelles.

\section{FAMILLE DES PECTINIDÉS}

\section{Genre Aviculopecten, M'Coy, 1851.}

\section{Aviculopecten Pomeli, Nov. SP.}

P1. IV, fig. 2.

L'mpreinte de valve que nous arons figurée, pl. IV, fig. 2, est celle d'un Aviculopecten très voisin de A. granosus Sowerby.

Elle a à peu près les mêmes dimensions que l'espèce de Sorrerby. La surface est garnie de côtes rayonnantes moniliformes, mais tandis que Avic. granosus n'oflire que deux sortes de côtes de grosseur différente, alternant régulièrement, notre espèce du Morvan en présente 3 sortes.

Ce cinactire suffit pour la distinguer de celle de Sowerby.

Gisiment. - Domaine des Roches (Morvan).

Nous dédions cette espece at notre éminent compatriote, M. Pomel, directeur honoraire de l'Ecole superieure des sciences d'Alger.

II. Aviculopecten Bosquetianus, DE KONINCK, 1851.

Ninl ligmir.

Corrorteress spécifirnes. - Conpille de taille médiocre, subsemieireulaire allongin; la valve ginnehe refoulierement convexe; la valve droite inconnue

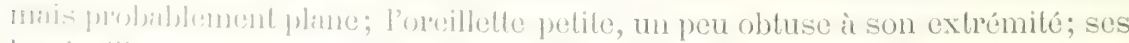
bonds libmes forment an angle aigna l'oreillotle postérieure beaucoup plus

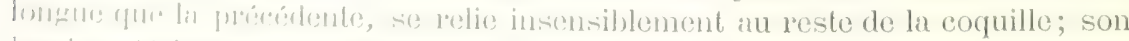

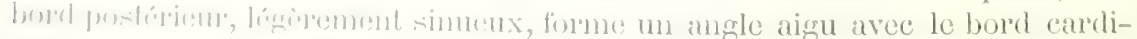

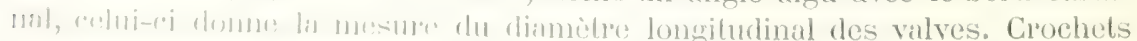

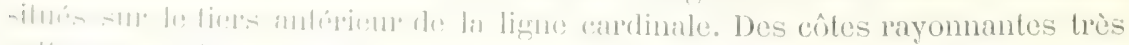

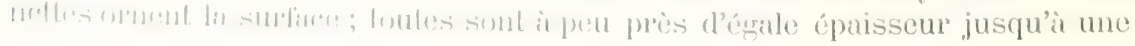


certuine distance des erochets; d'antres côtes, un peu plus minces, prenment alors naissance et s'intercalent entro les premieres, pour aboutir au borel ventral; le tout est traversé prex une quantité de fines stries concentriques d'accroissement très serrées, ondulées et peu visibles à l'ouil nu.

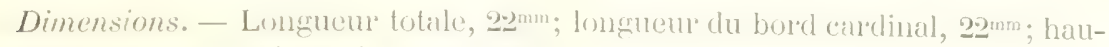
teur, 1.1mm. - J. limipont in de Kininck (1).

Gisement. - Neronde; nous possidons une valve gatuche unique.

III. Aviculopecten ingratus, DE KoNisck, 1885.

Pl. IV, fig. 3, 4.

Caracteres spécifiques. - Coquille un peu plus longue que haute. Dans le jeunc âge la surface est garnie de côtes rayounantes simples et de même forme, tandis que chez les adultes ces côtes sont de trois diametres différents et disposées symétriquement en gloupes de :3, exceptionnellement en groupes de 8 , suivant les diverses bifureations qu'ulles ont subies. Elles sont traversées par des stries d'aceroissment concentriques, incgalement distantes les unes des antres.

Dimensions. - Longueur maximum, 46mm; longueur du bord curdinal, $44^{\mathrm{mm} \text {; }}$ hauteur, 42mm. - J. Frixipont in tle Foninck (2).

Gisement. - Domaine des Roches.

Erplication des figures. - PI. IV, fig. 3. Enupreinte cxterne d'me valve droite d'un jeme inclividu.

Fig. 4. Moulage de li mine.

IV. Aviculopecten cœlatus, M'Cor, 1811.

Pl. X, fig. 8 .

Caracteres spécifiques. - Coquille de smande taille, suborbienlaire, subérquilatérale. Les oreillettes proportionnellement petites et semblables, il extrémilé arrondie; les postérieures un peu plus longnes et un pen plus hantes que les antérieures. Il existe sous l'oreillotte antériemo de la valve droite une putile échancrure du byssus. Le bord candinal est prospue moitio plus comet que le diamètre longitudinal; il présente st1 la valve droile et an niveau du crechet une courte lamelle interne ayant servi it la réception du liganent. Le crenchel

(1) Loc, cii., p. 215. P1. XXXV, fig. 봉, 30. 1885.

(2) Loc. cit., I. 221. I'l. XXXV, fig. 12, 13, 16, 17, 33, 34. 1885. 
est petit et submédian sur les deux valves. La surface est couverte de côtes rayonnantes, de deux épaisseurs différentes qui n’alternent pas toujours régulièrement; elles sont traversées par des lamelles d'accroissement ondulées et imbriquées que M'Coy considère comme formant le caractère distinctif de l'espèce.

Dimensions. - Longueur du plus grand spécimen, $77^{\mathrm{mm}}$; longueur du bord cardinal, 45 $5^{\mathrm{mm}}$; hauteur, $72^{\mathrm{mm}}$. - J. Fraipont in de Koninek (1).

Givement. - Rẻgny.

Explication des figures. - Pl. X, fig. 8. Fragment de valve.

Le Musée royal d'histoire naturelle de Belgique en possède un échantillon très beau, inserit sous le $n^{\circ} 3000$, et que nous lui avons donné sur la demande de M. de Kioninck. La nême vitrine renferme des spécimens de la méme espèce provenant du Yorkshire, de Beith, de Corrieburn et de Campsie (Ecosse), identique à celui de Régny.

V. Aviculopecten plagiostoma, DE KoninCK, 1885.

P. X, fig. 7 .

Caracteres spreifiques. - Coquille de taille moyenne, suborbiculaire, presque aussi haute que longue; la valve gauche régulièrement bombée; son oreillette antérienre plus courte que la postérieure et presque moitié moins haute; son bord intiricur est sinuenx et son extrémité arrondie; l'oreillette postérieure ast anssi sinnense an arriere at arondie il son extrémité. Le bord cardinal est mu ruart plus cont que le diamitre longitudinal de la valve; le crochet se trouve

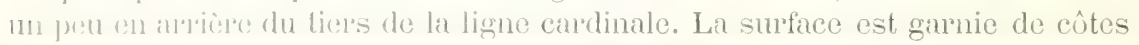
mỵnnantes, trìs nellos, séparées par des sillons relatirement larges, au milieu desfunls suresissent, en certuins points de la surface, de nouvelles côtes plus minces allenmant avec les premieres; toutes les côtes sont traversées par de

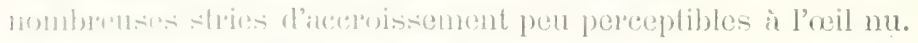

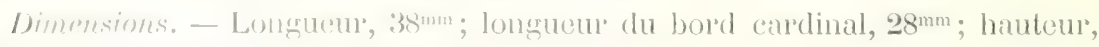

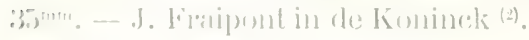

bisiment. - trignty

Erpliartion des figures. - IP. X, fig. 7. Fragment de valve.

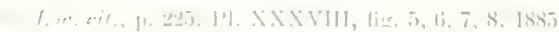

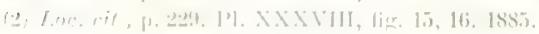


VI. Aviculopecten biornatus, DE KONINCK, 1885.

Non figuré.

Caractères spécifiques. - Corfuille dr taille moyenne, suborbiculaire, un peu plus longue que haute; la valve gaturhe finiblement bombés; les oreillettes petites, étroites; l'antériene un pen plus courte que la postérieño, arrondie cn arant; la postérieure faiblement excavéc en arlière. Le bord cardinal mesure un peu plus de la moitié de la longuemr totale de la coquille; crochets submédians; la surface est ornée de larges côtes rayonnantes, plus apparentes sur le côté antérieur et traversćes par un grand nombre de fines côtes concentriques très rapprochées les unes des autres.

Dimensions. - Longueur, 35 $5^{\mathrm{mm}}$; Iongueur du bord cirdinal, 20 ${ }^{\mathrm{mm}}$; hauteur, $30^{\mathrm{mm}}$. - J. Fraipont in de Koninck (1).

Gisement. - Domaine des Roches.

VII. Aviculopecten subfimbriatus, DE VERNEUIL, 1815.

Non figuré.

Caractères spécifiques. - Cette coquille est orbiculaire, symétrique, subúquilatérale et peu convexe; les côtes longitudinales, dont lit surfice est oruée, sont au nombre de 40 à 42 ; elles sont étroites, rapprochécs, équidistantes, arrondies, régulières et égales aux intervalles on sillons qui les śparent. Lne multitude de stries fines, légèrement écailleuses, les traversent en formant des fiestons dont les angles rentrants, pur rapport aux bords, sont placés sur le milieu des côtes, et les angles saillants dans leur's intervalles. Dans un espace de 5 millimètres, on compte de 8 ì 9 de ces stries concentriques.

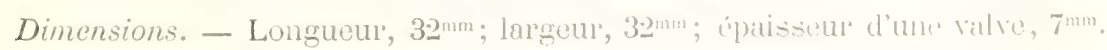
- De Verneuil (2).

Gisement. - L'Ardoisière; très rare.

(1) Loc. cit., 1. 233. Pl. XXXIV, fig. 19, 20, 21. 1585.

(2) Paleontologie de la Rusin. p. 327. Ml. XXI, fig. 5) a, b. 155. 
VIII. Aviculopecten? dupliciradiatus, DE KONINCK, 1885.

Pl. X, fig. 9.

Caracteres spécifiques. - Petite coquille, oblongue, plus longue que haute, oblique d'avant en arrière, subéquivalve, régulièrement convexe; les deux oreillettes peu développées, déprimées transversalement, anguleuses à leur extrémité libre. Le diamètre longitudinal est double de la longueur du bord cardinal; crochets submédians, nettement recourbés et bien définis. La surface est ornée de côtes rayonnantes obliques, groupées deux à deux, chaque groupe séparé par un large sillon. Ces côtes et les sillons sont traversés par des stries concentriques nombreuses, fines et ondulées, rendant les côtes légèrement noneuses.

Dimensions. - Longueur, 18 $8^{\mathrm{mm}}$; longueur du bor d cardinal, $9^{\mathrm{mm}}$; hauteur, $14^{\mathrm{mm}}$; ćpaisseur, $7^{\mathrm{mm}} \cdot-J$. Fraipont in de Koninck ${ }^{(1)}$.

Gisencut. - Régny.

Explication des figures. PJ. X, fig. 9. Valve ganche.

GEXRE ENTOLIUM, MEEK, 1864.

Entolium Witryi, DE Konisck, 1885.

!'. II. 1i.....

Corracteres spécifiques. - Coquille de taille moyenne, ovale, un peu plus haute que longue, équilatirile, très faiblement convexe; les oreillettes semblables ontre elles, aliformes, séparées du corps de la coquille par un sillon peu frofond; crochels nédians. La surface est ornée d'un grand nombre de fine cótes concentriques de différentes épaisseurs. On aperçoit de plus, à l'aidre d'mus brome loupe, les l'aces de fine's côtes rayomnantes, irrégulièrement distriburas it la surfare el pureeptibles principalement vers les bords.

Dimresionss. - Longuteur, 35 ${ }^{\mathrm{mm}}$; hauleur, 38 ${ }^{\mathrm{mm}}$. - De Koninck (2).

fisirmunt. - Siguret.

Eapliention des figures. - IJ, IV, fig. 5. Empreinte interne d'une valve.

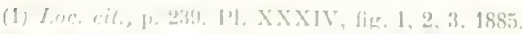

(1) 


\title{
CLLASE DES BRIGIII)PODES
}

\author{
ORDRE DES ARTIGULES
}

\author{
FAMILLE DES PRODUCTIDES
}

Genre Productus, Sowerby, 1812.

I. P. giganteus, IV. MARTin, 1809 .

Pl. XII, fig. 1, 2, 3, 4, 5.

Pl. XIII, fig. $1,2$.

Pl. XIV, fig. 6, 7 .

P. XV, fig. 1.

Historique. - Cette espèce a été figurée pour lin promione fois par Mnetin Lister, en 1688, duns un atlas célebro intitulé: "Historice sive synopssis methodica conchyliorum. - Appendix ad librum III de Conchytis sioe lapidibus, cui quandam similitudinem cum conchis murinis habeune ". Pl. CDLXV, fig. 25, $b$, et pl. CDLXVII, fig. 26, $b$.

Lister a figuré deux valves ventrales de dimensions différentes. La première a $135^{\mathrm{mm}}$ de long et $230^{\mathrm{mm}}$ de large; la deuxieme, appartenant a un autre individu, a $145^{\mathrm{mm}}$ de long et $165^{\mathrm{mm}}$ de lange. Ce sont les deux premières figures parues de eette grande espèce; olles ne sunt point arecompagnéses de texte.

Le véritable créateur de l'espèce est WVillam Martin. Cet anteur a donné dans son ouvrage intitulé Petrafacta derbiensia..., onvarge resté célibre malgré lat date ancienne de sa publication, les diagnoses de denx sprécimens de rethe

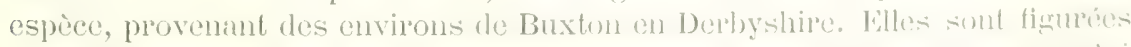
pl. XV, fig. 1, sous lo nom de Anomites giganteus, et pl. XVI, tig. 2, sums colui

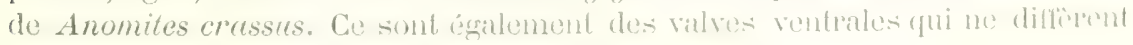


que par les dimensions de celles de Martin Lister; ainsi la première a $120^{\mathrm{mm}}$ de long et $180^{\mathrm{mm}}$ de large, la seconde a $125^{\mathrm{mm}}$ de long sur $120^{\mathrm{mm}}$ de large. Voici du reste les diagnoses qui les accompagnent:

" Pl. XV, fig. 1. Conchyliolithus Anomites (giganteus) transversim oblongus, ad latera dilatatus, longitudinaliter sulcatus striatusque: striis scabriusculis irregularibus, valva altera gibbosa. ")

"Pl. XVI, fig. 2. Conchyliolithus Anomites (crassus) subrotundus, longitudinaliter sulcatus striatusque: striis tuberculatis, valoa altera subglobosa."

Depuis W. Martin, cette classique espèce du terrain carbonifère a été décrite tantôt sous son rrai nom spécifique, tantôt sous des noms divers, considérés aujourd'hui comme de simples synonymes (1) par plus de vingt auteurs différents, depuis Sotrerby qui en a déerit un spécimen sous le nom de Productus scoticus en 1812, puis d'autres exemplaires sous ceux de $P$. giganteus, $P$. personatus, $P$. hemisphericus, jusqu'à Davidson (1861).

Il nous semble inutile de domner une nouvelle diagnose pour l'espèce du Plateau Central, et nous préférons placer sous les yeux du lecteur, comme nous le ferons du reste pour la plupart des Brachiopodes, la diagnose si claire et un même temps si brève du célèbre auteur anglais.

Curcuties spécifiques. - Coquille grande, variant de forme et de striation suivant l'âge et l'échantillon, quelque peu transversalement ovale; ligne cardinale droite, depassant la largeur de la coquille, et parfois (quoique moins communément) possédant une aréa cardinale étroite et rudimentaire dans la plus grande valve.

Valve ventrale fortement épaissie, spécialement ver's le milieu, mais moins ver's le crochet et vers le bord; plus ou moins gibbeuse et souvent très dilatée aux oreillettes qui sont enronlées semi-evlindriquement et plus ou moins délimitées. Crochet modérément développé, incurvé et dépassant de son extrémité sculement la liqne ardinalo. Extérieur uniformément convexe ou plus ou moins profondément ef irrofulierement sillomé, la surface étant couverte d'un nombre consiglénble de stries flexuenses, longitudinales, qui varient suivant l'âges et le spécimen, trois on davantage occupant habituellement la largeur d'une ligne (2mm256), vers lo milieu du bord des valves. Les stries sont aussi prifois contluentes, bifurquées, ou bien disparaissant et apparaissant soudainement, of croissint en nombre vers le bord des valves. Elles sont également contiguës ou séparérs par des sillons ou des intervalles de largeur variable. A des intervalles irremlines, les stries domnent issue it un petit nombre d'rpinus arences, cylindriques el courtes qui sont plus nombreuses sur les omeilledlus des valves.

Vabredorsilo ninee, concave, frousant la courbure de la ventrale et semblahlemront sculphie, la portion viscerale et les oreillettes étant aussi quelquefois $1 \times 57$.

11) Voir font let synonymes, In. Koninck. Monograplie des genres Productus et Chonetes, p. 34. Liègo. 


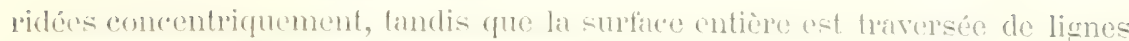
d'aceroissoment fines of concentrigues.

Appareil interne. - Dans l'intéricur de la ventrale, les empreintes musculaires du diducteur sont situées immédiatement au-dessous et en dehors de celles des adducteme; et, plus bas, vers le centre de la valve, il existe deux profondes dépressions subspirales. Dans l'intérieur de la valve dorsale, sous le processus cardinal trilobé et en forme de $\mathrm{T}$, se troure une côte longitudinale étroite qui se prolonge jusqu’a la muitié environ de la longueur de la valve, et de ehaque colé sont situées les impressions ramifies et dendritiques du muscle adductrur, tandis que en dehors et au front de celles-ci sont situées les deux " impressions réniformés». La surface interne est couverte d'imnombrables aspérités, et l'épaisseur de la coquille traversée par des canaux. Les dinensions sont variables. - Davidson (1).

Suivent les dimensions de spécimens anglais que nous n'avons pas à reproduire.

Gisements. - L'Ardoisière, près de Vichy, vallee du Sichon. Regny (Ecole des Mines et collection Jourdan).

Explication des figures. - PI. XII, fig. 1, a. Empreinte de la surface externe de la dorsale.

b. Empreinte de la surface interne de la ventrale.

c. Empreinte de la portion inférieure du muscle diducteur.

d. Fragment de la valve ventrale aree ses ornements extérieurs.

Fig. 2. Belle empreinte de la dorsale d'un autre spécimen : $a$. Trous des épines.

Fig. 3. Empreinte du bord cardinal d'une dorsale montrant les trous des épines sur les oreillettes.

Fig. 4. Id. Autre spécimen; le cỏté frontal de cette empreinte est représenté pl. XIII, fig. 1.

Fig. 5 et 6 . Moulage d'une cavité interne de $P$. giganteus de l'Ardoisière.

Fig. 5. Empreinte interne du crochet et des parties avoisinantes, vue du cóté de la face dorsale.

b. c. Empreintes des muscles adducteurs.

d. Septum médian.

a. Retour de la coquille.

Fig. 6. La même, vue du côté de la face ventrale.

a. b. Empreintes des muscles adducteurs.

c. d. Empreintes des muscles diducteurs.

Nota. - La fig. 6 est représentée le crochet en haut, tandis que la fig. 5 a été renversée par mégarde, de sorte que le crochet est en bas et le retour de la coquille brisée a en haut.

Pl. XIII, fig. 1. Empreinte de la dorsale vue du côté frontal. Le bord cardinal du méme spécimen est représenté fig. $4, \mathrm{pl}$. XII.

Fig. 2. Autre spécimen. Empreinte d'une dorsale.

(1) British Carboniferous Brachiopode. Part. V, p. 141. 1'l, XXXvil, fig. 1-4; pl. XXXVIll, lig. 1; pl. XXXIX, fig. 1-5; pl. XL, fig. 1-3. 
Pl. XIV, fig. 6. Moule interne de la ventrale.

$\alpha$. Empreinte du muscle dislucteur.

b. Empreinte du muscle adducteur.

Fig. 7. Le même échantillon, vu de dos, offrant l'empreinte des aspérités de la face interne de la même valve.

PI. XV, fig. 1. Empreinte du bord cardinal d'une valve dorsale.

a. Empreinte de pyyidium do Griffichides Eichwaldi, Fischer. Sur cet échantillon se roit nettement l'empreinte de l'aréa droite et très étroite de la ventrale, contiguë dans toute sa longueur an bord rectiligne de la dorsale.

Obsercution. - Le Productus giganteus n'existe qu'i l'Ardoisière. Bien que nous ayons recueilli des milliers de spécimens de fossiles divers a Régny et dans les autres gisements du Plateau Central, de l'ige de Dinant, nous n'y avons jamais découvert ce Productus. Toutefois des Productus gigunteus de provenance de Régny ont été signalés par M. de Koninck dans les collections de l'Ecole des Mines de Paris et dans la collection Jourdan at Lyon. Voir á ce sujet: Réunion extraordinaire de la Société géologique de France à Roanne. 1873. Page 451 (Compterendu de la course ficite ì Régny).

\section{Productus margaritaceus, Pinllips, 1836 (1).}

Sim figmin.

Carccteres spécifques. - Coquille mince, presque circulaire ou transversalement somi-rireulaire, rarement plus longue que large.

Ligne cardinale plutôt moindre que la largeur de la eoquille.

Valve ventrale rógulierement convexe, sans sinus. Crochet exigu, recourbe. Oreilletles étroites et à peine discernables de la convexité générale de: lin valve. Surtace ornée de nombreuses côtes épaisses, obtusúment arrondies ou aplaties, siparées par des sillons peu profonds. Les cotes se bifurquent sommul pris du burd, landis que toute la surface est traversée par des stries imbrigures, concentrifues at lris serrées, qui, à intervalles produisent des interruptions lioliaréns fortement marcquées ou lignes d'aceroissement.

Les inpues sont pen nomburenses, mais il y en a 4 ou 5 fortes qui saillent

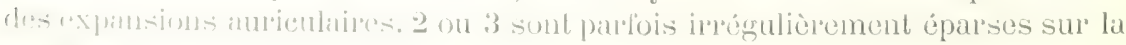
-1110litice.

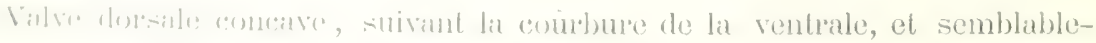

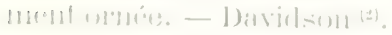

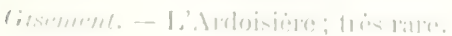

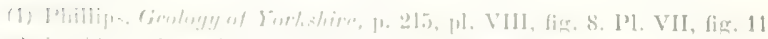

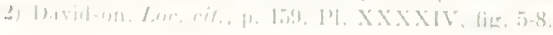


III. Productus Gora, D'Urbigny, 1842.

\author{
Pl. VII, fig. 7,8 . \\ Pl. X, fig. 1. \\ Pl. XI, fig. 1, 2. \\ Pl. XIV, tis. 1, 2, 3.
}

Historique. - D'apres M. de lioninck, le Productus Corre aurait été figuré au siecle dernicr par Walch sous le nom étrange de "Das schecurtze Schiff"). Voir" Naturforscher Stück XIV, pl. 1, fig. 2, p. 33; nous n'avons pu nous procurer cet ouvrage.

L'espèce a été en réalité crééc par Alcide d'Orbigny diuns Puléontologie du coyage dans l'Amérique méridionale, pl. V, fig. 8, 9, 10, 18.12, sous le nom cidessus. Presque en même temps l'espèce était déconverte et décrite par de Koninck sous le nom de Productus comoides (1813), sous celui de Producte corrugatr par N'Coy (18.4.), et enfin sous les noms de Productus Neffedieci et Productus tenuistriatus par de Verneuil (1845).

Voici la diagnose de d'Orbigny :

"Testì subrotundati, elevati, gibbosi, longitudinaliter tenuiterrye sulcati, sulcis exiguis, cequalibus ornati; calvi superiore inflate, producti, tuberculis raris, irregulariter sparcis, armati, curdine recto, lateraliter auriculato.,

"Localités. - Au-dessus de Patapani, village appartenant à l'une des îles du las: de Titicaca, sur le plateau bolivien, et à Yarbichambi. »

Comme pour le Pr. giganteus, en raison de la grande extension et de la beauté de cette coquille, nous croyons devoir reproduire la diagnose de Davidsoll.

Caracteres spécifiques. - Coquille mince, longitudinalement ovale on semicylindrique, habituellement plus longue que large. Ligne cardinale environ aussi longue que la largeur de la coquille.

Valve ventrale gibbeuse, uniformément convexe, on légèroment aplatie le long de la partie médiane. Crochet large et recourbé. Oreilles exiguës et généralement traversées par quatre ou cinc plis ondulés ou larges rides qui s'étendent à quelque distance au-dessus des portions latérales des valvos. Surfuce couverte de stries nombreuses, longitudinales, droites ou légèrement flexueuses, étroites, semblables ì des fils et rondes, avee des sillons vu des interespaces de largeur plutôt moindre. Des stries plus fines sont souvent intercalées entre les plus larges (caractère signalé pour la première fois dans la diagnose de M'Coy). Les cotes sont aussi régulièrement traversées par des 
lignes concentriques exiguës. Un petit nombre d'épines sont dispersées avec économie sur la surfuce, mais plus nombreuses sur les oreillettes.

Valve dorsale épousant la courbure de la ventrale et similairement ornée.

Dimensions variables. - Quelques échantillous ont atteint $21 / 2$ pouces de Iongueur et $31 / 2$ de largeur. - Davidson (1).

Gisements. - Régny, Saint-Germain-Laval, Montmain, l'Ardoisière, Thizy.

Explication des figures. - Pl. VII, fig. 7. Empreinte de la surface externe d'une valve dorsale d'un spécimen de petite taille provenant de Régny.

Fig. 8. Autre spécimen. Cet échantillon a été figuré parce qu'il offre nettement ce caractère des stries altemativement plus grosses et plus fines sur lequel a particulièrement insisté $\mathbf{M}^{\prime} \mathrm{Coy}^{(2)}$. Ce spúcimen provient également de Régnny.

Pl. X, fig. 1. Spécimen de Saint-Germain-Laval; empreinte externe d'une dorsale.

Pl. XI, fig. 1. Moule externe d'une dorsale. Petit spécimen de Montmain.

Fig. 2. Id. Mème localité.

Pl. XIV, fig. 1. Magnifique spécimen d'une empreinte de la valve dorsale vue du cóté de Ia Charnière. - L'Ardoisière,

Fig. 2. La même valve vue du còté frontal.

Cet échantillon est assez bien conservé pour permettre de compter les stries : à $20 \mathrm{~mm}$ du croshet, dans un espace de $20^{\mathrm{mm}}$ pris au milieu de la corfuille, on peut compter 45 stries, tandis que dans le Pr. giganteus on n'en compte que 35.

Fig. 3. Limpreinte externe d'une valve ventrale d'un autre spécimen. - L'Ardoisière.

Notu. - Ce dernier gisement seul renferme les gros échantillons de cette espèce.

IV. Productus undatus, DEFrance, $1826^{(3)}$.

Nim firmuti.

Crurretêres spécifiques. - Coquille un peu suborbieulaire ou légèrement fransverse. Liene cardinate un pen moindre que la largeur de la eoquille.

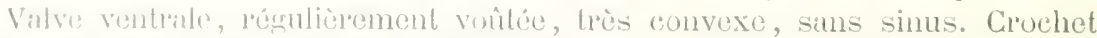
minre, anondi, incurvé, ne dépassant pas de beaucoup la ligne cardinale. Orrillettes petites. Surfices couverte de plis ou ridos, nombreux, irréguliers on intermmpes, subparalleder, onduleux el coneentriques, qui augmentent de

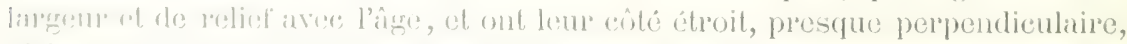

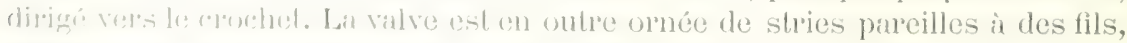

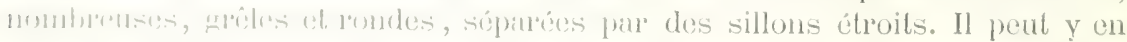

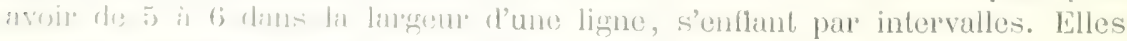

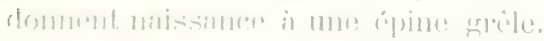

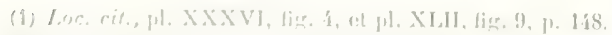

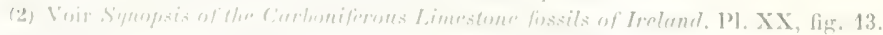

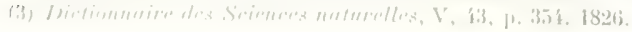


Valve dorsale concave, suivant la courbure de l'autre ralve, et ornée exactement de même.

Dimensions variables. - Davidson (1).

Gisement. - L'Ardoisière; rare.

V. Productus semireticulatus, MARTiN, 1809.

PI. I, fig. 1, 2, 3, 4, 13 a.

Pl. I11, fig. 4, 5, 6.

Pl. XI, fig. 6.

Pl. Xlll, fig. 8.

Historique. - L. de Koninck a retrouvé cette espèce classique figurée dans de vieux antemrs. La première figure est domnée par Du Costa, sous la rubrique: "Polyginglymon or multarticulate anomia, fossil, of which the living species, still remains undiscovered ", dans "Elements of Conchology ", page 293 . Pl. VI, fig. 10 .

La seconde figure, intituléo Gryphita, est due à Baumer, Hist. nat. regni mineral, 1780, p. 102. Pl. IT, fig. 29. L'espèce a dé créée par IV. Martin, sous le nom de "Anomites semireticulatus ». (Petref. derb., p. 7. Pl. XXXI, fig. 1 et 2 , et pl. XXXIII, fig. 4.)

"Semiorbiculatus, calcù planà decussatem. striatè, alterì gibbosù longitu"dinaliter striatì, natem versus transwersim rugosì."

Une autre espèce de Martin, figurée pl. XXII, fig. 1, 2, 3, sous le nom de "Anomites productus ", a été reconnue pour être une simple variété de la première.

Depuis IV. Martin, le Pr. semireticulatus a été décrit, comme le Pr. gigantens, par de très nombreux auteurs et sous plus de dix noms différents. On pourra consulter, dans la Monographie de L. de Koninek, l'interminable synonymie de cette espèce classique. Nous nous contenterons eneore, commenoms l'avons déjà fait, de reproduire la dernière diagnose due ì Davidson, qui a fixé les caractères définitifs de cette espèce et de ses variétés.

Caractères spéciffues. - De forme très variable, transwersalement ovinle, subeylindrique ou allongée. Ligne cardinale aussi longue ou it peine plus conte que la largeur de la coquille.

Valve ventrale gibbeuse et variablement voutéc avee un sinus très peu profond. Oreillettes modérément développeses. Crochet rouge, recourbé, habituellement convert de pides onduleuses, irroulierres at ancentripues, plus larges et plus profondes sur les oreillettes, pendant que lar surlite cuntione de

(1) Loc, cit, p. 161. P1. XXX15, fig. 7-13. 
la coquille est ornée de maintes stries rayonnantes, longitudinales, arrondies, dont le nombre s'accroît vers le bord par bifurcation et par inter'calation, et desquelles émanent à des distances variables des épines tubulaires de longueur quelquefois considérable.

Valve dorsale modérément concave, suivant la courbure de la valve opposée et semblablement sculptée.

Dimensions variables; quelques spécimens atteignent jusqu'à 3 pouces de long sur 4 de large. - Davidson (').

Productus Martini et Pr. concinnus. - Cette diagnose est suivie d'observations critiques relatives aux variétés de cette espèce. Nous en retiendrons seulement les points qui visent le $P r$. Martini et le $P r$. concinnus de Sowerby, parce que ce dernicr ust la forme dominante à Régny et à l'Ardoisière : “Je suis également tout prêt à admettre qu'il y a une différence sensible entre " le $P r$. semireticulatus Martin et le Pr. Martini Sowerby, au point de vue » de la forme, qui est simplement circulaire dans le premier et génieulée dans ) le sceond; mais ees deux formes extrềnes sont intimement reliées entre » elles par d'insensibles nuances, it tel point que très souvent il serait impos) sible de déterminer à laquelle des deux espèces appartiennent certains » spécinens. " Et plus loin : "Productus concinnus Sowerby n'est évidem" ment autre chose (qu'une plus petite forme ou synonyme de la même."

Gisements. - Le Productus semireticulatus traverse tout le carbonifère marin : Domaine des lioches, près Savigny-Poil-Fol, et la Varville (Morvan); Régny, Montmain, l'Ardoisière. Dans ces trois derniers gisements, c'est la forme concinnus qui domine.

Explicution des figures. - Pl. I, fig. 1. Empreinte externe d'une valve ventrale. La Varville.

Notu. - Comme dans tous les ćchantillons du Morvan, l'empreinte est ocrouse et se détache vivement sur le fond de conleur mastic de la roche, qui est un schiste décalcifié.

Fig. 2. Contre-empreinte du bord frontal d'une valve ventrale. - Mème gisement.

Fir. 3. Autre fragment. - Domaine des Roches.

lirg. 2. Linzreinte de valve dorsale. - Domaine des Roches.

Fir. 13 \&. Limpreinte interne d'me valve ventrale. - La Varville.

IP. VII, figg. 4. Valve ventrale, vue du côté du crochet et brisée à la partie viscérale. - Rimens.

Cat echantillon rappelle, par sa courbure, la fig. 9 de la pl. XLIII, de Daridson, du Pr. rencrinums.

Fig. 5. Valve dorsatle de lá même variéte. - Régny.

Firg. 15. Valve ventrale, colorée en rose - ce qui est fréquent á Régny - do la même varibtis conciunus.

[N. XI, lir. 6. Eumpreinte externe de dorsale de Pr. semireticuletus, var. concinnus. Mentmitin.

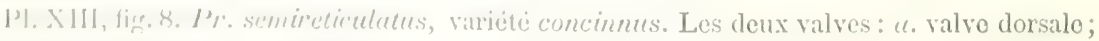
h. valve ventrater - L'Ardoisiere.

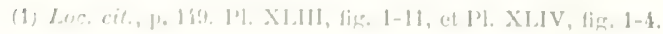


Obser'tations sur le Productus semireticulatus du Morten. - Dans sa note sur le terrain carbonifère du Morvan, insérće dans les Bulletins de Licadémie royule de Betgique, $3^{\circ}$ série, t. $1 \mathrm{X}, n^{\circ} 5,1885$, L. de Koninck, a qui j'arais soumis un grand nombre de fossiles recueillis par moi dans le Morvan, avait rapporté le Pr. semireticuletus de la Varville et du domaine des Roches an $\mathrm{P} r$. tortilis $\mathrm{Nl}^{\prime} \mathrm{Coy}$, toutefois avec hesitation. Il maintenait ainsi, a cette époque, l'espèce de l'auteur irlandais. Cependant, en 1847, L. de Koninck n'acceptait pas le Pr. tor'tilis et l'attribuait, avec doute il est vrai, an Pr. semireticulutus (1).

En 1854, M'Coy, revenant sur l'espèce crééc dix ans auparatvant par lui, crut deroir la maintenir (2).

La mème année, de Semenow (3) l'assimilait au Pr. undatus Defrance.

En 1861, Daridson, qui s'était lirré sur place à un examen approfondi de lia collection de Richard Griffith, autrefois décrite par M'Coy, et qui avait étudié le spécimen origrinal, confirma lattribution faite par de Semenow du Pr. tortilis N'Coy au Pr. undatus Dofrance (4).

Quels que soient les doutes qui peuvent planer encore sur la réalité de l'espèce irlandaise, créée et maintenue par M'Coy, il est facile de s'assurer, it l'aide d'un examen attentif, que les spécimens du Morvan n'ont rien de commun ni avec le $P^{\prime}$. tortilis, ni arec le Pr. undatus. Il suffit pour cela de lire les diagnoses de ces denx espèces. Seuls les caractères de $P r$ semireticulatus Iartin s'appliquent exactement aux Productus que j'ai recueillis it la Varville et au domaine des Roches.

TI. Productus pustulosus, Phillips, 1836.

Pl. VII, fig. 1, 2, 3.

Pl. IX, fig. 1 .

Pl. X, fig. 2.

Pl. XII, fig. 7 .

Pl. XIII, fig. 3, 4.

Historique. - Cette espèce, créée par Phillips, avait été figurée déjà dans l'ouvrage de Chemnitz "Martini's neues syst. conch. cabinet. Vol. VII, p. 301. Pl. LXIII, fig. 605 et 606 , et vol. VIII, p. 69. 1784 m. Ces figures, tris bonnes du reste, sont accompagnées de la diagnose suivante: "Pyxis transver'sim striata, testa planoconoexa, in medietate valuule convexe ab apice ad marginem insigniter sinuata et depressa, transverse arcuatim striate, striis latis lavibus, intersticiis seu sulcis striarum transoersarum dense crenulatis : - fossilis $"$.

Phillips créa l'espèce sous le nom de Producta pustulosa ${ }^{(5)}$ à latquelle on

(1) Voir Monograplie des genres Productus et Chonetes, p. 2:38.

(2) British Paleozoic rocks and fossils. Sedgwick and M Coy, 1854, 1. 4it.

(3) Die Fossilien des Schlesischen Kohlentiallis, 185', ए. 66.

(4) Loc. cit., Part. V, p. 161. P., XXXIV, fig. 13.

(5) Geology of Yorlishire. Vol. II, p. 216. Pl. VII, fig. 15. 
réunit aujourd'hui son Producta ovalis ${ }^{(1)}$, que l'on ne peut séparer de l'espèce ci-dessus. Les diagnoses sont assez courtes pour que nous les reproduisions.

Producta pustulosa. - Coquille carrée-arrondie, gibbeuse. Oreilles angulaires, sillonnées, privées d'épines. Rides transverses onduleuses, offrant de nombreuses épines éparpillées, qui se pressent de plus en plus en s’approchant des bords.

Producta ovalis. - Coquille oblongue; sinus dorsal léger avec des sillons concentriques faibles et de nombreux points spinuleux.

Comme pour les autres Brachiopodes, nous allons reproduire la diagnose de Davidson, qui est la dernière en date.

Caractères spécifques, - Coquille mince, carrée-arrondie, plus large que longue, rarement ovale et plus longue que large. Ligne cardinale plus courte que la plus grande largeur de la eoquille.

Váve ventrale régulièrement arrquée et gibbeuse, divisće longitudinalement par un sinus do dimensions variables. Crochet modérément développé et recourbé, ne dépassant guère la ligne cardinale de son extrémité atténuée. Oreilles larges presque rectangulaires et aplaties. Bord offrant un léger sinus au front. Ornementation extéricure variant quelque peu d'apparence dans les diffirents spécimens, mais habituellement constituée par des rides onduleuses, nombreuser, continues ou interompues dans leur parcours transversal. De nombreuses pustules ou tubercules allongés, portant des épines grêles, tubulaires, en quinconce ou en séries irrégulices sur toute la surface.

Valve dorsale légèrement coneave avee une petite élévation médiane ou bourrelet; de nombrenses rides transverses, des fossettes tubereuleuses et de courtes épines couvent la surfice. - Davidson ${ }^{(2)}$.

Givenents. - Reigny, Nironde, Saint-Germain-Laval, l'Ardoisière.

Exprliontion des figures. - 1'1. VIl, fig. 1. Valve ventrale. - Régny.

Fïg. ¿. Id. Mime gisement.

Fìg. 3. Valve dorsale. Bel échantillon du mème gisement.

P. IX, firg. 1. Fragment de ventrale. - Néronde.

[']. X, fir. 2. Noule interne d'une valve dorsale. - Saint-Germain-Laval.

[P]. XII, ficr. 7. a. P’ortion de valve ventrale. - L'Ardoisière.

b. Valve ventrale montrant nettement le sinus.

c. Empreinte de la valve dorsale avec le bourrelet encore indiqué, malgré l'érasement.

12. XIII, figg. 3. Valve vontrale. Tres belle empreinte, bien que les rides concentriques

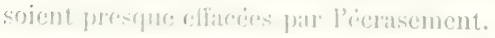

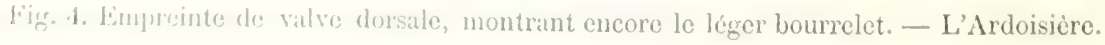

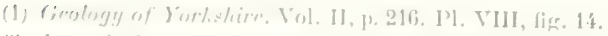

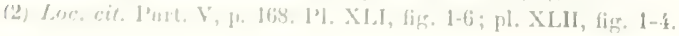


VII. Productus punctatus, Martin, 1809.

Pl. Y'II, fig. 9.

Pl. XI, fig. 3, 4.

Pl. XII, fig. 8.

Pl. XIT, fig. 4, 5.

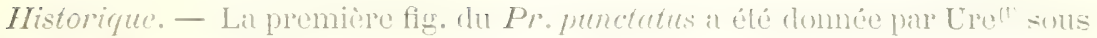
le nom de Conche pilosce.

Martin ${ }^{(2)}$ est le véritable créatem de l'espece, mais il a en le tort de confondre sous le mime nom le Productus fimbriatus de Sowerly, espèce étroitement allice à celle-ci, mais facile à distinguer.

Diagnose de Murtin. - "Rotundatus, transwersim striatus; stritis remutis; intersticiis puncutis, calou altere gibbosa, cardine subcurto. "

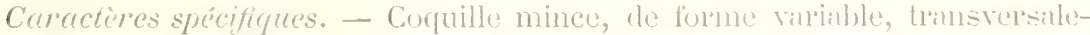
ment carrée-arrondio, ou légèrentent allongéc-oviale. Ligne carelinale plus courte que la largene de la coquille.

Valve ventrale conrexe, un freu gibbense, aree un sinus faible commenrant ¿̀ une courte distance de l'extrémité du crochet, et s'chlendant jusiru'an front. Crochet recourbé dépassant le bord cardinal de son extrémité atténtuce. Oreilles aplaties, mais vaguement délinitées. Surfice couverte de nombrensen bandes ou côtus concentriques, subrégulières, qui croissent en nomber at en largeur on s'éloignant de l'oxtrémité du crochet; mais dans les corpuiles adultes, elles se rapprochent et se resserrent pros du bord frontal. Cus bandes (dans la valve ventrale) sont legèrement souleveses vers leur horrl inférienr at séparécs d'une manière abrupte l'une de l'autre par mu espace lisise, itroit, après lequel il existe une rangée assez régulière de tubereules allongés on d'épines tubulaires, grềles et brillantes, et de nouveau, au-dessons de celles-ci, l'espace restant est rempli d’épines ténues, éparpillées, formant des parquets serrés, se recouvrant les umes les autres et conchés sur la valve.

Valve dorsale modérément concave, aree un léger bourledet, of ornéce comme la rentrale, mais les bandes sont légèrement concaves. - Davidson ${ }^{(3)}$.

Gisements. - Régny, Montmain, Thizy, l'Ardoisière.

Explication des figures. - Pl. VII, fig. 9. Empreinte d'une valve dorsule. - licgny.

Pl. XI, fig. 3. Empreinte très effacé, mais néamoins recomaissable; "u, trous des ipines.

- Montmain.

(1) The History of Rutherglen and East-Isilbride by Dacid Lre, 1793, 11. 316. 1'1. XV. 1ig. 7

(2) Petref. Derb., p. 8. Pl. XXXv11, fie. bi (fig. 7 et 8 exclusis).

(3) Loc. cit. Part. Y, 17. 172. 1'1. XLIV, fig. 9-16. 
Fig. 4. Autre fragment de valve. - Thizy.

Pl. XII, fig. 8. Ventrale. - L'Ardoisière.

Pl. XIV, fig. 4. Empreinte externe de dorsale, très beau spécimen; on voit á gauche de la coquille l'empreinte d'un paquet d'épines aussi fines que des cheveux, ainsi que l'avait observé il y a un siècle David Ure, et dont la longueur atteint $16^{\mathrm{mm}}$. - L'Ardoisière.

Fig. 5. Empreinte externe de dorsale. - L'Ardoisière.

VIII. Productus elegans, N'Coy, 1844.

Cette élégante espèce a été créée par M’Coy en 1844. Voici la diagnose qu'il domne dans le Synopsis :

"Coquille formant les $3 / 4$ d'un cercle, convexe; neuf ou dix sillons concentriques; lo bord rostral des larges sillons avec deux ou trois rangées d'incisions courtes, profondes et onduleuses; lo bord frontal arec trois ou quatre rangées concentriques de piqûres placées en quinconce. Crochet exigu. Ligne cardinale droite. Cette jolio petite coquille ressemble plus au jeune de Productus fimbriatus Soverby, qu'ì toute autre espèce du même geme. Elle est cependant beaucoup plus déprimée. Le crochet est large et saillant dans cette dernière coquille mais très petit dans la nôtre, et enfin les côtes ou plis sont beaucoup plus nombreux dans la présente espèce et poncturés sur le bord fronLal, au lieu du bord rostral, comme dans le Pr. fimbriatus. Les oreilles du Pr. clegrens sont très aplaties, la valve ventrale semblable à la dorsale mais plus aplatic.

"Dimensions. - Longueu1, 5 lignes; largeur, 6 lignes(1). ”

Obsercations. - Lál création de cette espèce n'a pas rencontré l'assentiment unanimo des auteurs. De Koninck, dans la Monographie des Productus, dit en dépivant lo Pr. punctatus: " M'Coy en décrit deux jeunes individus sous les noms ale $P^{\prime} r$. elegrens ot de Pr. laciniatus. ")

1)ilvidarnn l'este dans l'indécision. "Le professeur M'Coy, dit-il, constate dans son wuve British Paleoroic fossils que cette espèce est intermédiaire dans lons ses cantuteres, entre Pr.punctutus et Pr. fimbriatus, mais constitue une apree parfinitoment distincte des deux. Elle s'aceorde avee la première par les langes nombruses et non uniques de spinules sur chaque bande concen-

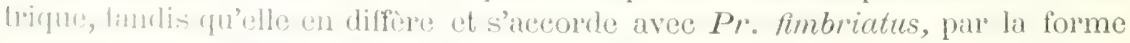
allungée-rante, l'absence de sillon médian el la très grande gibbosité de la valve vantrate. lille end difore cependant par sa taille exiguë, le plus grand nombre de bundes concentriques dans un uspace donné à la même distance du crochet,

(1) Synupzis of the Ciurb. Limest. of Iret., 1. 108. PI. XVIII, fig. 13, ot British Paleoz. fossils., M'Coy 11. 461), 1'l. 111 (H), 1iz. \&. 
et par la présence de plusieurs rangs de fines picqures, au lieu d'une rongée unique de tubereules allongés sur chaque bande. "

Suit la mention do nombreuses localités où $P_{r}$. punctatus et $P_{r}$. elegrans se rencontrent simulturément.

Nous n'avons point osé nous prononeer sur l'attribution de l'un on de l'nutre de nos assce nombreux spécimens du Platenu Central, au Pr. eleguns, car toutes nos empreintes sont aplaties, le plus souvent incompletes, comme on peut s'en assurer en examinant nos fignues; peut-ètre le spécimen de Pégny, pl. VIII, fig. 9, et celui de Thizy, pl. XI, fig. 4, pourraicnt-ils etre rapportés à l'espèce de M'Coy? Dans ce cas, le Pr. elegans serait plus abondant que le $P r$ punctatus dans le Pliteau Central, où nous n’avons jamais reneontró les grands spécimens de cette dernière espèce.

IX. Productus scabriculus, MARTIN, 1809.

Non figuré.

Caractères spécifiques. - Coquille à pourtour earré-arpondi, généralement plus large que longue, bords latéraux subparalleles; arrondie ou lixgèrement entaillée au front. Ligne cardinale plus conrte que la plus grande largeur de la coquille. Valve ventrale convere avec une dépression médiane laroge, peu profonde; crochet incurvé, ne dépassant pas la ligne cardinale si ce n'ost par son extrémité atténuée; oreilles petites, aplaties. Surface converte de nombrenses stries subrégulières, s'enflant par intervalles en tubereules oblongs, disposés parfois en quinconce et desquels s'élèvent des épines minees, incur'vées, de moins d'un demi-pouce de longueur; de faibles rides concentriques traversent quelquefois la valve et sont surtout marquées sur les oreilles. Valve dorsale concave, près du bord, avee une légère élévation médiane commençant non loin du front; surface couverte de nombreuses rides concentriques, de tubercules creux, courts, déprimés, et d'épines minces. Dimensions variables. Davidson (1).

Gisements. - Domaine des Roches, La Varville, Sizuret.

(1) Loc. cit., p. 169. PI. XLII, fig. 5-8. 1861. 
X. Productus fimbriatus, J. DE C. Sowerby, 1824 .

Pl. XI, fị. 5.

Pl. Xili, fig. 5, 6, 7.

Historique. - Déjà figurée par IV. Martin, sous le nom de Anomites punctatus (1), l'espèce a été distinguée et créée par Sowerby ${ }^{(2)}$ : "Coquille subhémisphérique, muni de 6 ou 8 sillons concentriques, crénelés et épineux. Crochet peu saillant ${ }^{(3)}$. ")

Caractères spécifiques. - Longitudinalement ovale ou ovol̈de; ligne cardinale un peu plus comrte que la largeur de la coquille.

Valve ventrale uniformément convexe, gibbeuse, et grandement arquée en profil. Crochet très recourbé, dépassant la ligne cardinale de son extrémité atténuée. Oreilles petites et seulement légèrement marquées. Surface régulièrement traversée par de $110 \mathrm{mb}$ reuses bandes ou sillons concentriques, saillants ou subrégulier's, aree des esplaces interposés aplatis. Une rangée de tubercules allongés, convint chaque bande, lesquelles projettent des épines longues, tubulaires, cylindriques.

Valve dorsale presque plate, ou modérément concave, traversce par de nombreuses bandes concentriques avee des intervalles concaves. Une rangée d'épines courtes, pressées l'une contre l'autre, naît de chaque bande. Davidison (i).

Obsercationn. - Cette espèce n'atteint pas les proportions du Pr. punctatus, est moins viniable dans sil forme, n'a point de sinus, et se distingue particulièrement pall la rangée simple d'épines eylindriques qui s'élève de chaque rangíe de tubercules allongés, comme il est ei-dessus déerit.

Gisement. - Propieres? L'Ardoisiere; Thizy.

Eirpliation des figures. - 11. XI, fig. 5. Valvo ventrale. - Propières. Fragment d'une tré mansaise conservation, comme tous ceux, du reste, que j'ai découverts dans les schistes do wetre: Incalitit.

P'. XIll, fir. 5. Eimpreinte interne d'une valve dorsale. - L'Ardoisiere.

lïg. 1;. bun minte externe de lat meme valve.

liğ 7. Limpreinte externe do la ventrale. - Même localité.

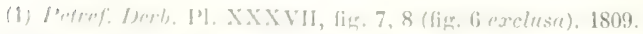

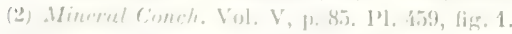

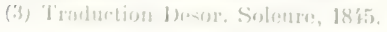

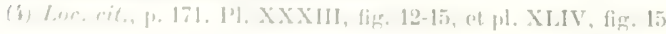


XI. Productus aculeatus, MARTix, 1812.

Non fignur.

Espèce décrite ef figuréc pour la première tois par IV. Martin, sons le nom de Conchyliolithus (Anomites) aculeatus.

Voici la diagnose qu'il en a domnée et les observations à la suite qui la complètent :

"Subrotundus, valvi concavâ leet, alteri gibbosì retrorsum aculeatì: "aculeis raris adpressis brecissimis, cardine subcurto.

"Observations. - Coquille imperforéc, avee l'une des ralres concares, l'au") tre convexe et gibbeuse. Charnière droite, mais moins allongée que dans ") la plupart des autres Anomites de la mime division (Syst. G. b. Anomite bb). "La valve convexe pleine de piquants. Lés épines sont en petit nombre, épar") pillées, très fines, courtes, appliquées contre la surface, mais dirigeant leurs " pointes en arrière, c'est-à-dire vers le crochet. Le crochet étroit et recourbé." - Martin (1).

Gisement. - L'Ardoisière; très rare (20 on 3 specimens).

GENRE GHONETES, Fischer DE IVALDHEIM, 183\%.

I. Ghonetes concentrica, DE KONINCK, 18.t7.

Non figuré.

Caractieres spécifiques. - Cette espece ost de toutes ses congumines him

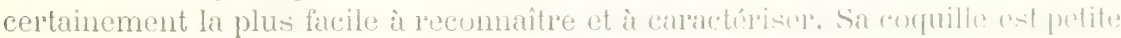

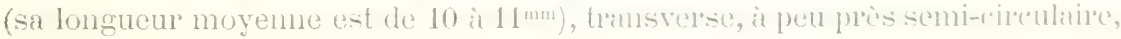
déprimée.

Valve ventrale garmic de 12 it 15 plis concentriques an erochet; ars plis, dont le nombre et la largeur augmentent progressirement arec l'age, sont tout it fait lisses, faiblement carénés ì leur partie antéricure, et légèroment disposís un terrasse; les sillons qui séparent ces plis sont assez protonds, mais ils sont

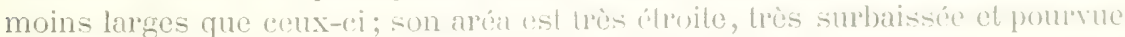

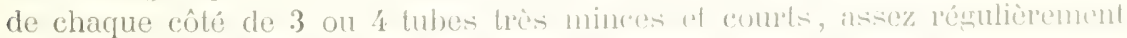

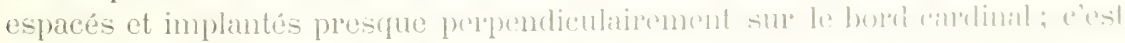

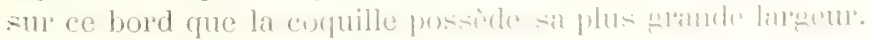


La valve dorsale ressemble tout ì fait à la ralve opposće; clle est tries peu concare et lrès rapprochéc de l'autre, de sorte qu'elle ne laisse entre elle et cette dernière qu'un espace trùs étroit pour le logement de l'animal.

L'intéricur des valves, sur lequel se reproduisent parlaitement les plis extérieurs, n’offre rien de remarquable, si ce n'ost qu'on n'y constate pas la présence des petites pointes qui hérissent la surface interne de la plupart des autres espèces, et que les plis y sont marqués de légères stries d'accroissement, qui ne se trourent pas à l'extérieur. - De Koninck (').

Cette espèce se trouve exclusivement dans l'horizon de Visé. Elle a été recueillie par de Semenow ì Hausdolf, oì elle n'est pas rare dans une assise du même îge, et probablement aussi en Eeosse.

Gisement. - L'Ardoisière; très rare.

Nous arons indiqué cette petite espèce, bien qu'clle ait été détruite, arec beaucoup d'autres, dans un incendie. Mais clle avait été soumise auparavant ì M. de Koninek, qui l'avait reconnue comme appartenant á l'espèce ci-dessus décrite, et nous l'arions figurée sur des planches inédites; elle a, clu reste, été formellement indiqquée dans la liste que ce savant auteur a publice des fossiles de l'Ardoisière, recueillis par nous dès 1872 (2).

\section{Chonetes papilionacea, Phlllips, 1836.}

P. VII, fig. 12b, 13 .

1'l. X. 11 ... 3.

P. XI, lig. 12, 13, 14.

Pl. XY, fig. 2, 3.

Mistorique. - ligurie pour la première fois par Lister en $1688^{(3)}$, sous la rubricue: "Pectinites fubelliformis, tuberculosa commissura, seu pectinites semicircularis compressior, minute ad modum striatus. "

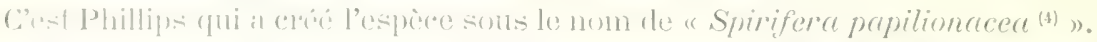
Veirei la diugnose qu’il en al domée: "Lxtremement large, semi-elliptique, avec

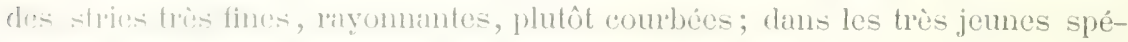

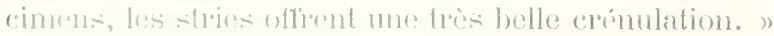

Corrutimess spécifiques. - Coquille minee, parfois un peu épaissic, transver-

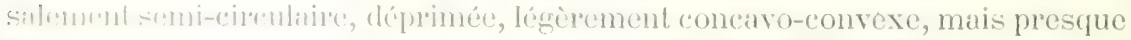

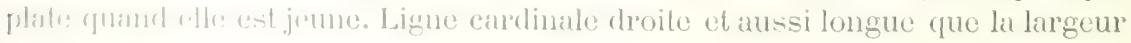
dis lis corpuilles.

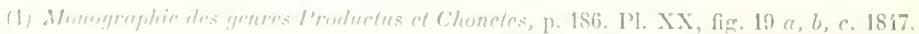

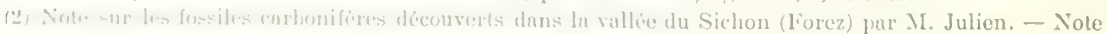

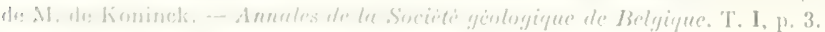

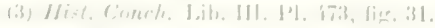

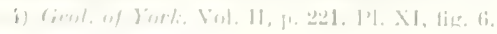


Vilve ventrate liomement convexe au erochet, mais tris aplatic sur les côtéx, et surtout press du bord cardinal. Crochet exigut et ne dépassant pas le bond carrimal.

Valve dorsale très faiblement concave au milieu, tandis que les parties latérales sont tris aplaties.

Une aréx bien dilimitie existe sur chargue valve. Celle de la ventrale est lat phus large. Elle est divisée an milien par un formnen triangulaine, partiellement vonté au-dessus par un pseudodeltidimn, lo preessus carelinal de la valve opposée remplissant le reste de l'ourerture.

La surtuce des deux ralves est couverte de nombreuses stries fines, filiformes, droites on Huxuenses, dont le nomlnes s'accroit par le moyen de bifurcations et de stries interealaires à des disaneos viniables des crochets. Ces stries et les espaces qui less séparent sont tratrersés lun des lignus serrées d'aceroissoment, nombruses, fincs ef concentriques, qui déterminent une belle crónulation. Des épines ténues émergent des stries à des distancess variables, et une rangée de 20 à 30 éprinces conptes se dressent sur le bord cardinal de la valve rentrale. Valves fortement articulées a l'aide de dents of de fossettes. Détails intérieurs imparfuitement connus.

Dimensions variatbles.

C'est peut-être lia plus grande ot lin plus helle des espices de cee geme, at elle est remarquable par sa forme alégante et sa structure. - Davidison (").

Gisements. - Régny, Saint-Gernain-Latal, Nontmain, Proprieres, Néronde, Ferrières, l'Ardoisière.

Explication des figures. - PI. VII, fig. 12 b. Jeune Chonetes papilionacea; valve ventrale. - Régny.

Fig. 13. Talve ventrale d'un autre spécimen. Une partie du test mancue ct lasise roir l'empreinte interne de cette valve. - liégny.

Pl. X, fig. 3. Coquille entière vue par la valve dorsale. On y distingue trè nettement le bord cardinal de la rentrale muni d'épines, la double arca et le pseudodeltidimm. lille pat en partie prritisce. - Saint-Germain-Laval.

Pl. XI, fig. 12. Empreinte externe de denx valves ventrales. - Montmain.

Fig. 13. Empreinte externe d'une ralve. - 1'popières.

Fig. 14. Enpreinte externe d'une valve. - propieres.

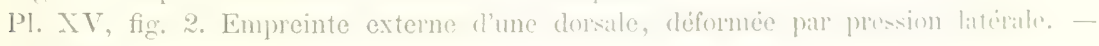
L'Ardoisière.

Fig. 3. Masnifique empreinte interne d'une valve dorsale, montrant les innombrables petits creux laisses sur le moule par les asjuerités dont la surfice interne de lat value wat hérissce. - L'Ardoisière; très commmn.

(1) Lue. cir, 1. 182. P1. XLVI, tig., 3-6. 
III, Chonetes Murchisoni, Nov. SP.

Pl. VII, fig. 14.
Pl. VIII, fig. 1.

Jolie espèe signalée nulle part ailleurs, et possédant les caractères suivants:

Coquille très aplatie, à bord semi-circulaire, angle de réunion du bord cardinal au bord frontal roisin de $90^{\circ}$. Les épines du bord eardinal sont au nombre de 20,10 de chaque côté. Test orné de stries excessivement fines; à $10^{\mathrm{mm}} \mathrm{du}$ crochet, dans $n 11$ espace de $10^{\mathrm{mm}}$, on en compte 54, tandis que le Ch. papilionacer n'en présente jamais plus de 44 .

Dimensions. - Longueur, $12^{\mathrm{mm}}$ environ; largeur, $20^{\mathrm{mm}}$; rapport de la longucur ì la lerrgeur, $100: 166$.

Gisements. - hégny, Néronde.

Tous dédions cette espèce as sir R. Murchison, en souvenir de la découverte de fossiles marboniferes su'il a faite dans la vallée du Sichon, en 1851.

Explication des figures. - Pl. VII, fig. 14. Valve rentrale.

Dans cet exemplaire on roit les épines courtes ( 2 ou $\left.3^{\mathrm{mm}}\right)$. Le bord frontal présente 180 stries environ, soit 2.j dans un espace tle $5^{\mathrm{mm}}$. - Régny.

Pl. VIII, fig. 1. Id. - Régny. Le méme échantillon de roche renferme des empreintes de Productus punctatus cur. cleguns? de Cypricurdella Julieni, Kon., deus radioles de Archeociduris. sp.? une plaque du mème oursin et une Modiole.

Sprécimens non fingués. - $a$. Un spécimen incomplet de Régny a présenté ume longueur de grm sur une largeur de 15); proportion : 100: 166. Les cotes ont le même degré de line-se, nous n'avons pu les compter.

6. Autre spécimen de Régny : $5 \dot{2}$ còtes dans un espace de $10^{\mathrm{mm}}$, a $10^{\mathrm{mm}}$ du crochet.

c. Lichantillon de Veronde : de 5.4 it 56 còtes it $10^{\mathrm{rmm}}$ du crochet, sur une longueur de $10^{\text {man }}$; sur le bord frontal, dans le meme espace, 5.1 cotes et sur tout le bord, environ 200.

1. Autre specimen de Nironde : longueur, $12 \mathrm{~mm}$; largeur, $20^{\mathrm{mm}}$; rapport de la longueur à lat largetur, 100$): 168$; nombre de stries it $10^{\mathrm{mm}}$ du crochet dans un espace de $10 \mathrm{~mm}, 54$.

Cette ispece nouvelle est relativement assez abondante dans les deux gisements ci-dessus. 
1V. Ghonetes comoïdes, Sowrrir, $1 \$ 23$.

11. VII, ti... 111, 11, 12,

Pl. IX, fig. 2, 3, 4, 5 .

Pl. XI, fig. $7,8,9,10,11$.

Historique. - Cette espèce a été décrite parr J. Sorrerby, suus le nom de Productus comoüdes (1). Voici lat diagnose qu’il "1l a donnée: "Cuguille semi-cireulaire, renflée, finement strice. Bord cardinal droit, aussi long que la coquille elle-même ${ }^{(2)}$.

Le traducteur ajoute: "Lir finesse des stries, jointe ir la forme de cette coquille, lui donne l'apparence d'un chignon; de là son nom spécifique. Il y a entre les crochets un espace uni qui ne se trouve point ordinairement dans ce genre. Outre les stries verticales de la surface, on remareque plusieurs grands sillons irréguliers. La coquille est très épaisse et rugueuse à la face interne. p

Curactires spécifirues. - Coquille larese, trinswersalement semi-cirenlaire, concaro-envexe. Ligne candinale droite, anssi longue ou un pen plus compte que lir plus grande linserem de la coquille. Valves solidement artieulées au moyen de dents et de fossettes.

Valve ventrale convexe; crochet plus ou moins développé et recourbé, mais ne dépassant pas le bord cardinal.

Talve dorsale concave, épousant la courbure de l'autre. Chaque valve est pourvue d'une aréa ì bords subparalleless, de plus ou moins grande lingeur, mais toujuurs plus étroite dans la petite valve. Au milieu de cette dernière, il existe un processus cardinal saillant, trilobé, qui pénètre et remplit presque une fissure trimgulaire eorrespondante dans l'aréa rentrale, ef qui étrit probablement couverte par un pseudodeltidium hombé. La surface externe de la coquille est finement striée. - Davidson ${ }^{(3)}$.

Gisements. - Régny, Néronde, Montagny, Ferrières (dans lo grès), Propières (dims le marbre), Cheval-Rigon, près Ferrières (dans le grès grossier siliceux.

Explication des figures. - Pl. VII, fig. 10. Grande et belle valre ventrale, vue du coité extérieur. Le test est rosé, comme cela se présente dans beancoup d'échantillons du méme gisement. - Régny. Très commun.

Fig. 11. Id.

Fig. 12 «. Valve ventrale montrant les épines de la ligne cardinale. - Meme givenent.

Pl. IX, fig. 2. Valve ventrale montrant des fragments du test et une partie de l'empreinte interne de la méne valve. - Néronde. Excessivenent commun; dans une carrière aban-

(1) Min. Conch. Vol. IV, p. 31. I'l. CCCXXix.

(2) Truluction Desor, 1. 359.

(3) Loc. cil., P. 180. Pl. XLV. fig. 7. 
donnée depuis de longues années, j'en ai compté plus de 200 faisant saillie á la surface d'un bane incliné.

Fig. 3. Empreinte interne de la valve ventrale, montrant les impressions musculaires semblables à celles figurées dans Darridson. (Appendix Pl, LV, tig. 8). - Néronde.

Fig. 4 a. Empreinte externe de la rentrale. - Néronde.

b. Empreinte interne de la mêne valve.

Fig. ว. Empreinte inteme de la ventrale. - Néroncle.

Pl. XI, fig. 7. Coquille isolée, vue du ceité de la dorsale. On voit nettement les deux aréas. Une partie rle la dorsale est empitée par la roche. - Montagny.

Fig. S. Valve ventrale. - Ferrières, un peu avant l'entrée du village, dans le grès, ì ganche de la route.

Fig. 9. Id. - Mème gisement.

Fig. 10. Valve rentrale, dans le marlbre de l'ancienne carrière, depuis longtemps abandonnée; un bloc, de la grosseur de la tète, renfermait une cinquantaine de spécimens. Propieres.

Fig. 11. Fragment d'empreinte de la surface interne d'une ventrale. - Cheval-ligon, près Ferrières, dans un grès grossier passant au poudingue.

Antre spécimen non fígurê. - Une empreinte de dorsale de Régny m'a donné les caractères suivants:

Longueur, 2.-2min ; largeur, 40

Tombre de stries it $10^{\mathrm{mm}}$ du crochet, dans un espace de $10^{\mathrm{mm}}$, 32 ; nombre de stries au hord frontal, dans le meme espace, 26 ; en tout, 150 enriron.

\section{Ghonetes Dalmaniana, DE KonINCK, 1843.}

13. Vil, tig. 15 at 16 .

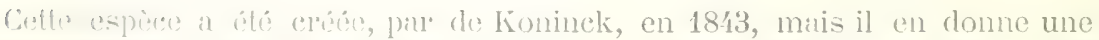
diagnosid ditinitive dans sil monormphie des genres Productus et Chonetes de 1847. Nons ne retiendrons de colle description, un peu longue, que les carac-

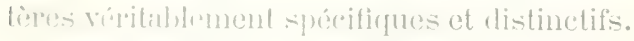

"Dimensionss. - I to longrueur moyeme de ce Chonetes est de 20 à $22^{\mathrm{mm}}$. Le

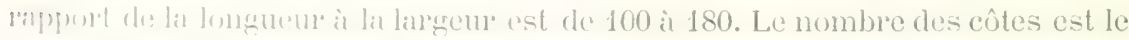

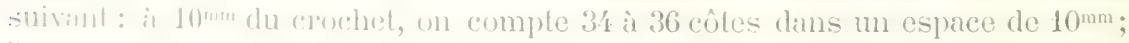

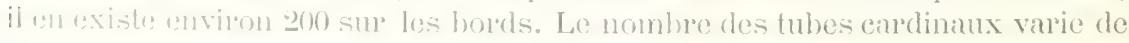

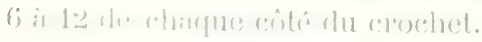

" Il se distingur: du jeune itge du Ch. pupilionacea, par sa plus forte coul-

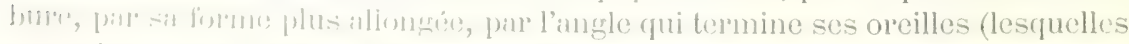

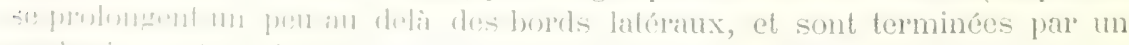

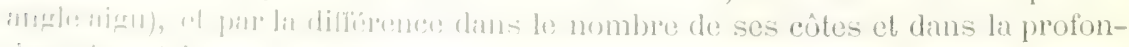

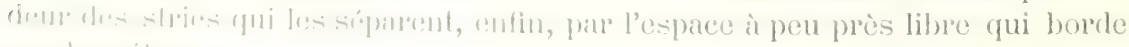

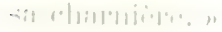


2I. de loninck a constaté son existence dans le carbonifüre marin du Sun-

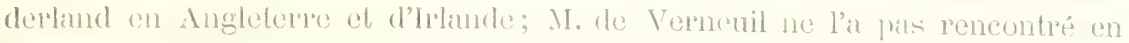
Rusicic.

Gisements. - Régny, Neronde, Montmain, Eerrières? l'Ardoisière.

Explication des figures. - Pl. VII, figr. 15. Vilvo ventrale. - Riegny.

Fig. 16. Un specimen de phus grande taille. Emupeinte interme de rentrale. - Régny. Exessirement commun dans ce gisement. 184.

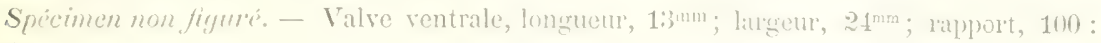

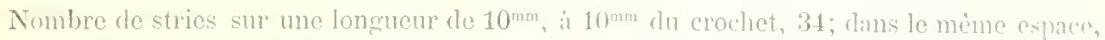
sur le bord frontal, 31 stries. Nombre total des stries, 110. 24 épines, environ, sur la ligne cardinale; 12 de chaque coté. - Rigny.

11. Ghonetes Laguessiana, DE Konisck, 1843.

\section{Non ficuré.}

Ce Chonetes, dédié à M. Luguesse, sous-ingúniemp au enpus des mines de

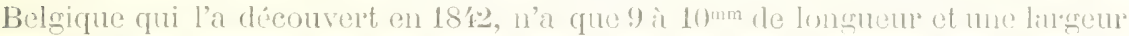

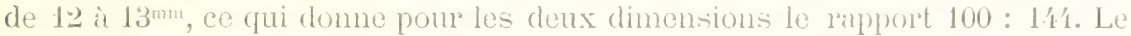
nombre des cotes au pourtour est de 105 environ. Les ipines curdiuales sont au nombre de 8, 4 de chaque côté.

Gisements. - Régny, l'Ardoisière, Peu commun, sauf à Régny.

Analyse de quelques spécimens. - Nous possédons une valve ventrale de hégny dont les dimensions sont les suivantes:

Longueur, $7^{\mathrm{mm}}$; largeur, $10^{\mathrm{mm}}$; rapport de la longueur it la liargeur, 100): 1.13.

Epines, $8 ; 4$ de chaque cité.

Nombre de stries sur lo bord frontal, 105 à 110 environ.

No 2. Un autre spécimen de Régny a une longueur de $10^{\text {mun }}$ environ, et une largeur de $14^{\mathrm{mm}}$; rapport, $100: 140$; lo nombre cles stries au bord frontal est d'un peu plus de 100.

$N^{\prime \prime} 3$. Enfin un dernier spécimen a exactement 105 cótes all pourtour.

Observations relatives anx deux especes précélentes. - I) avidson no parait pas dispose à accepter les espèces créées par l'auteur belge. Élles lui semblent intermédiaires par leur forme et leurs caractères entre Ch. papilionareu et Ch. Hurllensis, Phillips, et, en ellet, si l'on met en regard les chiffres relatifs anx trois espèces, on arrive facilement it comprendre les hésitations du célèbre auteur anglais.

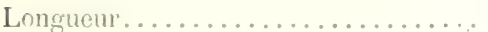

Largeur. . . . . . . . . . . . . . . .

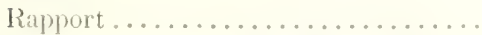

Nombre de stries au bord frontal.....

Iotal des épines. . . . . . . . . . . . . .

\begin{tabular}{|c|c|c|}
\hline Ch. Diluaniana & Cir. Harduexsis & Cir. Lagunsitaxa \\
\hline $20 \therefore 2^{2}$ & $15 i 16^{\mathrm{mm}}$ & 9) $\mathrm{a} 1 t^{\mathrm{mm}}$ \\
\hline $36^{\mathrm{mm}}$ & $21 \div 25 \mathrm{~mm}$ & 12 it $13^{++m}$ \\
\hline $100: 180$ & $100: 15 \pi$ & $100: 1.14$ \\
\hline (00, environ & 120 an plus & 105 \\
\hline $12 \therefore \because 4$ & ¿) i ! & 8 \\
\hline
\end{tabular}


On roit que $C h$. Hardrensis est bien intermédiaire par les caractères ci-dessus entre $C h$. Dalmaniana et Ch. Laguessiana, considérés comme formes extrêmes d'une espece ínínemment vuriable. Or, nous n'arons jamais rencontré dans nos gisements du Plateau Central, cette forme intermédiaire, pas plus que M. de Koninck en Belgique, aussi nous sommesnous décidé à conserver les deux espèces belges, fortifié du reste dans cette résolution par l'identité remarquable de la faune générale des deux pays. Cela ne saurait étonner si l'on se rappelle que la mer carbonifère venue du nord dans nos régions, avait réellement ses rivages dans le Platean Central.

VII. Chonetes variolata, D'ORBIGNY, 184\%.

Pl. 1, fig. 6, 7 .

Pl. XI, fig. 15.

Iristorique. - Figuré pour la premiere fois par Ure en $1793^{(1)}$, sous le nom de Pecten, accompangé d'me tris courte description.

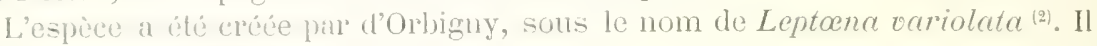
en a domné la diagnose snivante :

"L. testì transcersi, depressi, substriati, minute punctatì; fironte late

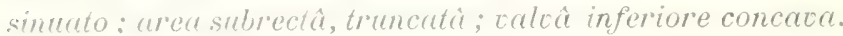

"Dimensions. - Angle apjicial, 160\%; hauteur (longueur), 10 mm; largeur,

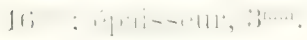

"Coquille beancoup plus liırge que haute, très déprimée, à valves très inégales, la valve supuriene un pen convexe, l'autre concave; le sommet très conrt, ne dépassunt pas la ligne de l'aréa. Aréa presque droite, formant un angle de 1600, offrant à ses extrémités la plus grande largeur de la coquille. De co print, les bords convergent vers le front, qui est très convexe, arrondi, mais felsancer au milien par le sinus. Lil valve dorsale (ventrale), offre trois dépressions : l'une, módinne on sinus très marqué près du front; les deux autres sur les côfís près du sommet, et s'évanouissant vers le bord. La surface ontien do la valve dortale olfre, avee quelques stries à peine apparentes ì la

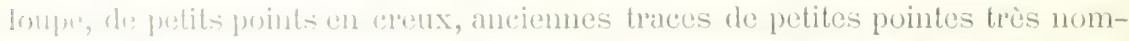

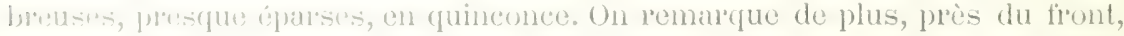

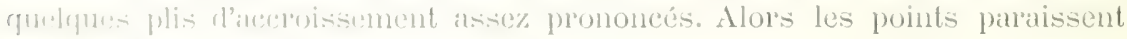

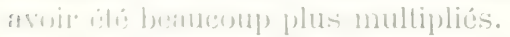

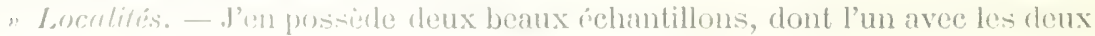

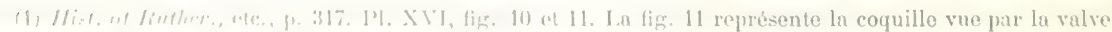

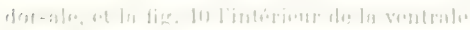

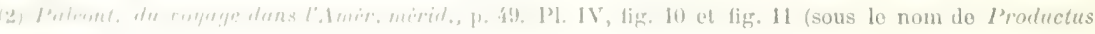
rerritulutin 
valves. Je les ai recueillis dans le terrain carbonifère de Yarbichambi (Bolivie). )

M. de Koninck, dans sa Monographie, en a donné (p. 206) une description beancoup plus complete, car cette espèce est très abondante à Tournai.

Nous n'en retiendrons que la mention de la forme générale, qui est nettement subrectangulaire, et celle des tubereules qui hérissent la surface interne sur la partie médiane et antérieure; les dimensions qui sont les suivantes: longueur moyenne, $10^{\mathrm{mm}}$; largeur, 15m; d'où le rappolt $100: 150$, et le nombre total des côtes qui est de 75 , environ, sir le bord frontal.

Gisements. - Domaine des Roches, Siguret, La Varrille (Morvan), Propières. Excessivement commun.

Explication de's figures. - Pl. 1, tig. 5. Deux Chonctes: l'un est représenté par une empreinte externe de la valve dorsale surmontée de l'aréa de la ventrale, c'est le Chonetes Giraudi, nov. sp. ; l'autre est représenté par une empreinte interne de la dorsale, montrant très nettement les fossettes causées par les asperités de la face interne, surtout abondantes vers le bord frontal, et appartient au Chonetes cariolate. - La Varville.

Fig. 6. Empreinte interne d'une dorsile, montrant les impressions des adducteurs et le septum. - Domaine des Roches.

Fig. 7. Empreinte externe de dorsale. - La Varville.

Pl. XI, fig. 15. Empreinte externe de dorsale, montrant l'aréa de la ventrale. - Propières.

Spécinen non figuré. - Un spécimen de La Viurville montre nettement les épines.

\section{Ghonetes Giraudi, NOV, sP.}

\section{Pl. I, fig. 5.}

Au milieu des $C h$. variolata, qui se rencontrent par milliers dans le gisement de La Varville, on peut reconnaître des spécimens, en nombre assez considérable, d'un autre petit Chonetes, qui, par sa forme, doit être distingué du premier. L'un d'oux a été figuré dans la planche I, fig. 5. Mágrée le maurais état de ces empreintes, on peut fixer quelques caractères distinctif's qui serviront à le faire reconnaître.

Caracteres spécifiques. - Coquille concavo-convexe, sub-canrie-inromdie; ligne cardinate égale à la plus grande largeur de lir coquille et de mème dimension que la longueur.

Valve ventrale renflée vers le sommet, et munie d'un sinus tres alphréciable; oreillettes aplaties; angle de rémion des oroillettes et de la ligno cardinale, droit.

Valve dorsale munie d'un bourrelot allant du sommet au hord frental of correspondant au sinus de la ventrale. 
Aréa très surbaissée et étroite.

Surface des deux valves ornée de stries fines au nombre d'une centaine, environ, sur le bord frontal.

Dimensions moyennes. - Longueur, 12m ; largeur, 12mm; rapport, $100: 100$.

Aucun spécimen n'a pu nous permettre de compter les épines, dont l'existence est pourtant certaine.

Gisenents. - Domaine des Roches, La Varville (Morvan).

Explicution des figures. - PI. I, fig. 5 (en haut et à gauche de la figure). Empreinte de valve dorsale, surmontée de l'aréa de la ventrale.

Voici ses dimensions:

Longueur, $11^{\text {nin }} ;$ largeur, $11^{\text {min }}$; rapport : $100: 100$.

Cet échantillon est trop mal conservé pour qu'on puisse compter le nombre des stries.

Spécimens non figurés. - No 1 . Empreinte interne de ventrale.

Longueur, 12mm ; largenr, 12mm .

№ 2. Empreinte externe de ventrale, un peu déformée.

Longneur, $10^{\text {mar }}$; largeur', $10^{\text {mm }}$.

On ne peut compter les stries it cause d'une mince pellicule d'ocre qui les masque en partie.

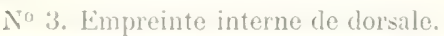

Longueur, 12mm; largeur, 12 amm.

No 4. Limpreinte externe de dorsale.

L.ongueur, $12^{\text {mun }}$; largeur, $12^{\text {mur }}$.

Nombre de stries dans $10^{\mathrm{mm}}$ :tu milieu du bord frontal, 40, soit 100 environ sur tout le bord frontial.

N゙ 5. Empreinte externe de dorsale.

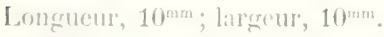

On ne peut compter les stries.

Nons sommes heureux de dédier cette espéce á M. J. Girand, préparateur de géologie á la Faculté des erieness, qui a su la distinguer an milieu d'imnombrables spécimens de $C h$. ouriolutu.

\section{Chonetes crassistria, M'Cor, 1844}

P. I, fig. 8, 9, 10.

Dirmposs de M'Con: Coquille semi-cinculaire convexe, la longueur est les

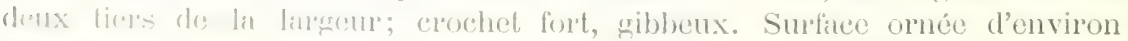

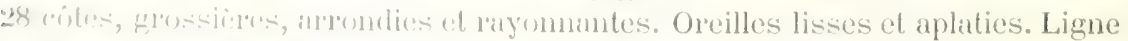

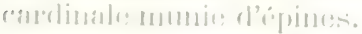

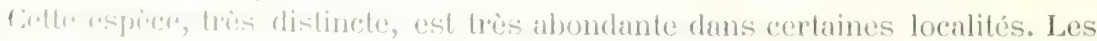

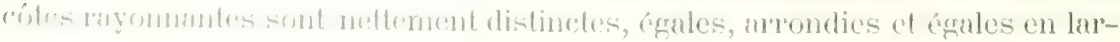


gemr anx espaleos qui les séparent. Les oreilles sont lisses et très aplaties. Il y a environ 12 épines courtes, grêtes et courbes sur la ligne cardinale.

Longueur, 5 lignes 1/2; largeur, 7 lignes $1 / 2$.

Observutions. - N'Coy a décrit cette espèce sous le nom de Leptena crassistria (Synopsis, p. 119). A la page 126, il décrit un autre Chonetes sous le nom d'Orthis sulcuta, figuré pl. XX, fig. 6. A notre avis, de Koninck assinile à tort ces deux cspèces bien distinctes de l'auteur irlandais, et les décrit sous le nom de Chonetes sulcata dans la Monographie, p. 196.

Davidson fuit du L. cressistria, M'Coy, une simple varisté du Ch. Buchiana de Koninck, aree quelques réserves toutefois (").

Notre petit Chonetes du Morvan nous paraît identique au Ch. crassistria de M'Coy, c'est pour puoi nous le déerivons sous ce dernier nom.

Gisements. - Siguret, près Sarigny-Poil-Fol (Morvan).

Explication des figures. - Pl. I, fig. 8. Fragment de roche présentant 2 spécimens:

a. Ventrale.

b. Empreinte externe d'une dorsale, surmontée de l'aréa ct de la rentrale.

Fig. 9 et 10. Les mémes grossis 4 fois. La figure 10 est mal éclairée et la coquille parait concave.

\section{Ghonetes Jourdani, Nov. SP.}

Pl. VII, fig. 17.

Joli petit Chonetes dont nous avons découvert l'unique spécimen figuré à Régny. Nous ne possédons que la valve ventrale. Elle est très convexe; la ligne cardinale, presque droite, est un peu plus courte que la plus grunde largeur de la coquille. Les dimensions sont à peu près les suirantes:

Longueur, 6 $6^{\mathrm{mm}} ;$ largeur, $7^{\mathrm{mm}} ; r^{2}$ apport, $100: 116$.

La valve est ornée d'environ 40 ì 45 côtes sur le bord frontal, en admettant dans ce nombre les côtes très courtes, qui prennent naissance tris près du bord, surtout vers les oreilles. Les cîtes sont simples tout pris du crochet, mais elles se dichotomisent toutes assez rapirlement.

Rapports et différences. - Se rapproche du Chonetes elergans, do Kon., lin le nombre des còtes qui est sensiblement lo mêne, mais s'en distingue par sil plus petite taille, par l'absence do rugosites sur ses colles, qui sont patraitoment lisses.

Gisement. - Régny, un seul échantillon.

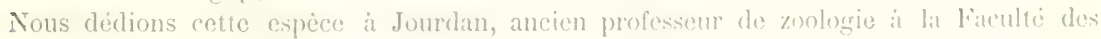
sciences de Lyon, qui a le premier étudie la fame do Rigny.

(1) Brit. Carb. Brach., P. $18 \pi$. 


\title{
FAMILLE DES STROPHOMENIDÉS
}

\section{GENRE STROPHOMENA, RAFINESQUE, 1820.}

I. Strophomena rhomboidalis, Whalengerg, 1821; var. analoga, Phillips, 1836.

\author{
Pl. III, fig. 8, 9 .
}

Caractères spécifiques. - Coquille plus ou moins transversalement semicirculaire ou sub-carrée; valves géniculées; ligne cardinale droite et aussi longue que la plus grande largeur de la coquille, avec des angles cardinaux arrondis, parfois prolongés en forme d'ailes déployées.

La valve ventrale est légèrement convexe au crochet, elle s'aplatit ensuite à whe certnine distance, et se courbe alors brusquement, le plus souvent à angle droit. Le bord frontal est ondulé, concave près des angles cardinaux; il s'incurve ensuite latéralement pour former au front une légère courbe extérieure. Sur la portion aplitie du disque, il existe un nombre variable de rides concentriques, ligerement onduleuses et parfois interlompues, qui convergent ver's la ligne cardinale st suront ainsi la combure du bord. La surface entière est aussi converte de nombreus's stries l'ayomnantes, filiformes; on observe généralement un petit finanen circulare près de l'extrémité du erochet ou sur le crechet in mo certain hige, mais il s'oblitire et se cicatrise chez l'adulte.

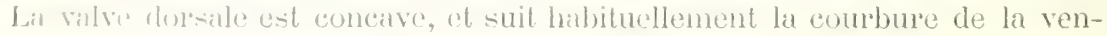
tratr, alle est semblablement ridée et striéc. - Davidson (1).

Dims les observalions qui suivent la diagnose, Davidson fait remarquer que Less st. thermhoidalis, du Silurien, St. depressa, du Dévonien, et St. analoga, du Cont)snifëre, n'offrent (que des différences variétales et non spécifiques. C'est fonly cotte mison qu'il conserve le nom de Strophomena rhomboidalis Wahlen-

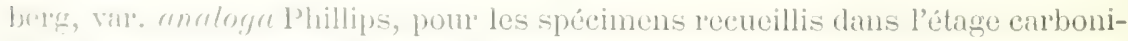
fïp, onlrant ainsi dans les vues du plus grand nombre des palcontologistes.

risements. - Láa Viarville, l’Ardoisière.

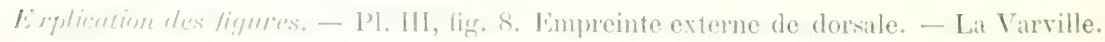

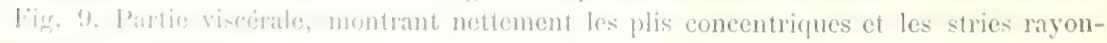
mante... Lat Visville.

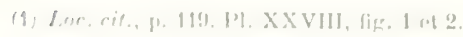


(iFNRE ORTHOTETES, F. DE IVALDHEIM, 1830.

I. Orthotetes crenistria, Phit.IPs, 1836.

\author{
1. III, ti!. 万, 6,7 . \\ Pl. VIII, tign. 3. \\ Pl. IX, fig. $7 a$. \\ Pl. XI, fig. $17,18,19$. \\ Pl. XV,fig. 6.
}

Historique. - Espèce créée par Phillips, sous le nom de Spirifer crenistriu, avec la diagnose suivante: "Surface des valves couverte de stries fortes, rayonnantes. Elles sont crénclées par les lignes d'acroissement. "

Caractères spécifiques. - Cuquille de forme tris rariable, transwersalement ou longitudinalement semi-circulaire. Ligne cardinale droitr, excédant lómèrement la plus grande largeur de la eoquille, ou quelque peu plus courte. Angles cardinaux arrondis ou prolongres en terninaisons aiguës; area ventrale plate, variable en largeur, et divisée par une fissure couverte par un psendodeltidium: aréa dorsale linéaire.

Valve ventrale de courbure variable, légèrenent convexe an crochet, plate an milieu et partiellement concave près du bord et au bord meme, on plus ou moins régulièrement convexe dans tonte son étendue. Crochet itroit, saillant et souvent tordu ou irrégulièrement incliné verse l'un des côtés.

Valve dorsale modérément on extrèmement ennvexe. Surfure des deux valves eonverte de stries nombreuses, forles, ravonuantes, arpondies avec des intrevalles aplatis de largeur variable, particllement oceupés par une on denx stries plus petites; les côtes et les intervalles étant en mème temps croisés par de fines lignes ou stries concentriques, très serrées, donnant it la surface de la coquille une apparence crénclée.

Les dimensions de ectte coquille sont très variables. - Davidison (1).

Gisements. - La Varville, Siguret, Régny, Saint-Germain-Laval, Néronde, Montmain, Propières, Thizy, l'Ardoisière, Le Gonget.

Explication des figures. - Pl. III, fig. 5. Fimpreinte de la valye dorsale. - La Virville.

Fig. 6. Id.

Fig. 7. Id.

Pl. VIII, fig. 3. Valve ventrale. - Régny.

PI. 1X, fig. 7 a. Valve ventrale. - Neronde.

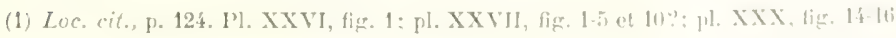


Pl. XI, fig. 17. Empreinte d'une portion de ventrale. - Propières, dans le schiste.

Pl. XI, fig. 18. Empreinte externe avec fragment de test de la dorsale. - Montmain.

Pl. XI, fig. 19. Enpreinte interne de rentrale. - Propières, dans le schiste.

Pl. XV, fig. 6. Empreinte d'un grand spécimen. - L'Ardoisière.

II. Orthothetes Sharpei, Morris, 1843.

Cette coquille, découverte par Sharpe dins les couches carbonifères de Kendal (Irlande), a été publiće, sous le nom ci-dessus, par Morris (1) qui, sans en domer de description, l'a néanmoins identifiće avee l'Orthis umbraculum L. de Buch, provenant d'Enniskillen (Fermanagh) que Portlock a figuré et décrit minutieusement (). De Verneuil ì qui Sharpe en avait donné un autre exemplaire provenant aussi de Kendal, en a donné une très courte diagnose que nous reproduisons: "Quatre ou six stries adventices, beaucoup plus fines que celles entre lesquelles elles sont interposées. Le fond des sillons est légèrement ridé par des lignes d'aceroissement. Valve ventrale concave, valve dorsale convexe.

"Dimensions. - Longueur, 67mm; largeur, 84 $\mathbf{4}^{\mathrm{mm}}$; épaisseur, 9 à $10^{\mathrm{mm}\left({ }^{(3)}\right.}$. "

Nous arons découvert plusieur's spécimens d'un Orthothetes de grande taille que nous rapportons ì cette espice; l'un d'eux mesure $11^{\mathrm{cm}}$ de large; une autre a une longueur de $75^{\mathrm{mm}}$ sur $64^{\mathrm{mm}}$ de large. On peut eompter 6 stries adventices.

Gisement. - L Ardoisiere.

GFNRE ORTHIS, DALMAN, 1828.

I. Orthis Michelini, LÉveIllé, 1835.

P. III, firs. 1, 2, 3, 4, 16, 17 .

Diregnese de Léreille : "Celle eofuille, très rapprochée de $O$. resupinata, en

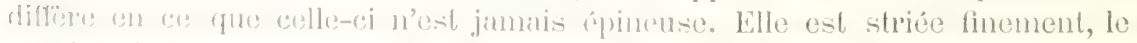

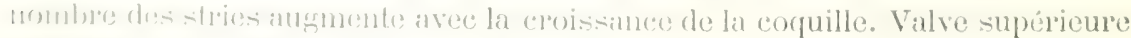

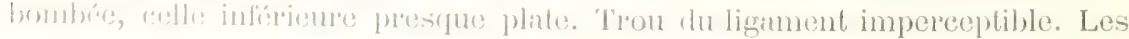

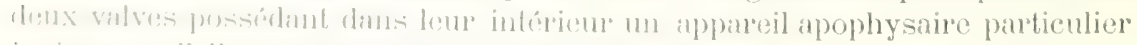

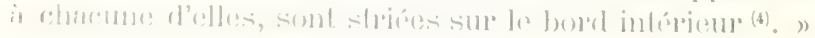

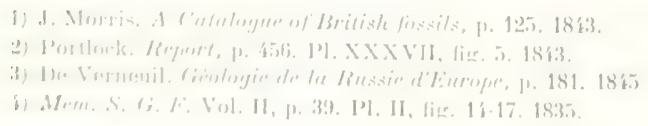


Caracteres spécifiques. - Coquille déprimée, presque cireulaire ou sub-trigone, aussi large ou plus large que longue, avec sa plus grande largeur près du bord frontal. Celui-ci olfre une légère courbure extérieure on intérieure.

Ligne cardinale très attínuée, ne dépassant pas le tier's de la largeur de la coquille, quelquefois moins. Arér ventrale petite, mais sensiblement plus grande que celle de la valve opposée, et divisée par une fissure qui est presque entierement remplie par le processus candinal de la valve dorsale.

Valve rentrale ou modérément convexe, avee une légère dépression commençant ver's le milieu et s'itendint jusqu'an bord frontal, ou aplatie tout du long, it l'exception du crochet qui est petit, légèrement ineurvé et saillant.

Valve dorsale modérément et uniformément convexe, ou féiblement déprimée verśs le front.

Surface des deux valves couverte de stries serrées, rondes, rayomantes of filiformes, qui croissent en nombre grâce à de nombreuses intercalations of bifurcations, tandis que de loutes les petites côtes émergent des épines crenser, semblables à des cheveux, plus étroitement accumulées vers les bords.

La structure intime de la coquille est perforée par de petits canaux dont les orifices extérieurs, sous forme de ponctuations, couvrent la surfince entière des valves. - Davidson (1).

Gisement. - La Varville.

Explicution des figures. - Pl. III, fig. 1. Empreinte externe de la valve dorsale, avee un fragment de test. - La Varrille.

Fig. 2. Munlage de la même.

Fig. 3. Empreinte externe. - La Varville.

Fig. 4. Moulage de la mème.

Fig. 16. Empreinte de la cavité viscérale, vue par la face dorsale. - La Varrille.

Fig. 17. La même, vue par la face ventrale.

II. Orthis resupinata, Martin, 1809.

Pl. VIII, fig. 2.

Pl. IX, fig. 6.

Pl. XI, fig. 16.

Pl. XV, fig. $4 a, b, c, 5$.

Historique. - Espèce créée par Martin sous le $110 \mathrm{~m}$ de Conchyliolithus anomites resupinatus. Transversim ovalis, longitudinaliter striatus : striis confertis cequalibus minutis, valca imperforati conocxiore (2).

(1) Loc. cit., p. 132. Pl. XXX, fig. 6-12.

(2) Loc. cit. 1'. XLIX, fig. 13, 14. 1809. 
Caractères spécifiques. - Coquille transversalement ovale ou elliptique. Valves convexes, parfois gibbeuses. Ligne cardinale droite, beaucoup plus courte que la plus grande largeur de la coquille, avec des angles cardinaux arrondis.

La valve dorsale, généralement la plus profonde, est régulièrement et uniformément convexe, ou légèrement aplatie et déprimée le long du milieu du front. Aréa étroite.

Valve ventrale de courbure variable, modérément convexe d'un bout à l'autre ou à la portion cardinale seulement, devenant plate ou légèrement concave près des bords latéraux et frontal. Le bord frontal offre une courbe uniforme ou ondulée, échancrant plus ou moins le bord de la valve opposéc. Crochet petit et modérément incurvé. Aréa triangulaire avec un foramen ouvert.

A l'extérieur, les valves sont couvertes de stries fines et serrées, filiformes, arrondies, rayonnantes, qui croissent en nombre par intercalations et bifurcations à des distances variables des crochets. Par intervalles, les stries ellesmêmes augmentent de hauteur' et d'épaisseur', donnant ainsi naissance à des épines tubulaires, filiformes, petites, creuses, qui deviennent plus nombreuses vers le bord. La structure intime de la coquille est perforée par d'innombrables canaux, lont les orifices extérieur's, en forme de fines ponctuations, couvrent la surface entière des valves. - Davidson (1).

Gisements. - La Varville (Morvan), Régny, Néronde, Montagny, l'Ardoisière.

Explicution des figures. - PI. VIII, fig. 2. Empreinte interne de la ventrale. - Régny.

Pl. 1X, fig. 6. Valve dorsale. - Néronde.

Pl. XI, fig. 16. Valve ventrale écrasée. -- Montagny.

Pl. XV, fig. 4. Empreinte de la carité viscérale vue par la face ventrale.

a. b. Fragments du test qui recouvrent encore le moule interne $c,-$ L'Ardoisiere.

Fig. 5. Le mème spécimen, vu par la face dorsale.

(1) Loc cit, 1, 130. P1. XXIX, fig. 1-6; pl. XXX, fig. 1-5. 


\title{
FAMILLE DES SPIRIFERIDES
}

\author{
GENRE SPIRIFER, SOWERBY, 1815.
}

1. Spirifer duplicicosta, Phlllips, 1830.

Pl. VIII, fig. 7, 8, 9 .

Pl. IX, fig. 10,11 .

PI. X; fig. 5 .

Pl. XI, fig. 20 .

Historique. - Espèce crécéc par Phillips qui en donne la diagnose suivante: "Bourrelet anguleux, côtes rayomantes nombrenses, bifurquées ver's le bord (1). ")

Caractères spécifiques. - Transversalement subrhomboïdale quand elle est adulte; plus longue que large ou prosque cirenlaire quand olle est tout à fait jeune.

Valves modérément convexes avec un bourrelet plus ou moins saillant sur la dorsale et un sinus correspondant sur la ventrale. La ligne cardinale est plus courte que la largeur de la coquille; aréa de lingenr modérée, crochets recourbés. Valves ornées de plis rayonnants nombreux, qui angmentent rapidenent à des distances variables des crochets, par interealation ansi bien que par bifurcation. - Davidson ${ }^{(2)}$.

Gisements. - Régny, Néronde, Saint-Germain-Laval, Propières, Montmain, Ferrières et l'Arcloisière.

Explication des figures. - Pl. VIII, fig. \%. Vilve ventrale montrant nettenent la trifurcation des plis, indiquée dans la diagnose de M'Coy du Spirifer firsciculeter(3) consideré comme symonyme du Spirifer duplicicosta par Davidson. - Régny.

Fig. 8. Valve dorsale. - Régny.

Fig. 9. Id.

Pl. IX, fig. 10. Valve ventrale, identicue an speimen de higny, jl. Vill, fig. \&. Néronde.

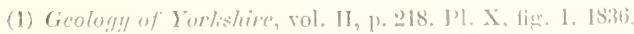

(2) Loe. cit. I’at V, p. 2.5. 1'l. III, lig. 7, I0; 11. IV, lig. 3, 5-11.

(ii) Brit. P'uleñ. foss., p. 116. 1'I. III (1)), tig. 25. 
Fig. 11. Echantillon très mal conservé et brisé au bourrelet par suite d'écrasement latéral. - Néronde.

Pl. X, fig. 5. Valve ventrale. - Saint-Germain-Laval.

Pl. XI, fig. 20. Empreinte interne déformée d'un échantillon de la mème espèce? - Propières.

II. Spirifer Tornacensis, DE KONINCK, 1883.

Pl. I, fig. 11 .

Pl. II, fig. 1, 2, 3, 4, 5, 6, 7, 8.

Pl. III, tig. 1 .

Historique. - C'est en 1883 que M. de Koninck a distingué et dénommé cette espèce, si abondante a Tournai, qu'il avait jusqu'alors confondue avec le Spirifer cincus Keyserling. En 1887, il a publić une diagnose définitive de cette espece, fue nons allons reproduire. Cette coquille, quelle que soit sa taille, qui pent etre assez torte, est toujour's transverse et beancoup plus large que longue. La largeur de son aréa représente son plus grand diamètre transverse. Cette arét n'est pas très derée et ses bords sont subparallèles sur la majeure partic de son étenclue; sa surface est striée en travers. L'ouverture deltoïde est Lriangulatire, large et ouverte sur toute sa hautenr.

Lil valve dorsale est beancoup moins profonde que la valve opposée. Son brommetet nost presque pas saillant et peu distinet; ses bords se confondent prespue insensiblement avee les parties adjaeentes. Il en est de mème du sinus de Jar valve ventrate, Jepuel est peu profond et inal défini. La surfaee de chacune

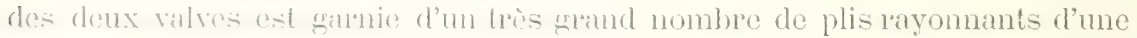

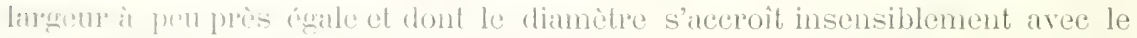
devaloppement de la conquille; ees plis, qui sont au nombre de 10 à 12 pour le bromelet el le sinus, se bifmequent rurement. Ils sont arrondis, et, chez les individus de bonme conservation, converts de lamelles d'aceroissement, imbrigurus ut refremenent minees, qui les rendent m pen ruyeux. Je n'y ai jamais

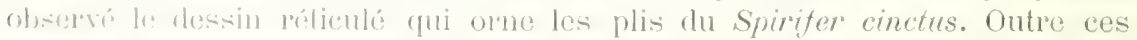
lamethes, on y obsersed de distance en distance, un sillon transversal, indiquant

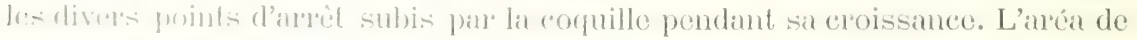

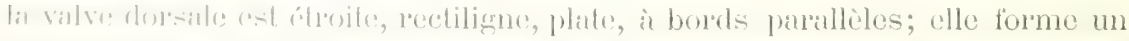

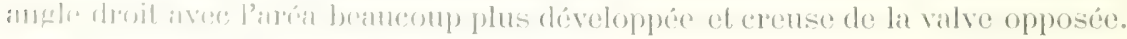

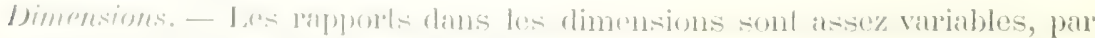

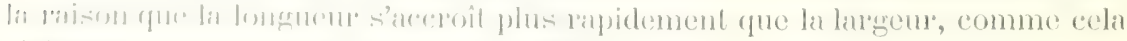

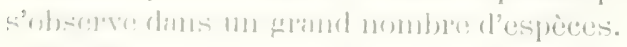

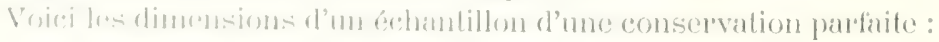

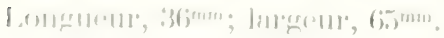


Rirpports et différences. -. J'ai pendant longtemps confondu et identifié cette espèce avec les Sp. cinctus, keyserling, dont je la considerais comme variété. De Koninek (").

Nota. - De 1812 i 1883, M. de Koninck avit égralement confondu, le Sp. cinctus Jui-mène, arre le S\%. Mosquensis, Fischer, et les avait réunis sous le $120 m$ de Sp. Sowerbyj, le hon.; mais la constance des canactères observés sur une quantité considerables d'echantillons ot surtout la différenee existant dans la eonformation intriene des valves des deux coquilles, l'avait convaineu qu’elles étaient spécifiquement différentes.

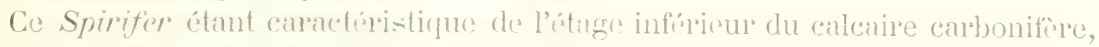

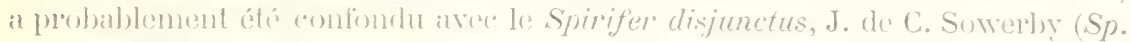

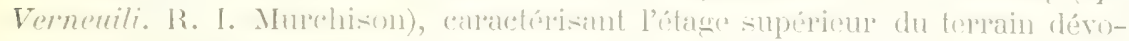
nien, par certains antrur- qui on ont indiqué la présence dans lo terrain carbonifere. Ce dernier an diffère cependant par la simplieité des plis des ailes, la plus grande finesse of la dichotomisation de eenx du bourrelet et du sinus; par la limitation nette du lobe ef du sinns de ses valves, et phus eneore, par la forme plus haute et plus rigubirement triatgrulaire deson aréa.

Giscments. - Domaine des Roches, La Varville (Morvan).

Erplication des figures. - Pl. I, fig. 11. Empreinte ale la portion cardinale des deux valves; les deux aréas sont trés visibles et l'on y remarçue nettement la striation de l'aréa rentrale. C"est un jemne spécimen. - La Varville.

Pl. II, fig. 1. Empreinte en crenx de la surface externe d'une ventrale. La figure, mal éclairée, semble incliquer une valve en relief. - Li Tarville.

Fig. 2. Moulage de la méme. L'échantillon est un pen dèjeté par pression latérale.

Fig. 3. Empreinte en creux de la partic umbonale dr la corpille. - La Varville; speciunen plus argé que colui de la pl. I, tig. 11.

Fig. 4. Moulage dn meme.

Fig. J. Autre spécimen adulte, écrasé, de manvaise consersation, montrant la dorsale, l'aréa de la rentrate aree son formen et l'empreinte interne de la rentrale. - lat larrille.

Fig. 6. Autre specimen montrant nettement les dens areas. - La Virrille.

Fig. 7. Empreinte de dorsale. - La Varville.

Fig. 8. Moulage de la meme.

Pl. III, fig. 14. Spécimen de grande taille, completenent aplati et déformé par presion; moulage de dorsal le. - La Varville.

Le Sir. Tomnensis se rencontre par milliers dans le gisement de Ia Viurville, il en est l'espece la plus abondante arec lo Chonetes corvolutu, Ch. Givandi, Spirifir lineutus et onthis Mirhelini.

Ohserpretions critiques. - Co spirifer, malere la nettete de ses caracteres distinetifs, it été aussi méconnu d'une manière étrange par MI. de Koninck, qui, dans nu examen trop rapide de la faune du Morvan que jo lui arais sommise, l'a assimile an Spirifer cotcomous

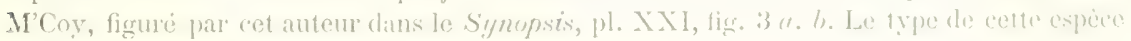

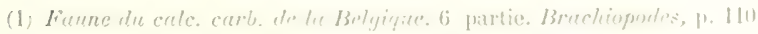


est le Sp. calcarcitus du Devonshire. Ce Spirifer a été décrit par J. Sowerby (1) d'après des spécimens recueillis á Barnstaple dans le Dévonien supérieur du conté de Deron, par Sedgwick et Murchison. Un examen critique de cette espèce dévonienne a été fait par divers anteurs, qui tous se sont accordés it reconnaitre que l'espéce de Sowrerby ne pouvait ètre que le jeune du Spirifer Terneuili. C'cst, en premier lien, l'opinion de Phillips, qui a montré l'estrème analogie du Sp. calcaratus Sow., avec le Sp. Verneuili Murch. ${ }^{(2)}$.

L'année strirante, de Koninck ${ }^{(3)}$ a exprimé la mème opinion; puis c'est le tour de Rœmer(t). L'illustre de Verneuil(5) s'appuie également sur l'opinion de de Koninck; Schnur( ${ }^{(0)}$, le savant auteur de la Monographie des Brachiopodes de l'Eifel, exprime la mène opinion. N'Coy lui-mème(i), dans sa belle ourre relative aux fossiles britanniques, publiec en collaboration arec Sedgrick, se prononce ì son tour sur l'espèce de Sowerby, et la considère comme étant le jeune de Sp. Verneuili, Murch. jusqu'a la taille de 1 pouce de long. Enfin, de Semenow(s), dans les tableaux synonymiques qu'il donne à la suite de sa publication sur les fossiles de Silésie, rejette le Sp. culcaratus, en tant qu'espèce carbonifère.

Ainsi le Sp. culcurrutus Sow., du conté de Devon a disparu de la science, en se confondant avec le Sp. Terncuili alont il represente le jeune aige.

sp. culcuratus de l'Irlunde. - On est done en droit de se demander ce que peut bien étre le specimen de la collection de R. Griflith, décrit par N'Coy, et assimilé par lui à l'espéce de Sowerby; serait-ce eneore un jeune Sp. Ferneuili qui aurait survécu dans cette region jurqu'i l'éporgue oì s'épanouissait la luxuriante fame carbonifère irlandaise, qui peut rivaliver par l'abondance et la variété des tỵes avec celle qui se développait en Belgique?

La réponso il cette question a été donnée par Daridson ${ }^{(9)}$ : "La coquille figurée comme Sp. calcurutes par le prof. MCoy dans le Synopsis, pl. XXI, fig. 3, et pl. VII, fig. 4 de mos planches, n'apjartient pas à l'espece ainsi nommée par Sowerby; elle est probablement une déformation du Sp. bisulecutus. ")

Sp. rulcurutus de Lion. du Morecun ${ }^{(10)}$. - M. de Koninck a assimilé, en notre présence, à la suite d'un examen superficiel et troj) rappirle, nos immombrables Sp. Tornucensis de La Varville, a l'espece irlantaise, rayée de la science par Davidson. Or, les caractères de la cogpille du Morvan ne permettent de la rapprocher ni du jeune áge du Sp. Verneuili, ni meme dn Sp. bisulenus. Leur étude alprofondie nous a conduit, au contraire, à l'assimiler l'une manicre alsolue au Sp. Tornuensis du carbonifere inférieur de Belgique, et cette ditermination se troure amplement justifiéc par l'étude de la riche faune qui l'aceompagne of rui est exclusivement tommaisienne.

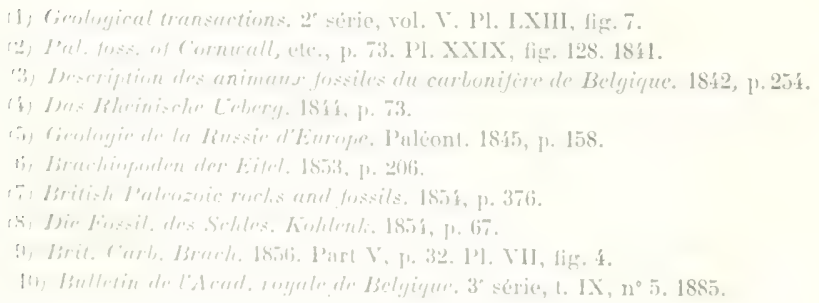


III. Spirifer Rømerianus, DE KonINCK, 184?.

I'. I, 1i-1. 1:, 1:3\%.

Caracteres spescifiruese - Coruille de taille movenne, de forme trimngulaire. L'aréa assez étroite, ì bords subparalleles, assez tranchants, oceupant la plus

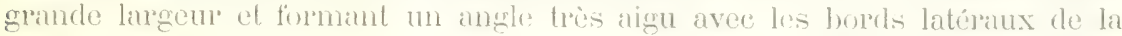
coquille.

La valve ventrale est plus profomde que la dorsale et garnie d'un sinus relativement assez large, borelí de chaque côté d'un pli plus épais et plus saillant que ceux qui garmissent le reste de la surface. Ceux-ci sont an nombre de S ou 10 de ehargue côté du sinus et du lobe médian de la valve dorsite.

J'ai remareque que plusieur's spécinens ont leur sinus grarni d'un pli médian rudimentaire (voir Pl. XXIX, fig. 23 et 27), qui se traduit souvent par un sillon ou par un simple aplatissement au sommet du bournelet de la valve dorsale. Le erochet de la valve ventrale est assez aigu et fort reconter. La surfince des bons échuntillons est couverte d'une quantite innombrable de fines lamelles d'aceroissement, imbriquées, concentriques.

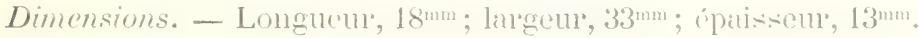

Gisements. - Cette espere ne se trouve gue dans le caleschiste de Tournai, oir elle est assez rare. - De Koninck (1). - La Varville (Morvan).

Explication des figures. - Pl. I, fig. 13 b. Empreinte en crens de la partie umhonate des valves. - La Varville; assez rare.

Fig. 13. Moulage de la mème.

IV. Spirifer crassus, DE KonINCK, 1843.

Non fignné.

Caracteres spécifiques. - Transversalement ovale; valves presque sigalement converes of quelque peu ponthes; ligne eardinale plus eomple que lat plus grande largeur de la eoquille; aréa triangulaire de langenr modérée, angles cardinam aremdis. Crochet petil, incurve, peu prominent. Le boureted médian n’est que peu élexé an-dessus des portions latérales de la valve; sinus

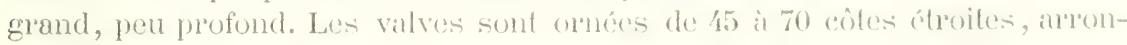

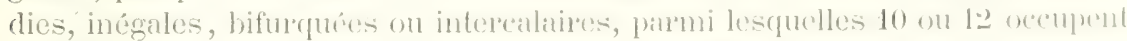
la surface du bourrelet at du sinus. - Davidson (2).

Gisement. - L'Ardoisiere; tress mare.

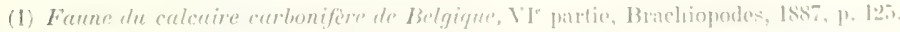

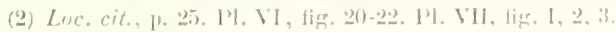


V. Spirifer bisulcatus, SOWERBY, 18:5.

PL. IT, fig. 8 et 9 .

Historique. - Espèce créce par J. de C. Sowerby, sous le nom de $S_{p}$. bisulcatus (Mineral Conchology, pl. CDLXLI, fig. 1 et 2, 1825), mais déerite d'abord sous le nom spécifique de Sp. trigonalis Sowerby (non Martin), et figurée dans Min. Conch., pl. CCLXV, fig 2 et 3, 1820.

Diagnose de Sotcerby : Coquille somi-circulaire, renflée, sillonnée verticalement. Bord inférieur légèrement saillant, avee un sillon de chaque côté du bourrelet. Charnière longue, droite. Crochets très rapprochés (1). ”

Curacteress spécifiques. - Coquille semi-circulaire ou subrhomboïdale, ordinairement plus large que longue, avee des valves presque également convexes. Li ligne cardinale est, en général, plus longue que la plus grande largeur de la copquille, les extrémités cardinales étant arrondies en formant des angles d'ouverture variable. Aréa modérément larege, divisée par une fissure triangulaire, qui est 'n partie termée par un pseudodeltidium. Crochets recourbés et parfois connidénblement rapprochrs. Le sinus présente une profondeur modéréc; le bonrrelet, rigulièrement arrondi, n’est pas très élevé et ne dépasse grupro lr nivean des partics latérales. Chaque valve est ornée de 30 à 40 côtes obtusiment anrondies; (elles sont simples et rarement bifurquées, mais s'ac-

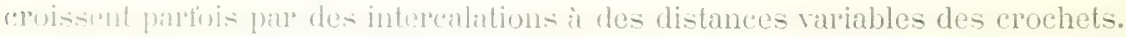
Luscoles du boureret sont disposées en trois groupes, séparés par des sillons plus protionds.

Dinnensinns tres variables. - Davirtson (2).

Gísement. - L'Archisiere; trés commun et de grande taille. Il y est associé it d'énormes

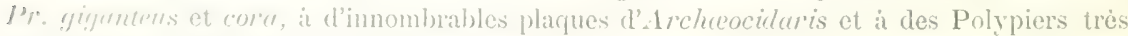
nembrenx. - Regny, Saint-Germain-Laval.

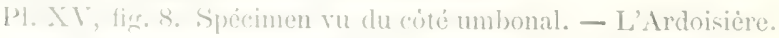

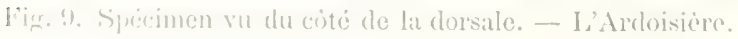

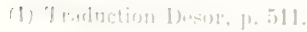

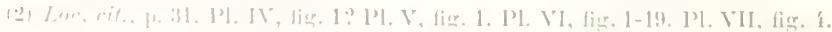


V. Spirifer distans, J. DE C. Sowerny, 1825.

P. I, tig. $16,17$.

Mistorique. - Espice eréée par Sorverby : "Coquilic semi-cireulaire, enflée, sillomée verticalement. Aire médiane de la valve droite enflée, concave au milien, et s'étendant jusqu'au crochet. Crochets recourbés, distants. Esparer entre les crochets triangulaire, arqué (1). ")

Caracteres spécifiques. - Très variable de forme et de proportions, le phu souvent imparfaitement rhomboidale et transverse, avec des valves incigalement conrexes. Ligne cardinale aussi longue que la plus grande largenr de lir coquille.

Valve dorsale plus ou moins convexe, mais non gibbeuse, en ginéral; semicireulaire et quelque peu cehancrée au front. Les bords latiraux forment mure courbe convexe, à partir d'une courte distance des angles cardinam, lesfuels sont sourent prolongés en temminisons aiguës. Le bonrelet est large, mais peu élevé; sa surface supérienre étant très aplaties avee un sillon Iongitudinal s'étendant le long de son milieu ou tont à fait jusqu'au linont, ou se convertissant bientôt en une côte centrale arrondie. Les parties latérales de la coquille, de chuque côté du bourrelet, sont ornés de 14 à 15 côtes petites, simples ou bifurequées.

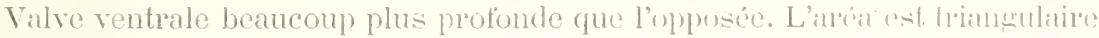
et variable dans ses proportions aussi bien que dans son développenonent. Tantôt elle présente une grande surfice aplatic, à angle droit arece le nivean de la valve dorsale, tantôt elle est plus on moins concave ab blevie, montrint ou non une portion du crochet antedessus et an delit de sa forminatison angulaire. La fissure est itroite ot en grande partie converte par un preudodelticlium eonvexe.

Le sinus est de profondeur modérée, et, en général, montre le longr du centres une côte bien dúlimitée qui est plus grande que celles qui ornent les deux

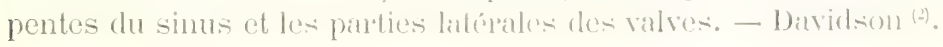

Gisement. - Le Varville; tress rare. Sur plusients millier's d'empreintes fossiles de cette localité, je n'ai recueilli que les teux spécimens figurés.

Explication des figures. - P’. I, fig. 16. Fragment de l'arcia et du foramen. - Lal Varville.

Fig. 17. Moulage de l'aréa de la ventrale d'un autre specimen. Cet cichantillon est iston-

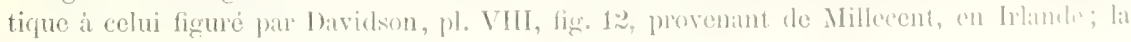

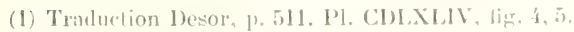

(2) Loc, cit., p. iti. Pl. VIII, tig. 1-17. 
hauteur et la largeur de l'aréa sont les mêmes, ainsi que la hauteur et la largeur à la base du foramen. Il en est de mème de la courbure du crochet.

Nota. - En Belgique, M. de Koninck ne le signale qu’à Drehance (étage II), où il est très rare.

VII. Spirifer integricosta, Phillips, 1836.

Pl. VIII, fig. 9 bis.

Historique. - L'espèce a été créée par Phillips, mais elle avait été figurée précédemment par Martiu sous le nom de Conchyliolithus anomites rotundatus (1).

Diagnose de Phillips : "Crochets très raprochés; côtes rayonnantes, très obtuses, entieres (2). $)$

Caracteres spécifiques. - Transrersalement ou longitudinalement ovale, presque cireulaire quand elle est jeune; ligne cardinale plus courte que la plus grande largeur de la coquille.

La valve dorsale n'est pas tout ì fait aussi profonde que la valve opposée, et onnée de 21 ì 25 cites arrondies, simples ou bifurquées, dont ies trois plus mpandes, on cites centrales, composent le bourrelet, qui n'est que peu saillant nu-dessus de la convexitó rérgulièe de la valve, excepté au voisinage du front.

Salve ventrale convexe; erechet de dimensions, proportions et ineurvation modirécs. Lin sultiue est ornée de 20 à 24 côtes arrondies; le sinus s'étend de l'extrimiti du crochet jusqu'an front, et varie à la fors de profondeur et de linmentr dans les diffirent- spécimens. Aréa plus laroge que haute, divisée par

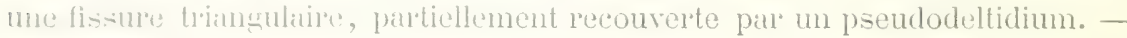
Disilsill 3 .

Gisements, - Saint-Grmain-Laval, liégny, l’Ardoisière, Néronde.

Érplimution des figures. - Pl. VIIt, fig. 9 bis. Valve klorsale. - Régny.

VIII. Spirifer Urii, l'LEMNi, 18:28.

I'l. I, Ii: 11,15 .

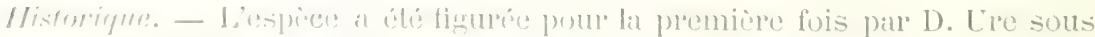

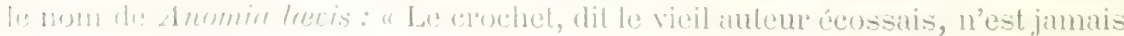

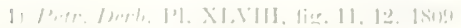

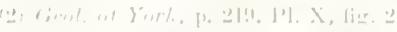

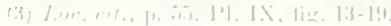


perforé et le bord cardinal est droit. De très beaux spécimens de cette coquille existent en très grand nombre dans une carrière de pierre ì chaux, ouverte sur la rive gauche de l'Aven, un peu plus bas que Strathaven. Ailleur's, autant que j'ai pu m'en assurer', ils sont très rares (1). ")

L'espèce a été créée par Fleming (2), et dédiće par lui à Ure. Voici la diagnose qu'il en a donnée:

"Coquille lisse, un sillon médian sul chaque valve, se terminant au front " par une étroite ondulation du hord; valve imperforée, presque plate, avec " une étroite gibbosité au crochet. Valve perforée, pourvue d'un erochet "gibbeux, allongé et recourbé. Charnière (hinge) épaisse, courte. "

Davidson a donné une excellente description de cette espèce; on peut y l'ecolurir (3).

Gisement. - La Varville; très rare.

Explication des figures. - Pl. I, fig. 14. Valve ventrale. - La Varrille.

Fig. 15. Valve ventrale. - La Varville.

GFARE MARTINIA, M'CoY, 1811.

I. Martinia glabra, MARTin, 1809 .

Pl. VIII, fig. 5 .

Pl. XV, fig. 7 .

Historique. - L'espèce a été crécée par IV. Martin sous le nom de "Conchyliolithus anomites glaber, transversim ovalis, levois, natibus approximatis, margine sinu obtuso(4). "

Le Sp. glaber a été décrit par tous les auteurs qui ont écrit sur la finune carbonifère: Sowerby, Phillips, Fleming, L'Coy, de Koninck, de Verneuil, ete. Nous nous bornerons à mettre sous les yeux du lecteur la diagnose de Davidson.

Caractères spécifiques. - Très variable de forme et de proportions; transversalement ovale, rarement aussi longue ou plus longue que large. Vialves presque également convexes, avec un bourrelet sur la dorsale et un sinus sur la ventrale. Ligne cardinale beaucoup plus courte que la plus grande largenr de la coquille; angles cardinaux arondis; crochets plutôt rapprochés; celui

(t) The Hist of Ruther, 1793, p. 313. Pl. XIV, fig. 12.

(2) Britiste animals, 1828, 1. 376.

(3) Loc, cit., [1. 58. 1'l. XII, fig. 13, 14.

(4) Petr. Derb. Pl. XLVIII, fie. $y$ et 10. 
de la ventrale proéminent, recourbé et de dimensions modérées. Aréa cardinale dans la dorsale; celle de la ventrale triangulaire et de dimensions modérées, avec des bords latéraux plus ou moins nettement limités; foramen partiellement recouvert par un pseudodeltidium.

Le bourrelet de la dorsale est, ou légèrement et uniformément convexe, s'élevant graduellement des parties latérales de la valve, ou brusquement dressé avec une dépression longitudinale le long de son milieu, qui se trouve parfois aussi reproduite dans le sinus de la ventrale. L'appareil spiral est grand et occupe la plus grande partie de l'intérieur de la coquille. La surface des valves est en général lisse, mais parfois quelques côtes obscures, arrondies, peuvent être observées sur les portions latérales. - Davidson (1).

Gisements. - Régny, Saint-Germain-Laval, Néronde, l'Ardoisière.

Cette espèce est excessivement commune ì Régny, et rare ì l'Ardoisière.

Explication des figures. - Pl. VIII, fig. 5. Valve ventrale montrant bien le sinus, engagée dans le marbre. - Régny.

PI. X V, fig. 7. Spécimen vu du cóté de la dorsale, et montrant toute la région cardinale. L'Ardoisière.

II. Martinia lineata, MARTin, 1809.

P. II, fig. 9, 10, 11, 12, 13.

Pl. VIII, tig. 6.

IIistorique. - Cette espèce est aussi très répandue dans le carbonifère marin du monde entier. Elle a été créée par W. Martin sous le nom de Conchyliolithus anomites lineatus dans son onvrage sur les fossiles du Derbyshire, pl. XXXVI, fín. 3. Voici la derepiption qu’il en domne:

"Transoersim ooulis, decussutim striatus : striis longitudinalibus confertis, subtilissimis; trunscersis remotioribus subelevatis majoribus, margine integro. ")

Davidson a reconnu deux variétés principales dans cette espèce :

Lne variéte $\alpha$. Sp.lineata Martin $=$ imbricata Sowerby $=$ Martini Fleming $=$ reticulata $\mathrm{I}^{\prime} \mathrm{Coy}$;

tet whe variété $\beta$, Sp. elliptica Phillips.

Je rowis que nous n'ivons pas la variété elliptice dans le Plateau Central; nous nous bormorons in reproduire la diagnose de la variété lineata.

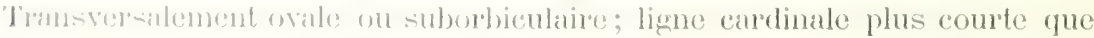

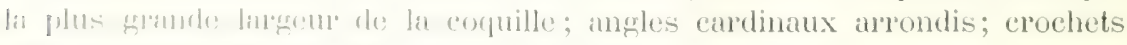

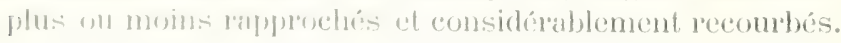

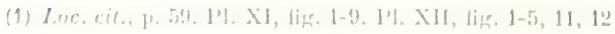


Valve ventrale modérément et uniformément convexe, montrant rarement ini bourrelet.

Vialve dorsale plutôt plus profonde que l'autre, uniformément convexe ou offrant une légère dépression, visible seulement au voisinage du front, ou s'étendant jusqu'i l'extrémité du crochet.

Aléa petite avee des bords latéraux obscurément limités. Foramen triangulaire, particllement recouvert par un pseudodeltidium.

Surface des deux valres marquée de lignes nombreuses, irrégulièrement imbriquées; les stries rayonnantes étant tantôt si rapprochées qu'on ne les distingue qu'avee difficulté, tantît variant dans leur degré de proximité, mais rarement séparées sur un point queleonque de plus d'une ligne (2mä20). Les lignes conecntriques varient ágalement de la même manière, mais sont, en général, plus largement séparées; difficiles à distinguer dans quelques spécimens, elies forment, dans d'autres, des côtes fortes, larges, aplaties, ou légèrement arrondies. - Davidson (1).

A cette deseription nous ajouterons les quelques lignes suivantes que Davidsun a consacrées au lineatus dans l'Appendice (2) : Lors de ma description du $S_{p}$. lineatus, je n'avais pas encore vu d'exemplaire dont la coquille fuit parfaitement conservée; mais la découverte ultérieure de quelques spécimens excellents d'Ecosse, ainsi que de l'Inde et d'Amérique, m'a montré que, à l'extérieur, la eoquille citait couverte de nombreuses cotes concentriques, rarement éloignées de plus d'une ligne, mais habituellement très serrées, et de chacune d'elles se projetaient des épines nombreuses, serrées, formant une série de franges épineuses, qui se débordaient l'une l'autre, sur toute la coquille. Lorsque les épines étiient absentes, ce qui est l'état général dans lequel on trouve la coquille, la surface semble marquée de lignes nombreuses, irrégulièrement imbriquées, les unes rayonnantes, provenant des petites élévations d'où sort chaque ápine, comme je me suis elforéé de le démontrer dims la figure grossie de la pl. LI, ce qui est très différent de la mancire irréguliere suivant laquelle les épines sont dissémines sur la sunfice de Sp. Urii, dont la fig. 15, pl. Li, est une reproduction."

Gisements. - Le Morvan (excessivement commun'; Regny (id.); l'Ardoisiere (assez rare).

Explicution des figures. - Pl. II, fig. 9 et 10. Moulage naturel de la carite interne d'une coquille complète.

Fig. 9. Vue du crité dorsal.

Fì. 10. Vue du còté ventral.

Fig. 12. Empreinte exteme vue du cité ventral.

Fig. 11. Moulage de la méme.

Les spécimens des fig. 9, 10, 11 et 12 sont déformés par pression; ils aplartiennent an mème individu.

(1) Loc, cit., 1. 62. 1'1. X111, fig. 1-13.

(2) Loc. cit., prart. V, Appentix, p. 225, 1'L, LI, tier. 15. 
Fig. 13. Empreinte externe d'un fragment de valve dont les bandes concentriques et les trous des épines sont trẻs nettement visibles.

Tous ces échantillons sont de la Varrille (Morran).

Pl. VIII, fig. 6. Coquille vue du côté de la face ventrale. - Régny.

I. Spiriferina cristata, variété octoplicata, J. DE C. Sowerby, 1827.

Non figuré.

L'espèce a été créée par Sowerby qui en a donné la diagnose suivante : "Coquille allongée, renflée, semi-circulaire, plissée, environ 8 ou 10 gros plis anguleux. Renflement médian de la valve droite uni; crochets distants. Dépression de la valve gauche triangulaire, recourbée (1). - Traduction Desor. »)

Voici en outre la diagnose de Davidson qui considère cette espèce comme une simple variété de Spiriferina cristata : "Transversalement subrhomboïdale. Valves presque également convexes et parfois plutôt gibbeuses. Ligne cardinale aussi longue que la plus grande largeur de la coquille. Angles cardinaux aigus ou légèrement arrondis. Aréa coneave, triangulaire et de variable épaisseur. Fissure partiellement courerte par un pseudodeltidium. Crochets petits et recourbés. Le bourrelet de la dorsale est le plus souvent composé d'une seule côte qui est beaucoup plus large que celles situées sur les parties latérales de la coquille; sa crête est en général arondie du crochet jusqu'à la moitié de sa longueur environ; alors elle s'aplatit de plus en plus à mesure qu'elle approche du borrl frontal, mais parfois elle reste anguleuse durant toute sa longueur aree une tendance a la formation d'un pli rudimentaire sur chacun de ses flanes, si bien ([ue dans ces cas plus rares, le bourrelet revêt vers le front une appurence obscurément triplissée.

" Le sinus de la ventrate est profond, aigu et généralement simple, mais anssi plus rarement interompu par une côte rudimentaire qui devient visible an voising gre du front. I.es valves sont ornées de 8 à 12 côtes anguleuses qui sont, comme le sinus ef le bourrelet, traversées par des lamelles concentriques Iré- lapprochées, disposées comme des écailles (2). )

Gisement. - I.Ardoisiere.

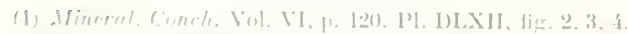

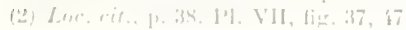


II. Spiriferina insculpta. PHillips, 1836.

Pl. XI, fig. 21.

Pl. IX, fig. 7 b.

Caractères spécifiques. - Coquille d'assuz petite taille, généralement subsemicirculaire, abstraction faite du crochet de la valve ventrale qui, d'ailleurs, s'élève peu au-dessus du niveau de la valve opposée; sa largeur dépasse souvent d'un tiers sa longuenr'; les deux dimensions sont dans le rapport de 100: 120. Ligne cardinale droite, occupant le plus grand diamètre de la coquille; aréa très développée, régulièrement triangulaire, plane ou très faiblement courbée; ouverture deltoïde large. Valves à peu près également convexes. La valve dorsale est assez généralement garnie de 5 gros plis carénés, dont celui du milieu, servant de bourrelet, est un peu plus développé que les autres et correspond au large et profond sinus de la valve opposée. La valve ventrale est ornée de 6 plis semblables, qui, de même que les préeédents, sont tous chargés de minces lamelles imbriquées et concentriques d'aecroissement, donnant aux valves un aspect particulier qui peut servir à faire reconnaître l'espèce. Le test est finement perforé. - De Koninck (1).

Gisements. - Régny, Néronde, Montmain, Ferrières (dans le grès), l'Ardoisière. Cette espèce est assez commune.

Explication des fiyures. - Pl. XI, fig. 21. Moulage d'une dorsale. Montmain; assez commune dans ce gisement.

Pl. IX, fig. 7 b. Echantillon de Néronde, présentant à cóté de Orthothcte's crenistria, une valve dorsale de Spiriferina inseulpta.

III. Spiriferina partita, PORTLOCK, 1843

Pl. II, fig. 14, 15.

Curactères spécifiques. - Petite coquille mesurant $7^{\mathrm{mm}}$ de long et $12^{\mathrm{mm}}$ de large. Valve ventrale avec un profond sinus, muni d'un léger pli au fond.

Valve dorsale avec un bourrelet arrondi, offrant an centre un sillon linéaire le rapprochant du Spirifer pingens. Les sillons entre le bourrelet et les plis latéraux sont fortement marqués. Le nombre de ces derniers est variable, il est tantôt de 3 , tantôt de 6 de chaque côté.

Forme générale arrondie, se rapprochant de celle du Spirifer speciosus.

(1) Brachiopodes, p. 99. P'l. XXII, fig. 51-55. 1887. 
Localité. - Kildress en Irlande. - Portlock (').

Obsercation. - De Koninck a cru devoir assimiler la Sp.partita Portl. à la Sp. acuticostata, qu'il dit avoir découverte, en 1843, dans le calcaire de Visé (Etage III), où elle est très rare (2).

Gisement. - Siguret.

Explication des figures. - Pl. II, fig. 15. Dorsale montrant nettement le sinus médian du bourrelet. - Siguret.

Fig. 14. Moulage d'une ventrale de Spiriferine probablement de Spiriferina partita.

\section{Spiriferina peracuta, DE KoNINCK, 1887.}

Pl. II, fig. 16.

Caractères spécifiques. - Coquille d'assez petite taille, beaucoup plus large que longue, de forme subrhomboïdale; ligne cardinale droite représentant lo plus grand diamètre transverse de la coquille; extrémité cardinale très aiguë; bords latéraux presque droits et formant de chaque còté avec le bord cardinal un angle terminal de 40 is $50^{\circ}$.

Valve dorsale moins profonde et moins convexe que la ventrale, garnie d'un bourrelet médian, le double plus épais que les plis adjacents qui sont au nombre de 7 de chaque côté et dont l'épaisseur diminue progressivement en se rapprochant des bords; ce bourrelet est ordinairement déprimé dans son milieu.

Valve ventrale beancoup plus profonde et un peu gibbeuse; son sinus est large et profond. Crochet pointu et fortement recourbé. Aréa ereuse et assez blevée; sa surface est presque lisse et marquée de quelques stries transversales peu visibles à l'oxil nu; la fente deltrïdale est assez étroite et bordée de chargue côté d'une petite rainure ayant probablement servi à recevoir un pseudodeltidium. La surfice ost garnie de minees et nombreuses lamelles concentriques, rógulièrement imbriquées.

Dimensions. - Longucur, 10mm ; largeur, 20mm ; rapport, $100: 200$.

Rirpports et differences. - Cotte jolie espece a de grands rapports avec Spiriferine octoplicate. Mais elle en differe par sa largeur relativement plus forte ut surlout par l'acuité deses angles cardinanx, ainsi que par la profondeur de (i) valve ventrale - De lioninck ${ }^{(3)}$.

'isiements. - La Varville, Sigurot (Morvan).

Erplication des figuress, - IPl. II, fig. 16. Valve ventrale. - La Varville.

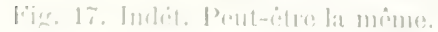

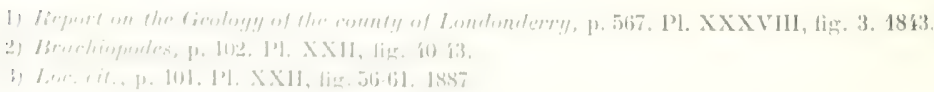


GeNRE RETZIA, KiNG, 1850.

I. Retzia ulothrix, DE Koninck, 1843.

Non figuré.

Caractères spécifiques. - Coquille de taille médiocre, à contour transversat lement ovale; valve dorsale moins convexe et moins profonde que la ventrale; celle-ci porte 10 ou 12 gros plis rayomants dont les deux médians sont les plus épais, les autres allant en diminuant de chaque côté jusqu'aux deux extrêmes, qui sont en partie efłacés. Los sillons qui séparent ces plis ont à peu près lì même largeur que les plis qui y donnent naissance; celui du milieu de la valve ventrale est le plus profond et tient lieu de sinus, tandis que le pli correspondant est un peu plus saillant que ses adjacents et fait fonction de bourrelet. Le crochet de la valve ventrale, assez fortement recoubé, ne dépasse pas le niveau de la valve dorsale; il est percé d'un foramen circulaire de moyenne grandeur'; son aréa est petite, triangulaire et étroite, son delticlium est peu apparent; li surface est souvent marquée de stries coneentriques ou de fines lamelles imbriquées, principalement sur les bords. - De Koninck (1).

Gisement. - La Varrille; un seul spécimen, non figuré.

II. Retzia Buchiana, DE KONINCK, 1843 .

Non figuré.

Carcctères spécifiques. - Très petite coquille allongée, sub-triangulaire, ì angles latéraux obtus et arrondis; valve ventrale un peu plus bombée et plus profonde que la dorsale, à crochet assez aigu, peu combé, presque droit, tronqué et terminé par une petite ouverture cireulaire; aréa petite, triangulaire, presque aussi haute que large; surface de la valve dorsale orkée de sept gros plis rayonnants dont le médian sert do bourrelet, et dont les autres conpespondent au même nombre de sillons de la valve ventrale; le sillon médian, fenant lieu de sinus, est un peu plus large et plus profond que les adjacents, qui vont en dimimuant vers les bords. - De Koninck (2).

Gisement. - L'Ardoisière; un seul specimen.

(1) Loc. cit., p. 92 . Pl. XX1I, fig. 1-4. 1887.

(2) Loc. cit., 1). 93. 1'l. XX11, fig. 59. 1887. 
III. Retzia (Acambona) serpentina, DE KonINCK, 1843.

PI. III, fig. 19 c.

Caractères spécifiques. - Coquille de taille moyenne, sub-ovale, oblongue, dont la plus grande largeur est située au tiers inférieur de sa longueur. Valves normalement convexes, et complètement dénuées de sinus et de bourrelet.

Valve ventrale, un peu plus profonde que la dorsale, terminée par un crochet peu recourbé, s'amincissant régulièrement, et dont les arêtes presque droites jusqu'au tier's inférieur', se recourbent et s'arrondissent à peu près en demicercle, en se joignant du côté du front. Le crochet est assez fortement tronqué et terminé par une large ouverture circulaire, en partie bordée par le deltidium, au-dessous duquel il existe une petite aréa triangulaire, dont la limite inférieure est due au bord supérieur des oreillettes qui terminent le sommet de la valre dorsale. La surface est ornée de minces côtes longitudinales aplaties, souvent superficielles, dont la largeur augmente lentement par l'accroissement, mais dont quelques-unes se bifurquent par division à une certaine distance de leur origine et finissent par se trourer au nombre de 60 ou 70 chez les individus adultes.

Le test est finement poncturé et les cônes spiraux sont courbés à leur base et composés de 11 ou 12 tour's de spire chez les individus ayant atteint une longreur de 20 à $22 \mathrm{~mm}$. - De Koninck (").

Gisement. - Siguret, près Savigny-Poil-Fol.

Explicution des figures. - Pl. III, fig. $19 \mathrm{c}$. Valve dorsale (figure mal éclairée, paraissant concave). Le spécimen présente une dépression médiane légère et très étroite, qui n'est pas indiquée dans la diagnose; ce caractère ne nous a pas paru suffisant pour séparer notre unique spécimen de Retzia serpentina.

Le méme échantillon présente en $\ell$ un Bellerophon sublicvis, et en $c$ des plaques nombrenses de Palwehinus Robineti.

GENIR ATHYRIS, M'CoY, 1844.

I. Athyris Royssii, Lévellé, 1835.

Pl. III, Iis. 16; 17 .

Dinegnesse de Líceille. - "Spririfer de Royssi : Coquille unie, valves relevées

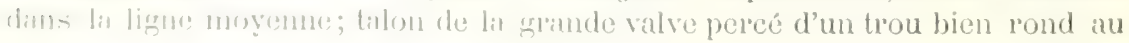


bord duquel vient se placer le sommet de la petite valve; spires intérieures très rapprochées l'une de l'autre dans la partie médiane et s'écartant ver's les extrémités (1).

Caracteres spécifiques. - Coquille circulaire, ou transversalement ovale, sub-globuleuse; crochet incurvé et tronqué par un petit foramen circulare, qui touche au crochet de la valve dorsale. Les valves sont presque également et uniformément convexes jusqu'à un certain âge, après lequel il se forme graduellement un large pli médian, plus ou moins élevé dans la valve dorsale, et un sinus correspondant dans la ventrale. Le bord frontal est, par conséquent, presque droit ou présente une courbure plus ou moins prononcée. La surface externe est rígulièrement couverte de nombreuses côtes écailleuses, concentriques, de chacune desquelles rayonnent des franges serrées, formées par des épines allongées, parfois aplaties.

Dans l'intérieur, la charnière est fortement articulée, les plaques dentales ou rostrales de la valve ventrale assurant, par leur position, beaucoup de solidité au crochet de cette valve. La plaque cardinale de la dorsale est perforée, près de son sommet, par une petite ouverture circulaire, au-dessous de laquelle se trouve l'extrémité des appendices spiraux réunis par un système de lamelles compliqué.

Dimensions et proportions relatives très variables. - Davidson ${ }^{(z)}$.

Gisement. - La Varville; très abondant.

Explication des figures. - PI. III, fig. 16, 17. Moule interne d'un spécimen.

Figg. 16. Vu du côté dorsal.

Fig. 17. Vu du côté rentral.

II. Athyris lamellosa, Lévellté, 1835.

Pl. III, figr. 15.

Historique. - Cette espèce a été coééc par Léroillé. Ello appartiont exclusivement à l'étage de Tournai; elle a été découverte également à Hook-Point, en Irlande, sur le même horizon, par de Koninck ${ }^{(3)}$.

Diagnose de Léveillé. - "Spirifor lamellosus : Coquille avee lames placées horizontalement dans le sens d'aceroissement. La grande valve inféricure presque toujours dépourvue de lames et ne conservant que de larges stries. Valves relevées comme dans le Spirffer (Athyris) Royssii, ver's la ligne

(1) Mín, de la S. G. de Frence. 'T. II, p. 39. I'l. II, fig. 18, 19, 20. 1835.

(2) Loc. cit., p. 84. P. XVHI, tig. 1-11.

(3) Arnales de la S. G. de Belyeique. T. 1X, p. 50, 188-82. 
moyenne. Trou du ligament bien rond, recevant à son bord le sommet de la petite valve. Spires intérieures semblables à celles du Sp. Royssii, paraissant attachées à la grande valve, dans laquelle Léveillé les a toujours vues (1). »

Caractères spécifiques. - Coquille d'assez grandè taille, à contour transversalement et assez régulièrement ovale; ligne cardinale presque droite; valves médiocrement convexes, à peu près également profondes; sinus de la valve dorsale ayant son origine à une certaine distance de l'extrémité du crochet. Peu marqué et peu profond d'abord, il se creuse el s'élargit assez rapidement à partir de la moitié de sa longueur et produit sur le front une courbe régulière et assez élevée chez les individus adultes; crochet petit et peu recourbé terminé par un foramen circulaire, reposant directement sur le sommet de la valve dorsale. Le bourrelet de celle-ci n'est pas bien limité, mais normalement convexe et non déprimé dans sa partie médiane. Toute la surface est couverte de lamelles concentriques, minces, presque également distantes les unes des autres sur la majeure partic des valves, à l'exception de celles des bords frontaux oì elles sont très nombreuses et très serrées chez quelques adultes. De Koninck (2).

Gisement. - La Varville.

Explication des figures. - Pl. III, fig. 15. Empreinte interne des deux valves.

Le même échantillon présente á cóté de la ventrale, une ventrale de Discinc nitida.

III. Athyris planosulcata, Phillips, 1836.

Pl. VII, fig. 19.

P'. VIII, figr. 10.

P. X, tig. (j.

Ilistorique. - Fapece crée pro l'hillips qui en a domé la diagnose suivante: "Forme pentagonale, cléprimée, milieu de chaque valve muni d'un sinus plial (3). )

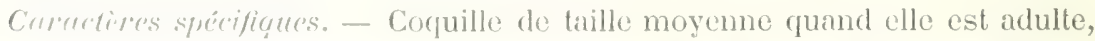
maí- qúnculement assoz petite et sublenticulaire, la longueur dépassant légèremmut la lirreur. Les valves sont fuiblement convexes et ì peu près également profinder, quelquefois légerement inflechies ou déprimées sur le front. Le

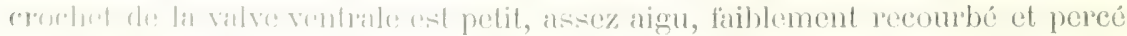

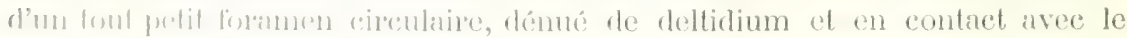

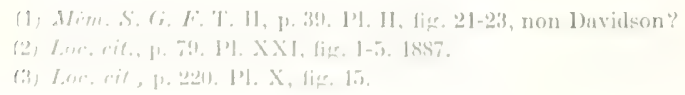


sommot de la valve dorsale. Cette ouverture, qui n'est pas toujour's bien distincte, semble quelquefois laire défaut, surtout chez les individus très adultes.

Lil surface de chacune des deux valves est chargée d'un grand nombre d'expansions lanelliformes, qui semblent avoir pour origine les petits vides concentriques dont la coquille reste couverte apres la disparition des expansions; celles-ci semblent aroir úté formées de membranes extrêmement minces, et probablement transparentes, servant à maintenir en place les longues aiguilles rayonnantes ef effilées qui entourent toute la coquille, à l'exception du sommet.

Dimensions très rariables. - De Koninek ${ }^{(1)}$.

Gisements. - Saint-Germain-Laval, Régny, Montmain, l'Ardoisière.

Explication des figures. - Pl. VII, fig. 19. Valve ventrale aplatie. - Régny.

Pl. VIII, fig. 10. Deux spécimens, l’inférieur vu du cóté de la ventrale, le supérieur du cóté de la dorsale. - Régny.

Pl. X, fig. 6. Spécimen de plus grande taille, vu par la ventriale. - Saint-Germain-Laval.

Observation. - Cetto espèce est très rejpandue à l'Aldoisière, oiz elle est associée à Athyris ambigua. Quelques spécimens ont conservé les expansions lamelliformes et fibreuses gui forment une large auréole it la coquille. Ces échantillons ont été détruits dans un incendie avec beaucoup d'autres, et n'ont pu ètre figurés.

IV. Athyris ambigua, J. DE C. Sowerby, 1822.

Non figuré.

Historique. - L'cspece a été créée prà Suwerby. Comme elle est excessivement répandue dans le monde entier, elle a été suceessivement déerite par plus de vingt auteurs différents. Nous nous bornerons à donner la diagnose du créateur et celle de de Koninck, qui est la dernière en date.

Diagnose de Sowerby. - "Coquille subpentagonale, gibbeuse. Valve droite (dorsale) renflée au milieu. Crochets saillants, perforés. Bord curdinal très court (2)

Diagnose de de Koninet ${ }^{(3)}$. - "Coquitle de taille moyeme, ì contour plus ou moins pentagonal, ordinairement un peu plus lirge que longue, modérément convexe; valve ventrale munie d'un sinus longitudinal médian, bicn prononcé, s'étendant du crochet jusqu'au bord frontal; crochet reeourbé, peu saillant, pereé d'un petit foramen circulaire, en contact immédiat avec le som-

(1) Loc. cit, p. 86. P1. XXI, lig. 16-32. 185\%.

(2) Loc. cit., trad. Desor, p. 397. 1'1. CCCLXXV1.

(3) Loc. cit., p. 75. P1. XIX, fig. 26, 27, 30-38. 1887. 
met de la valve opposée. Je n'ai pas aperçu de trace de deltidium. Un bourrelet moins prononcé et moins long existe sur la valve opposée, qu'il rend onduleuse, et quelquefois nettement trilobée; ce bourrelet est ordinairement divisé dans sa partie supérieure par un étroit sillon médian; front profondément ondulé. Surface presque lisse, et uniquement ornée de fines stries concentriques d'accroissement. »)

Gisement. - L'Ardoisière; assez abondant.

V. Athyris ingens, DE KonINCK, $188 \%$.

Pl. X, fig. 4.

Caractères spécifiques - Grande coquille ovale, beaucoup plus large que longue, gibbeuse; valves à peu près également profondes au centre; dans le jeune îge elles paraissent être assez régulièrement convexes l'une et l'autre, mais vers le milieu de leur croissance il se produit sur la valve ventrale un sinus médian plus ou moins large et plus ou moins profond, correspondant à un bourrelet plus ou moins elevé de la valve dorsale, dont les régions latérales sont alor's fortement inclinées vers les bords, surtout aux environs du front.

Le crochet de la valve ventrale est très épais et modérément recourbé; il est pereé d'un foramen circulaire asscz médiocre. La surface est à peu près lisse; on n'y aperçoit qu'à l'aide d'un instrument grossissant de fines stries longitudinales, très superficielles et ne domnant point lieu à la production d'ornements lamelliformes ni spiniformes semblables à ceux qui couvrent la surface des A. Royssii et ornuta. - De Koninck (1).

Gisenent. - Saint-Germain-Laval.

Erpulicution des figures. - Pl. X, fig. 4. Valve dorsale.

(Jbserection. - Cette espece a été découverte par M. Dupont dans le calcaire de Furfooz et des l'anjuys (étage de Waulsort).

(1) Brrechiupodes, p. 83. I'1. XX, fig. 1-10. 1887. 


\section{FAMILLE DES RHYNCHONELLIDES}

Genre RHYNGHonella, F. DE WAldheim, 1809.

I. Rhynchonella acutirugata, DE KONINCK, 1887.

Pl. III, fig. 10, 11, 12, 13.

Cette Rhynchonelle, spéciale à Tournai, a été confondue par de Koninck, jusqu'en 1887, avec Rh. Pleurodon de Phillips. C'est la seule que nous ayons trouvée dans le Morvan.

Caractères spécifiques. - Coquille de taille moyenne, lor'squ'elle est adulte, sub-rhomboïdale, ou plus ou moins ovale dans son contour, gibbeuse à tout âge; ses valves sont ordinairement gamies de 5 ou 6 plis fortement recourbés. Le centre de la valve ventrale est assez fortement déprimé et largement sinué; le fond du sinus est faiblement bombé et garni de 3 ou 4 plis rayounants, limités par la surface latérale des plis courbes adjacents, beaucoup plus développée que celle de tous les autres.

Le crochet de la valve ventrale est assez aigu, peu développé et peu recourbé; son ouverture est petite et semble dépourvue de deltidium. La valve dorsale est fortement bombée et plus profonde que la valve opposée; le bourrelet, peu distinct, est composé de 3 ou 5 plis rayonnants, dont celui du centre est un peu plus large et plus élevé que les autres. Tous les plis de la surface sont remarquables par l'acuité de leur bord, qui occupe toute leur étendue, ayant son origine ì l'extrémité même des crochets. - De Koninck (1).

Gisements. - La Varville, commune; Siguret.

Explication des figures. - Pl. III, fig. 10. Fragment de la valve dorsale.

Fig. 11. Empreinte en creux d'une valve ventrale.

Fig. 12. Moulage de la mème.

Fig. 13. Empreinte interne d'une ventrale.

(1) Brachiopodes. Appendix, f. 141. Pl. XVl, fig. 1-14. 


\section{Rhynchonella pleurodon, Phillips, 1836.}

Pl. VII, fig. 18.

Diagnose de Phillips. - Transversalement ovale, crochet saillant, côtes aiguës s'étendant jusqu'au crochet, côtís très profondément réfléchis et dentés (1). )

Caractères spécifiques. - Transver'salcment ovale, rarement plus longue que large, de forme très variable; valves plus ou moins convexes, quelquefois très gibbeuses; crochet modérément saillant, recourbé et montrant un petit foramen circulaire sous son extrémité anguleuse; il est entouré et légèrement séparé de la ligne cardinale par un deltidium; bourrelet large, presque carré et possédant sa plus grande hauteur près du front; il s'abaisse alors brusquement pour rencontrer le bord correspondant de la valve opposée. Sinus de la ventrale de profondeur modérée; côtes nombreuses et anguleuses s'étendant sur la surface entière, uu nombre de 10 à 24 environ sur chaque valve, dont 3 ì 9 pour lo bourrelet, et 2 à 8 pour le sinus, mais 5 pour le bourrelet et 4 pour lo sinus sont le cas général. Les plis qui couvrent les parties latérales de la dorsale sont tres courbes, tandis que ceux de la ventrale sont presque droits avec leur's extrémités recourbées ver's le haut. Les côtes ont une rainure longitudinale le long de leur partie médiane à quelque distance du bord, - Davidson (2).

Gisements. - Régny, l'Ardoisière.

Explication des figures. - Pl. VII, fig. 18. Valve dorsale. - Régny.

Cet exemplaire est identique î celui figuré par Davidson pl. XXIII, fig. 9, et représentant un jeune spécimen de Settle (Yorkshire).

\section{Rhynchonella pugnus, MARTin, 1809.}

P'. VIII, fig. 4.

I'I. IX, fig. 8 et 9 .

Mistorique. - L'espèce a été figurée et décrite pour la première fois par Martin, sulus lo. $110 \mathrm{~m}$ de Conchyliolithus anomites pugnus subrotundus, longitudinaliter sulcutns: suleis obsolescentibus, valoa imperforate, gibbosa, margine undertos: undri rninerueplicata ${ }^{(3)}$.

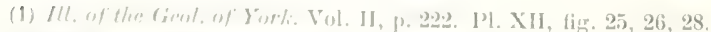

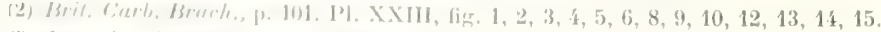

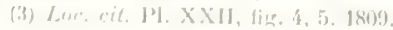


Caracteres spécifiques. - Coquille de forme très variable, transversalement ovale, on deltoïde aplatie, plus large que longue. Valve dorsale gibbense,

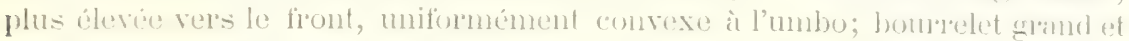
plus ou moins saillant.

Valve ventrale noins convexe que l'opposée, avec un sinus de profondeur modérée, commençunt ì une courte distance du crochet et s'étendant jusqu'au front. Crochet petit, très recourbé; for umen très petit, placé sous l'extrémité du crochet, rarement visible chez les coquilles adultes. Charque valve est ornée de 9 à 14 cotes qui deviennent obsolites en approchant des crochets; on en comprte 3 it 6 sur le bourrelet et le sinus. - Davidson (1).

Gisements. - Regny, Montmain, Néronde; assez commune dans tous ces grisenents. L'Ardoisiere.

Explication desfigures. - Pl. VIII, fig. 4. Valve dorsale montrant 3 plis au bourrelet; longueur de la coquille, $12^{\mathrm{m} m}$. Davidson a figuré pl. XXII, fig. 11, $11 a$, une jeune coquille de Clitheroe, identique à celle-ci. - Régny.

Pl. IX, fig. 8. Valve dorsale, identique á celle de Régny et de Clitheroe. — Néronde.

Fig. 9. Spécimen un peu plus grand (16 $\left.6^{\mathrm{mm}}\right)$, iclentique a celui de la fig. 14 de Davidson. Néronde.

\section{Rhynchonella angulata, LiNXÉ, $176 \%$.}

Non figuré.

Caracteres spécifiques. - Coquille de taille movenne, ordinairement plus: longue que large, subtrigonale ou cunéiforme, sa plus grande largeur se trouvant située à une petite distance de la ligne frontale. Les parties latérales des crochets des deux ralves sont fortement déprimées ef donnent lieu à la formation d'espaces arrondis plus ou moins creux, qui s'étendent jusqu'à environ la moitié de la longueur de la coquille. La valve ventrate est peu profonde et son sinus est composé d'un, deux ou trois gros plis anguleux; son crochet est pointu, peu recourbé et peréé d’un petit foramen, limité par un deltidium étroit. La valve dorsale est très convexe, ses côtés sont fort recourbés. La surface, dénuée d'ornements dans le principe, ne tarde pas à se ennvir de 6 ou 9 plis anguleux plus ou moins épais dont 2 on 3 forment le lobe médian, tandis cu'un, deux ou trois plis semblables, fortement recourbés, oceupent chacun les prortions latérales de da valve. - De Kionincki (2).

Gisement. - Rèny; très rare.

(1) Loc, cit., p. 97. Pl, XXII, fig. 1-15.

(2) Brachiogiodes, 1, 57. P1. XVI, fig. 39-60. 1887. 


\section{FAMILLE DES TEREBRATULIDÉS}

GENRE DIELASMA, KING, 1850.

I. Dielasma insigne, DE KonINCK, 1887.

PI. III, fig. 18.

Caractères spécifiques. - Coquille de taille moyenne, de forme ovale, plus longue que large et possédant sa plus grande largeur vers le milieu de sa longuems.

Valve ventrale peu profonde, régulièrement bombée, non sinuée; bold frontal faiblement tronqué; crochet peu recourbé, dépassant légèrement celui de la valve dorsale, tronqué presque horizontalement et percé d'une assez grande ouverture ovale, en forme de déversoir.

Valve dorsale, moins profonde que la valve opposée, mais aussi normalement bombée; commissure palléale ou frontale presque droite; commissures latérales faiblement et régulièrement courbées. La surface est presque lisse dans la majeure partie de son étendue et ne porte que de faibles stries concentriques d'accroissement ver's les bords. - De Koninck (1).

Gisenent. - La Varrille.

Explication desfigures. - PI. III, fig. 18. Empreinte, vue du cóté de la dorsale. Le crochet de la ventrale, intact au moment de la découverte, s'est brisé par accident avant la photographie. - La Varville.

\section{Dielasma sacculus, Martin, 1809.}

P. VII, fig. :20.

Ristorique. - Espuese figurée et décrite par WV. Martin sous le nom de Conchyliolithes unomites succulus, subscrotiformis lavis, margine obtuso, sinu , $1,1, \cdots$, 
Curactères spécifiques. - Coquille de taille généralement médiocre et n’atteignant jamais de grandes dimensions, de forme obovale ou subpentagonale, ordinairement un peu plus longue que large, plus ou moins profondément sinuée au front; valves plus ou moins renflées, ì peu près également profondes.

Valve ventrale munie d'un sinus médian concave, assez profond, ayant son origine vers le milieu de sa longueur, et ne s'étendant guère au delà du bord frontal de la valve dorsale; celle-ci est normalement convexe ou légèrement déprimée dans la région du front. Les commissures latérales sont obtuses et arquées; la commissure frontale est plus ou moins arquée selon la plus ou moins grande profondeur du sinus de la valve ventrale. Le crochet est modérément recourbé et percé d'un foramen assez étroit. La surface est lisse ou marquée de quelques lignes concentriques d'accroissement. - De Koninck (1).

Gisement. - Régny.

Explication desfigures. - Pl. VII, fig. 20. Valve ventrale; le sinus est très net, le spécimen est identique i celui de la pl. I, fig. 29 de Davidson, provenant de Park Hill, Longnor; Derbyshire. - Régny.

III. Dielasma hastatum, J. DE C. SowerBy, 1824.

Non figuré.

Caractères spécifiques. - Coquille allongée, de forme subovale, à bord frontal plus ou moins tronqué et faiblement échancré. Son grand diamètre transverse est situé vers le milieu de sa longueur. Valve dorsale régulièrement voûtée du côté du erochet et faiblement déprimée au côté opposé dans sa partie médiane, de façon à y produire un large sinus peu profond, limité à droite et à gauche par un léger renflement de la coquille. Valve ventrale semblable à la précédente, mais un peu plus épaisse et garnie d'un sinus médian un peu plus profond, ayant son origine près du erochet; la commissure latérale des valves ne produit qu'une ligne faiblement inftéchie du côté de la valve ventrale; la commissure frontale, légèrement échancrée en avant, a une direction à peu près droite vue de face. Le crochet de la valve ventrale est épais, rigulièrement recourbé et dépasse un peu celui de la valve opposée; comme celui d'un grand nombre d'espèces congénères, il est orné de chaque côté d'un móplat oblique, provenant d'une dépression latérale de ses bords; son ouverture, oblique d'avant en arrière, est assez grande et de forme longitudinalement ovale.

La surface est ornée de quelques rides concentriques plus on moins épaisses et plus ou moins irrégulières, dont les plus fortes se remarequent vers les bords. - De Koninck (2).

Gisement. - L'Ardoisière.

(1) Brachiopodes, p. 27. PI. VI, fig. 14-17, 19-26, 35-45, et P'. VII, fig. 60-77. 1857.

(2) Brachioporles, p. 9. Pl. III, fig. 1-26; Pl. IV, fig. 19-22, et Pl. IV, fig. 23-25 (variútes). 1857. 


\title{
ORDRE DES INARTIGULÉS
}

\author{
FAMILLE DES CRANIIDÉS
}

\section{GenRe GRANIA, Retzius, 1781.}

Nous avons trouvé un spécimen de ce genre, spécifiquement indéterminable. Gisement. - Régny.

\section{FAMILLE DES DISCINIDÉS}

\section{GENRE DISGINA, LAMARCK, 1819.}

\section{Discina nitida, Phillips, 1836.}

Pl. III, fig. 15.

Cette espèce a été créée par Phillips sous le nom de Orbicula nitida; voici la description qu'il en a donnée : "Ovale, glabre, finement radiée; valve inférieure plate, la supérieure conico-lenticulaire; sommet rapproché du bord, étroit (1).»

Caracteres spécifiques. - Coquille à contour circulaire ou ovale-allongée, la partie postérieure étant beaucoup plus étroite que l'antéricure. La plus grande valve ou valve libre at conoüle on patelliforme et plus ou moins élevée, le sommet saillant chant situé à des distances variables entre le centre et le bord pustérieur, mais il n'est pas toujours la portion la plus élevée de la valve. La surface est couverte de nombreuses rides ou stries concentriques, petites, irrégulières. La petite valve ou valve inférieure est parfois aplatie ou légèrement concave du côté du bord antérieur, elle offre un foramen ovale, entouré d'un bord ácé, convexe, qui s'étend du voisinage du centre de la valve jusqu'à une distance variable du bord postérieur. Cette valve est également ornée de côtes ru do rides nombreuscs, petites, irregulières et concentriques, séparées par des intervalles átroits, aplatis. - Davidson ${ }^{(2)}$.

Giscrument. - La Varville.

L. spúrimen que nous possédons est une valve inférieure, reproduite pl. III, fig. 15, à cóté de Alligris lamellosse.

(1) I.re. cil., fr. 2021. I1. XI, fig., 10, 11, 12, 13, 1836.

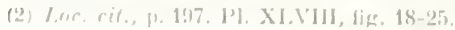




\title{
CLASSE DES BRYOZOAIRES
}

\author{
FAMILLE DES FENESTELLIDÉES
}

Genre FENESTELLA, Mrller.

I. Fenestella plebeia, M'Coy, 1844.

Pl. VI, fig. 1, 2.

Caractères spécifiques. - Colonie plate, étalée en éventail. Axes épais; fenestrules égales, de forme rectangulaire, deux ou trois fois plus longues que larges, largeur égale à celle des axes. Dissépiments minces, réguliers; 4 ou 5 cellules le long de chaque fenestrule.

Face opposée entièrement granulée et grossièrement sillonnée dans le sens de la longueur.

C'est une espèce très commune. - M'Coy (1).

Gisement. - La Varville.

Explication des figures. - PI. VI, fig. 1. Empreinte d'un fragment de colonie.

Fig. 2. Id.

Observation. - Cette espèce est excessivement commune chans le riche gisement cité plus haut. Les rameaux de la colonie sont ocreux et se détachent vivement sur le fond pále de la roche qui est un schiste décaleifie analogue à celui du domaine des Roches. Les cellules, indiquées par des points ocreux en saillie, se laissent compter avec la plus grande facilité, mais leur conservation réclame des soins très délicats, car ces points ocreux très friables disparaissent au moindre frottement, et l'étucle spécifique devient dès lors impossible dans le cabinet.

Les fragments représentés sont très petits, mais on peut s'assurer dans la carriere que cortaines colonies peuvent atteindre an moins $10^{\mathrm{cm}}$ de lanteur.

(1) Synopsis, p. 203. Pl. XXIX, fig. 3. 
II. Fenestella Morrisii, M'Coy, 1844.

Pl. VI, fig. 3.

Caractères spécifiques. - Colonie étalée, conique, parfois en forme de coupe; axes épais, arrondis, à dichotomie régulière; dissépiments très minces, se succédant à des distances égales; cellules grandes, séparées par des intervalles égaux à leur diamètre, entourées d'un bord saillant; environ 6 le long d'une fenestrule. Fenestrules légèrement irrégulières, habituellement quadrangulaires, un tiers plus longues que larges. - M'Coy (I).

Gisement. - La Varville.

Explication des figures. - Pl. VI, fig. 3. Echantillon de La Varville; rare.

III. Fenestella multiporata, M'Coy, 1844.

PI. XVII, fig. 5.

Caracteres spcifiques. - Colonie foliacée; axes minces se divisant d'une manière irrégulière, nettement carénés; dissépiments minces, distants; fenestrules grandes, très allongées, irrégulières; calices très nombreux, petits, à bord circulaire épaissi et au nombre de 7 ou 8 le long d'une fenestrule. La face postérieure régulièrement striée.

Le grand nombre de cellules cntre chaque dissépiment est la particularité la plus remarquable de cette espèce. Elle atteint fréquemment une longueur de 2 ou 3 pouces; il y a cnviron 3 axes par ligne. - M'Coy ${ }^{(2)}$.

Gisement. - L'Ardoisiere; rare.

Explication des figures. - PI. XVII, figc. 5. Fragment de colonie montrant les axes carénés et le nombe de pores indiqué diuns la diagnose.

IV. Fenestella ejuncida, M'Coy, 1844.

Non figuré.

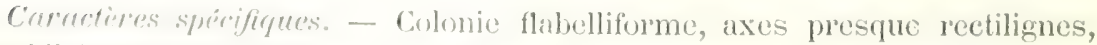
equidistants, frick minces; discépinents presque aussi épais que les axes, 
situés à des distances égales; fenestrules grandes, de taille et de forme presque égales, oblongues, légèrement plus longues que larges, environ trois fois plus larges que les axes; cellules petites, saillantes, placées alternativement une à l'origine de chaque dissépiment et quatre le longg du bord de la fenestrule: 7 à 8 axes dans l'espace de 3 lignes. - M'Coy (1).

Gisement, - L'Ardoisière; commune.

V. Fenestella membranacea, Phillips (2), 1836.

Non figuré.

Caractères spécifiques. - Colonie allongée, conique; axes droits, équidistants avec une carène centrale aiguë; dissépiments d'une épaisseur presque égale à celle des axes; ferrestrules allongées, un peu plus larges que les axes, presque trois fois aussi longues que larges; cellules petites au nombre de 3 environ le long d'une fenestrule; face opposée granulée.

L'espèce atteint souvent une longueur de 9 à 10 pouces; 5 axes occupant un espace d'une ligne environ. - M'Coy ${ }^{(3)}$.

Gisement. - L'Axdoisière; très rare.

Observation. - M. G.-W. Shrubsole ${ }^{(4)}$ a publié une rérision des nombreuses Fenestelles du carbonifère anglais; il n'en conserve que 5 espèces seulement, savoir :

Fenestella plebeia, M'Coy.

- crassa, M'Coy.

- polyporata, Phillips.

- nodulosa, Phillips.

- membranacea, Phillips.

La $F$. multiporata, est rapportée à $F$. polyporata. Quant à $F$. ejuncida M'Coy, il fait observer qu'on n'en possède encore que de petits fragments qui ne peuvent provisoirement être utilisés.

Nous avons cru devoir conserver les espèces des anciens auteurs, en raison du petit nombre, de la mauvaise conservation et de l'état fragmentaire de nos spécimens de la France centrale.

(1) Synopsis, p. 201. Pl. XXVVII, fig. 11.

(2) Geol. of York, p. 198. I'l. I, fig. 1-6.

(3) Synopsis, p. 202.

(4) Q.J. G. S. Vol. XXXV, n 138, p. 275. 1879. 
GENRE POLYPORA, M'COY, 1844.

Polypora Goldfussiana, DE Kosinck, 1842.

Non figuré.

Caractères spécifiques. - G. flabelliformi; ramis attenuatis, irregularibus; aperturis laxis, subocatis; cortice ad marginem dentatè.

L'espèce dont il est ici question nous a paru nouvelle, et c'est avec plaisir' que nous la dédions au savant professeur de l'Université de Bonn, à qui la science est redevable d'une foule de découvertes paléontologiques. Elle est surtout caractérisée par l'irrégularité avec laquelle ses rameaux s'étendent en éventail; l'eneroûtement corticiforme et mince, lisse au milieu et dentelé sur les bords. Elle est rare et nous ne l'avons trouvée qu'à Visé. - De Koninck (").

Observation. - M. de Koninck a reconnu formellement cette espèce parmi un lot de fossiles de l'Ardoisière, que je lui avais soumis en 1873, au début de mes recherches ${ }^{(2)}$. Cet échantillon unique a été perdu dans un incendie, et il m'est impossible aujourd'hui de l'examiner à nouveau.

Gisement. - L'Ardoisière; très rare.

\section{FAMILLE DES ACANTHOCLADIDÉS}

Genre Glaugonome, Lonsdale, 1843.

Glauconome pulcherrima, M'Coy, 1814.

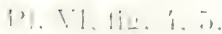

Conracteres spécifignes. - Tige portant des branches bipinnées; la tige et les principales loratches sont fortement carénées; les petites branches insérées lint sill lir lige? principale que sur les branches secondaires, s’atténuent régu-

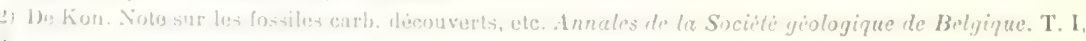


lièrement et sont privées de carène. Cellules grandes, à bord saillant, disposées sur une rangée de chaque côté do la tige et des rameaux secondaires et une rangée de très petites cellules sur la carcine; cellules des petites branches aussi grandes que celles des rameaux secondaires, mais plus rapprochées car il n'y a plus de carène entre les deux rangées.

Face opposée finement striée longitudinalement.

Gisement. - Blacklion, comté de Leitrim (Irlande). - $\mathrm{M}^{\prime} \mathrm{Coy}{ }^{(1)}$.

Gisement. - La Varville; assez commun.

Explicution des figures. - Pl. IV, fig. 4. Empreinte de plusieurs rameaux.

Fig. 5. Un des rameaux, grossi.

\section{FAMILLE DES PTILODICTYONIDÉS}

\section{GenRe PALAEOfLUSTRA, NOV. GeN.}

Palæoflustra Jolieti, Nov. SP.

Pl. XVII, fig. 4.

Nous avons trouvé à l'Ardoisière un Bryozoaire nouvenu que nous avons désigné sous les noms générique et spécifique ci-dessus, et qui nous paraît appartenir à la famille des Ptilodictyonida de Zittel.

Nous rappelons les caractères de cette famille:

Colonie comprimée, lamelleuse ou ramense, composée de deux couches de cellules tubuleuses, serrées, qui sont adossćes l'une à l'autre.

Notre spécimen ne pouvant rentrer dans aucun des genres connus de cette famille, doit par suite constituer un genre nouveau.

Diagnose. - Longueur du fragment de la colonie, 60 $0^{\mathrm{mm}}$; largeur, $7^{\mathrm{mm}}$; se dichotomisant deux fois d'une façon régulière, et les branches conservant la même largeur que la tige principale. Surface présentant de 12 it 14 sillons longitudinaux au fond desquels sont disposées les cellules. Dans un espaco de $5^{\mathrm{mm}}$ on compte 10 calices.

L'échantillon montre l'empreinte externe d'une des fiees du Bryozoaire; le

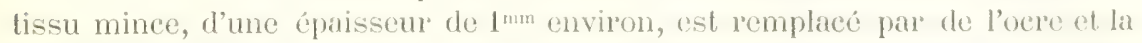
surface externe de cet ocre montre des calices de même grandeur et disprosís comme ceux de la face inféricure.

(1) Synopsis, p. 199. P1. XXvill, fig. 4. 
Gisement. - L'Ardoisière, un seul spécimen.

Je dédie cette espèce á mon regretté ami M. Joliet, ancien maître de conférences à la Sorbonne, dont la mort prématurée a interrompu les remarquables travaux qu'il avait commencés sur les Bryozoaires.

\section{FAMILLE DES CHETETIDÉS}

Genre MONTICULIPORA, A. D'Orbigny, 1850.

I. Monticulipora tumida, Phlllips, 1836.

PI. VI, fig. 9, 10.

Pl. XI, fig. 28.

Caractères spécifiques. - Polypier rameux, formé de branches cylindroïdes, ordinairement libres et divergentes, quelquefois anastomosées, de grosseur

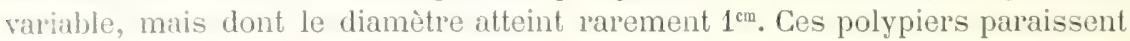
quelquefois comme encroûtants et formant plusieurs couches concentriques. Ordinairement les polypiérites ont leur origine au centre des branches et rayonnent obliquement vers la surface extérieure; comme ils sont prismatiques, il se fait que la forme de leur' calice affecte celle d'un losange ou d'un ovale, se disposant en quineonce dans les parties régulières du Polypier, surtout pendant le jeune âge; dans les parties, au contraire, où les polypiérites arrivent perpendiculairement it la surface, les calices ont une forme arrondie ou polyédrique plus régulière et nullement allongée, mais ils ont ordinairement alor's un diamitre tries variable.

Dans leur ctat normal, les calices ont leur bord légèrement épaissi; leur diamètre sist petit et n'a en moyenne qu'un quart de millimètre. - De Koninck (1).

\footnotetext{
Gismucnts. - Li Varville, Propières, l'Ardoisière.

Evplimetion des figures. - PI. VI, fig. 9. Empreinte d'un jeune individu. - La Var-

Fie. 10. Lat meme, grossie 4 fois.

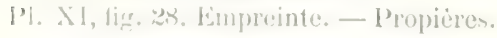
ville.

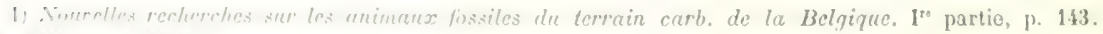

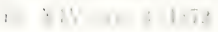


II. Monticulipora inflata, DE KonINCK, 1842.

Pl. XVII, fig. 12.

Caractères spécifiques. - Polypier de forme très variable, offrant ordinairement l'aspect de grosses branches irrégulièrement bosselées, à extrémités obtuses et arrondies. Quel que soit cependant le diamètre de ees branches, le Polypier proprement dit n'en occupe qu'une faible partie qui se présente sous la forme d'une couche très mince, d'une épaisseur égale dans toute son étendue, et n'atteignant généralement pas plus d'un $1 / 2 \mathrm{~mm}$.

Les polypiérites sont done extrêmement courts et ne possèdent pas cette disposition rayomnante qu'ils affectent dans l'espèce précédente. La surface du Polypier n'est nullement rugueuse et son aspect a quelque chose de celui du velours; elle est composée de la réunion d'une infinité de calices, de forme polygonale à angles plus ou moins effacés et qui, bien que ne présentant pas tous le même contour, ont très approximativement le même diamètre. Ce diamètre est très petit, et sur une ligne droite ayant $5^{\mathrm{mm}}$ d'étendue, j’ai compté 28 ('alices. - De Koninck(1).

Gisement. - L'Ardoisiere.

Erplication des, figures. - Pl. XVII, fig. 12. Empreinte.

(1) Loc. cit., p. 146. Pl, XIV, fig. 4, 1872. 



\title{
CLASSE DES ÉGHINIDES
}

\author{
ORDRE DES PÉRISCHOÉCHINIDÉS
}

FAMILLE DES ARCH EOCIDARIDÉS

Genre ARGH a

I. Archæocidaris Urii, FlenING, 1828.

Pl. XVI, plaques, fig. 8, 9, 10 .

Synonymie et références. - Cidaris Urii, Fleming (British animals, p. 478). 1828.

Vetusta? Phillips (Geol. of York, 1. 208). 1836.

Benburbensis Portlock (Report..., p. 352. PI. XVI, fig. 10). 1843.

Echinocrinus, Urii, N'Coy (Synopsis, p. 174. Pl. XXVII, fig. 1). 1844.

Historique. - Cette belle espèce a été découverte par David Uro dans les carriùres de Kilbride et do Craigenglen, paroisse de Campsie, ì l'état de plaques isolées et de radioles. L'une de ces plaques et un radiole ont été dessinés avec une grande exactitude par l'auteur sous le $110 \mathrm{~m}$ d'Échinus et d'Aculeus Echini (1).

En 1828, Fleming a donné une courte description de l'espeèe en la dédiant à Ure. Nous la l’eproduisons pour mémoire:

" Plaques isolées seules observées; tubercules antourés d'un double bour") relet. Bord des plaques granulé. Radioles finement striés longitudinalement " et couverts d'épines rer's l'extrémité. "

(1) History of Rutherglen. P1. XVI, fig. 7 et 8 . 
Caractères spécifiques. - Aires interambulacraires composées de 4 rangées de plaques grandes, hexagonales, excepté les plaques adambulacraires, qui sont un peu arrondies sur le côté externe et pentagonales. Elles portent un gros tubercule central sphérique, perforé au sommet, qui déborde au-dessus d'un anneau circulaire, dont il est séparé par une gouttière profonde. La surface de la plaque, autour de l'anneau du bouton, est divisée en deux parties inégales par un second anneau, faiblement quoique nettement indiqué. Ce second anneau, obscurément hexagonal, entoure le premier, dont il est plus rapproché que du bord de la plaque. Le bord extrême des plaques est orné d'une rangée de granules, d'où partent des côtes se dirigeant ver's le centre et s'arrêtant vers l'anneau périphérique, dont la surface interne reste absolument lisse.

Aires ambulacraires composées de deux rangées alternantes de plaques pentagonales petites, portant chacune une paire d'ambulacres disposés sur une ligne horizontale. Au côté externe et presque au point de réunion des deux zones ambulacraires, on remarque sur chaque plaque un granule de même grosseur que ceux qui ornent le pourtour des plaques interambulacraires, et d'autres granules plus petits. On compte de 12 à 14 plaques ambulacraires pour une plaque adambulacraire. Péristome et appareil apical inconnus.

Dimensions moyennes des plus grandes plaques de l'Ardoisière. - Longueur, 14 à $16^{\mathrm{mm}}$; hauteur, 8 à $11^{\mathrm{mm}}$. - A. Julien.

Gisement. - L'Ardoisière. C'est uniquement à l'Ardoisière, parmi tous les gisements du Plateau Central, que se trouve l'Archaocidaris Urii; on peut y recueillir des centaines d'empreintes de plaques interambulacraires isolées, disséminées dans toute l'épaisseur de la formation, qui compte plus de 100 mètres; les plaques ambulacraires sont excessivement rares.

Explication des figures. - Pl. XVI, fig. 8, 9, 10. Empreintes externes de plaques interambulacraires.

Un échantillon non figuré et unique, qui appartient it la Faculté des Sciences de Clermont, at offert une portion d'aire ambulacraire de $30^{\mathrm{mm}}$ de long, bordée d'une série de trois plaques adambulacraires. Ce spécimen nous a permis d'ajouter quelques caractères à ceux donnés par les auteurs.

()bsertation. - M. W. Keeping a figuré, en 1876, dans le Quat. Journ. of the Geol. Soc. (1), quelques plaques interambulacraires et ambulacraires qu'il attribue par erreur, á notre avis, it l'Archerocieleris Lrii litem. En effet, nous n'avons jamais trouvé de plaques d'A. Urii de fomo irreguliere is tubercule excentricue, à surface lisse, comme la plupart de celles qu'il ligure. Les platues ambulacraires, de forme particulière, qu'il représente, ne se rencontrent jamais dans l'esprece écossaise, ni dans les espèces américaines. Nous pensons que ces plaques deivent alpartenir it un grenre different du genre Archeocidaris.

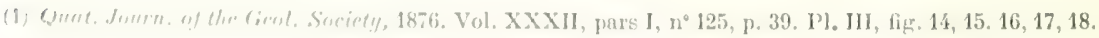


II. Archæocidaris Grüneri, Nov. SP.

Pl. XVI, fig. 11, 12.

Caractères spécifiques. - Jolie espèce, de dimensions plus faibles que $A$. Urii, Les plaques interambulacraires, de forme hexagonale arrondie, présentent un petit tubercule central sphérique, perforé, entouré d'un anneau circulaire étroit, presque aussi élevé que le tubercule. Autour de l'anneau se trouve une surface lisse très étroite, limitée par une crète très faiblement indiquée, de mème forme que la plaque. La partie externe de la plaque est marquée de fortes côtes rayonnantes émanant de granules qui bordent le pourtour. Ces côtes sont grossières, assez irrégulières, peu nombreuses (36 environ).

Les plaques ambulacraires sont munies de deux pores contigus aux plaques adambulacraires.

Dimensions. - Longueur moyenne des placques interambulacraires, 6 mm hauteur, $5^{\mathrm{mm}}$.

10 à 12 plaques ambulacraires sont adjacentes à une plaque adambulacraire.

Cette espèce se distingue de $A$. Urii par sa petite taille, ses côtes beaucoup plus fortes et moins nombreuses, le tubercule central moins fort et moins saillant, et la partie lisse entourant l'anneau plus restreinte. Enfin les plaques ambulacraires ne présentent pas d'ormementation.

Nous dédions cette espèce à Grüner, auteur de magnifiques travaux sur la Géologie de la Loire.

Gisement. - L'Ardoisière; assez rare.

Explication des figures. - PI. XVI, fig. 11. Empreinte d'une portion de test montrant deux rangées de plaques interambulacraires $(a)$ et l'aire ambulacraire adjacente $(b)$.

Fig. 12. Empreinte de deux plaques interambulacraires isolées.

RADIOLES DE L'ARDOISIÈRE.

Pl. XVI, fig. 14, 17.

Pl. XVI, fig. 13, 15, 16.

Pl. XVII, fig. 8, 10 .

Pl. XVII, fig. 9.

On trouve à l'Ardoisière un nombre considérable d'empreintes de radioles d'Archwocidaris, mais nous ne les avons jamais observées en connexion certaine avec les plaques; celit nous met dans l'impossibilité d'attribuer à l'une ou l'autre de nos deux espéces les piquants qui leur reviennent.

On peut les diviser en deux groupes: les uns sont a 4 rangcies longitudinales d'épines, les autres à 6 rangées. Nous allons en décrire quelques-uns figurés dans nos planches: 
a. Radioles à 4 rangées d'épines.

PI. XVI, fig. 14. Empreinte d'un fragment de radiole de $39^{\mathrm{mm}}$ de long, lisse sur le tiers inférieur; les épines sont très visibles.

Pl. XVI, fig. 17. Empreinte du même, vu sur l'autre face.

Pl. XVII, fig. 8. Empreinte de radiole offrant quatre rangées d'épines, dont 3 visibles.

Pl. XVII, fig. 10. Petit fragment de $20^{\mathrm{mm}}$ de long, également á 4 rangées. La figure est renversée par erreur.

\section{b. Radioles à 6 rangées d'épines.}

Pl. XVI, fig. 13. Empreinte d'un fragment de radiole, de $19^{\mathrm{mm}}$ de long et $2^{\mathrm{mm}} 5$ de large. Les épines sont nettement disposées en spirale; 4 rangées linéaires bien visibles, savoir : 2 rangées médianes et 2 rangées latérales, ce qui porterait le nombre total í 6 rangées si le radiole était vu dans son pourtour.

Fig. 15. Fragment de $25^{\mathrm{mm}}$ de long et $5^{\mathrm{mm}}$ de large. On voit nettement 2 rangées internes d'épines et 2 rangées latérales. Les épines, très rapprochées l'une de l'autre, sont placées suivant une ligne spirale.

Pl. XVI, fig. 16. Empreinte de radiole cassé : longueur, $54^{\mathrm{mm}}$; plus grande largeur á la hase, $4^{\mathrm{mm}}$; collerette très atténuée. On aperçoit sur l'empreinte 4 rangées d'épines, soit deux liatérales ef deux internes. La base est lisse sur les $12^{\mathrm{mm}}$ inférieurs.

Pl. XYII, fig. 9. Empreinte d'un fragment présentant 4 rangées d'épines visibles très rapprochées; l'une de ces épines, qui a laissé son empreinte, a $3^{\mathrm{mm}}$ de long.

III. Archæocidaris Nerei, MüNSTER, 1839.

P. VI, fig. 6,7 .

Carcectères spécifiques. - La plaque a un disque mamelonné circulaire. Quelques petits spécimens sont pógulièrement hexagonaux. Le mamelon hémisphririfue est perforé au sommet et entouré par un anneau saillant lisse. La portion restunte de la placpue est finement granulaire.

Los piquants, larges, longs, presque cylindriques, semblent être lisses, cinc co m'est qu'it l'aide d'une loupe qu'on remarque de tròs fines stries. Miïlistoly

En 1812, de: Koninck a rapporté à l'espèce de Münster des plaques, des madioles at des piemes buecales decouverts à Toumai. A la base du bourrelet il direrit un scorolicule tris ctroit, bordé par un anneau de granules allongés.

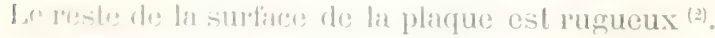

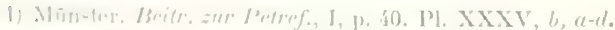

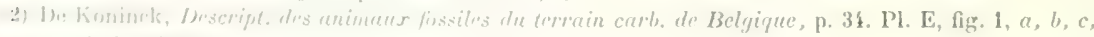
$1, \cdots, 1,11,1, i, 18,2-i i$ 
Les spécimens que nous avons découverts dans le Morvan sont identiques à l'esperee belge.

Giscment. - Domaine des Roches, La Varrille, Siguret.

Explicution des figures. - Pl. VI, fig. 6. Fragment de roche ofirant l'empreinte d'une douzaine de plaques et de radioles très fins, striés á la base et épineux á la partie supérieure.

- Domaine des Roches.

Fig. 7. Plaque isolée et incomplète, probablement de la mẻme espèce. - Siguret.

\section{Archæocidaris, SP.?}

Pl. VIII, tier. 1.

Sur l'échantillon de Régny, pl. VIII, fig. 1, qui présente une vinlve ventrale de Chonetes Murchisoni, on distingue encore une portion de plaque d'un Archceocidaris et un radiole, mal venus dans la figure. Le radiole est épineux et très fin.

C'est le seul indice de l'existence du genre Archaocidaris à Régny que nous ayons pu découvrir, nous le signalons ici pour l'indiquer aux chercheurs.

Nous avons trouvé également des traces, spécifiquement indéterminables, dans les carrières de Saint-Germain-Laval.

\section{FAMILLE DES MELONITIDÉS}

\section{Genre PALAChinus, Scouler.}

Voici ce que dit M'Coy, à propos de la création de ce genre, dont les caractères ont été fixés par le célèbre auteur irlandais lui-même:

"Le nom ci-dessus fut primitivement donné par le Dr Scouler à un échan" tillon de la collection de la Société Royale de Dublin, et à un autre de la „ collection du R. Fox. Ils furent exhibés à une réunion de la Société Géolo") gique de Dublin sous ce nom. A cette époque, les échantillons étaient très " masqués par la gangue, et on les consichrait comme uniques. Il fint impos" sible dès lor's de se livrer avee profit à un examen satistaisant de lem. "structure, et par suite aucun caractère ne pul êtro donné pour les séparrer "des autres genres fossiles on récents. La découverte ulterieure d'antres "spécimens, ainsi que celle de deux nouvelles espèces des sehistes du Nord 
» et du Sud de l'Irlande, m'ont permis d'établir la diagnose du genre. J'ai " adopté le nom proposé par le $\mathrm{D}^{\mathrm{r}}$ Scouler. "

Ainsi le nom a été proposé par Scouler, mais le genre a été scientifiquement établi par M'Coy dans les termes suivants que nous nous plaisons à reproduire: « Test sphéroïdal.

"Ambulacres composés de 2 rangées de plaques pentagonales; chacune " d'elles est perforée par 2 rangées de pores. Interambulacres composés de " 2 rangées de plaques pentagonales et de 3 ou plus plaques hexagonales.

"Les plaques sont couvertes de tubercules spiniformes privés de ligament " central; anus central, dorsal; plaques génitales comme dans le genre "Echinus ; bouche ventrale, centrale (1). "

\section{Palæchinus Lacazei, Nov. SP.}

Pl. XVI, fig. 3, 4, כ.

Caractères spécifiques. - Aire interambulacraire avec 4 rangées de plaques, les 2 rangées adambulacraires formées de plaques pentagonales, et les deux rangées intérieures de plaques hexagonales. Les plaques sont juxtaposées et ne chevauchent pas les unes sur les autres. Les plaques pentagonales ont une moyenne de $6^{\mathrm{mm}}$ de long et de $5^{\mathrm{mm}}$ de haut; les plaques hexagonales ont $7^{\mathrm{mm}}$ de long et $5^{\mathrm{mm}}$ de large.

La surfice extérieure des plaques, uniformément convexe, présente de nombreuses granulations de mème grosseur, mais très petites et ne méritant pas le nom de tubereules.

Aires ambulacraires composées de 2 rangées de plaques granuleuses, présentant chacune 2 paires de pores: une paire sur la plaque elle-même, l'autre pare sur une petite plaque marginale intercalaire; 6 plaques ambu lacraires correspondant ì une plaque adambulacraire.

Nous avons dédié cette espèce à notre illustre maître, M. de Lacaze-Duthiers, ancien Président de l'Acadómie des Sciences, le restaurateur des études zoologiques en France.

Gisment. - L'Ardoisiere.

Explication des figreres. - Pl. XVI, fig. 3. Fragment décrit ci-dessus.

Fig. 4. Fragment de test écrasé, présentant des plaques interambulacraires mèlées à des placines ambulacraires et a des pieces do la måchoire; une dent en particulier est très visiblo.

rig. 5. Uno portion d'aire ambulacraire. 


\section{Palæchinus Rutoti, Nov. sP.}

Pl. VI, fig. 8.

Empreinte externe d'un fragment de test, montrant 4 rangées de plaques interambulacraires qui se recouvrent mutuellement. La rangée adambulacraire compte 11 plaques visibles, de forme pentagonale. Leur surface paraît lisse et n'otfre, sur la mince couche d'ocre qui a remplacé le test, que quelques faibles rides transversales irrégulières, dues à la fossilisation.

Les plaques interambulacraires sont hexagonales. Elles ont une longueur moyenne de $5^{\mathrm{mm}}$ et une hauteur de $3^{\mathrm{mm}}$.

L'aire ambulacraire, visible sur une longueur de $30^{\mathrm{mm}}$, est formée par une double rangée de plaques se recouvrant en sens inverse des plaques interambulacraires. Chacune d'elles a 2 pores; quatre plaques ambulacraires sont contiguës à une plaque adambulacraire.

Nous avons dédié cette espèce à notre savant ami M. Rutot, conservateur du Musée royal d'Histoire naturelle de Bruxelles.

Gisement. - Domaine des Roches, près Savigny-Poil-Fol.

Palæchinus Robineti, NOv. sP.

Pl. III, fig. 19 b.

Caractères spécifiques. - Plaques adambulacraires pentagonales; celles des rangées moyennes, hexagonales; les unes et les autres ornées de tubercules milixires séparés par des granules plus petits et moins nombreux. Les plaques étant isolées ou les fragments de test incomplets, il nous est impossible de fixer le nombre des rangées de ces plarques.

Plaques ambulacraires petites, disposúes sur 2 rangées, chacune d'elles a 2 pores. Ces plaques portent du côté interne 2 ou 3 tubereules miliaires, de la grosseur de ceux des plaques interambulacraires.

Un spécimen nous a laissé voir 5 de ces plaques porifères correspondant exactement à une plaque adambulacraire.

Dimensions. - Plaques adambulacraires: longueur, $5^{\mathrm{mm}}$; hauteur, $3^{\mathrm{mm}}$. Plaques interambulacraires (rangée médiane) : longueur maximum, $7^{\mathrm{mm}}$; hauteur, $4^{\mathrm{mm}}$. Plaques ambulacraires: longueur, 2.5 à $3^{\mathrm{mm}}$; hauteur, $1^{\mathrm{mm}}$.

Rapports et différences. - L'ornementation des plaques interambulacraires est la même que celle de P. Kónigi M'Coy, mais les dimensions sont plus 
faibles pour toutes les plaques que nous avons découvertes; les tubercules miliaires sont également plus réguliers et les granules moins nombreux dans nos spécimens. Ces diverses raisons nous ont engagé à en faire une espèce nouvelle que nous dédions à notre ancien préparateur, M. Charles Robinet, actuellement professeur au lycée de Chartres.

Gisements. - La Varville, Siguret.

Explication des figures. - Pl. III, fig. 19 b. Fragment de test écrasé dont les plaques chevauchent l'une sur l'autre.

\section{Palæchinus gigas, M'Coy, 1844.}

Non figuré.

Caractères spécifiques. - Orbiculaire, déprimé. Aires interambulacraires formées de 6 rangées de plaques; 4 rangées de pores sur chaque côté des aires ambulacraires; 7 plaques ambulacraires ont la même hauteur qu'une plaque adambulacraire. Tubercules grands, égaux; chacun d'eux est entouré par un anneau. - $\mathrm{M}^{\prime} \mathrm{Coy}{ }^{(1)}$.

Gisement. - Régny.

Nous avons découvert une seule plaque interambulacraire d'une conservation parfaite, identique à celles que décrit M'Coy. Le fragment de roche est un calcaire gris-noiratre, intermédiaire entre le vrai marbre et les schistes enveloppants. C'est une espèce á rechercher.

\section{Palæchinus Konincki, Nov. sP.}

Pl. X, fig. 20.

Pl. XI, fig. 27.

Nous créons cette espèce pour un fragment d'oursin brisé, de grosse taille ef dont une aire interambulacraire a été recouverte par des fragments de deux autres aires qui ont chevauche sur la première et l'ont en partie recouverte. Les dimensions de: l'aire recouverte sont:

IJauteur, $30 \mathrm{~mm}$; Jargeur approximalive, $55^{\mathrm{mm}}$.

Sur les bred supéricur gauche de la figure qui représente l'aire recouverte, on wil te nombreuses plaques an connexion. Les plaques adambulacraires sont fentagenales el de plus grande taille, celles des rangées intermédiaires hexa- 
gonales et plus petites. En examinant avec attention l'échantillon, il semble reconnaître que le nombre des rangées est de 5.

Dimensions d'une grande plaque adambulacraire. - Hauteur, $8^{\mathrm{mn}}$; longueur, $11^{1} \cdot 1+11$.

Dimensions d'une plaque de la 2 rangée, près du sommet. - Longueur, $8^{\mathrm{mm}}$; hauteur, $6^{\mathrm{mm}}$.

L'ol'nementation est composće de tubereules miliaires, très serués, semblables à eeux de $P$. ellipticus $M^{\prime}$ Coy. Sur les plaques adambulacraires, les tubercules forment des séries linéaires horizontales, et l'on peut en compter de 8 à 10. Les tubercules miliaires paraissent plutôt disposés en quinconce sur les autres plaques.

Rapports et differences. - Il se distingue du P. ellipticus M'Coy par une taille au moins double et l'orientation des tubereules dans les plaques adambulacraires.

Gisements. - Régny, Néronde, Saint-Germain-Laval.

Explication des figures. - Pl. X, fig. 20. Spécimen décrit ci-dessus. - leégny.

Pl. XI, fig. 27. - Fragment d'un Palechinus que nous attribuons provisoirement ì la même espèce. - Néronde.

Nous dédions cette belle espèce à l'illustre paléontologiste belge, M. de Koninck.

\section{FAMILLE DES LÉPIDOCENTRIDÉS}

Genre Pholidocidaris, MeEk et Worthen, 1869.

Pholidocidaris Gaudryi, NOV. SP.

Pl. XVI, fig. 1, 2, 6, 7 .

Synonymes. - Lepidocentrus Mïnsterianus (1).

Melonites Gaudryi, nov. sp. (2).

L'Oursin que nous attribuons à cette espèce, n'est pas rare à l'Ardoisière; il est moins commun que l'Archceocidaris Urii, mais il l'est beaucoup plus que le Palcechinus Lacanei.

(1) Julien. Comptes-rendus de l'Académie des scienees, 5 janvier 187'.

(2) Id. 31 mars 1890 . 
Tous les spécimens sont exclusivement à l'état d'empreintes; nous en avons découvert 4 ou 5 ; mais ils sont tellement écrasés que leur étude est d'une difficulté extrême, et malgré tout le soin que nous y avons apporté, elle reste nécessairement incomplète, d'autant plus qu'on a fréquemment affaire à des moules internes ou à de simples fragments d'échantillons. On trouve également, avec une certaine fréquence, des plaques isolées et des empreintes de radioles. Il nous a fallu longtemps pour relier entre eux plaques et fragments et nous convaincre que nous avions sous les yeux non des espèces ou des genres distincts, mais bien les différentes parties d'un même Oursin. C'est en rapprochant ces diverses parties et grâce à l'heureux hasard de découvertes successires, que nous arons reconnu qu'il ne s'agissait ni d'un Melonites, ni d'un Lepidocentrus, mais bien d'une espèce nouvelle du genre américain Pholidocidaris, créé par Meek et Worthen.

Caractères spécifiques. - Aires ambulacraires : Nous avons découvert en 1873, l'empreinte externe d'un fragment d'aire ambulacraire, mesurant $3^{\mathrm{cm}}$ de large sur $3^{\mathrm{m}}$ de haut. Ces plaques ambulacraires, disposées sur 6 rangées, sont limitées de part et d'autre par' des plaques adambulacraires. Les premières sont hexagonales allongées, percées de deux pores plus rapprochées du bord inférieur; la longueur de ces plaques est de $4^{\mathrm{mm}}$ et leur hauteur de $1^{\mathrm{mm}} 5$ environ. L'empreinte interne de ces plaques ne laisse voir aucune ornementation, mais d'autres plaques, isolées ou groupées, vues du côté externe, offrent un gros granule, parfois associé à de petits granules miliaires.

Aires interambulacraires. - Le nombre des rangées de plaques interambulacraires nous est inconnu. Toutefois un spécimen horriblement écrasé et un autre représenté pl. XVI, fig. 2, nous permettent de penser qu'il est au moins de 3 dans le voisinage de l'apex.

Forme et ornementation de plaques interambulacraires. - Les plaques sont de forme très irrégulière et tout à fait semblables sous ce rapport à quelquesunce du Pholidocidaris irregularis M. et W. Ces deux observateurs font remarquer dans la description de l'espèce ci-dessus, que les plaques offrent une lelle variété de formes qu'il est à peine possible de tonner une idée exacte de leur contrur, sans les décrire l'une après l'autre ${ }^{(1)}$. On peut en dire autant de celles de l'Ardoisiere.

Quant ì l'ornementation, elle est également des plus variées; le nombre et la grosseur des granules et leur disposition tantôt symétrique, tantôt comme livece au hasard, rappellent encore les caractères de l’espèce américaine. Beaucroup mêne de ces plaques, absolument identiques, ne sauraient en être distinguées. Proxemple, une plaque de forme ovale ou quasi circulaire, présente un tubercule contral entouré d'une couronne de granules secondaires disposés prìs du brorl extériour. Nous dirons même que c'est la découverte d'une de ces

(1) rimetegical Sureey of Jllinots. Vol. V, p. 512. PI. XV, fig. 9. 1873. 
plaques, rappelant également l'ornementation du Lepidocentrus Münsterianus, de Koninck, qui nous avait fait croire ì la présence de cette espèce viséenne à l'Ardoisière. Nous avons recomnu plus tard notre erreur, grîce à de nouveaux spécimens montrant de telles plarues en comnexion avec de vraies plaques de Pholidocidaris. Du reste, l'espèce de Visé n'étant connue que par une seule plaque, l'assimilation avec le genre dévonien Lepidocentrus reste douteuse à notre avis.

Une autre plaque, de forme irrégulière, présente sur l'un des bords un tubercule plus gros, puis 2 ou 3 tubercules de deuxième ordre, placés en des points queleonques de la plaque, le reste de la surface étant couvert de granules miliaires. Une troisième plaque offie 8 ou 10 tubereules de second ordre au milieu desquels sont disséminés un grand nombre de granules miliaires. Une quatrième, elliptique, un peu plus grande, n’offre que des tubereules igaux de second ordre, etc. etc.

Radioles. - Les radioles sont de plusieurs grandeurs, ils sont identiques à ceux du Pholidocidaris irregularis. Ils ont une tête un peu renflée, s'effilent graduellement, sont arrondis, droits et marqués de stries longitudinales fines et serrées.

Gisement. - L'Ardoisière.

Nous dédions cette belle et rare espèce au célèbre paléontologiste qui a rendu tant de services à la science française, M. Albert Gaudry.

Explication des figures. - Pl. XVI, fig. 1. Fragment d'empreinte mal conservée, montrant l'aire ambulacraire à 6 rangées.

Fig. 2. Fragment d'un autre spécimen, montrant une portion de l'aire interambulacraire.

a. c. Plaques offrant l'ornementation.

b. Plaque où l'ornementation est effacée.

d. Plaque ambulacraire.

Fig. 6. Empreinte d'une très grande plaque mesurant $25^{\mathrm{mm}}$ de long sur $16^{\mathrm{mm}}$ de large. On y observe un gros tubercule perforé, excentrique, quelques tubercules secondaires et des granules miliaires épars sur le reste de la surface. Cette plaque est á rapprocher des plaques adambulacraires de la fig. $9 a$, pl. XV, de l'ouvrage de Meek et Worthen.

Fig. 7. Fragment montrant des plaques interambulacraires isolées dont l'ornementation est très visible. On remarque á côté des plaques ambulacraires et des piquants. 



\title{
(CLASSE DES CRINOIIDES
}

\author{
ORDRE DES EUCRINOÏDES
}

FAMILLE DES ACTINOCRINIDÉS

Genre AGtinoGrinus, Miller, 1821.

I. Actinocrinus icosidactylus, Miller, 1843.

Pl. V, fig. 1, 2.

Historique. - Cette espèce a été découverte par Portlock dans le calcairu carbonifère de Hook-Point, comté de Wexford (').

Caractères spécifiques. - Le calice, de moyenne taille, est de forme subconique. La surface externe de ses diverses pièces est ornée de petites côtes rayonnantes, extrêmement minces, simples.

Les pièces radiales sont assez petites; leur largeur va en diminuant; elles n'offrent rien de remarquable. De chaque côté du biseau de la pièce axillaire se trouve la première pièce brachiale, également axillaire, qui offre ceci de particulier qu'elle est située dans le même plan que celles qui la précèdent. Les quatre premiers articles des quatre bras auxquels domne naissance chacun des cinq rayons, sont très petits et disposés sur une seule rangée horizontale.

Les pièces interradiales sont généralement assez petites et au nombre de six. Pour chaque côté symétrique, le nombre des pièces anales est de dix, dont quatre grandes.

(1) Report, etc., p. 348. Pl. XV, fig. 7, 7 a. 
La base est assez épaisse et très courte; elle est entourée d'un bourrelet plat, divisé en trois parties égales par une petite rainure correspondant à la ligne de jonction des trois pièces qui concourent à sa composition. Sa surface articulaire est bordée d'une petite côte circulaire ornée de stries concentriques (1).

Gisement. - La Varville.

Explication des figures. - Pl. V, fig. 1 et 2. Deux fragments d'une empreinte externe de calice. - La Varville.

II. Actinocrinus triacontadactylus, Mrler, 1821.

Pl. $T$, fig. 5.

Caractères spécifiques. - Le calice est d'une forme conoïde et de taille moyenne. La surface externe de ses pièces est ornée de petites côtes saillantes, rayomnant du centre ver's les côtés et formant entre elles des angles plus ou moins aigus. Les côtes les mieux marquées et les plus visibles sont celles qui se dirigent du centre vers le milieu de chacun des côtés de chaque pièce. Par leur rencontre avec celles des pièces adjacentes, elles produisent un dessin rógulier composé d'un grand nombre de petits triangles isocèles sur les échantillons de parfaite conservation. - De Koninek ${ }^{(2)}$.

Suit la description eomplète que nous supprimons, n’ayant trouvé que très raroment des plaques isolées.

Gisement. - La Varville.

Explication des figures. - Pl. V, fig. 5. Une plaque.

PI. T, fig. 4. Actinocrinus, sp.? Empreinte interne de la base d'un calice, montrant les :3 pieces basales en connexion. - Domaine des loches.

(1) Recherches sur les Crinoüles du terrain carbonifure de la Belgique par L. de Koninck et H. Le Hon. 1853, p. 143. 1). II, lig. 4, ot pl. IV, fig. 6.

(2) Loce cit., p. 131. P'1. III, fig. 1. 18533. 
Genre Poteriocrinus, Miller, 1821.

I. Poteriocrinus spissus, IDE Koninck et LE IION, 1853.

Non figuré.

Caractères spécifiques. - Le calice est de forme conique, beaucoup plus long que large.

La base est formée de 5 pièces allongées, terminées par un angle d'environ $115^{\circ}$; clles sont géniculées et plus larges ver's le bord supérieur qu'au bord inférieur. Leur profil montre qu'elles sont un peu courbées en dehors, dans le sens de leur longueur. La surfice articulaire destinéc à recevoir le dernier article de la tige est très faiblement coneave, lisse dans son centre et boldée d'un cercle de petites stries rayomantes. Au centre elle est percée d'une ouverture pentangulaire assez grande. - De Koninck et Le Hon (i)

Gisement. - La Varville.

Nota. - Nous arons trouvé un calice uniquement composé des pièces basales; il a été déterminé par M. de Koninck (2).

\section{Poteriocrinus radiatus, Austin, 1842.}

Non figuré.

Nous arons trouvé une plaque isolée qui a été déterminée comme appartenant à cette espèce par M. de Koninck ${ }^{(3)}$.

Gisement. - La Varville.

(1) Recherches sur les Crinoüdes, p. 94. Pl. I, fig. 9, 9 "

(2) Note sur le terrain carbonifere du Moreun, 1., 8 .

(3) Note sur le terrain carbonifere du Morcen, 1. 7. 


\section{Poteriocrinus plicatus, Austin, 1842.}

Pl. V, fig. 3.

Nous avons figuré pl. V, fig. 3, une empreinte de plaque radiale se rapportant tris probablement au $P$. plicatus, Austin.

Gisement. - La Varville; très rare.

IV. Poteriocrinus crassus, Miller, 1821.

Pl. XVII, fig. 6 et 7 .

Caractères spécifiques. - Le calice de ce Poteriocrinus est subconique à base très large. Toutes les pièces sont plissées aux angles, et leur surface est couverte de petites granulations parfaitement perceptibles à l'oil nu. Base composée de 5 pièces pentangulaires un peu plus larges que longues, très épaisses, ayant chacune à leur intérieur deux petits appendices qui, par la réunion de toutes les pièces, donnent lieu à une ouverture pentaphylle ayant la forme d'une croix grecque à 5 branches.

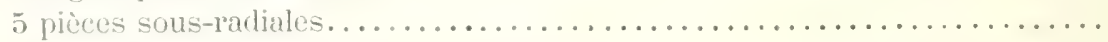

Lá tigo est très grosse et trè̀s longue. Du côté du sommet et jusqu’à une certáne distance de cette extrémité, elle est composée de la réunion d'un nombre

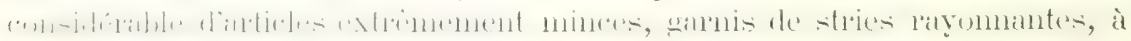
mowerture: tres large. A une certaine distance du sommet les articles sont un [mu plus épais mais ils conservent les mêmes caractères. - De Koninek ${ }^{(1)}$.

Gisements. - Règny, Saint-Germain-Laval, Néronde, Létra, Evaux, l'Ardoisière.

Esplicution des figures. - P1. XVII, fig. 6. Mloule interne du canal d'une tige. - L'Ardusciere.

fign. 7. Id .

Jans tous les gisenents les empreintes internes et externes de tiges de Poteriocrinus et mime de: Patycrinus sont imnombrables et pourraient donner lieu it une étude intéressante.

(1) Recherches sur les Crinoüdes, 1. 57. 1)1. I, fig. $10 a, b, c, d$. 


\title{
CLASSE DES ANTHOZOAIRES
}

\author{
ORDRE DES ZOANTHAIRES
}

GROUPE DES TÉTRACORALLIAIRES

GENRE LITHOSTROTION, LIVYD, 1699.

Lithostrotion junceum, Fleming, 1828.

Pl. XVII, fig. 14.

Caractères spécifiques. - Polypiérites minces, allongés, cylindriques, inégalement rapprochés, fortement courbés au-dessus de leur point d'origine, subparallèles entre eux, inégalement distants, recouverts d'une forte épithèque dont les prolongements latéraux sont susceptibles d'attacher quelques Polypiérites les uns aux autres, ce qui, en l'absence de cloisons, peut les faire confondre avec certaines espèces de Syringopor $\alpha$. Celte épithèque est fortement plissée en travers; lor'squ'elle disparait, on aperçoit les côtes cloisomnaires.

Le calice, qui est assez profond, est orné de 16 à 18 cloisons principales alternant avec un égal nombre de plus petites, et s'étendant jusqu'à la columelle; celle-ci, relativement forte, est comprimée latéralement; les planchers sont distants d'environ $1^{\mathrm{mm}}$ les uns des autres.

Le L. junceum se distingue de tous ses congénères par la laxible dimension de ses Polypiérites (2 ou $\left.3^{\mathrm{mrn}}\right)$. - De Koninck (1).

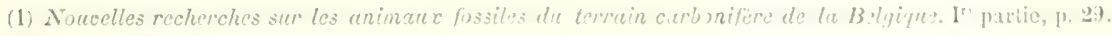
Pl. III, fig. 1. 1872. 
Gisement. - L'Ardoisière; non rare.

Explication des figures. - Pl. XVII, fig. 14. Empreinte d'un fragment de Polypier montrant en $a$ un calice conservé.

II. Lithostrotion irregulare, PHillips, sP.(1), 1836.

Non figuré.

Caractères spécifiques. - Polypier en touffes dendroïdes très considérables; Polypiérites très longs, cylindriques, un peu flexueux, surtout ver's la partie inférieure sur laquelle on observe souvent des bourgeons en partie avortés et soudés aux Polypiérites voisins. Leur diamètre est de 4 à $5^{\mathrm{mm}}$. La columelle est assez peu saillante et peu comprimée. Le nombre des cloisons varie de 16 à 24, suivant l'âge; elles sont minces, assez écartées les unes des autres et s'étendent presque jusqu'au centre, ete. - De Koninck (2).

Gisement. - L'Ardoisière; abondant.

Obsercation. - Nous rappellerons que M. Edouard Dupont a rencontré cette espèce dans un grand nombre de localités belges et l'a signalée comme établissant un horizon dans l'étage de Visé qui, dans beaucoup de circonstances, peut servir de guide dans la classification des couches calcaires au milieu desquelles elle est souvent intercalée sous forme d'un banc distinct de 15 is 20 (m) d'épaisseur.

GENRE DIPHYPHYLLUM, LONSDALE, 1845.

Diphyphyllum concinnum, LoNSDALE, 1845.

Non figuré.

C'urecteres spécifirues. - Polypier fasciculé formant de grandes colonies, conpuse du I'olypicutes tres longs, subeylindriques, se multipliant par bourfremmoment latrial ot se dirigrant presque parallèlement les uns aux autres.

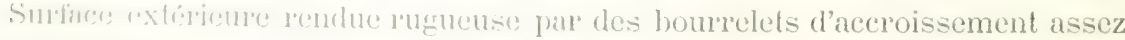
promenterses.

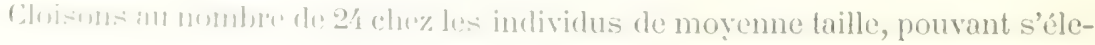

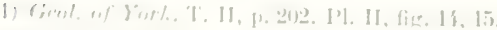

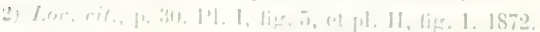


ver jusqu'i 36 chez les adultes; ces cloisons prineipales alternent aver des cloisons rudimentaires de grandeur moitié moindre. Les cloisons prineipales s'étendent jusqu'à la muraille interne et forment par leur ensemble une aire extérieure qui se détache facilement de la partie centrale. Celle-ci, dont le diamètre égale environ la moitié du diamètre total du Polypiérite, est composée de la réunion d'un grand nombre de planchers lisses, à bords relevés et plissés dont l'ensemble, isolé, représente une petite colonne cannelée sur haquelle on observe aisément les joints de chaque plancher; ces plancher's sont assez lóguliers et distants les uns des autres d'environ $1^{\mathrm{mm}}$. Le calice paraît avoir été assez profond. Les Polypiérites dont le diamètre moyen ne dépasse pas 8 à $10^{\mathrm{mm}}$, semblent pouvoir atteindre une très grande longueur, $50^{\mathrm{cm}}$, d'après Ludwig. - De Koninek (1).

Gisement. - L'Ardoisière; on trouve parfois de beaux spécimens de ce Polypier.

GENRE GLISIOPHYLLUM, DANA, 1848.

\section{Glisiophyllum turbinatum, N'Coy, 1855.}

Non figuré.

Caractères spécifiques. - Polypier turbiné, légèrement courbé rer's son origine, ordinairement assez court et trupu, mais prenant quelquefois anssi une forme allongée et relativement grêle, reconvert d'une forte épithèque à bourrelets d'aceroissement bien prononeés et irréguliers.

Calice circulaire, médiocrement profond, à bor'd minee et faiblement recourbé en dehors. Cloisons principales au nombre de 54 à 60 , dont la moitié environ atteint le centre, les cloisons sont minces et alternent avec autant de cloisons rudimentaires.

Les grands échantillons ont 5 à $6^{\mathrm{cm}}$ de hauteur et autant de diamètre, lecquel chez d'autres individus se réduit jusqu'à la moitié. La saillie de la partic de la cloison principale qui forme une fausse columelle a jusqu'ì $8^{\mathrm{mm}}$ d'étendue. De Koninck ${ }^{(2)}$.

Gisement. - L'Ardoisière; très rare.

(1) Loc, cit., p. 36. 1'. II, fig. 4. 1872.

(2) Loc. cit., p. 39. Pl. III, fig. 2. 1872. 


\section{GenRE GYATHOPHYLLUM, GoldFuss, 1826.}

Gyathophyllum multiplex, Keyserling, 1840.

Non figuré.

Caractères spécifiques. - Polypier allongé, subcylindrique, faiblement courbé, un peu comprimé latéralement, garni de bourrelets d'accroissement très prononcés, distants entre eux d'environ $5^{\mathrm{mm}}$.

Calice subovale. Cloisons au nombre d'environ 150; elles sont ordinairement droites ou fuiblement courbées, très minces, serrées, et sensiblement égales entre elles et s'étendent jusqu'au centre. Les planchers sont très petits et assez distants l'un de l'autre. Les traverses vésiculaires sont très petites et excessirement nombreuses; elles sont presque aussi hautes que larges.

Hauteur du Polypier, environ 15 ${ }^{\mathrm{cm}}$; grand diamètre du calice, $4^{\mathrm{cm}}$; petit diamètre, $3^{\mathrm{cm}}$. - De Koninek (s).

Gisement. - Cussy-en-Morvan, dans le marbre.

L'échantillon risé, qui comprend plusieurs calices, a été soumis à M. de Koninck qui, après une étude approfondie, l'a rapporté d'une manière certaine á cette espèce tournaisienne.

GENRE AMPLEXUS, SOWERBY, 1814.

Amplexus coralloides, Sowerbr, 1814.

1\%. VI, tin. 15.

1’. XVII, fig. 13.

Carrertipes spécifiques. - livme eylindrique ou conique très allongée, bourrelets d'aceroissment peu prononcés, épithèque minee, finement striée en tra-

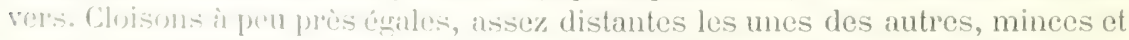
tout is fuit marginales. I cour nombre est très variable et dépend de l'âge et du dimmitre du l'olypier. Nomble des choisons très variable, pouvant atteindre 64

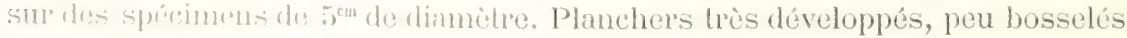
ret is surtiter lissos sur prostue fonte leur étendue. Leur nombre est considé- 
rable et ils sont assez rapprochés l'un de l'autre; fossette septale large et peu profonde. Calice peu profond, à bord droit et à cloisons minces et peu apparentes. - De Koninck (1).

Gisements. - La Varville, Rẻgny, Ferrières, Propières, L’Ardoisière.

Explication des figures. - Pl. VI, fig. 15. Empreinte externe, l'épithèque manque. - La Varville.

PI. XVI, fig. 13. Empreinte externe de plus grande taille. - L'Ardoisiere.

GENRE ZAPHRENTIS, RAFinesque et ClifFord, 1820.

Caractères génériques. - Polypier simple, allongé, libre et finement pédicellé, entouré d'une épithèque complète. Calice plus ou moins profond; pas de columelle; une seule fossette septale très développée, située au devant de la cloison primaire; cloisons nombreuses et en général bien développées, ̀̀ bord dentelé et s'étendant à la surface des planchers jusqu'au centre de la chambre viscérale, ou à peu près. - De Koninck (2).

Gisements. - Les Zaphrentis sont assez communs dans la plupart des giscments du Morvan et du Plateau Central, notamment it La Varville, Régny, Le Gouget, Ferrières, L'Ardoisière. Mais ces Polypiers se trouvent toujours à l'état d'empreintes ou tellement encroultés qu'ils sont spécifiquement indéterminables, et que nous devons nous borner à signaler l'existence du genre.

Genie GyathaXonia, Michelin, 1816.

\section{Gyathaxonia, SP.?}

Pl. VI, fig. 11, 12, 13.

Les trois empreintes de calice figurées sur cette planche, nous paraissent appartenir incontestablement au genre Cyathaxonia, en raison de la columelle centrale styliforme et très saillante. Le nombre des cloisons dans les trois paraît être le même que celui de $C$. Konincki, c'est-ì-dire 26 cloisons principales et 26 plus petites, soit 52 en tout; mais la taille do nos calices est un peu plus considérable. Pour cette dernière espèce, M. de Koninck aceuse une laille de 5 à $6^{\mathrm{mm}}$ pour le plus grand diamètre, alors que les échantillons figurés atlei-

(1) Loc cit., p. 65. Pl. IV, fig. 12. Pl. V, fig. 1; pl. Vi et pl. VII, tig. 1. 1872.

(2) Nourelles recherches, ete., p. 80. 1872. 
gnent $12^{\mathrm{mm}}$. Nous n'osons ni les attribuer à cette espèce ni en faire une espèce nouvelle, ne possédant que des empreintes de calice.

Gisement. - La Varville.

Nota. - Les deux espèces de Cyathaxonia belges appartiennent exclusivement à l'horizon de Tournai.

Dans la me̊me planche, fig. 14, nous avons figuré un Polypier indéterminable de Siguret.

GENRE SYRINGOPORA, GOLDFUSS, 1826.

I. Syringopora ramulosa, GoldFuss, 1826 .

Pl. XVII, fig. 11.

Caractères spécifiques. - Polypiérites très allongées, flexueux, subparallèles, distants entre eux d'environ $2^{\mathrm{mm}}$ et couverts d'une épithèque fortement plissée en traver's; les tubes de connexion assez robustes et distants d'environ $1^{\mathrm{cm}}$ les uns des autres. Le diametre des Polypiérites peut atteindre exceptionnellement jusqu'ì $3^{\mathrm{mm}}$; d'ordinaire il n'en a que 2 à 2,5. - De Koninck (1).

Gisements. - Régny, Saint-Germain-Laval, Néronde, Montmain, Montagny, ChevalRigon, l'Ardoisière. Très commun partout, formant parfois des masses de 40 a $50^{\mathrm{cm}}$ de diamètre.

Explication des figures. - Pl. XVII, fig. 11. Bloc de roche montrant un grand nombre d'empreintes de Polypiérites. - L'Ardoisière.

II. Syringopora distans, FISCHER, 1828.

Son figuré.

Crurcetères spécifiques. - Polypiérites allongés, ordinairement assez droits, ruglruefinis un peu contournés et géniculés, très grêles, entourés d'une forte épilherque ornée d'un grand nombre de stries d'aecroissement bien marquées; ces Polypirites sont áloignes les uns des autres de 4 à $\mathbf{6}^{\mathrm{mm}}$, et nont euxménes qu’un diamedro de 1 à $2^{\mathrm{mm}}$. Les tubes de comnexion sont généralement distunts de 5 it 8 mrn ou plus. - De Koninek ${ }^{(2)}$.

Gisement. - Domaine des lioches.

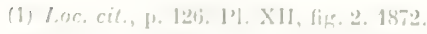

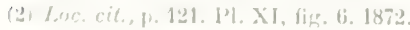


Genre GLAdochonus, M'Coy, 1844.

I. Gladochonus Michelini, Milne Edwards et Haime, 1851.

Non figuré.

Caractères spécifiques. - Polypier rappelant la forme d'une pipe, courbé d̀ une faible distance au-dessous du calice et se prolongeant en un pédicelle très grand, droit et subulé; au point de courbure naissent un ou plus souvent deux éperons qui divergent l'un de l'autre. Les stries costales sont petites, et il paraît y en avoir une vingtaine?

Longueur du Polypier, $15^{\mathrm{mm}}$; diamètre du calice, 3 ou $4^{\mathrm{mm}}$; diamètre du pédicelle, $1^{\mathrm{mm}}$. - Milne Edwards et Haime (1).

Gisements. - Siguret, très rare; Propières, dans le schiste.

\section{Gladochonus Heribaudi, Nov. SP.}

Pl. XI, fig. 29 et 30.

Caractères spécifiques. - Polypier ayant le port du Cladochonus crassus (Janira crassa) M'Coy (2). L'épithèque est ridée transversalement; les calices, de forme conique allongée, offrent une vingtaine de cloisons denticulées très conites.

Les dimensions sont les suivantes:

Longueur des Polypiérites, $6^{\mathrm{mm}}$ à $6,5^{\mathrm{mm}}$; diamètre des calices, $1,5^{\mathrm{mm}}$ à $2^{\mathrm{nmm}}$.

Gisements. - Régny, Néronde, Montmain, Saint-Germain-Laval, Cheval-Rigon près Ferrières, l'Ardoisière.

Nota. - Ce Cladochontes, comme on le voit, est très répandu dins le Plateau Central, dans les horizons de Dinant et de Visé; on peut done s'étonner ì bon droit de ne voir aucume espèce de ce genre signalée dans les mêmes lorizons belges.

Explication des figures. - Pl. XI, fig. 29. Empreinte offrant it la fois le moulage interne et le monlage externe par suite de la disparition de l'épithèque. - Néronde.

Fig. 30. Moulage d'un autre spécimen du même gisement.

Nous dédions cette espèce à M. lo Frère Héribaud, professeur au pensionnat de ClermontFerrand, lauréat de l'Institut, bien comm par ses travaux sur la flore du Plateau Central.

(1) Polypier's fossiles, p. 310. Pl. XVII, fig. 8

(2) Synopsis, 1), 197. Pl. XXVII, lig. 4. 



\section{VÉGÉTAUX}

Dans le cours de nos longues recherches, nous avons pu constater la présence dans beaucoup de carrières, de nombreux débris de végétaux, tantôt transportés des terres voisines par les courants, tantôt fossilisés in situ. Ainsi, au domaine des Roches, à La Varville, où ces débris sont indéterminables. A l'Ardoisière, nous avons trouvé une empreinte de Sphenopteris sp.? et une belle empreinte de Bornia transitionis. A Régny, les dalles de schiste sont parfois couvertes d'empreintes d'algues; l'algue elle-même est souvent transformée en charbon. Nous avons également observé de larges empreintes identiques au Cancellophycus scoparius des terrains jurassiques. La recherche et l'utilisation de ces débris exigeraient le concour's d'un spécialiste.

Toutefois en explorant près de Joux et à Valsonne les schistes vert-sombre, devenant bruns par altération à l'air, qui recouvrent immédiatement le poudingue superposé aux couches marines, nous avons découvert des empreintes bien conservées de la flore enfouie à la base du grès anthracifère.

Nous avons soumis ces empreintes à M. l'abbé Boulay, professeur à l'Institut catholique de Lille. Avec une amabilité parfaite, dont nous tenons ici ì lui exprimer notre gratitude, le savant paléophytologiste a bien voulu examiner nos spécimens et nous envoyer le résultat de ses observations que nous reproduisons intégralement.

No 1. Gardiopteris polymorpha, Schmper: Traité de Paléontol. I, p. 5亿2.

Cyclopteris polymorpha, Gœppert: Ueber die Fossile Flora der Silurischen, der Deoonischen, unteren Kohlenformation, 1859, p. 502, taf. XXXVIII, fig. 5" ; Schimper: Mém. de la Soc. des sc. nat. de Strasbourg, t. V, liv. 22 et 3, 1). 339. Pl. XXV, fig. 1 et 2.

L'unique foliole ci-contre concorde bien avec celles des figures citées de Schimper et de Goeppert. 
No 2. Sphenopteris Schimperiana, Gappent; Schimper : Terr. de transit. des Vosges, dans Mém. Soc.sc. nat. de Strasbourg. Pl. XXVII, fig. 3.

L'échantillon est tellement fruste qu'une approximation quelconque restera toujours très douteuse. Celle que je propose me semble la mieux justifiée.

No 3 et 3 bis. Rhodea patentissima, STur : Culm-Flor des MahrischSchlesischen Dachschiefer's, 1875, p. 36, t. IX, fig. 1-9 (voil en particulier fig. 5); Hymenophyllites patentissimus Const. V. Ettingshausen, Fossile Flora des Mcehrisch-Schlesischen Dachschiefer's, 1875, p. 36, t. IX, fig. 1-9 (voir en particulier fig. 5); Hymenophyllites patentissimus, Const. V. Ettingshausen, Fossile Flor a des Mcehrisch-Schlesischen Dachschiefers, 1865, p. 26, t. VII, fig. 4.

Les pinnules du $n^{\circ} 3$ sont encore plus grêles que sur les figures citées; cependant quand on compare le mode de découpure et la direction des lobules, on arrive à se convaincre de l'identité.

N. B. - On peut rattacher cette espèce à l'ancien genre Sphenopteris, toutefois Goppert arait nommé un Sphenopteris patentissina de la formation rhétique.

No 4. Sphenopteris Schimperi, Geeppert; Schimper : Traité de Paléontologie. I, p.408. Mém. de la Soc. des se. nat. de Strasbourg. T. V, liv. 2-3, p. 341. Pl. XXXVil, fig. 1-5.

Votre spécimen est bien imparfait; on ne voit que les sommets des pinnules ramper le long de la cassure. Cependant la concordance est remarquable avec certaines portions des spécimens figurés par Schimper, et aussi avec le Rhodea Moravica, Stur, Culm-Flora des Mchrisch-Schlesischen Dachschiefers, 1. X, fig. 4 et 6. Mais je ne suis pas convaineu que son Rhodea Moravica soit identique an Trichomanes Moravicum, Ettingshausen, Culm-Flora, ete, p. 24, 1. H, fig. 4. Stur voit dans ce dernier le résultat d'une macération qui n’a respecté que les nervures. Il vaudrait mieux dans ce cas nommer l'espèce à nouve:111.

Le $n^{\circ}$ \& bis semble êtro plus sûrement encore une sommité de penne de Sphenopteris Schimperi phutôt que du S. Moracica. Cette espèce appartient aussi au grenre Rhoden de Slur. Je n'ai pas examiné s'il y a lieu d'admettre ce genre.

No 5. Todea Lipoldi, STur, Culm-Flora des Mwhisch-Schlesischen Dachschiefers, J1.71, 1. XI, fig. 8.

Tranes insuffisuntre pour assurex la détermination de l'espèe. Il y a cepen-

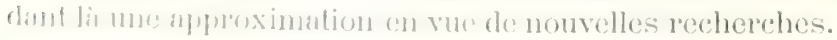

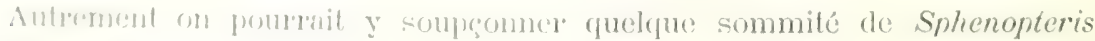
Sichimperit. 
$\mathrm{N}^{\circ}$ 6. 'Traces tres imparfaites de grandes pinnules de fougères. Au premier' abord clles ressemblent ì certaines folioles axiles des Neuropteris.

Est-ce encore une foliole de Cardiopteris, peut-être du Ch. frondosa, Sch.?

No 7. Archæocalamites radiatus, Stur, Culm-Flor a des Methrisch-Schlesischen Dachschiefer's, p.:2, t. I-V.

Bornia radiata, Sch. Traté de Paléont. I, p. 334.

Bornia transitionis, $\mathrm{I} . \mathrm{-A}$. Roemer.

Calamites radiatus, Brot.

Calamites transitionis, Goeppert.

Malgré l'imperfection du spécimen, on peut cepentant vérifier avee certitude le caractère de l'espèce, la continuité des côtes d'un article au suivant.

No 8. Stigmaria ficoides (radicelles). C'est me simple présomption obtenue par voie de comparaison. Il faudrait l'axe principal avee ses cicatrices pour assurer la détermination.

Le Stigmaria ficoides est d'ailleur's fréquent ì ce niveau.

$\mathrm{N}^{\circ}$ 9. Cet axe pointillé me semble appartenir à un pétiole de fousiere. Ces organes présentent fréquemment un aspect de ce gemre. 



\title{
ÉTUDE CRITIQUE
}

I)ES

\section{FATNES CARBONIFÈRES MIARINES DU MORVAN ET DU PLATEAU GENTRAL}

\author{
POSITION STRATIGRAPHIQUE DES ASSISES QUI LES RENFERMENT
}

\section{Considérations préliminaires.}

La formation carbonifère marine de la France centrale est disséminée en innombrables lambeaux, sur une vaste étendue qui ne comprend pas moins de six départements, savoir : la Saône-et-Loire, le Rhône, la Loire, la Niève, l'Allier et la Creuse. Elle s'étend de Cussy-en-Morvan, au nord, jusqu'à Néronde et Saint-Germain-Laval, dans la Loire, au sud; et de la vallée de la Saône et du Rhône, à l'est, jusqu'aux environs d'Evaux et de Chambon, dans la Creuse, à l'ouest. Cette portion nord-est du Plateau Central se laisse diviser en deux, à l'aide d'un axe qui a joú, à l'époque du dépôt du terrain carbonifère, un rôle capital dans la répartition de ses principales assises. C'est l'axe de dépression Digoin-Chagny qui de nos jour's encore ser't d'assiette au Canal du Centre et sépare le Morvan du Plateau Central proprement dit.

Partout où on l'observe, dans chaque lambeau sans exception, quelque limité qu'il soit, le terrain carbonifère se compose de deux termes : un terme inférieur d'origine marine, formé de roches très diverses, et un terme supérieur, qui recouvre uniformément les dépôts marins sous-jacents. Ce dernier lerme est composé de poudingues et de grès anthraeifère, intimement liés ì des porphyres contemporains et à des tufs porphyritiques. Chacun de ces deux termes forme-t-il un seul étage, ou peut-il êtro subdivisé en plusicurs? Et, dius ce dernier cas, à quels horjzons du carbonifore belge, pris comme ćlalon, 
peut-on rapporter les subdivisions possibles de notre carbonifère de la France centrale?

C'est la réponse à cette question qui fait l'objet de cette partie de l'ouvrage, lequel a pour but précisément le développement et la justification de la Note succincte que nous avons présentée à l'Académie des Sciences, dans la séance du 31 mars 1890, sous le titre suivant: " Résultats générqux d'une étude d'ensemble du Carbonifère marin du Plateau Central. "Ce n'est done pas une description complète et détaillée de ce terrain, avec carte géologique à l'appui, que nous allons exposer. Il faudra, pour atteindre ce résultat bien désirable, de nombreuses années encore et une légion d'observateurs; car telle est la division à l'infini; tel est le moreellement extrême offert par cette formation, qu'une carte géologique comprenant l'ensemble des lambeaux carbonifères ne pourra être dressée qu'au fur et à mesure de la détermination complète des autres terrains de cette vaste région. Notre ouvre n'a d'autre prétention que de tracer une large esquisse, que de dessiner un cadre aussi complet que possible aujourd'hui, renfermant les prineipales divisions et subdivisions de l'assise marine inférieure et de l'assise supérieure ou terrestre; cadre dans lequel viendront s'inscrire graduellement à leur place, ainsi préparée à l'avance, tous les faits de détail, au fur et à mesure de leur acquisition future.

Pour atteindre ce but, nous avons dû employer la seule méthode applicable dans nos rógions, par trop déshéritées, par un métamorphisme intense, provoqué soit par l'intrusion d'innombrables roches éruptives ou par l'imbibition d'ranx thermales, soit par l'effort des mouvements orogéniques. Cette méthode a consisté simplement, en faisant abstraction du temps illimité qu'elle exigeait, en raison de l'extrème rareté et du déplorable état de conservation des fossiles, it constituer assez de famnules, sur toute la surface du territoire carbonifère, pour découvir et déterminer avec précision tous les horizons distincts de notre formation. Or, ees faunules devaient, sous peine d'être inutilisables et sans portée stratign'aphique, contenir nécessairement, au milieu d'éléments indifférents, c'ost-ì-dire d'espèces d'une grande longévité, grâce à laquelle elles ont pu traverser lout le carbonifère, des espèces à longévité brève, nettement caractéristiques des assises qui les renferment. Quel résultat attendre, par exemple, do lia diécouverte de Crinoïdes, dont les tiges ou les articles pullulent, on d'espices, comme Orthotetes crenistria, Productus semi-reticulatus, Amplexus corallö̈des, que l'on trouve à chaque pas? Ne sait-on pas que ces fossifer si conmuns se rencontrent de la base au sommet, à Tournai aussi hien qu'i Viso? Mais il n’en ost pas de mêne, en revanche, d'autres espèces procienses, avidenent recherches par le stratigraphe, telles que : Spirifer tornurensis ; Porcellire Puñ); Schizostoma crateriforme; Spirifer striatus ; Eurmpluntus pentangulutus; Chonetes papilionacea; Enomphalus crotalosfomn ; Lithostrotion, juncerum; Posidoniella vetusta, etr., qui déterminent, suns

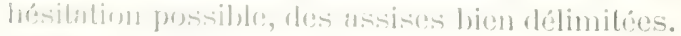

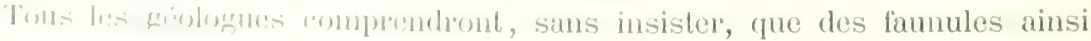


composées de spécimens suffisamment conservés pour être déterminables, comparées avec un soin minutieux aux célèbres faunes belges, illustrées par les grands travaux de L. de Koninck, suffisent largement à constituer des points de repère lumineux, de véritables phares capables de projeter sur tout ce vaste ensemble une éclatante lumière.

C'est la méthode que nous avons suivie, et que nous allons justifier en démontrant sa nécessité absolue, en raison des difficultés exceptionnelles qu'offre l'étude du terrain carbonifère de la France centrale; difficultés telles en effet, que, faute d'avoir appliqué la méthode préconisée, tous les géologues qui l'ont abordée, depuis Grüner jusqu'aux observateur's les plus récents, ont échoué dans leurs tentatives.

$1^{\circ}$ Je signalerai d'abord le morcellement extrême de notre carbonifère marin, qui est disloqué, fragmenté en imombrables lambeaux. Ces lambeaux, tantôt limités par des failles, tantôt à l'état d'enclaves dans nos immenses éruptions de porphyre, sont isolés les uns des autres et sans lien entre eux; d'où la nécessité absolue de s'appuyer sur les fossiles, car le lien paléontologique est le seul qui permette de les rapprocher et de suivre à travers l'espace la continuité des horizons.

$2^{\circ}$ Mais je ferai remarquer de suite l'extrême rareté des fossiles dans la plupart des gisements, et leur très mauvais état de conservation qui est dû principalement à l'intensité du métamorphisme des couches fossilifères.

Il faut consacrer de longues heures dans les carrières en activité, le plus souvent abandonnées, car le calcaire destiné au four à chaux est rare el de faible importance pour découvrir quelques empreintes utilisables. Il faut même de longues semaines, en travaillant de l'aube au crépuscule, pour arriver à la constitution d'une faunule, d'un ensemble offrant une réelle valeur stratigraphique.

La plupart des fossiles, quand il en existe, sont laminés et déformés. Mais ils ont le plus souvent disparu par suite de la dissolution du test par des caux thermales acides, et à leur place subsistent autant de cavités, la plupart aplaties par le laminage de la roche, sur les parois internes desquelles toutefois se dessine assez souvent leur empreinte externe. Quelquefois, dans ces cavités, à la Varville, à Propières, à Cheval-Rigon, à l'Ardoisière, à Néronde, ete., se meuvent librement des moules internes, rarement bien conservés ; par excmple, des moules de calices de Crinoïdes à la Varville, de Productus giganteus ou d'Orthis resupinata à l'Ardoisière. Quelquefois, et cela est heureux, le test disparu a été reconstitué après coup, comme par un artiste délicat ct habile, par de nouvelles eaux thermales qui ont déposé dans les parties vides laissées par les anciens tests, de l'oxyde de fer ou de fine argile ocreuse; exumple, l'Ardoisiere.

Les fossiles intacts, engagés dans les marbres ou dans les calcairos noirs 
crinoïdiques, sont presque impossibles à dégager. Il résulte de ces faits que, si la plupart des Brachiopodes aux caractères externes ou internes si facilement reconnaissables, si les Bryozoaires, si les tests ou les plaques isolées d'Echinides tessellés, si les Syringoporas, ete., s'offrent un peu partout avee des caractères même spécifiques, déterminables, en revanche, les Gastéropodes et les Lamellibranches qui parfois pullulent comme à Régny, à l'Ardoisière et à la Tarville, les Polypier's surtout, échappent presque en totalité à toute possibilité de classement sjécifique, voire générique. Régny, par exemple, nous a fourni, grâce à des mois de recherches, 70 espèces seulement, et l'Ardoisière, 81. Mais chacun de ces gisements en aurait livré plusieurs centaines, si d'aventure les fossiles s'étaient trouvés aussi bien conservés qu'en Belgique.

3० L'observateur est en outre singulièrement dérouté par la variété des facies lithologiques de ce terrain.

Aux roches carbonifères classiques de la Belgique, qui constituent de précieux repères, viennent s'adjoindre, dans la France centrale, des roches incomues dans le Nord, mais dont les débris fossiles qu'elles récèlent, révèlent sans erreur possible, leur caractère marin non moins que leur âge carbonifère. Je mentionnerai, à ce point de vue : les Calschistes noirs tournaikiens de Siguret, près Savigny-Poil-Fol, dans la bande ouest du Morvan; le marbre noir de Régny, si semblable à celui de Belgique, fait qui avait déjà frappé Alléon-Dulac; les marbres blanes veinés de bleu à Stromatoporoïdes de l'Ardoisiere, du Gouget, de Ferrières et d'Evaux, identiques au marbre waulsortion des Paucuys, par exemple, et qui ont dérouté tous les géologues français qui ne connaissent pas le Carbonifère belge; le calcaire orangé ì cherts pâles et ì grosses tiges de Crinoïdes (VI $a$ de la classification de M. E. Dupont) que nous avons découvert à Saint-Germain-Laval, à la base du lambeau rarbonifère de ce gisement, au-dessous des schistes calcaires noirs à Paléchinides. Comme roches spéciales au centre de la France, je citerai les schistes jaunes décalejfiés du domaine des Roches et de la Varville, près Arrée, confondus in tort aver des schistes déxoniens; les grès calcaires bleuâtres, ou décalcifiés et ccroux de l'Ardoisière, que nous avons découverts des 1872, et qui l'enferment une superbe faune de Visć, la plus belle de France à coup sûr; les arkosos lianlinisies marines de Ferrières et les gres siliceux de ChevalRigron qui suyportent le véritable grès anthracifère d'origine terrestre, avec lerpuel on les a récenment confondus; le grès anthracifère lui-même ì Lepi-

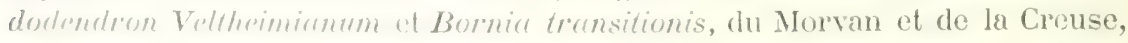

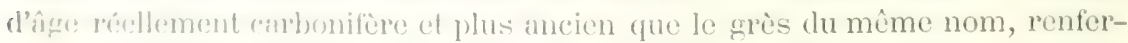
mant he mimes vergatax fossiles du Beanjolais, du Lyonnais, du Forez et du l3onthonntis, dont l'age ost compris cntre l'assise de Vise et le millstone-grit.

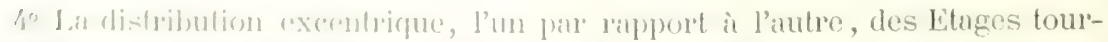

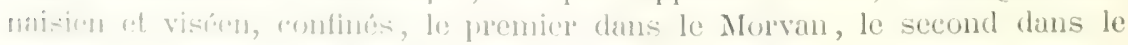


Plateau Central; distribution qui ublige le géologue à étudier les deux régions, s'il veut siasir l'enchânement des phénomènes et découvir les oscillations du sol qui ont suceessivement déplacé les rivages de la mer à cette époque.

$5^{\circ}$ La superposition directe, par transgressivité ou par failles obliques, des schistes carbonifères sur les schistes cambriens ou cristallophylliens supérieurs, par suite de l'émersion de la France centrale aux époques silurienne et déronienne. Cette superposition et, pour ainsi dire, cette continuité dans le sens vertical des deux étages extrêmes du terrain primaire, a facilité et perpétué leur confusion depuis Dufrenoy et Fournet jusqu'à la date la plus récente.

C'est done par une recherche patiente des restes fossiles, en si mauvais ètat qu'ils soient, que l'on peut triompher de ces difficultes que nul autre terrain ne présente à un pareil degré. Mais cette recherche serait vraiment fastidieuse et rebutante, car elle doit s'aceomplir dans une région montagneuse, le plus souvent déshéritée des moyens de trimsport et des commodités ordinaires de la vie, si le géologue, livré à lui-mème, sans nul encouragement dans cette étude longue, aride et si difficile, n’était intérieurement sontenu et réconforté par le sentiment profond, par la conviction absolue de l'importance extrême des résultats qui doirent couronner ses efforts.

La connaissance du carbonifère marin de la France centrale n'implique pas seulement l'établissement du parallélisme de ses assises avec les assises synchroniques de la Belgique, de Tournai à Visé inclusivement; mais cette connaissance, si importante qu'clle apparaisse, une fois acquise, permet à l'esprit d'envisager des horizons nonvenux. Elle devient comme la préface d'investigations plus lointaines auxquelles ne saurait satisfaire le pays belge. Dans le Nord, l'absence de roches éruptives à cette époque est complite, et l'on sait que les mouvements du sol y sont peu accusés. On constate toutefois, uprès T'ournai, l'affaissement lent de la région à l'est et à l'ouest du Centre belge; affaissement qui a permis à la mer viséeme de recouvrir le Boulomais et le pays de Liège, précédemment émergés.

Dans le Morvan, au contraire, les mouvements préliminaires anté et posttournaisiens, c'est-ì-dire famenniens et chanxhiens, sont fort aceusés, et d'autres non moins importants se sont produits dans le Platean C.nntral, dès le début de l'époque chanxhicnne jusqu'à la finn du viséen.

Ces mouvements, prélude des grandes oscillations hepeynionnes do l'ápoque houllère moyenne, qui dominent toute l'histripe de notre carhonilère, ont en assez de puissance, à leur dernière phase, pour amener la disparition complète de la mer et l'émersion totale dlu sons-sol marin, plus complètement qu'en Belgique. La région du Centre nous offre dans le mème temps dex límoignages grandioses d'un prodigieux développement de l'activité interne du stobe, activité toute comparable ir celle qui a donné liou au colossal épanchenent des granites et des granulites des époques silurienue et dévonienne. Les roches 
porphyriques si variées, qui oftrent un si vaste champ d'études au lithologiste, s'intercalent dans la formation carbonifère ou lui succèdent sans intervalle, de telle sorte que l'âge respectif de leur's puissants massifs ou de leurs innombrables filons peut se déduire, avec une certitude presque mathématique, de leurs étroites relations de contact avec les sédiments marins ou terrestres, caractérisés par leur faune ou leur flore.

Chose singulière! Par l'effet d'un enchaînement logique, qui s'impose à la conviction d'un esprit attentif, de phénomènes accomplis à une aussi faible distance, si notre faune carbonifère se révèle comme une dépendance, un prolongement par un bras de mer pénétrant jusqu'au cœur de la France, de cello qui peuplait l'océan du Nord; en revanche, les mouvements oscillatoires contemporains de la Belgique et du nord de la France nous apparaissent à leur tour comme un corollaire nécessaire des phénomènes éruptifs et orogéniques dont le Plateau Central et le Morvan ont été le thêâtre. Comment, en effet, se représenter le retrait graduel de la mer carbonifère belge dans la direction du pôle à l'époque houillère inférieure, retrait qui fait succéder à la haute mer des lagunes d'abord, puis la terre ferme, bientôt couverte de luxuriantes forêts, sinon comme le résultat du soulèvement général de la France centrale, se propageant au loin jusque par delà les bassins de la Loire et de la Seine?

Cu soulèvement prodigieux substituait, dans nos régions, à une terre basse aux livages indécis une contrée alpestre, bientôt couverte d'innombrables orifices éruptifs, d'où allait s'épancher un vrai déluge de porphyre en fusion. Ces deux régions étaient done bien réellement unies, à cette époque, par les liens l'une étroite soliclarité. Leur histoire géologique se complète et s'éclaire mutuellement, et l'observateur est mis dans la nécessité d'en étudier les chapitres simultanément.

Toutes les publications belges relatives aux temps primaires doivent être familiures aux géologues de la France centrale. Et réciproquement l'on peut, je crois, affirmer sans témérité, que les géologues belges ne possèderont la slof des oscillations do leur sol dans la même période, qu'en les envisageant à hat lumiere des grands phúnomenes orogéniques et éruptifs, qui se sont déroulís dans notre pays, car l'identité des roches at des fossiles que nous avons constatín à l'epoque carbonifère, se manifeste encore à une époque plus anciemene.

Ainsi lo récif dévonien supérieur de Dion (Allier) est identique, à tous les prints do: vue, roches et fossiles, au récif contemporain de Frasne et érigé dans In minn: mas. Les schistes à Oldhamia de llaybes se retrouvent également idtrntiques it Néburat, au pied oecidental de la chaine sud des voleans à cralieres dn lemy-rle-1)ôme.

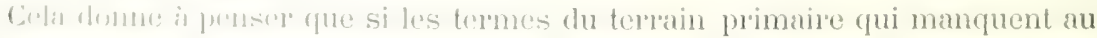

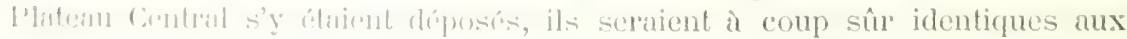

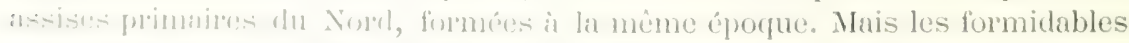

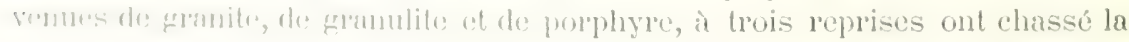


mer de nos régions, et empéché le dépôt des assises siluriennes et dévoniennes inférieures et moyentnes. Nous avons tenu à exposer ces rapprochements qui ont échappé ì nos devanciers, et qui sont le fruit de longues observations et de patientes recherehes.

En résumé, pour ne pas prolonger davantage ces considérations préliminaires, quelle marche devons-nous suivre, après l'exposition de la partie paléontologique de cet ouvrage, qui nous fait connaître la faune que nous avons recueillie et pu déterminer?

Nous devons établir la répartition de cette faune en faunules distinctes, provenant de chacun des 17 gisements que nous avons explorés. Puis, nous devons faire l'étude comparative de chacune des faunules avec ses voisines d'abord, avec les faunes et faumules belges ensuite. Nous arriverons ainsi, par comparaison, à la détermination précise de nos niveaux stratigraphiques et à la classification générale de nos assises carbonifères. Mais pour faciliter l'exécution de ce plan, il nous paraît convenable de rappeler d'abord les classifications successives du terrain carbonifère de Belgique qui sert d'étalon au carbonifère de l'Europe, et de mentionner ensuite, à titre de complément d'enquête, les décourertes accomplies dans ce pays, dans le cours de ces dernieres années, en tant qu'elles peuvent trouver leur application directe dans le Plateau Central.

\section{Historique des classifications du terrain carbonifère belge.}

La première division de l'étage carbonifère marin est due ì André Dumont. Dès 1830, ce savant géologue montra qu'il se compose d'une assise inférieure, caractérisée par le calcaire à Crinoïdes ou petit-granite - d'une assise moyenne, formée par la grande masse dolomitique, enfin d'une seconde: assise calcaire. Ces trois subdivisions figurent dans la légende de la carte géologique dans laquelle cet étage est indiqué comme formé de calcaire à Crinoïdes, de Dolomie et de calcaire à Productus. M. Dewalque a fait remarquer, il y a longtemps, que A. Dumont considérait la Dolomie comme ayant le plus de rapports avec le calcaire à Crinoïdes, ce qui permettait une division en deux sous-étages seulement ${ }^{(1)}$.

En 1860, M. Gosselet, dans un mémoire resté célèbre, rattacha la Dolomie au calcaire de Visé, contrairement à ce que pensait A. Dumont, et il établit les assises suivantes dans les deux étages ${ }^{(2)}$.

(1) G. Dewalque. Prodrome d'une description géologique de la Bolcique, p. 85, 1’aris, 1880.

(2) J. Cosselet. Mémoire sur les termins primuires de la Bolgique, des environs didvesnes et du BonIonnais, in-8". Paris, 18060. 
$1^{\circ}$ Etage houiller.

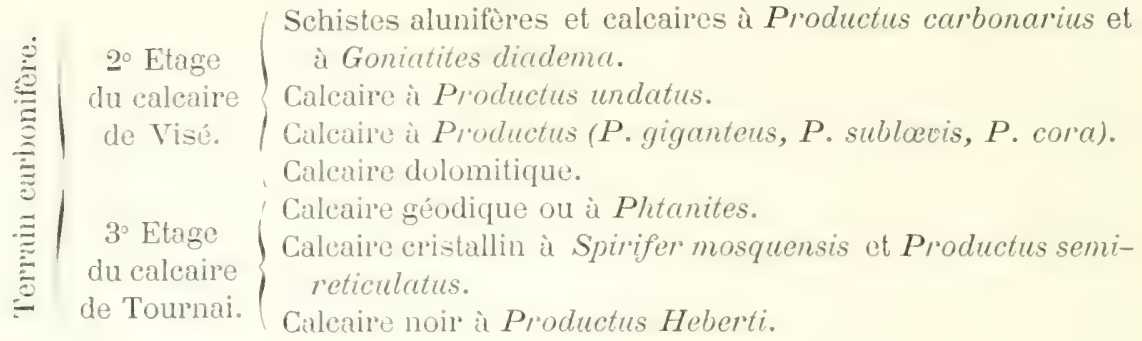

C'est l'année suivante que M. E. Dupont commença la belle série de travaux qu'il a suceessivement élaborés sur le mème sujet. Dès 1865, il publiait une Echelle stratigraphique du calcaire carbonifère de Belgique en six assises désignées par le nom de la localité où il les avait reconnues le mieux caractérisćes ${ }^{(1)}$.

Les roici énumérées dans leur ordre de succession :

Assise VI de Visé.

Assise V de Namur.

Assise IV de Waulsort.

Assise III d'Anseremme.

Assise II de Dinant.

Assise I des teanssines.

C'est dans ce travail que M. E. Dupont fit comnaitre, pour la première fois, les marbres construits Waulsortiens, dont la rraie nuture avait échappé à ses devanciers.

Il reennut au microscope qu'ils étaient formés par deux Stromatoporoïdes "gu'il désigna sous les noms de Stromatocus bulbacens et de Ptylostroma fibroste. Stimule par les observations critiques auxquelles cette classification dumna lieu, M. L. Dupunt lir perfectionna et publia sous le nom d'Echelle stratigraphique de la carte géologique de Belgique, une classification nouvelle que unts reproduisons ci-dessous.

Lo termin curbunifere y cost sublivisé en trois étages et en cinq assises, sitiriI" :

Silige superrienr on de Visc..., Assise supérieure de Visé V2.

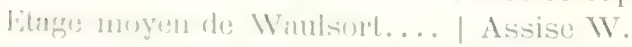

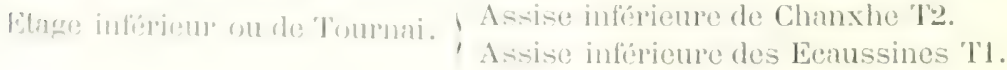


Voici le détail des assises:

ETAge viséex à Chonetes comoides et Productus undatus.

\section{Assise de Visé V2.}

V2d. Calcaire gris, bleu marbré, noir et gris bleu avec lits d'anthracite: Productus gigunteus.

$\mathrm{V}_{2} e$. Brèche et calcaire bréchiforme.

$\mathrm{V} 2 \mathrm{~b}$. Calcaire gris et noir compact ou bleu grenu a Lithostrotion irregulare, calcaire bleu marbré à Productus undatus.

V2a. Calcaire blanc et gris avec grains eristallins: Productus cor $\ell$, Chonetes papilionacea.

\section{Assise de Dinant V1.}

V1h. Calcaire très compact noir et gris avee lits de dolomie.

V1 $g$. Calcaire gris alternant avee de la dolomie: Productus subloctis.

V1f. Dolomie grise à larges paillettes.

V1e. Dolomie noire géodique à grains fins.

V1d. Dolomie brune à grains moyens et erinoüdes.

Vle. Calcaire noir, bleu et gris avec dolomie.

V16. Calcaire noir compact avec bandes de phtanites noirs (calcaire à carreaux de Dinant).

Vla. Calcaire gris violacé et noir sub)-compact uvee des bandes et des rognons de phtanites gris.

\section{Etage Waulsortien it Spirifer cuspiclatus.}

Wp. Calcaire bleu et dolomie à crinoïdes avec larges bandes de phtanites blonds (remplissage des chéneaux des récifs).

Wo. Dolomie bigarrée ou non (calcaire à stromatoporoüdes on amorphe altéré).

Wn. Calcaire gris et blane sub-compact (sable corallique).

Wm. Calcaire blane veiné de bleu (récifs de stromatoporoïdes).

\section{Etage tournaisien it Spirifer tornacensis.}

\section{Assise de Chanxhe T2}

T2a. Caleaire et dolomio ì crinoüdes. 


\section{Assise des Ecaussines T1}

T1e. Calcaire bleu à crinoïdes avec bandes de phtanites noirs (calcaire d'Yvoir).

T1d. Calchistes noirs (calcaire à chaux hydraulique de Tournai).

T1c. Calcaire bleu à crinoïdes et schistes intercalés à la base (calcaire des Ecaussines).

T1b. Schistes vert-sombre à Spirifer octoplicatus.

T1 $a$. Calcaire bleu à crinoïdes avec lits de schistes intercalés.

A ce tableau nous ajouterons la mention de quelques fossiles qui d'après M. Dupont caractérisent expressément quelques-uns des horizons belges établis par lui. Nous les avons colligés en lisant attentivement le texte explicatif des feuilles de Dinant, de Ciney, de Clavier, de Modave, d'Yvoir et d'Hastières.

Sous-assise T1b. Elle est caractérisée par Spirifer octoplicatus. Disons ici que cette espèce a été supprimée par L. de Koninck car elle n'existe que dans l'étage de Visé et qu'elle a servi à former deux nouvelles espèces qui l'ont remplacée, savoir Spiriferina Malleri et Spiriferina peracuta.

Sous-assise Tlc. Est caractérisée par Spirifer cinctus. - Michelinia favosa et Syringopora geniculata.

Sous-assise T1d. (Calschiste de Tournai) renferme Spirifer tornacensis Porcellia Puzo, - Schizostoma crateriforme, etc.

Sous-assise Tle. (Calcaire d'Yvoir) renferme Phillipsia gemmulifera, - Spirifer cinctus, - Michelinia favosa.

Sous-assise IVp. Spirifer subcinctus.

Sous-assise Wo. Gyroceras gibberosum.

Sous-assise Wn. Idem.

Sou-assise W $\mathrm{Tm}$. Dans les poches des récifs : Spirifer cuspidatus, - id. striatus, - id. convolutus, - Rhynchonella pugnus, - Euomphalus pentangulatus,

Platyschisma helicoides, - Productus Flemingi, - Bellerophon Lohestce, ir. excucatus, - Phymatifer cariniferts, - Gyroceras giblerosum, - Nautilus extensus, - id. mutabilis, - id. cariniferus, - id. normalis, - id. discors, - id. lyriostomus, - id. orthoceras, - id.fandum, - Amplexus coralloüdes, - Orthis voisin de resupinata. - Nombreuses Fenestelles, ete.

Srus-issise V1b. (Calcaire à carreaux de Dinant). Euomphalus catillus, id. crotrulostomus. - Chonetes comoides.

Sous-assise V1c. Chonetes papilionacer, - id. comoides, - Productus semireticulatus, var. concinnus, - irl. plicatilis, - id. Cora (caractéristique de tout l'útage vistens), - Bellerophon gigunteus, - Goniatites vittiger, - AviculopecIen dissimilis, - Phillipsia derbyensis, - Straparollus mitis, - Platyschisma ovoriden, - Spririfer bisulcatus, - Syringopora ramulosa (caractéristique de l'éluge vistrent), - Éuomphulus crotulostoma. 
Sous-assise V1f. Chonetes papilionacea, - id. comoides, - Euomphalus crotelestomun.

Sous-assise V1g. Productus sublcevis (caractéristique de la zone), - id. cora. - Euomphalus crotalostoma.

Sous-assise V2 $a$. Productus cora. - Chonetes papilionacea.

Sous-assise V2b. Productus undatus. - Cyrtocer'us unguis. - Nautilus planotergatus. - Orthoceras approximatum, - id. giganteum. - Goniatites obtusus, - id. mutabilis, - id. striatus. - Lithostrotion Junceum, - id. irregulare.

Sous-assise V2d. Productus giganteus de grosse taille. - Spirifer bisulcatus, id. - Lithostrotion Junceum, - id. irregulare.

Cette classification si détaillée, et que nous avons complétée pour la facilité de nos études personnelles par l'adjonction d'espèces caractéristiques de certains horizons, signalées par M. E. Dupont lui-même dans ses cuvres, a été modifiée assez récemment par la nouvelle Commission de la carte géologique de Belgique, organisée par Arrêtés royaux du 31 décembre 1889 et du 3 janvier 1890. Voici la classification qui doit servir de légende et qui, malgré quelques modifications, est pour ainsi dire établie d'après les mêmes principes qui ont guidé M. E. Dupont.

\section{CALCAIRE CARBONIFËRE.}

Etage viséen (V).

Vg. Calcaire à Productus giganteus.

$\mathrm{Vf}$. Brèche calcaire.

Ve. Calcaires gris et noir (marbre bleu belge).

Vd. Caleaire à grains cristallins foncés. Productus cora. - Chonetes papilionacere.

Vc. Dolomies de Namur avec calcaires subordonnés.

Vb. Marbre noir de Dinant.

Va. Calcaire gris et violacé avee Cherts (phtanites) gris et blonds.

Vn. Calcaires stratifiés crinoïdiques gris ou bleus, dolomies à crinoïdes, Cherts pâles.

Vm. Calcaire massif, blanchâtre veiné de bleu, souvent dolomitisé.

\section{ETAGE TOURNAISIEN ('T).}

Tn. Calcaires strutifiés erinoïdiques, gris ou bleus, dolomies à crinoïdes, Cherts pâles.

Tm. Calcaire massif, blanchâtre veiné de bleu souvent dolomitisé.

$\mathrm{T} f$. Calcaire et dolomie à crinoïdes de Chanxhe. 
Te. Calcaire à crinoïdes d'Yvoir, avec bandes de Chert noir.

$\mathrm{T} d$. Calschistes noirs à chaux hydraulique de Tournai.

Te. Calcaire à crinoïdes des Ecaussines, avec schistes intercalés à la base.

$\mathrm{T} b$. Schistes vert sombre à Spiriferina octoplicata.

$\mathrm{T} a$. Calcaire bleu à crinoïdes; calcaires avec schistes intercalés à Phillipsia.

\section{Facies Waulsortien (W).}

Wn et Wm. Massifs coralliens qui n'ont pu être rapportés ni au Viséen ni au Tournaisien.

Cette classification n'a pas rencontré l'assentiment unanime des géologues plus particulièrement voués à l'étude des terrains carbonifères belges. Quant à nous, qui nous plaçons strictement sous le point de vue exclusif qui a dirigé nos études dans notre propre pays, nous émettrons le regret que les savants géologues qui l'ont formulée se soient cantonnés sur un terrain si étroitement local, si exclusivement lithologique. Ils ont trop oublié, à notre avis, que le carbonifère belge, avec sa prodigieuse faune composée de miliiers d'espèces en grande partie décrites par l'un des plus grands paléontologistes du siècle, arec ses assises marines ininterrompues, les seules qui offrent ce caractère en Europe; que le carbonifère belge, dis-je, est typique, et doit servir d'étalon à fout lu carbonifère européen, au même titre, par exemple, que le cambrien du pays de Galles, que le silurien d'Angleterre et de Bohême, et autres formations classiques, pour les étages similaires; et que cette classification, d'où la paléonfologie est exclue, est malheureusement inutilisable à l'Etranger, où les faciès lithologiques sont en grande partie différents. C'est la méthode de A. Dumont, le grand stratigraphe, il est vrai, mais aussi le grand contempteur de la paléontologie, qui semble se perpétuer dans cette œuvre, à laquelle on ne peut prédire qu’une durée éphémère. Qu'on nous permette de le répéter à notre tour : toute classification géolugrique doit être désormais basée sur l'évolution biologique des faunes et sur leur caractère bathymétrique, en même temps que sur les os-cillations de l'écorce terrestre qui ont amené le déplacement des rivages. I.: caractere lithologirque doit être banni avee soin et n'intervenir que dans la descriptirn détaillée du terrain.

Depuis trois ou quatre ans, il est vai, un vigoureux effort dans ce sens s'est houreusement produit en Belgique même. Une brillante pléiade de géologues, sous l'inspiration des vrais principes établis jadis par W. Smith, Al. Brongniart, Ciressly, Hebert et leurs disciples, ont introduit dans la classification belge des proints de vue nouvraux at des horizons paléontologiques inédits, grâee ì de

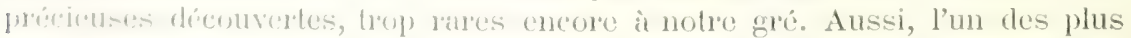
distingues, 11. le chanoine Dorlodot des Essarts, vient-il de proposer tout

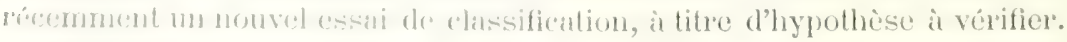

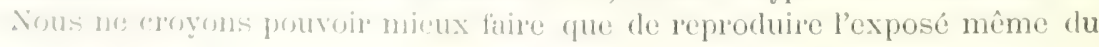
savant groshlogue: 
CLASSIFICATION DE M. DORLODOT DES ESSARTS.

“ Nous proposons de diviser le calcaire carbonifère de Belgique en trois " étages. L'étage inférieur ou tournaisien comprendrait les divisions T $a$, T $b$, " T $c$ et $\mathrm{T} d$ de la nouvelle légende officielle, c'est-à-dire la partie inférieure du " tournaisien de M. Ed. Dupont jusqu'au calschiste de Toumai inclusivement. "L'étage moyen ou chanxhien se composerait de toutes les formations com" prises entre le calschiste de Tournai et le marbre noir de Dinant ( $\mathrm{V} b$ de la "légende officielle) exclusivement. Le marbre noir de Dinant deviendrait ainsi " la base de l'étage supérieur ou viséen; nous proposons de réintroduire dans " ce dernier la division en viséen inférieur et viséen supérieur, telle qu'elle a " été établie par M. Dupont.

"Notre étage chanxhien comprend les formations waulsortiennes de M. Du" pont, ou du moins la plupart d'entre elles (le récif de Biron excepté, synchro" nique de V1b d'après les récentes recherches de M. Devalque), l'assise de " Chanxhe, le calcaire d'Yvoir, avec ou sans cherts blonds, que l'on avait considéré jusqu'ici, à la suite de M. Dupont, comme la base du viséen. Cet "étage est composé de roches fort diverses; néanmoins, d'après leur grou"pement le plus fréquent, l'on peut distinguer dans l'étage chanxhien deux "faciès typiques :

" $1^{\circ}$ Le faciès chanxheux, qui est le plus répandu, présente, au-dessus de " couches foncées à crinoïdes sporadiques et cherts noirs (T $e$ de la lógende ") officielle), la puissante formation de calcaires à crinoïdes, exploité sur les " bord de l'Ourthe et du Hoyoux. Dans les régions qui ne sont pas trop éloi" gnées des calcaires construits waulsortiens, la partie supérieure de l'étage est " constituée par le calcaire subcompact à cherts blonds (V1a de M. Dupont, " $\mathrm{V}$ a de la légende officielle);

" $2^{\circ}$ Le faciès waulsorteux présente ordinairement, au-dessus de quelques

" couches foncées à crinoïdes sporadiques et cherts noirs Te, des masses de

" calcaires construits à Stromatocus et Ptylostroma, transformés ou non en

" dolomies (W m et Wo massifs), qui forment d'énormes nodules au milieu

" de roches stratifiées, désignées dans la notation de M. Dupont par les lettres

"Wp, Wn ou V1 $a$ et une partie de Wo.

") Le calcaire subcompact (Wn et $\mathrm{V} 1 \alpha$ ) domine souvent à la partio supé" rieure, et il passe alors vers le haut, au marbre noir de Dinant, qui constitue " pour nous la base du viséen. Les raisons qui nous portent à proposer un " étage moyen ainsi constitué, sont les suivantes:

" $1^{\circ}$ Les roches construites à Stromatoens et Ptylostromar sont très dére" loppées entre les limites que nous avons assignées à ect étage; elles font " complètement défaut plus bas et ne se trouvent qu'exceptionnellement ì un " niveau supérieur ;

„La faune viséenne, mélangée aree la faune propre des réciĺs apparait daus 
") les calcaires construits immédiatement au-dessus du calschiste de Tournai ; » ce qui semble exiger que l'on place à ce niveau une séparation d'étages.

"D'autre part, la faune tournaisienne se rencontre dans le petit granite de ") l'Ourthe et du Hoyoux juscu'au contact du marbre noir de Dinant, dont la ") faune est exclusivement viséenne. Il faut donc admettre également une sépa" ration d'étages à la base de ce marbre noir. Au point de vue paléontologi» que, l'étage chanxhien est donc caractérisé par l'apparition, dès la base de cet " étage, de la faune viséenne mêlée à des espèces spéciales, dans certaines " parties limitées du bassin; tandis que dans le reste du même bassin, la faune » tournaisienne se conserve jusqu'au sommet de l'étage. Dans le viséen, au " contraire, la faune tournaisienne a disparu et la faune viséenne s'est répandue " sur toute l'étendue du bassin;

" $3^{\circ}$ La nécessité de séparer du viséen les couches VIa de M. Dupont pour " les rattacher aux formations de Waulsort et de Chanxhe ressort de consi") dérations stratigraphiques. La base du marbre noir de Dinant constitue, en effet, un excellent horizon. Au contraire, les couches V1 $\alpha$ de M. Dupont, qui sont d'ailleurs impossibles à distinguer des couches waulsortiennes Wn, "présentent une allure des plus capricieuses. Il n'est pas douteux qu'elles se trouvent souvent dans le prolongement latéral des formations waulsortiennes; tandis que nous ne connaissons aucun fait qui établisse le passage latéral du V1a au VIb de M. Dupont. Ajoutons que la présence, d'ailleurs fort rare dans les couches V1 a, d'une espèce viséenne, le Spirifer bisulcatus, qui a déterminé M. Dupont à rattacher ces couches au viséen, a perdu toute "valeur depuis que l'on a reconnu l'apparition de nombreuses espèces "viséennes dans les formations waulsortiemmes, immédiatement au-dessus du " ealschiste de Tournai (1). ")

Enfin, pour terminer cette revue rapide, nous mentionnerons, par ordre de date, les principales découvertes faites dans les trois ou quatre dernières années, en nous bornant à celles qu'il nous a été donné d'utiliser.

1891-1892. - M. M. Lohest découvre le banc de calcaire à Echinides, Palcerhinus gigus, ete., qui forme un précieux horizon à la base même de l'assise de Dinant. Puis, MMI. Lohest et H. Forir, continuant leur's recherches, retrouvent le même niveau dans la bande carbonifère de la Meuse. M. Paul Destinez donne la faune de ce banc, recueillie par lui à Poulseur.

1892. - M. H. de Dorlodot signale pour la première fois le faciès waulsortien dans le bassin do Namur; et M. Dewalque, à son tour, décourre le récif de birron (Ciney), rui repose dans cette localité sur le marbre noir viséen.

189-1893. - MM. de La Tallée-Poussin et II. de Dorlodot démontrent que

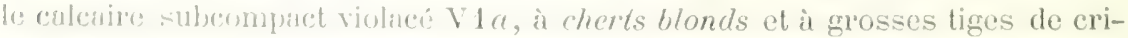
urödes, se rattache dateralement au calcaire gris et blane waulsortien à titre de farcies hétírotrigue, at gu'il doit être enlevé à l'assise de Dinant pour

(1) Arin. de la Sore. Givol. de Belgirur, tome XX, 1" livraison, pages 35-38. 1892-1893. 
descendre d'un degré, contrairement à l'opinion de M. Dupont, qui se basait sur la présence dans ce calcaire du Spirifer bisulcatus.

1893-1894. - M. P. Destinez donne la liste des fossiles de la carrière de Pair (Clavier), que l'on croyait ouverte dans V1 b et qui s'est révélée chanxhienne. Il en est de même de la faune du marbre noir viséen V1b de Petit-Modave, publiée en 1895 par le même savant. Reconnue également chanxhienne, elle doit être retirée du viséen.

1893-1894. - M. Lohest démontre l'âge tournaision des dolomies situées au bord sud du bassin de Namur, à Ampsin, à l'est de Huy, considérées jusqu'alors comme viséennes. Faisant remarquer l'importance considérable de cette découverte, M. Lohest dit: "Plusieur's géologues à la suite d'André »Dumont, croyant que dans le bassin septentrional le calcaire carbonifère " inférieur n'était plus représenté à l'est de Namur, avaient émis l'idée que " pendant la période carbonifère inférieure, une séparation existait encore en " partie, entre le bassin de Dinant et celui de Namur. Cette séparation aurait ") rendu compte des différences que présentent les sédiments de cette époque » de part et d'autre du Condroz. Nous voyons qu'à Huy, ces différences "n'existent pas et que la totalité de la bande carbonifère est comparable, " comme composition, à une bande du Condroz. )"

1895. - Enfin, tout récemment, M. G. Soreil recueille la faune de Denée, composée de 38 espèces, laquelle caractérise la base de V1b, immédiatement au-dessus du calcaire à Echinides qui lui-même recouvre V1 $a$. Cette note, parue il y a quelques mois à peine, trouve immédiatement son application dans le Plateau Central, comme nous le verrons plus loin.

Ainsi, chacune des découvertes qui se fait en Belgique, dans le terrain carbonifère, éclaire d'une lumière vive nos gisements du Plateau Central, et nous montre la solidarité étroite qui existe entre les deux pays. 


\section{MORVAN}

Les gisements que nous avons étudiés d'une manière spéciale dans cette région sont au nombre de quatre. Trois d'entr'eux répartis à une faible distance, autour de Savigny-Poil-Fol, appartiennent à la bande carbonifère qui longe à l'ouest le pied du Morvan. Ce sont les gisements de la Varville, du domaine des Roches et de Siguret.

Le quatrième, celui de Cussy-en-Morvan, est surtout connu depuis la mention qu'en a faite M. Collenot, en 1873, dans la Description géologique de l'Auxois. Nous les étudierons successivement.

\section{LA VARVILLE.}

La Varville est située à peu près à 1 kilomètre au sud d'Avrée, canton de Luzy (Nièvre) ${ }^{(1)}$. C'est une chaumière isolée, au toit de chaume, élevée sur le bord d'un chemin creux. Elle est facile à découvrir, car elle est indiquée sous ce nom sur la carte d'état-major.

Le proprićtaire possède attenant à sa pauvre demeure un champ établi sur des schistes décalcifiés, formant talus sur le bord du chemin, et remplis d'empreintes fossiles. Grâce à son obligeance, j’ai pu faire des fouilles considérables dans lo champ. Ces schistes de couleur jaunâtre, sont à la partie supérieure de la formation carbonifère, et directement recouverts à quelques mètres de là par le grè̀s anthracifüre. Les fossiles y sont tous à l'état d'empreintes ou même de moules internes. Le test des coquilles a disparu, dissous entièrement par les caux thermales, et a été remplacé par une minee couche d'ocre brune, qui tranche par sa teinte foncée sur la couleur claire du schiste.

Un laminage intense s'est produit, et bien souvent les empreintes sont déformés el réduites ì la minceur d'une feuille de papier.

Ca grisement, d'une richesse extraordinare, que j’ai fouillé pendant près d'un mois uvec l'aide de mon préparateur M. Charles Robinet, m'a donné plusieur's milliers de spécimens. On doit regretter que l'état de conservation soit si mauvass, car la faune de la Varville pourrait rivaliser avec la célebre faune de 'Tr)

(1) Consulter la fouillo d'Autun do la carto fróologiquo détaillóo do la Franco. 
Au lieu d'une cinquantaine d'espèces seulement que j'ai pu reconnaître avec certitude, c'est par centaines que l'on compterait les espèces tournaisiennes du Morvan.

Voici la liste complète des espèces et des genres de ce gisement. Ils sont au nombre de 53. Nous avons indiqué leur position dans les étages tournaisien, waulsortien et viséen de Belgique par les lettres initiales T, W, V en nous servant des travaux de M. de Koninck. Les espèces non accompagnées de ces lettres sont nouvelles pour la science ou n'ont pas encore été découvertes en Belgique.

Liste DES ESIÈCES C.ARBONifÈres DE LA VARVILLE.

1. Griffithides seminiferts, Phillips T.

2. Phillipsia Barrandei, nov. sp.

3. Brachymetopus Duponti, nov. sp.

4. Entomis concentrica, de Kon.

5. Bairdia, sp.

6. Orthoceras Martinianum, de Kon. T.

7. Naticopsis propinqua, de Kon. T.

8. Loxonema acutum, de Kon. T.

9. Loxonema Lefeborei, de Kon. T.

10. Portlockia pygmeed, de Kon. T.

11. Straparollus laevigatus, Lev. T.

12. Schizostoma crateriforme, de Kon. T.

13. Phanerotinus serpula, de Kon. T.

14. Bellerophon sublectis, P. et Mich. T.

15. Capulus uners, de Kon. T.

16. Lepetopsis Leforti, nov. sp.

17. Sanguinolites inconspicuus, de Kon. T.

18. Parallelodon bistriatus. Portl. T.

19. Leiopteria Van den Brœeki, n. sp.

20. Productus semireticulatus, Mart. var. Martini. T. V.

21. Productus scabriculus, Mart. T. V.

22. Chonetes variolata, d'Orb. T.

23. Chonetes Giraudi, n. sp.

24. Strophomena analoga, Phill. T. V.

25. Orthothetes crenistria, Phill. T. W. V.

26. Orthis Michelini, Lév. T. V.

27. Orthis resupinate, Mart. T. V.

28. Spirifer Tornacensis, de Kon. T.

29. Spririfer Roemerianus, de Kon. 'I'.

30. Spirifer distans, Sow. IV. 
31. Spirifer Urii, Flem. T.

32. Spiriferina peracuta, de Kon. T.

33. Martinia lineata, Mart. T. V.

34. Retzia ulothrix, de Kon. T.

3ว. Athyris Roissyi, Lév. T.

36. Athyris lamellosa, Lér. T.

37. Rhynchonella acutirugata, de Kon. T.

38. Dielasma insigne, de Kon. T.

39. Discina nitida, Phill. T.

40. Fenestella plebeia, M'Coy, T. V.

41. Fenestella Morrisii, M'Coy.

42. Glauconome pulcherrima, M'Coy.

43. Monticulipora tumida, Phill. T. V.

44. Archceocidaris Nerei, Münst. T.

45. Palcechinus Robineti, n. sp.

46. Actinocrinus icosidactylus, Portl. T.

47. Actinocrinus triacontadactylus, Miller T.

48. Poteriocrinus spissus, de Kon. et Le Hon. T.

49. Poteriocrinus radiatus, Austin T.

50. Poteriocrinus plicatus, Austin T.

51. Amplexus coralloides, Sow. T. V.

32. Zuphrentis, sp.

53. Cyathaxonia, sp.

\section{DOMAINE DES ROCHES.}

Ce gisement a été découvert par M. Michel Léry. Il est indiqué par le signe conventionnel sur la feuille d'Autun de la carte géologique détaillée de France, au lieu dit: Champ de la Barette. Voici ce que l'honorable Directeur du service dit de ce gisement dans la légende de la feuille d'Autun à la mention Quartzites dévoniens : "Entre Savigny-Poil-Fol et Cuviny, un gris argileux " jaunitre contient des fragnents écrasés d'Enerines, de Spirifer's indétermi"n nables. Ce niveau fossilitere correspond aux lentilles calcaires de Diou au "sud, de Cussy-en-Morvan au nord. "

Les foruilles que nous y avons faites, pendant plusieurs jours, nous ont permis do ríunir la faunule ci-dessous.

FACNE DU WOMAINE DES ROCHES OU DU CHAMP DE LA BARRETTE.

1. Brirdire,sp.?

2. Corpulus fimbriatus, de Kon. T.

3. Arieulopercen Pomeli, nov. sp. 
1. Aviculopecten ingratus, de Kon. T.

5. Aviculopecten biornatus, de Kon. T.

6. Parallelodon bistriatus, Port. T.

7. Productus semi-reticulatus, Mart. var. Martini T. V.

8. Productus scabriculus, Mart. T. V.

9. Chonetes variolata, d'Orb. T.

10. Chonetes Giraudi, nov. sp.

11. Spirifer tornacensis, de Kon. T.

12. Martinia lineata, Mart. T. V.

13. Archoocidaris Nerei, Münst. T.

14. Palcechinus Rutoti, nov. sp.

15. Actinocrinus, sp.?

16. Syringopora distans, Fisch. T.

Nous avons également indiqué par des lettres initiales conventionnelles la position dans les étages belges des espèces communes aux deux régions.

\section{SIGURET.}

Le troisième gisement est celui de Siguret, ou plutôt, un point situé à 100 mètres environ à l'ouest du hameau de ce nom qui est voisin de SavignyPoil-Fol. Ce sont des schistes noirâtres, non décalcifiés, qui affleurent sur quelques mìtres carrés seulement. On peut se figurer par la situation de ces trois gisements combien sont difficiles les recherehes paléontologiques dans cette bande carbonifère du Morvan. Il n’y a point de carrières. Celles-ci sont ouvertes, soit dans le lias pour la fabrication de la chaux, soit dans les quartzites cambriens pour les matériaux d'empierrement ou de construction. Le sous-sol carbonifère, sans relief, est couvert de prairies et de bois, et le paléontologiste n'a pour ses recherches que les talus peu élevés des chemins creux, les berges des ruisseaux ou les parois de la voie ferrée de Rémilly à Luzy située en tranchée dans les schistes carbonifères.

Dans le gisement de Siguret, faible surface que la culture a délaissée, tous les tests ont disparu. Tout y est à l'état de moules creux ou d'empreintes. Nous $\mathrm{y}$ avons toutefois, en y consacrant le temps nécessaire, recueilli vingt-cinq espèces dont la liste suit :

FAUNE CARBONIFÈRE DE SIGURET,

1. Griffithides seminiferus, Phill. T.

2. Bairdia, sp.

3. Loxonema vitutum, de kon. 'T. 
4. Loxonema Lefebrrei, Lév. T.

5. Scalites humilis, de Kon. T.

6. Straparollus concolutus, de Kon. T.

7. Schizostoma crateriforme, de Kon. T.

8. Phanerotinus serpula, de Kon. T.

9. Porcellia Pũ̃o, Lév. T.

10. Bellerophon sublevis, Pot. et Mich. T.

11. Parallelodon bistriatus, Portl. T.

1.2. Parallelodon meridionalis, de Kon. T.

13. Entolium Witryi, de Kon. T'.

14. Productus scubriculus, Mart. T. V.

15. Chonetes curiolata, d'Orb. T.

16. Chonetes crassistria, M'Coy.

17. Orthotetes crenistria, Phill. T. IV. V.

18. Spiriferina partita, Portl.

19. Spiriferina peracuta, de Kon. T.

20. Martinu lineata, Mart. T. V.

21. Retria serpentina, de Kon. T.

22. Rhynchonella acutirugata, de Kon. T.

23. Archceocidaris Nerei, Münst. T.

24. Palechinus Robineti, nov. sp.

25. Cladochonus Michelini, M. Edw. et J. Haime. T.

Un examen eomparatif de ces 3 faunules entr'elles démontre qu'elles sont synchroniques. Si l'on examine, par exemple, les faunes de la Varville et du domaine des Roches, on troure la liste suivante:

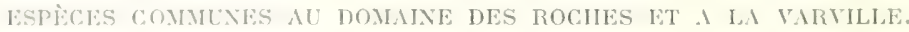

Parralletorlen listriatus, Portl. 'T.

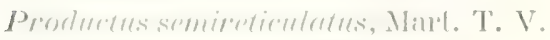

Productus screbriculus, Mint. 'T. V.

Chonetes carioluta, d'Orb). 'T'.

Chonetes gurandi, nov. sp.

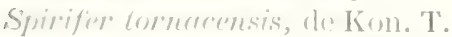

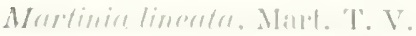

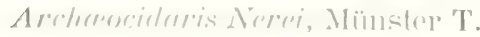

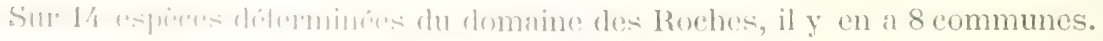


ESPèces COMMUNES A LA VARVILLE ET A SIGURET.

Griftithides seminiferus, Phill.

Bairdia, sp.

Loxonema Lefeborei, de Kon.

Schizostoma crateriforme, de Kon.

Phanerotinus serpula, de Kon.

Bellerophon sublavis, Pot. et Mich.

Parallelodon bistriatus, Portlock.

Productus scabriculus, Mart.

Chonetes variolata, d'Orb.

Orthotetes crenistria, Phill.

Spiriferina peracuta, de Kon.

Martinia lineata, Mart.

Rhynchonella acutirugata, de Kon.

Archceocidaris Nerei, Münster.

Palcechinus Robineti, nov. sp.

Sur 24 espèces de Siguret, 14 se trouvent à la Varville.

espèges cominnes a siguret et aU Domaine des roches.

Bairdia, sp.?

Parallelodon bistriatus, Portlock T.

Productus scabriculus, Mart. T. V.

Chonetes variolata, d'Orb. T.

Martinia lineata, Mart. T. V.

Archceocidaris Nerei, Münster' T.

Sur 14 especes aux Roches, il y en a 5 communes avee Siguret.

Ces trois faunes sont done synchroniques et leur contemporanéité se déduit encore de leur comparaison avec les fuunes belges. Examinons-les it ce point de vue.

LIUNE DE LA VAIRVIILL.

Cette fauns, lix plus importante des trois, analysie an se basint sur los hat vaux de L. de Koninck, se compose des 5 déments suivints: 


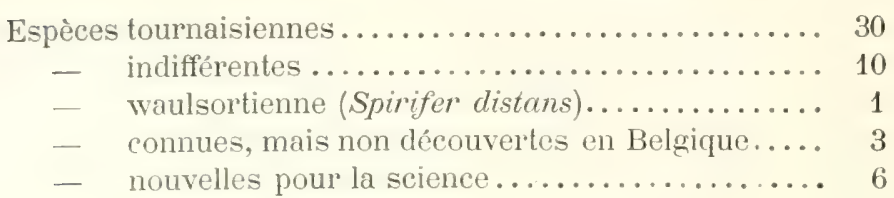

Le trait le plus frappant qui ressort de cet examen comparatif est que la faune de la Varville ne renferme aucune espèce exclusivement viséenne.

NIVEAU STRATIGRAPHIQUE DE LA FAUNE DE LA VARVILLE.

C'est une faune absolument tournaisienne. Elle correspond dans son ensemble aux assises $\mathrm{T} a, \mathrm{~T} b, \mathrm{~T} c, \mathrm{~T} d$ de la classification belge. Sans doute nous n'avons pas le terme Tc dans le Morvan, mais nous devons admettre la correspondance avec tout l'ensemble de l'étage de Tournai. En effet, le terme Tb désigne les schistes vert-sombre à Spiriferina octoplicata, mais l'on sait que L. de Koninck a supprimé cette espèce, exclusivement viséenne, et qu'elle est devenue entre ses mains Spiriferina Mölleri et Spiriferina peracuta. Or, cette derniere est relativement commune à la Varville et ì Siguret. D'autre part, tous les autres fossiles se trouvent parmi les plus earactéristiques de Tb. Exemple : Porcellia Pũo. - Schizostoma crateriforme. - Naticopsis propinqua. - Chonetes carioluta. - Spirifer tornacensis. - Rhynchonella acutirugata, Archceocidaris Nerei, ete., ete.

Ce résultat est capital, car c'est la première fois que la faume de Tournai est découverte en Franee (abstraction faite du département du Nord). Il est confirmé par l'examen des deux autres faunes dont les gisements sont peu rloignés de la Varville.

FAUNE DU DOMAINE DES ROCHES.

Celte faune se compose de 4 ćléments, savoir :

Especes trumaisiennes.................... 8

- indifférentes...................... 3

nemvelles........................... 3

Gionnes non déterminés spécificpuement.......... 2

Total................ 16

Ah-rine romplietr d'espices visemes proprement dites. Les espèces tourmancmes se rencontrent toutes duns le calschiste de Tournai. 
FAUNF DE SIGURET.

La faune de Siguret comprend:

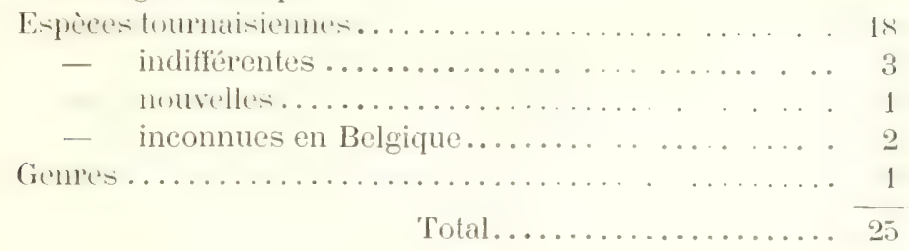

L'analyse détaillée à laquelle nous avons soumis trois faunes de la bande carbonifère ouest du Morvan nous permet done d'affirmer non-seulement leur synchronisme, mais encore leur âge exclusivement tournaisien; car parmi les milliers de spécimens recueillis à la Varville, s'il s'était trouvé dans le nombre, des espèces viséennes, nous aurions facilement constáté leur présence malgré le mauvais état de conservation des fossiles. Ce résultat est de la plus haute importance. Outre qu'il nous révèle l'existence de l'étage tournaisien dans le cœur de la France, il nous permet encore d'assigner au grès anthracifère du Morvan à Lepidodendron Veltheimianum et ì Bornia transitionis sa place véritable. Il est contemporain du calcaire d'Yvoir Tle ou de l'assise de Chanxhe T2. A aucun tître, il ne saurait être considéré comme le prolongement du grès anthracifère du Beaujolais ot du Forez qui repose sur les sédiments à faune viséenne. La confirmation de ce résultat ressort définitivement de la comparaison de nos trois faunules avee les faunes chanxhiennes de Pair (Clavier) et de Petit-Modave, récemment découvertes; la première, par MN. Dewalque et P. Destinez, la seconde par M. P. Destinez. Ces firunes, typiques pour l'assise pélagique de Chanxhe, contemporaines des amas d'Hydrozocires veculsortiens du bassin de Dinant sont formées du mélange d'espèces tournaisiennes, en voie de disparition et d'espèces viséennes qui apparaissent ì ce moment et vont bientôt se développer d'une manière exclusive.

EXAMEN COMPARATIF DE LA FAUNE DE PAIR ET DES FAUNULES DU MORVAN.

Nous donnons ici, d'après M. P. Destinez, la liste des espèces recucillies par lui à Pair et publiée dans les Amnales de la Société géologique de Belgique (1).

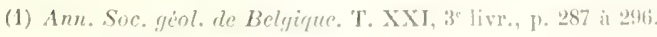


LISTE DES FOSSILES DU MARBRE NOIR (Vb) DE PAIR (CLAVIER).

\section{Poissons.}

Cladodus? (dent).

- cf. Springeri, John et Worthen T.

Physonemus (lanyon de nageoire) $\mathrm{T}$.

\section{Céphalopodes.}

Cyrtoceres Pusosianum, de Kon. T.

ignotum, de Kon. T.

Gyroceras serratum? de Kon. T.

Orthoceras neglectum, de Kon. T.

ef. discrepans, de Kon. T.

Goniatites Belvalianus, de Kon. T.

\section{Gastéropodes.}

Bellerophon Mïnsteri, d'Orb. T.

umbilicatus, de Kon. T.

Bucunia cleyans, d'Orh). T.

Cf: Tropydoeychus Duchasteli, Lév. T.

Worthenia Münsteriana? de Kon. T.

Loxonema Lefeborei, Lév. T.

Mourloniu, spenow.

$$
\text { pulcherrimum, M'Coy, V. }
$$

Ptychomphalus Benedenianus, de Kon. T.

Rhinesderma firagile, de Kon. V.

Strapremollus levigatus, Lex. T.

ef. coelatus, de Kon. V.

Netiropsis at. ocoidea, do Kon. T.

i). $110 \mathrm{~s}$.

Leguetopsis Busselerinenus, de Ryekholt V.

\section{Lamellibranches.}

Acieulopecten? anisotus, I'hillips. V.

ct: Bosquetirenus, de Kon. V.

exryuisiues, de Kon. T.

inegratus, de lion. T. 
Aciculopecten? Knochonniensis, M'Coy.

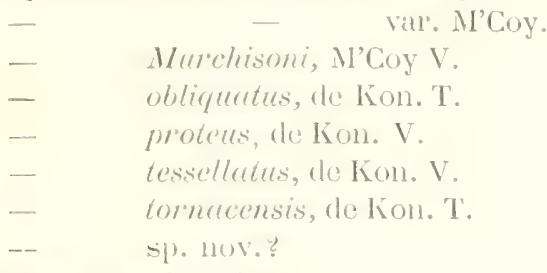

Meleagrina rigida, M'Coy. pulchelle, M'Coy.

Posidonia ef. costata, M'Coy.

Entolium? cet. tenue, de Kon. WV.

- sp. $110 \mathrm{v}$.

- Witryi, de Kon. T.

Tellinomya pusilla, de Kon. T?

Parullelodon eleyentulus, de Kon. V.

- meridionalis, de Kon. T.

- bistriutus, Portlock T.

Parallelodon, sp. nov.

Conocardium ef. Nysti, de Kon. 'T'.

- interlineatum, de Kon. IV.

- herculeum, de Kion. T.

\section{Brachiopodes.}

Productus semireticulutus, Martin T. V.

- marginalis, de Kon. V.

- Deshuyesianus? de Kion. V.

- plicatilis, Sowerby T. V.

- Nystianus, de Kon. V.

- tessellatus? de Kon. V.

- scabriculus, Martin V.

Chonetes variolata, d'Orb. T. undatus, var., Defi. V.

- Buchiana, de Kon. V.

- $\quad$ - val. Hardrensis, Phillips V.

- concentrica, de Kon. V.

- Laguessiuna, de Kon. V.

- tuberculata, M'Coy, V.

Athyris membranacer, de Kon. 'T'.

- Roissyi, Liv. 'T.

Orthis Michelini, I, iv. 'T'. 
Orthis resupinata, Martin V.

- ??

Orthotetes crenistria, Phillips T. IV. V.

- - var. radialis, Phill.

- - var. arachnoidea, Phill. V.

Lingula mytiloides, Sow, var. elliptica, Phill. V et $\mathrm{H}$.

Discina Davreuxiana, de Kon. T.

Crania (orbicula) quadrata, M'Coy.

Spirifer tornucensis, de Kon. T.

- Ramerianus, de Kon. T.

- Urii, Fleming.

- triradialis, Phillips V.

- neglectus? Hall. T.

Spiriferina insculpta, Phillips V.

- laminosa, M'Coy, T.

- Malleri, de Kon. T.

- peracuta, de Kon. T.

Dielasma corrugatum, de Kion. T.

insigne de Kon. T.

Retivia radialis, Phillips $\mathrm{V}$.

\section{Bryozoaires.}

Polypora verrucosa, M'Coy.

Vinculariandroides, M'Coy

Vineularia rarirostu, M'Coy.

Ptylopora pluma, N'Coy.

Glauconome pulcherrima, M'Coy.

Aconthocladia? grandis, M'Coy.

Ichthyorachis Newenhami, M'Coy.

lienestella carinata, M'Coy.

- ejuncida, M'Coy.

formosa, M'Coy.

frutex, M'Coy.

multiporata, M'Coy.

oculuta, $\mathrm{M}$ 'Coy $\mathrm{T}$.

plebeia, M'Coy, T'. W. V.

quardradecimalis, M'Coy.

corricrosu, M'Coy.

ef. Morrist, M'Coy.

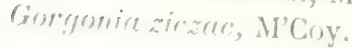




\section{Polypiers.}

Amplexus coralloides, Sow. 'T. IV. V.

- cornu-arietis, de Kon. 'T.

- cornuformis, Ludwig T.

- Henslowi, Edw. et Haime V.

Hadrophyllum Edwardsianum, de Kon. T.

sp. nov. (12 à 13 côtess).

$Z$ aphrentis cornu-copice, Michelin ' $\mathrm{T}$ '.

- cyathina, Edw. et Haime T.

- Delunouei, Edw. et Haime T.

- intermedie, de Kon. 'T.

- Edourdsiana, de Kon. T.

- Nystiana, de Kon. T.

- Omaliusi, Edw et Haime T. IV. V.

- vermicularis, de Kon. T.

- cf. vermicularis, an. sp. nov. T.

Monticulipora tumida, Phill. V.

Favosites parasitica, Phill. T.

Petraia? ef. Benedeniana, de Kon. T.

Cyathaxonia cormu, Michelin T.

Koninchi, Edw. et Haime T.

Menophyllum tenumarginatum, Edw. et Haime 'T.

Pentaphyllum cariophyllatum, de Kon. T.

Pyrgia Labechei, Edw. et Haime.

Lophophyllum breve, de Kon. T.

Palceacis compressa, Neek et Worthen T.

- cyclostoma, Phill. 'T.

Syringopora ramulosa, Goldf. T.

Cladochonus Michelini, Edw. et Haime T.

\section{Crustacés.}

Sp. $110 \mathrm{v}$.

Dithyrocaris Scouleri, var. M'Coy.

Leperditia Dewalquei, R. Jones et Kirklsy.

Phillipsia coelata (tête), M'Coy.

- gemmulifera, Phillips sp. V el T.

- granulifera, Phill. - P. Derbyensis, Martin. V. et 'T'.

- pustulata, Sohl., var. truncatula, Phill. 'T'. 


\section{Paléchinides.}

Palachinus gigas, W'Coy.

- Konigii, M'Coy.

- eleguns, M'Coy.

Archceocidaris Urii, Fleming.

Cidarites Munsterianus, De Kon. V.

\section{Crinoïdes.}

Actinocrinus dorsatus, De Kon. et Le Hon. T.

Platycrinus granulatus, Miller. T.

Cf. Millerianus, De Kon. et Le Hon. T.

Si nous examinons la répartition de ces espèces à Tournai et à Visé, d'après es traviux de De Koninck, nous arrivons aux résultats suivants:

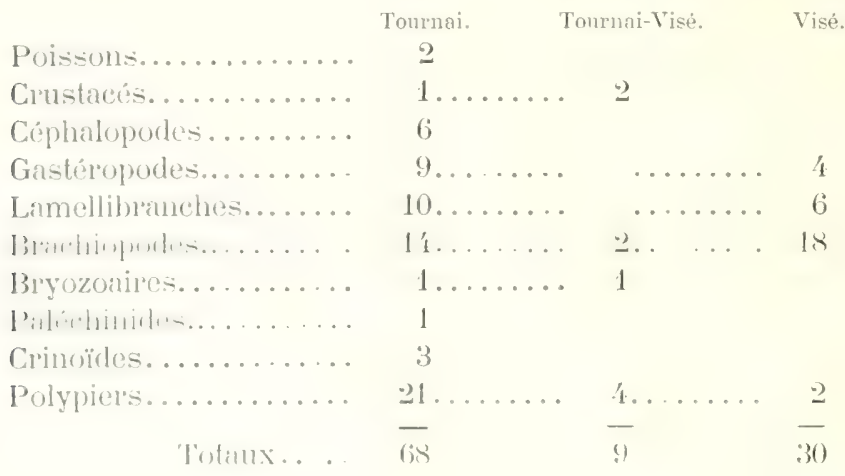

Si nous recherehoms les especes commmes it Pair et à la Varville, nous ohtenons la listes suivante:

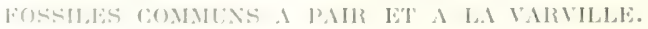

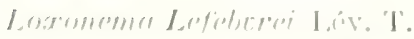

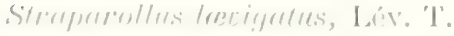

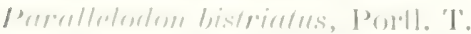

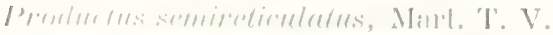

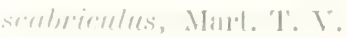


Chonetes variolata, ('Or'). T'.

Orthotetes crenistriu, Phill. T. WV. V.

Orthis Michelini, L, L. T.

- resupinutu, Mint. 'T. V.

Sprifer tornacensis, De Kon. 'T.

- Remerianus, De Kon. T'

- Nerei, T.

Spiriferina peracuta, de Kon. 'I.

Athyris Roissyi, Lév. T.

Dielasma insigne, De Kon. T.

Glauconome pulcherrima, M'Coy.

Monticulipora tumida, M'Coy. T. V.

Fenestella plebeia, M'Coy. T. IV. V.

- morrisii, M' Coy.

Amplexus corculloides, Sow. T. W. V.

Sur 53 espèces : 20 se trouvent à Pair, dont 11 spéciales à Tournai; 7 traversent tout le carbonifère; 2 n’ont pas été signalées jusqu’à la découverte de la faune de Pair, en Belgique. Pas une seule espèce spéciale à Visé.

Les faunules du domaine des Roches et de Siguret contiennent aussi des espèces communes, ainsi que le montrent les deux listes suivantes:

ESPẼCES COMMUNES A P.IIR ET AU DOMAINE DES ROCHES.

Aciculopecten ingratus, De Kon. T.

Parallelodon bistriatus, Portl. T.

Productus semireticulatus, Mart. T. V.

- scabriculus, Mart. T. V.

Chonetes variolata, d'Orb. T.

Spirifer tornacensis, De Kon. 'I'.

Sur 16 espèces déterminées, 6 sont à Pair, savoir : 4 tournaisiennes et 2 indifférentes.

ESPİCES DE SIGURET COMMUNeS A PAIr.

Loxonema Lefeborei, Lév. T.

Parallelodon bistriatus, Porti. T.

meridionalis, De Kon. 'T'.

Entolium Witryji, De Kon. 'T.

Productus scabriculus, Mart. T. V. 
Chonetes variolata, d'Orb. T.

Orthotetes crenistria, Phill. T. W. V.

Spiriferina peracuta, De Kon. T.

Cladochonus Michelini, Edw. et J. H. T.

Sur 24 espèces, 9 se trouvent à Pair, savoir : 7 tomrnaisiennes et 2 indifférentes.

Ainsi, en négligeant les espèces nouvelles spéciales au Morvan, ou nouvellement découvertes en Belgique, ainsi que les espèces indifférentes, sans valeur stratigraphique, les espèces communes sont toutes tournaisiennes. Nos trois faunes morvandelles sont réellement plus anciennes que la faune pélagique chanxhienne de Pair.

La comparaison avec la faune de Petit-Modave va nous donner le même résultat.

Fossiles du MARbre NoIr viséen $b$ DE PETIT-ModAVE (i).

\section{Crustacés.}

Leperditia Deuculquei, R. Jones et Kirkby.

Cf. Bairdia curtus, M'Coy.

Entomis biconcentrica, Jones.

Phillipsia pustulata, Schloth. T.

- Derbyensis (Yeux), Martin. T.

- truncutulus, d'après Phillips.

\section{Céphalopode.}

Orthoceras (indéterminable).

\section{Gastéropodes.}

Raphistoma of. redians, De Kon. T.

Naticopsis cf. Sturii? De: Kon. V.

Éromplialus mammula, De Kon. V. exreltutus, Do Kon. V. (ass. V.).

Lepotopsis Philligsi? I) Kon. (Pertella sinuosa, Phill.). V. 


\section{Lamellibranches.}

Posidonomya oblique, De Kon. V.

? constricte, De Kon. V.

Aciculopecten cf. tornacensis, De Kon. T.

Parallelodon bistriatus, Portl. T.

Coneardium alatum, De Kon. V.

- incrmatum, De Kon. T.

- ef. Plillipsi, De Kon. on sp. nov.? T.

\section{Brachiopodes.}

Orthis Michelini, Léveille. T.

Productus semireticulatus, Martin. 'T. IV. V.

- marginalis, De Kon. V.

- cf. tessellatus, De Kon. V.

- Nystianus, De Kon. V.

- plicatilis, Sowerby. T. et V.

Chonetes Dalmaniana, De Kon. V.

- variolata, d'Ob. T.

- Hardrensis, Phill. V.

Strophomena analoga, T. et V.

Spiriferina insculpta, Phill. V.

Spirifer tornacensis, De Kon. 'T.

- clathratus, M'Coy.

- cf. convolutus, Phill. V.

Streptorhynchus crenistria, Phill. (O. caduca, M'Coy). T. V.

Athyris Roissyi, Lév. T.

- ef. planosulcata, Phill. V.

Dielasma avellana, De Kon. V.

\section{Bryozoaires.}

Fenestella plebeia, M'Coy. T. W. V.

- quadradecimalis, M'Coy.

- varicosa, M'Coy.

- oculata, N'Coy.

- crassa, M'Coy.

Polypora papillate, M'Coy.

- verrucosa, M'Coy.

Glauconome pulcherrima, M'Coy.

Vincularia raricosta, I'Coy. 


\section{Echinides.}

Palechinus elegans, M'Coy.

- Tonigi, W'Coy.

- gigus, M'Cuy.

\section{Polypiers.}

Amplexus comuformis, Ludwig. T.

Petraia Benedenana, De Kon. T.

Cyathrexonia cornu, Mich. T.

Zaphrentis cermicularis, T.

Si l'on compare cette liste avec celle que nous venons de publier des mêmes couches de Pair (Clavier), on remarquera que tous ces fossiles sont signalés, sauf les sept espèces suivantes:

Spirifer clathrutus, M'Coy, qui n'avait pas encore été indiqué en Belgique.

Fenestella crassa, M'Coy.

Polypore papillata, M'Coy.

Conocardium alatum, De Koninck.

- inarmatum, De Koninck.

- Cf. Phillipsi, De Koninek.

Entomis biconcentrica, R. Jones.

Voici la lépartition de ces espèces dans les étages de Tournai et de Visé, d'uprès les travaux de De Koninek:

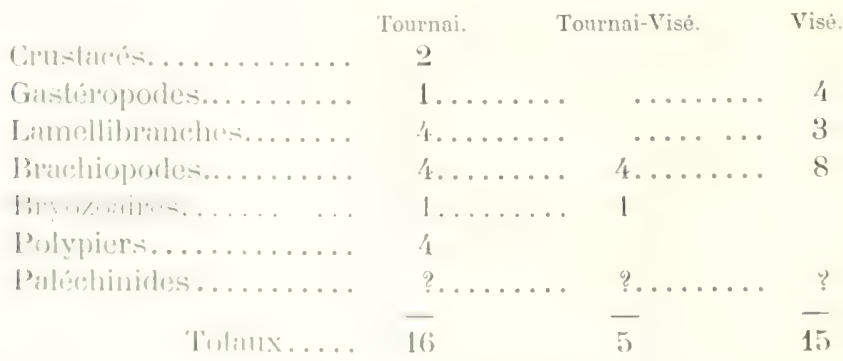

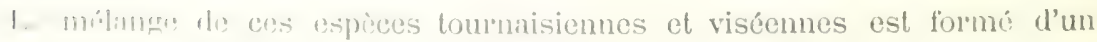
momhln: presifue ingal. 
ESPİES DE IA VARVILLE COMMUNES A PETIT-MODAVE.

Entomis biconcentrica, Jones.

Bairdiu, sp.

Parallelodon bistriatus, Portl. T.

Orthis Michelini, Léveillé, T.

Productus semireticulutus, Nart. T. V.

Chonetes variolata, d'Orb. T'.

Strophomena analoga, Phill. T. V.

Sprifer tornucensis, De Kon. T.

Orthotetes crenistria, Phill. T. V.

Athyris Roissyi, Lév. T.

Fenestella plebeia, M'Coy. T'. V,

Glauconome pulcherrima, M'Coy.

Sur 50 espèces déterminées, il y en a 11 qui se trouvent à Petit-Modave, soit : 5 tournaisiennes, 4 indifférentes, 2 nouvelles pour la Belgique et provisoirement sans valeur stratigraphique.

IAPLCES COMMUXES IU DOMAINE DES ROCHES ET A PETIT-MODAVE.

Parallelodon bistriatus, Portl. T.

Productus semireticulatus, Mart. T. V.

Chonetes variolata, d'Orb. T.

Spirifer tornacensis, De Kon. T.

Sur 14 espèces déterminées, 4 se trouvent il Petit-Nlodave, savoir : 3 tournaisiennes of 1 indifiérente.

ESPÈCES COMMUNES A SIGURET ET A PETIT-NODAVL.

Buirdic, sp.

Parallelodon bistriatus, Portl. T.

Orthis Michelini, Lév. T.

Productus semireticulatus, Mart. T. T'.

Chonetes variolata, d'Orb. 'T'.

Orthotetes crenistria, Phill. 'T. V.

Sur 24 espèces déterminées, il y en a 5 communes, saroir : 3 tournaisiennes ot 2 indifférentes. 
Ainsi se trouve établi, d'une manière définitive, le synchronisme des faunes de la bande carbonifère occidentale du Morvan, avec l'assise inférieure de Tournai de T $1 b$ à T $1 d$ de la classification de M. Dupont, ou T $b-T d$ de la classification officielle.

\section{GISEMENT DE CUSSY-EN-MORVAN.}

Ce curieux gisement de quelques mètres carrés de surface seulement a été signalé depuis longtemps par M. Collenot dans sa Description géologique de l'Auxois. Voici en quels termes :

"Nous devons signaler aussi sur la partie médiane du Morran, an milieu " des porphypes, au village de Cussy-en-Morvan, un dépôt de calcaire mar" neux bleuâtre dans lequel nous avons rencontré un Cyathophyllum. La " pierre (qui est employée à faire de la chaux devient parfaitement blanche par " la cuisson, ee qui indique que sa coloration en bleu foncé provient de " matières organiques. Le calcaire de Cussy nous paraît être de même origine

" que celui des bords de la Loire ${ }^{(1)}$."

Et plus loin :

"Le limbeau de calcaire fétide de Cussy-en-Morvan nous a paru faire partie " de l'élage dévonien, comme étant analogue au caleaire des bords de la Loire; " il est (lone peu probable qu'il appartienne à l'étage carbonifère (*). ")

Ainsi lo marbre de Cussy-en-MYrvan était considéré au début, par M. Collenot, anmue dévonien et synehronisé par lui avee le récif coralligène de Dion et de Gilly-sur-Loire, dont nous avons déterminé plus tard l'âge frasnien. Mais la díconverte d'un Lophophyllum dans ce marbre, pendant l'impression de son ourrage, le conduisit à modifier son opinion. Voici, en effet, ce que nous lisons plus loin encore: " De nouvelles observations et la similitude qui - existe putre lo calcuine de Cussy aree d'autres roches bien caractérisées sur le - virsinn sud-est du Morvint ${ }^{13)}$ nous portent à ranger ce calcaire dans l'étage - andunitere. Nous y sommes conduit encore par la détermination du seul - lissile recueilli jusqu'ici it Cussy, lequel appontiont a la classe des zoophytes - ma1111:.

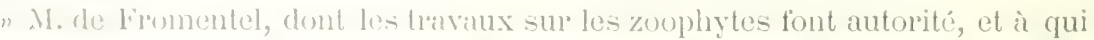

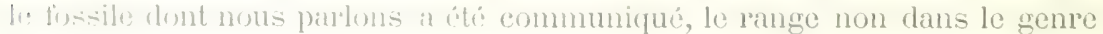
C'yathophyllum, comme: nuns l'avions dit en décrivant le calcaire de Cussy

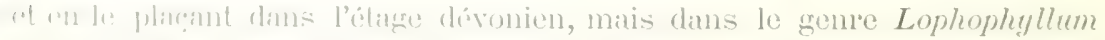

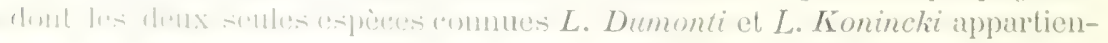

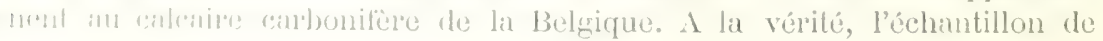

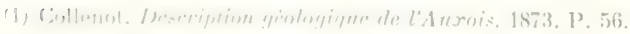

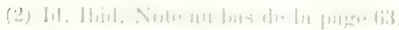

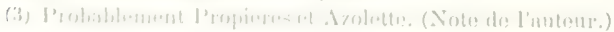


"Cussy, qui semble appartenir à une espèce nouvelle, est trop incomplet pour " être déerit spécifiquement, mais comme le genre auquel il appartient, n'a pas "été trouvé en dehor's de l'étage carbonifère, nous eroyons avoir été dans l'er" reur, quand sur l'autorité de MM. Dufrénoy et E. de Beaumont (Expl. de la "Carte géologique de France, t. I, p. 506 et 507), nous arons déclaré, en par"lant du terrain carbonifère, que, dans la France centrale, ce terrain ne porte

" pas de trace d'origine pélagienne (1). "

Nous donnerons encore deux autres citations du même volume qui montrent bien que M. Collenot s'était définitivement rallié à l'opinion exprimée par M. de Fromentel. " La disposition en coin entre deux murs de porphyre, du calcaire »noir et fétide de Cussy-en-Morvan (l'érosion a détruit le mur septentrional, "sans quoi le vestige de Cussy noyé dans le porpyhre resterait probablement " inconnu) est également la conséquence du même phénomène de dislocation. "Si ce gisement, conservant encore des fossiles qui doivent le faire ranger " dans le calcaire carbonifère, a moins souffert dans sa structure, il n'en a " pas moins subi des frottements et des modifications au contact des masses ") encaissantes... (2). ")

« La chaux carbonatée, si précieuse aux sols siliceux, est extrêmement „ rare; on ne la trouve qu'à Cussy-en-גIorvan, dans une carrière de calcaire " carbonifèré, aujourd'hui abandonnée et enclavée dans les roches cristal") lines ${ }^{(3)} \cdot$ )

En 1879, M. Michel Lévy attribua le marbre de Cussy au Dévonien, comme on peut s'en assurer par cette citation : "Les schistes de Champ-Robert et du " Puits, qui contiennent des lentilles de marbre blane, nous paraissent se rap" porter à cet Etage carbonifère, tandis que nous considérons les lydiennes ” et les calcaires foncés de Cussy-en-Morvan, et de l'Huis-Pernelle, près Plan" chez, comme appartenant à la formation dévonienne, car la granulite les tra"v verse et la formation tuffacée précédente les disloque ${ }^{(4)}$. »

Observation. - A propos de la granulite, nous demanderons la permission de reproduire cette autre citation du même auteur :

“ Dans le Morvan, la granulite paraît avoir été la formation éruptive domi" nante pendant une longue période géologique; toujour's postérieure au gra"nite, elle a probablement métamorphisé et certainement pereé des couches " dévoniennes (Bourbon-Lancy, Cussy-en-Morvan). Mais clle avait déjà sans " doute antérieurement formé de vastes épanchements plus anciens que tous ") les terrains stratifiés voisins ${ }^{(5)}$."

Nous ne partageons aucune des idées de M. Miehel Léry relativement ì ces deux localités; nous démontrerons plus loin que le marbre de Cussy-en-1Ior-

(1) Collenot. Loc. cit. Note rectificative au bas de la page 154.

(2) Collenot. Loc, cit, , P. 358.

(3) H. Hid., p. 557 .

(i) B. S. G. 1'. Réunion extreortinate it Semur, T. V"II, p. 939.

(5) Il. Hid., p. 761 . 
van est tournaisien. La granulite n'a traversé, d'après nous, que les schistes cambriens du voisinage. Il en est de même à Bourbon-Lancy, que nous avons étudié d'une manjère spéciale et qui offre une belle coupe de terrain cambrien, sans aucune trace de Dévonien. Nous ajouterons encore à ce propos que le vaste lambeau de terrains primaires qui s'étend entre le Frasnien de Gilly-sur-Loire et Chalmoux, sur la feuille de Charolles, lequel est traversé également par la granulite, loin d'être carbonifère marin, comme l'a colorié sur cette feuille M. Michel Lévy, est au contraire, à notre avis, nettement Cambrien.

Par conséquent, nous sommes convaincu que la granulite vient s’intercaler entre le Cambrien, qu'elle a traversé de longs et minces filons, ou de puissants massifs, et le récif frasnien de Diou (Allier) et Gilly-sur-Loire. Elle est done post-cambrienne mais ante-frasnienne, et, à plus forte raison, ante-carbonifère, sans qu'il soit possible, jusqu'à présent, de fixer d'une manière plus précise l'époque de sa sortie. C'est là notre conviction, basée sur nos recherches persomnelles, et qu'on nous permettra de ne pas passer sous silence.

En 1883 (1), M. Stanislas Meunier a fait connaitre quelques Foraminifères appartenant aux genres : Saccamina, Sars; Cameroconus, nov. gen.; Climaccamina, Brady; Endothyra, Phillips; Septammina, nov. gen.; Archcediscus, Brady; qui sont renfermés en grand nombre dans le marbre très compact, d'un roir profond, de Cussy. Il se base sur la présence de ces Foraminifères pour attribuer ce marbre, au calcaire de Visé des géologues belges, et il signale la préscnce de cet horizon, pour la première fois, sur le territoire français: "En résumé, dit ce savant, bien que les Fusulines soient très rares dans le calcaire de Cussy-en-Morvan, les fossiles que renferme cette roche suffisent pour révéler la présence en France d'un horizon stratigraphique considéré jusqu'ici comme étranger à notre pays ${ }^{(2)}$. )

Nous ne savons ce que veut dire par là M. Stanislas Meunier. Il y a de longues années, en effet, que l'on connait le carbonifère marin de l'étage de Visé à Régneville, à Sablé, à Plancher-les-Mines, à Régny et dans la Montagne-Noire. Nous-même avons publié en 1874, dans les Comptes-Rendus de l'Académie des sciences, le gisement de l'Ardoisière, près de Vichy. Quant au calcaine it Fusulines, facies marin du Houiller et non point du Viséen, car on n'a jumais décourert de Fusulines à Visé, il n’existe nulle part en France.

Etrange assertion de M. M. Fayol. - A propos de la mer à Fusulines, qu'on nous pernelte une digression, ì propos d'une assertion étrange de M. H. Fayol,

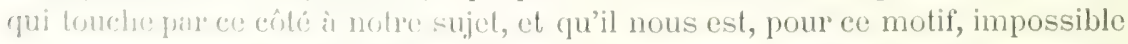

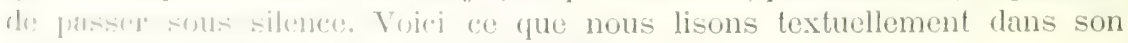
ouvrage sur le bassin houilled de: Commentry.

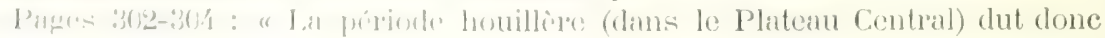

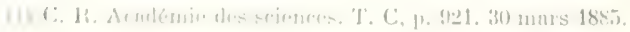

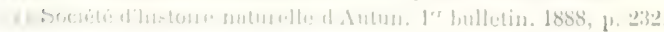


") s'ourrir sur un sol très accidenté. Le rivage de la mer était à Buxières.... „ La sédimentation commença simultanément dans les lacs (y compris celui "de Commentry) et dans l'anse marine de Buxieres; elle fut rapide sur cer"tains points, lente sur d'autres. Tous les lacs furent comblés, et il vint un ») moment où la formation houillère ne se poursuivit plus qu'à Buxières. Le lac "de Commentry fut un des premiers remplis........................ "Comme la formation houillère, la formation permienne s'est effectuée d̀ la "fois dans un grand nombre de laes et sur le littoral marin. Les dépôts lacus" tres ont pris fin avee le remplissage des lacs; les dépôts marins se sont pour" suivis sans interruption jusqu'ì l'époque jurassique, qui se trouve reprísen" tée au nord du département de I'Allier, par une série de roches où domine ") le calcaire (1). ")

2 Page 330 : "Aueun vestige de glacier n’a été p’encontrú dans le bassin de "Commentry. La mer, d'ailleur's, était it peu de distance, 2 k kilomètres, ì "Buxières, et les sommets voisins de Commentry ne semblent pas avoir ") dépassé à ce moment l'altitude de 1,000 mètres (). )

$3^{\circ}$ Enfin, page 345 : "Formation permienne de Buxières-la-Grue. A Buxières, " la formation permienne, très puissante, se présente sous la forme de couches " bigarrées argilo-marneuses qui reposent en concordance sur les couches " houillères (sous-jacentes)....... ct la sédimentation s'y pour'suit sans inter"ruption dans la mer ${ }^{(3)}$."

Ainsi, suivant M. H. Fayol, et les citations précédentes prouvent chez lui une conviction bien arrêtée, pendant que le lac de Commentry, gouffre de 800 mètres de profondeur ${ }^{(4)}$, entouré de montagnes ne dépassant pas 1,000 mètres, se remplissait en 170 siècles ${ }^{(5)}$ par voie d'apport fluvial, le terrain houiller de Buxières, distant de 24 kilomètres seulement, se déposait dans la mer, et dans cette mer permanente et immuable, continuaient à s'entasser sans interruption couches houillères el permiennes, couches triasiques et jurassiques! D'où provenait done cette mer? et quelle était-elle? Mais, une mer au sein de laquelle se déposait du houiller, ne pouvait être que la mer à Fusulines, dont M. H. Fayol qui semble même en ignorer le nom, nous certifie ainsi, à notre véritable stupéfaction, la présence à deux pas de l'Auvergne, en plein Bourbonnais! Or, cette mer ne pouvait être que la continuation do la mer viséenne de l'Ardoisière et de Régny, respectée par les premier's soulèvements hereyniens et reléguée à Buxières-la-Grue! Chose non moins singulière! L'honorable directeur de Commentry ne se préoceupe pas plus de l'absence totale de fossiles marins à Buxiùres, que de l'origine si radica-

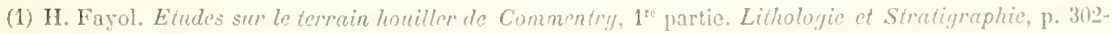
301. Soint-Etienne, 1886.

(2) Id. Ibid., p. 330 .

(3) Id. Ibid., p. 345 .

(4) Id. Ibid., p. 305.

(5) Id. Hsid., p. 321. 
lement incompatible qu'il attribue à deux bassins, quasi contigus, synchroniques, offrant mème composition, même structure, même flore et même faune. Ici, à Commentry, on aurait un delta fluvio-lacustre; et là, à Buxières, un dépôt pélagique pur, exclusif, sans mélange, où sans doute, devaient, dans cette conception, s'aceumuler et se superposer sans trève ni repos, Faune houillère à Fusulines, Faune permienne à Céphalopodes, Faune pélagique du Trias! On nous permettra de ne pas insister davantage sur l'étrangeté d'affirmations qui n'ont rien de commun, ni avec l'observation, ni avec la science acquise la plus élémentaire, et de rappeler simplement que la mer carbonifère, chassée de France, d'Angleterre, de Belgique et d'Allemagne, après l'époque de Visé, n'est revenue dans nos régions qu'à la fin du Trias. C'est à M. Pellat que l'on doit la découverte, en 1876, des premières traces de fossiles marins amnonçant le retour de la mer. Il s'agit des coquilles littorales, Natices, Myophories, Avicules, qui gisent dans le keuper de la montagne de Drevin, près de Couches-les-Mines (Saône-et-Loire) (1). Un peu plus tard, la mer Infraliasique, pénétrant jusqu'à Saint-Amant-Montrond, non loin de Commentry, déposait des banes d'Ostreea sublamellosa, signalés sur la colline du Grand-Tertre par M. Dagincourt. Entre les couches marines de Régny et de l'Ardoisière d'ume part, et les niveaux fossiliféres que je viens de rappeler d'autre part, y compris la zone à Acicula contorta, tout dans la France centrale, honiller, permien, grès bigarré est, de l'aveu unanime des géologues français, d'origine terrestre et continentale.

L'historique de Cussy-en-Mlorvan ctant terminé, nous ferons connaître, d̀ notre tour, notre opinion personnelle sur cet intéressant lambeau de marbre crinoïdirque enclavé dans le porphyre.

Dans une cxcursion à Cussy, nous avions réussi à recueillir, in situ, au sommet de la carrière abandonnée, un bloc rempli de beaux Polypiers. C'est le Cyathophyllum multiplex, Leyscrl. Nous avions tenu à le soumettre à M. de Roninck qui venait de publicx tout récemment son mémoire sur les Polypier's fossiles du teranin carbonifère de la Belgique. C'est à lui que nous devons la détermination précise de notre spécimen du Morvan. Le Cyathophyllum multiplex ext exclusirement tournaisien. Il se reneontre ì Toumai dans les banes canlcailes intercalés dans le culschiste. Sa découverte vient done confirmer l'attribution it cet étage, faite jadis par M. de Fromentel, aprés eximen du Lophofhyllum de M. Collenot. Cussy-en-Morvan appartient, sans conteste, aux

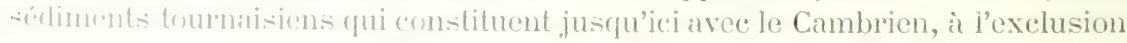

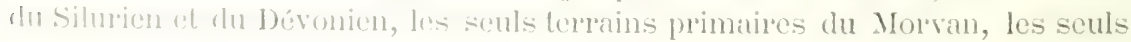

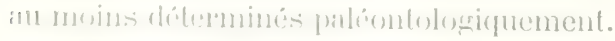

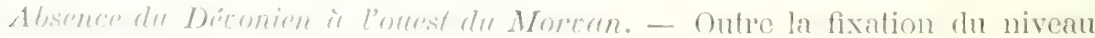

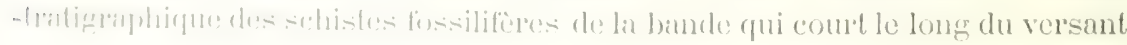

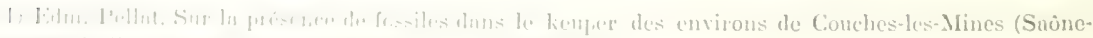

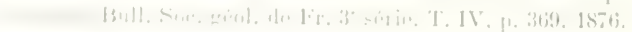


oceidental du Morvan, entre Rémilly et Luzy, nous avons pu nous convainere qu'il n'y existe aucune trace de terrain dévonien. Il suffit, pour s'en assurer, de faire la coupe transversale de la bande entre ces deux localités, soit le long de la voie ferrée, soit un peu plus au sud, entre Prairiau st Siguret, par lo domaine des Roches et Savigny-Poil-Fol.

\section{COUPE DE PRAIRIAU A SIGURET (NIEVTRE).}

Onest.

Siguret

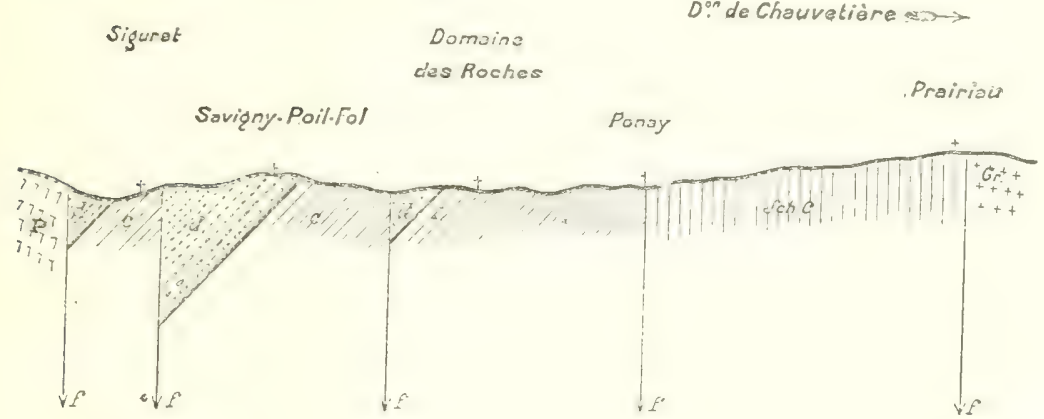

Gr. Granite.

$P$. Porphyre.

Sch.C. Schistes cambriens.

a. Schistes gréseux et caverneux it la base, jaumes-rougeàtres sans fossiles, passant graduellement aux schistes fossilifères $b$.

b. Schistes décalcifiẻs, fossilifères.

c. Schistes gris-noiràtres, fossilifères (mèmes fossiles (qu'en $L$ ).

d. Poudingue et grès anthracifère.

f. Failles.

Quand on arrive de Luzy et qu'on a contomrué la montagne granitique de Chauvetière, on aborde à Prairiau la bande des quartzites et des schistes cambriens, de couleur noire, plissés et azoïques. Ces schistes sont, on plusieurs points, traversés par des filons de granite on de pegmatite. Ils sont limilés, a l'ouest, au domaine de Ponay, par une faille très nelte, visible sur les deux talus de la route. Cette première faille est dirige N. $55^{\circ} \mathrm{L}$. et on peut la suivere dans le nord jusqu'à la route de Toulon-sur-Arroux, au pont du Veurdre, où elle coupe cette route entre Luzy el Lanty, it 4k600 à l'ouest de Luzy.

Un peu plus loin, une nouvelle faille, parallete it la premiere, va du domaine des Roches au Moulin-Neuf, contigu is la vole ferrée de Never's à Chagny. 
Les schistes compris entre ces deux failles ont une direction générale N.-S., ils sont inclinés ver's l'ouest de $40^{\circ}$ à $50^{\circ}$.

Ces schistes jaunâtres ou rougeâtres d'abord, parfois caverneux et gréseux, deviennent bientôt comme argileux et satinés. Les preuves d'un laminage intense se laissent fréquemment observer. Sans fossiles vers la base, ils présentent à la partie supérieure, dans le voisinage du poudingue et du grès anthracifere qui les surmonte, de nombreuses empreintes, par exemple dans une épaisseur de 5 à 6 mètres, au-dessous de la base du poudingue.

C'est là que nous avons recueilli la faune du domaine des Roches, mentionnée plus haut. Nous attribuons à la faille et à la sortie d'eaux thermales, la décalcification de ces schistes et la coloration brun-rouge du poudingue et des grès. Au delà de la faille qui interrompt les poudingues, les schistes reprennent leur coloration gris-noirâtre; ils sont tout aussi fossilifères et nous ont donné, par exemple, de nombreux spécimens de Rhynchonella acutirugata, etc. Savigny-Poil-Fol est bâti sur le poudingue, qui est beaucoup plus épais en ce point. Au delà du village, une nouvelle faille interrompt la stratification et le hameau de Siguret est bâti sur des schistes également noirâtres, remplis d'empreintes ou de moules de fossiles, parmi lesquels ceux dont nous avons donné précédemment la liste. Ces schistes disparaissent rapidement sous les poudingues et les grès habituels, traversés par un filon de porphrre qui termine la coupe.

Ainsi cette bande paléozoïque qui affleure au pied occidental du Morvan, limitée entre le granite de Chauvetière et le porphyre de Siguret, ne laisse aperecroir, à notre avis, aucune trace de ce terrain dévonien qui a été figuré sur la feuille d'Autun. Elle est exclusivement constituée par les quartzites cambriens de Luzy ì l'est, et à l'ouest par les schistes tournaisiens, avec une faune caractéristique, qui viennent buter contre les premiers par faille. Les schistes tommaisiens sont recouverts dans le voisinage des failles par le grès inthracifère du Morvin, devenant poudinguiforme à la base.

Cette coupe temoigne en ontre qu'entre ces schistes fossilifères, prolongement des calschistes de Tournai dans le coeur de la France, et le grès superprosí, aucune assise nouvelle ne vient s'intercaler. Quelles conséquences en tirer relativenent à l'âge du grè̀s anthracifère du Morvan? C'est que ce grès secupe stratigraphiquement la position du calcaire d'Yvoir et de l'assise de Chanxhe. Nons y reviendrons plus loin, au chapitre consacré au grès anthracifiens. 


\section{PLATEAU CENTRAL}

Les gisements fossilifères que nous avons étudiés dans le Plateau Central sont au nombre de 12, savoir : Régny, Montagny, Néronde, Montmain, Le Gouget, Saint-Germain-Laval (Loire), Thizy, Propières, Létra (Rhône), Ferrières, en y comprenant Cheval-Rigon, l'Ardoisière (Allier), Evaux (Creuse).

Tous ces gisements sont connus et ont été décrits par des auteurs différents, Grüner, Fournet et moi-même.

Nous commencerons leur étude par Régny dont la faune est plus considérable et peut servir de type.

\section{REGNY.}

La faune de Régny a été recueillie dans toute l'épaisseur de la formation, schistes et marbres, et dans toutes les carrières en activité ou abandomnées qui entourent cette localité célèbre.

\section{FAUNE DE RÉGNY.}

1. Cochliodus acutus, Ag.

2. Psammodus, sp.

3. Dithyrocaris, sp.

4. Bairdia, sp.

5. Nautilus sulciferus, Phill. V.

6. Cyrtoceras Pusosianum, de Kon. V.

7. Macrochilina Pireti, n. sp.

8. Loxonema priscum, Goldf. V.

9. - propinquum, de Kon. V.

10. Straparollus Dionysii, D. de Montf. V.

11. Euomphalus amœnus, de Kon. V.

12. Phanerotinus nudus, Sov. V.

13. Baylea spirata, de Kon. V.

14. Bellerophon scalifer, de Kon. V.

15. Waagenia Ferussaci, d'Orb. V. 
11. Lepetopsis, cf. Busscherianus, de Ryck. T.

17. Dentalium, sp.

1r. Edmondia orbiculata, de Kon. V.

1!) - scalaris, M'Coy, V.

201. - filigrana, de Kon. V.

21. - amabilis, de Kon. V.

2-2. - - ? selecta, de Kon. V.

-3. - ? ameren, de Kon. V.

2y. Sanguinolites tricostatus, Portl. V.

2.). Solenopsis Bielawskii, 11. sp.

26. Cypricardella? Julieni, de Kon.

2-. Nucula, sp.

ㄱ․ Nuculana, sip.

2!). Tellinomya, sp.

30. Parallelodon argutus, Phill. V.

31 . - - comoides, de Kon. V.

: 3 .2. $\quad-\quad$ mytiloides, de Kon. V.

33. Modiola chneiformis, de Kion. V.

3'. Leiopteria hirindo, de lion. V.

:3). - lunulute, Phill. V.

:3i. Aviculopecten colatis, M'Coy V.

$\therefore$ : - plagiostoma, de Kon. V.

:ir. - dupliciradiatus, de Kon. V.

3:1. Productus giganteus, Mart. V.

il). corte, d'Orb. V.

11. - semireticulatus, Mart, var. concinnus, V.

12. - pustulosus, Phill. V.

13. punctutus, Mart. $\mathrm{V}$.

í. elegans, M'Coy, V.

is. Chometes popilionacen, Phill. V.

i6. Murchisoni, n. sp.

\{. - Comoides, Sow. YT.

18. Dalmuniuna, de Kon. V.

1: L Lagruessiuna, de Kon. V.

at. Jourdenti, 11. sp.

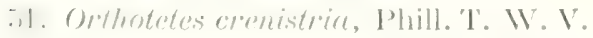

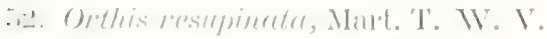

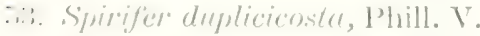

in. bisulcretus, Sow. V.

… - interfricostre, Phill. I'.

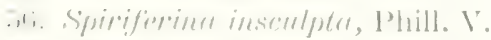

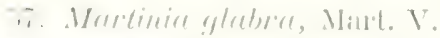

a. limenter, Minl. I'. 
39. Athyris planosulcata, Phill. V.

60. Rhynchonella pleurodon, Phill. V.

61. - pugnus, Mart. V.

62. - angulata, Limné $\mathrm{V}$.

63. Dielasma sacculus, Mart. V.

64. Crania, sp.

65. Archeocidaris, sp.

66. Palcechinus gigas, N'Coy V'.

67. - Koninchi, n. sp.

68. Poteriocrinus crassus, Miller. V.

69. Amplexus coralloides, Sow. T. W. V.

70. Zaphrentis, sp.

71. Syringopora ramulosa, Goldf. V.

72. Cladochonus Heribaudi, n. sp.

EXIMEX BATHYMÉTRIQUE DE LA FALNE DE RÉGYY.

On est frappé quand on explore les schistes arec lentilles de marbre noir des carrières de Régny, de l'abondance inusitée des traces laissées par les algues marines. Souvent les empreintes de ces végétaux remplacées par la matière charbonneuse ont une épaisseur appréciable et l'on est amené à penser que la hauteur de la mer était faible, que la faune devait revêtir un caractère franchement littoral ou sublittoral, compris dans le balancement des marées et ne dépassant pas la zone des Laminaires. Cette conviction est fortifiée par l'examen de la faune. Ainsi les bivalves des genres Solenopsis, Tellinomya, Parallelodon, Modiola, Leiopteria, Aviculopecten sont prédominants. Ce caractère se maintient jusqu'au contact même du poudingue qui scrt de base au grès anthracifère. Le rivage méridional de la mer carbonifère ne pouvait done être bien éloigné.

Examinons maintenant la valeur stratigraphique des espèces en nous référant aux travaux exécutés dans ce sens pour les fossiles belges par M. de Koninck. Nous y serons aidés par les notations T. W. V. qui accompagnent les espèces communes aux deux pays et qui conservent la même signification que précédemment.

Si l'on excepte les dix genres que l'on n'a pu déterminer spécifiquement i cause du maurais état de conservation, il reste soixante-deux especes qui se répartissent dans les cinq groupes suivants :

$1^{\circ}$ Especes de Toupnai............. 1 (Lepetopsis ef. Busscheriunus.)

2 Espèces viséennes............. 19

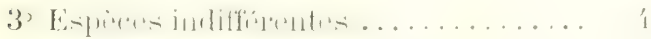

4 Especes nouvelles............. 7

$5^{\circ}$ Espèces encore incommues an Belgique 1 (Cochliodus acuuss). 
Si nous faisons abstraction de Lepetopsis ef. Busscherianus, nous voyons que la faune de Régny est tout à fait différente des faumes tournaisiennes du Morvan, avec lesquelles elle n'a de communes que les quatre espèces du 3 e groupo ou espices traversant tout le carbonifère, savoir: Orthotetes crenistria, Orthis resupinata, Martinia lineata et Amplexus coralloides.

C'est une faune plus jeune, une faune viséenne, offrant non point les caractìres de l'assise de Visé à Productus giganteus proproment dite, mais ceux de l'assise de Dinant de M. E. Dupont. Si nous recherchons, en effet, dans cette liste les espèces que M. Dupont a désignées nominativement comme caractérisunt certains niveaux particuliers de cette assise, nous relerons les noms suivints:

Productus semi-reticulatus, var. concinnus. Mart., caractéristique de V1 $b$.

Chonetes comoides, Sow., caractéristique de V1b, Vle, Vif. papilionacer, Phill., caractéristique de V1b, V1e, V1f.

Productus cor'l, d'Orb., caractéristique de V1g.

Nous pouvons ajouter maintenant Palcechinus gigas M'Coy, qui, d'après les décourertes récentes, est situé à l'extrême base de l'assise Vlb.

Il n'est pas saus intérêt de comparer la faune de Régny avee une faune dinanlienne de position bien déterminée que M. G. Soreil qui l'a découverte a publiéc il y a quelques mois à peine. Je veux parler de la faune de Denée composée anjourd'hui de trente-huit espèces, et qui caractérise la base de V1b. Elle git immédiatement au-dessus du calcaire à Echinides (Pal. gigas) qui lui-même reconvre l'assise V1a, faciès latéral des marbres waulsortiens à Stromatoporoides. Au reste, la comparaison de chacune de nos faunules dinantiennes avec Denée nons paraît si instruetive que nous n'hésitons pas ù reproduire intégralement cette faune belge. 
Listl des fossiles du Marme Nolr di: Dexée.

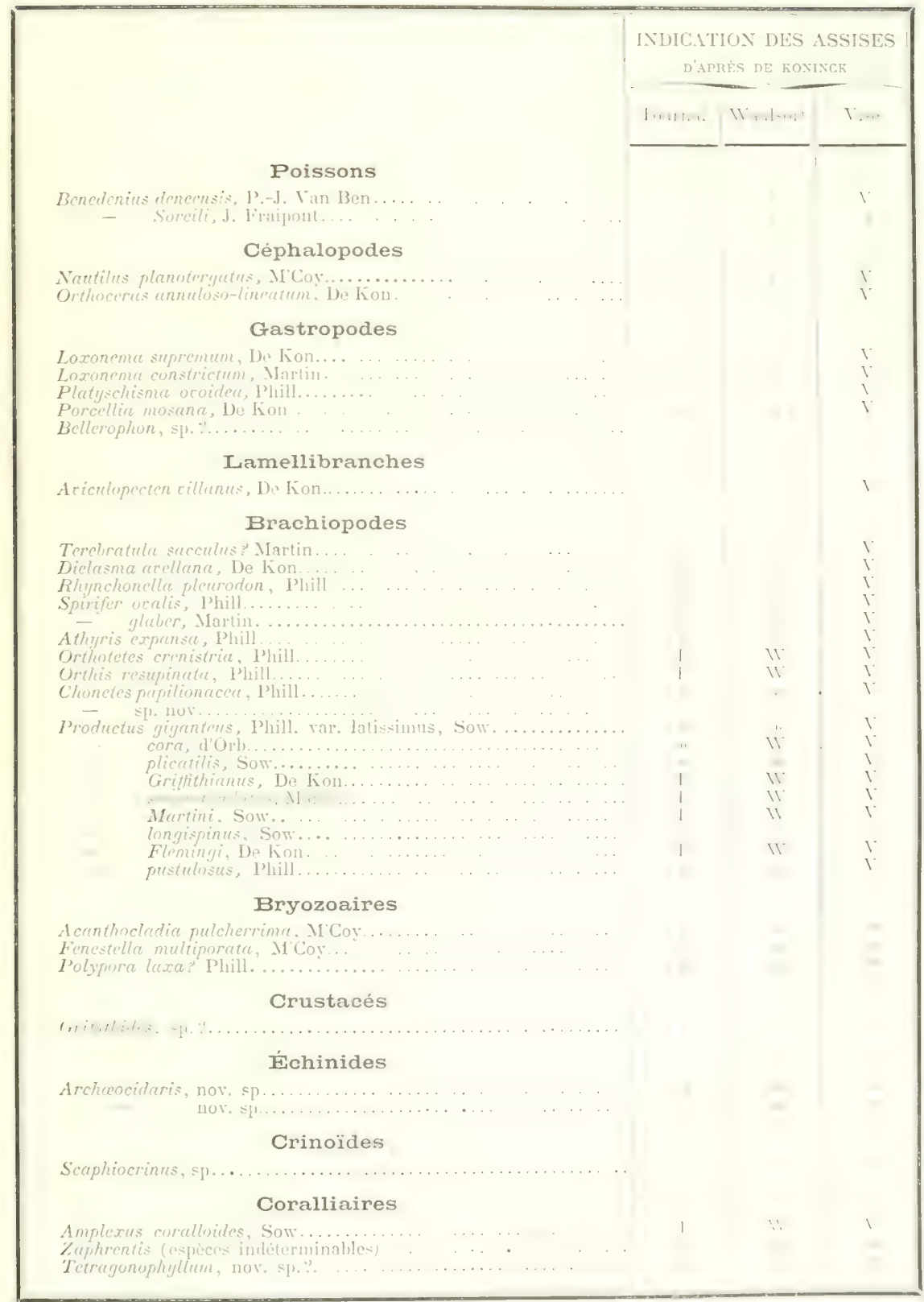


La comparaison des faunes de Régny et de Denée nous donne la liste suivante :

ESPLCES COMIUUNES A RÉGNY ET I DENÉE.

Dielasma sacculus, Mart. V.

Rhynchonella pleurodon, Phill. V.

Martinia glabra, Mart. V.

Orthotetes crenistria, Phill.

Orthis resupinate, Mart.

Chonetes papilionacea, Phill. V.

Productus cora, d'Orh. V.

- semi-reticulatus, Mart.

- pustulosus, Phill. V.

Amplexus coralloides, Sow.

Malgré le nombre r’estreint d'espèces communes, nombre que des recherches persévérantes accroîtront d'une manière certaine, les six espèces exclusivement viséennes confirment le caractère franchement dinantien de la faune carbonifère de Régny.

Conclusion. - Régny colrespond dans tout son ensemble à l'assise de Dinant V1b à V1h de M. E. Dupont, y compris à la base l'horizon des Paléchinides. Nous observerons, en outre, d'une manière toute spéciale, l'absence absulue au sommet du gisement de la faune dite de Visé à gros Productus gigunteus et Cor'u et à Polypier's isolés ou constructeurs. La faune de Régny reste bien homogìne avee son caractere exclusivement littoral ou sublittoral de la base jusqu'au contact des poudingues qui supportent le grès anthracifère.

l'our' ne pas nous répéter indéfiniment, voici la marche que nous suivrons daus nos analyses. Nous domnerons sucessivement la liste des faunes des antres gisements; nous examinerons leur composition par rapport aux lanues lodge, puis nous domerons le lableau des espèces communes avee les givenents voisins d'abord, avee Dinant et avee Denée ensuite. Cela fait, nous dirdurrons de cette comparaison les eonelusions relatives : $1^{\circ}$ à leur synchro-

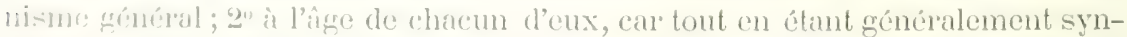
chronirpues le hasand des érosions ou des oseillations a pu modifier légèrement

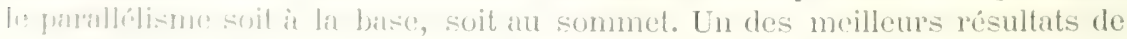

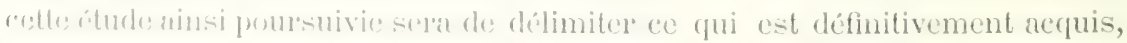

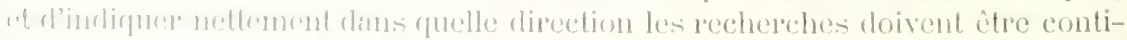
$1111+\cdots+2$ 


\section{NERONDE:}

"En allant de Balbigny à Néronde, dit Grüner" dans la description géologique " de la Loire, page 326, on rencontre les premières masses calcaires sous les " nur même de la ville; mais les carrières en exploitation sont situées plus ") à l'est, entre la chapelle qui domine la ville et le bois de la Chaux. Le calcaire » est divisé en banes d'une faible épaisseur. Il est criblé de tiges d'enerines, " mais ne semble renfermer aucun autre fossile. "

Voici la liste des fossiles que malgré leur très mauvais état de conservation, nous avons pu réussir à déterminer. Ils ont été recueillis dans toutes les earrières en activité ou abandonnées et à tous les niveaux de la formation.

Bairdia, sp.

Loxonema propinquum, de Kon. V.

Flemingia Hisingeriana, de Kon. V.

Straparollus pileopsideus, Phill. V.

Eumphalus crotalostomus, M'Coy V. - cutilliformis, de Kion. V.

Baylea spirata, de Kon. V.

Edmondia? augresta, de Kon. V.

Cypricardelle? Julieni, de Kon.

A ciculopecten Bosquetianus, de Kon. V.

Productus pustulosus, Phill. V.

Chonetes papilionacea, Phill. V.

-- Murchisoni, 11. sp.

-- comoides, Sow. V.

- Dalmaniana, de Kon. V.

Orthothetes crenistria, Phill. T. IV. V.

Orthis resupinatr, Mart. $T . \mathrm{V} . \mathrm{V}$.

Spirifer duplicicosta, Phill. V.

- integricosta, Phill. V.

Spiriferina insculpte, Phill. V.

Martinia glabra, Mart. V.

Rhynchonellu pugnus, Mart. V.

Palechinus Koninchi, n. sp.

Poteriocrinus crassus, Miller. V.

Syringopora ramulosa, Goldf. V.

Cladochonus Heribuudi, n. sp.

Si l'on excepte le geme Bairdia non déterminé spécificquement, il reste 24 espèces qui se décomposent ánsi : 


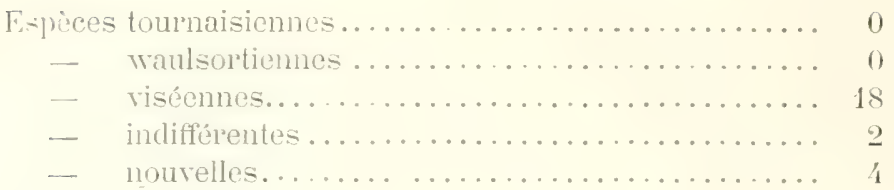

La fanne de Néronde est viséenne.

Elle est synchronique de celle de Régny, car sur vingt-quatre espèces déterminées, dix-neuf existent à Régny. En roici la liste :

FOSSILES COMMUNS A NERRONDE ET A RB̈GYY.

Loxonema propinquum, de Kon.

Baylea spiratu, de Kinn.

Cypricardellu? Julieni, de Kion.

Productus pustulosus, Phill.

Chonetes pupilionucen, Phill.

- Murchisoni, nov. sp.

- comoides, Sowerby.

Datmanianu, de Kon.

Orthotetes crenistria, Phill.

Orthis reselpinuter, Nart.

Spirifer deplicicoste, Phill.

- intergricosta, Phill.

Sprififerine inseculpte, Phill.

Mertinia ylubrer, Mart.

Rlignchoneller puesmess, Mart.

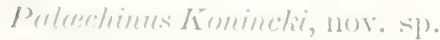

Pesteriorrinus cretsistes, Miller.

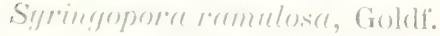

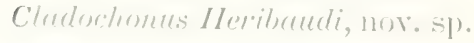

I,

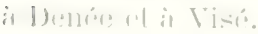

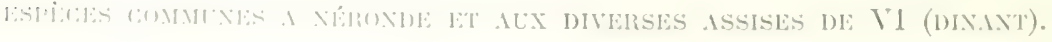

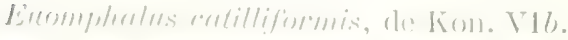

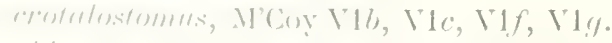

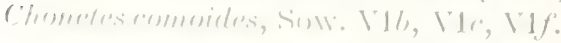

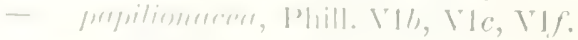


Productus corel, d'Or\%. Vis.

Palechinus Koninctit, nov. sp).

L'E. crotulostomus N'Coy est une espece rare et des plus caractéristiques. Nous arons fait figurer une portion du moule interne que nous avons en l'heureuse chance de recucillir.

Le Chonetes comoides est excessivement abondant dans une vicille carrière abandonnée, où on l'observait par centaines faisant saillie ì la surface des banes délaissés, supportant la faible lentille calcaire enlevée. Le Pal. Konincti est relativement abondant dans cette carrière; nous y soupcomnons la présence de $\mathrm{Pal}$. gigas mais les plaques de cet oursin tessellé sont si mal conservées, que nous avons préféré nous abstenir de le citer.

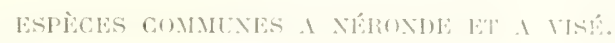

Flemingia hisingeriana, de Kon.

Straparollus pileopsideus, Phill.

Bayleu spirata, de Kon.

Spirifer duplicicosta, Phill.

ESPÈCES COMNUYES A NŔRONUE ET A IDENÉ.

Productus pustulosus, Phill.

Chonetes papilionacea, Phill.

Orthotetes crenistria, Phill.

Orthis resupinatre, Phill.

Martinia glabra, Mart.

Euomphalus catilliformis, de Kon.

POSITION STR.ITIGR.APIIQUE DE LA FAUNE DE NÉRONDE.

Elle est identique à celle de liégny et ello correspond ì tout l'ensemble de l'assise de Dinant, de V16 it Vhh, y compris le nivenu it Paléchinides de l'extrême base.

\section{MONTMAIN.}

Le gisement de Montmain qui a proemó los fossiles menlionmés ci-dessons appartient à la bande carbonifère de Néronde. Grüner an al donné la compe page 328 de son ouvrage. C'est dans la curriere onverte judis en ce point, pour 
l'extraction d'une mince lentille de calcaire que l'on a constaté la présence d'un filon de stibine traversant les couches carbonifères, lesquelles butent par faille contre les schistes cristallophylliens de Sainte-Agathe.

Grüner ne cite aucun fossile. Et cependant leur recherche était intéressante, car d'après cet auteur, le calcaire de Montmain occupe un niveau beaucoup plus inférieur que les autres gisements. Voici ce qu'il dit:

"La coupe dont nous venons de nous occuper nous montre le calcaire de " Montmain à une distance assez grande du grès à anthracite; il doit passer à " plus de 100 mètres au-dessous du poudingue de Sainte-Colombe.

„ Par contre, à Régny, Thizy et Montagny, il est presque à la limite même "du terrain supérieur. On est en droit, ce me semble, de conclure de là que le n calcaire existe dans le groupe calcaréo-schisteux à plusieur's reprises diffé"l'entes, et qu'il y forme plutôt une série de masses cunéiformes, limitées en " direction et en profondeur, qu'un ensemble de banes réguliers et continus. "Dans tous les cas, le calcaire de Montmain ne s'étend pas au loin. A un " kilomètre de la carrière, au sud-est du hameau Le Rey, il est déjà moins " puissant et surtout moins pur"; au delà, il disparaît tout à fait ou se trans" forme en grès dont le ciment même est à peine effervescent. Ainsi à Violay, "où la succession des roches s'observe pourtant bien, il n'y a plus aucune trace " de calcaire."

LISTE DES FOSSILES DE MONTALIN.

Cypricardella? Julieni, de Kon.

Productus corea, d'Orb. V.

- semireticulatus, Mart., var. concinnus V.

- punctutus, Mart. V.

Chonetes papilionacen, Phill. V.

- Dulmaniana, de Kon. V.

Orthotetes crenistria, Phill. 'T. W. V.

Spirifer duplicicosta, Phill. V.

Spiriferina insculpta, Phill. V.

Athyris plano-sulcate, Phill. V.

Rhynechonella purgnus, Mart. V.

Sigringroporatramulosa, Goldi. V.

Cladorhomes Heribaudi, nov. sp.

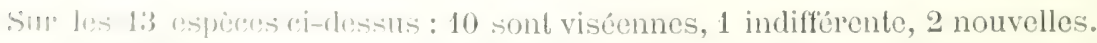

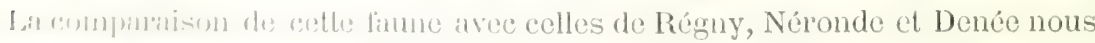

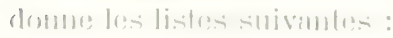




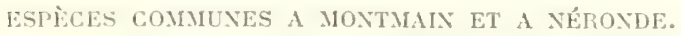

Cypricardella? Julieni, de Kon.

Chonetes papilionacea, Phill.

- Dalmaniana, de Kon.

Orthotetes crenistria, Phill.

Spirifer duplicicosta, Phill.

Spiriferina insculpta, Phill.

Rhynchonella pugnus, Mart.

Syringopora ramulosa, Goldf.

Cladochonus Heribandi, nov. sp.

Total : 9 .

Sur 13 espèces, Montmain en a 9 de communes avec Néronde.

ESPÈCES COMMUNES A MONTMAIX ET A RÉGYY.

Cypricardella? Julieni, de Kon.

Productus $\operatorname{cor}^{2} a$, d'Orb.

- semireticulatus, Mart., var. concinnus.

- punctatus, Mart.

Chonetes papilionacea, Phill.

- Dalmaniana, de Kon.

Orthotetes crenistria, Phill.

Spirifer duplicicosta, Phill.

Spiriferina insculpta, Phill.

Athyris planosulcata, Phill.

Rhynchonella pugnus, Mart.

Syringopora ramulosa, Goldf.

Cladochonus Heribaudi, nov. sp.

Les 13 espèces de Iontmain se trouvent toutes à Régny. Identité absolue.

ESPÈCES COMMUNES A MONTMAIN ET A DENÉL.

Productus cora, d'Orb.

- semireticulatus, Mar't.

Chonetes papilionacea, Phill.

Orthotetes crenistria, Phill.

Sur 13 espèces, 4 communes. 
POSITION STRATIGRAPHIQUE.

Montmain est synchronique de Régny, de Néronde et de Dinant, et doit avoir même notation $\mathrm{V} 16-\mathrm{V} 1 h$.

\section{LE GOUGET.}

Le Gouget est situé à 1,500 mètres du bourg d'Affoux (Rhône). Il a été reconnu par Grüner qui en a donné une bonne coupe ${ }^{(1)}$. Le marbre bleu micacé forme quelques banes intercalés dans un schiste argileux, reposant sur des schistes cristallophylliens. Le marbre est pétri d'encrines et de polypiers, parmi lesquels nous avons reconnu la présence de Zaphrentis. Le métamorphisme a fait disparaître à peu près tous les fossiles des schistes argileux; toutefois nous arons été assez heureux pour y découvrir une empreinte nette d'Orthotetes crenistilit.

Le marbre est waulsortien. Il est identique à celui de Ferrières, de l'Ardoisière et d'Eraux qui sont également waulsortiens. Nous avons là, sous les yeux, dans ce gisement comme dans les localités précédentes, les couches les plus inférieures de la formation carbonifère. Dans le Plateau Central elles ne descendent jamais jusqu'au tournaisien.

\section{MONTAGNY, COMBRES ET THIZY.}

La zone carbonifère qui suit la route de Roanne à Thizy est, dit Grüner, richement pourvue de calcaire bitumineux. On l'exploite dans les communes de Nuntagny, Combres et Thizy. Le calcaire occupe le flane droit de la vallée du Rhodon, au sud de Montagny, passe au nord du bourg de Combres et se dirige de là sur Thizy, dans le département du Rhône. Les banes caleaires y sont nombreux el faciles ì exploiter (2). Les fossiles inclus dans le calcaire ou dans les schistes sont en très mauvais état de conservation et fréquemment défintrués jur pression.

Montregny et Combres. - Nous avons recueilli dans ces deux localités les 3 expreces suivantes:

Chometes comorides, Sow.

Orthis resupincete, Mart.

Sigringoporece remulose, Goldt.

(1) ripiner. Lence ril, [1. 131.

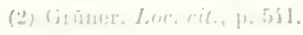




\section{POSITION STRATIGIAPHIQUE.}

Ces trois espèces assez abondantes, en particulier Chonetes comoides ef Syringopora ramulosa, en touffes qui attirent immédiatement le regard, démontrent le synchronisme des schistes et des marbres de ces deux gisements avec Régny.

Thizy. - Ce gisement nous a fourni:

Productus cor'a, d'Orb.

- punctatus, Mart.

- elegans, M'Coy.

Orthotetes crenistria, Phill.

Même âge que le précédent et que Régny.

\section{PROPIÈRES.}

Nous avons vu dans l'historique que le calcaire carbonifère a été signalé dès 1825 par Valuy à Propières et à Azolette dans le Beaujolais. Les ancicmes carrières ouvertes dans ces deux localités sont depuis longtemps abandonnées, et à Azolette elles sont comblées. Mais on en exploitail une nouvelle au pied du village de Propières au moment de notre visite. Dans l'ancienne carrière, nous avons trouvé des traces de Bryozoaires, d'Encrines et de Fossiles indéterminables. Toutefois, nous avons recueilli un bloc rempli de Chonetes comoides, Sow. Dans la nouvelle carrière, nous avons découvert dans le marbre exploité ainsi que dans les schistes enveloppants la faumule suivante:

Cypricardella? Julieni, de Kon.

Productus fimbriatus? Sow. V.

Chonetes papilionacea, Phill. V. - variolata, d'Orb. T.

Orthotetes crenistria, Phill. T. W. V

Spirifer duplicicosta, Phill. V.

Monticulipore tumide, Phill. T. V.

Amplexus coralloides, Sow. T. W. V.

Cladochonus Michelini, M. E. et J. H. T.

Il résulte de ces découvertes que l'ancienne carrière abandomnée de P'lopières était ouverte sur du calcaire dinantion. La nouvelle est, an contrare, ouverte sur une lentille comprise dans les schistes chanxhiens.

Si nous examinons la faune, nous la voyons composée, en effel, de trois 
espèces différentes : d'une espèce encore inconnue en Belgique, et de deux espèces franchement tournaisiennes. Ce mélange d'espèces viséennes et tournaisiemnes indique le niveau de Chanxhe. Il y aurait un grand intérêt à poursuivre ces recherches, car c'est dans cette région que l'on peut espérer recueillir une faune analogue à celle de Pair ou de Modave. Malheureusement, l'extraordinaire maurais état de conservation des fossiles ne s'y prête guère. Les schistes ont subi, soit par pression, soit par voie hydrothermale, un métamorphisme énergique. Les tests ont disparu, les empreintes sont déformées. Les vides laissés par la disparition des fossiles sont recouverts de très nombreux petits cristaux de Pyrite cubique, et il faut être très familiarisé avec cette faune pour tirer quelque profit de ces débris. On peut, du reste, juger de l'état de conservalion déplorable des fossiles par l'examen de nos figures.

\section{LÉTRA.}

Le gisement de Létra, dans la vallée de l'Azergue, est d'une valeur presque nulle. Il consiste en un lambeau de schistes, avec faible banc de marbre cristallin intercalé reposant transgressivement sur le terrain primitif comme tout le carbonifère de la région, et recouvert par le grès anthracifère.

Les schistes sont fortement comprimés et métamorphisés, ce qui a déterminé la disparition presque totale des fossiles, sauf des encrines, dont les empreintes d'articles sont toujours visibles.

Le marbre bleu cristallin, d'aspect waulsortien, nous a offert une grosse tige que nous attribuons à Poteriocrinus crassus. Là encore on se troure à la base de la formation carbonifère qui se révèle chanxhienne, comme à la nouvelle carrière de Propières, comme au Gouget dans le Rhône, à Ferrières dans l'Allier, à Evaux dans la Creuse.

Nota. - Il faut bicn se garder de confondre ce petit lambeau carbonifère avec le marbre blanc que l'on observe en face, au Mont-Jone, près de Ternant. Ce dernier n'est autre chose que du cipolin interealé dons les séricitoschistes ot dans les schistes chloriteux et amphiboliques avec masses bacillaires d'ipiclote et nids d'asbeste, du terrain primitif. Les schistes carbonifères et le grès anthracifere qui les recourent, fortement inclines, viennent buter contre la faillo qui suit lo thalweg de la vallée de l'Azorgue, et qui met en contact le termin carbonifère d'une part, le ter'ain eristallophyllien d'autre part. Cette erreur in b́é commise pan foumet. Voici un extrait de l'ouvrage de Drian qui vise celinit: " M. Fonmet, qui a ctudié avec soin ce gisement, a reconnu son extension depuis le Mont-Jone jusque sous Ronzières; le village de Ternant est bâti sur ses couches. Il admet que ce systeme se trouve ì la base du terrain carbonifirn qui so développe sucecssivement à l'ouest. Il regarde même comme 
probable qu'il n'est autre chose qu'une partie des calcaires des environs de Tarare, dont le métanorphisme s'est trouvé très avancé par suite de circonstances locules (1). $n$

\section{SAINT-GERMAIN-LAVAL.}

Les anciennes carrières de Saint-Julien-d'Odde sont aujourd'hui comblées. On en a ouvert d'autres à droite de la route, sur le prolongement de la même lentille calcaire.

La description qu'a donnée Grüner' du calcaire exploité jadis s'applique exactement aux nouvelles carrières:

"Le calcaire est de couleur grise tirant sur le bleu, d'une nuance moins » foncée que les calcaires analogues de Régny et de Néronde. Il est compact, " à cassure esquilleuse et peu r'iche en bitume. Les encrines y sont abondantes ) et apparaissent dans les cassures transversales sous forme d'écussons spa" thiques, d'une nuance plus claire que le reste de la roche. On y trouve aussi ") quelques térébratules. Le mur est formé de schistes; le toit, de grauwackes "fines. Les deux roches sont tendres et se désagrègent facilement. Dans lo „v voisinage de la masse calcaire, elles sont elles-mêmes imprégnées de carbo" nate de chaux. Les schistes sont verts, gris, couleur lie de vin ou bleuâtres; » ils renferment des encrines lorsque le ciment est plus ou moins calcaire. »Le grès du toit est gris tirant sur le bleu ou passant au vert; les fossiles ) semblent y manquer (2). )

Le gisement de Saint-Germain-Laval est réellement un des plus intéressants du Plateau Central.

A la suite de recherches patientes, continuées pendant plus d'une semaine en compagnie de notre ancien préparateur, M. Charles Robinet, agrégé de l'Université, professeur au Lycée de Chartros, nous avons pu réunir une intéressante faunule qui, grâce aux découvertes récentes faites en Belgique, vient éclairer d'une vive lumière l'âge de ce précieux giscment.

Nous y avons découvert les espèces suivantes:

1. Griffithides globiceps? Phill. V.

2. Bairdia, sp.

3. Cypricardella? Julieni, de Kon.

4. Parallelodon comoides, de Kon. V.

5. Modiola fusiformis, de Kon. V.

6. Productus cor'a, d'Orb. V.

7. - pustulosus, Phill. V.

(1) Drian. Mineralogie et Giologie des encirons de Lyon, p. 268. Lyon, 1859.

(2) Grüner. Loc, cit., p. 387. 
8. Chonetes papilionacea, Phill. V.

9. Orthotetes crenistria, Phill. T. W. V.

10. Spirifer duplicicosta, Phill. V.

11. - bisulcatus, Sow. V.

12. - integricosta, Phill. V.

13. Martinia glabra, Mart. V.

14. Athyris planosulcata, Phill. V.

15. - ingens, de Kon. W.

16. Archaocidaris, sp.

17. Palachinus Konincki, n. sp.

18. Poteriocrinus crassus, Miller. W. V.

19. Syringopora ramulosa, Goldf. V.

20. Cladochonus Heribaudi, n. sp.

Cette faune réclame un examen attentif. Elle est composée de vingt espèces, distribuées ainsi :

2 genres non déterminés spécifiquement;

12 espèces viséennes;

1 espèce indifférente;

1 espèce waulsortionne (Athyris ingens);

1 espèce à la fois waulsortionne et viséenne (Poteriocrinus crassus);

3 especes encore incomnues en Belgique (Cypricardella Julieni, Palachinus Konincki, Cladochonus Heribaudi).

C'est donc une faune viséenne.

La comparaison aree les faunes de Régny, Néronde et Montmain démontre leur partait synchronisme, comme en témoignent les tableaux suivants:

LSPLECES COMMUNES A SAINT-GERMAIN-LAVAL ET A RÉGNY.

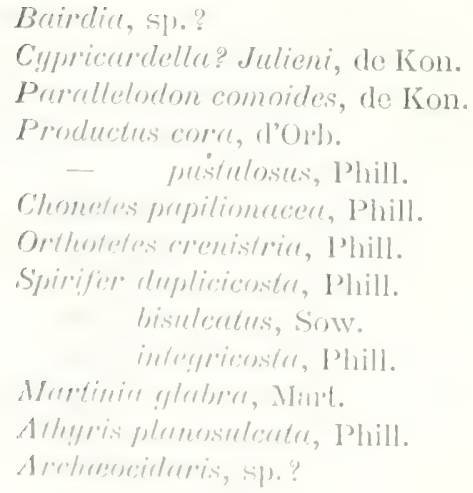


Palechinus gigas, M'Coy.

Konincki, nov. sp.

Potopincrinus crassus, Miller.

Syringopora ramulosa, Goldf.

Cladochonus Heribuedi, nov. sp.

Sur 20 espèces, Saint-Germain-Laval en a 18 communes avec Régny.

ESPÈces COMMUNES A SAINT-GERMAIN-LAVAL ET A NéRONDE.

Bairdia, sp.

Cypricardella? Julieni, de Kon.

Productus pustulosus, Phill.

Chonetes papilionacea, Phill.

Orthotetes crenistria, Phill.

Spirifer duplicicosta, Phill.

- integricosta, Phill.

Spiriferina insculpta, Phill.

Martinia glabra, Mart.

Palcechinus Koninchi, nov. sp.

Poteriocrinus crassus, Miller.

Syringopora ramulosa, Goldf.

Cladochonus Heribaudi, nov. sp.

Total : 13 espèces.

Sur 20 espèces, Saint-Germain-Laval en a 13 communes avec Néronde.

ESPÈCES COMnUNES A SAINT-GERALIN-LAVAL ET A MONTMAIN.

Cypricardella? Julieni, de Kon.

Productus cora, d'Orb.

Chonetes papilionacea, Phill.

Orthotetes crenistria, Phill.

Spirifer duplicicosta, Phill.

Athyris planosulcata, Phill.

Syringopora ramulosa, Goldf.

Cladochonus Heribaudi, nov. sp.

Total : 8.

Enfin, nous trouvons les cing espèces communes ¿t Saint-Germain-Laval, Denée et Dinant. 
Productus cora, d'Orb.

- pustulosus, Phill.

Chonetes papilionacea. Phill.

Orthotetes crenistria, Phill.

Martinia glabra, Mart.

Ce qui caractérise spécialement le lambeau carbonifère de Saint-GermainLaval et lui donne son véritable intérêt, e'est qu’à la base on observe des bancs de calcaire subcompact à cherts blonds et violacé à grosses tiges de crinoïdes. Ces calcaires sont identiques à ceux de Belgique V1a. Ces derniers sont, comme l'a démontré surtout M. le professeur Dorlodot, synchroniques des marbres waulsortiens à Stromatoporoïdes dont ils constituent un faciès hétérotopique. Ils sont done à Saint-Germain-Laval par voie d'analogie, synchroniques de tous les marbres waulsortiens de la région du Forez et spécialement de celui de Ferrières. Saint-Germain-Laval et Ferrières se trouvent donc reliés entr'eux par l'établissement de ce synchronisme, comme d'autre part SaintGermain-Laval et Régny sont liés par leur faune dimantienne. On voit ainsi comment les récents progrès accomplis en Belgique éclairent d'une vive lumière le carbonifère marin de la région si tourmentée du Plateau Central.

C'est là un des plus saisissants exemples de l'influence heureuse et décisive qu'exercent sur deux r'égions similaires les progrès géologiques accomplis dans l'une d'elles. Mais les calcaires V1a ne sont pas seuls. Ils supportent les schistes noir's à Paléchinides. A Régny on trouve réunis sur les mêmes échantillons de roches Pal. gigas et Pal. Koninchi. A Saint-Germain-Laval j'ai découvert Pal. Konincki dont les plaques sont abondantes, mais je ne doute pas qu'on n'y trouve également Pal. gigas, car bien des plaques dont l'ornementation est malheureusement effacée paraissent lui appartenix. Ces couches it Paléchinides sont intercalées entre les calcaires à cherts blonds ou les calcan lies le-vin à grosses tiges de crinoïdes V1a et les calcaires gris-bleu ì faune de Dinant. La eoupe de Saint-Germain-Laval est done en définitive la suivante. Au sommet: schistes et calcaires à faune de Denée ou dinantienne; an-duscus schistes noir's à Puléchinides (base du Dinantien); en bas calcaires V1a chanxhiens. Sanint-Germain-Laval constitue le trait d'union entre Ferrières d'une part, el lígny, Néronde et Montmain d'uutre part. Les couches extrêmes de la bas correspondent de même synehroniquement et hétérotopiquement au mathe wanlsortien du Gouget. Comme au surplus, les marbres waulsortiens du Grouget de lerrieres, les caleaires Vla de Saint-Germain-Laval reposent lrinsgressivonent sur los terans cristallophylliens, on peut affirmex qu'il n'existe nulle trane de l'étnge toumaisien dans le Plateau Central comme cela a

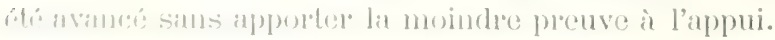




\section{FERRIL̈RES.}

Le gisement de Ferrisres a été signalé par Boulanger dès 181́. Voici comment s'exprime cet ingénieur : "Le lambeau le plus considérable de terrain de " transition existant dans le département de l'Alliel' est celui qui s'étend le " long de la vallée du Sichon, depuis les environs de Cusset jusqu'au pied du "Nontoncelle, sur plus de deux myriametres de longueur, mais e'est aussi le " plus tourmenté; entourées de tous côtés par le porphyre rouge quartzifère " et traversées en divers sens par des filons de cette substance, les couches " de ce terrain ne présentent pas de direction constante ni d'ordre de superpo") sition bien déterminé (1).

"A Ferrières même, au milieu des schistes, se trouve une masse calcaire ") sans stratification bien apparente et qui a une épaisseur assez considérable. »C'est un calcaire saccharoïde, gronu, d'un gris blanc sale; on l'exploite en " moellons pour faire de la chaux et pour les constructions (2). ")

Le terrain carbonifere repose en stratification transgressive sur lo terain cristallophyllien et peut-être sur le cambrien. Ce substratum est surtout formé de schistes amphiboliques passant à la colnéenne et de schistes mâclifères. Le terrain carbonifère est représenté: $1^{\circ}$ par des poudingues durs à galets de quartz, de lydienne, de schistes identiques à ceux de l'Al'doisiere; 2 par des masses lenticulaires de marbre cristallin blanc, veiné de bleu en relation avec les poudingues comme celui de l'Ardoisière. Le mirthe offre parfois des géodes tapissées de jolis scalénoèdes de calcite; $3^{n}$ enfin par des grès supérieurs, en général altérés et tendres près de Ferrières et siliceux et très durs à Cheval-Rigon. Ils passent au poudingue ì la base vers le manbre. Ces grès sont franchement marius. A partir de ce dernier hameau sulement, on observe le véritable grès anthracifère d'origine continentale. Féfuemment traversé par des filons de porphyre, il se poursuit régulièrement dans liz direction d'Aronne (canton de Cusset), où une faille le fait buter contre les ardoiscas cambriennes. Celles-ci se développent à leur tour avee de nombreux plissements jusqu'au lambeau carbonifère de l'Ardoisière. Nous pensons que la déconverte des premiers fossiles marins à Ferrières est due à Jourdan. I) is 1874, connaissant notre intention d'étudier'a rallée du Sichon, il nous invitait à explorere surtout les grès de Ferrières où il avait découvert des moules de grandes belphinules (lisez Euomphales).

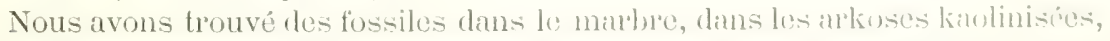
a 100 mètros en avant des premières maisons et dans les frrès silicuse de Cheval-Rigon.

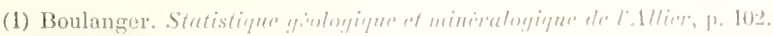

(2) Id. Hoid, , r. 10t. 
FAUNE DU MARBIR.

C'est un marbre encrinitique. Mais outre les articles isolés et les fragments de tiges l'encrines, on y constate encore la présence de polypiers. Dans un bloc exposé depuis longtemps à l'air, nous avons observé un calice de Zaphrentis.

Ce marbre est fréquemment d'un aspect identique au marbre waulsortien des Pauquys. Nous n'hésitons pas à lui attribuer le même âge.

\section{FAUNE DE L'ARKOSE.}

L'Arkose altéréce est visible et facile à explorer sur le talus gauche de la ronte de Cusset à Ferrières, en venant de Cusset. A 100 mètres des premières maisons du village, nous avons découvert un assez riche gisement fossilifère, peut-être le mène qui avait fourni les Delphinules à Jourdan. Les fossiles sont tous à l'état d'empreintes ou de moules, colorés par l'oxyde rouge de fer. Voici la liste de eeux que nous avons pu déterminer :

Chonetes papilionacea, Phill.

- comoides, Sow.

- Dalmaniana? de Kon.

Spirifer duplicicosta, Phill.

Spiriferina insculpta, Phill.

Orthis, sp.?

Athyris, sp)?

Archecociduris, sp.?

Amplexus coralloides, Sow.

Cladochonus IIeribundi, nov. sp.

Zuphorentis, spl)?

RALNE IDES GRLS SHLICIFIES DE CHEVIL-RIGON.

Productus, sp.? moules externes eneroûtés, spécifiquement indétermimables.

Chometes rommosides, Sow.

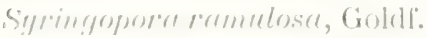

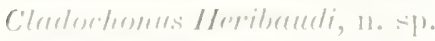

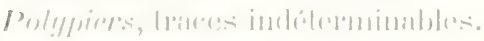

Artirlese d'omrinums. 
Ces deux fiamules sont d'une tres hante importance. lilles nous démontrent d'abord que les assises marines du carbonifère existunt bien réellement a Ferrières, car les empreintes sont nombreuses et avec du travail on arriverait eertainement à les augmenter. De plus, elles sont d'mne manière cer'taine de l'âge de Dinant. En laissant de côté Euomphalus catilliformis, trouvé par Jourdan et que nous avons rappelé dans les diagnoses, Chonetes comoides, Chonetes papilionacea, Syringopora ramulosa sont suffisants pour le démontrer. Ajoutons-y encore Spirifer duplicicosta et de jolies empreintes de Spiriferina insculpta, identiques à celles que l'on recueille si abondamment à Montmain, à Néronde, à Régny et à l'Ardoisière.

Il ne pourrait y avoir de doute que pour le marbre; mais ce marbre est en stratification discordante avee le Cambrien du voisinage et est intimement lié au grès marin qui le recourre; et le polypicr et les tiges d'encrines que j'y ai recueillis sont bien carbonifères. M. de Koninck en était convaincu. Lors d'un premier voyage, vers 187'́, au début de mes études, j’avais recueilli de très jolies tiges d'encrines dans ce marbre. Je les avais soumises à cette époque à M. de Koninck qui n’hésita pas à les considérer comme appartenant à des espèces carbonifères. La position de ce marbre à la base des grès marins et son identité lithologique aree le marbre waulsoltien des Pauquys, nous donne la conviction que e'est bien l'horizon stromatoporique, synchronique de l'assise de Chanxhe, que nous avons sous les yeux à Ferrières et que nous devons le rapprocher des marbres identiques d'Eraux, du Gouget, de Létra, comme témoins de la limite de la transgressiun carbonifère dans cette partie du Plateau Central.

Il ne nous parait pas inutile de mentionner comme nous l'avons fait jusqu'ici, les espèces que Ferrières et Cheval-Rigon, malgré le petit nombre que nous avons recueilli, ont de communes avec nos autres gisements du Plateau Central. La lecture de ces listes achevera de convaincre le lecteur du parfait syrnchronisme de toutes ces localités carbonifères.

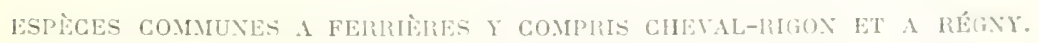

Chonetes papilionacen, Phill.

- comoides, Sow. (Ferrieres et Cheval-Rigon).

- Dalmaniana? de Kon.

Spirifer duplicicostre? P'lill.

Spiriferina insculptu, l'hill.

Amrlexus coralloides, Som.

Syringopora ramulosa, Goldi. (Cheval-1Rigon).

Cladochonus Heribuadi, nov. sp. (Cheval-Rigon).

Sur 9 espèces déterminées, 8 communes sauf Euomphalus catilliformis? de Kon. 
ESPÈCES COMMUNES A FERRIẼRES ET SAINT-GERMAIN-LAVAL.

Chonetes papilionacea, Phill.

Spirifer duplicicosta, Phill.

Syringopore romulosa, Goldt.

Cludochonus Heribaudi, nov. sp.

Le synchronisme des deux faunes permet d'en conclure celui des marbres inféricur's stromatoporiques de Ferrières et des calcaires violacés ou à cherts blonds Vla de Saint-Germain-Laval. La position stratigraphique de ces deux catégories de roches est identique, ce dont il était impossible de se douter avant la découverte des faunes marines qu'elles précèdent immédiatement. Cet exemple nous permet encore une fois de saisir l'intérêt suprême qu'offrent les recherehes paléontologiques pour débrouiller les terrains anciens du Plateau Central et l'impossibilité d'établir des cartes géologiques exactes avant d'aroir fait ces études préliminaires.

\section{RSPÈCES COMMUNES A FERRIËRES ET $A$ NÉRONDE.}

Enomphalus catilliformis? de Kon.

Chonetes papilionacea, Phill.

- comoides, Sow.

- Dalmaniana? de Kon.

Sprivifer duplicicosta, Phill.

Spiriferina insculpta, Phill.

Syringopora ramulosa, Goldf.

C'ladochonus Heribaudi, nov. sp.

Sur 9 ospèces déterminces à Ferrières, 8 sont à Néronde.

ESTMCES COMMUNES A FLIRIMIRS HT I MONTMLIN.

Chonetes papilionacen, Plitl.

Dralmennirnate, do Kons.

Sivirifere depplicirosta, Phill.

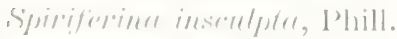

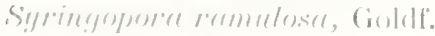

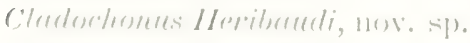

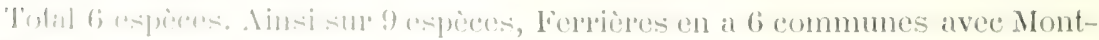
miniil.

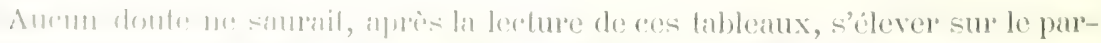

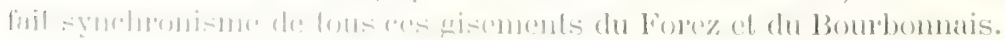




\section{L'ARDOISIERE.}

C'est dans l'automne de 1873 que nous arons décontert ee magnifique gisement. Nous cherehions ì cette époque à retrouver le peint mal défini, où Murchison en 1850 avait trouvé pour la première fois, dans la vallée du Siehon, des fossiles carbonifères qu'il fit déterminer par de V'erreuil (1). C'est au cours de ces recherehes pendant lesquelles j'errai longtemps dars les ardoises cambriennes que Murchison croyait aussi d'âge carbonitiene, à la poursuite de fossiles introuvables que je finis par' découvrir des empreintes dans les talus d'un chemin d'exploitation récemment ourert. C'est lo chemin qui part du pont américain jeté sur le Sichon à 1 kilomètre en aval du r’estanrant de l'Ardoisière.

Avant de commencer l'étude de la faune, nous croyons devoir donner la coupe de ce gisement célibre, le plus beau gisement marin du Plateau Central.

COUPE DU GISEMENT DE L'ARDOISIERE, PRES VICIIY.

Iii.. II.

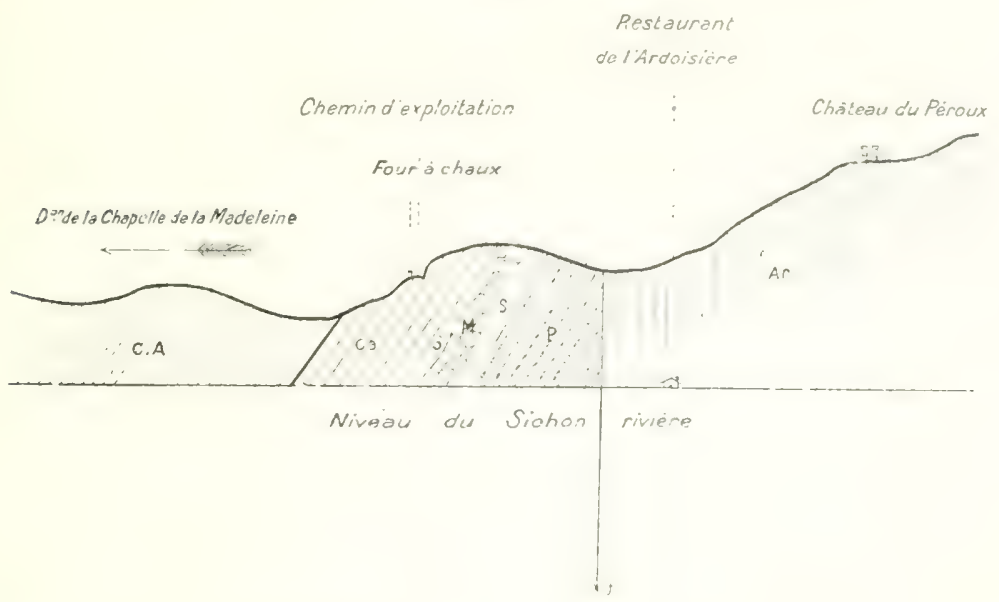

Ar. Schistes ardoisiers cambriens.

$P$. Poudingues.

S. Schistes verditres chanxhiens.

M. Marbre a Stromatoporoides intereali.

$C^{\prime} a$. Calschiste gréseux fossilifère.

G. A. Grès anthracifere,

f. Faille.

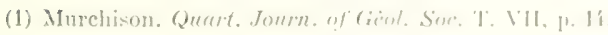


Le lambeau carbonifère marin de l'Ardoisière, compris entre les ardoises cambriennes à la base et le grès anthracifère au sommet, débute par un puissant poudingue à galets de quartz, de lydienne, de schistes, de granite, etc. Ces poudingues alternent à la purtie supérieure avec des schistes verdâtres, tendres, renfermant une lentille de marbre cristallin, exploitée pour l'amendement des terres. Au-dessus se trouve la puissante formation des grès calcaires fossilifères. Ces grìs bleuâtres, tròs durs, d'une centaine de mètres d'épaisseur, sont superficiellement altérés, ou plutôt décalcifiés et ocreux. On peut suivre les progrès de la décalcification de l'extérieur à l'intérieur en brisant des bloes. La roche intacte bleuâtre fait seule effervescence avec les acides. Dans la partie altérée ocreuse les tests ont été dissous, mais les empreintes externes ou internes des fossiles étant devenues par cela même très visibles, il est facile alor's d'en étudier les plus fins détails, comme on peut s'en assurer en examinant les photogravures. Les tests sont parfois remplacés par de l'oere qui a pris avec le temps la consistance du calcaire. J'ai constaté le fait fréquemment pour Productus giganteus, Spirifer bisulcatus, Orthis resupinata, des Fenestelles, ete. Ces bancs fossilifères ont été clivés comme les poudingues de la base par les mouvements orogéniques puissants dont la région a été le théâtre. C'est pour cela qu'on les désigne souvent sous le nom de calschistes gréseux.

On perd ainsi souvent de superbes échantillons qui se divisent selon ces plans de clivage, comme les galets pugilaires de quartz ou de granite des poudingues que l'on ne peut extraire intacts de leur alvéole.

A la partie supérieure du gisement les grès calcaires marins passent au grès anthracifère sans intercalation de poudingue, comme ì Régny par exemple.

F.JUE DES SCHISTES VERD.ITRES INFÉRIEURS.

Dans les schistes tendres de conleur verdâtre, qui alternent plusicur's fois avec les poudingues supérieurs de la base, nous n’uvons recueilli dans nos frouilles que deux moules oxternes de liges d'enerines, probablement de Platyrrints:

LIIINE DU MALIBHL.

La mantme frisente peu d'empreintes, mais en examinant attentivement la

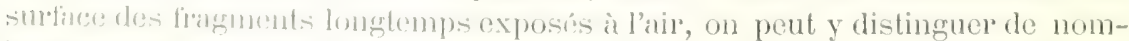
broux potils andieles d'oncrines, des traces de polypiers indéterminables et

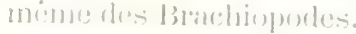

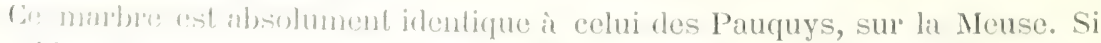

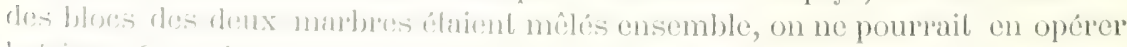

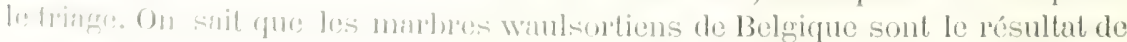


l'agglomération de deux Hydrozoaires découverts par M. E. Dupont, savoir le Stromatocus bulbacens qui forme le calcaire gris-blane sub-compact et le Ptylostroma fibrosa, qui apparaît sous l'aspect de bandes bleues radiées. A ces orgunismes destructure mieroscopique, que l'on ne peut étudier que dans les coupes minces, s'issocient de nombreuses Fénestelles, lont les expansions foliaccos simulent de fines dentelles, des Polypiers surtout représentés par Amplexus corulloides, des Encrines et des coquilles diverses disséminées avec parcimonie dans la masse, mais partois agglomérécs en nombre immense daus les cavités du calcaire stromatoporique.

J'avais apporté en 1889 au Musce royal d'histoines naturelle de Bruxelles d'énormes bloes de marbre provenant de l'Ardesisiere. Quel ne fut pas mon étonnenent en constatant une identité parfaite avee le marbre des Pauguys. Plusieurs naturalistes de eet établissement, MM. Rutot, Van den Broeck, Nourlon et M. Dupont lui-mème furent tellement frappés de cette ressemblance que j’obtins facilement du sarant directeur du MIusée royal l'autorisition de faire exécuter des coupes minces de notre marbre, jour les comparer au microseope avec les milliers de coupes de mulf)res waulsortiens, réunies dans les collections du Musée par les soins de M. E. Dupont.

L'examen microscopique démontra que l'identité des caractères extérieurs se poursuirait juscule dans la masse interne. Une seule distinetion fut rérélée; c'est que le marbre de l'Ardoisiere, qui souvent présente à la surface des petits grains roulés de quartz, en partie empâtés, est égralement pénétré à l'intérieur d'une fine poussiere du même minéral. Il en résulte une difficulté triss grande pour la préparation des plaques minces.

L’identité absolue des marbres des Pauquys et de l'Ardoisière signalée ici pour la première fois est un fait capital, parce qu'il jette une lumière éclatante sur des roches restées jusque-là énigmatiques, parce qu'il nous apprend (pue nos lentilles de marlore sont bien d'âge curbonifère et sont le représentant dans la France centrale des récifs d'Hyadrozonires waulsortiens de la Belgique. Sans doute il y a quelques difrérences d'uspert entre nos divers mathere de l'atrdoisière, de l'errières, d'Evaux, du Gouget, de Létra; mais ces differences sont du mème ordre, peut-être moins considérables que eelles qui distinguent les marbres des Pauguys, d'Anseremme, de Vaulsort et d'antres localités belges du mèrne âge.

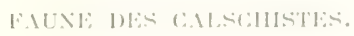

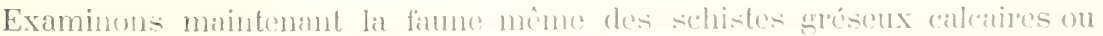

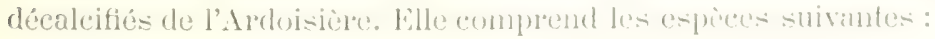

1. Petalodus IIastingsice, ()wen.

2. Dithyrocaris, sp.

3. Phillipsia Eichoreldi, Visch. 
4. Bairdia, sp.

5. Nautilus sulcatus, Sow. T. V.

6. - subsulcatus, Phill. T. V.

7. - globatus, Sow. V.

S. - costellatus, M'Coy.

9. Orthoceras Goldfussianum, de Kon. T. V.

10. Cyrtoceras unguis, Phill. T. V.

11. Naticopsis planispira, Phill. V.

12. Loxonema nanum, Kon. V.

13. - constrictum, Mart. V.

14. Turbonitella biserialis, Phill. T. V.

15. Flemingia Hisingeriana, de Kon. V.

16. Platyschisma helicoides, Sow. T. V.

17. Straparollus pileopsideus, Phill. V.

18. Euomphalus acutus, Sow. T. V.

19. Schizostoma catillus, Mart. V.

20. Ptychomphalus, sp.

21. Dentalium, sp.

2.2. Solenopsis pelagicus, Goldt.

23. Conocardium minax, Phill. V.

2'. Nucule, sp.

25. Nuculana, sp.

26. Tellinomya, sp).

27. Parallelodon Lacordaireanus, de Kon. T. V.

28. comoides, de Kon. V.

29. Aviculopinna spathula, M'Coy:

30. Posidoniclla cetustu, Sow. V.

31. Aciculopecten subfimbriatus, de Virn.

32. Productus gigantells, Mart. V.

$33 . \quad$ - maryarituceus, Phill. V.

3.. - Corel, (l'Orb). V.

:3. - - undutus, Defr. V.

3ti. - semiretioulutus, Mart. T. V

37. pustulosus, Phill. V.

$38 . \quad$ - munctulus, Matr. V.

39. - - Rimbriatus, Sow. V.

i). - aculeatus, Mart. V.

11. Chonetes concentrira, de Kion. V.

往. - papilionurese, l'hill. VT.

ii) - Dommanirana, de Kon. V.

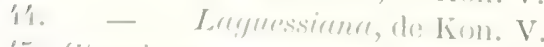

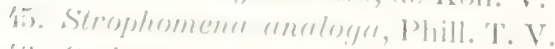

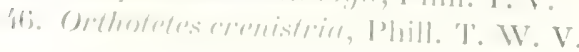


i7. - Sharpei, Morris V.

is. Orthis resupinate, Mart. T. W. V.

19. Spirifer duplicicoste, Phill. V.

j). - crassus, de Kon. V.

i. - bisulcatus, Sow. WV. V.

52. - integricosta, Phill. V.

53. Spiriferina cristata, Sow. V.

51. - insculpte, Phill. V.

55. Martiniu glabre, Mart. V.

j). - lineutu, Mart. T. V.

57. Retzia Buchiana, de Kon. V.

5. Athyris planosulentr, Phill. V.

59. - ambigua, Sow. V.

60. Rhynchonella pleurodon, Phill. V.

6i. - purgnus, Mart. V.

(i2.) Dielasma hastatum, Sow. W.

63. Fenestella multiporata, M'Coy.

(5't. - ejuncide, M'Coy.

(i.). - membranacea, Phill.

i6i. Polypora Goldfussiana, de Kon. V.

i7. Palceoftustra Jolicti, nov. gen., nov. sp.

is. Monticulipora tumida, Phill. T. V.

(i9.) - inflata, de Kon. V.

7). Archceocidaris Urii, Flem. V.

71. - Grïneri, 11. sp.

7-2. Palcechinus Lacazei, n. sp.

73. Pholidocidaris Gaudryi, n. sp.

7'́. Poteriocrinus crassus, Miller V.

7.). Lithostrotion junceum, Flem. V.

76. - irregulare, Phill. V.

77. Diphyphyllum concinnum, Lonsd. V.

78. Clisiophyllum turbinatum, M'Coy V.

79. Amplexus corculloides, Sow. T. WV. V.

\&i). Zaphrentis, sp.

81. Syringopora ramulosa, Goldf. V.

8.2. Cladochonus Heribaudi, n. sp.

Sur les 82 espèces qui composent cette faune, dont le nombre s'élèverait it plusieurs centaines, n'était le mauvais état de conservation des fossiles qui rend impossible la détermination d'une multitude d'empreintes, nous comptons: 


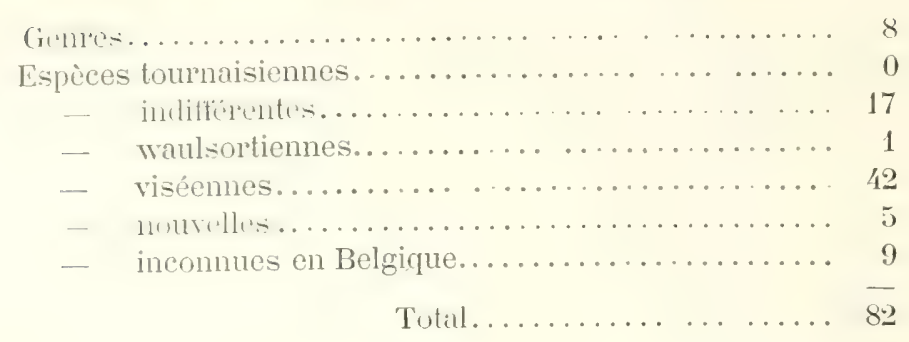

Quelques-unes de nos espèces indiquées sous la rubrique « inconnues en Belgique n ont été déconvertes dans ces derniers temps. Ce qui ressort surtout de cet examen, c'est l'absence complète d'espèces tournaisiennes et le nombre élevé, 42 d'espèces exchusivement spéciales à Visé. Si l'on recherche les gisements belges de ces espèces, on voit qu'elles se trouvent toutes sans exception dans le célèbre massif de Visé, situé entre Liège et Maestricht dont notre gisement de la vallée du Sichon reproduit l'exacte physionomie biologique. La seule différence appréciable consiste dans le changement du faciès lithologique de la roche, entièrement différente du marbre noir de. Visé.

Comme à Visé, nous royons réunis dans cet admirable gisement les gros Productus giganteus et cora, les gros Spirifer bisulcatus, les Lithostrotion junceum et irregulare, lo Diphyphyllum concinnum et l'Archceocidaris Urii. Il n’est pas jusqu'à l'absence de stratification bien nette, qui ne rapproche ces deux localités sours.

Nous pouvons aujourd'hui comparer la belle faune de l'Ardoisière avec les faunes carbonifères du Plateau Central, ce que nous ne pouvions fare à l'époque de notre découverte de ce gisement. Les relations stratigraphiques qu'il possède avee eux ne pouvaient pas davantage r'ésulter d'un examen sur le terrain, car l'Ardoisière, comme Ferrières, comme Saint-Germain-Laval, comme une foule d'antres, sont ì l'état de lambeaux isolés, limités par des failles, cnclavés de toutes parts soit dans les terrains primitifs ef cambriens, soit dans les porphyres et les granites. Le rapprochement paléontologique que nous pouvons réaliser aujourd'hui, va fare luire à nos yeux ces relations inconnucs jusque-lia et projeter une éblouissante lumière sur notre carbonifère marin de la Fronce centrale si inconnu, si dédaigné, et pourtant d'un intérết si puissint.

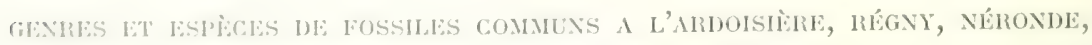

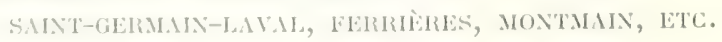

Dithyrocoris, sp. - lichly.

Bruirdir, sp) - Irégny, Saint-Genmain-Laval, Néronde.

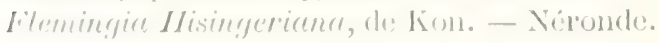


Straparollus pileopsideus, Phill. - Néronde.

Dentalium, sp. - Régny.

Nucula, sp.-Régny.

Nuculana, sp. - Régny.

Tellinomya, sp. - Régny.

Parallelodon comoides, de Kon. - Régny, Saint-Germoin-Lrval.

Productus giganteus, Mart. - Rigny.

- cora, d'Orl). - Régny, Thizy, Montmain, Sinint-Germain-Laval.

- semireticulatus, Mart. - Régny, Montmain.

-- pustulosus, Phill. - Régny, Néronde, Saint-Germain-Laval.

- punctutus, Mart. - Rigny, Thizy, Montmain.

- fimbriatus, Sow. - Thizy, Propières.

Chonetes papilionacea, Phill. - Régny, Néronde, Montmain, Propières, Saint-Germain-Laval, Cheval-Rigon.

Chonetes Dalmaniana, de Kon. - Régny, Néronde, Montmain, Ferrières.

- Laguessiana, de Kon. - Régny.

Orthotetes crenistria, Phill. - Régny, Thizy, Néronde, Montmain, Le Gougel, Propières, Saint-Germain-Laval.

Orthis resupinata, Mart. - Rêgny, Montagny, Néronde.

Spririfer duplicicosta, Phill. - Régny, Néronde, Montmain, Propières, SaintGermain-Laval, Ferrières.

Spirifer bisulcatus, Sow. - Régny, Saint-Germain-Laval.

- integricosta, Phill. - Régny, Néronde, Saint-Germain-Laval.

Spiriferina insculpte, Phill. - Régny, Néronde, Montmain, Ferrières.

Martinia glabre, Nart. - Régny, Néronde, Saint-Germain-Laval.

- lineuta, Mart. - Régny.

Athyr is pluno-sulcatu, Phill. - Régny, Montmain, Saint-Germain-Laval.

Rhynchonelle pugnus, Mart. - Régny, Néronde, Montmain. pleurodon, Phill.

Monticulipora tumida, Phill. - Propières.

Poteriocrinus crassus, Niller. - Néronde, Létra? Saint-Germain-Laval Evaux (Creuse).

Amplexus coralloides, Sow. - Rémny, Propières, lierrières.

Zaphrentis, sp.? - Régny, Le Gouget, Ferrières.

Syringopora ramulosa, Goldf. - Régny, Montagny, Néronde, Nontmain, Saint-Germain-Laval, lerrières.

Cladochonus Ireribaudi, nov. sp. - Régny, Néronde, Montmain, Suint-Germain-Laval, Ferrières.

Il résulte de ee tableau que l'Ardoistère a de commun avee nos plincipaux gisements le nombre d'espeeces suivant: 
Nombre total d'espèces communes bien déterminées.... 28

Nombre commun avec Régny................ 24

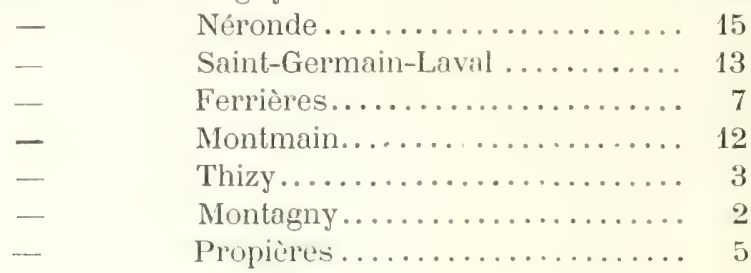

Le gisement de Régny est celui qui présente dans l'état actuel de nos recherches le plus grand nombre d'espèces, soit 24. Ces nombres s'augmenteront, à coup sûr, avec des recherches plus complètes.

Examinons maintenant l'Ardoisière et Régny, afin de nous éclairer sur les faciès, c'est-à-dire sur les circonstances bathymétriques des faunes. Pour cela, nous devons, abstraction faite des fossiles communs, envisager l'ensemble des espèces spéciales des deux gisements.

ESPÈCES SPÉCIALES A RÉGNY ET GISEMENTS DU MÊME AGE (47).

Cochliodus acutus, Ag.

Psammodus, sp.

Nautilus sulciferus, Phill.

Cyrtoceras puzosianum, de Kon.

Macrochilina Pireti, nov. sp.

Loxonema priscum, Goldf.

- propinquum, de Kon.

Straparollus Dionysii, Denys de Montfort.

Euomphalus amenus, de Kon.

Phanerotinus nudus, Sow.

Breylea spirata, de Kon.

Bellerophon scalifer, de Kon.

Weregenia Fierussaci, d'Orb.

Lepetopsis Busscheriunus, de Ryek.

Eitmondia orbiculatu, de Kon.

- scalaris, M'Coy.

filigrrena, de Kon.

remulitis, de Kon.

selectre? de Kon.

amrence? de Kon.

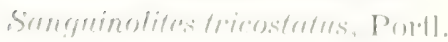


Solenopsis Bielatostii, nov. sp.

Cypricardella? Julieni, de Kon.

Parallelodon argutus, Phill.

- mytiloides, de Kon.

Modiola cuneiformis, de Kon.

Leiopteria hirundo, de Kon.

lunulata, Phill.

Aviculopecten ccelatus, M'Coy.

$$
\text { - plagiostoma, de Kon. }
$$

Chonetes Murchisoni, nov. sp.

- comoides, Sow.

- Jourdani, nov. sp.

Rhynchonella angulata, Linné.

Dielasma sacculus, Mart.

Crania, sp.

Archceocidaris, sp.

Palachinus gigas, N'Coy.

Konincki, nov. sp.

Euomphalus crotalostomus, M'Coy.

- catilliformis, de Kon.

Aciculopecten Bosquetianus, de Kon.

Griffithides globiceps? Phill.

Modiola fusiformis, de Kon.

Athyris ingens, de Kon.

ESPËCES SPÉCIALES A L'ARDOISIÈRE (44).

Petalodus Hastingsice, Agas.

Phillipsia Eichwaldi, Fisch. de Wald.

Nautilus sulcatus, Sow.

- subsulcatus, Phill.

- globatus, Sor.

- costellatus, M'Coy.

Orthoceras Goldfussianum, de Kon.

Cyrtoceras unguis, Phill.

Naticopsis planispira, Phill.

Loxonema manum, de Kon. constrictum, Hart.

Turbonitella biserialis, Phill.

Platyschisma helicoides, Sow.

Euomphalus acutus, Sow. 
Schizostoma catillus, Mart.

Ptychomphalus, sp.

Solenopsis pelagicus, Goldf.

Conocardium minax, Phill.

Parallelodon Lacordaireanus, de Kon.

A viculopinna spatula, M'Coy.

Posidoniella cetusta, Sow.

Productus margaritaceus, Mart.

- undatus, Defrance.

- aculeatus, Mart.

Chonetes concentrica, de Kon.

Orthotetes Sharpei, Molris.

Spirifer crassus, de Kion.

Spiriferina cristata, Sow.

Athyris ambigna, Sow.

Dielasma hastatum, Sow.

Fenestella multiporata, N'Coy.

- ejuncida, M'Coy.

- membranacea, Phill.

Polypora Goldfussiana, de Kon.

Palcenfustra Jolieti, nov. gen., nov. sp.

Monticulipora inflata, de lion.

Archerocidaris Urii, Flem.

- Grïneri, nov. sp.

Pal. Laca rei, nov. sp.

Pholidocidaris Gaudryi, nov. sp.

Lithostrotion junceum, lilem. irregulare, Phill.

Diphyphyllum concinnum, Lonsd.

Clisiophyllum turbinatum, M'Coy.

Envisagée au point de vue bathymétrique, nous avons déjù vu que la faune de liérny était une faune littorale à faciès vaseux. Elle paraît en partie comprisu dans les limites du balaneement des marées, ce qui est indiqué à la fois par l'association des genres prédominants et par l'abondance des empreintes d’algues. Eille doit meme deseendre jusqu'ì la zone des Laminaires.

Il n'en cest pas ainsi de la faune de l'Ardoisiere. Cette dernière offre un carac-

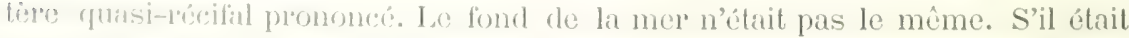
surtrot viseux à liegny a ailleurs, ce qui indique la prédominance des sehistes argilonx, il est formé it l'Ardoisière d’un sable quartzeux fin, plus tard cimenté

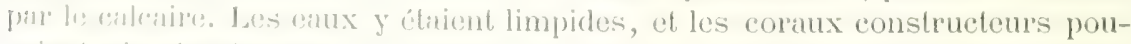

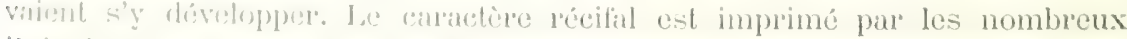
Polypirs glus l'on y obsenve, Polypiers constructeurs, tels que : Lithostrotion 
junceum et irregulare, Diphyphyllum concinnum, Syringopora ramulosa; parfois en touffes énormes, associés à des Polypiers libres, tels que: Amplexus coralloides, de plus grande taille qu'ailleurs; Clisiophyllum turbinatum, Lonsdaleia rugosa, Zaphrentis, Cladochonus, ete. Des Hydrozoaires et de nombreux Bryozoaires se mêlent à eux: Polypores, Fenestelles, Monticulipores. Des Oursins aux genres variés, Archeocidaris, I’alcechinus, Pholidocidaris anx affinités américaines, sont répandus à profusion. On ne peut donner un coup de pic sans faire apparaître immédiatement une plaque ou un radiole. D'autre part, les gros Brachiopodes remplaçant les Diceras jurassicques ou les Hippurites crétacés, pullulent dans toute l'épaisseur du gisoment. A l'Aroloisière seulement se reneontrent los énormes Productus gigunteus et Pr. cora, le gros Spirifer bisulcatus, le grand Orthotetes Sharpei. N'est-ce pas lit un faciès récifal? Et ce gisement ne réunit-il pas ces aspeets caractéristiques des récifs jurassiques qu'Etallon avait désignés sous le nom de Glypticien, de Zonnthairien et de Dicératien?

L'ensemble de la faune, tout en présentant un véritable fond commun avee Régny, Néronde et Saint-Germain-Laval, en diftère done par ce caractère bathymétrique. On ne voit pas, il est vrai, de vrai calcaire construit ì l'Ardoisière. La roche normale, non altérée et décalcifiée, n'a rien de commun avec le marbre construit de l'assise frasnienne de Diou, par exemple, ou de tel autre récif coralligène. Aussi ne pouvons-nous pas affirmer que l'Ardoisière est un vrai récif; mais l'examen de sa faune nous oblige à l'envisager comme constituant une tentative de récif, ou comme formée dans le voisinage immédiat d'un récif aujourd'hui disparu. C'est une faune quasi-récifale.

La découverte de ce caractère nous paraît être décisive par les conséquences qu'il est permis d'en tirer l’elativement à son âge, si nous le comparons à celui des gisements de la Loire. Telle est, en effet, la question nouvelle à résoudre. Quel est l'âge relatif de l'Ardoisière et de nos gisements dinantiens du Plateau Central, tous synchroniques entre eux et de position V1, tandis que Vise ou l'Ardoisière belge appartient à l'assise supérieure V2? Eh bien! nous sommes arrivé à la convietion que l'Ardoisière et Régny, ainsi que les autres localités dinantiennes de nos régions, sont parfitement synchroniques, et ne constituent que des faciès distincts d'un même horizon stratigraphique.

Examinons d'aboled, au point de vue de la stratigraphie comparce, les deux gisements de l'Ardoisière et de Ferrières, situés aux deux extrénités de l'ellipse carbonifêre, entourée de porphyre, qui oecupe la vallée du sichon, gisements séparés par le puissant massit intermédiaire cambrien d'Aromne.

A l'Ardoisière, l'assise de Dinant proprement dite it f'ume de Régny, c'ést-ìdire l'assise V1 de la classification de M. Dupont, fait absolument défaut. Cette assise devrait s'interealer entre le marbre waulsortion do la base et les calschistes fossilifères qui supportent directement le gròis anthracifiere. Or', il n'en est rien. Cette lacune est-elle le rosultat d'une émersion produite ì ce moment 
ou d'une faille qui aurait englouti dans la profondeur les assises dinantiennes? Aucune de ces hypothìses ne saurait être admise.

La liaison entre les schistes verdâtres dans lesquels le marbre de la base est intercalé, avec les assises si fossilifères qui les recouvrent, est parfaite. Il y a continuité de sédimentation et nulle trace de faille, ou du moins nous n'avons pu en constater.

A Ferrières, c'est l'inverse. Les marbres exploités dans le village sont en continuité parfaite avec les arkoses supérieures à faune de Dinant bien curactérisée. Mais, les grès marins de Cheval-Rigon supportent immédiatement les bancs de grès anthracifère normal, sans intercalation aucune de l'horizon de l'Arduisiore V2. Quelles conséquences tirer de ces faits? Il me semble qu'étant donne cet état de choses, l'équivalence latérale du gisement semi-récifal viséen de l'Ardoisière ot des sédiments littoraux et dinantiens de Ferrières doit s'imposer à l'esprit d'une manière absolue.

Mais nous arons déjà établi le synchronisme de toute la bande carbonifère de hil Loire avec les arkoses de Ferrières et les grès siliceux de Cheval-Rigon! D'autre part, nous avons constaté la superposition directe du grès anthracifère, dans cette région, sur les schistes à lentilles de calcaire noir de ces divers gisements, car nulle part, malgré nos recherches, nous n'avons pu saisir l'infercalation de l'horizon viséen V2. L'Ardoisière est mique dans le Plateau Contral. La conséquence absolue est que l'équivalence latérale de ce récif isolé ne s'applique pas seulement à Ferrières, mais comporte une généralisation absolue. L'Ardoisière et Régny sont done du même âge. L’assise supérieure V2 doit être abolie, tout au moins dans la France centrale.

En ast-il de même en Belgique? C'est une question que nous soumettons à l'oximen des géologues belges. Nous sommes tout disposé à le croire en nous pappelant l'identité palcontologique absolue de notre faune de l'Ardoisière avoe celle du petit massif si célèbre de Visé. En outre, à Visé, ect horizon propse directement sur le calcaire dévonien. M. Dupont a depuis longtemps constaté qu'il n'offre aucune apparence de stratification ("), et j’ai pu m'assurer stur place de la réalité de ee fait. La question serait tranchée dans notre sens, depuis longtemps sans doute, si le massif de Visé reposait, comme celui de l'Ardoisiòre, non plus sur le dévonien, mais sur des roches waulsortiennes.

\section{LVAUX (CREUSE).}

An sind do sinint-Julien-de-Gencte, sur lit rive gauche du Chat-Cros, les whistug ot gries gui reposent transgressivement sur le stéaschiste cristallophyllien renforment un bane de marbe cristallin, gris-bleuâtre, identique 
d'aspect à celui de l'Ardoisière et des autres gisements waulsortiens ci-dessus décrits. Un article d'encrine est le seul fossile qui y ait été jadis découvert (1). Nous avons visité ce gisement, et nous y avons recueilli, à notre tour, deux beaux articles de Poteriocrinus crassus. Les schistes encaissants sont malheureusement si métamorphisés que les fossiles en ont totalement disparu.

La position stratigraphique du marbre d'Evaux a done pour symbole $\mathrm{T} 2=\mathrm{II}$.

Maintenant que nous avons analysé minutieusement tous les faits découverts dans les seize gisements que nous avons explorés, en nous tenant constamment sur le ferme terrain d'une comparaison attentive de nos faunes entre elles, et avec les faunes classiques de la Belgique, nous résumerons ci-dessous tous les r'ésultats acquis, de manière à les intercaler, dans un prochain chapitre, dans un tableau de synchronisme qui permettra de les embrasser d'un coup d'œil, ainsi que l'âge varié de nos grès anthracifères, que nous arons à dessein laissés provisoirement de côté :

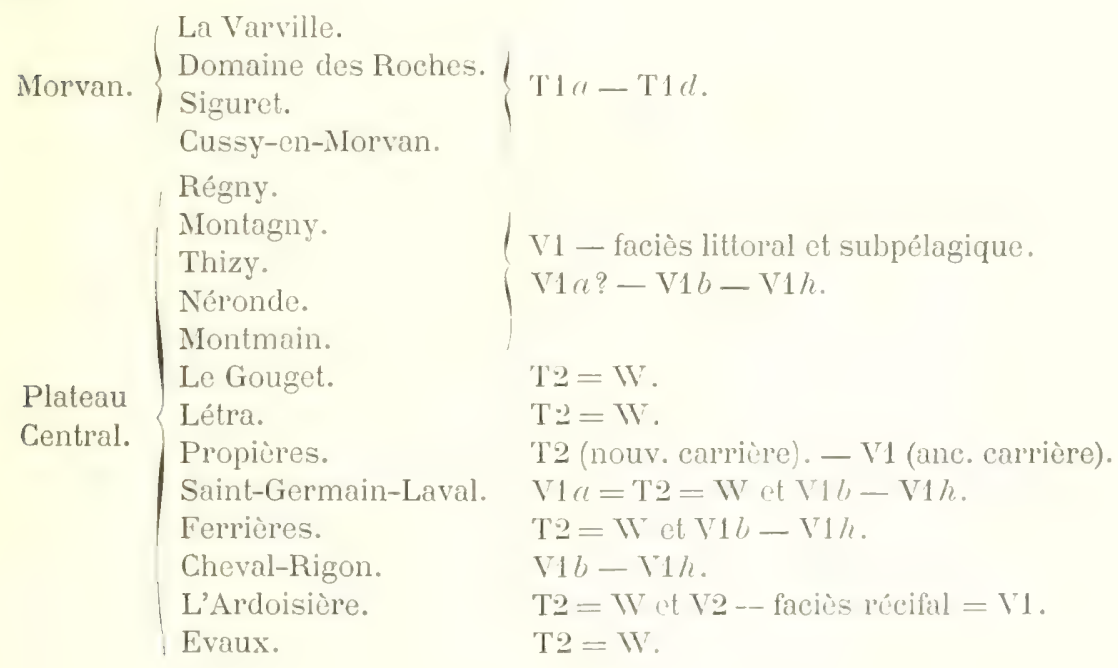

(1) Grüner. Etude des bassins houillers de la Crense, p. 12. 


\section{AGE DU GRÈS ANTHRAGIFÈRE}

Nous avons laissé de côté, on examinant nos gisements de calcaire carbonifère, le grès anthracifère, sur l'âge duquel des opinions diverses ont été émises. Pour ne pas ralentir la discussion relative à l'âge de nos faunes marines, nous avons préféré en constituer un chapitre spécial, dans lequel nous rappellerons d'abord les découvertes de plantes dans nombre de localités disséminées çà et là dans toute l'étendue de la formation, de manière à constituer un tableau complet de toutes les espèces qui ont été recueillies et publifes. Nous ferons ensuite l'historique des opinions relatives à l'âge de cette formation, et nous donnerons nos conclusions, qui seront résumées, ainsi que les résultats auxquels nous a conduit la discussion des gîtes marins, dans un tableau général de synchronisme de la formation carbonifere du Plateau Central et du Morvan.

P.ILÉONTOLOGIE DU GRÈS ANTHRACIİ̀RE.

L'existence du charbon dans le grès anthracifère était connue du temps d'Alléon-Dulac, qui en signale à Crémeaux, à Saint-Maurice, à Villemonteix, à Lay dont l'exploitation remonte ì 1762 , dans les paroisses de Régny et de Montagny, et dans celle des Sauvages. Le vieil auteur l'appelle que, suivant d'anciens Mémoires tirés du Trésor des Chartes du Beaujolais, il y a cu une mine de charbon de terre en exploitation dans la paroisse de Saint-Cyr-deChatoux.

Passinges et Valuy donnent à leur tour des détails intérossunts sur les mines d'anthracite de la même formation. Nous ne retiendrons des écrits de ces deux observateurs que l'assertion de Passinges mentionmant lia découverte de plantes à Saint-Maurice-en-Roannais, paree qu'elle nous partit ctre la premitre en date concernant des végétaux distincts:

"Très près du bourg, au sud, dit l'ancien profossour de l'Ecolo Centrale do "Roanne, on a découvert des liges de bambou longues de 9 is 10 pouces, un " peu arquées, ayant des stries longitudinales, et envilon 2 ponees de dimmetro. „Elles ont été découvertes daiıs une vieille vigne que l’on défonçat pour la 
» renouveler; elles étaient enfouies dans une roche de grès qui ressemble „ parfaitement à un granite grossier; il faut l'examiner de bien près pour se ") persuader que c'est un grès, car il peut laisser un doute sur l'époque de la " formation de certains granites. "

Il est probable que les tiges de bambou qu'il cite ne sont autre chose que le Bornia transitionis découvert pour la première fois dans le Plateau Central.!

Il faut aller jusqu'en 1836 pour avoir l'indication d'une nouvelle découverte. Nous faisons allusion aux plantes recueillies par Fournet dans les déblais des deux puits de Valsonne et soumises par lui à l'examen de Brongniart, qui les assimila aux plantes du terrain houiller.

En 1841, Grüner découvrit de belles empreintes à Neulize. Il signala aussi à Naconne de nombreuses empreintes de Calamites qui gisaient dans un grès fin, schisteux, reposant sur le poudingue de la base, et recouvert immédiatement par le grès porphyrique.

A la Mure, dans la vallée de l'Azergue, où l'on sait que le grès anthracifère est très développé, Ełray a recueilli un certain nombre de végétaux fossiles, ainsi qu'à Valsonne et ì Joux, près de Tarare, dans des conditions de gisement identiques à ceux de Nacomme.

lin voici la liste ${ }^{(1)}$ :

Calamites radiatus, syn. Bornia transitionis, Goepp. - Gare de Tarare, La Mure.

Cyclopteris polymorpha, Goepp. - Valsonne.

- Koechlini, Schimp. - Valsonne.

Sphenopteris Schimperiana, Gœpp. - Joux.

Cyclopteris? gigantea, Ebray. - La Nure.

Stigmaria ficoides, Brgt. - Valsonne, Avenas, Joux, La Mure.

Sagenaria Weltheimiana. - Beaujeu, Joux, Valsonne.

Nous rappellerons iei les plintes que nous-mêmes avons recueillies dans les deux dornicrs gisements et qui ont été déterminées par M. l'abbé Boulay:

Cordiopteris polymorpha, Schimper.

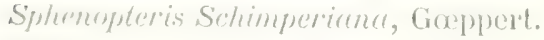

Reluodere putentissima, Stur.

Todere Lipordeli, Stur.

Arehereculamites radiatus, Stur.

Stigmarin fieroides, Brot. 
Le grès anthracifère du Roannais a oflert ì M. Grand'Eury les plantes énumérées ci-dessous (1):

Lepidodendron Weltheimianum, Presl.

$$
\text { - } \quad \text { tetragonum, Stern. }
$$

Lepidostrobus Rhodomnensis, Grand'Eury.

Sphenopteris elegans, Bi'gt.

- filifera, Stur.

- Göperti, d'Ettingsh.

- Dacallioides, Goepp.

Clepsydropsis duplex, Unger et Williams.

Palceopteris, sp.

Bornia transitionis, Gopp.

Stigmaria levis, Goepp.

Syringodendron, sp:

Outre ces espèces, M. l'ingénieur Maussier en a découvert d'autres dans des fragments roulés de quartz lydien (Kieselschiefer), provenant des environs de Combres. Ces galets ont été arrachés à un banc d'une puissance de 30 à $40^{\mathrm{cm}}$ reconnu en place sur une longueur de plusieurs kilomètres. Il est interealé entre des couches d'anthracite.

Ces cailloux renferment des frogments de Clepsydropsis duplex, Unger et Will.; et de Lepidodendron (2).

A Anost, dans le Morvan, M. Renault a découvert dans le grès anthracifere( ${ }^{(3)}$ :

\section{Lepidodendron Weltheimianum. \\ Cardiopteris polymorpha.}

Bornia.

En 1890, Mni. de Launay et de Grossouve ont découvert des Bornice et des $C y c l o p t e r i s ~\left(C\right.$. polymorpha) dans le grès anthracifère de Brégeroux ${ }^{(3)}$.

Déjè Mallard avait trouvé des Borniu dans l'anthraeite des bords du ChatCros; et des végétaux indéterminés avaient été signalés dans le même grès, à Taleix, commune de Fontanières, près d'Evaux, par Grüner (5).

(1) Flore carbonifire du dipurtement de la Loire et du centre de la France, passim.

(2) B. Renault. (Comple-rendu de l'Académie des Seiences. 15 octobre 1887.)

(3) Societé d'Mistoire naturalle d'Autur. 2' Bulletin, 1889, p. $50 \mathrm{t}$.

(4) B. S. G. F. $3^{\text {e }}$ série, t. XVI, p. 108's.

(5) Description des bassins houillers de la Crerrse, 1. 10; et Bulletin de la Creuse, 1. 111, 1., 18 t. 
Nous réunissons, pour la commodité du lecteur, dans le tableau suivant, toutes les plantes bien déterminées trouvées dans le grès anthracifère de la France centrale :

\section{PLANTES DU GRÈS ANTHRACIFÈRE.}

RÉGIONS.

\begin{tabular}{|c|c|c|}
\hline \multicolumn{2}{|c|}{ Morvan............... } & $\begin{array}{l}\text { Lepidodendron Weltheimianum, Presl. } \\
\text { Cardiopteris polymorpha, Schimper. } \\
\text { Bornia transitionis, Gœepert. }\end{array}$ \\
\hline \multirow{4}{*}{$\begin{array}{l}\text { Plateau, } \\
\text { Cintral. }\end{array}$} & Beaujolais. & $\begin{array}{l}\text { Cyclopteris polymorpha, Gceppert. } \\
\text { Syn. Curdiopteris polymorpha, Schimp. } \\
\qquad-\quad \text { Kuchlini, Schimp. } \\
\text { Sphenopteris Schimperiana, Gceppert. } \\
\text { Stigmaria ficoides, Brgt. } \\
\text { Lepillodendron Weltheimianum, Presl. } \\
\text { Bornia transitionis, Gopp., syn. Archooca- } \\
\text { lamites radintus, Stur. } \\
\text { Rhodea patentissima, Stur. } \\
\text { Todea Lipoldi, Stur. }\end{array}$ \\
\hline & Roannais. & 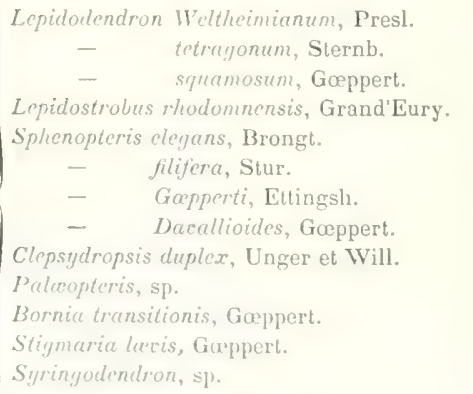 \\
\hline & Allier. & $\begin{array}{l}\text { Bornite transitionis, Gorpert. } \\
\text { Sphenopteris Schimperi, Gorppert } \\
\text { Bois de tougere. }\end{array}$ \\
\hline & 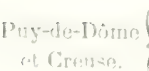 & $\begin{array}{l}\text { Cyclopteris polymorpha, Goeppert. } \\
\text { Bornia transitionis, Gorpert. }\end{array}$ \\
\hline
\end{tabular}

LOCALITES.

Anost.

-

VaIsonne.

Joux.

Joux, Valsonne.

La Mure.

Valsonne, Joux

Valsonne, Joux, Arenas, La Mure.

Valsonne, Joux, Beaujeu.

Tarare, Joux, La Mure.

Joux.

Joux.

Régny, Naconne,

Kieselschiefer de Combres, etc.

L'Ardoisière.

Brégeroux.

Bords du Chat-Cros.

L'exnmen do ce tableau nous révile la parfaite unité de la flore découverte jusigu'is co jour. Aucun mélange avece la flore houillere proprement dite comme mela alin prour lo Millstone-gril. C'est me flore plus ancienne d'âge carbonifire froplement dit. Mais avant de préciser tout à fait l'âge relatif des grès

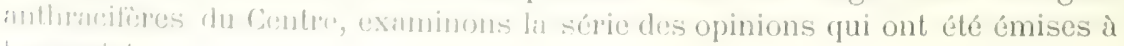
b.11P sujet. 
1830. Dufrénoy n'a pas distingué le grès anthracifère et l'a compris d'une manière vague dans le terrain de transition des Anglais (1).

1840. Grüner fait comme nous l'avons vu dans l'introduction, du grès anthracifère du Roannais, l'étage supérieur du Silurien ${ }^{(z)}$.

1841. Dufrénoy méconnaissant d'une manière étrange les relations du grès anthracifere avec les schistes carbonifères inférieurs, assimile le grès anthracifère au Dévonien, en continuant à laisser, à l'exemple de Grüner, le marbre de Régny dans le Silurien.

1855. Fournet se rapproche davantage de la vérité en ne faisant qu'une formation du grès anthracifère et du marbre inférieur, mais trompé par la soidisant identité des empreintes de Valsonne avec celles du terrain houiller, il proclame le synchronisme de Régny, de Saint-Etienne et de Rive-de-Gier.

On découvre même dans le singulier paragraphe auquel nous faisons allusion, le motif pour lequel Fournet attribuait une origine marine aux bassins houillers de la France centrale, ainsi que la première indication du mouvement orographique grandiose qu'à la suite de M. Mareel Bertrand, nous désignons en France sous le nom de Systeme hercynien. "On le voit en effet, le carboni») fère existant au nord comme au midi, à l'est aussi bien qu'au centre et à ") l'ouest de la France, il n'y avait plus lieu à refuser d'accorder à son terrain " houiller l'origine admise pour les couches anglaises et belges. Si, dans cer") tains cas, la jonction n'est pas toujours aussi complite chez nous que dans " les pays sus-mentionnés, si, par exemple, le dépôt de Saint-Etienne et de "Rive-de-Gier est jeté de côté par rapport à Régny et à Roanne, cela tient à un "simple mouvement du sol, qui, survenant entre les deux périodes, a déplacé " quelque peu la masse des eaux de la mer en créant de nouveaux rivages (3). ") 1857. Grüner recomnaissant les erreurs qu'il avait commises dans la classification de 1840, à la suite de l'examen de la collection des fossiles marins rassemblés par Jourdan, et de la proclamation de l'existence du carbonifère marin à Régny, par de Verneuil, mais ne sachant où placer le grès anthracifère, l'assimile au Millstone-grit des Anglais.

1873. M. Douvillé, dans la réunion extraordinaire de la Société géologique de France à Roanne, rattache le grès anthracifère aux ctages houller's inferricur ef moyen de Belgique, en le considérant comme antéricur au plissement lrès important qu'il venait de signaler à l'Académie des sciences, et qui avait affecté l'écorce terrestre depuis la Saxe jusqu'aux Ardennes. "Le terrain anthracifère "du Roannais, en connexion et en concordance avec les schistes et marbres " carbonifères de Régny, constitue le terrain houiller inférieur. Les conches ) de Rive-de-Gier et de Saint-Etienne, dans leur position indépendante ef leur " passage dans le haut aux couches permiennes, constituent le terrain houiller

(1) Mim. pour sercir... T. I, p. 2fl (Consilerations générales sur le llatean Central do la lirance)

(2) Annales des Mines, $3^{\circ}$ série. 'T' XIX, p. 5i3.

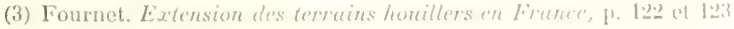


„) supérieur. Au premier, se rattachent les bassins de la Ruhr et de la Bel"gique; au second les bassins de Sarrebrück et tous ceux qui dépendent du " Plateau Central de la France. Le mouvement qui s'est produit a été un mou"vement violent, car il a été accompagné d'éruptions porphyriques et de la » formation de puissants conglomérats. Sa direction E. $25^{\circ} \mathrm{N}$. est connue "depuis la Saxe jusqu'aux Ardennes. Grüner avait lui-même signalé depuis : longtemps comme de la même époque la faille de Régny et les failles-limites ") de direction, de Saint-Etienne, dont la première ouverture a donné naissance " à la vallée houillère (1). ")

1879. M. Maussier, directeur des mines de Lay, partage l'opinion émise par 1I. Douvillé. " La formation anthracifère du Roannais, dit-il, est du même âge ") que les bassins houillers anciens de la Belgique, du nord de la France et du " pays de Galles. Tous ces bassins reposent sur la même base qui est le caln caire carbonifère signalé par Jourdan et Grüner dans l'arrondissement de »Roanne (2). )

1877. M. Grand'Eury se basant sur la nature de la flore, empreinte d'un caractère général de haute antiquité carbonifère, déclare que le grès porphyrique à anthracite du Roannais appartient r’éellement “ à l'Etage du culm, peut-être même à sa partie moyenne ${ }^{(3)}$. " Dans son bel ouvrage, M. Grand'Eury, à l'exemple des géologues allemands, subdivise le calcaire carbonifère en deux sous-étages qui se retrouveraient superposés dans le même ordre, culm en haut, calcaire carbonifère en bas dans plusieur's bassins carbonifères d'Europe, par exemple dans le Harz et la Moravie. Aujourd'hui cette expression de culm ne représente plus un étage. Une connaissance plus approfondie de l'Allemagne a appris qu'elle désignait exactement un faciès particulier du calcaire carbonifère tout entier. C'est ce que M. de Lapparent met en lumière, avec toute raison, dans le chapitie intitulé : " Le système carboniférien entre la Meuse et la "Russie. - Ainsi tout le long de ce qu'on peut appeler l'axe de l'Europe cen"trale, l'époque dinantionne (comprenant tout le earbonifère marin, du Tour" naisien au Visćen inclusivement) a vu se déposer autour des massifs anciens "une suecession de sédiments surtout arénacés, dont l'énorme épaisseur, "14,000 metres d'apris .I. Stur en haute Silésie et en Moravie, contraste avec la puissanco mokérée du calcaire carbonifère, formé au même moment dans D des canx moins agitées et sans doute près d'une côte plus stable, par l'acti") vití des ormanismes (n). ") Nous ne sommes done pas mieux renseignés sur la prosilion récle du grès anthracifère quavee les opinions qui ont précédé celle de. M. Grand'Lury.

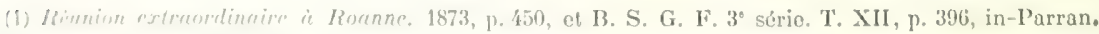

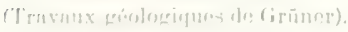

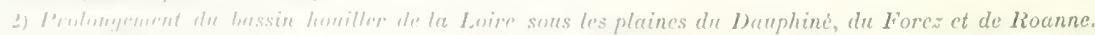

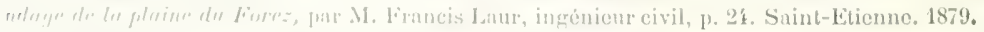

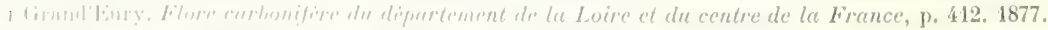

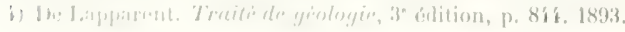


L'étude paléontologique quu nous avons pu faire ì l'aide des milliers de fossiles recueillis par nous dins le substratum marin du gres anthracifure, nous permet de démontrer que le grès anthracifòre s'est formé pendant toute la durée du carbonifère nurin, à partir de la fin du Toumaisien, et que ce grès a un âge différent suivant la région où on le considère. Il nous parait superflu d'insister sur la méthode suivie par nous et qui n'a pas besoin de justification.

Grès du Morvan. - Le grès à anthracite du Morvan recourre directement les calschistes tournaisiens. Il débute par un poudingue, vérituble cordon littoral, qui recouvre directement les couches les plus élevées du calschiste ainsi qu'on peut le voir dans la coupe de Savigny-Poil-Fol, que nous avons domnée plus haut (voir page 189). Le grès du Morvan est done Chanxhien, et le début de sa formation coïncide exactement avec culle du caleaire d'Yroil Tle de la classification de M. E. Dupont.

Grès de la Creuse. - Le grès anthraciferre de lit Creuse, qui recourre directement les schistes d'une faible épaissenr dams lesquels su trouve intercalée la lentille de marbre waulsortien des bords du Chat-Cros, s'ost formée au début de l'étage viséen.

Grès it anthracite du Beaujolais, du Roannais et du Bourbonnais. - Reste le grès à anthracite du Beaujolais, du Roannais et des environs de Ferrières et de Cusset. Le grès anthracifère qui couvre d’immenses ćtendues dans ces trois régions est partout de même ûge, postérieur au Viśen. Il est dans la classification belge l'équiralent du Namurien inférieur', c'ust-ì-dire de l'assise de Loverval. Il ne monte pas jusqu'i l'assise d'Andenne équivalent du Millstonegrit anglais. Dans la classification anglaise, le grès anthracifè̀re est l'équivalent de la série d'Yoredale. La flore de cette série est, en effet, plus ancienne que celle du Millstone-grit, laquelle se compose d'un mélange de plantes carbonifires plus anciennes et de plantes houillères plus l'eentes. Dans la classification allemande, il correspond ì la Iüngste Grauwacke ou au k'loctzleerer Sandstein.

En un mot, la position stratigraphique du gris anthrareifere est dommée en chaque point de la France centrale oì on le considere, par l'ige des conches marines qui le supportent. La nature des régétaux fossiles onfouis chans son épaisseur achève de la préciser. On pent résmer ce cheppitre chl aftirmant que la formation des grè̀s anthracifères, loin d'être partout du même tign, n'il eosisé de se produre depuis la elôture de l'ème tommaisieme. litle se déplatcait ef se répartissait, au gré des oscillations du substratum, quui se déroulaient dans lo Morvan et dans le nord du Platean Central au fur et it mosure do homp émersion. Cetle discussion achevée, nous pouvons la résumer dans le tableau de synchronisme général qui suit: 


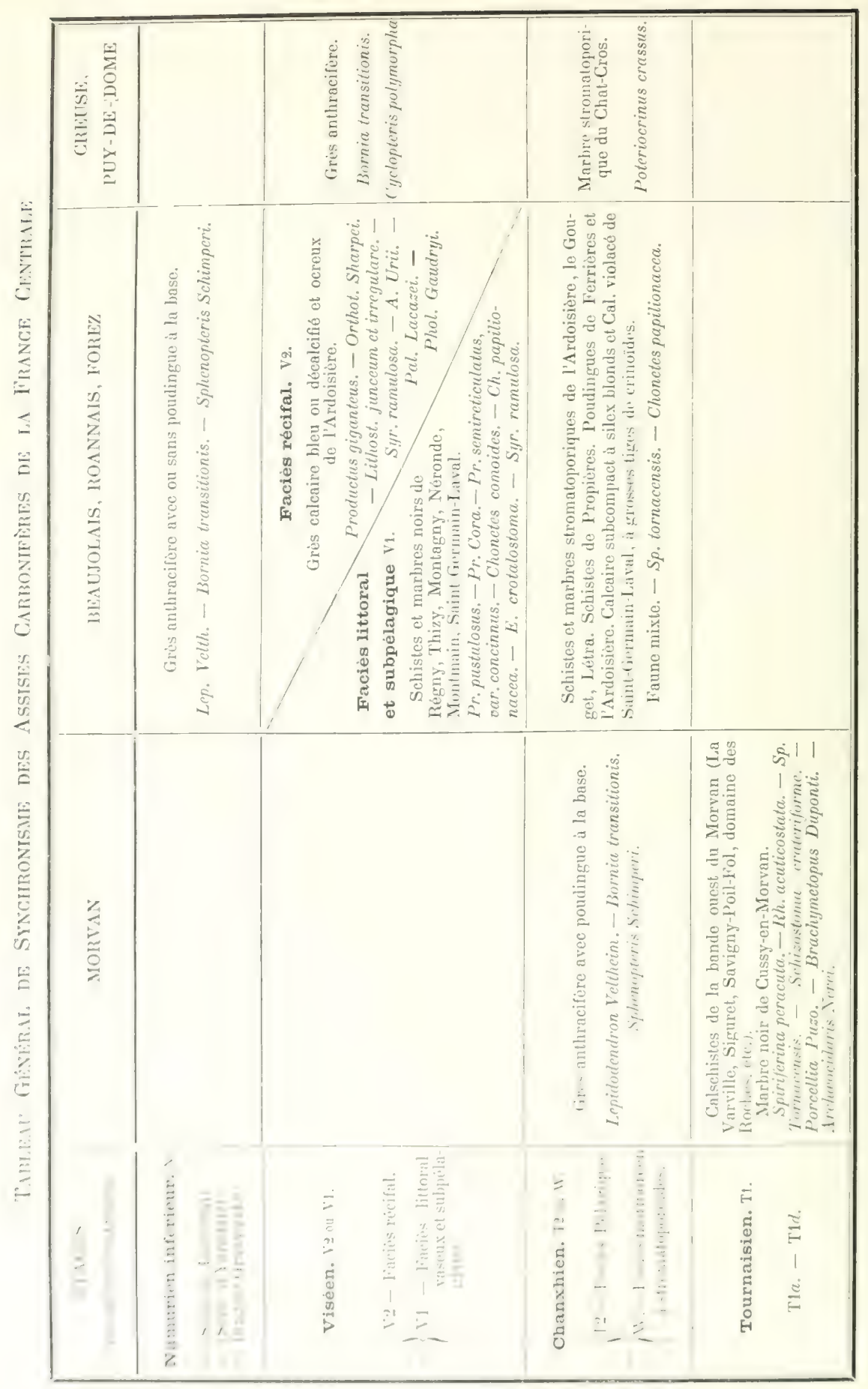




\title{
EXAMEN GRITIQUE
}

\author{
DES FAUNES CARBONIFERES MARINES DE LA FRANCE \\ ET DE QUELQUES LOCALITÉS LTRANGËRE
}

La connaissance du curbonifüre marin de la France centrale serait incomplète si, à la comparaison attentive que nous en avons faite avec la mème formation typique de Belgique, nous n'ajoutions dans une revue d'ensemble une semblable comparaison avec les faunes similaires découvertes dans les massif's montagneux du territoire français et dans quelques localités étrangères. Cet examen nous procurera en outre un double profit. Il nous permettra de réunir en un même chapitre tous les faits paléontologiques qui nous occupent, épar's dans des publications diverses. En seeond lieu, de ce rapprochement avec la France centrale et la Belgique, se dégagera avec une netteté saisissante la notion de la transgression générale de la mer carbonifère, un des phénomènes les plus intéressants de l'histoire de la Terre, comparable ì tons les points de vue aux grandes transgressions, classicques aujourd'hui, des mers calloviemne et cénomanienne à l'áporque secondaire et de la mer helvétienne à l'époque tertiaire. Or', malgró l'importance extrême de eo phrinomène dont la science devra, dans un état de perfection plus avancé, rechereher les canses profondes et mystérieuses, il est resté tout ì finit en dhohos des préoceupattions des savants belges. L'étude du Platean Central qui, par m houreux hasard, possède les deux étages toumaisien el visćen, metha fincilement ef naturellement en évidence ce beau phénomène do transegresion, nt nons permettra d'en fixer la date avee une rare procision. Nons alloms donr examiner, dans ce but, les localités tommaisiennes d'Trande of de Rustie d'abond, les

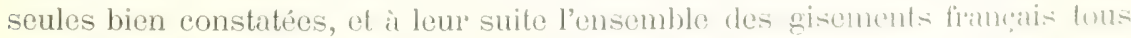
d'âge exclusivement visíen. 


\section{LOCALITÉS TOURNAISIENNES.}

\section{Hook-Point (Irlande).}

Il y a déjà plusieur's années que de Koninck a contribué à l'illustration du célèbre gisement d'Hook-Point, au sud du comté de Wexford en Irlande, en démontrant le synchronisme de sa faune avec celle de Tournai. Avant d'indiquer les espèces communes qu'il y a signalées, je reproduirai avec plaisir la description de cette localité donnée par M. John Kelly et qui est peu connue en France.

"Hook Head or Hook-Point, in Wexford, is situated on the east side of "Waterford Harbour, and the shore here affords a fine section of the lower "part of the carboniferous rocks. First, red conglomerates and red sandstone, "I with some beds of yellow in ascending southwards, and a few beds of red " or blue shale; the upper part all yellow or gray, and a little calcareous, and " it is in these the fossils first appear's. Next comes a series of thin beds of " limestone, and black shale alternating. The limestone rises in large flags, of " which both sides are covered with a profusion and rariety of fossils. The is " on the townland of Portersgate. Over it lie yellow sandstone and calcareous " gray sandstone, and this again is succeded by thin beds and afterwards thick "beds of limestone alternating with shale, for more than a mile along the "shore, but nearly level. Next, towards Hook-Point, the whole becomes limes" tone, with but a ferr and rery thin beds of shale. In the whole Peninsula, the - rock is so well exposed along the sca-shore that it is one of the finest locali- ties in Irelund for fossils. Very fine specimens of erinoüd heads have been " found near the l'oint. They are usually got in the thin beds of shale which ". separate the limestone beds, where the action of the waves carries away the , soft matter of the shale and leaves them standing in relief on the surface of

"the berl of limestone. In considering them, on the spot, the idea is suggested thrit they grew on the surfinee of a bed of limestone in the sea - as scaweed now deses - that a flood came over the place, charged with sand and mud, " ind kilher the animals, which, therefore, lay dead, and ware buried in the mordely deposit left by the walers, which is usually foum three to six inches llikk lutwern the berls of limetone. The heads and stems, for some feet in

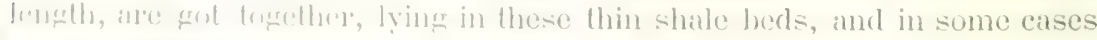

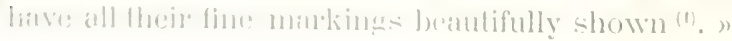

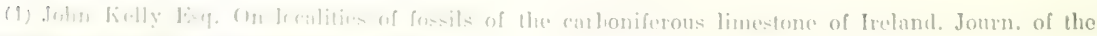

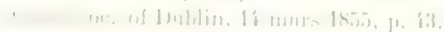


M. Kelly a fait suivre cette description intéressante de la liste complète des fossiles de Hook-Point. Nous ne la reproduirons pas car cette liste doit préalablement être révisée et mise au niveau des progrès de la paléontologie moderne; mais nous rappelierons que dans son voyage à Dublin, de Koninck en examinant cette collection, y a trouvé suffisamment de fossiles pour en établir le synchronisme avec la fame tournaisienne. Ce sont les espèces suivantes:

Actinocrinus triacontadactylus, Miller.

Michelinia megastoma, M'Coy.

- facosa, Goldi.

Polypora fastuosa, de Kon.

Ptylopora pluma, M'Coy.

Orthis Michelini, Lév.

Athyrts Roissyi, Lév.

- lamellosa, Lév.

Spirifer tornacensis, de Kon.

- laminosus, M'Coy.

Conocardium fusiforme, M'Coy.

Schizostoma crateriforme, de Kon.

Conularia formosa, de Kon.

Phillipsia pustulata, Schl.

Le Morvan a des relations fauniques aussi étroites avec les calschistes de Hook-Point qu'avec ceux de Tournai, car nous pouvons citer les espèces communes suivantes:

Actinocrinus triacontadactylus, Miller.

Orthis Michelini, Lév.

Athyris Roissyi, Lév.

- lamellosa, Lév.

Spirifer tornacensis, de Kon.

Schizostoma crateriforme, de Kon.

MaLowka-Murajewna (Russie).

A l'extrémité orientale de l'Europe se trouve lo calcaire de Malowkin-Murajewna. P. de Semenow et WV. von Möller ont poursuivi son développement it partir des frontières méridionales du cercle de Riäshsk, at l'est, jusqu'aux frontieres du eerele d’Odö̈ew, avec la province de Kalouga dans l'ouest. Én ee qui concerne le développement de ce même calcaire du sud au nord, on remarque ru'il vient au jour sur la ligne de Dankow, sur les limites méridio- 
nales de laquelle on rencontre les assises dévoniennes typiques avee Spirifer disjunctus (Verneuili), Rhynchonella livonica, et d'autres espèces fossiles. La faune de ce calcaire a été l'objet de controverses infinies de la part d'un grand nombre de géologues. Les uns l'ont rangée dans le dévonien; d'autres, au contraire, dans le carbonifère marin. Parmi ceux qui ont considéré le calcaire de Malowka comme dévonien, nous citerons le général de Helmersen (i) qui a émis le premier cette opinion en 1841, Murchison, de Verneuil et de Keyserling (2), Iérémeieff ${ }^{(3)}$, Barbot-de-Marny ${ }^{(3)}$, Romanowsky ${ }^{(5)}$, de Semenow et W. von Möller ${ }^{(6)}$.

Les géologues qui ont rangé cette formation dans le carbonifère sont Trautschold (") et J. Auerbach ${ }^{(8)}$.

De Koninck, après une étude approfondie de cette faune, a fait connaître son opinion en 1874 dans un mémoire intitulé : Etude de la faune de MalowkaMurajeuna ${ }^{(9)}$.

Plus récemment, lors de la publication de son grand ouvrage sur la faune carbonifère de Belgique, le savant belge a indiqué les espèces suivantes du mémoire de de Möller et de Semenow dont il a rectifié la diagnose :

Spirifer aculeatus, Schnur = Spiriferina Mölleri, de Kon.

Terebratula bursa, Sem. et Möll. = Dielasma tenerum, de Kon.

Rhynchonella Punderi, Som. Mö̈l. = Rhynchonella acutirugata, de Kon.

Retzia prominula, C. I'. Roemer = Acambona serpentina, de Kon.

Spirifer inftatus, Schnur = Spirifer glaber, Mart. (de petite taille).

Michelinia rossica, Sem. et Möll. = Michelinia favosa, Goldf.

Si les determinations de M. de Koninck qui n'ont pas du reste été faites d'apres les objet eux-mímes, mais d'après les planches du mémoire de de Semenow ot Möller, acquièrent droit de cité dans la seience par l'acreptation des paléontologistes russes, il sera établi que l'horizon de Tournai ast représenté sur x prints en Europe, savoir Tournai lui-même, auquel nous ajoutons naturellement los autres gisements de la même région y eompris ceux du nonel de la linuee, misuite Hook-Point en Irlande, la Varville dans le Mor-

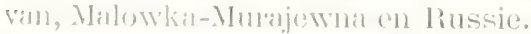

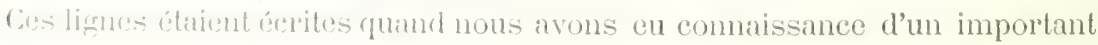

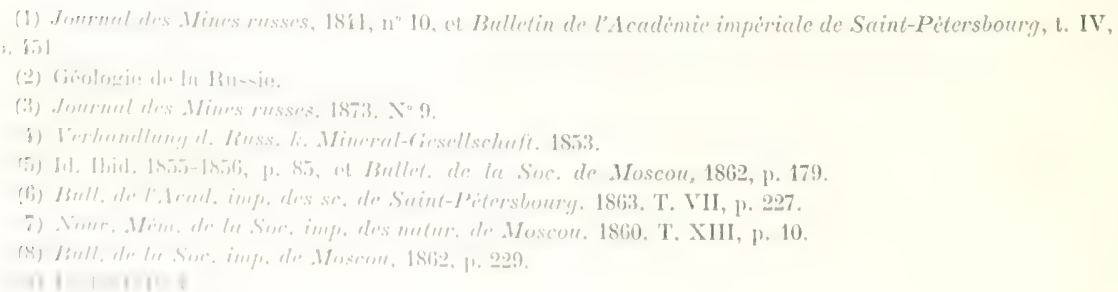


mémoire de M. H. de Peetz sur l'élage de Malowkat ${ }^{(1)}$ des parties méridionales des gouvernements de Riazan et de Toula. Toici les conclusions du savant géologue russe:

L'étage de Nalowka réellement intermédiaine aux systèmes dévonien et carbonilère, se subdivise en deux assises qui sont de haut en bas:

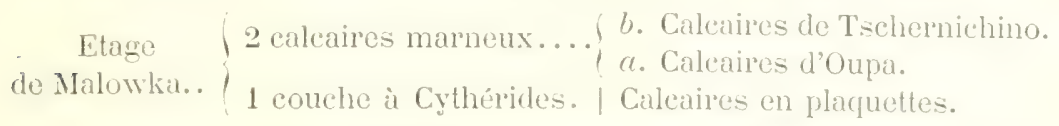

La couche à Cythérides renferme une faune en général dévonienne; tontefois l'abondance des restes de poissons appartenant aux genres Psammodus, Orodus, Helodus, Cladodus, Hybodus, Osteolepis et Palceoniscus, la présence en grande quantité de la Spiriferina octoplicata et du Productus Panderi (forme proche du $P r \cdot \operatorname{cor} a$ ) indiquent une affinite avec la faume du cubbonifere.

La faune de l'assise supérieure (des calcaires marneux) so distinsur en quelques points de la précédente. La faune mixte des calcaires d'Oupa est réritablement intermédiaire entre celle des calcaires en plaquettes et celle tout ì fait carbonifere des calcaires de 'Tschernichino.

Les Cythérides se rencontrent très rarement. On y troure une grande quantité d'espèces du genre Athyris qui n'existent pas dans l'assisa inférieure et en outre Martinia glabra, Orthis resupinata, Productus fallux, Pr. Panderi, Rhynchonella Panderi (Syn. acutirugata, de Kon.), Zaphentis Koninchii, Michelinia megastoma.

Enfin les calcaires marneux s'appauvissent peu à peu en formes déroniennes, de telle sorte que les calcaires de Tschernichino renferment une faune d'un caractère tout à fait carbonifère.

Au-dessus seulement viennent les couches à Pr. gigunteus. Ainsi les conclusions du travail de M. de Koninck se trouvent vérifiées par les études tontes récentes de $\mathrm{M}$. de Peetz.

\section{Amérinle du Nord.}

Pour retrouver la faune de Tounai il faut se transporter dans l'Amerigue du Nord où l'horizon tournaisien est représenté par le calcaire de Burlington dins l'Iowa, de Quincy dans l'Illinois, d'Hamnibal dans lo Missouri et dans le 'Tennessee.

Voici, en effet, les fossiles signalés par M. de Koninck, ct qui citablissent lo synchronisme avee l'étage tournaisien. Orthis Michelini, var. Burlingtonensis; Burlington, Quincy, Hannibal. - Productus Filemingii, var. Burlingtonensis;

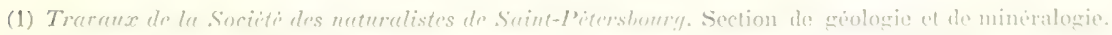

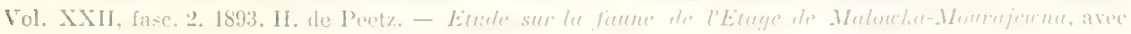
211. (réstumé p. 103). 
Burlington, Quincy. - Spirifer Grimesii, J. Hall, 1854 = Spirifer cinctus, Keyserl., 1846, Burlington, Quiney. - Spirifer Forbesi, Norw. et Pratten, 1854; Spirifer imbrex, J. Hall, 1858; Burlington. D’après de Koninck, ces deux Spirifers sont des formes représentatives de Spirifer tornacensis, surtout le premier. - Porcellia nodosa, Hall, 1860; forme représentative de P. Pũo, Lév.Pernopecten Shumardianus, Winchell, 1865; forme représentative de Entolium Witryi, de Kon.

Ce caractère tournaisien de la faune du calcaire de Burlington, de Quincy, etc., est confirmée par la grande abondance des Crinoïdes aux genres variés, et l'apparition d'Echinides tessellés des genres Palcechinus et Archceocidaris.

\section{Relations des gisements du Plateau Central avec les autres gisements français.}

Il nous a semblé du plus haut intérêt de comparer nos gisements du Plateau Central avec ceux du reste de la France. Nous laisserons de côté les petits lambeaux des environs d'Avesnes et du Boulonnais, si bien étudiés par M. Gosselet, parce qu'ils sont une dépendance visible de la grande formation belge.

Nous n'examinerons que les faunes mises à jour dans les lambeaux du Cotentin et du grand Massif breton, dans ceux des Vosges, de la Montagne Noire et des Pyrénées. Cet examen montrera l'identité parfaite de toutes ces faunes et nous permettra d'en tirer des conclusions précises relativement à la transgression de la mer carbonifère dans notre pays. Nous n'entrerons dans aucun détail stratigraphique. On les trouvera facilement en recourant aux références qui sont toutes indiquées. Nous nous contenterons de citer les localités fossilifures, de donner les listes de fossiles connus et publiés et de mentionner le nom des savants qui les ont découverts ou déterminés.

En $u$ mot, nous nous bornerons à mettre sous les yeux des lecteurs le tableau complet des faunes carbonifères de la France et à en tirer les conclusions qui en découlent relativement à leur âge.

\section{Cotentin.}

Le culcaniro carbonifère a été recommu dans cette région en 1854, par EugèneEurles Destongchampes (1). On peut l'observer à Régneville, au sud-ouest de Coutinece- (Manche), dans la petite vallée de la Malliance.

Los doux erisoments découverts sont ceux de Montmartin et de Régneville. Lo calcaire est 131 marlse d'aspect gris-char saceharoüde, fort compacte, ayant tons les cantatires dess marbles waulsortiens belges reposant en stratification diseremelantes stre le Silurien. 
Voiei la liste des fossiles recueillis (1) :

Euomphalus, esp. indét.

Conocardium hibernicum, Sow. IV.

Posidonomya vetusta, Sow: V.

Avicula, très grande, indét.

Spirifer striatus, Sow. IV.

Productus giganteus, très abondant, Sow. V.

- semireticulatus, Mart. T. V.

- punctatus, Sowerby V.

Chonetes papilionacea, Phill. V.

- comoides, Sow. V.

- Dalmaniana, de Kon. V.

Orthis resupinata, Mart. T. IV. V.

Orthis, très grande.

Leptona depressa?? Phill. T. IV. V.

Cyathophyllum mitratum, Schlot.

Débris de Crinoïdes.

$$
\text { plicatum, Goldf. }
$$

Observation. - L'ensemble de cette faune caractérise, il est vrai, l'étage belge de Visé, en particulier : Chonetes papilionacea, Ch. comoides, Ch. Dalmaniana, Productus giganteus, $\mathrm{Pr}$. punctatus. Mais la présence de Spirifer striatus, et de Conocardium hibernicum qui, d'après de Koninck, sont des fossiles essentiellement waulsortiens, indique au début une faune chanxhienne transformée graduellement en viséenne.

La conclusion, c'est que la mer carbonifère est arrivée dans le Cotentin à cette époque chanxhienne et qu'elle y a séjourné pendant toute la durée de la période de Visé.

\section{MASSIF BRETON.}

Le massif nous offre le calcaire carbonifère à l'est et à l'ouest. A l'ouest, il s'étend en une longue bande étroite courant de Châteaulin par Carhaix jusqu'ì Uzel. A l'est, la bande carbonifère constitue le bassin de Laval et s'étend jusqu'i Sablé.

Dans le Finistère, d’après M. Barrois (2), le carbonifère présente de bas en haut des poudingues et tufs porphyriques, des tuls porphyritiques, et les

(1) Paul Dalinier. Stratigraphic des terrains primaires dans la presqu'ile du Cotentin (thise), p. 109. 1861.

(2) B. S. G. F. Réunion extraordinaire de la Soe. géol. 1886. Structure géoloyique du Finistere, 1), 661. 
schistes de Châteaulin formés de couches alternantes de schistes, d'ardoises, de psammites.

Ces schistes et psammites contiennent de mauvaises empreintes végétales ver's Carhaix, à Plouyé; les psammites ont fourni quelques fossiles marins.

Enfin des lentilles calcaires inter'stratifiées ver's la base de la série contiennent :

Phillipsia derbyensis, Mart. V.

Productus semireticulatus, Mart. T. V.

A Plouyé, dans les psammites, on trouve:

Spirifer striatus, Sow. W.

Strophomena rhomboidalis, Phill. T. V.

Phillipsia derbyensis, Mart. V.

Productus semireticulatus, Mart. T. V.

Nous voyous ici le même mélange que dans le Cotentin. Spirifer striatus est waulsortien, mais Phillipsia derbyensis est viséen, et il existe déjà dans les calcaires interstratifiés de la base; Productus semireticulatus se trouve dans toute la série carbonifère, ainsi que Strophomena rhomboidalis qui, on le sait, est sans valeur stratigraphique, car il se rencontre depuis le Silurien jusqu'au sommet du carbonifère marin. On sait que l'Atrypa reticularis a aussi vécu aux époques silurienne et dévonienne, et que ces deux espèces d'une longévité si extraordinaire ne peuvent servir qu’à caractériser des formations paléozoïques et $110 n$ point un étage, encore moins une assise.

La mer' a done fait son apparition en Bretagne à l'époque chanxhienne alors que vivait Spirifer striatus et a continué à y séjourner. L'invasion de la Bretagne a done cu lieu en même temps que celle du Cotentin et du Plateau Central.

\section{Qúxon.}

En 1890, M. Lebesconte a filit comnaître un petit lambeau carbonifère, situé ù Dnónchn en Saint-Aubin-d'Aubigné (Ille-et-Vilaine) (1). Ce lambeau est composé

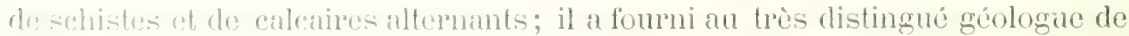
lhonnes los fossiles snivants qui ont été déterminés par M. Barrois:

Phillipsire gemmulifere, Phill. 'T'. V.

- Derbyensis, Mant. V.

Spririfer striatus, Mant. IV.

denplirierseste, Phill. V.

- bisulereluss, som. l.

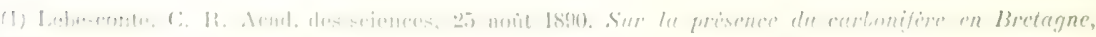


Spirifer oceani, d'Orb. V.

- concolutus, Phill. V.

- laminosus, de Kon. T.

- lineatus, Mart. T. V.

- glaber, Mart. V.

Productus aculcatus, Mart. V.

- semireticulatus, Mart. T. WV. V.

- scabriculus, Mart. T. V.

- Flemingï, Sow. T.

Chonetes hardrensis, Phill. V.

- papilionacea, Phill. V.

Streptorhynchus crenistria, Phill. T. W. V.

Leptcena depressa, Phill. T. IV. V.

Orthis Michelini, Lér. T.

- resupinata, Mart. V.

Rhynchonella pleurodon, Phill. V.

Terebratula hastata, Sow. V.

Euomphalus Dionysii, Montfort V.

Conocardium alceforme, de Kon. T.

Cypricardina squamifera, Phill. V.

Syringopora, sp.

M. Bezier(1) a recueilli dans la même carrière les espèces suivantes déterminées par M. Ehlert:

Phillipsia truncatula, Phill. 'T.

Brachymetopus Mac'Coyi?

Productus semireticulatus, Mart. T. W. V.

- pustulosus, Phill. V.

Parallelodon bistriatus, Portl. T.

Straparollus cequalis, de Kon. V.

Spirifer bisulcatus? Sow. IV. V.

Conocardium, sp.

Chonetes, sp.

Soit un total de 32 espèces déterminées, provenant de la méme carrière, formé d'un mélange d'espèces appartenant aux trois horizons de Tommai, Waulsort et Visé. Les espèces de Tournai sont: Spirifer laminosus, M'Coy; Productus Flemingii, de Kon.; Orthis Michelini, Lév.; Phillipsia truncatula, Phill. (cette demière espèce n'est pas de Tournai, mais de Hook-Point); Parallelodon bistriatus, Portl. Une espèce appartient à l'horizon de? Wiulsort, Spiri-

(1) Bezier. C. R. Acadénie des sciences, 1' sept. 1890. Sur un gisemunt curbonifuere de l'ärge de Visé, reconnu a Quenon en Saint-Aubin-d'Aubigne (llle-et-Vilaine), p. 403. 
fer striatus, Phill. Toutes les autres sont viséennes, en particulier Spirifer bisulcatus, S. duplicicosta, S. Oceani, Chonetes papilionacea, Euomphalus Dionysii, Phillipsia derbyensis, ete.

Cette faune est comparable dans une certaine mesure à celle de Pair et de Modave. Arec Pair, elle offre les espèces communes suivantes:

Phillipsia gemmulifera.

- Derbyensis.

- truncatula, récemment découvert en Belgique (note additionnelle).

Productus scabriculus.

Chonetes Hardrensis.

Orthotetes crenistria.

Orthis Michelini.

- resupinata.

Phillipsia Derbyensis.

Arec la faune de Petit-Modave :

Spirifer convolutus.

Orthotetes crenistria.

Productus semireticulatus.

Chonetes Hardrensis.

Orthis Michelini.

Leptena depressa.

Parallelodon bistriatus.

En résumé, le gisement de Quénon dans lequel se rencontrent 12 espèces communes aux faunes chanxhiennes de Pair et de Modave est d'âge chanxhien. Il est plus récent que nos gisements tournaisiens du Morvan. Il est plus ancien que nos belles faunes viséennes de Régny et Néronde. Il représente le fuciès pélagique de nos marbres waulsortiens du Plateau Central et du Cotentin. Il est une nouvelle preuve que la mer carbonifère, venue du Nord, a envahi simultanément la Bretagne et le Plateau Central.

\section{SABLÉ.}

"En 1839, dit M. d'Arehiac (1), M. de Verneuil soutint, contre l'opinion reçue " silor's, (fure te véritable calcaire carbonifère existait dans le voisinage de Sablé, " crractirise par les Euomphalus pentangulatus et Calyx et par un gros Pro"dnctus, wisin du P. hemispharericus (giganteus) (2). Peu après, ayant visité cette 
" Localité avec notre savant ami, nous manifestûmes la même manière de " roin (1). Mais ce ne fut que lors de la réunion de la Société Géologique, sur " ce point, que les observations combinées de MM. Triger et de Verneuil, dans " la coupe si souvent décrite de Sillé-le-Guillaume à Sablé, démontrèrent que, " de cette ville à Brûlon, le calcaire carbonifère, avec des schistes et des " veines anthraciteuses subordomées présentant un double plissement de " chaque côté d'un axe médian anticlinal, se trouvait compris, au nord comme " au sud, entre des schistes et des calcuires dévoniens.

"Le groupe carbonifère inférieur ainsi constitué est recouvert, à stratification » discordante, par le supérieur, à Saint-Pierre-la-Cour, à l'ouest de Laval, et » s'étend sur une longueur de 50 à 55 kilomètres de l'E.-S.-E. à l'O.-N.-O.

» de Juigné, près Sablé, à Asnières et Poillé, la Bazouge, Argentré, etc. »

De Verneuil publia, à cette époque (2), une liste de fossiles d'après les éléments qu'il avait recueillis lui-même, et ceux des collections de MM. Guéranger, Dayoust, de Lorière.

Nous reproduisons ci-dessous une liste plus complète publiée récemment par $^{2}$ I. Guiller ${ }^{(3)}$ :

Phillipsia gemmulifera, Phill. T. V. derbyensis, Mart. V.

Nautilus Cordieri, Desportes.

Orthoceras, sp.?

Eumphalus pentangulatus, Sow. WT.

- Dionysii, D. de Montf. V.

- helicoides, Sow. T. WV.

- catillus, Sow. V.

Turbo tiara, Sow. V.

cequalis, Sow. V.

Capulus vetustus, de Kon. V.

Bellerophon Corriei, d'Orb.

- Soccerbyi, d'Orb.

- bicarenus, Lév. 'T.

- hiulcus, Sow. V.

- costatus, Sow. V.

- solesmensis, Guér.

Cypricardia squamifera, Phill.

Conocardium fusiforme, N'Coy. T.

hibernieum, Sow. IV.

(1) B.S.G.F., 1841, vol. XII, p. 480.

(2) B. S. G. F., 2 éérie, t. VII, 1850. - Réunion extraordinaire au Muns, p. 776.

(3) Guiller. Géologie de la Surthe, 1. 87, 1856.

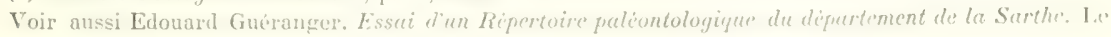
Mans, 1853. 
Productus giganteus, Sow. V.

- plicatilis, Sow. V.

- semireticulatus, Mart. T. V.

- pustulatus, Phill. V.

- punctatus, Sow. V.

Chonetes papilionacea, de Kon. V.

- comoides, Sow. V.

Lepteena arachnoidea, Phill.

Orthis crenistria, Phill. T. WV. V.

- resupinata, Phill. T. W. V.

- subarachnoidea, d'Arch. et de Vern.

Atrypa acuminata, Sow.

Spirifer glaber, Sow. V.

- cuspidatus, Sow. IV.

- striatus, Sow. WV.

- bisulcatus, Sow. WV. V.

Terebratula sacculus, Mart. V.

Palechinus Verneuili, Guéranger.

Amplexus coralloides, Sow. T. W. V.

$Z$ (ip)hentis Phillipsii, M. E. et J. II. T.

- Guerangeri, M. E. et J. H. T. H 1.

- excazata, M. E. et J. T.

- cylindrica, M. E. et J. H. T.

Cyathaxonia tortuosa, Michelin. T.

Syringopora parallela, Fisch. T.

$$
\text { - distans, Fisch. T. }
$$

Micheliniu tenuisepta, Phill. T. V.

Si nous examinnns cneore cette liste au point de vue de la classification belge, nous constatons, comme dans les gisements précédents, un mélange d'especes des trois éliges, mais avec prédominance des espèces viséennes. Ainsi, Tommi peut relimer Phillipsia gemmulifera, Bellerophon bicarenus, ot un centain nombre de Polypiors, tels que : Zaphrentis Phillipsii, Z. excaculu, Z. aylindrice, Cyuthaxoniu tortuose, Syringopora parallela, Syringopored distuns. Qunnt an Michelinia temuisepta, De Koninek le signale à Tournai

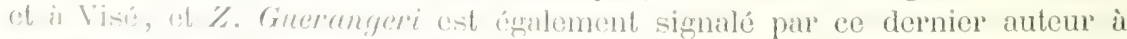

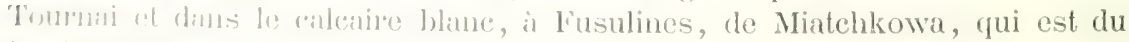
honilher manth.

las aprefes de: Wanlsonl soml les suivantos: Euomphalus pentangulatus, E. helienides, Comocardium hibernicum, Spirifer striatus, S. cuspidatus.

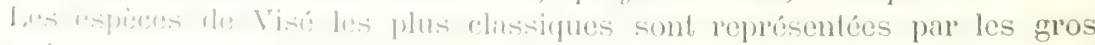

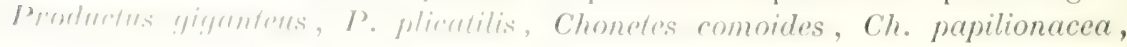


Euomphalus Dionysii, E. catillus, E. cequalis, Bellerophon hiulcus, B. costatus, ctc.

De ce mélange il risulte que la faune appartient réellement ì l'horizon de Chanxhe avec continuation du séjour de la mer pendant toute la duréc de l'étage visúen. Nous arrivons aux mêmes conclusions que celles qui l’ésultent de l'examen des faunes précédentes. Ce mélange que nous constatons dans toute la péninsule bretonne nous proure, une fois de plus, qu'il n'y a réellement que deux horizons carbonifères : celui de Tournai et celui de Tisé, aree des couches de pissige entre les deux, celles de Chanxhe-Vaulsort, et que la transgression de la mer carbonifere, dans l'ouest de la France, s'est opérée à la même époque que dans le Plateau Central.

La seule différence qu'il nous semble exister entre la Bretngne et le Plateau Central, c'est que la Bretagne est plus favorisée en fossiles chanxhiens, à l'exclusion des marbres stromatoporiques qui caractérisent plus spécialement cet horizon dans la France centrale; de telle sorte que les assises de la base affectent un caractère plus pélagique ou plus chanxhien en Bretagne; tandis qu'elles offrent un caractère plus coralligène ou waulsortien dans le Rhône, le forez et la Narche.

\section{SAINT-Roch-PRÈS-ChiNgḱ.}

Ce nouveau gisement a été découvert par M. Daniel Ghlert (1), (qui y a reconnu les espèces suivantes:

Phillipsia Derbyensis, Mart.

Cypricardia squamifera, d'Orb.

Terebratula hastata, Sow.

Rhynchonella pugnus, Mart.

Spirifer glaber, Nart.

- lineatus, Mart.

- crispus, de Kon.

Leptena depressa, D'Orb.

Productus cor $\ell$, d'Orb.

- semireticulatus, Mart.

- punctatus, Mart.

Pentremites ellipticus, Sow.

Toutes sont visémes et ne domnent lien à ampune ofservation.

Dans une Note du plus hant intérét, insérée dans les Comptes-rendus de

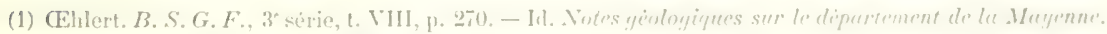
(Extrait du Bulletin de la Societe d'Eudes scientifinues d'.1myers. 1852.) 
l'Académie des Sciences (1), M. Ehlert a déroulé l'intéressant tableau des oscillations qui se sont produites pendant la période primaire dans le bassin de Laval. On nous permettra d'en donner l'extrait suivant:

« A l'époque carbonifère, par suite d'une oscillation descendante, la mer " envahit de nouveau cette région (qu'elle avait abandonnée à la fin du Dévo" nien inférieur ) et vient battre les falaises du grès dévonien; c'est à ce moment " que se remplit la grande dépression correspondant au bassin de Laval, en " même temps que sur certains points, notamment à Changé, Louverné, "Argentré au nord de Laval, et à Lhuisserie au sud, les eaux, profitant des " fractures produites dans les crìtes de grès, envahissent la région dévonienne, " amenant ainsi une transgressivité et une discordance entre les dépôts infé"rieurs et les différentes assises de la série dévonienne. Ces fiords sont remplis exclusivement par les couches inférieures du carbonifère, schistes, grès "grossiers et poudingues avee anthracite, ayant un faciès détritique et indiquant des dépôts littoraux dont les éléments sont empruntés aux diverses roches "du Déronien.

„ Pur suite d'un exhaussement continuel du sol, les dépôts suivants occupent ") seulement le centre du bassin de Laval. Ce sont les calcaires noirs à Pro"ductus giganteus, dans lesquels j'ai trouvé plus de 50 espèces appartenant à "la faune de Visé, qui supportent des schistes, des phthanites et des grès avec ") traces chrirbonneuses, représentant sans doute les couches d'anthracite de "Poilcé (Sinthe), signalées par De Verneuil au-dessus du calcaire de Sablé; " enfin des grauwackes renfermant une faune analogue au calcaire sous-jacent, " des calcaires amygdaloïdes, souvent colorés en rouge et en vert, et des "schistes argileux, formant un ensemble (calcaires et schistes de Laval), qui " peut ĉtre assimilé au houiller inférieur proprement dit. C'est sur' ces couches, "relevées verticalement, que se sont déposés, en stratification discordante, "les banes de Saint-Pierre-la-Cour, qui appartiennent au houiller supérieur. ”

\section{Missif V'osgiex.}

Le ealcaire carbonifere marin forme deux lambeaux situes, l'un, au sud des Vosges, entro Faucogney et Thann; l'iutre, au nord, dans les environs de Schirmeck.

PMTIE MERHONALE - PMNCHER-LAS-MINES.

Las promiens fossiles carboniteres ont été découverts par Jourdan (2). Ce ¿nvant u'a rimp publir sur cosujet; il s'ost contenté de citer P'roductus giganteus

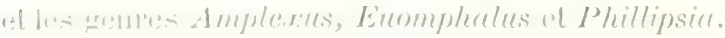

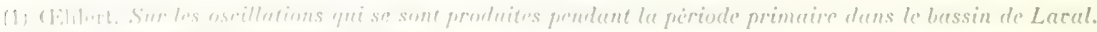

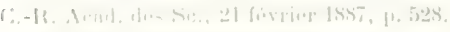

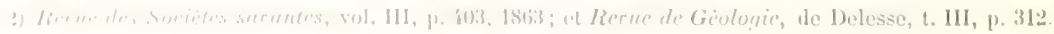


C'est du Visé. Fournet a publié une liste plus complète, à laquelle nous renvoyons le lecteur (1).

Mais des gisements nouveaux ont été découverts par M. Keller, ingénieur des mines, et, dans la suite, par MM. Bleicher et Mieg; tous ces gisements sont dans les environs d'Oberburbach, dans la Haute-Alsace. Ce sont des schistes jaunâtres passant en bas à une grauwacke métamorphique grise, riches en fossiles. Cet ensemble est recouvert do grauwackes détritiques, d'argilophyres, enfin de porphyres.

Nous réunissons en une seule liste tous les fossiles publiés par MM. Bleicher et Mieg (2) et déterminés par M. de Koninck:

Phillipsia Eichwaldi, Fisch.

Cythere (Cypridina) inornata? M'Coy.

Goniatites sphcericus, Mart.

Nautilus sulcatus, Sow.

Straparollus Dionysii, Montf.

Tychonia Omaliana, de Kon.

Macrochilina Newberryi? Stevens.

- vois. de ventricosta, de Kon.

- vois. de monodontiformis, de Kon.

Worthenia vois. de Waageni, de Kon.

Platyschisma glabrata, de Kon.

Euomphalus pentagonalis, Sow.

Bucania textilis, de Kon.

Ptychomphalus sulcifer, de Kon.

$$
\text { - } \quad \text { vois. de variatus, de Kon. }
$$

Raphistoma junior, de Kon.

$$
\text { vois. de glans, de Kon. }
$$

Naticopsis elegans, de Kon.

$$
\text { - planispira, Phill. }
$$

Murchisonia amona, de Kon.

Baylea spirata, de Kon.

Phanerotinus nudus, Sow.

Loxonema, vois. de priscum? Goldt.

Entalis ingens? de Kon.

$$
\text { - acumen? de kion. }
$$

Chonetes papilionacer, Phill.

- tuberculata? M' Coy.

- vois. de Dalmaniana, de Kon.

(1) Fournet. Etudes sur l'extension des torecins houillers on Frunere, p. 114. 185.5.

(2) C. R. Acad. des Se., 13 févrin 1882, 26 juin 1882, 28 novenbre 1857; (4. B. S. G. F., 3e súrie, 1. X. p. 50'; t. XII, P. 107; t. XIII, 1. 113. 
Productus giganteus, Mart.

- $\quad$ - var. hemisphericus.

- $\operatorname{cor} a$, d'Orb.

- fimbriatus, Sow.

- undatus, Defi.

- scabriculus, Sow.

- semireticulatus, Mart., var. Martini.

- vois. de rugatus, Phill.

Atrypa, sp.

Orthotetes crenistria, Phill.

Orthis resupinate, Nart.

Spirifer duplicicosta, Phill.

- bisulcatus, Sow., passage au trigonalis.

- lineatus, Miart.

- glaber, Nart.

- sp.?

Rhynchonella pugnus, Mart.

Aciculopecten semicircularis, M'Coy.

$\begin{array}{ll}\text { - } & \text { vois. de dissimilis, M'Coy. } \\ \text { - } & \text { Soucrbyi, M'Coy. } \\ \text { - } & \text { tumidus? de Kon. } \\ \text { - } & \text { vois. de spinulosus, M'Coy. } \\ \text { - } & \text { Knockoniensis? M'Coy. } \\ \text { - } & \text { rugulosus, M'Coy. } \\ \text { - } & \text { nov. sp. }\end{array}$

Schizodus nuculoides? de Kon.

Palcearca squamosa? de Kon.

- vois. de costellata, M'Coy.

Cardiomorpha, 11. sp). suleate, de Kon.

Tellinomya, 11. sp.

Edmondia, n. sp).

Mytilus ou Modiola, vois. de M. ungaloba, M'Coy.

Monticulipore tumida, de Kon.

M. Bleicher a en outre déterminé les espèces suivantes:

I'pymatifer purgilis, Phill.

Loxumeme pulcherrimum, M'Coy.

Murchisonire neren, de Kism.

Nulicopsis elegrans, de Kon.

Turbinilopsis Hoeningheresirmus, de Kon.

11. sp. 
Capulus Ehlerti, de Kon.

Entalis cyrtoceratoides, de Ron.

Rhynchonella pleurodon, Phill.

Spiriferina insculpta, Phill.

Palcechinus ellipticus, M'Coy.

Cidaris, sp.?

Pecten variabilis, M'Coy.

Aviculopecten, n. sp.

Conocardium alceforme, Sow. armatum, Phill.

Isocardia (Edmondia) unioniformis? de Kon.

Nucula, vois. de Palmee, Sow.

Aviculopecten variabilis, M'Coy.

\section{- lunulatus? de Kon.}

- hemispharicus? de Kon.

et genres Palcarca, Pterinea, Cardiomorpha, Mytilus; enerines, radioles d'Archaocidaris; Foraminifères (Endothyra).

M. Meyer a signalé dans les mêmes gisements:

Productus cora, d'Orb.

Chonetes papilionacea, Phill.

Spirifer ovalis, Sow.

- bisulcatus, Sow.

- cf. laminosus? M'Coy.

Conocardium alceforme, Sow.

Cf. Solen siliquoides, de Kon.

Toutes ces espèces sont viséennes.

P.RTIE SEPTENTRIONALE.

M. Vélain a étudié le terrain carbonifère dans les Vosges septentrionales (1). Cette formation s'étend, dit-il, au pied du Donon, dans lir partie septentrionale des Hautes-Vosges, sur les deux versants de la Bruche.

Elle est formée de puissants massifs calcaires, le plus souvent marmenréens et exploités comme tels dans les vallons latéraux qui, de part et d'aulre, se rendent à la valkée principale. Ce sont d'abord, smo ho the droit, coux de Schirmeck, de Wackenbach, de Framont et de la Cracho; puis, sur lo vorsint opposé, celui de Russ; enfin, plus au sud, celui moins important de Rothau.

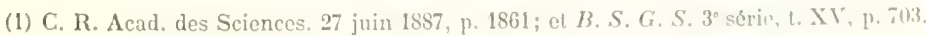


Voici les espèces trouvées à Schirmeck par M. Vélain, et déterminées par M. Ehlert:

Productus cora, d'Orb.

Spirifer lineatus, Mart.

- bisulcatus, Sow.

- Cheiropterix, d'Arch. et de Vern.

Dielasma hastata, Sow.

Schizophoria resupinata, Mart.

Rhynchonella cuboides, Sow.

Acroculia Ehlerti, de Kon.

Turbonellina lepida, de Kon.

Naticopsis elegans? de Kon.

Straparollus planorbiformis, de Kon.

Toutes ces espèces, sauf Straparollus planorbiformis qui est waulsortien, apparticment ì l'étage de Visé. Nous ferons toutefois exception pour Rhynchonella cuboides, fossile caractéristique du Frasnien, et dont la mention ne doit pas être due à une erreur de détermination, mais sans doute à un accident qui a mêlé cette Rhynchonelle dévonienne à un lot de fossiles carbonifères.

L'invasion de la mer, grâce à la présence du Straparollıs, remonte donc à la même époque que dans la France centrale et la Bretagne, peut-ĉtre, au pis aller, un peu plus tard, vers la fin du chanxhien!

Nous n'acepterons pas cependant les conclusions par lesquelles notre excellent ami, M. Vélain, termine sa note; à savoir que le golfe vosgien de Schirmeck, après avoir côtoyé la bordure orientale du Morvan et celle du Plateau Central, devait venir se relier avee la mer largement ouverte qui occupait alors les régions méditeranéennes. Car le Morvan, primitivement recouvert par la mor de 'Tournai, était exondé à l'époque de Visé ainsi que la moitié méridionale du Plitcau Central, it purtir du parallele de Clermont-Ferrand. Cette partie, réunic aux $A$ peses, devait former un barrage transversal.

La communication entre lit mel carbonifère du Nord et celle de la Montagne Noire devait in notre avis s'effectuer de préférence par l'ouest. Le carhonifère marin axiste en Vendée, mais il n’a eneores été l'objet d'ancune étude. 


\section{MONTAGNe Nolre.}

C'est Marcel de Serres qui a découvert pour la première fois des Productus dans le midi de la France (1).

En 1840, Graft' et Fournet(2) étudièrent les environs de Neffiez et recueillirent un certain nombre de fossiles qui furent déterminés par du Vernenil. En voici la liste :

Productess giganteus.

- Edelburgensis.

- latissimus.

-

- semireticulatus.

Spirifer integricoster.

- lineatus.

Euomplealus acutus.

Caninia aft. gigantea.

Lithostrotion floriforme.

Lithodendron fusciculutum.

Bellerophon hiuleus.

Ces dépôts ont été étudiés depuis par MI. de Rouville, Frech el Bergeron. M. Bergeron (3) a recueilli dans les nombreux îlots de calcaire à Productus situés entre Neffiez et Cabrières, figurés du reste dans la carte géologique du département de l'Hérault de notre vénéré collègue, M. de Rouville, les espèces suivantes:

Phillipsia gemmulifere, Phill.

- aff. Eichualdi, lisch.

Loxonema rugiferum, Phill.

Scalites aff. curbonarius, de Kion.

Bellerophon att'. Mënsteri, d'Orb).

Murehisonia nana, de kion.

Prorluctus giganteres, Nirrt.

- striatus, Fisceh.

- corll, d'Orh).

- semireticulutus, Nint.

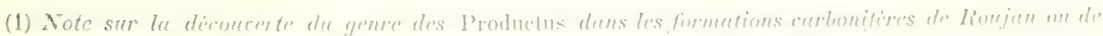

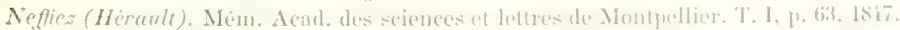

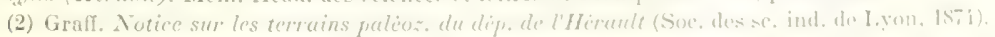

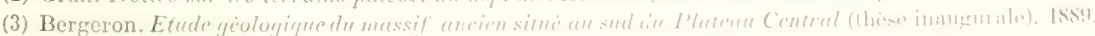


Spirifer bisulcatus, Sow.

- glaber, Mart.

Rhynchonella angulata, Linné.

Terebratula reftexa, de Kon.

Et les genres:

Euomphalus.

Naticopsis.

Straparollus.

Turbonitella.

Macrochilina.

Murchisonia.

Conocardium.

Edmondia.

Aviculopecten.

Cyathophyllum.

Amplexus.

Favosites.

M. Freeh avait signalé, en 1887, d'assez nombreux fossiles (1).

Phillipsia gemmulifera, Phill.

Loxonema rugiferum, Phill.

- fecundum, de Kon.

Straparollus Dionysii, Montf.

Phymatifer pugilis, Phill.

Euomphalus catillus, Mart.

- crotalostoma, M'Coy.

- letus, de Kon.

Murchisonia nana, de Kon.

Rhynchonella cordiformis, Sow.

Sprivifer bisuleatus, Sow.

$$
\text { angulate, Limné. }
$$

- planicosta, Phill.

Derlyere senilis, l'hill.

Orthis restupinute, Mart.

Prorluctus giganteus, Mart.

striatus, risch.

Nucnelanu attenuala, Finom.

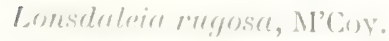

Lithesteretion ireregulare, Phill.

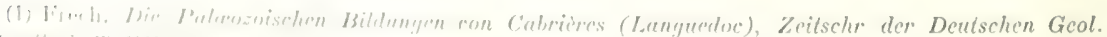

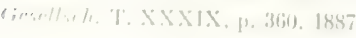


L'examen de ces listes nous permet d'étendre les résultats déjà acquis dans la France septentrionale et centrale à la Montagne Noire. Sauf deux espèces waulsortiennes, Rhynchonella cordiformis et Bucania exilis, toutes les autres appartiennent à l'étage de Visé; nous y constatons même les espèces les plus caractéristiques des deux assises de Dinant V1 et de Visé V2, confondues ensemble dans le mème gisement; ainsi nous voyons Euomphalus crotalostomus si caractéristique de Dinant V1, associé à de gros Productus giganteus, ì Pr. striatus et latissimus de Visé V2, ce qui nous démontre une fois de plus l'équivalence latérale de l'Ardoisière et de Régny. Rappelons encore ici l'association à Régneville (Manche) des gros Productus giganteus et du Chonetes comoides.

C'est à l'époque chanxhienne que la mer carbonifère a envahi le midi de la France.

\section{PyréNÉES.}

La faune des assises carbonifères pyrénéennes est paurre, ou plus exactement, à coup sûr, les auteur's de sa découverte n'ont, par suite de leurs trouvailles restreintes, signalé qu'un très petit nombre de genres ou d'espèces toutes viséennes. En première ligne, nous citerons notre excellent collègue et ami, M. Louis Lartet, qui a fait connaître le premier ce grand horizon (1).

M. Lartet signale dans les schistes de Larbont, dont l'aspect rappelle de très près celui des calschistes de l'Ardoisière, Productus giganteus, P. latissimus, $P$. striatus, etc., des Trilobites du genre Phillipsia, des Spirifers et d'autres Brachiopodes, des Bryozoaires, des Polypiers et de nombreux crinoïdes. Dans les vallées voisines, les Bivalves et les Gastéropodes paraissent l'emporter sur les Brachiopodes, les Productus y étant même parfois assez rares. La publication de cette faune serait évidemment du plus haut intérêt, à cause de sa ressemblance avec celle de l'Ardoisière. Il nous semble qu'elle apporterait encoro une preuve de plus du synchronisme des deux assises de Dinant et de V'isć que nous considérons résolument comme deux faciès latéraux.

II. Joseph Roussel (2) a publié, de ce mème gisement de Larbont, les fossiles suivants :

Productus giganteus.

- semireticulatus.

- $\operatorname{cor} a$.

- latissimus.

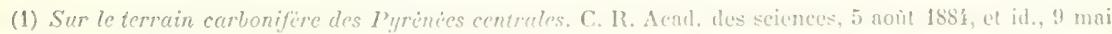
1887.

(2) Roussel. Eitude stratigraphique des I'yrences (thise), 1893, p, 280. 
Cette formation serait, d'après ce savant, représentée dans toute l'étendue de là chaîne,

Le carbonifère a été également signalé dans la haute vallée d'Ossau, entre Laruns et Gère-Bélesten, par M. Seunes ${ }^{(1)}$; la scule espice déterminée, Goniatites crenistria est de l'étage de Visé.

\section{Résumé général.}

Cette revue rapide, mais dans laquelle cependant nous nous sommes efforcé de n'oublier aucun des fossiles signalés, nous montre les progrès accomplis dans la connaissance du terrain carbonifère depuis 18:0, époque à laquelle de Yerneuil admettait qu'en France on n'a de calcaire carbonifère bien caractérisé que sur les frontières de la Belgique, à Sablé et à Régny. Nous voyons au contraire que la mer carbonifère a recouvert pour ainsi dire toute la France; mais, sauf le Morvan, tous les gisements connus jusqu'à ce jour n’offrent que des fossiles de l'étage de Visé avec quelques rares fossiles tournaisiens mélangés vers la base. L'identité de toutes les faunules découvertes et que nous avons réunies aussi complètement qu'il nous a été possible de le faire, nous démontre leur synchronisme parfait sur tout le territoire, toujours le Morvan excepté. Ainsi Régneville, Plouyé, Quénon, Changé, Sablé, Schirmeck, Plancher-lesMincs, Thann, Burbach, Neffiez, Larbont, etc., sont synchroniques de Régny, Néronde, Montmain, Saint-Germain-Laval, Propières, Ferrières, l'Ardoisière, dans la france centrale.

Le carbonifère ne présente donc que deux étages en France, l'étage tournaisien dans le Morvan, l'étage viséen dans le reste du territoire. A la base de ce dernier étige sont des dépôts d'aspect varié, représentant les assises de Chanxhe ou assisces de mélange, à faciès continental dans le Morvan, plus spécialement stromatoporicue dans le Plateau Central et le Cotentin, à faciès de culm ou prufois sub-pélagique en Bretagne, dans les Vosges et la Montagne Noire.

Il y avait, (on le voit, un puissant intérêt à comparer nos gisements du Platean Central aver les lambeaux carbonifères découverts depuis 1840 sur tout lo territuine françis, car los observations auxquelles ils ont donné lieu se trouvent rlispersées dans des publications variées et n'ont jamais été l'objet l'une abule d'onsemble. On ne pouvait done tirer de ees etudes partielles et Fints lim chutrelles ancune déduction générale.

II n'mulpe pis dans notre plan de fane le même travail pour le carbonifère,

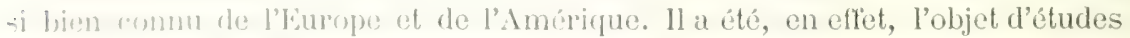

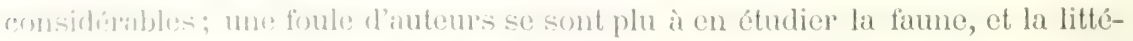
mature cartunifino ost une des plus riches qui existent. En Angleterre, David 
Ure dis 1793, William Martin, anteur du Petrefacta Derbyensia, J. Soworby et J. de C. Sowerby, son fils, ainsi que le duetem likming, ont decrit les premières especes connues et en ont figuré $n$ eertain nombro. J. Phillips, en 1836, fit commatre dans son célibre ouvrage Geology of Yorkshire, qu'il faut tonjour's consulter, 96 formes de Brachiopodes carboniferes, dont 63 nouvelles. Les deux années 1843 et 1814 virent paraitte presque simultanément quatre acures considérables, savoir: $1^{\circ}$ Geological Report on Londonderry and parts of Tyrone and Fermanagh, du colonel Portlock (1843); 2 Synopsis of the curboniferous limestone fossils of Irelend, de M'Coy (184t), où l'auteur irlandais décrit la fame la plus riche et la plus variéc de l'Europe; auvie précoce pent-ĉtre, mais dont les défaillances ninsi que le. constate Davidson lui-mêne, ont été largement réparés dans le savint ef précicux ourrage du même auteur: On the British Puleosic fossils in the Geologicul Wuseum of the University of Cambridge (1855); 3 Description des fossiles carboniferes de la Belgique, de L.-G. de Koninck (1813), œuvre non moins capitale, qui dès son apparition eut un grand retentissement et dissipa le chaos dans lequel était encore plongée la paléontologie du carboniềe; 4 onfin le bel et fondamental ouviage de de Vermenil, Puléontologie de lu Russie (1815), dont la partic qui a trait à la période carbonitère fit aceomplir de nomveaux progrès à la connaissance de la faune qui nous oceupe. Plus recemment, Thomat Davidson a porté juscu'a la prerfection la comnatssance de la partie de la fanne carbonifire des Iles Britamiques qui a trait anx Brachiopodes.

En Belgique, de Koninck, dont nous avons cité rquelques lignes plus haut l'ourre magistrale, continua it faire paraitre une foule d'études relatives it lir finune carbonifère et entreprit clans les Annales du Musée roygl d'histoire natu-

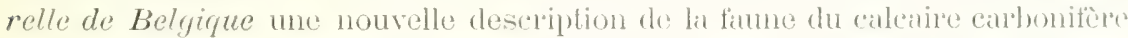
de la Belgique. Le chiffe énorme de 1,573 espèces fut dérit par cet illustre paléontologiste avee la collaboration de M. Julien Fraipont pour les Lancllibranches ef réparti dans les trois itages de Tounnai, de Wanlsort ef de Visí, établis par M. Edouard Dupont. Lit mont soule est cortellement vonue interrompre cette ouvre monumentale. Nous citerons figalenent le cont mémone du Dr Léveillé, qui a trait à des fossiles elassiques de Tommai of les tratraux du baron de Ryckholt qui ne sont point sams merite. Ljontons que do nos jomers, le carbonifère marin est étudié avee noe ardeme nouvelle par me pléinde de

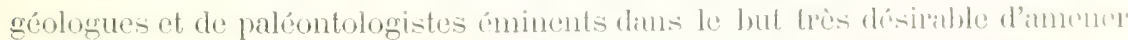

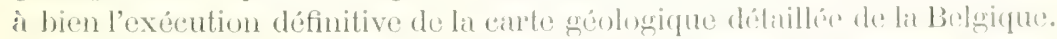

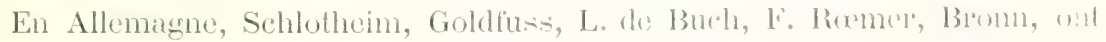

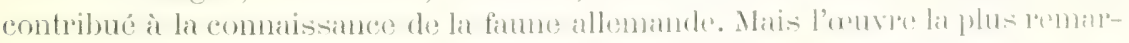

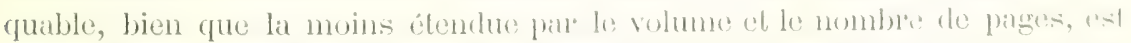
colle de Von Sunenow "Ueber die Fossilien des Schesischen Köhlentadtiess" (1854).

En Russie, do 1830 i 1837 , lischer von Waldhein publia l'Oryctogrephice du

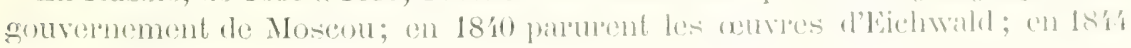


celles de Fahrenkohl; l'année suivante, le grand ouvrage de Murchison, de Verneuil et de Keyserling. Ce dernier décrivit également le carbonifère de la Petchora, en 1846. Mentionmons aussi les ouvres de MM. Trautschold (1876), Inostranzeff, etc.

Enfin I'Espagne elle-même a été l'objet de travaux de la part de Paillette et de Verneuil, et, il y a un petit nombre d'années, de notre savant collègue II. Charles Barrois.

Si nous quittons l'Europe pour jeter un coup d'oeil sur les travaux relatifs au Nouveau Monde, nous derrons citer en première ligne le Voyage dans l'Amérique méridionale, d'Alcide d'Orbigny (1842), et les grandes publications de Shumard, J. Hall, Meek et Worthen, ete.

On voit par cette énumération sommaire à quelles publications nombreuses ut considérables a donné lieu à l'étranger l'étude de la faune carbonifère mal’ine, alors qu’en France, pendant toute cette longue période, cette faune si intéressante restait dans un oubli complet.

Nous constatons ainsi une lacune des plus regrettables dans la littérature paléontologique de notre pays, mais pour cette partic de l'histoire de la terre sculement, car les oeurres de Burrande, de d'Archiae, de Deshayes, d'Alc. d'Orbigny publićes ì la même date, constituent une ouvre grandiose digne du pays de Lamarek, de Curier et d'Ad. Brongniart, les créateurs illustres de la palćontologie animale et végétale.

Or, toutes ces recherehes relatives à la faune carlonifère de l'Europe péla qrique, coralligène ou ì faciòs de culm, montrent qu'elle appartient intégralement à l'étage de Visć. Toumai n'est représenté d'une manière certaine, comme nous l'avons vu, que dans quatre régions, la Belgique et le nord de la Franee, la comté de Wrexford en Irlande, Malowka-Murajewna en Russie, et le Morvan (m) Finee.

Tout est donc viséen an linrope; toutes los fumules viement se grouper antrun rles grand- gisements que nous ont fait comnaitre dans chaque pays les antoursénumérés plus haut, tels que Bolland et Settle, Visé, Hausdorf, Ratin-

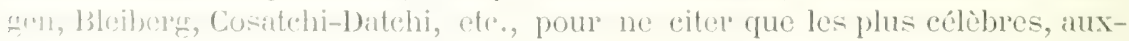

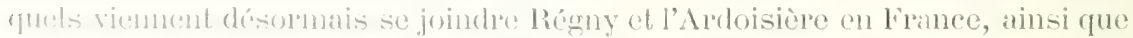
lins sations de moindere importance gui an forment lo coltigne. 


\title{
TRANSGRESSION DE LA MER GATBBONIFERE
}

\author{
DANS LE MORVAN ET LE PLATEAU CENTRAL, EN FRANCE ET EN EUROPE.
}

Nous avons mentioné snecessirement les faits qui démontrent l'invasion de la mer carbonifère, en notant arec précision la date de ce phénomène en chaque point soumis à l'analyse. Tous ces faits de détail, s'ils restaient isolés dans leur dissémination, perdraient une grande partie de leur signification et de leur valeur. Il est done indispensable de les réunir et de les synthéticer, afin de bien mettre en lumiere le tableau d'ensenble et les particularites de cette rapide et générale invasion de la mer carbonifère, l'un des plus remarquables phénomènes de l'histoire géologique de notre pays. Ce grand événement acquerrait en outre un relief bien ylus net s'il était exposé ì sa raie place, à sa date chronologique, et encadré dans me vue, même suceinete, des faits qui l'ont précédé ou suivi. Enfin ajoutons ceci, que si les merveilleux progrès de la géologie, dans ce dernier quart de sicele, ont mis en Iumière d'autres grandes invasions marines, d'autres rastes déplacements des mer's, par exemple aux époques callovieme et cénomanienne, il est un de ces cnvahissements, celui de la mer helvétienne qui, dans sa manière d'ètre au début, comme dans son évolution générale, comme dans le tormidable cortège des manifestations orogéniques et éruptires qui l'ont accompagné, évorque irrésistiblement à l'esprit le tableau non moins extraordinaire de lil transigression carbonifere. Nous croyons done bien faire, et ne pras sortir du calle do eet ouvrage, en consacrant un derniel ehapitre ì un rapide aperegu des divers points de vue que nous renons de signaler.

TERRAINS PRIMITIFS ET PRIMUIRES DE LA FRINCL CKNTRMLL.

Terrains primitifs. - Les terrains primitifs de la funce contrate sont repré-

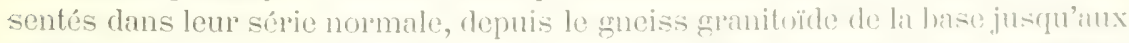

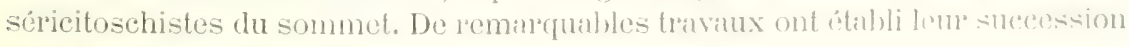
sur divers points (1).

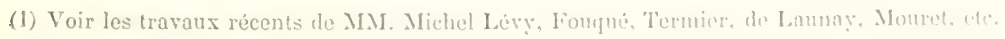


Terrains primaires. - Des lacunes considérables se manifestent, au contraire, dans l'ensemble des terains primaires. Les assises cambriemnes, bien qu'apparaissant disloquées de touts part, couvrent une vaste étendue.

C'est l'étage le plus important du Plateau Central, après le carbonifère marin, ef e'est pourtant le moins connu. Aucun fossile, sauf des traces peu ou point déterminables, n’y a été décourert jusqu'à ce jour. Formé d'une suceession de roches direrses, tantôt prises pour du terrain primitif, tantôt confondues avec le dévonien on le carbonifère, il mérite une étude spéciale qui permettrait d'en relier les lambenux disséminés dans le Puy-de-Dôme, l'Allier, la Loire, la Nièrre, l'Yonne, la Côte-d'Or', lin Saône-ct-Loire et le Rhône; d'y établir la nature et l'ordre de suecession des assises qui le composent, et très probablement d'y découvrir des fossiles. C'est, at mon avis, le grand desideratum de la géologie du Platean Central.

Jusqu'ici, le Silurien tout entier, ainsi que les étages inférieur et moyen du Déronien, font défaut. Il ne pariût pas, jusqu’à présent du moins, et sous récerve des décourertes fulures, que la mer, à ees deux grandes époques, ait pénétré dans la france centrale.

Cutte région s'est exhaussée ver's la fin de la période cambrienne, période marine qui parait aroir légné sur une grande étendue de l'emplacement actuellement wecupé pirr le Morvan et le Platenu Central. Toutes les formations de eet âge que l'on ý rencontre (Silurien et Dévonien) sont d'origine éruptive. C'est la grande période d'émission des roehes granitiques. Ces roches ont disloqué ou traversé le terrain primitif ot le cambricn, ef en ont emballé d'innombrables blocs, parfois même des ilots de plusieurs kilomètres d'étendue, témoin les lambeaux du be l'Avelat et de Nébouzat, dans la région sud des volcans ì cratères du p’uý-rle-Dome. Mais leur venue paraît bien antérieure à l'époque frasnienne.

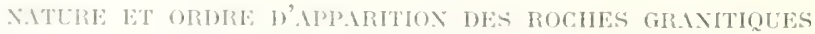
D.INS LAE PLATLIL CENTHAL.

I a plus ancionne est le Granile porphyroüde at gros cristaux d'orthose.

polis vioment suecessirement:

1) Ciranite it grans movens;

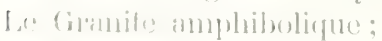

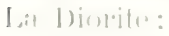

in cilnmulitu:

Lil Pentmalite.

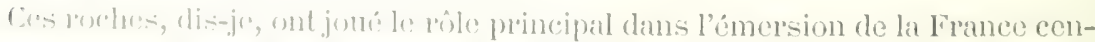

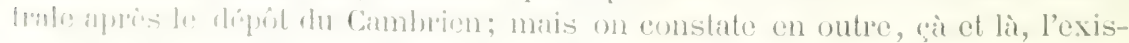

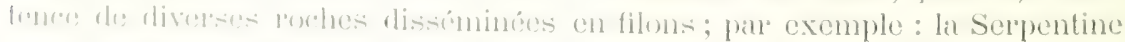

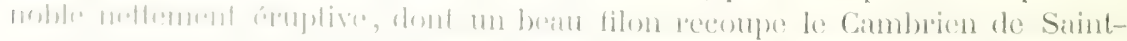

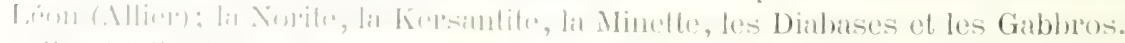

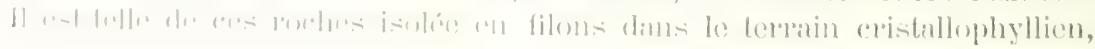


dont il est impossible de fixer l'âge relatif ou absolu. Nais, pour celles de la première catégorie, allant du granite porphyrö̈le à la pegmatite, les faits sont assez multipliés et assez coneordants pour justifier l'orde de suecession que nous arons étubli plus haut. Ainsi, le granite porphrroütle, qui forme le soubassement du plateau supportant les voleans ì cratère à l'ouest de Clermont, est traversé sur nombre de points par des filons de granite à grains moyens ou par des épanchements de granite amphibolique. Le granite amphibolique est également traversé par des filons de diorite, dans les environs du lac d'Aydat par exemple.

La granulite traverse tontes les roches préédentes et purticulierement des filons de diorite à Recolène, près de Nébonzat. Enfin, la pegmatite à grands éléments traverse toujour's les filons de grmulite, lorsque ces deux roches sont en contact, par exemple, entre Thedde? et Saint-Genes-Champanelle, près Clermont. Partout, en dehor's du Puy-de-Dône et de l'Allier, oì nous avons pu constater les relations de ces grandes roches éruptives, nous avons toujours vérifié le mêne ordre d'apparition. La sortie de ces roches paraît s'être accomplie en deux grandes périodes : la plus ancienne comprenant les granites et les roches amphiboliques; la plus réeente, les granulites et les pegmatites. Toutefois, l'absence du Silurien et du Dévonien inférieur et moven ne permet pas de séparel ces deux phuses, comme cela a été fait dans d'autres massifs montagneux. Tout ce que nous savons, éest que la première a cloturé la période cambrienne, dont elle a partout brisó et emballé les assises, et que lir seconde était terminée à l'époque carbonifère, voire même frasnienn.

Le carratère des éruptions de cette époque est très net. Le granite se distingue par des épanchements gigantesques. Il a donné naissance à d'énormes massifs démantelés et arrondis par l'érosion. Les filons ne font cependant pari défuut; on les observe sur diver's points. Les granulites et les pegmatites forment, au contraire, de plus rares massifs; mais leurs filons sont innombrables. Le Plateau Central s'est fendu comme une glace à leur sortir, et c'est par les milliers de fractures de grande longueur qui l’ont sillonné que se sont injectées ces roches plus récentes. Il serait bien désirab)le de déconvirir quelques fossiles siluriens dans le Plateau Central, au moins dans la partie nord, pour dater ces grandes éruptions et séparer les deux groupes éruptit's.

Frasnien. - C'est à l'époque frasnienne que la mer revient dans le Platean Central, comme le démontre lo beau récif coralligine qui est coupé par la Loire a Diou (Allier). Ce lambeau de terrain devonien, formé de poudingues quantzenx it la base, aree Cyathophyllum cespitostum, tiges l'cuerines et Spirifer's eneope: indéterminables, de schistes de couleur vert sombre ou brun rougeâtre, de grés, de banes de dolomie, enfun de matbre construt identique à celui de lírisne,

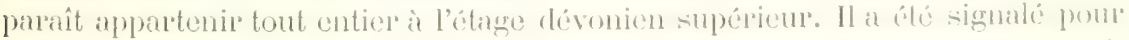

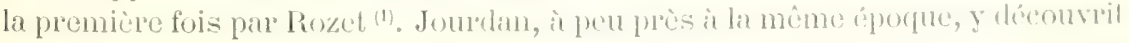

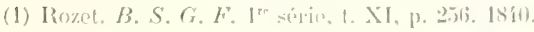


quelques fossiles, parmi lesquels Atrypa reticularis, espèce sans valeur stratigraphique, en raison de son extrême longévité, qui lui a permis de traverser les époques silurienne et dévonienne, et que l'on recueille par centaines à Diou. Ces fossiles sont conservés au Musée Saint-Pierre, à Lyon, où j’ai pu les examiner il y a plus de quinze ans, grâce à l'obligeance de M. le docteur Lortet. Les spécimens sont étiquetés de la main même de Jourdan et rapportés it l'époque carbonifère.

Moi-même, à plusicurs reprises, j’ai recueilli dans le marbre, près d'un millier dé fossiles, dont je publierai la liste complète quand l'étude en scra acherée. Dans une communication faite au Congrès de Clermont en $1876^{(1)}$, et dans une note à l'Académie des Scienees ${ }^{(2)}$, j'attribuais le récif de Diou au déronien moyen. Plus récemment, je suis parrenu à y découvrir Spirifer Verneuili, Rhynchonella cuboides, Strophalosia productoides, Productus subaculeatus, etc., qui me permettent de fixer définitivement l'âge des marbres de Diou et de Gilly sur les deux rives de la Loire, à l'époque frasnienne inférieure. Ce massif isolé est entouré de toute part par le Cambrien.

Cette invasion de la mer frasniemne, au nord du Plateau Central, a coinncidé avec la submersion d'une partie de la France, comme en témoignent la découverte de Rhynchonella cuboides à Cop-Choux ${ }^{(3)}$ et la présence d'une faune dévonienne supérieure à l'extrémité méridionale des Vosges, entre Chagey et Chéncbié (Haute-Saône) (i).

Frasnien supérieur et Famennien. - Le Frasnien supérieur à Cardium polmatum et le Famennien font défaut.

TR.MNGRESSION DE LA MER CARBONIFERE.

Nons arivons cufin à la grande transgression de la mer carbonifère dans le contre de lit France, ì laquelle l'arrivée momentanée de la mer frasnienne avait servi de prélude. Dans la région qui nous occupe, le terrain tournaisien est le premier que l'on l'encontre. Le Morvan s'affaisse tout entier. La mer carlonifète vente du nord dépose des sédiments identiques aux ealsehistes

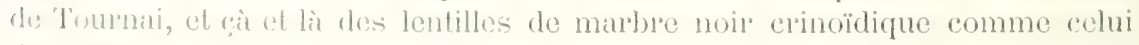
de Cirs.y. Le Platean Central reste émergé; la séparation des deux régions enïncide concore de nos jours avee la dépression qui les separe, et que l'on antilisés pour l'établisscment du canal du Centre.

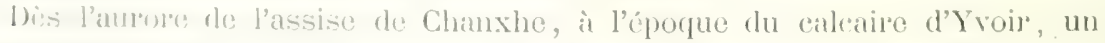
menvement de bascule sacemplit. Le Morvan se souleve; une formation

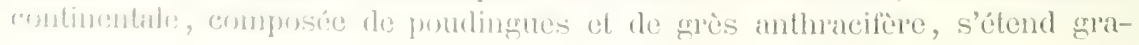

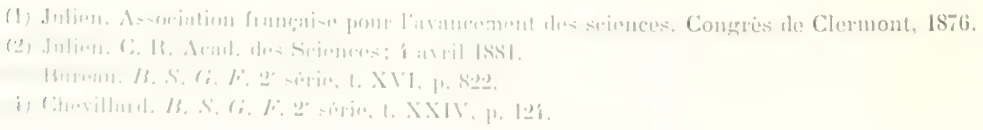


duellement à la surface des calsehistes marins émergés qu’elle recourre entièrement. Le Plateau Central, situó au sud de l'axe Digoin-Chagny, s'affaisse it son tour, el la mer de Chanxhe-Visé le recourre jusqu'au parallèle de Clermont-Ferrand. Les rivages de cette mer dessinent une grande courbe it concavité tournée vers le nord, courbe julonnée sur son parcour's par Evaux (Creuse), Ferrières (Allier), St-Germain-Laral et Néronde (Loire), et le Gougret (Rhône). Des récifs d'hydrozoaires identiques aux récifs belges du même âge, tels que les Pauquys, Waulsort, Anseremme, sétablissent çà et lì. La Creusce se relève apres l'édification du récif du Chat-Cros, et se conve it son tour d'un voile superficiel de poudingues et de grès. Nais li mer persiste partout ailleurs et se maintient jusqu'i la fỉn de la période carbonifère marine. Ce n'est qu'après le développement des helles faunes de l'Al'doisière et de Régny que le Plateau Central s'exhausse, s'émerge et que l'érosion étale ì sa surface la puissante formation du grès anthracifère du Beaujolais, du Roannais et du Boulbonnais.

Cette transgression de la mer se révele avee une netteté saisissante quand on examine le substratum qu'elle a recouvert de ses dópôts. Dans la Nièvre, les calschistes s'appuient sur les quartzites et les schistes cambriens. Dans la Cieuse, à Evaux, le marble waulsortien et les schistes qui l'encaissent sur les rives du Chat-Cros, reposent directement sur les stérschistes du terrain primitif. Dans le Beaujolais, dans le Rhône et dans liı Loire, le substratum est formé par les schistes, micacés, chloriteux ot amphiboliques; enfin, dans l'Allier', par les ardoises cambriennes de l'Ardoisière et de Frrrières.

Cette transgression a-t-elle dépassú de buancoup la limite des rivages gur? nous avons tracée plus haut? Cela nous parait peu probable, car nous retronverions certainement des roches carbonifères en grand nombre, dans les brèches et poudingues des bassins honillers de la france centrale, ce qui n'est pas. Il semble done que la partie septentrionale du Pluteau Central ait scule átí immergée.

L'affitissement du Plateau Central s'est propagé dans toute la France dès l'époque chanxhienne. Nulle part, en effet, comme nous l'avons vu plus haut, on n'a trouvé de traees de la faune pure et exclusivo de Tommai, sans ancun mélange de fossiles viséens. La faune chanxhienne apparait pantout ì la base. Partout, en même temps, on constate le maintien de lin mer jusc(u'it la fin du viséen inclusivement. La transgressivité des dépôts carbonifères il été sur tous les points signaléc par MM. Dalimier, Barrois, Lhtert, Bergeron, ete. Elle se montre partout aussi nette que dams nos légions.

Mais eette transglession ne s'est pas bornéc at la linance. Elle s'est effectuéc en même temps et à la même date dans l'burope cultiere, en Anghleterré, en Allemagne et en Russic. C'est lik l'un des plus beaux exemples d'invasion marine que nous offre l'histoire de la géologie. I a belgigne cllo-mêne n'y " pas échappé. Les depnières recherehes ont annené, comme nous l'avons déjai vu, la découverte de l'Etage tournaisien dans les environs de IIug, de lelle sorte 
que la mer' au début recourrait sans discontinuité tout le pays belge compris entre Tournai à l'ouest et Huy à l'est. Mais à Boulogne et à Visé, le Viscen seul existe, et il lopose transglessivement sur le Díronien.

La transgression se manifeste donc iei par l'extension de la mer à gauche, of ì droite du golfe touranisien jusqu'à ces localités. Notre opinion diffère, comme on le voit, de celle récemment exposéc par M. l'abbé Bourgeat au Congrès de l'Association française de Caen (1894). Il nous est impossible, en nous basint sur la connaissance du Carbonifère marin de la France centrale, de considérer le Tournaisien et le Viséen comme des formations sỵnchroniques et équivalontes. Ces deux étages soat réllement distincts. Ils se sont suceédé dans le temps. Yous sommes pleinement d'aceord arec les géologues belges sur ce point. Mais oì nous nous éloignons d'eux et où nous nous rapprochons partiellement de l'idée émise par le sarant professenr de l'Institut eatholique de Lille, e'est en domant notre pleine et entière adhésion au synchronisme du Carbonifère marin du Boulonnais et du petit massif de Visé, ainsi que nous nvons été amené à le faire dans la France centrale pour l'Ardoisière et Régny, et notre opinion recoit un appui décisif de l'existence à Visé même de fossiles Eminemment chanxhiens on waulsortiens tels que Spirifer striatus et Euomphalus pentungrelutus.

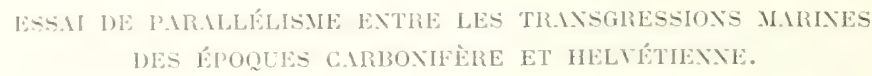

Le parallólisme que nous allons tenter d'établir entre ces deux grandes transiressions repose sur les quatre ordres de faits suivants :

1. Morle d'invarion de la mer':

20 Cause idenlicpue qui a amené son refoulement;

3.' Concomilance de l'autivité éruptive;

1" Appration de graciens dans les Apes hereyniennes et pliocènes.

Morstr d'incresion de le mer. - 1) même que la mer helvétienne n'a pas

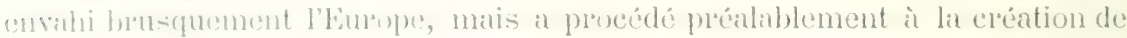

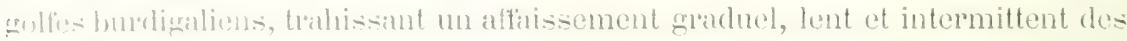

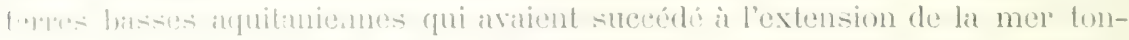

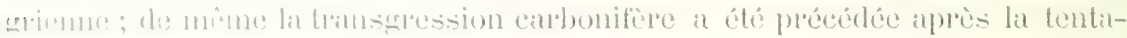

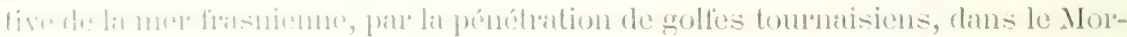

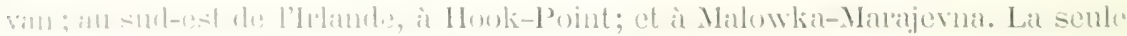

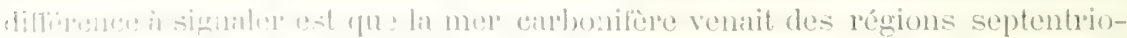

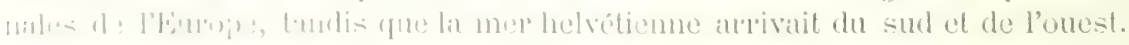

Comere due refoulement des mers. - Dans les deux cas, colte canse est due it

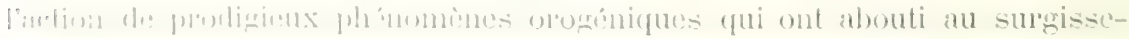

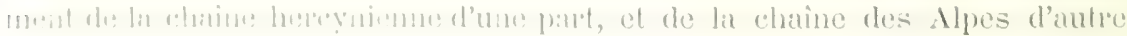


part. Nous nous bornons iei, bien entendu, à signaler les effets visibles de cette action, seuls accessibles à l'observation, sans en aborder la eause profonde, toujour's la même sans doute et toujour's agissante, mais cause aussi mystérieuse dans l'état actuel de la science que l'origine même de la vie et de son évolution à travers les îges.

Concomitance des phénomènes éruptifs. - Dans les deux cas encore, Ie paroxysme de l'activité éruptive s'est joint au paroxysme orogénique. Il a semé l'Europe à l'époque carbonifère de la nombreuse variété des Porphyres, avee émission de puissantes eaux thermales qui ont apporté de la profondeur tant de minerais variés; et à l'époque tertiaire, il a créé ces innombrables voleans aujourd'hui éteints, formés de l'accumulation des 'Trachytes, des Phonolites, des Andésites, des Domites et des Basaltes.

Apparition de glaciers. - Enfin, si des hautes altitudes atteintes par les Alpes pliocènes et les grands volcans éteints, n'ont pas tardé à rayonner d'immenses glacier's qui ont au loin déposé leur's moraines et créé par leur fusion la colossale formation des Alluvions anciennes avec enclaves morainiques ef bancs de lignite; en retour, des sommets de la chaîne hereynienne et spécialement du chaînon alpestre du Plateau Central, de pareils glacier's se sont étalés jusqu'au pied et ont domé naissance au terrain houiller supérieur de cette région. Mais ces alluvions carbonifüres, à enclares de brèches glaciaires et de banes de houille sont aujourd'hui considérablement réduites par l'érosion. Ce ne sont plus que des lambeaux plissés, faillés, ou parfois engloutis sous le gneiss et le mieaschiste renversés sur eux, pendant que la chaîne aux sommets alpestres a disparu à son tour.

Je ne reproduirai pas ici les preuves de l'origine glaciaire des formidables brèches de nos lambeaux houillers supérieur's de la krance centrale. Je crois les avoir suffisamment exposées dans des publications antérieures (1). Mais on me permettra d’insister néanmoins sur la l’éelle existence, au début de la période houillère supérieure, des conditions nécessaires ì l'apparition et à l'extension de puissants glaciers.

\section{CONDITIONS NÉCESSAIRES A LA GRÉATHON IET AU DÉVELOPPEMENT DES GLACIERS EN GĹNERAL.}

Ces conditions sont au nombre de trois, savoir : $1^{\circ}$ Un climat chaud, écal ef très humide; 2 de vastes mers constituant l'appareil évaporateur nécessaire; $3^{\circ}$ de très hautes montagnes formant l'appareil condenseur et subsidiairement l'existenec dans les hautes altitudes des chaînes, de vastes hassins de réeep- 
tion et d'accumulation des neiges. Or, toutes ces conditions étaient largement réalisées au début de la période houillère supérieure.

Climat de l'époque houillère supéricure. - L'uniformité générale el l'extrême humidité du climat houiller nous sont révélées par la profusion inouïe des Fougères arborescentes, seuls végétaux anciens que nous pouvons comparer à In flore actuelle. Pour établir ce résultat aux yeux des géologues, nous croyons ne pouvoir mieux faire que de consulter le savant ouvrage de Grisebach, sur la végétation du globe. Voici ce que nous y lisons, par exemple, dans les chapitres consacrés à la flore de l'Inde orientale, de Java et de la Jamaïque:

"Les Fougères arborescentes exigent un climat possédant des pluies abon") dantes et une chaleur tropicale uniforme...... A partir des jungles de l'Hi" malaya indien, les Fougères arborescentes accompagnent les climats humides ) de l'Inde postérieure jusqu'à la zone équatoriale de l'archipel où elles aug" mentent en variétés. A Java, elles habitent, variant en espèces, les massifs "montagneux aussi hauts qu'ils sont boisés, c'est-à-dire jusqu'aux sommets 390-2,631 mètres). De même dans les Philippines, elles ne commencent à se " montrer qu'au delà du niveau de 325 mètres, notamment dans les jungles où "l'humidité atmosphérique est très considérable. Dans la majorité des cas, "leur trone svelte a peu de hauteur et n'atteint guère la couronne des essences " dicotylédonées par lesquelles il est ombragé. Les vaisseaux rayés propres "aux Fougères et qui remplissent leur corps ligneux contribuent, sans doute, ¿̀ la flexibilité élastique de ce dernier' qui est d'ailleurs assez solide. L'espèce la plus fréquente dans la région inférieure de Java, figurée par M. Iunghuhn (Alsophila contaminans), n'atteint que 3 à 5 mètres de hauteur, et sa rosette terminale, composće de pennes finement foliacées, s'étend en are doucement voûté pour former un dôme dont le diamètre égale la hauteur du trone. Il est toutefois remarquable que ce soit préeisément une des plus grandes Fougères arborescentes, mais à trone fort mince par rapport à une hauteur de 13 ì 16 mètres (Alsophila lanuginosa), qui se trouve positivement limitée à la région forestière supérieure de Java (2,271-2,923 mètres). Comme à cette allitude la température n'a plus qu'environ $10^{\circ}$ el qu'au-dessus de la région des nuages (2,136 mètres) l'humidité diminue également, nous bous trouvons ici devant une valeur-limite climatérique atteinte par les Fougères arborescontes, valeur qui, correspondant ì la présence de cette forme de plantes sous des latiludes plus devées de l’hémisphipe austral, doit être prise en ensideration quand il s'agrit d'applécion les conditions physiques qui ont dû

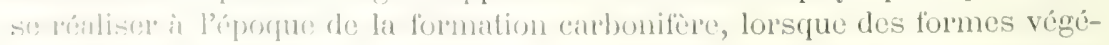
tabes d'uns organisalion analogne prédominaient sur le globe entier. La pré-

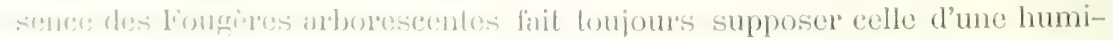

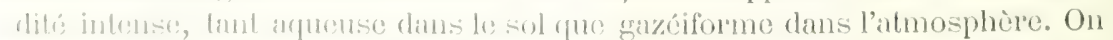

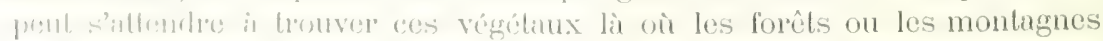

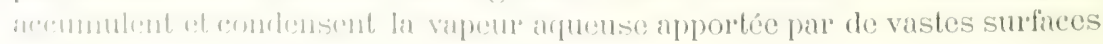


" maritimes et où domine cette température uniforme qui rend possible une "végétation non interrompue, tandis que les degrés de température qu'exige " cette végétation varient dans des limites plus étendues (1). )"

Et plus loin, à propos de la végétation de l'île de la Jamaïque : "Tandis que » la température baisse, l'humidité va toujour's en croissant en sens vertical, " jusqu'à l'altitude que la région des nuages atteint dans les montagnes Bleues » (1,527-2,143 mètres) où, pendant toute l'année, chaque jour les vapeurs "aqueuses se condensent après les heures de la matinée, et se précipitent en " pluie après midi. Alors ce ne sont que les sommets les plus élevés qu'on " voit pereer ectte couche de nuages dans le domaine desquels la température " descend déjà au-dessous de $15^{\circ}$, grâce aux obstacles qui s'opposent à l'inso") lation. Nous trouvons au même niveau où la culture du Cafier est encore ") pratiquée, une ceinture forestière séparée (1,218-1,818 mètres), consistant " presque exclusivement en Fougères arborescentes. On voit, à la vérité, des » individus isolés descendre plus bas dans la forêt ì essences angiospermes, " même sur les collines de la côte septentrionale; mais ce n'est qu'à ces alti" tudes qu'elles se réunissent en masses parfaitement délimitées, où les trones " les plus grands alteignent une hauteur de 16 à 20 mètres. M. Ersted fait " observer que, sur le globe entier peut-être, il n'est point d'endroit où les "Fougères arborescentes se présentent à l'état social comme ici, et où, en »refoulant tout le reste de la végétation, elles reproduisent pour ainsi dire le " tableau des époques anciennes du monde passé. Elles ne sont accompagnées " que par deux conifères (Juniperus barbadensis et Podocarpus coriaceus), par » quelques arbustes tels que des individus isolés d'Ericées, de Mélastomacées » et d'un Viburnum, ainsi que par un genre endémique voisin des Cornées "(Fadyenia). En outre, les Epiphytes ne font point défaut, et cependant, parmi " ceux-ci également prédominent les Fougères herbacées et les Lyeopodiacées. "Les orchidées ne sont représentées que par dos formes à petites fleur's (Le"panthes, Stelis) (z)."

Ces citations du célèbre ouvrage de l'ancien professeur de Göttingue, nous font exactement connaître les vraies conditions d'existence des Fougùres arborescentes dans le monde actuel. Elles mettent fin à la légende si populaire qui nous montre la forêt houillère s'étendant à perte de vue sur des terres basses, marécageuses, le plus souvent inondécs, sans montagnes à l'horizon.

L'uniformité et l'extrême humidité du climat brûlant de la période élant ainsi établies, il faut en rechercher la cause. Or', celle-ci ne peut résider comme à l'époque actuelle que dans l'existence de vastes mer's. Nais tout le monde sitit qu'il y a vingt ans à peine, on ignorait d'une manière absolue l'existence et par' suite l'emplacement de l'océan houiller.

(1) A. Grisebach. La eigétation du globe, etc. Traduction P. de Tchilatehef. T. II, 1. 20-22 (1)ommine indien des moussons). 1878.

(2) Id. Ibid. T. II, p. 513. (Indes occidentales, Jumaique). 1878. 
Océan houiller. - La découverte de la mer houillère dont les rivages sont encore loin d'être connus, est done une des plus récentes et des plus belles conquêtes de la seience moderne. Elle a été révélée en France en 1881 seulement par M. C. Grand'Eury dans une Note à l'Académie des seiences qui produisit à son apparition une réclle émotion dans le monde géologique ${ }^{(1)}$. L'année suivante, notre distingué collègue, M. Ch. Barrois faisait connaître les dépôts marins houillers d'Espagne dans son bel ouvrage sur les Asturics. Puis M. Gemmellaro annonçait bientôt la découverte dans la vallée du Sosio, près de Palerme, de dépôts pélagiques permiens à Fusulines et à Céphalopodes. II r'ésulte encore, tant de découvertes faites antérieurement à la Note de M. Grand'Eury et qui avaient passé inaperçues que d'autres faites postérieurement, que l'Océan houiller enveloppait l'Europe centrale et occiclentale transformée en région alpestre. Ainsi les dépôts houillers marins ont été découverts par Tictze en Carinthie, par Stache dans la Carniole et les Alpes méridionales, par' Teller' ì l'île de Chio, par Neumayr en Asie-Mineure. L'Ocćan houiller a encore été signalé au Caucase, en Perse, en Asie à Wladiwostock, dans les provinces de Shansi et de Yunnan en Chine, au Japon, à Sumatra et à Bornéo. Ajoutons à toutes ces domées la connaissance du Carbonifère russe portéc au Congrès de Paris en 1878 par M. de Moeller; puis la mention des lits marins à fossiles littoraux ou pélagiques interealés dans le houiller moyen de Belgique ef du nord de la France, d'Angleterre et de Westphalie, ce qui indique ì coup sûr l'existence de la mer dans les régions septentrionales voisines, et l'on admetlra sans peine, par la seule considération de cette vaste mer entoul'ant l'ile alpestre allant de la Bretagne à la Bohême, de la Belgique au Plateau Contral at ax Pyrénées, et sans faire appel à de vaines hypothèses, les sources véritables de eelte humidité extrême, de ces précipitations abondantes qui régnaicnt dans le climat chaud et uniforme de l'époque houillère supérieure.

Alpes hercyniennes. - Reste onfin l'appareil condenseur. Il était formé par les Alpes hereyniemes auxquelles on n'accordait qu'une allention distraite il y a vingt-cinq ans, mais dont les magnifiques travanx d'Edouard Suess, de Neumayl et de H. Mareel Bertrand ont désolmais mis en relief l'importance capitale. A quelle hauteur s’élevail le chainon du Plateau Central? Aneme étude n’a áténcone réaliséc daus cette direetion. Mais si l'on en juge par l'énorme puissénee dos matriaux détritiques arrachés par l'érosion, l’altitude devait être de plusions milliors de mètres.

Nos limubeaux houillers cont en effet des épaisseur's énormes dont on peut se faile une idere par les chiffes suivants:

Bussin houller de Sint-Etienne....... 2.450m d'épaisseur.

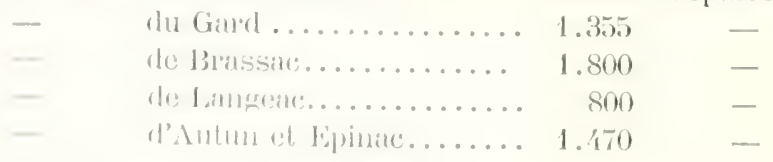

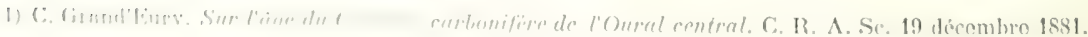


Mais nous comnaissons déjà la hauteur du chânon hereynien belge. Elle a été évaluée par MMI. Briart et Cornet à 5,000 ou 6,000 mètres (i). Dans la chaine des Apalaches, d'après M. de Margerie, les anticlinaux des terrains primaires devaient atteindre 6,7 et 8 kilomètres au-dessus de leur base ${ }^{(2)}$.

Les Apes du Plateau Central devaient posséder une hauteur comparable. Toutes les conditions nécessaires et indispensables à la production de glacier's de montagnes se trouvaient done rémies à un haut degré dans le Plateau Central au début de la période houillère supérieure. Or, la preuve de ces glaciers est faite, sans doute possible par l'existence de brèches formidables à caractères exclusicement glaciaires, ¿i la base ou enclavées dans l'épaisseur et au même niveau de tous nos lambeaux houillers. 'Toutefois, on peut se demander' encore si l'élévation de la température à l'époque houillère n'était pas un obstacle à l'existence de tels glaciers. Un coup d'œil jeté sur le monde actuel nous démontrera la nullité d'une objection de cette nature.

MM. Briart et Cornet font remarquer dans leur beau mémoire que les monts Kénia et Kilimandjaro, situés dans l'Afrique équatoriale, entre l'équateur et le quatrième degré de latitude sud, sont, grâce à leur altitude de 5,500 mètres et de 6,000 mètres couverts de neiges éternelles. - L'absence de glaciers tient seulement à l'absence sur ces montagnes isolées et coniques de bassins de réception des neiges. D'après Humboldt, sur le versant nord de l'Himalaya, sous le $30^{\mathrm{e}}$ parallèle, celui du Caire, la limite inféricure des neiges éternelles se trouve à 3,900 mètres. Les glaciers immenses qui s'en échappent descendent jusque dans le voisinage des forêts de palmiers. D'après Darwin, à la Terrede-Feu, par $46^{\circ}$ latitude sud, celle de Genève, des glaciers nombreux dont un de 25 kilomètres de long et de 15 kilomètres de large, atteignent le golfe de Pénas et produisent par leur rupture des icebergs. - Enfin d'après Lindsay, à lir Nouvelle-Zélande, dans l'île méridionale sur la côte de Canterbury, à l'ouest, par $43^{\circ}$ latitude sud, celle de Florence en Italie, le grand glacier' de Cook descend des Alpes zélandaises jusqu'à 163 mètres au-dessus du niveau de la mer. Son front pénètre dans une forêt de Myrtacées avec Fougères arborescentes et Cordyline. Le Palmier Areca n'en est pas éloigné non plus. On ne saurait done s'étonner de l'existence dans la France centrale de puissants glacier's à l'époque houillère supérieure, car les conditions nécessaires à leur appration, à leur maintien et à leur extension s'y trouvaient toutes réalisées.

Du reste, les géologues pourront se convaincre de leur róalité en allant étudier sur place ces brèches, ef particulierement celles du mont Crépon, que Grüner considérait en 1847 comme des brèches d'éboulement, dans l'ignorance où lui-même et tous ses contemporains d'alor's (je parle des géologues frangrais) étaient à cette époque de la nature des caractéres glaciaires. Celui qui de nos

(1) Comet et Briart. Sur In folief du sol en Butyique apres les temps pulturvïques. Mem. Soc. G. I3. T. IV 1877 .

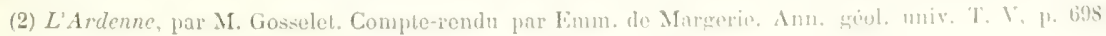
699. 1888 . 
jours rejetterait à priori l'origine glaciaire des brèches colossales enclavées dans nos lambeaux houillers de la France centrale, malgré la netteté de ces caractères, se verrait, à notre avis, acculé à la nécessité de rejeter la permanence et la continuité des lois physiques dans le passé de la Terre. Il serait obligé de nier l'abaissement graduel de la température suivant l'altitude, ou bien de nier encore la transformation de la neige en névé et du névé en glace. Nous refusons d'admettre que de telles négations puissent trouver longtemps crédit à une époque où la géologie est cultivée avec une ardeur aussi passionnée par un nombre de plus en plus grand d'observateurs, ennemis déclarés des vieux préjugés d'école, avides de toute vérité nouvelle.

Ici se terminent les observations que nous avions à présenter relativement à nos glacier's houiller's, l'un des phénomènes les plus grandioses de la période carbonifère.

Nous n'ajouterons plus qu'un mot pour terminer. La mer, chassée du Plateau Central par le soulevement hereynien n'y est revenue, arrivant cette fois de l'est, qu'à la fin de la période triasique. La date de son retour a été fixée, grâce à une heureuse découverte accomplie en 1875. Un très distingué géologue, M. Pellat, a constaté comme nous l'avons vu dans le cours de cet ouvrage, des traces de fossiles marins, Natices, Myophories et Avicules, coquilles littorales gisant dans un bane de dolomie rose, vers le haut du keuper de la montagne de Drevin, près de Couches-les-Mines dans la Saône-et-Loire.

IIIN. 


\section{TABLEAUX REGAPITULATIFS}





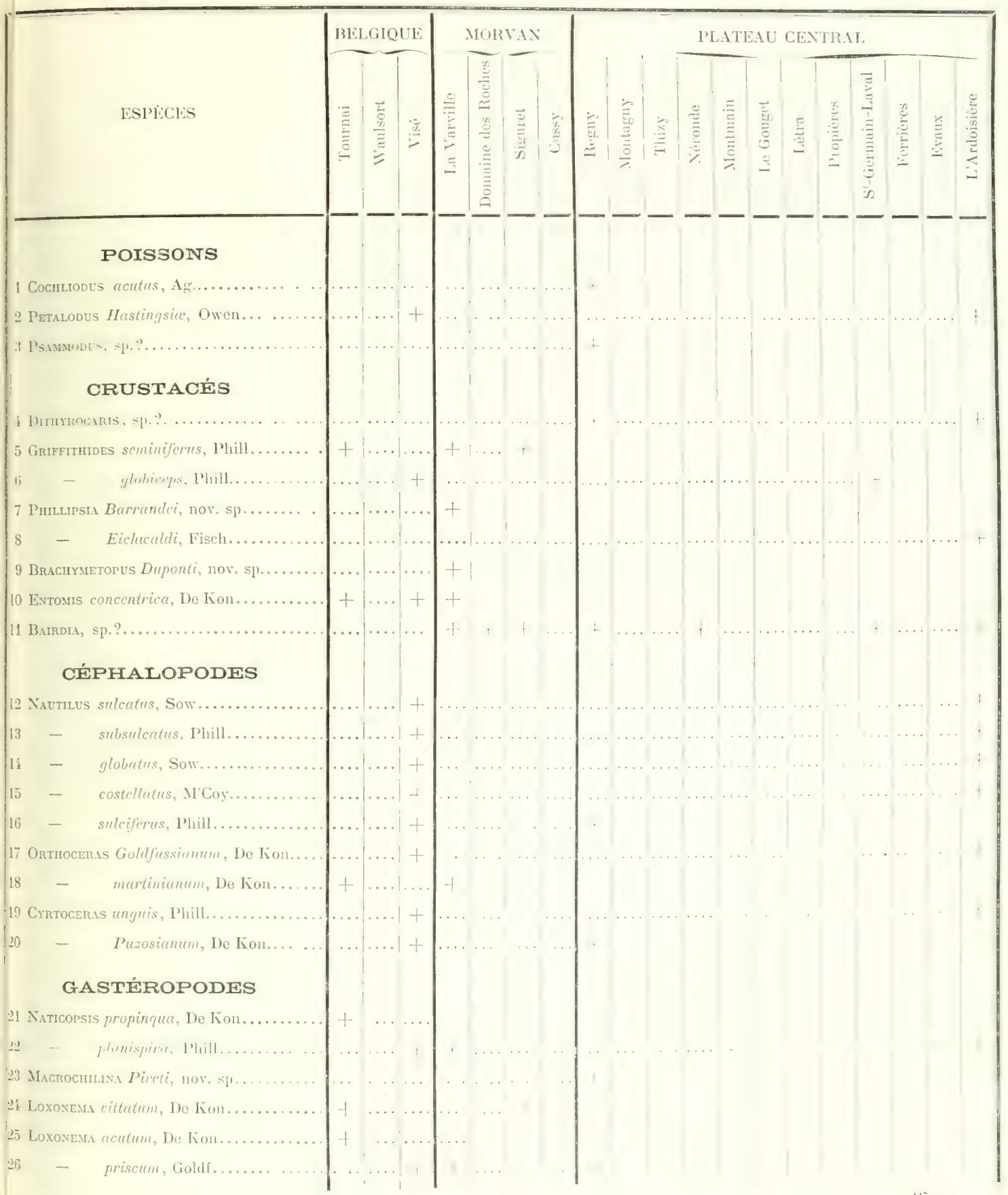




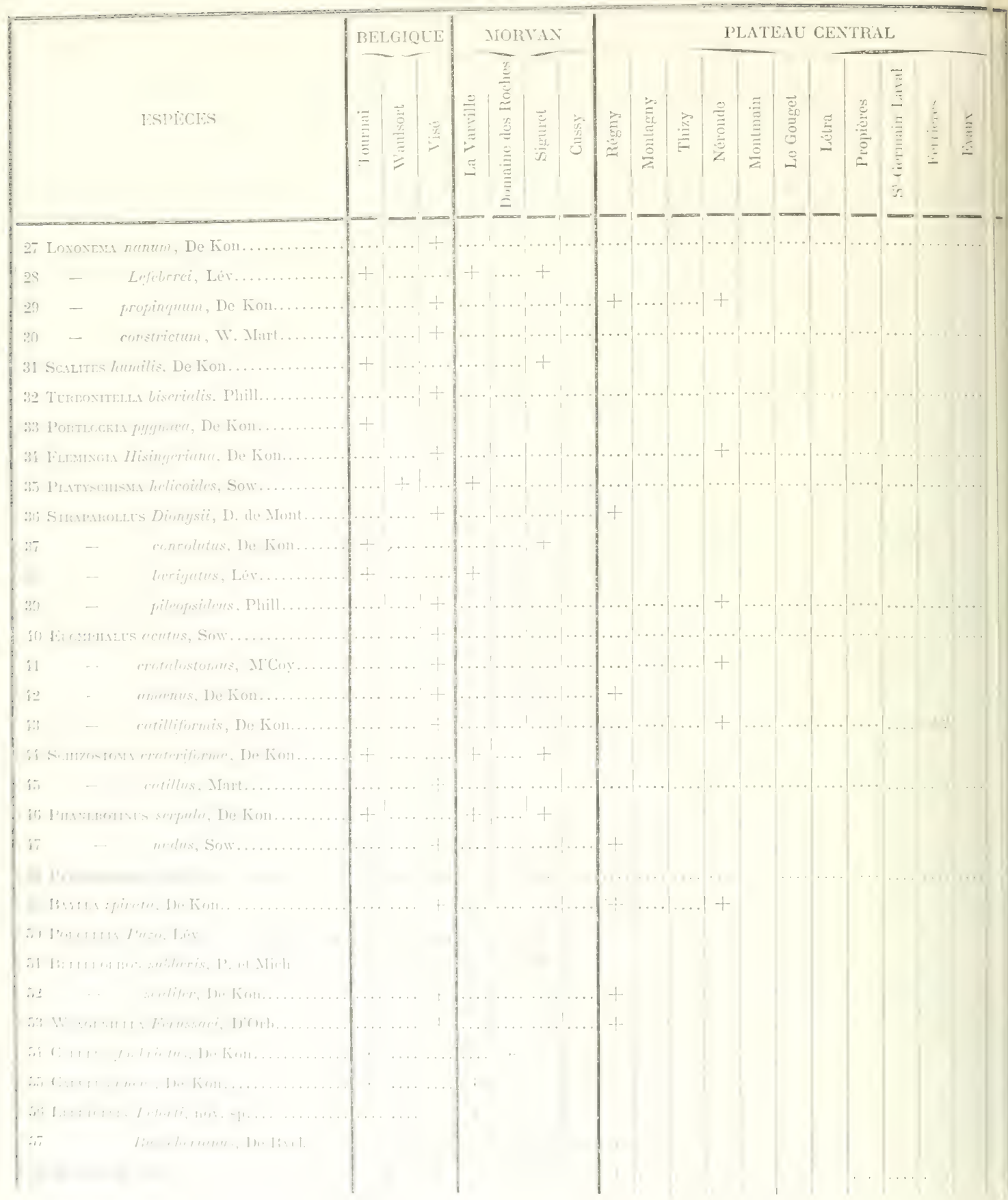




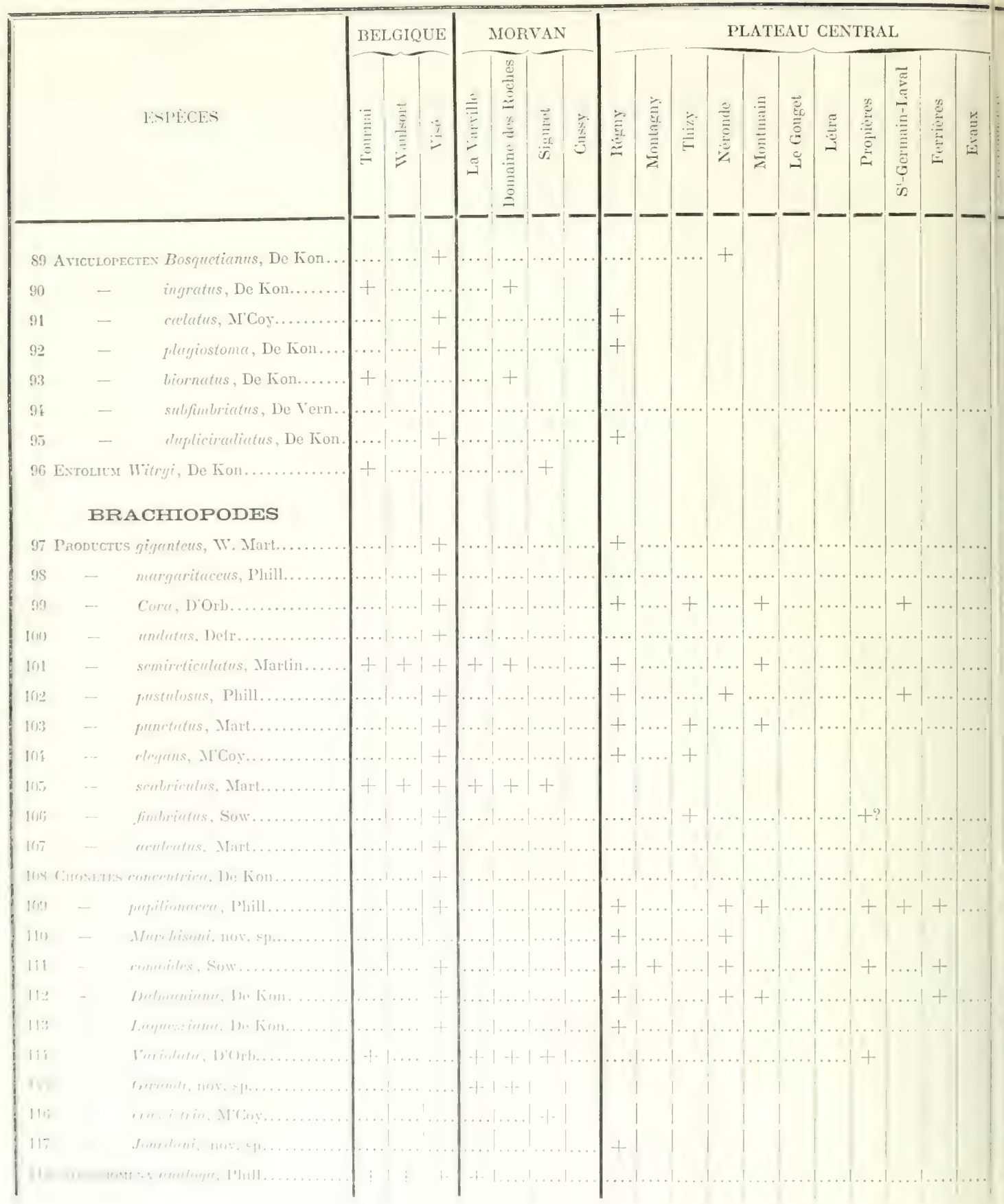




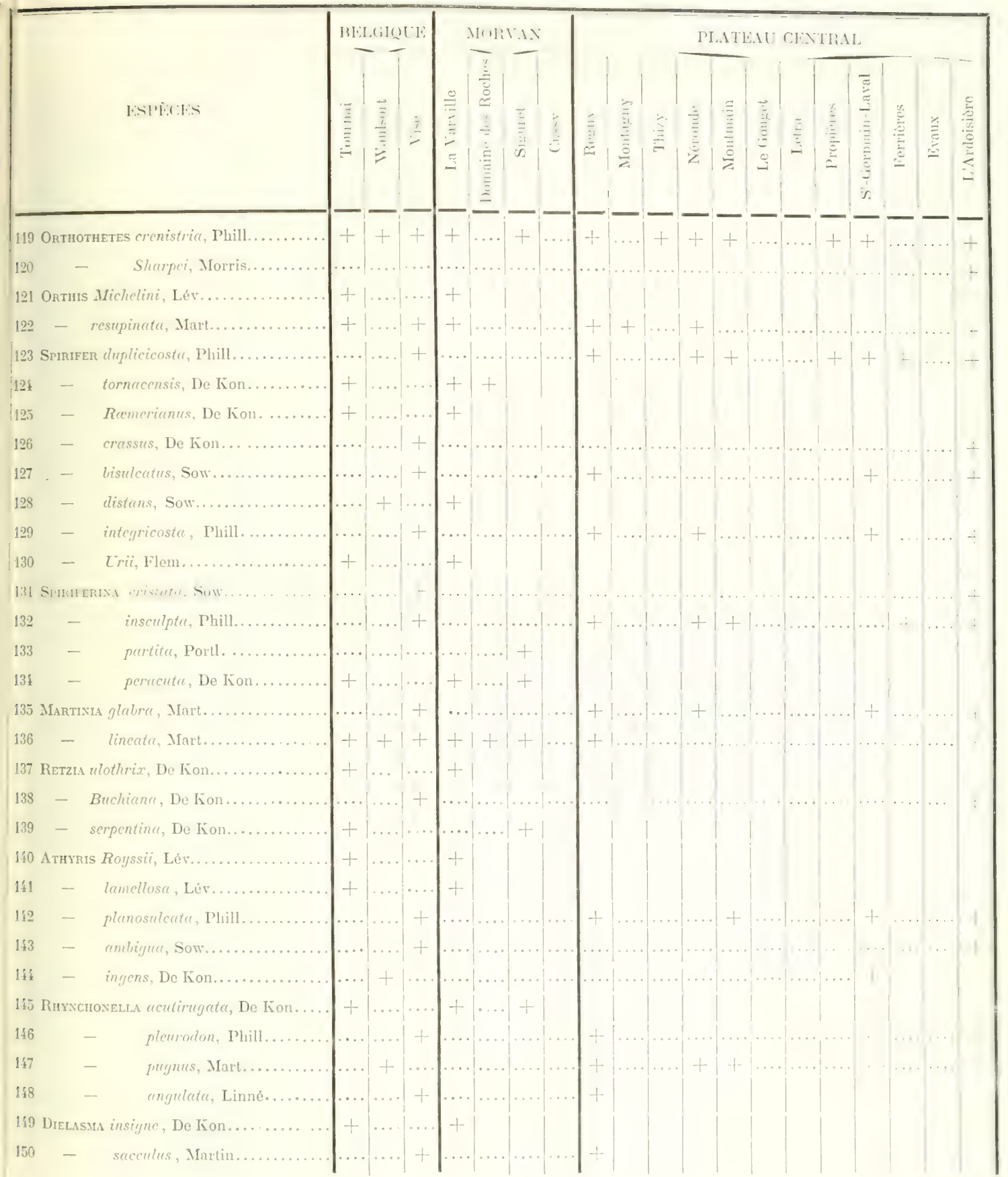




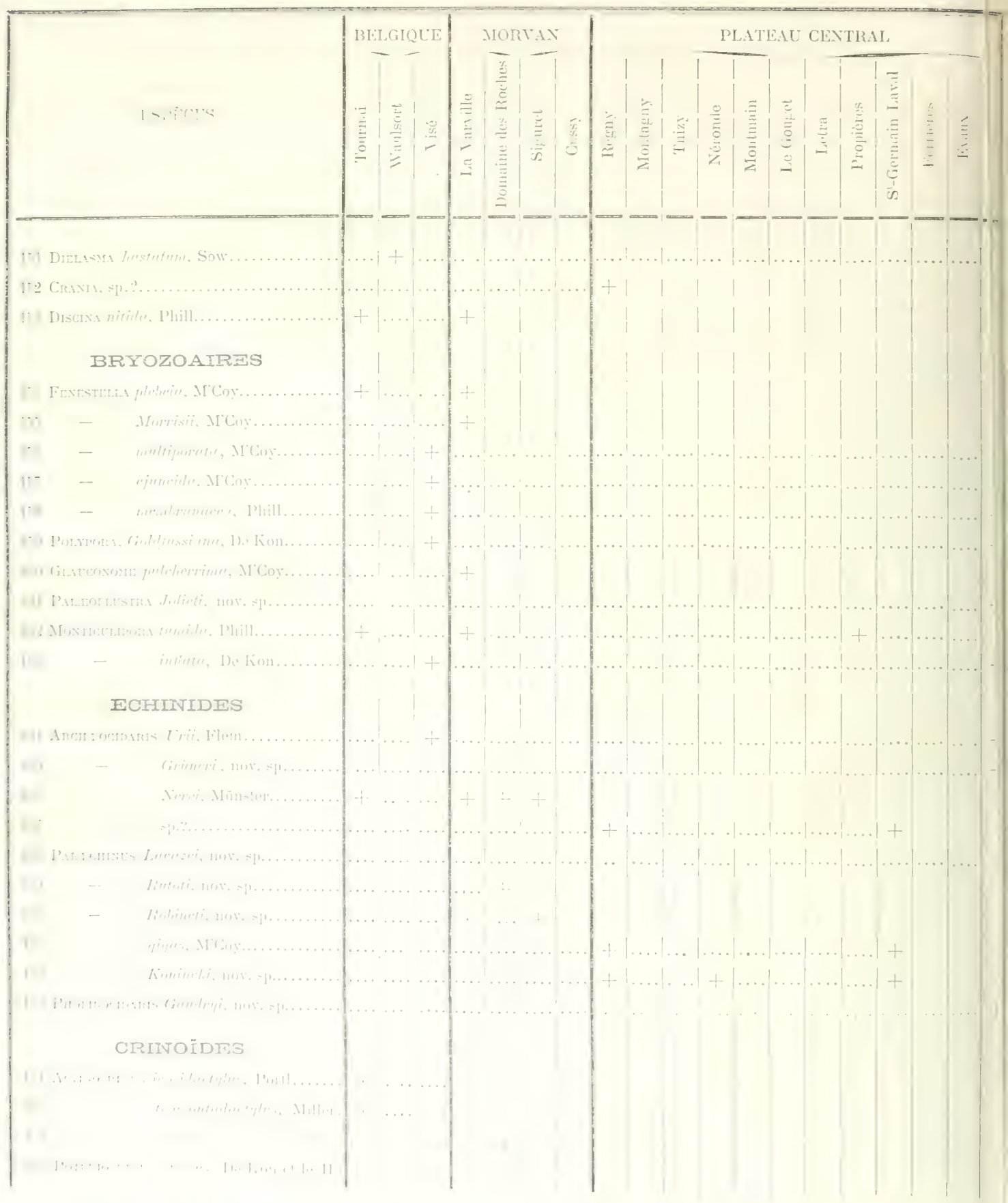




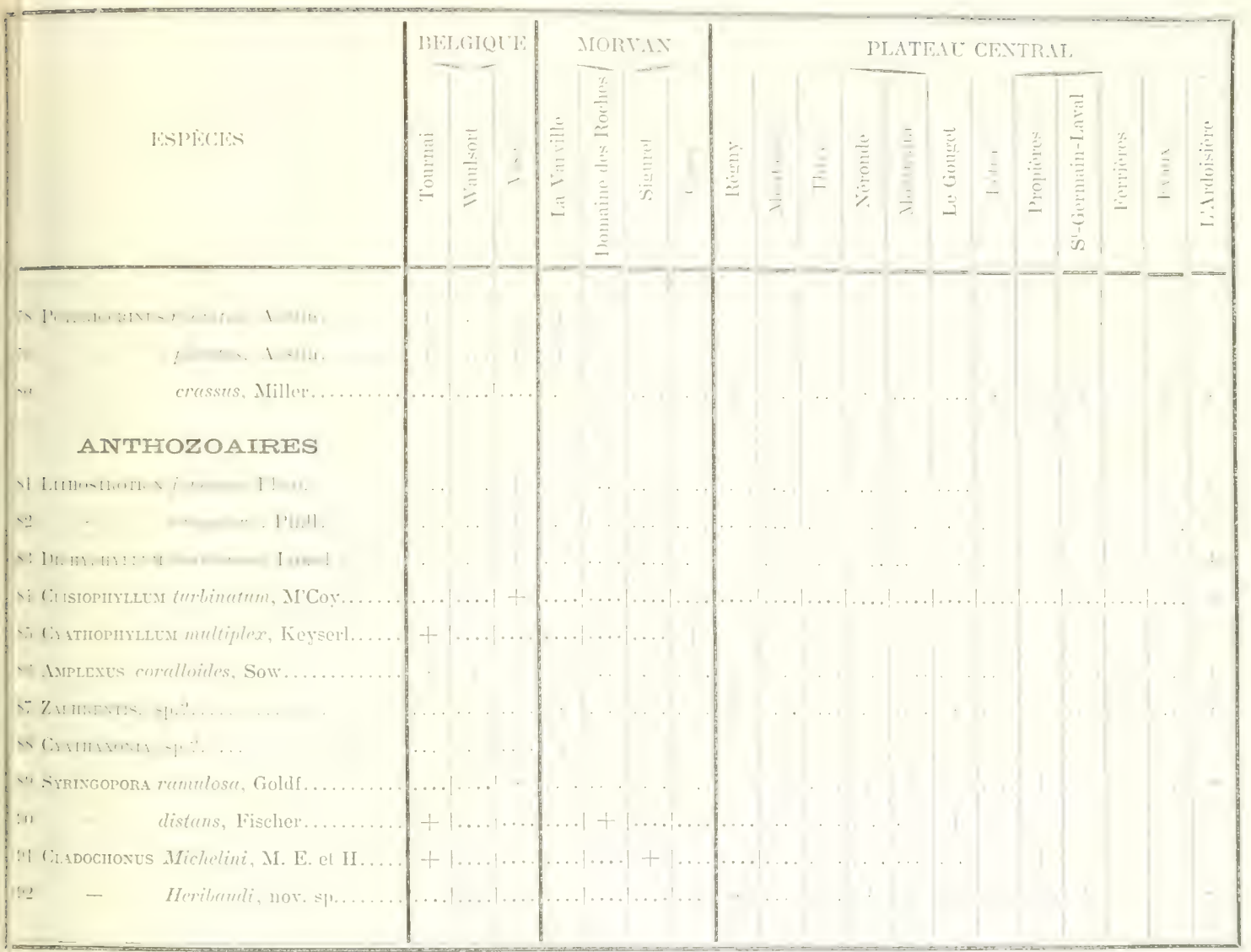




\title{
INDEX BIBLIOGRAPHIQUE
}

\author{
OUVRAGES CITÉS OU CONSULTÉS.
}

1601. Schwexchfelt (C.). Stirpium et fossilium Silesiæ Catalogus. Lipsice. 1 vol. in-8.

1685-1692. MArtin Lister. Historia sive synopsis methodica conchyliorum et tubularum marinarum. 4 yol. in-folio cum tabulis (Eneis.

1699. Luidus (Ed.-Lwyd). Lithophylacii britannici Ichnographia. Lipsice. In-12, 14 pl.

1720. Volkmaxs (G.-A.). Silesia subterranea. Franckfurt. 1 vol. in-4으. 33 pl.

1751. Dargenville. Enumerationis fossilium quxe in omnibus Galliæ Provinciis reperiuntur Tentamina. Paris. In- $8^{\circ}$.

1755. Dargenville. L'Histoire naturelle éclaircie dans une de ses parties principales, l'Oryctologie qui traite des terres, des pierres, des métaux, des minéraux et autres fossiles. Paris.

1762. WALch. Das Steinreich systematisch entworfen. Halle.

1763. Baumer (J.-IV.). Naturgeschichte des Mineralreichs, mit besonderer Anwendung auf Thüringren. 2 vol. in-12 mit Kupfern.

1765. Altéos-Dulac. Histoire naturelle du Lyonnais, Forez et Beaujoliais, 2 vol. in-12. Id. Edition de 1795. 4 vol.

1771. DE Hüpsch. Nouvelles découvertes de quelques testacés pétrifiés rares et inconnus, pour servir à l'histoire naturelle de la Basse-Allemagne et enrichir les collections du Règne animal (traduit de l'allemand avec figures). Cologne, Froncfort et Leipzig, chez Metternich, libraire.

1776. MENdes DA Costa (Emmanuel). Elements of conchology: or an introduction to tho knowledge of shells, with seven plates. London.

1780. DE WViTRy. Mémoire sur les fossiles du Tournaisis ot les pétrifications en général, relativement ì lear utilité pour la vie civile. MIém. de l'Ac. I. et li. des sciences et belleslettres de Bruselles, t. III, p. 15-41, 4 planches de fossiles.

1781. Von Hürscn. Naturgeschichte des Niederdentschlandes und anderer Gegrenden. Nïremberg.

1781. Burtin (M.-X.). Oryetographie de Bruxelles, ete. Bruxelles. 1 vol. in-folio, 32 pl. 1785. Chevnitz (J.-H.). Neues systematisches Conchylion-Cabinet. Nïrembery.

1793. URE (Rev. David). The history of Rutherglen and East-Kilbride published with a view to promote the study of Antiquity and natural IIistory. 11 plates of fossils. 
1797. Passinges. Mémoire pour servir à l'histoire naturelle du département de la Loire. Journal des Mines, t. VI et VII. 1797. - Voir aussi Annales de l'Auvergne, 1840. T. XIII, p. 359 et 378 .

1808-1820. Parkinson (James). Organic remains of a former world. London. 3 vol. in- $4^{\circ}$, 50 pl. col.

1809. MARTix $\left(\mathrm{TT}^{\mathrm{T}}\right.$.). Petrefacta derbiensia or figures and description of petrifications collected in Derbyshire. Wigan. In-4ㅇ, t. I, fig. color.

1814-1829. SowerBY (J.). The mineral Conchology of Great Britain or coloured figures and descr. of those remains of testaceous animals. 6 vol. with 609, coloured plates. London.

1820. Lamouroux (J.). Exposition méthodique des genres de l'ordre des polypiers. Paris, 1820.

1820. Vox Schlotheim (E.-F.). Die Petrefactenkunde. Gotha, 1820. In-8o avec pl.

1823. Drapiez. Coup d'œil minéralogique et géologique sur la province de Hainaut. Bruxelles. In-4 4 pl.

1825. Fischer de Waldieim (G.). Notice de la Choristite, genre de coquilles fossiles du gourernement de Moscou. Moscou.

1825. Cauchy. Mémoire sur la constitution géologique de la province de Namur. (Couronné par J'Académie de Bruxelles.)

1825-1836. VALuY. Notes sur le terrain primitif et de transition des environs de Lyon, insérées dans les Annales de la Société linnéenne de Lyon pour 1836 avec notes de M. Leymerie.

1826. Cumberland. (G.). Reliquire conservate from the primitive materials of our present globe, with popular Descriptions of the prominent characters of some remarkable fossils. Encrinites and their connecting links. 1 vol. Bristol.

1826-1814. Goldfuss (A.). Petrefacta Germaniæ, oder Abbild. u. Beschreib. d. petrefacten Deutschlands, u. d. angrenz. Länder. Leipzig.

1826-1828. I)EFRANCE. Dictionnaire des sciences naturelles.

1828. FLEMrig (J.). History of British animals. Edinburgh. In-8 .

1829. de CALvoNt. Essai géologique sur le départenent de la Manche. 1 rol. in- $8^{\circ}$.

1829. Fischer de Wadienem (G.). Notice sur le système apophysaire ou sur la charpente osseuse des 'Térébratules. Aloscou.

1830. Dufréxoy et de Benumont (Elie). Mémoires pour servir à une description gẻologique de la lirance. Puris.

1830. Dumont (А.). Mémoire sur la constitution géologique de la province de Liège. (Mém. cour, de l'Ac. roy. de Belgique, t. VIII.)

1831. Desinutes (G.-l’.). Description des coquilles caractéristiques des terrains, 1 vol. avec pl. Paris.

1831-1817. Müxsten. (Gralf zu). Beitrige zur Petrefaktenkunde. Bayreuth.

183:3. Javrate (C.-J.). Lissai sur la constitution géognostique de la province de Liège. Brueleses. In-1", 9 pl.

1833. 'Tumat. Statistique minéralogrique et géologique du clópartement de la Haute-Saóne.

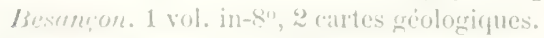

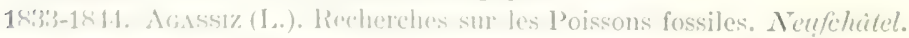

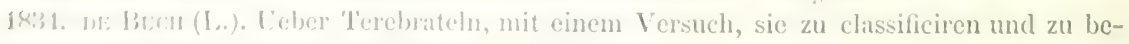
schreiben. Mem. de l'Ace roy des se. de Berlin.

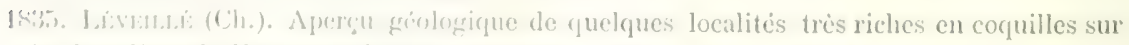

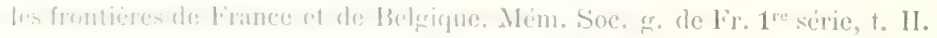


1836. Pintlirs (Jolm). Illustrations of the Geology of Yorkshire. London. In-4 ${ }^{\circ}$.

1836. Deshixes (G.-P.). Histoire naturelle des animaux sans vertèbres, par J.-B. de Lamark. Paris, $2^{e}$ édit.

1836. Fourvel. Etude des gîtes houillers et métallifères du Bocage vendéen.

1837. Blavier. Essai de statistique minéralogique et géologique du département de la Nayenne. Paris. In-8 .

1837. Lexaerie. Notice sur le terrain de transition du département du Rlổne et des parties adjacentes du département de la Loire (non compris le terrain houiller), B. S. G. F. $1^{\text {re }}$ série. 'T'. VIII, p. 310.

1837. DE Buch (L.). Ueber Delthyris oder Spirifer und Orthis. Mémoire de l'Ac. royale des sciences de Berlin.

183\%. Fischer de Walduen (G.). Oryctographie du gouvernement de Moscou.

1837. Gilbertson. A catalogue of fossils from the mountain limestone of Great Britain. Preston.

1838. DE BuCn (L.). Essai d'une classification et d'une description des Térébratules, traduit de l'allemand par Henri le Cocq. Extrait des Mém. de la Soc. géol, de France. T. III, mém. $n^{0} 6$.

1838. Potiez et Michaud. Galerie des mollusques on Catalogue méthodique, descriptif et raisonné des mollusques et coquilles du Muséun de Douai. 2 vol. Paris.

1840. Rozer. Mémoire géologique sur la masse des montagnes qui séparent le cours de la Loire de ceux du Rhóne et de la Saóne. M. S. G. F. $1^{\text {re }}$ série. T. IV.

1840. DE Buch (L.). Essai d'une classification et d'une description des Delthyris ou Spirifers et Orthis, traduit par H. le Cocq. Mém. Soc. g. Fr. $1^{\text {re }}$ série. T. IV.

1840-1847. Michelin (H.). Iconographie zoophytologique. Description des Polypiers fossiles de la France. 1 vol. Paris. In-40 (texte), et 1 vol. in-4 $4^{\circ}$ (79 planches).

1840-1858. Austiv (Th.). Esq., and Th. Austin junior. A monograph of recent and fossil Crinoïdea. Bristol.

1841. Dufrenoy et Beaumont (E. DE). Explication de la carte géologique de France. Paris. 2 vol. in $-4^{\circ}$.

1841. DE Koninck. Mémoire sur les Crustacés fossiles de Belgique. Nouv. Mím. Ac. roy. de Belg. T. XIV. 1 pl.

1841. Grüner. Mémoire sur les terrains de transition et los porphyres du département de la Loire. Annales des Mines. $3^{\circ}$ série. T. XIX.

1841. Phillips (J.). Figures and descriptions of the paleozoic fossils of Cornwall, Devon and West Sommerset.

1842. Quenstedt (Fred.-Aug.). Handbuch der Petrefactenkunde. 1 rol. in-8 texto et 1 vol. in- $8^{\circ}$ de $62 \mathrm{pl}$. Tubingen.

1842. DE Bucru (L.). Ueber Productus oder Leptrena. In-4" mit Kupfertafeln. Mém. de l'Ac. roy. des sc. de Berlin.

1842-1844. De Koninck. Description des animanx fossiles qui se trouvent dans le termain carbonifère de Belgique. Liège. In-4. 1 vol. 650 p. et atl. de 69 pl.

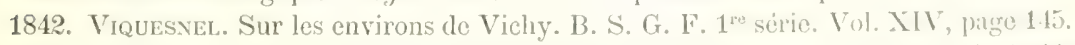

1842. d'Orbigny (A.). Voyage dans l'Amérique méridionale. 'I'. III, 1' partie (palćont.); in- $4^{\circ}$ avec $\mathrm{pl}$.

1842. Griffith (Richard). Notice respecting the fossils of the mountinin limestone of Ireland, as compared with those of Great Britain and also with the Deronian system. Dublin. 
1842. D'Archiac et DE Verneuil. Memoir on the Fossils of the older deposits in the rhenish provinces. 1 vol. Paris.

1843. Pontlock. Report of the Geology of the county of Londonderry and of parts of Tyrone and Fermanagh. Dublin. In- $8^{\circ}$.

1843. Morris (J.). A catalogue of British fossils. 1843. Id. Edition de 1854.

1844. M'Coy (F.). A Synopsis of the characters of the carboniferous limestone fossils of Ireland. Dublin.

1844. Boulanger. Statistique géologique et minéralurgique du département de l'Allier. Moulins. In-12 avec carte.

1844. REMER. Das rheinische Uebergansgebirge.

1845. Sowerby (James). Conchyliologie minéralogique de la Grande-Bretagne, 1825, traduit de l'anglais par Ed. Desor, avec un arant-propos et des notes contenant de nombreuses additions et rectifications par L. Agassiz. 1 vol. texte, 1 vol. pl. Soleure.

1845. DE Strzelecki (P.-E.). Physical Description of New Southwales and ran Diemen's Land, accompanied by geological map., sections, and diagrams and figures of the organic remains. London.

1846. Vox Keyserlixg (Al.-Graf). Wissenschaftliche Beobachtungen auf einer Reise in das Petschora-Land im Jahre 1843. Saint-Péter'sbourg. Texte et pl.

1846. Geinitz (H.-Bruno), Grundriss der Versteinerungskunde. 1 vol. avec pl. Dresden und Leip zig.

1846. GrïNer. Analyses de divers calcaires de transition du département de la Loire. Ann. des Mines, $4^{\mathrm{e}}$ série. T. X.

1847. De Serres (Marcel). Note sur la découverte du genre des Productus dans les formations carbonifères de Roujan ou de Neffiez (Hérault). Mém. Ac. des sciences et lettres de Montpellier. 'T. 1 .

1847. Maxis (W.). Statistique minéralogique, géologique et minéralurgique du département de Saóne-et-Loire. Macon.

1847. de Kosisck (L.-G.). Monographie du genre Productus. Mémoire Soc. roy, des se. de Liège. 'T. IV. 208 p., $19 \mathrm{pl}$.

1847. DE Koninck (L.-G.). Recherches sur les animaux fossiles. $1^{\text {re }}$ partie. Monographie des genres Productus et Chonetes. Liege. In- $4^{\circ}$.

18.17. Grüver. Notice sur la constitution géologique du département de la Loire, in-8 ${ }^{\circ}$. Saint-Eitienne.

1847. DE Rycknol.t. Résumé géologique sur le genre Chiton. Bruxelles. In- $8^{\circ} .4$ planches.

18.18-1819. Bronx, Göprefte und von Meyer. Index Paleontologicus oder Uebersicht der bis jetzt bekannten fossilen Organismen. Stuttgard. 2 vol. in-8 ${ }^{0}$

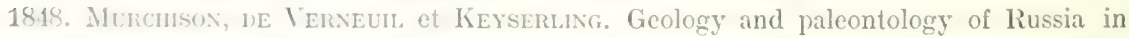
Europa and the Ural Mountains. 2 vol. with 7 maps, 12 views and 50 plates. London, 18.18.

18.19. In: Vimexelit. Sur les fossiles des terrains anciens de Nefliez et de Roujan (Hérault). B. S. (;. F. z' serie 'T' VI.

1850. H: Vinexeru. Réunion extraordinaire de la Soc, géol. de France au Mans. Bull. Soc.

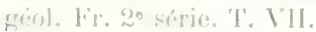

1850). 1,()mbivy (A.). Prodrome de paléontologie stratigraphique universelle des animaux mollusquies et rayonnes. P'aris. 3 vol. in- $8^{\circ}$.

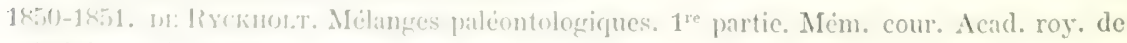

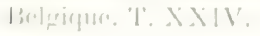


1851. Edwards (Milne) et Uame (J.). Monographie des Polypiers fossiles des terrains paléozoïques. Archives du Muséum d'histoire naturelle. T. V.

1851. DE Koninck (L.-G.). Description des animaux fossiles qui se trouvent dans lo terrain carbonifère de la Belgique. Supplément. Liège. In-40. 1 vol. 66 p., 5 planches.

1851-1856. Bronn (H.-G.) und Remer. Lethea greognostica oder Abbildungen und Beschreibungen der für die Gebirgs-formationen bezeichnendsten Versteinerungen. Stuttgard. 3 vol. in-8 ${ }^{\circ}$ et atl. 124 pl.

1851. Murcinson (R.-I.). On the slaty liocks of the Sichon and on the origin of the mineral springs of Vichy. Quart. journ. of the Geol. Soc. of London. T. VII.

1852. De Beaumont (E.). Notice sur les systèmes de montagnes, p. 263. Lettre de de Verneuil relative aux fossiles carbonifères de Jourdan.

1852. Murchison. Mémoire pour démontrer que l'extrémité nord de la chaîne du Forez ou que les roches ardoisières du Sichon doivent itre rapportées á l'époque carbonifère (traduit par M. Alluard). Extrait des Annales scientifiques, littéraires et industrielles de l'Auvergne. T. XXV.

1852. Munne-Evwards et Haime. A Monograph of the british fossil corals. London.

1852. DE CESSAC. Statistique minéralogique et géologique du département de la Creuse.

1853. Guéranger (Ed.). Essai d'un répertoire paléontologique du département de la Sarthe. Le Mans.

1853. DE Koninck et LE IION (H.). Recherches sur les Crinoides du terrain carbonifère de la Belgique. Mém. Acad. royale de Belcrique. In-40. T. XXVIII. 215 pages, 7 pl.

1853-1869. Erchwald. Lethea Rossica ou Paléontologie de la Russie décrite et figurée. 3 vol. de texte et atlas de $113 \mathrm{pl}$. in-folio. Stuthard.

1853. Davidson (Th.). Observations on the Chonetes comoides, Sowerby. Proccedings of the Geological Society. 14 décembre 1853.

1854. Von Semenow (P.). Die Fossilien des schlesischen Kohlenkalks.

1855. Founnet (J.). Etudes sur l'extension des terrains houillers en France. Lyon.

1855. Desor (Ed.). Synopsis des Echinides fossiles. Paris. In- $8^{\circ}, 37$ pl.

1855. KeLLy (John). On localities of fossils of the carboniferous limestone of Ireland. Juurnal of the geol. Soc. of Dublin. 14 mars.

1855. M'Cor (Frederick). Deseription of British palœeozoie Fossils in the University of Cambridge. 1855 .

1856. Deslongcinamps (Eug.-Eudes). Calcaire carbonifère de Régneville. Mém. Soc. linn. de Normandie. Vol. X, p. 53.

1856. Davoust (Abbé Frédéric). Quels sont parmi les corps organisés fossiles recucillis en France, ceux qui n’ont encore été trouvés que dans le département de la Sartho? Bull. Soc. agric., sc. et arts de la Sarthe. 2 série. T. III. Volume XI.

1856. Davidson (Th.). Introduction i l'histoire naturelle des Brachiopodes vivints et fossiles (trad. de l'anglais par MM. Eudes Deslongchamps et Eugène-Eudes Deslongchamps.) Extr. du $x^{\text {e }}$ vol. des Mém. de la Soc. linn. de Normandic. Cuen.

1856-1860. Davinson (Th.). A monograph of the British carhoniferous Brachiopodia. Paleontographical Society. 1856, 1857, 185s, 1859, 1860.

1857. Grüner. Deseription géolostique et minéralogique du département de la Loire avec carte et atlas. Paris.

1858. Hofmann (E.). Trilobiten Russlands. Suint-Pétersbou'?.

1858. Hale (James) et Vhitwey (J.-D).). lieport on the Geological Survey of the state of lowa. Vol. I. 185s. Part. I, (ieology ; l'art. II, Palwontology. 
1859. DE Kioninck (L.). Traduction avec notes et additions du mémoire sur les genres et les sous-genres de Brachiopodes munis d’appendices spiraux, par Davidson. Mém. Soc. sc. Liene. T. ATl. $\approx$ pl.

1859. Procès-verbaux des séances de la session extraordinaire de la Société géologique á Lyon. Du $1^{\text {er }}$ au 11 septembre 1859.

1859. Drian. Minéralogie et pétrologie des environs de Lyon. 1 vol. in- $8^{\circ}$.

1860. Gosselet (J.). Mémoire sur les terrains primaires de la Belgique, des environs d'Avesnes et du Boulonnais. Par'is. In-8".

1860. Murchison (R.-I.) Les terrains paléozoïques des provinces Rhénanes et de la Belgique. Extr. de la Revue universelle. T. VII.

1861. Dalmifr (Paul). Stratigraphie des terrains primaires dans la presqu'ile du Cotentin (thèse). Paris.

1861. Fournet (J.). Géologie lyomaise. 1 vol.

1861. Le Hardy de Beaulieu (Ch.). Guide minéralogique et paléontologique dans le Hainaut et l'Entre-Sambre-et-Meuse. Liege.

1861. Dupont (E.). Notice sur les gites de fossiles du calcaire carbonifére des bandes de Florennes et de Dinant. Bull. Ac. sc. de Belgique. T. XII, p. 293.

1862. Kaemin-Schlumberger et Schimper (WV.-Ph.). Mémoire sur le terrain de transition des Vosges. Strasbourg. 1 vol. in-4". 31 pl.

1863. DE Kioxrixck (L.). Notice sur quelques Brachiopodes carbonifères recueillis dans l'Inde par M. le Dr Fleming et WV. Purdon et décrits par Ch. Davidson (traduction). Mém. Soc. roy. sc. de Liège. T. X VIII, 17 p., 4 pl. 1863.

1863. DE Koxisck (L.). Mémoire sur les fossiles paléozoïques recueillis dans l'Inde par M. le [) Fleming d'Edimbourg et décrits par L. de Koninck. Mém. Soc. roy. sc. de Liège. T. XVIII. 8 pl.

1863. I) unowt (E.). Sur le calcaire carbonifère de la Belgique et du Hainaut français. Bull. Ac. sc. de Belgique. T. $\mathrm{XV}, \mathrm{p} .86$.

1863. Horton (Ch.). Sur les terrains primaires des environs de Visé. B. S. G. F. T. XXI, p. $\tau 6(j-\tau) \% 0$.

1861-188:3. Satter (J.-W.). A monograph of the British Trilobites of the mountain Limestone, Deron, Silur, etc. London. 1 vol. in-4․ $31 \mathrm{pl}$.

1861. Jefont (E.). Notice sur le marbre noir de Bachant (Hainaut français). Bull. Ac, des se. de Belyique. T. XVII, p. 181.

18fi5. Beyran (L.). Leber eine Kohlenkalk Fauna von Timor mit dreï Küpertafeln. Berlin. 1865.). 1)trext (EA.). Essai d'une carte géologique des environs de Dinant. Bull. Acad. de

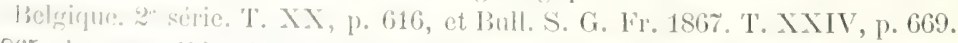

186is. Juprowe (Ed.). Echelle stratigraphiçue du cal. carbonifère de la Belgique. Bull. Ac. roy. de ludwicule. T' XX.

18(5). (ince (Johm). Bingraphical Notice of the Rev. l)avid Ure, with an Examination, critical and detailed of his History of Rutherglen and East Kilbride. Contains a list of the foseils figured by Tre.

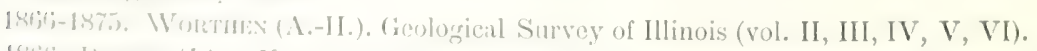

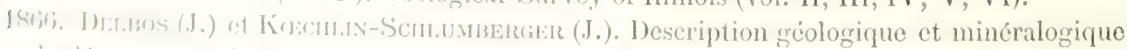

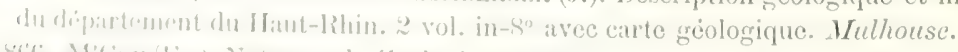

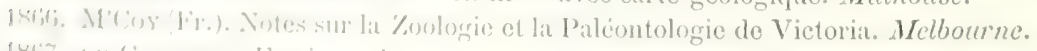

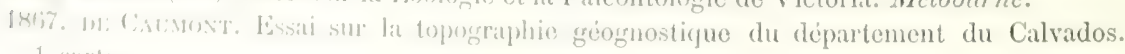
1 (arls. 
1867. Guéranger (Ed.). Album paléontologique du département de la Sarthe. Le Mans. 1 vol. in-4". $45 \mathrm{pl}$.

1868. Julikv (A.). Sur l'existence d'anciens glaciers dans le Puy-de-Dóme et le Cantal et sur l'origine véritable des conglomérats ponceux de la colline de Perrier. Comptes-rendus Ac. sc. 28 décombre 1868.

1868. Ebray (Th.). Végétaux fossiles du terrain de transition du Beaujolais.

1868. Dewalque. Prodrome d'une description géologique de la Belgique. Liège. In-So.

1868. D'Archiac (A.). Rapport sur les progrès de la palćontologie en France. 1 vol. in- $8^{\circ}$.

1868. GrüNER. Description des bassins houillers de la Creuse.

1869. Juliex (A.). Des phénomènes glaciaires dans le Plateau Central de la France, en particulier dans le Puy-de-Dóme et le Cantal. Paris, J.-B. Bailliere.

1869. de Rouville (P.). Session extraordinaire de la Société géolog. de France á Montpellier. 1 vol. in-8 .17 pl., 1 carte géolog.

1869. DE Koxinck (L.G.). Sur quelques Echinodermes remarquables des terrains paléozoïques. Bull. Ac. roy. de Belg. 2e série. T. XXXVIIl, 1 pl.

1870. Chavtre (E.). Notice historique sur la vie et les travaux de Fournet. 1 broch. Lijon.

1871. Dupont (Ed.). Observations sur la constitution du calcaire carbonifẻre de la Belgique. Bull. Ac. roy. de Belgique. T. XXXI, p. 147-176 avec coupes.

1872. DE Koninck. Nourelles recherches sur les animaux fossiles du terrain carbonifẻre de la Belgique (coralliaires). Mém. Acad, roy. Belgique. In-1. T. XXXIX, $15 \mathrm{pl}$.

1872. Douvilté. Sur la discordance qui existe entre le houiller inférieur et le houiller supérieur. C. R. Ac. sc. 20 mai.

18\%. Rigaux. Géologie du Boulonnais. 1 pl.

1873. Raborsson (l'abbé). Note sur la découverte du T'errain carbonifẻre faite à Saint-Nicolas-de-Rougemont. Bull. Soc. géol. Fr. 3e série. T. II, p. 121.

1873. DE Kioninck (L.-G.). Recherches sur les animaux fossiles, 2 e partie. Monographie des fossiles carbonifères de Bleiberg en Carinthie. Bruxelles. In-4 ${ }^{\circ} .1$ vol.

1873. Douvilué. Age du grès anthracifère. Réunion extraordinaire de la Soc. géol. de France à Roanne.

1873. Collenot (J.-J.). Description géologique de l'Auxois. Semur. 1 vol. in-S。.

1873. Gosselet et Bertaut. Etude sur le terrain carbonifère du Boulonnais.

1873. Toula (F.). Kohlenkalk-Fossilien v. d. Südspitze v. Spitzbergen. Vienne.

1874-1884. Joves (R.), Kirby (J.-IV.) et Brady. A monograph of the British fossil Bivalved Entomostraca from the carboniferous Formations, London. In-40. $7 \mathrm{pl}$.

1874. DE Koninck. Sur les fossiles carbonifères découverts dans la vallée du Sichon (Forez) par M. Julien. Annales de la Société géol. de Belgiqque. T. I, p. 3.

1874. Graff. Notice sur les terrains paléozoïques du département de l'Hérault. Ann. de la Soc. des sc. indust. de Lyon.

1874. Julien (A.). Sur une faune carbonifère marine découverte aux environs de l'Ardoisière dans la vallée du Sichon (Forez). Comptes-rendus Ac. des sc. 5 janvier 1874.

1875. Pellat. Sur la présence de fossiles dans le kenper des environs de Couches-les-Mines (Saòne-et-Loire). B. S. G. F. 3 érie. T. IV, p. 369.

1875. Dupont (Ed.). Sur le calcaire carbonifere entre Tournai et les environs de Namur. Bull. Ae. roy. de Belgique. T. XXXIX, p. 26 6-311 atrec. 2 pl. de coupes.

1875. Dupont (Ed.). Rapport sur le projet de publication d'une nouvelle carte gréolorique de la Belgique, proposition faite par M. Dewalque dans la séance du 5 juin 1875 . Bull. Ac. roy. de Belg. 'T. XL, p. 291-307. 
1875. DE Koninck (L.-G.). Notice sur le calcaire de Malowka et sur la signification des fossiles qu'il renferme. Bull. Soc. Nat. de Moscou. T. XLVIII, $2^{e}$ partie.

1875. Toula (F.). Kohlenkalk Fauna der Barents-Inseln (Nowajasemlja) mit 6 Taf. Wien.

1875. Grisebach (A.). La végétation du globe d'après sa disposition suivant les elimats (traduit par P. de Tchihatchef). 2 tomes. Paris.

1876-1883. Rever (Ferd.). Lethra paleozoica. Stuttgard.

1876. Keepring (W.). Notes on the paleozoic Echini. Quarter. Journal of the geol. Soc. Vol. XXXIl. Part. I, pl. III, fig. 14-18.

1876. Keeping (IV.). On the discovery of Melonites in Britain. The quarterly Journal of the geological Society. Vol. XXXII. Part. 4, nº 128, p. 395.

1876. Armstrong (James), Youvg (John) and Robertson (David). Catalogue of the Western Scotsh fossils. Glasgow.

1876. De Tromelin et Lebesconte. Observations sur les terrains primaires du nord du département de l'Ile-et-Vilaine et de quelques autres parties du massif breton. B. S. G.

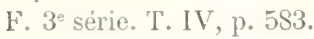

1876. Juliex (A.). Etude sur les faunes paléozoïques de la France centrale. Assoc. franç. pour l'avancement des sciences. Clemont-Ferrand.

1877. Cornet (F.-L.). el Briart (A.). Sur le relief du sol en Belgique après les temps paléozoïques. Mém. de la Soc. géol. de Belgique. T. IV.

1877-1878. DE Kioxinck (L.). Recherches sur les fossiles paléozoïques de la nouvelle Galles du Sud (Australie). Mém. Soc. royale de Liège. T. VI et VII.

1877. Gravd'Eurr (C.). Flore carbonifére du département de la Loire et du centre de la France. Paris, Imprimerie nationale.

1877. Rexiult (B.). Sur les débris organisés contenus dans le quartz et le silex du Roannais (lettre adressée à M. Dumas). C. R. Ac. des sc., 15 octobre.

1877. De Tromeln et Lebesconte. Observations sur les terrains primaires du nord du département d'Ille-et-Vilaine et de quelques autres parties du massif breton. B. S. G. F. 3 séric. T. IV.

1877. de 'Trometin (G.) et DE Grasset (C.). Etude sommaire des faunes paléozoïques du Bas-Langnedoc. Le Havre. Congrès de l'Association française pour l'avancement des sciences,

1877. Jubiex (A.). Note sur la découverte d'un gisement de plantes permiennes à Coulandon (Allier). Assoc. frane. pour l'avancement des sciences. Congrès du Harre.

1877. Juniex (A.). Sur l'existence de filons de bitume dans le granite des environs de Clermont-lerrand. Comptes-rendus Ac. sc. 9 avril.

187S. Kimmix (G.-II.). Manual of geology of Ireland. London. In-8 with illustrations and mitp,

187S. Bursby (J.-J.). Thesaurus Devonico-carboniferous. The flora and fauma of the Devonian and the Cinboniferous periods. Londent. In-4".

1878. m: Matulin. Sur la composition et les divisions générales du système carbonifère. Compres international de greologrie tenu ì Paris du 29 au 31 août et du 2 au 4 septomulnet.

1878. 1m: Koxmck. Faune du calcairo cambonifere de la Belgique. $1^{\text {re }}$ partie. Poissons et fonter Nintile. In-folis, 152 p., 31 pl. Annales du Mus. roy. d'hist. nat. de Belgique. I'. 11.

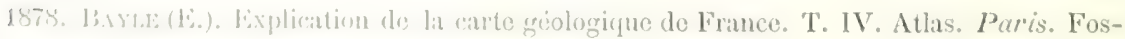
ites principanx desterains do la lirance. 
1878. Juidex (A.). Les voleans de la France centrale et les Alpes. Anmuaire du Club alpin français. $5^{\text {a }}$ vol.

1879. Sirubsole (G.-IV.). On the British carboniferous Fenestellide. The quart. journ. of the geol. Soc. Vol. XXXV. Pirt. 2, no 138, p. 275.

1879-188\%. Collenot. Catalogne de la collection du Mnsée de Semur (Cóte-d'Or). Extr. des Bull. de la Société des seiences historiques et naturelles de Semur.

1879. Von Kanen. Die Kulm-Fauma von Iferborn. In-8. Planches.

1880-1881. Mourlon (Michel), Géologie de la Belgique. Bruxelles. 2 vol, in-So. Pl.

1880. Dewalque (G.). Prodrome d'me description géologique de la Belgique. Paris.

1880. Gehlekt (D.). Note sur le calcaire de Saint-Roch i Changé, près Laval. B. S. G. F. 3 série. T. VII, p. 270.

1880. DE Koninck. Faune du calcaire carbonifére de la Belgique. $2^{\circ}$ partie. Céphalopodes (suite). In-folio. 133 p. et $19 \mathrm{pl}$. Annales du Mus. roy. d'hist. nat. de Belgique. T. V.

1880. Juliex (A.). La Limagne et les bassins tertiaires du Plateau Central. Structure gréologique, architecture, climat, fiume et flore. 1 pl. de coupes. Ann. du Club alpin franç. itril.

1881. Purvis.s. Sur la délimitation et lia constitution de l'étage houiller inférieur de la Belgique. Bull. Ac. roy. sc. de Belgique.

1881. Malmenbe (Renier). Note sur l'horizon de l'étige inférieur du système houiller. Annales de la Soe. géol. de Belgique. T. IX. Liège.

1881. Arcelix (Adrien). Explication de la carte géolog. des deux cantons nord et sud de Micon. Extrait des Annales de l'Académie de MIicon.

1881. Dagincourt. Note sur la géologie des environs de Saint-Amand (Cher). B. S. G. F. 3e série. T. IX.

1881. Gosselet (J.). Esquisse géologique du nord de la France et des contrées voisines.

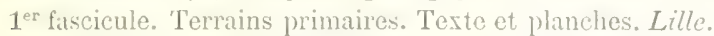

1881. Grand'Eury. Sur l'àge du calcaire carbonifère de l'Oural central. C. R. Ac. se, 19 décembre.

1881. Julien (A.). Sur l'existence et les caractères du terrain cambrien dans le Puy-deDóme et l'Allier. C. R. Ac. sc. 21 mars.

1881. Juliex (A.). Sur la nature et l'ordre d'apparition des roches éruptives ancionnes que l'on observe dans la région des volcans it cratères du Puy-de-Dime. C. R. Ac. se. 28 mars.

1881. Julien (A.). Sur Ie terגain déronien de Diou (Allier) et de Gilly (Stóne-et-Loire). C. R. Ac. se. 4 arril.

1881. Jutrex (A.). Sur l'existence du terrain cambrion is Saint-Lion et Chitel-perron (Allier). C. R. Ac. sc. 30 mai.

1851. Julien (A.). Sur la faune carbonifere de Rény (Loire) et ses relations avee celles de l'Ardoisière (Allier). C. R. Ac. sc. 13 juin.

1881. Jubiex (A.). Sur Ie synchronisme de la faume carbonifére marine de l'Ardoisière (Allier) et de la flore anthracifère du Roannais et du Beanjolais. (C. R. Ac. sc. 11 juillet.

1881. Michel-Lévy et Vébarx (Ch.). Notes et comptes-rendus d'exeursions. Réunion extraordinaire de la Soc. géol. de France it Semur en Auxois (texte). Puris.

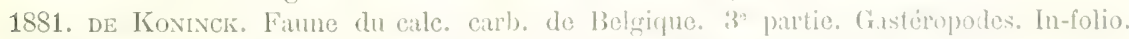
170 pages, 21 pl. Anı. du Mus. roy. d'hist. nat. de Belgique. 'T. VI.

1881-1882. Laur (Francis). Gúologie et Iydrologie de la plaine du Forez. Butule par les sondages. 4 livr. avee planches. Suint-Étienne. 
1882-1884. Dupont (E.). Explication des feuilles de Ciney, Dinant, Clavier, Natoye, Modave, Hastières. Bruxelles.

1882. Bleicher et Ming. Sur le carbonifère marin de la Haute-Alsace. C. R. Ac. sc. 13 fér. et 26 juin.

1882. Bletcher et Mieg. Note sur le carbonifère marin de la Haute-Alsace et ses relations arec le culm. B. S. G. F. $3^{e}$ série. T. X.

1882. CEhlert (D.). Notes géologiques sur le département de la Mayenne. Extrait du Bulletin de la Société d'études scientifiques d'Angers. 1 vol. in-8. 1 carte géologique.

1882. Barrols (C.). Recherches sur les terrains anciens des Asturies et de la Galice. Mém. de la Soc. géol. du Nord. T. II. Lille.

1882. de Koxixck (L.-G.). Notice sur la famille des Bellerophontide. Ann. se. g. de Belg. T. IX.

1883. Woodward (H.). A Monograph of the british carboniferous trilobites, London. 1 vol. in $-4^{\circ} .10 \mathrm{pl}$.

1883. Dupont (Ed.). Sur les origines du calcaire carbonifère de lia Belgique. Bruxelles. In- 8 .

1883. Stache (G.). Kohlenkalk-fauna a. d. Geb. der West-Sahnra, nit 7 'Taf. Wien.

1883. Iiutot (A.). Les plténomènes de la sédimentation marine, étudiés dans leurs rapports avec la stratigraphie régionale. Bulletin du Musée royal d’hist. nat. de Belgique. T. II.

1883. DE Kosixck. Notice sur le Spirifer mosquensis et sur ses affinités avec quelques autres espèces dn méme genre. Bull. Mus. roy. d’hist. nat. de Belgique. T. II.

1883. DE Kioxrvck. Faune du calcaire carbonifère de la Belgique. $4^{\mathrm{e}}$ partie. Gastéropodes (suite et fin). In-folio. 256 p., 36 p)l. Annales du Mus. roy. d'hist. nat. de Belgique. T. VIII. 1883. 1): Koxinck. Notice sur la distribution géologique des fossiles carbonifères de la Belgique. Bull. Mus, roy. d'hist. nat. de Belgique. T. II.

1883. Jerien (A.). La théorie des voleans et le Plateau Central. Historique, théories actuelles, vues nouvelles. Annuaire du Club alpin français. $10^{e}$ rol.

1883-1593. Zrtel (Karl-ג.). Traite de paléontologie. Traduit par Ch. Barrois. 4 vol.

1881. Renevier (E.). Les faciés géologiques. Archives des se. phys, et nat. de Genève. 3 période. Tome Xil.

1881. Bureac (Ed.). Recherches sur la structuro géologique du terrain primaire de la BasseL.nire. H. S. G. I'. 3" série. 'T'. XII.

1884. Lartiat (L.). Sur le terrain earbonifère des Pyrénées centrales. C. R. Ac. se. 5 août 1881 et ! mai 1887.

18s. Mr.mentr et Mn:ci. Note sur la palcontologie du terrain carbonifére de la Haute-

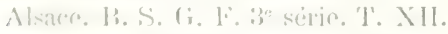

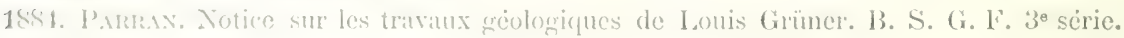
'1. Xil.

1841. Muver (fi.). Heitrag zur Kentnise des cum in den südichen Vogesen. Abhandlungen

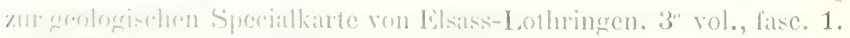

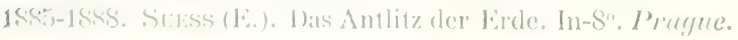

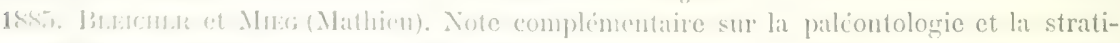

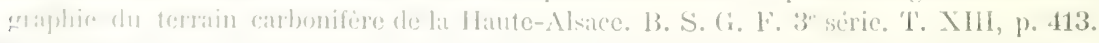

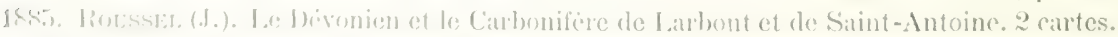

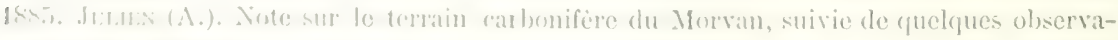

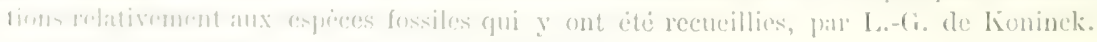

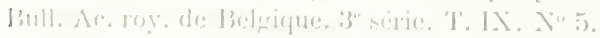


1885. be Koxrxck. Paune du calcaire carbonifére de la Belgique. $5^{\circ}$ partie. Lamellibranches. In-folio, $280 \mathrm{p}$. et $41 \mathrm{pl}$. (en collaboration avee le $\mathrm{D}^{\mathrm{r}} \mathrm{J}$. Fraipont). Annales du Mus. roy. d'hist. nat. de Belgique. 'I'. XI.

1886. Gulder (ג.). Géologie du département de la Sarthe et profils géologiques des routes. Le Muns. 1 vol. in-1․

1886. Masson (Louis) et Bevort (Félix). Notice géologique sur le lépartement du Rhóne. Lyon.

1886. DE Rouville. Monographie géologicue de la conmune de Cabrières (Ilérault). Mém. do l'Acad. des sc. et lett. de Montpellier.

1856. Barrors. Structure géologique du Finistère, B. S. G. F. Réunion extraordinaire en Bretagne.

1856. DE Koninck (L.-G.) et Lomest (Maximin). Notice sur le parallélisme entre le calcaire carbonifere du nord-ouest de l'Angleterre et celui de la Belgique. Bull. Ac. roy. de Belg.

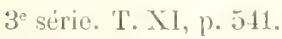

18S6. Juliev (A.). Brèches voleaniques et Moraines dins la France centrale. Ann. du Club alp. franc. 13 vol.

188\%. Bertrand (Mareel). La chaine des Alpes et lit formation du continent européen. B. S. G. F. 3 série. T. $\mathbf{X} V$.

1S57. Frecn (Fr.). Die palroozoischen Bildungen von Cabrières (Languedoe) mit Karte und Fig. Berlin.

1857. Faty (J.). Le Poudingue houiller. Ann. Soc. géol. de Belg. T. V, p. 10i)-110. Liège, 1878. - Id. T. XIII, p. 183. Liege, 1887.

1857. Rutot (A.). Origines et modes de formation des Roches sédimentaires de la Belgique. Bruxelles.

1887. CEnlert. Sur les oscillations qui se sont produites pendant la période primaire dans lo bassin de Laval. C. R. Ac. sc.

1887. DE Rouville. Sur les formations paléozoïques de la région de Cabrières.

1887. Mourlon (Michel). Notice sur les travaux paléontologiques de L.-G. de Koninck. Annales du Mus. roy. d'hist, nat. de Belgique.

1887. DE Koninck. Faune du calcaire carbonifère de la Belgique. $6^{\circ}$ partie. Brachiopodes. In-folio, 153 p., 37 pl. Ann. Mus. roy. d'hist. nat. de Belgique. T. AIV.

1887. Fischer (Dr Paul). Manuel de conchyliologie et de paléontologie conchyliologique suivi d'un appendice sur les Brachiopoles, par D. P. QEhlert. 1 vol. grand in-So arec $23 \mathrm{pl}$. Paris.

1887. Bleicher. Sur la découverte du carbonifẻre á fossiles marins et ì plantes aux environs de Raon-sur-Plaine. C. R. Ac. sc. 28 mars.

188\%. Lebesconte (P.). Constitution générale du Massif breton cumpare it celle du Finistère. B. S. C. F. $3^{\circ}$ série. 'T. XIV.

1887. Vérain (Ch.). Le Carbonifère dans la région des Vosgres. B. S. G. H. 3 série. 'T. XV.

1888. Gossetet (J.). L'Ardenne. In-4 $4^{\circ}$ de $S 81$ pages, xxvi planches photographices, 1 carte, xi planches de coupes et 24 figures sur bois dans le texte. P'uris.

1888. de Margerie (Enm, de). Compte-rendu de l'Ardenne de M. Gosselet. Amuaire géologicute universel. T. V, p. 683.

1888. Meuvier (St.). Examen paléontologique du calcaire à Saccamina de Cussy-en-Morvan.

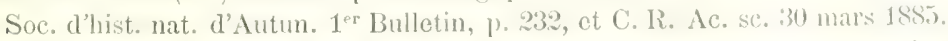

188S. Bertrand (Marcel). Sur la distribution géographique des roches éruptives en Lurope. B. S. G. F. $3^{\circ}$ série. T. XVI. 
188s. CEhlert (D.). Notice nécrologique sur M. de Koninck. B. S. G. F. $3^{e}$ série. T. XVI. 1889. Frech (F.). Das Französische Central-Plateau. Eine Skizze seiner geologischen Entwickelung. Zeitschift der Gesellschaft für Erdkunde zu Berlin. Vier-und-zwanzigster Band. Zweites und drittes Heft.

1889. Tschervichew. Notes sur le rapport des dépóts carbonifères russes avec ceux de l'Europe occidentale. Annales Suc. géol. du Nord. T. X VII.

1889. Fraipont (J.). Laurent-Guillaume de Koninck. Sa vie et ses ouvres.

1859. Bergerox. Etude géologique du massif ancien situé au sud du Plateau Central. Thèse inaugurale. Puris.

1889. CEhlert (1).). Notes sur les terrains paléozoiques des environs d'Eaux-Bonnes. B. S. G. F. $3^{e}$ série. 'T. XVII.

1S90. Le Verrier. Note sur les formations géologiques du Forez et du Roannais. Bulletin des services de la carte géologique détaillée de la France. No 15.

1830. Foųcé. Le Platean Central de la France (mémoire lu à la séance générale des 5 Académies en 1890). Rerue scientifique. T. XXXXVI, p. 546.

1890. Jubiex (A.). Résultats généraux d'une étude d'ensemble du carbonifère marin du Plateau Central. C. R. Ac. sc. 31 mars.

1890. de Ladiay. La dislocation du terrain primitif dans le nord du Plateau Central. B. S. G. F. 3 sćrie. T. XVI.

1890. De Launay. Note sux le terrain anthracifère du Puy-de-Dòme. B. S. G. F. $3^{e}$ série. T. \11.

1890. Lebescunte. Sur la présence du carbonifère en Bretagne. C. R. Ac. sc.

1890. Bézn r. Sur un gisenent carbonifère de l'étage de Visé, reconmu à Quenon en SaintAubin d'Aubigné. C. R. Ac. sc.

1890. ine la Taliée Poussin (Ch.). Compte-rendu de la session extraordinaire de la Soc. de Belgique tonce it Dinant les $1,2,3$ et 4 septembre 18s8. Ann. S. g. Belg. T. XVI, 2. live.

1891. De la Vallé Polssin (Chr.). Note sur les rapports des étages tournaisien et viséen de M. Dupont avec son étage waulsortien. Ann. S. g. Belg. T. XVIII, $1^{\text {re }}$ livraison.

1891-1892. Lonest (M.). Sur la présence d'un bane de calcaire á Echinides à la partie supéricue du calcaine il Crinoides exploite (presentation). Ann. S. g. Belg. T. XIX, $2^{\circ}$ livia1s(11.

1891-189:. Stanim (X.). Matériaux pour la flore et la faune du houiller de Belgique. Ann. Soc. teren. Belg. T. XIX, 3 livr.

1891-1892. de ta Varlée Poussix (Ch.). La coupe de la Chapelle a Hastière (présentation).

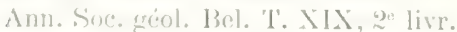

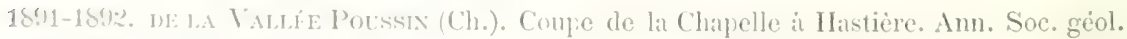
de bieler. 'T'. XIX, 3" livr.

1891-1852. 19: 1)ontonot (H. Résultats d'me excursion at la Chanelle (Hastière) et aux

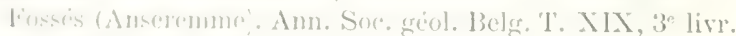

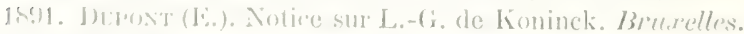

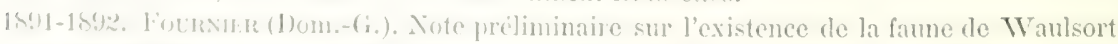

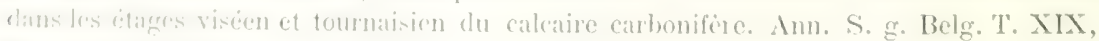
\%live. (Mnmoires).

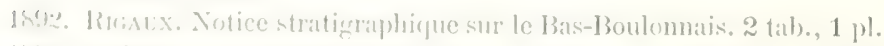

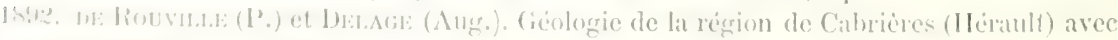

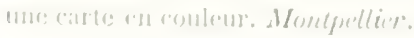


1892-1893. ne Donlodot (H.). Découverte du Waulsortien dans le bassin de Namur. Ann. S. g. Belg. 'T. XX, $1^{\text {re livr. }}$

1892-1893. Dewalque (Gr.). Sur quelques fossiles carbonifères du niveau $\mathrm{Vb}$ it Sprimont. Ann. S. g. Belg. T'. XX, $1^{\text {re livr. }}$

1892-1893. DE DORLODot (H.). Essai de classification du calcaire carbonifère de Belgique. Ann. S. g. Belg. T. XX, $1^{\text {re }}$ livr. (Bulletin).

1892-1893. Dewalque (G.). Sur le calcaire carbonifère de la carrière de Paire (Clavier). Ann. S. g. Belg. 'T'. XX, $1^{\text {re }}$ livr.

1892-1893. De Dorlodot (II.). Quelques réflexions á propos de la faune de Paire (Clavier).

Ann. S. g. Belg. T. XX, $1^{\text {re }}$ livr.

1892-1893. Dewalque (G.). Récif waulsortion de Biron (Ciney). Ann. S. g. Belg. T. XX, $1^{\text {ru }}$ livis.

1892-1893. Lonest (M.). Observation au sujet de la communication de G. Dewalque sur le récif waulsortien de Biron (Ciney). Amn. S. g. Belg. T. XX, $1^{\text {re }}$ livr.

1892-1893. Bayet (L.). Le calcaire carbonifère de Beaumont. Ann. S. g. Belg. T. XX, : livi.

1892. Dupont (E.). Les calcaires et schistes frasniens dans lia région de Frasnes. Bull. Soc. belge de géologie, paléontologie et hydrologie. Bruxelles. T. VI, fasc. III et dernier, 1). $1 \pi 1$.

1892. Meuvier (Stanislas). Etude micrographique sur le calcaire à Saccamina de Cussy-enMorran. Le Naturaliste. 15 aont 1892. Revue illustrée des sciences naturelles. $14^{e}$ année. No 131.

1893. de Lapparext. Traité de géologie. $3^{e}$ éd. Paris, Savy. In-8 .

1893. Rousser (J.). Etude stratigraphique des Pyrénées (thèse).

1893. Seunes. Le carbonifère dans la haute vallée d'Ossan. B. S. G. F. (3o séric). T. XXI. 1893-1894. de Donlonot (H.). Un dernier mot sur la coupe de Pierre-Pétru. Ann. S. g.

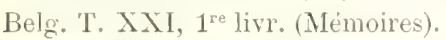

1893-1894. Lohest (M.) et VELGE (G.). Sur le niveau géologique du calcaire des Écaussines. Ann. S. g. Belg. T. XXI, $3^{\circ}$ livr.

1893-1894. Destinez (P.). Nouveaux fossiles des calcaires de Paire (Clavier). Ann. S. g. Belg. T. XXI, $3^{e}$ livr. (voir t. XX. Bulletin, p. Lxxin. Dewalque).

1893-1894. Lonest (M.). De la présence du calcaire carbonifère inférieur au bord sud du bassin de Namur, à l'est de Ifuy, et de ses relations avec le calcaire carbonifère inférieur du bassin de Dinant. Ann. S. g. Belg. T. XXI, $3^{\circ}$ livr. (voir observations de II. de Dorlodot à la page 281).

1893. Julien (A.). Sur l'origine glaciaire des brèches des bassins houillers de la lirance centrale. C. R. Ac. sc, 24 juillet.

1893. Julien (A.). Sur la géogénie et la stratigraphie des bassins houillers de la lirance centrale. C. R. Ac. sc. 21 aout.

1893-1894. Briart (A.). Géologie des environs de liontaine-l'Evêque et de Landelies (avee 2 pl.). Ann. S. g. Belg. T. XXI, $1^{\text {re }}$ livr. (Mémoires).

1893. De Peetz (II.). Etude sur lit faune de l'étage de Malewka-Mourajevnia. Tratsaux de la Société des naturalistes de Suint-Pétersbourg. Section de géologie et de mineralogio. Vol. XXII.

1894. Frecu (F.). Die Karnischen Alpen. 1 vol. Halle.

1894. Dormal (V.). Quelques mots sur le caleaire carbonifère dans la vallée de la Méhaigne. Ann. S. g. Belg. Procés-verbal (projet) de la séance du 21 janvier 1S91, p. Xurv. 
1894. Bourgeat (Abbé). Classification du carbonifère du Nord. Association française pour l'avancement des sciences. $23^{\circ}$ session. Caen.

1894. Bertrand (Marcel). Lignes directrices de la géologie de la France. C. R. Ac. sc. T. CXVIII, p. 258.

1894. Dollfus (G.). Recherches géologiques sur les environs de Vichy (Allier) avec 5 pl. Paris.

1894. Julien (A.). Sur le synchronisme des bassins houillers de Commentry et de SaintEtienne et sur les conséquences qui en découlent. C. R. Ac. sc. 15 janvier.

1894. Jublex (A.). Anciens glaciers de la période houillère dans le Plateau Central de la France. Annuaire du C. A. F. $21^{e}$ vol.

1895. Lohest (M.). Sur l'íge du calcaire de Lens et de la dolomie de Cambron. Ann. S. g. Belg. T. XXI, $1^{\text {re }}$ livr.

1895. Lonest (M.). Sur le parallélisme entre le calcaire carbonifère des environs de Bristol et celui de la Belgique. Ann. S. g. Belg. T. XXII, $1^{\text {re }}$ livr.

1895. Dewalque (G.). Sur Spirifer mosquensis Auct. Procès-verbal de la séance du 17 février.

1895. Destinez (P.). Sur les fossiles du calcaire à Paléchinides de Poulseur. Ann. S. g. Belgo. Procès-verlal de la séance du 17 mars (projet).

1895. Destinez ( $\mathrm{P}_{\text {. }}$ ). Recherches sur les fossiles du marbre noir viséen de Petit-Modave. Procès-rerbal de la séance du 19 mai. Bull. S. g. Belg. (projet).

1895. Forir (II.) et Lonest (MI.). Découverte du niveau à Paléchinides dans la bande carbonifere de la Meuse. Procés-verbal de la séance du 16 juin. Bull. Soc. géol. de Belgique (projet).

1895. Soreir, (G.). Note sur la faune du marbre noir de Denée Vb. Soc. géol. de Belgique. Procès-verbal de lat séance dı 20 juillet 1895 . Bull. S. g. Belg. (projet). 


\title{
TABLE DES GENRES FT ENPECLS GARBONIFERES
}

\author{
DE LA FRANCE CENTRALE
}

MENTIONNÉS OU DÉCRITS DANS L'OUVRAGE - trieconterductylus. Miller......... 130 Amplexus corculloides, Sow............... lit Archeocidaris Grüneri, nov, sp........... 12.3

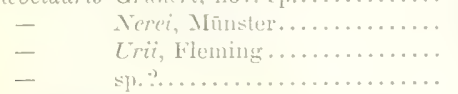
Athrris ambrigur, J. de C. Sow. .............. - ingets, de Fon.

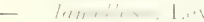

- planostelcutre I’hill.

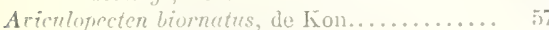

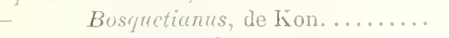

- colutus, M'Cor................

- dupliciratiatus, de lion.......

- infrectus, de Kion..............

- plagiostoma, de Kion..........

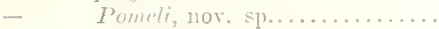

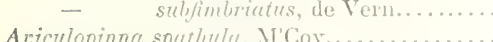

Bairdia, MCoy ....................... II

Baylea spiruta, de lion.................. in

Bellerophon scalifer, de lion. ............. 3:

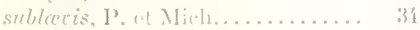

Brachymetopus Duponti, nox, s1.............

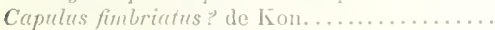

- uncus? de Fion....................

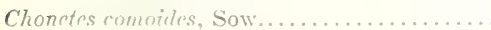

- concentrica, de Kon...............

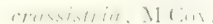

- Delmuniuna, de Kion.

- Gircudi, nov, spr.................

- Jourdani nov. sp me

- Luquessiuna, de Kon...............

- Murchisoni, nov. sp.................

- papilioneres, Phill................. rerrivlute. d1 1, h.

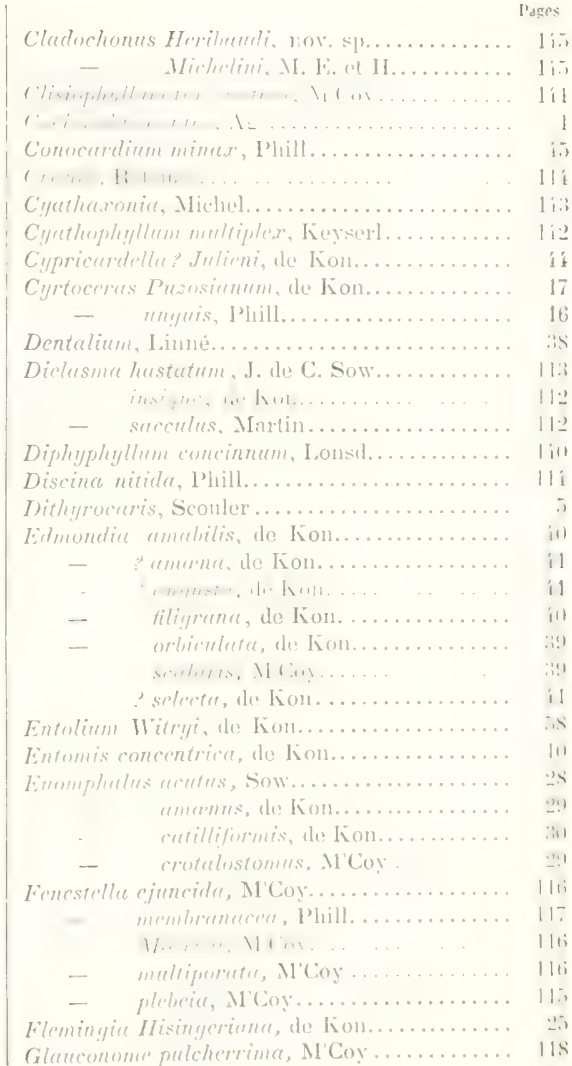




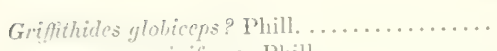
seminiferts , Plill..

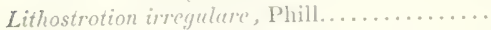
junceum, Flem.

Lciopteria hirundo, de Kon...

Lunulata, Phill.

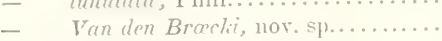

Lepetopsis Busscherianus, de Ryck.........

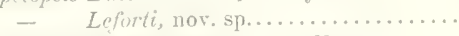

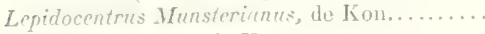

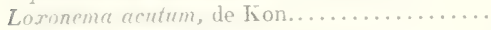

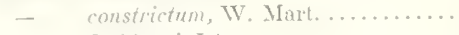

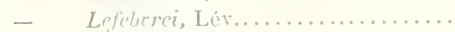

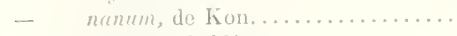

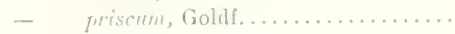

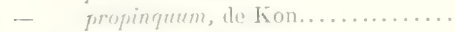

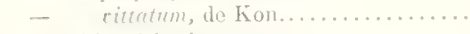

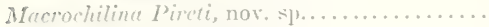

Marinie glabra, Martin................

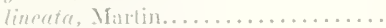

Melonites Gumelryi, Julien...............

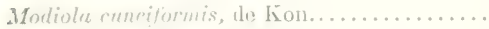

- fusiformis, de Kon...

Monticulepora inlletra, de Kon............. tumide, Phill..

Nucula, do Lam...................

Nuculune, I.ink. .

Nuticopisis funispire, Phill..

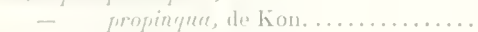

Nantilus astellatus, M'Coy....

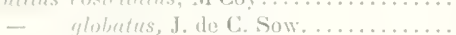

- subsuleretris, Phill..

- sulmotus, Sow.....

Drthoestus Gombliessianum, do Kon........... Mrerinimum, do: lion...

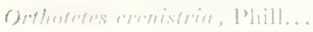

- Mresmpimuter, Mart...

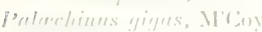

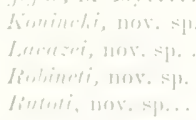

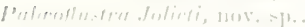

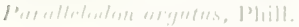

biarrientes, l'oril..

Lambides, dre Kint1......

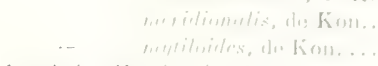

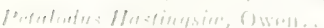

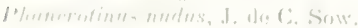

seryoule, is: Kinn

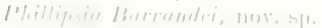

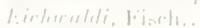

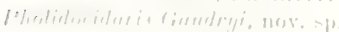

P'uges. | 1'icile.

Platyschisma helicoides, J. de C. Sow.........

Polyporra Goldfissiana, de hon............. IIs

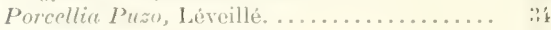

Portlockia pytgmeer, de Kon............... 25

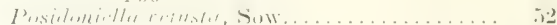

Poteriorrinus crusius, Miller............. 1:34

plicatus, Austin............. 138

frrliutus, distin............ 1:37

spissus, de lion. et Le II....... $1: 37$

Productus aculeutus, Martin. ............... .3

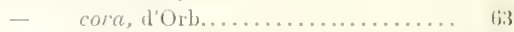

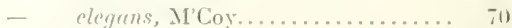

- fimbriutus, J. de C. Sow.......... 72

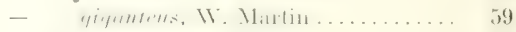

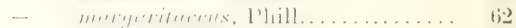

- punctatus, Martin ............. 159

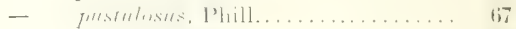

- undetus, Defr................ bit

- semireticulatus, Mart. ........... i.

- scabriculus, Martin............ il

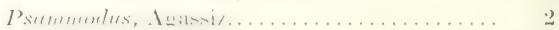

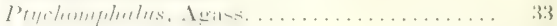

Retaiu Bucliunn, de Kron. ................ 1013

- serpentince, de lion................ wi

- vlothrix de Kon.................. bis

Rhinchonella usutirugute, de kion........... 1199

- anguluta, Linné............ 111

- pleurodon, Phill.............. 111

- pergnus, Martín............ 1110

Sanguitrolites inconspicutes, de Kion......... 12

- tricostutus, Portl............ 12

Sculites humilis, de lion................ ㄴt

Schirostome cutillus, Martin................ 31

- creteriforme, de Kion......... 30

Solenopis Bielenestii, nov. sp............ i3

- pelugicus, Goldf............. it

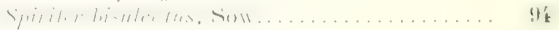

- cremutus, de Rion................ 91

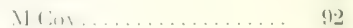

sur................

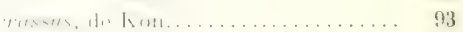

- distarts, J. de C. Sow.............. 9.

- duplicicoste, l'hill. .............. \$9

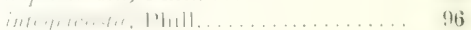

- Limentumes, de lion.............. 93

- Tornuerensts do Kon............... 90

Irii, lilnm................... 99

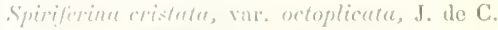

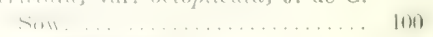

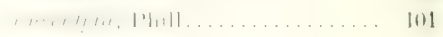

- purtiti, Portl................ 161

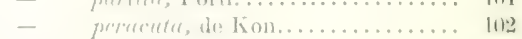

Streprerolles concoluters, to lion............ 27 1)ionysii, D), to Montfort........

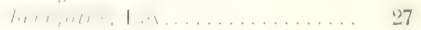
pilmpsidens, Jhill.......... 28

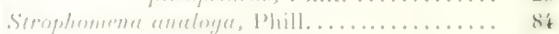

Syringupare distens, Fischer............... tí 


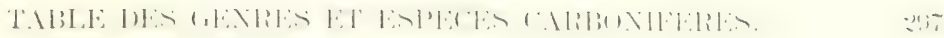

Syringopore iramelose, Golde.

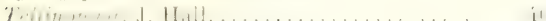

Turhonitelle biserirelis, Plill.

Waagenia Ferussuci, d'Orb.

Zaphrentis, Raf. et Cliti..

\section{I'LANTES}

Archuocratemites reuliutus, Sum

Bornia radiata, Sch.......... . . Ii!t

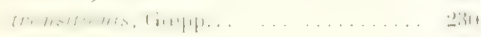

Calamites transitionis, Gepp............ 1i!!

Cardiopteris? gigantea, Ebray . . . . .............

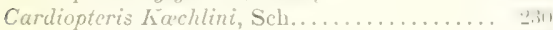
frondose, Sch. .............. li!! polymorpha, Schimp............ lit

Clepsydropsis duplex, Unger et Vill. Schimp. 23n
I't,

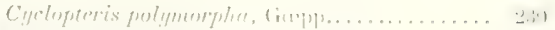

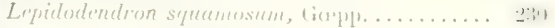

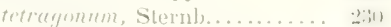

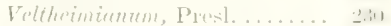

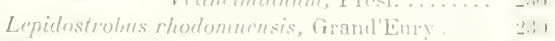

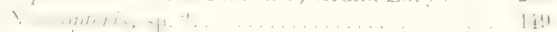

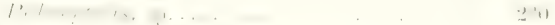

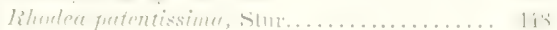

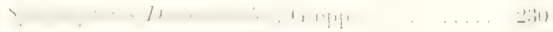

- Gopperti, Eltiness _. 23,

- deguns, brong............... 2..t

- filifere, stur...............

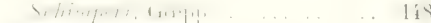

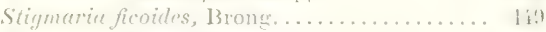

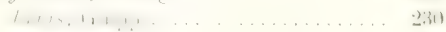

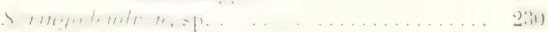

Tortmo Lipoldi, stur . _ _......... lis 


\title{
TABLE GÉNÉRALE DES MATIYRES
}

\author{
INTROIUCHION
}

(im-ideratum- pritinimain.

Historique. - 1751, Desalliel d'Argenville. - 1795, Alléon-Dulae - 1797, Passinges. 1825, Valuy. - 1830, Dufrénoy. - 1837, Leymeric. - 1810, Rozet. - Id., de Verneuil. - Id., Grüner. - 1811, Jufrénoy. - Id., Elie de Beammont. - 1812, Viquesnel. - 1844, Bonlanger. - 1846, Grïner. - 1847, Jourdan. - 1848, de Verneuil. - 1849, Drian. - 1851, Murchison. - 1855, Fournet. - 185\%-1859, Grüiner. - 1868, Grüner (Creuse). - 1872, A. Julien. - 1873, de lioninck et Jourdan.

I'lin

\section{DESCRIPTION IDES FOSSILES}

Classe des Poissons.................................... 1

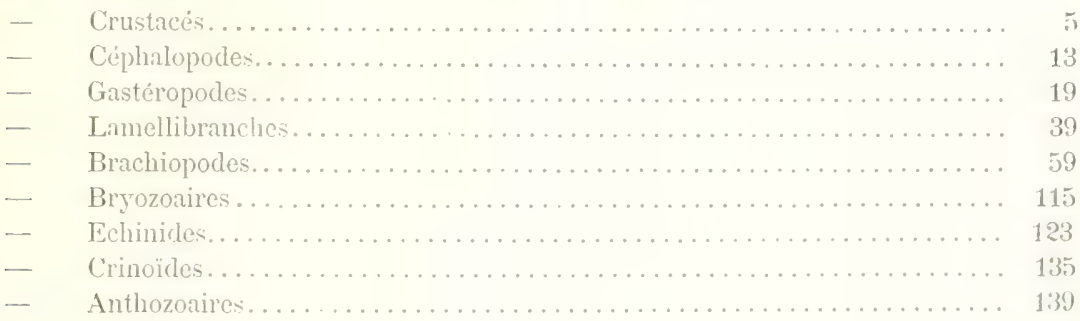

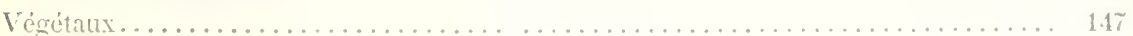

ETUDE CRITIQUE DES FAUNES C.IRBUNIEERLS MARING DL MOTR T. ET IUT PLATEAL CEYTRAL

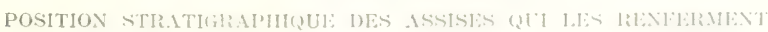

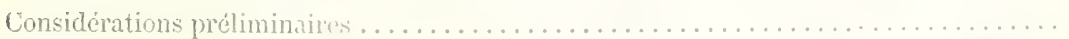

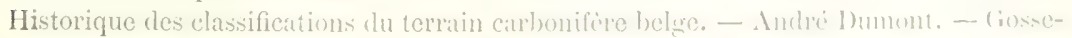

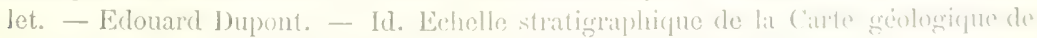

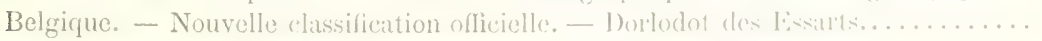

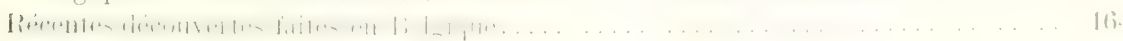




\section{MORVAN}

La Varville :

Liste des cspèces carboniféres de La Varville...................... 16 .

Domaine des Roches :

Faune du domaine des Roches ou du champ de la Barrette.................. 1lis

Siguret :

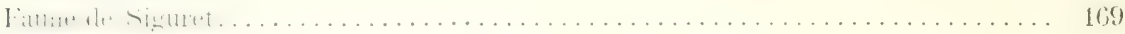

Espèces communes il La Varville et au domaine des Roches............... 170

et à Siguret........................ 1 \%

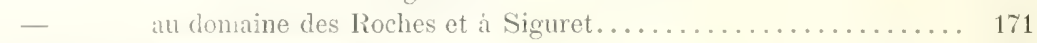

Ces trois faunes sont synchroniques............................ 1 .1

COMPARATSON AYEC LES FAUNES BETGES

Fame de La Vartille. Analyse................................ 1 1

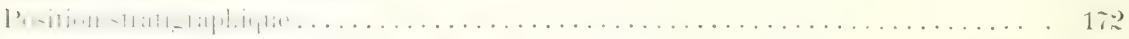

liane du Domaine des lioches, Analyse, etc.......................... 172

l'

FX.MEX COMARATI" DE LA FAUNE DE PAIR ET DES FAUNLLES IUU MORYAN

Liste des fosiles du marbre noir tb de Pair (Clavier).................. 1it

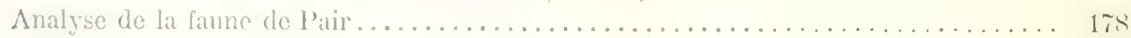

Espéces communes it Pair et it Ia Varville........................... 1is

- $\quad$ - et an clomaine des Roches................... 179

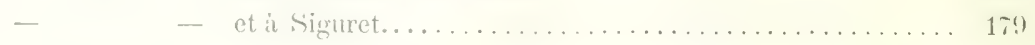

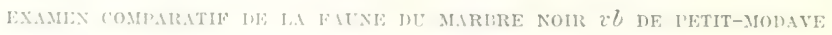

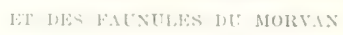

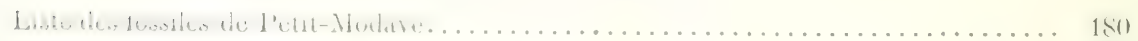

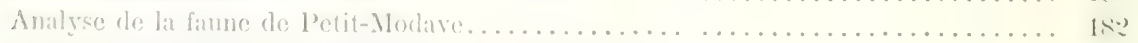

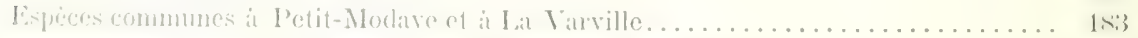

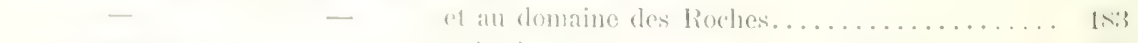

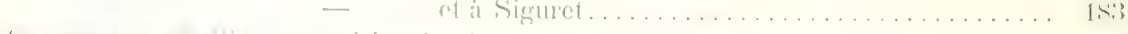

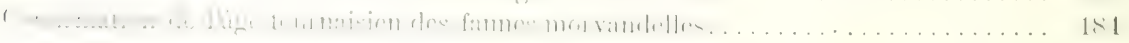

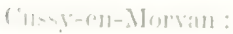

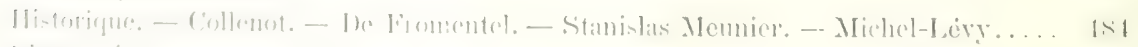

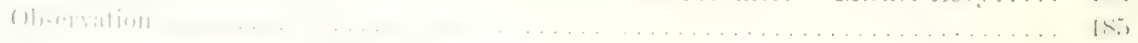

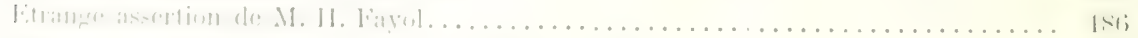

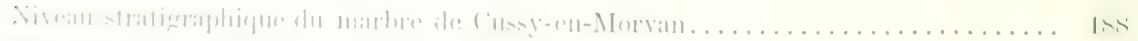

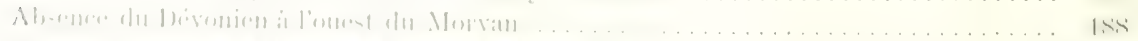

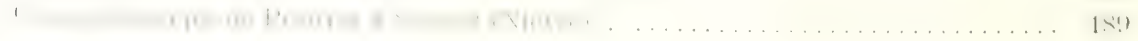


PIAEAU CHNTRAL.

Régny :

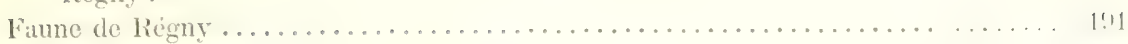

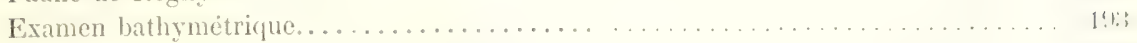

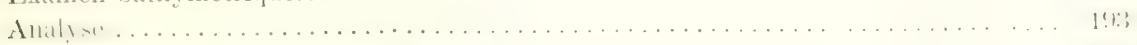

Espèces communes à Régny et à Dinant......................... 191

Conparaison des faunes de Régny et du nurbre noir de Denée............. $1: 1$

Liste des fossiles du marbre noir de Dene. . . . . . . . . . . . . . . . . . . . 1!n

Espèces communes it Régny et it Denee.......................... 1!n;

Conclusion. - Position stratigraphique de Regny.................... Ini

Néronde :

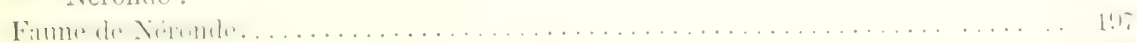

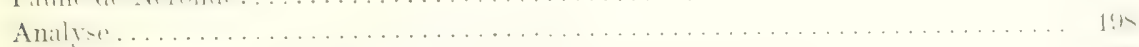

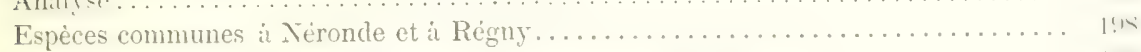

\begin{tabular}{|c|c|c|}
\hline - & -. & et aux diverses assises de Dinant............ \\
\hline & - & et it Visé... \\
\hline & - & $\ldots \ldots \ldots \ldots \ldots$ \\
\hline
\end{tabular}

Position stratigraphique de la faune de Neronde..................... 1:m

Montmain :

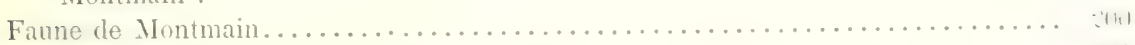

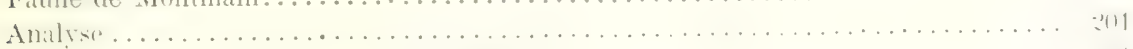

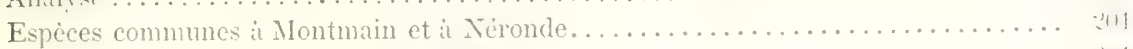

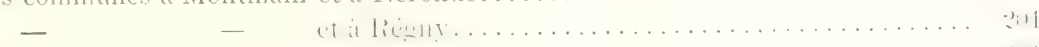

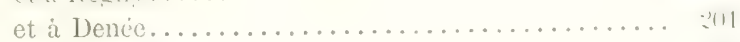

Position stratigraphique de la faune de Montmain....................

Le Gouget :

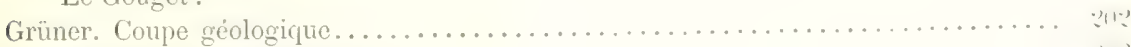

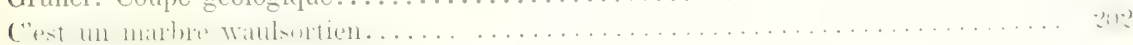

Montagny, Combres et Thizy :

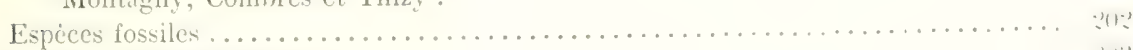

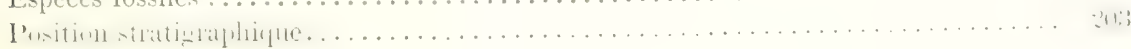

Propières :

1 ble

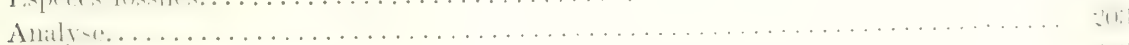

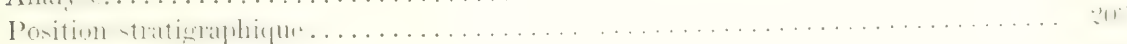

Letra:

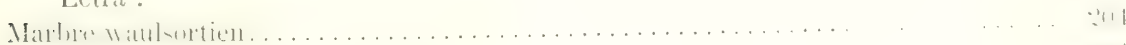

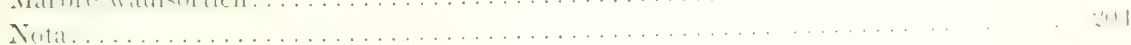

Saint-Germain-Laval :

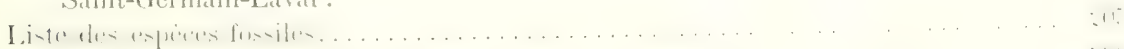

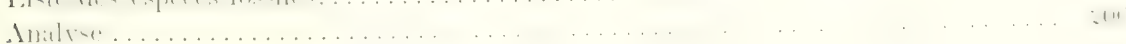


Especes communes à Saint-Germain-Laval et à Régny....................

\begin{tabular}{|c|c|c|}
\hline - & -- & et à Néronde... \\
\hline & - & 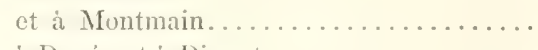 \\
\hline
\end{tabular}

Saint-Germain-Laval relie stratigraphiquement Ferrières et hegny . . . . . . . . 208

Cinp

L'étage tournaisien n'existe pas dans le Plateau Central.................. 208

Ferrières :

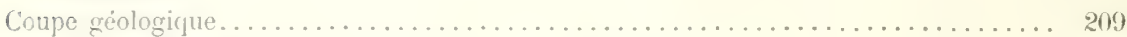

Faune du marbre. Il est waulsortien............................... 210

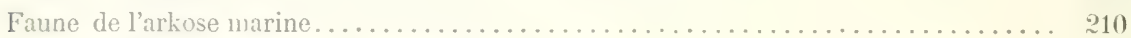

Faune des grès silicifiés de Cheval-Rigon ........................... 210

Espèces communes à Ferrières, y compris Cheval-Rigon et Régny............. 211

- $\quad$ - et Saint-Germain-Laral.... 212

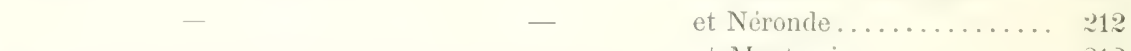

Syruhnonismo hes gisenuents earbonifines dh Tort

et Montmain ............ 212

L.Ardoinie :

IIistorique. - Murchison, 1850. - A. Julien, 1872..................... 213

Coupe grelogrigue du gisement de l'Ardoisière, près Vichr.................. 213

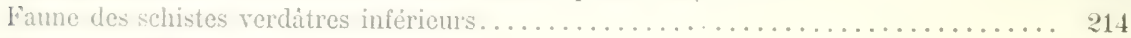

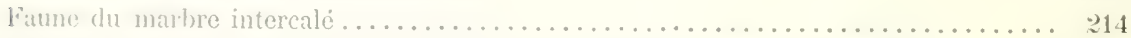

Identité absolue du marbre de l'Ardoisiero et du marbre belge des Pauquys........ 215

Wane des calschistes gréseux................................ 215

Analyse de lat fame des calschistes............................. 218

Identite de la faune des calschistes et de la faune belge de Vise............... 218

Genres et espieces de fossiles communs it l'Ardoisière, Régny, Néronde, Saint-Ger-

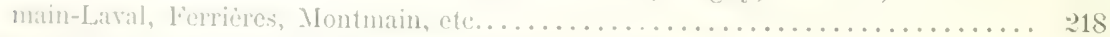

lispeces speciales at Rigny et autres gisenents du mème ige.............. 290

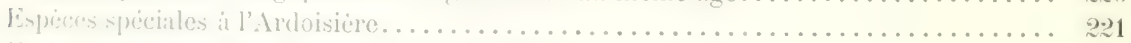

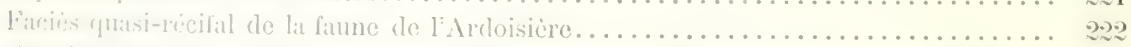

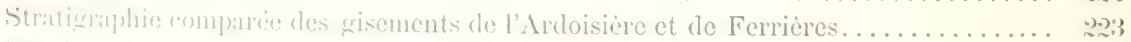

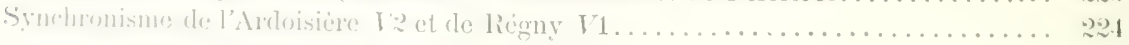

livanx ( (19mise):

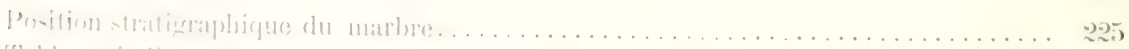

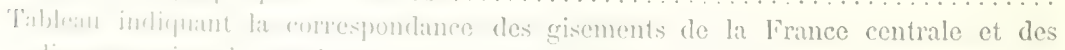

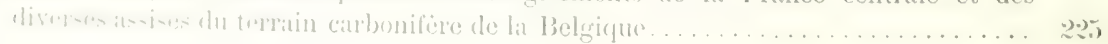

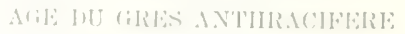

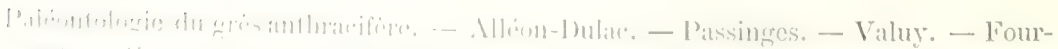

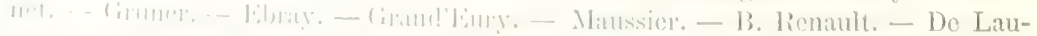

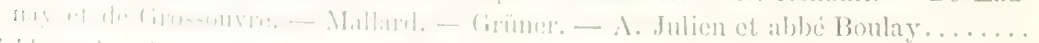

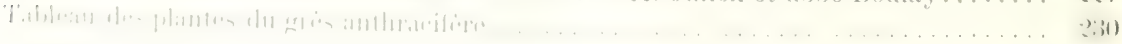




\section{POSITION STRATIGIRAPHQUE DU GRES ANTHRACIFERE}

Ilistorique. - 1830, Dufrénoy. - 1840, Grüner. - 1841, Dufrénoy. - 1855, Fournet. - 1857, Grüner. - 1873, Douvillé. - 1879, AIaussier. - 187\%, Grand'Eury. . æ231 Age du grès antluracifère du Morvan .............................

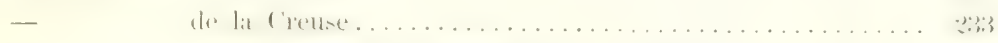

'Tableau général de synchronisme des assises carbonifères de la France centrale....... 2.31

\section{EXAMEN CRITIQUE ILS FAUNES CARBONIFERES MARINES DE LA FRANCE ET DE QUELQUEN LOCALITES ETRANGERES}

Localités tournaisionnes:

Hook-Point (Irlande), John Kelly. - M'Coy. — De Koninck. . . . . . . . . . . . . . . Malowka-Murajewna (Russie), P. de Semenow et W. von Möller. - De Koninck.

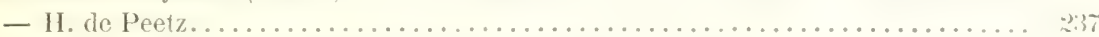
Amérique du İord. - Calcaire de Burlington (Iowa), de Quincy (Illinois), d'Hannibal

(Missouri) et Tennessee ..........................................

\section{RELATION DES GISEMENTS DU PLATEAU CENTRAL AVEC LES AUTRES GINEMENTS} FRANCAIS

Cotentin :

Montmartin et Régneville. - Eugène Eudes-Deslongehamps (185t . . . . . . . . . :

Massif breton :

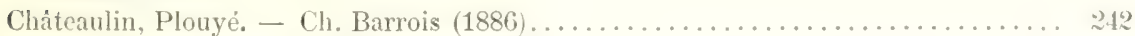

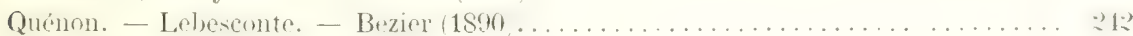

Sablé. - De Verneuil (1839), — Guiller $(1886) \ldots \ldots \ldots \ldots \ldots \ldots \ldots \ldots \ldots \ldots \ldots \ldots$

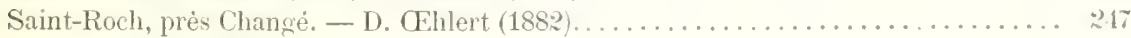

Massif vosgien :

a. Partie méridionale.

Plancher-les-Mines. - Fournet et Jourdan (1855) ................... 2.1s

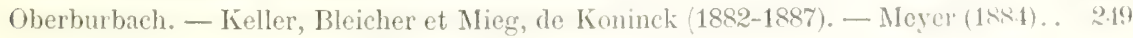

6. Partie septentrionale.

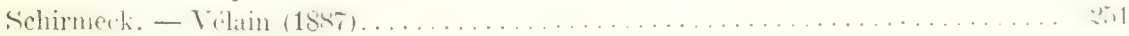

Montagne Noire:

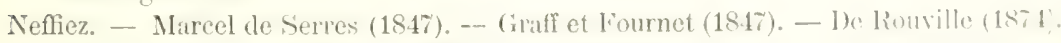

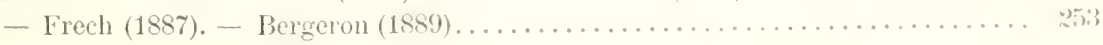

Pyrénées:

Larbont. - Lonis Lartet (18\$1 et 1887). - Roussel $(1893) \ldots \ldots \ldots \ldots \ldots \ldots \ldots \ldots$. 25.

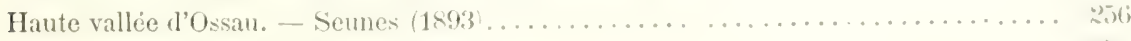

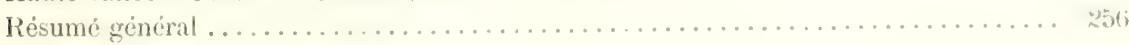


TR MSGRESSION DE LA MER CARBONIFERE DANS LE MORVAN ET LE I'LATEAU CENTRAL, EN FRANCE ET EN EUROPE

Terrains primitifs et primaires de la France centrale.................. 259

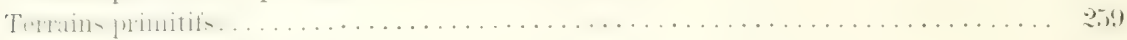

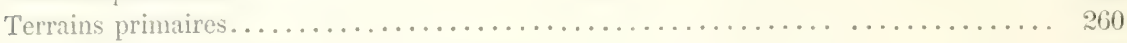

Nature et ordre d'apparition des roches granitiques dans le Platen Central........ 260

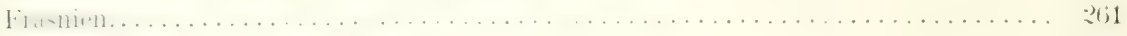

Frasnien supérieur et Famennien............................ 262

Transgression de la mer carbonifère............................ 262

\section{FASAI DE PARALLELISUE ENTRE LES TRANSGRESSIONS MARINES IDES EPOQUES CARBONIFERE ET HELVÉTIENNE}

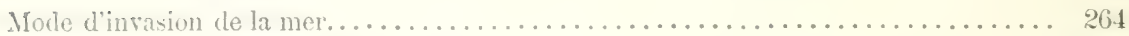

Canse du refoulenent des mers.............................. 204

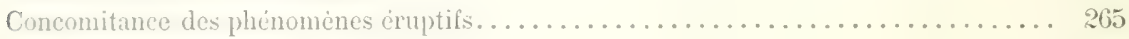

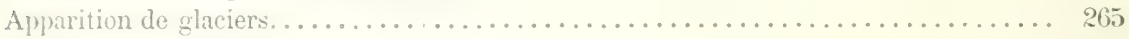

\section{CONITTIONS NECESSAIRES I LA CREATION ET AU DETELOPPEMEXT} DES GLACILRS EY GEXERAL

Cés conditions sont au nombre de trois. Elles étaient réalisées au début de la période

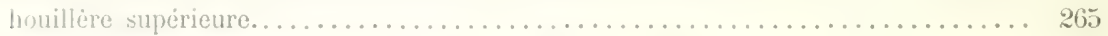

Climat de l'ípoque houillere supéricure. - A. Grisebach................ 266

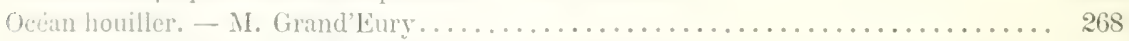

Alpes hercyniennes. Leur hauteur dans lo Platean Central, en Belgique et dans les

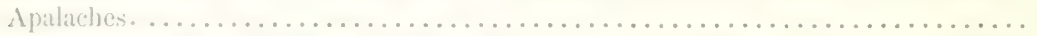

formidables brèches houillères du Plateau Central, a caractères exclusivement gha-

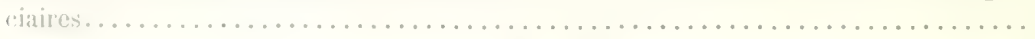

L'éciation de la temperature a l'épocue houillere, n'était pas un obstacle í l'existence

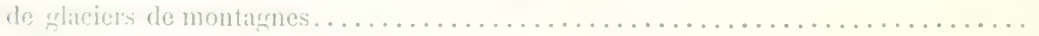

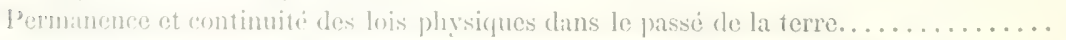

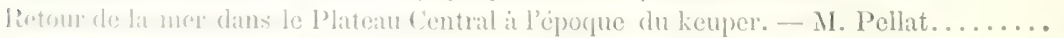

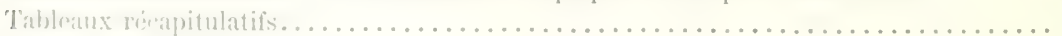

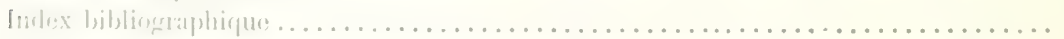
T'able des gemes et espeecs carbonifères de la lirance centrale, nentionnés ou décrits

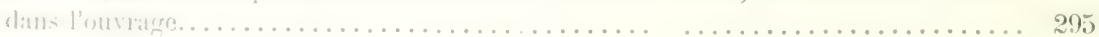

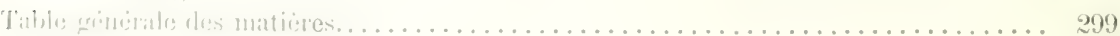

FIX I)I: LA TABLI:

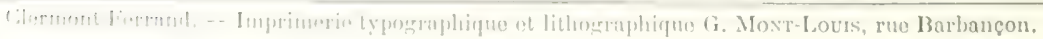




\section{LEGGEDES DES PLANCHES}




\section{I.EGENDE DE LA PLANCHE I}

Fis. 1. Productus semireticulatus, Martin. Valve ventrale. Lat Varville.

Fig. 2. Productus semireticulatus, Martin. Valve ventrale, bord frontal. La Varville.

Fin. 3. Proluctus semireticulatus, Martin. Valve ventrale. Domaine des Roches.

Vi.. 4. Productus semireticulatus, Valve dorsale. Donaine des Roches.

Fir. i). Chonetes Giraudi, nov. sp. Empreinte d'une valve dorsale. La Varville.

Frr. 6. Chonetes rariolata, d'Orbigny. Empreinte interne d'une valve dorsale. Les Roches.

Iir. 7. Chonetes curiulutu, d'Orbigny. Empreinte externe de valve dorsale. Propières.

Firi. S. Chonetes crassistriu, MCOy. a ventrale; b dorsale sumontée de l'aréa de la ventrale. Siguret.

Fis, g et 10. Chonetes crussistria, M'Coy. Les memes grossies 4 fois. La figure 10 est mal éclairée et parait concave.

Fir. 11. Spirifer tornacensis, de Kon. Empreinte de la partie cardinale des deux valves. La Varrille.

Fis. 12. Spirifer Romeriunus, de Kon. Moulage le l'empreinte de la figure XII. La Varville.

"Enpreinte interne d'une rentrale de $1 \%$ semireticulatus, Martin.

Fu, 13, ! Enpreinte en creux de la partic umbonale des ralves de Sp. Roumerianus.

ic ().thotetes, sp.?

d Aviculopecten, sp.?

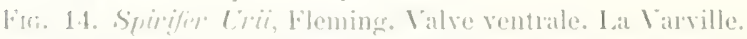

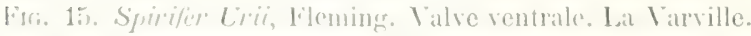

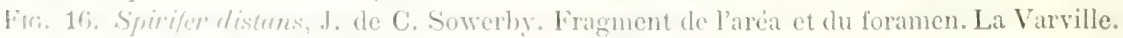

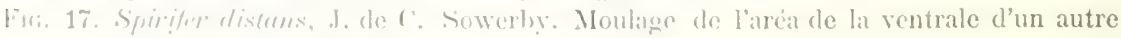
yniciment. Ial liaville. 

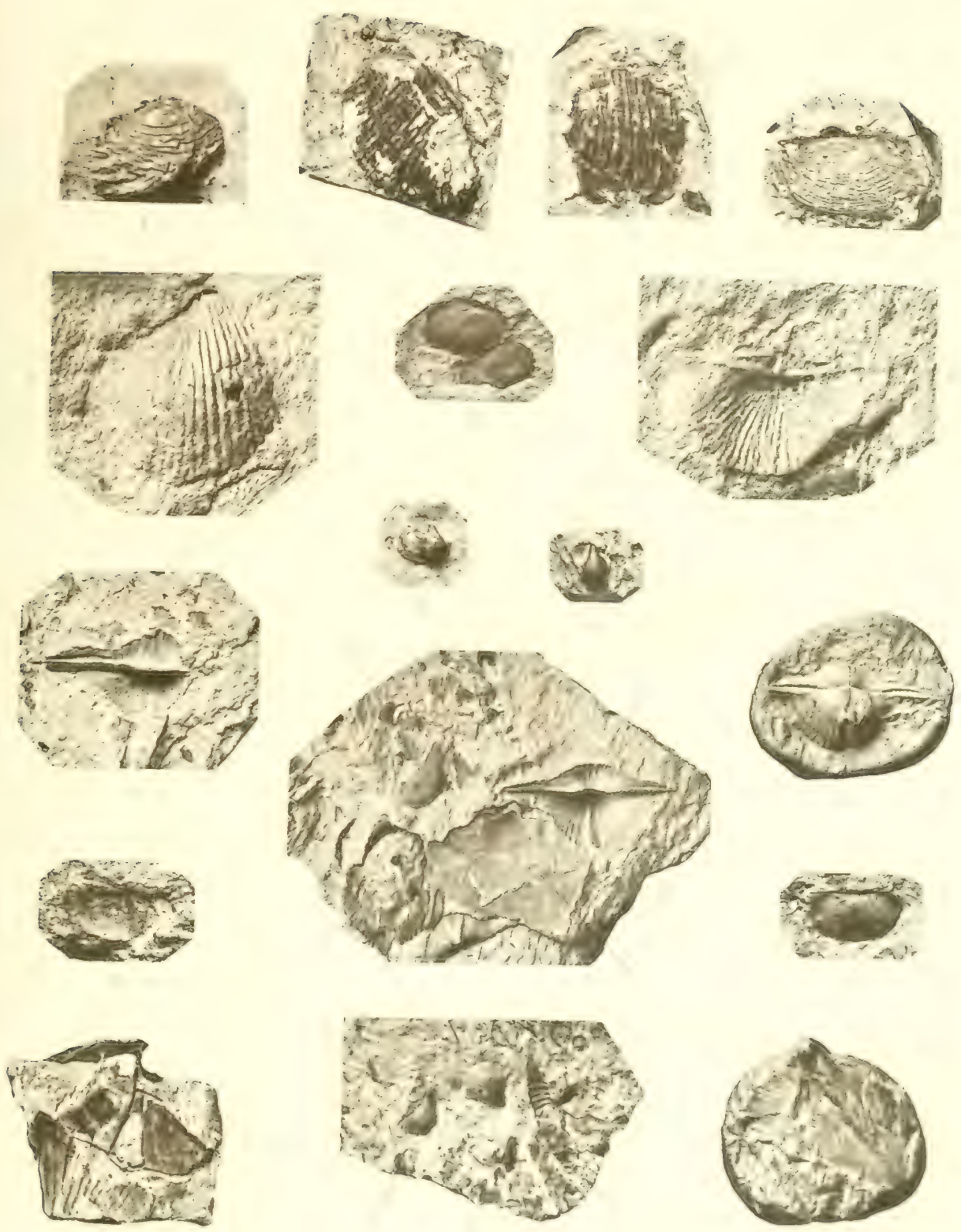




\section{I.EGENDE DE LA PLANCHE II}

Fig. 1. Syirifer tornacensis, de Kon. Empreinte en creux de la surface externe d'une ventrale. La figure mal éclairée semble indiquer une valve en relief. La Varville.

Fic. 2. Spirifer tornacensis, de Kon. Moulage de la même.

FIG. 3. Spirifer tornacensis, de Kon. Empreinte en creux de la partie umbonale de la coquille. La Varville.

Fig. 4. Spirifer tornacensis, de Kon. Moulage de la même.

Fı. 5. Spirifer tornacensis, de Kon. Autre spécimen. Même localitè.

Fis. 6. Spirifer tornacensis, de lion. Autre spécimen montrant nettement les deux aréas.

La Varville.

Fig. 7. Spirifer tornacensis, de Kon. Empreinte d'une valve dorsale. La Varville.

Fig. 8. Spirifer tornacensis, de Kon. Moulage de la mème. La Varville.

FIs. 9. Murtiniu lineutu, Martin, sp. Moulage naturel de la cavité interne d'une coquille complète. Vu du cóté dorsal. La Varville.

Fìis. 10. Martinia lineata, Martin, sp. Vu du côté ventral.

Firi, 11. Martinia lineata, Martin, sp. Moulage de la figure XII.

Fici. 12. Martinia linecuta, Martin, sp). Enpreinte externe vue du cóté ventral.

Fig. 13. Martinie linecta, Martin, spr. Empreinte externe d'un fragment de valve dont les bandes concentriques el les trous des épines sont trés nettement visibles.

Fis. 14. Spiriferinu pertitu, Portlock. Moulage d'une ventrale. Siguret.

Fir. 15. Spiriferinu pertiu, Portlock. Valve dorsale. Siguret.

lini. 16. Spriviferinu peracuete, de lion. Valve ventrale. La Varville.

Fis. 17. Espece incleternince. Peut-etre la meme? I a Varville. 

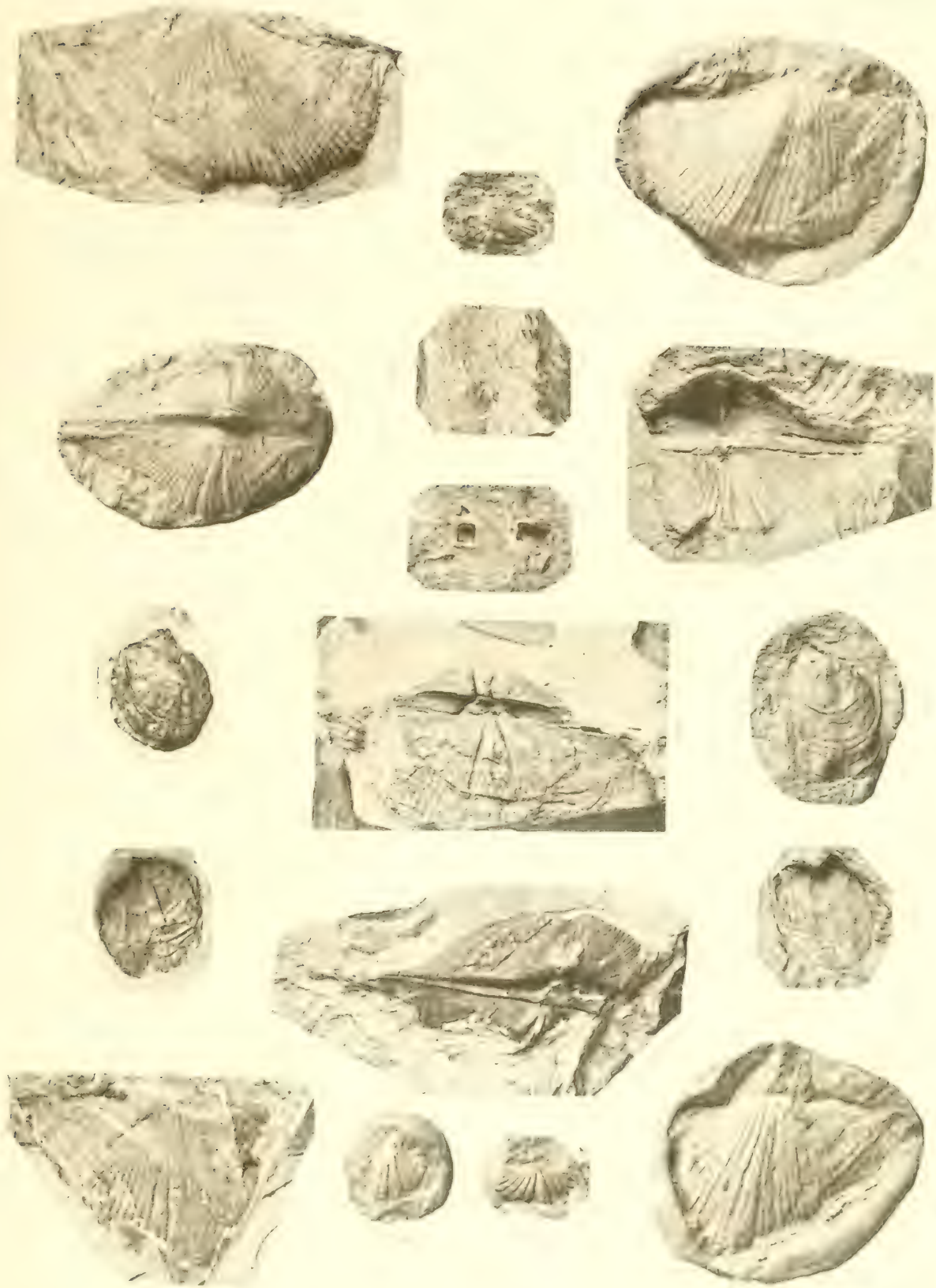




\section{I.ÉGENDE DE LA PLANCHE III}

Fiti. 1. Orthis Michelini, Lév. Empreinte externe de la valve dorsale avec un fragment de test. La Varville.

Fis. ?. Orthis Míchelini, Ióv. Moulage de la mème.

FIG. 3. Orthis Michelini, Lèv. Empreinte externe d'un autre spécimen. Mème gisement.

FIss. 4. Orthis Michelini, Lév. Moulage de la même.

Fig. ․ Orthotetes crenistria, Phill. Empreinte de la dorsale. La Varville.

FIG. i). Orthotetes crenistria, Phill. Empreinte de la dorsale. La Varville.

FIs. T. Orthotetes crenistria, Phill. Lmpreinte de la dorsale. La Varville.

l'Ir. s. Strophoment phomboidalis, Wahlenb., var. analoga, Phill. Empreinte externe d'une valve dorsale. La Varville.

Firs. 4. Strophomena thomboidulis, Wahlenb. Partie viscérale. La Varville.

Firi. 11. Rhynchonelle acutirugata, de Kon. Fragment d'une valve dorsale. La Varville.

Firf. 11. Empreinte en creux d'une valve ventrale. La Varville.

Fíri. I:. Moulage de la même.

Fir. 13. Empreinte interne d'une valve. Li Varville.

Firi. 11. Spirifer tornacensis, de Kon. Moulage d'une valve dorsale aplatie et déformée par pression. La Varville.

l'ur. Li. Athyris lamellosu, Léveillé. Empreinte interne des deux valves. La Varville.

Firi. 16. Athypts Roissyi, Léveillé. Moule interne vu du cóté dorsal. La Varville.

11.. 17. Altupris Rerssyi, Léveille. Vu du coté ventral.

l'ki. 1- Diedusmu insigne, de Kion. Empreinte vue du cóté de la dorsale. Le crochet de la ventrale a été brisé. I a Varville.

Fir: I'. Lietziu serpentince, de Kon. Valve dorsale. Siguret (figure mal éclairée, paraissant roncave). Lo méme fragment de roche fossilifère montre Bellerophon subleveis. Pot. et Mich., of P'uluedumes Riobineti, nov. sp. 

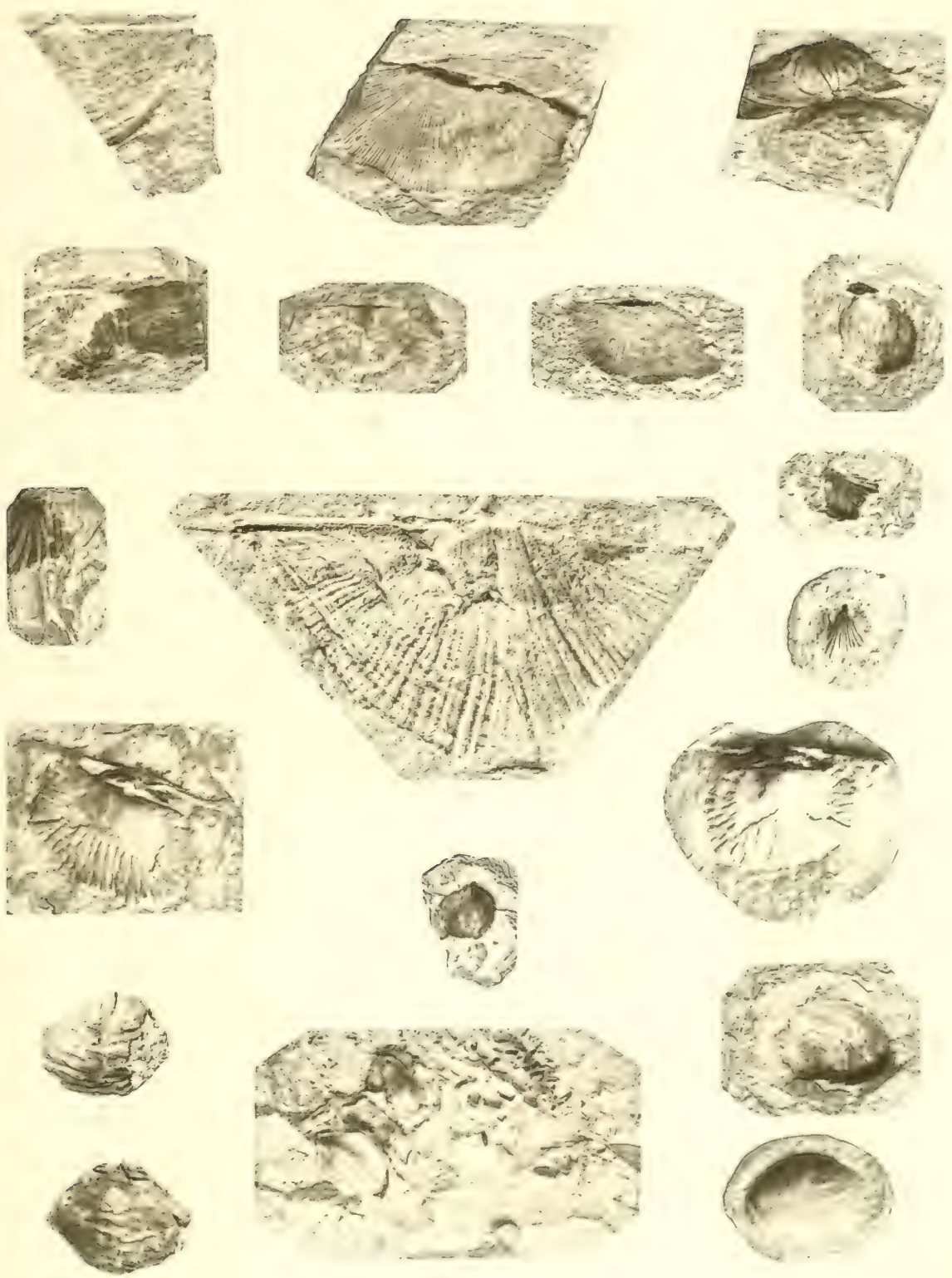




\section{LÉGENDE DE LA PLANCHE IV}

Frg. 1. Orthoceras Murtinimum, de Kon. Empreinte d'un fragment. La Varville.

F1G. 2. A viculopecten Pomeli, nor. sp. Empreinte esterne. Domaine des Roches.

Fig. 3. Aviculopecten ingratus, de Kon. Empreinte externe d'une valve droite de jeune individu. Domaine des Roches.

Fiss. 1. Aviculopecten ingratus, de Kon. Moulage de la mème.

FIci. 5. Entolium Witryi, de Kon. Empreinte interne d'une valve. Siguret.

Fis. 6. Leiopteriu Van den Broecki, nov. sp. Valve droite. La Varville.

Fro. i. Purallelorlon bistriutus, Portl. Valve droite à demi engagéedans la roche. La Varville.

Virs. \. Purallelodon bistriutus, Portl. La même grossie quatre fois.

Firi. 9. Lepetopsis Leforti, nov. sp. La Varville.

Fici. 11. Bellerophon sublecuis, Potiez et Michaud. La Varrille.

Fú. 11. Porcellia Puso, Léreillé. Empreinte externe. Siguret.

FIfi. 12. Porcelliu Puzo, Léveillé. Moulage de la mème.

Frri. 1:. Schizustama crateriforme, de Kon. Fragment d'un spécimen montrant les 4 premiers tours. Siguret.

Fir. 11. Schizostomu cruteriforme, de Kon. Moulage d'un autre spécimen. La Varville.

Fin. 1.. Strapurollus hevigutus, Léveillé. Spécimen vu par la partie inférieure. La Varville.

Firi. 1‘. Sculites humilis, de Kon. liragment montrant les 3 premiers tours de spire. siguret.

Fin. 12. Phunerotinus Serpulu, de Kon. Eimpreinte d'un fragment montrant les 3 premiers tours. Siguret.

Fin. 1s. Copulus uncus? de Kon. Empreinte interne. La Varville.

Fis, 19. Vuticopsis prupinque, de Kon. Limpreinte externe d’un spécimen écrasé. La Varville.

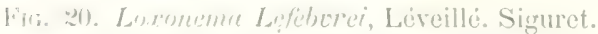

livi. 21. Lotronemu "utum, de Kon. Monle interno. La Varville. 

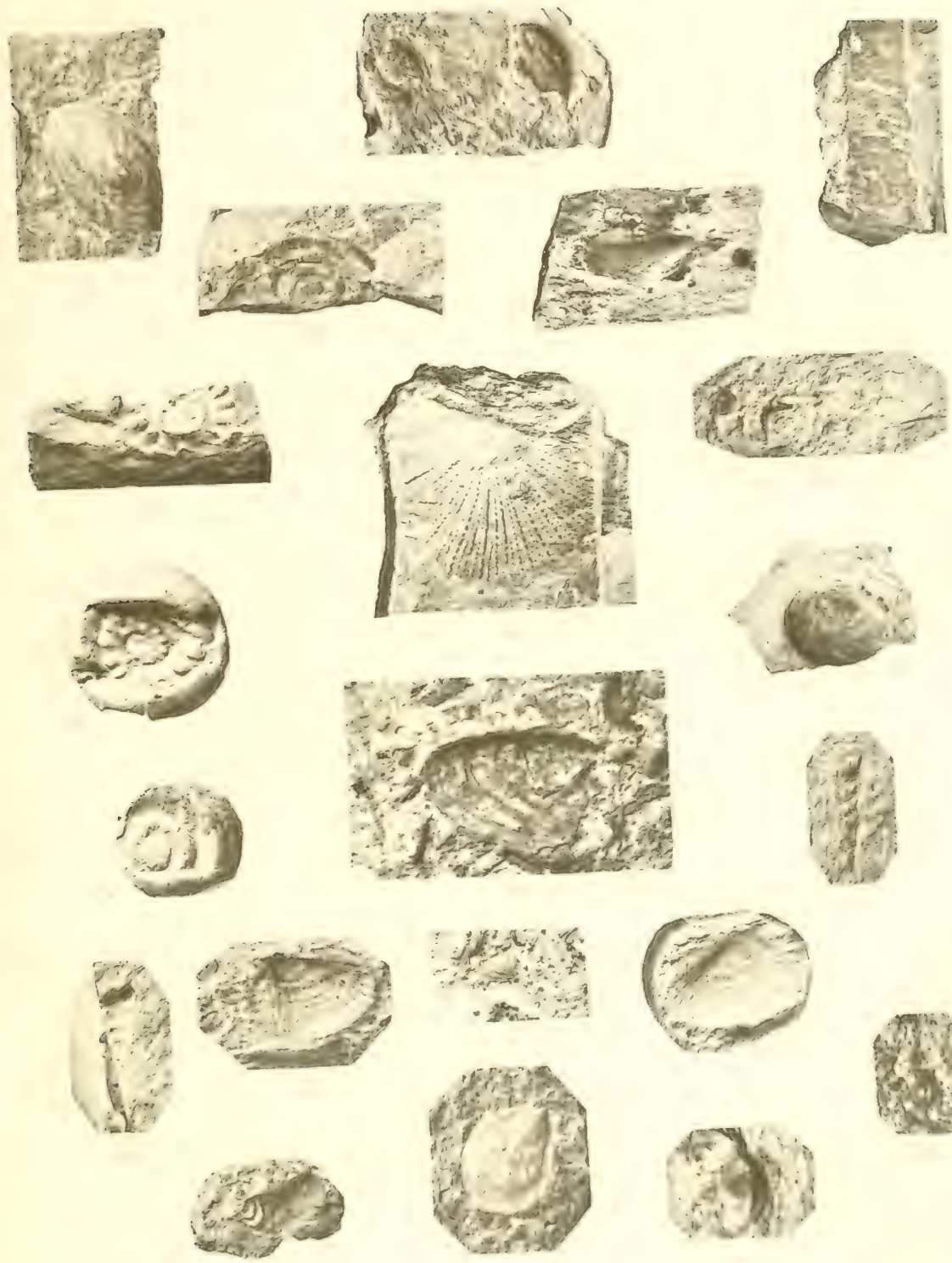




\section{LÉGENDE DE LA PLANCHE V}

FIG. 1. Actinocrinus icosidactylus, Portl. Empreinte externe de calice. La Varville.

FIG. 2. Actinocrinus icosidactylus, Portl. Autre fragment du méme spécimen.

Fig. 3. Poteriocrinus plicatus, Austin. Empreinte d'une plaque radiale. La Varville.

Fig. 4. Actinocrinus, sp.? Empreinte interne de la base d'un calice. Domaine des Roches.

Fig. 5. Actinocrinus triacontadactylus, Miller. Une plaque. La Varville.

FIG. 6. Brachymetopus Duponti, nov. sp. Tète grossie 4 fois. La Varville.

FIg. 7. Brachymetopus Duponti, nov. sp. Pygidium, grandeur naturelle.

Firi. 8. Brachymetopus Duponti, nov. sp. Empreinte externe, grossie 4 fois. Cette empreinte, qui est en creux, parait en relief.

Fig. 4. Braclumetoptes Duponti, nor. sp. Pygidium, grossi 4 fois.

FIs. 111. Griffithides, sp.? Glabelle déformée. La Varville.

Frf́, 11. La même, grossie 4 fois.

Fıs. 12. Phillipsia Barrandei, nov. sp. Portion de tête. La Varville.

Fır. 1:3. Phillipsia Barrandei, nov. sp. La même, grossie 4 fois.

Frs. 11. Griffithides seminiferus, Phillips. Pygidium. La Varville.

Fic. 15. Griffithides seminiferus, Phillips. Grossi 4 fois.

Frs. 16. Autre spécimen. Grossi 4 fois.

Frs. 17. Plitlipsia Barrandel? nov. sp. Empreinte d'une portion de thorax. La Varville.

Fir. 18. Phillipsiu Barrandei? nov. sp. Le mème, grossi 4 fois.

Frri. 19. Entomis concentricu, de Kon. Valve grossie 4 fois. La Varville.

Fici. 20. Fragnient de roche montrant al la surface de nombreux ostracodes $(a, b, c)$. La Varville. 

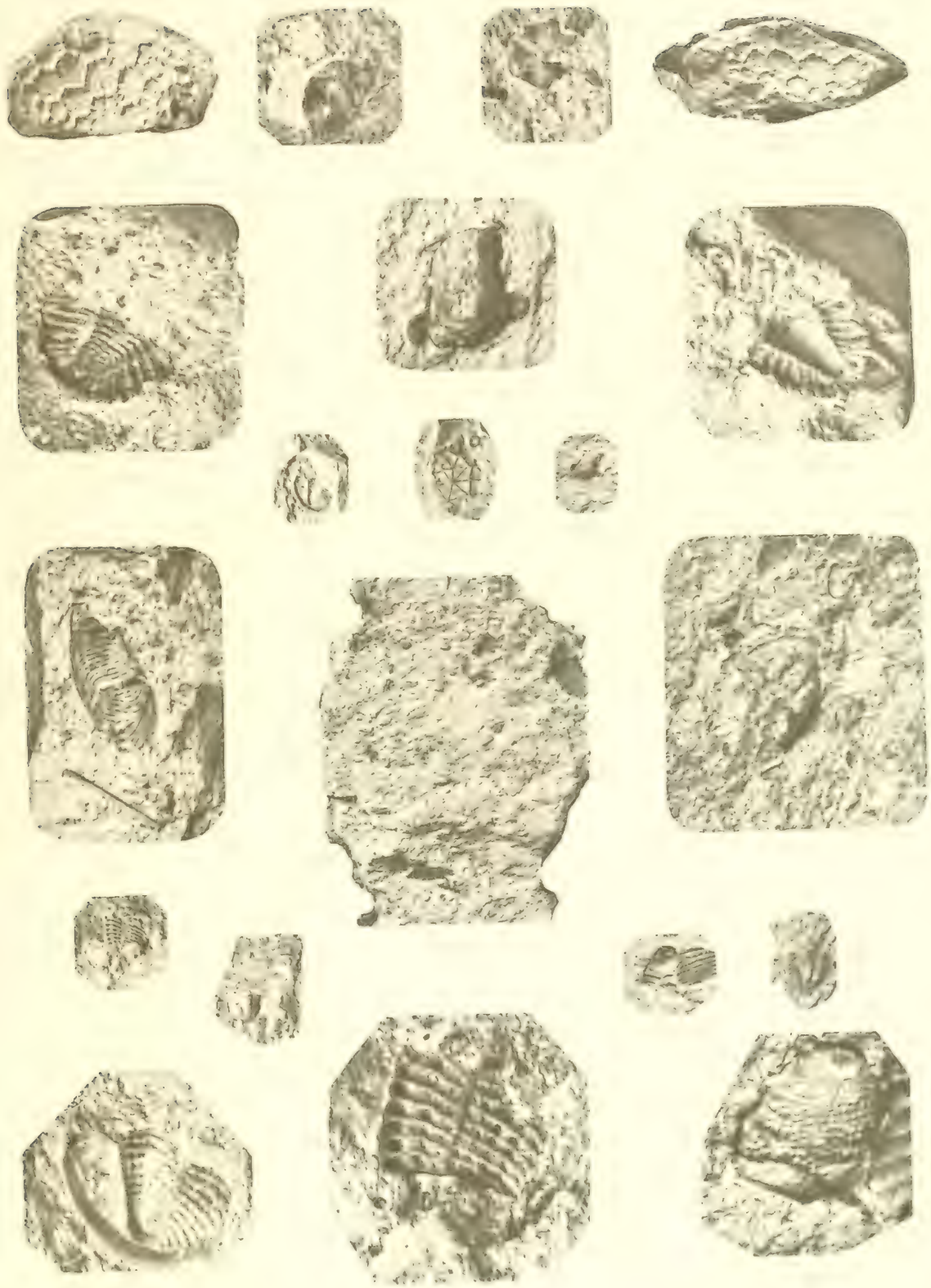




\section{LÉGENDE DE LA PLANCHE VI}

Fig. 1. Fenestella plebeia, M'Coy. Empreinte d'un fragment de colonie. La Varville.

Fig. 2. Fenestella plebeia, M'Coy. Empreinte d'un fragment de colonie. La Varville.

Fig. 3. Fenestella Morrisï, M'Coy. Empreinte de la Varrille.

FIg. 4. Glauconome pulcheryima, M'Coy, Empreinte de plusieurs rameaux. La Varville.

FIG. 5. Glauconome pulcherrima, N'Coy. Un des rameaux grossi.

FIG, 6. Archeocideris Nerei, Münster. Fragarent de roche montrant l'empreinte de plaques interambulacraires et de radioles. Domaine des Roches.

Fig. $\pi$ Archaocidaris Nerei, Münster. Plaque isolẻe et incomplète. Siguret.

FIG. S. Pulcechinus Rutoti, nor. sp. Empreinte d'ure portion de test. Domaine des Roches.

Fig. 9. Monticulipor'u tumida, Phill. Empreinte d'un jeune individu. La Varrille.

FIG. 10. Monticulipora tumida, Phill. La même, grossie 4 fois.

FIG. 11, 12, 13. Cyathaxonice, sp.? La Varville.

Fig. 14. Polypier indéterminable. Siguret.

Fis. 15. Amplexus coralloides, Sowerby. Empreinte externe. La Varville. 

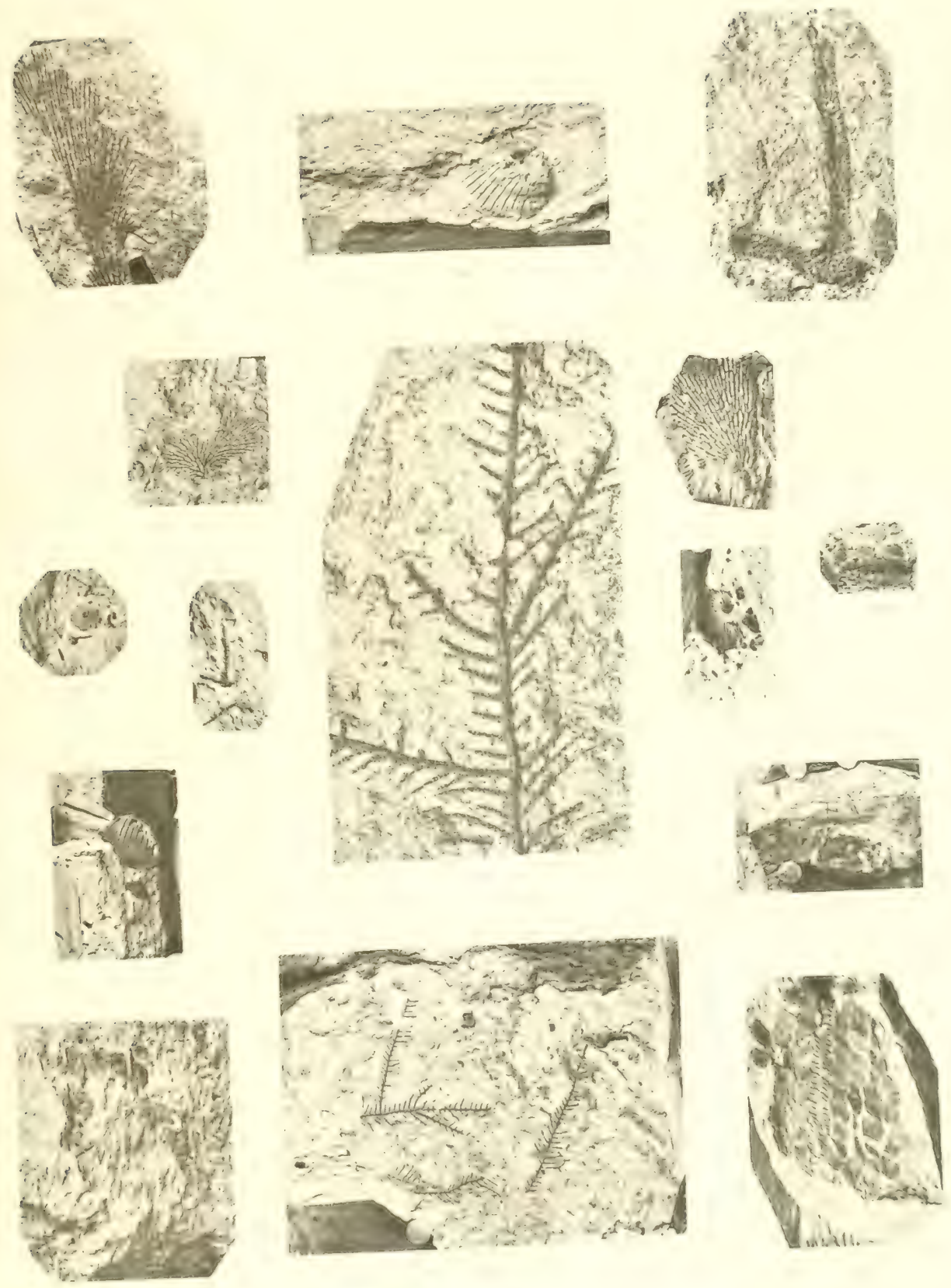




\section{LÉGENDE DE LA PLANCHE VII}

Fig. 1. Productus pustulosus, Phill. Valve ventrale. Régny.

Fig. 2. Productus pustulosus, Phill. Même gisement.

Fir. 3. Prorluctus pustulosus, Phill. Valre dorsale, même gisement.

Fro. 4. Productus semireticulatus, Martin. Var. concinnus. Valve ventrale. Régny.

Frs. 5. Productus semireticulutus, Martin. Var. concinnus. Valve dorsale, même gisement.

Fiss. i. Productus semireticulatus, Martin. Var. concinnus. Valve ventrale, me̊me gisement.

Y... i. Productus $\operatorname{con}^{2} u$, d'Orbigny. Empreinte externe d'une valve dorsale. Régny.

Fis. ¿ Producus cor $\ell$, d'Orbigny. Empreinte externe d'une valve ventrale. Régny.

Fin. ¿ Prorluctus punctatus, Martin. Empreinte de dorsale. Régny.

Firi. 1". Chonetes comoides, Sowerby. Valve ventrale. Régny.

Firr. 11. Chonetes comoides, Sowerby. Valve ventrale, même gisement.

Fifr. 19 ( 1 ). Chonetes comoules, Sowerby. Valve ventrale. Régny. (b) Valve ventrale de jeune Chonetes papilionacea, Phillips.

Firi. 13. Chonetes papilionaceu, Phill. Valve ventrale. Régny.

Firi. 11. Chonetes Murchisoni, nov. sp. Talve ventrale. Régny.

Firi. 1.. Chonetes Dalmaniana, de Koninck. Valve ventrale. Régny.

Prri. I'i. Clunetes' Dalmanianu, de Koninek. Empreinte interne de valve ventrale. Mêmo grisement.

Firi, 17. Chonetes Jourduni, nov. sp. Valve ventrale. Régny.

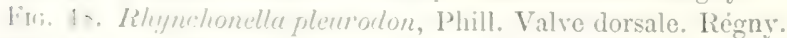

Fir: 19. Athyris plunosulcatu, Phill. Valve ventrale aplatio. Régny.

Fri. si). Didersma succulus, Martin. Valve vontrale (a). Régny. 


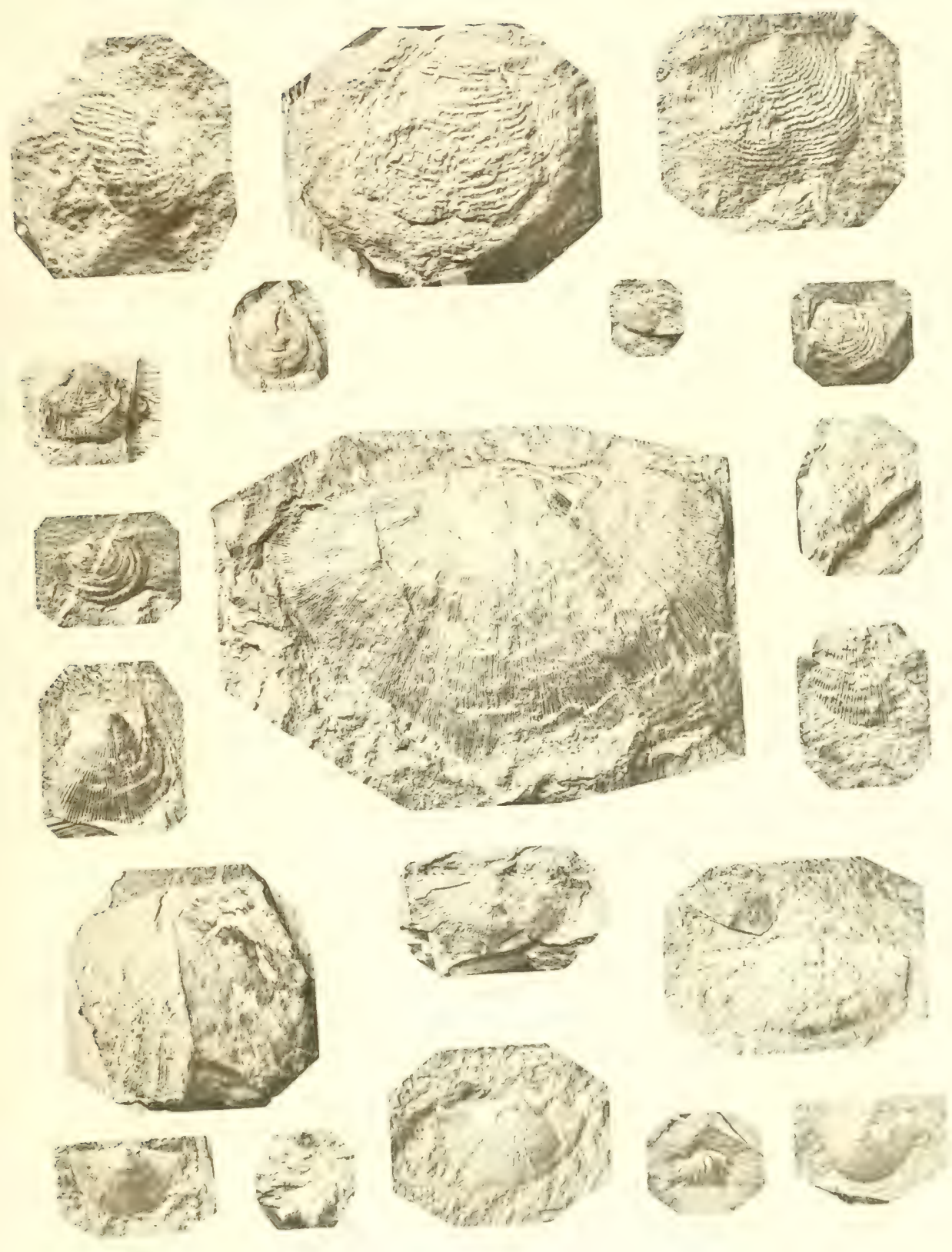






\section{LÉGENDE DE LA PLANCHE VIII}

Fugi. 1. Chonée's Murchisont, nov, sp. Valve ventrale, montrant les épines. Régny.

Fiss. 2. Orthis resupinutu, Mart. Empreinte interne de la ventrale. Régny.

FIc. 3. Orthotete's crenistria, Phill. Valve ventrale. Régny.

Fis. 4. Rhynchonelle pugnus, Martin. Valve dorsale. Régny.

Fis. 5. Murtinue gledera, Martin. Valve ventrale. Régny.

Fics. i. Murtinu linecete, Martin. Vue du cóté de la face ventrale. Rẻgny.

Fisi. 7. Spirifer duplicicosta, Phill. Valve ventrale. Régny.

Firi. Sprifer duplicicoste, Plitll. Valve dorsale. Régny.

Firi. :. Spririfer duplicicosta, Phill. Valve dorsale. Même gisement.

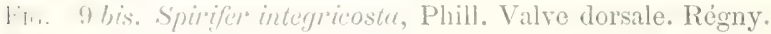

1... 10. Athyr is plunosuleutu, Phill. Deux spécimens. Régny.

Fir. 11. Lepetopsis ef. Busscheriunus, de Rychiholt. Régny.

Firs. I?. Wenergeniu Fertessuei, A. d'Orbigny. Fragment de la face dorsale. Régny.

Fiss. 1:3. Belleprophen seculifer', de Koninck. Spécimen un peu déformé, vu de dos. Régny.

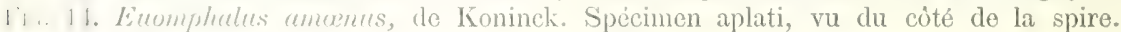
Rérny.

Firs. 1.i. Plenerotinus nuelus, J. de C. Sowerby. Régny.

Fisc. 1ri. Struperrollus Dionysii, D. de Montfort. Spécimen aplati, vu du côté de la spire.

liki. 1\%. Lo fonemu priseum, Goldfuss. Moule interne. Régny.

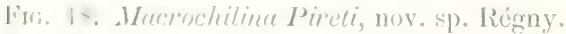

Firi. 1'. Esdmondiu amabilis, de Kon. Valve droite. Régny. 

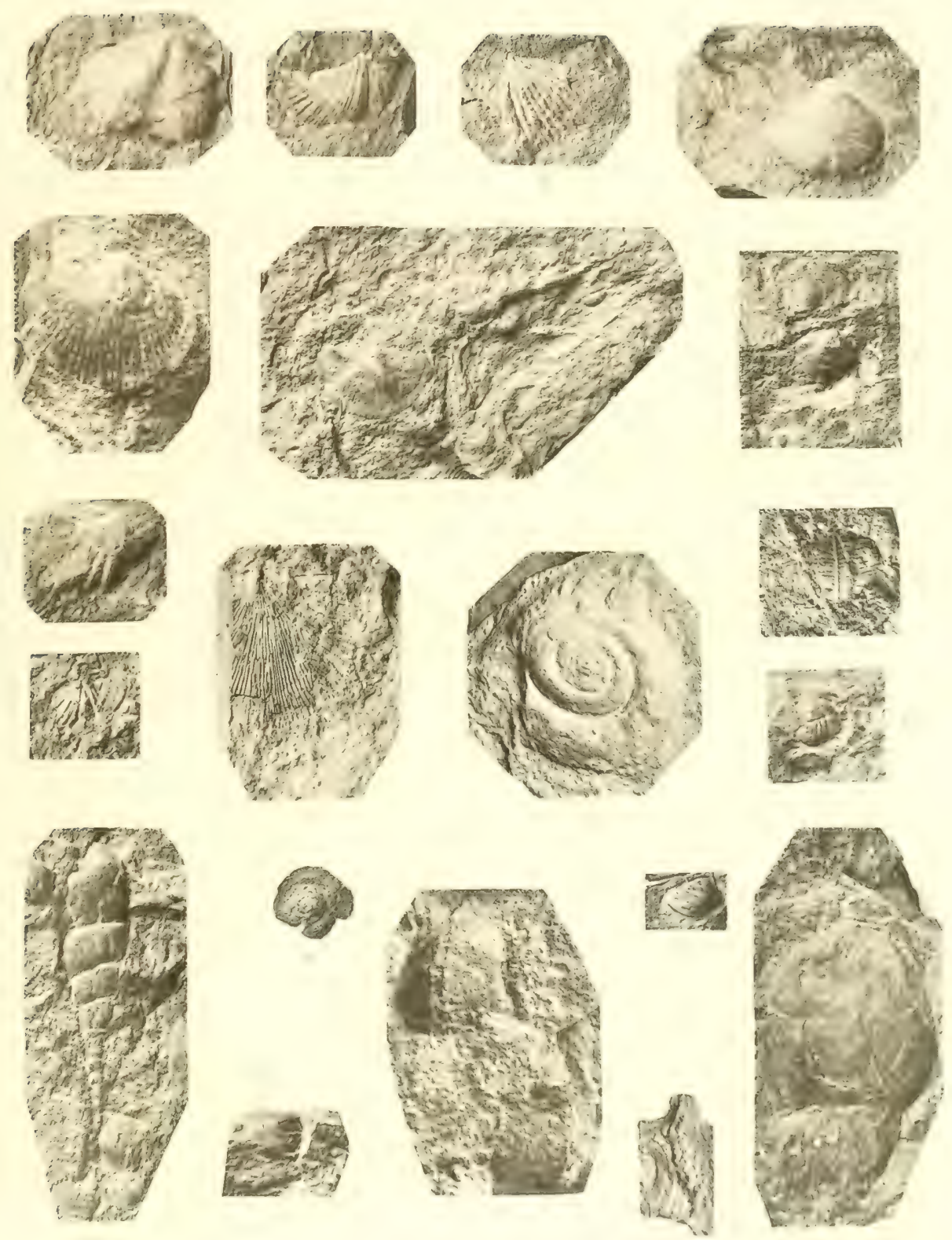




\section{LÉGENDE DE LA PLANCHE IX}

Fiti. 1. Productus pustulostes, Phill. Fragment de ventrale. Néronde.

Fi.. Chonetes comoides, Sowerby. Valve ventrale. Néronde.

Fir. :. Chonetes comoides, Sowerby. Empreinte interne d'une ventrale. Même gisement.

FIr. 1. Chonetes comoides, Sowerby. (a) Empreinte externe d'une ventrale; (b) empreinte interne de la même ralve. Même gisement.

Fis. 5. Chonetes comotides, Sowerby. Empreinte interne d'une autre valve ventrale. Méme givement.

Fir. 1i. Orthis resupinutu, Martin. Valve dorsale. Néronde.

Fui. i. Orthotetes crenistria, Phill. Valve ventrale $(\alpha)$. Néronde.

FIg. - Rhynchonellu pugnus, Martin. Valve dorsale identique à celle de Clitheroe figurée par Datvidson, pl. XXII, fig. 11. Néronde.

Fss, :'Rhynchonella pugnus, Martin. Spécimen un peu plus grand, identique à celui de la fig. 14 de la même planche XXII de Davidson. Néronde.

Firi. 11. Spirifer duplicicosta, Phill. Valve ventrale. Nérunde.

Fisr. 11. Spirifer cluplicicostu, Plilll. Autre spécimen déformé. Même gisement.

Fis. 1:. Euomphalus crotalostomus, M'Coy. Moule interne d'un fragment du dernier tour, vu de profil, et dont une partic seulement a été figurée. Néronde.

Fri. 13. Énomphulus crotalostonus, M'Coy. Le mème, vu de dos, pour montrer la carène. 

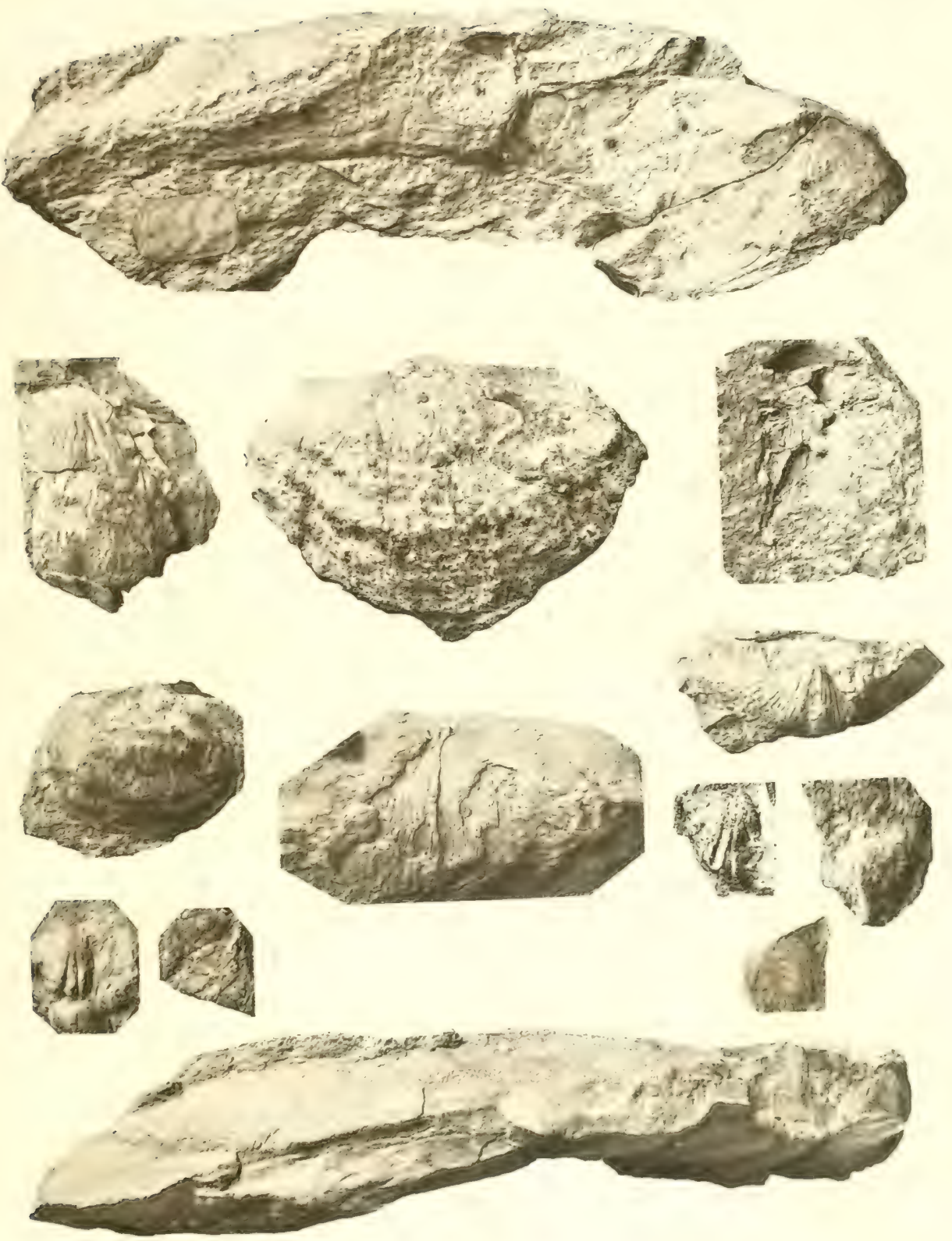




\section{LÉGENDE DE LA PLANCHE X}

Fis. 1. Productus Cor'u, A. d'Orbigny. Empreinte externe d'une valve dorsale. SaintGermain-Laval.

Fig. 2. Productus pustulosus, Phill. Moule interne d'une valve dorsale. Saint-GermainLaval.

Fin. 3. Chonetes papilionucea, Phill. Vu par la valve dorsale. Saint-Germain-Laval.

Fili. 4. Athyris ingens, de Kon. Valve dorsale. Saint-Germain-Laval.

Fisi. 5. Spirifer duplicicostu, Phill. Valve ventrale. Saint-Germain-Laval.

lirci. (j. Athyris planosulcata, Phill. Spécimen ru par la ventrale. Saint-Germain-Laval.

Fir. 7. Aciculopecten plagiostoma, de Kon. Fragment de valve. Régny.

Fis. S. A ciculopecten relatus, M'Coy. Fragment de valve. Régny.

FIg. 9. Aciculopecten? dtupliciradiatus, de Koninck. Valve gauche. Régny.

Frs. 10. Modiola fusitormis, de Kon. Saint-Germain-Laral.

Fis. 11. Leciopteria himando, de Kon. Enıpreinte d'une ralve gauche d'un jeune individu. Rérrny.

Vici. 12. Purallelodon comoides, de Kion. Talve droite. Régny.

Firi. 13. Solenopsis Bielauskiii, nov. sp. Fragment de la valve droite. Régny.

1'vi. 1.1. Sanguinolites tricoslatus, Portlock. Valve droite. Régny.

Fivi. 15. Edmondia? ameenu, de Kon. Valve gauche. Régnt.

Firi. 16. Edmondien secularis, M'Coy. Valve droite. Régny.

Fin. 17. Edmomelie orbiculate, de Kion. Valve droite. Regny.

Lini, 18. Eilmondiu? selecte, de lion. Valve droite. Régny.

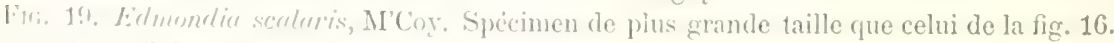
Regny.

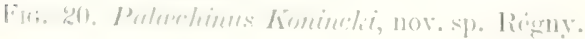



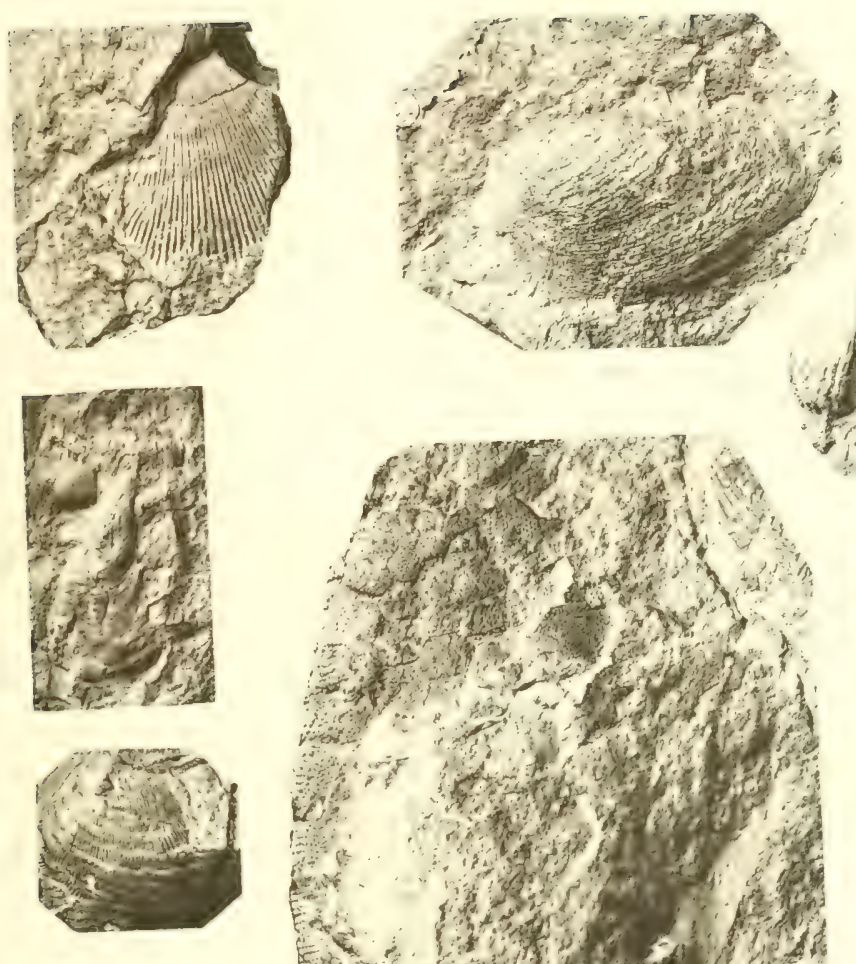

4.

$\because+3, a y+4,4$

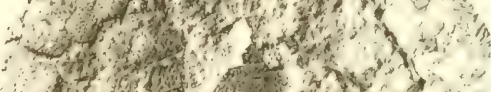

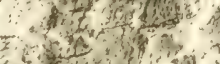

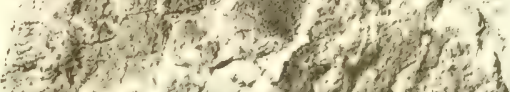
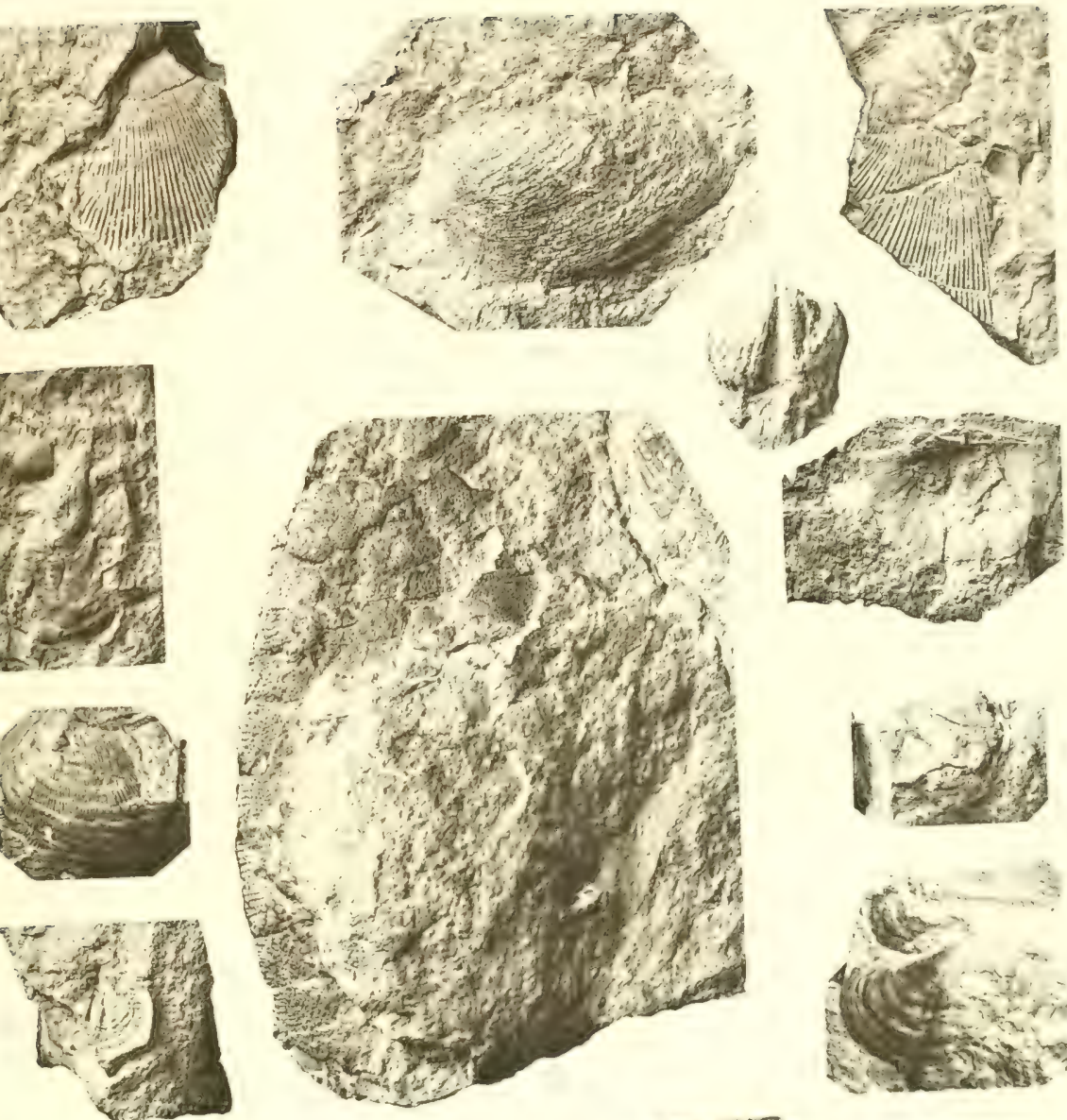

c.

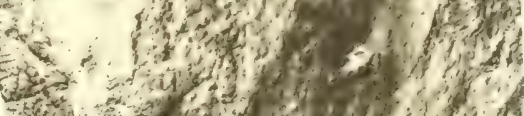

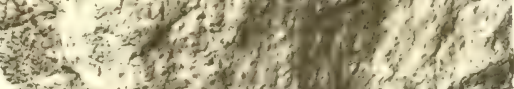
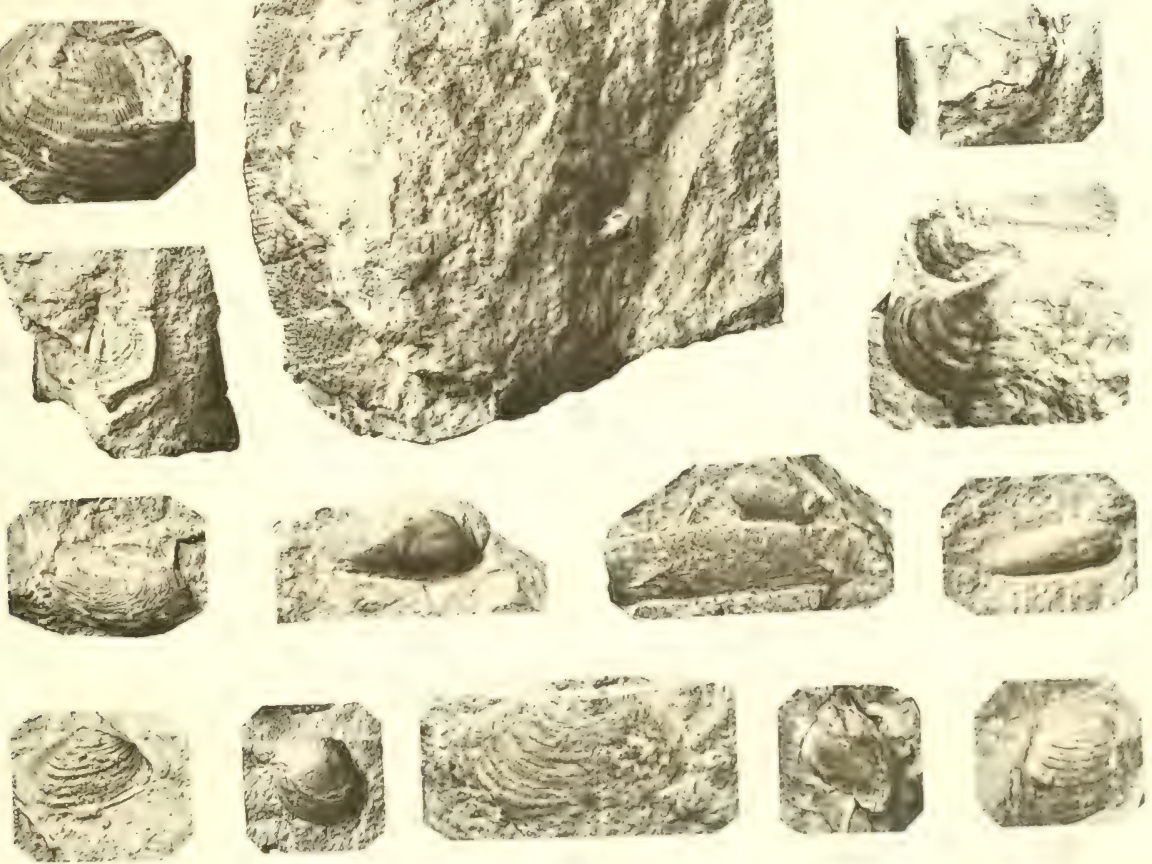




\section{L.EGENE DE LA PLANCHE XI}

Fii. 1. Productus coru, A. d'Orbigny. Moule externe d'une valve dorsale. Montmain.

Firi. $\because$ Productus $\operatorname{cor}^{2} a, A$. d'Orbigny, Moule externe d'une valve dorsale. Méme localité.

Firi. :3. Iroductus punctutus, Martin. Empreinte très effacée; $(\alpha)$ trous des épines. Montmain.

Fur. 1. Productus punctatus, Martin. Autre fragnent de valve. Thizy.

11.. .. Productus fumbriutus? J. de C. Sowerby. Fragment de valve ventrale d'une très mauvaise conservation, comme tous les spécimens de cette localité. Propières.

lini. i. Productus semireticulutus, Martin. Var. concinnus. Empreinte externe de dorsale. Montmain.

Firs. i. Chonetes comoides, Sowerby. Vu du coté de la dorsale. Montagny.

Fili. ․ Chonetes comoides, Sowerby. Valve ventrale. Ferrières.

Fic. ¿. Chonetes comodides, Sowerby. Valve ventrale. Ferrières.

Fir. 11. Chonetes comoules, Sowerby. Valve ventrale. Marbre de Propières.

Fivi. 11. Chonetes comoides, Sowerby. Fragment d'empreinte de la surface interne d'une valve. Cheral-Rigon.

11. 1:. Chonetes pupilionacer, Phill. Empreinte externe de deux valves ventrales. Montnuan.

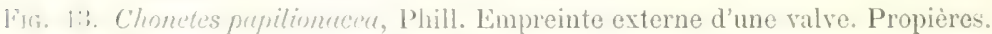

1.r. 11. Cleonetes pupilionuere, Phill. Empreinte externe d'une valve. Propières.

Fir. 15. Chonetes variolatu, d'Orb). Empreinte externe de la dorsale montrant l'aréa de la ventrale. Propieres.

Iisc. Mi. Orthis restipinuta, Mart. Valve ventrale écrasée. Montagny.

Fin: 1: Orthotetes crenistru, Phill. Empreinte d'une portion de ventrale. Propières.

Firi. In Crthotetes crenistriu, lohill. Limpreinte externe aree fragment de test de la dorsale. Nontmain.

1.1. 1'. Mrthetetes crenistrin, Phill. Limpreinte interne de ventrale. Propières.

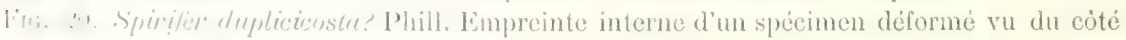
de la valve dorsale. Propières.

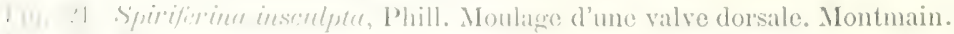

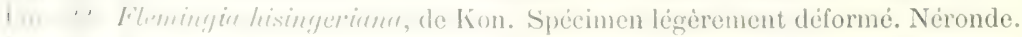

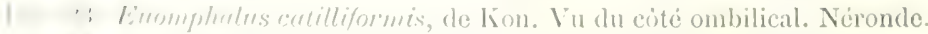

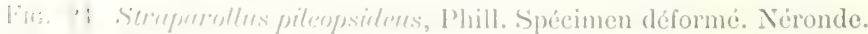

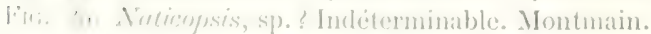

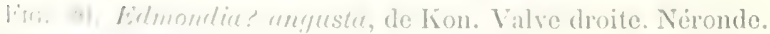

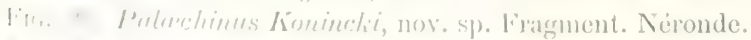

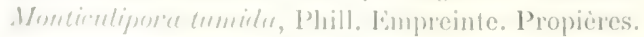

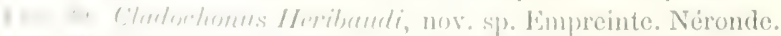

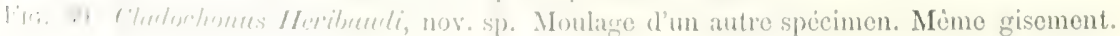



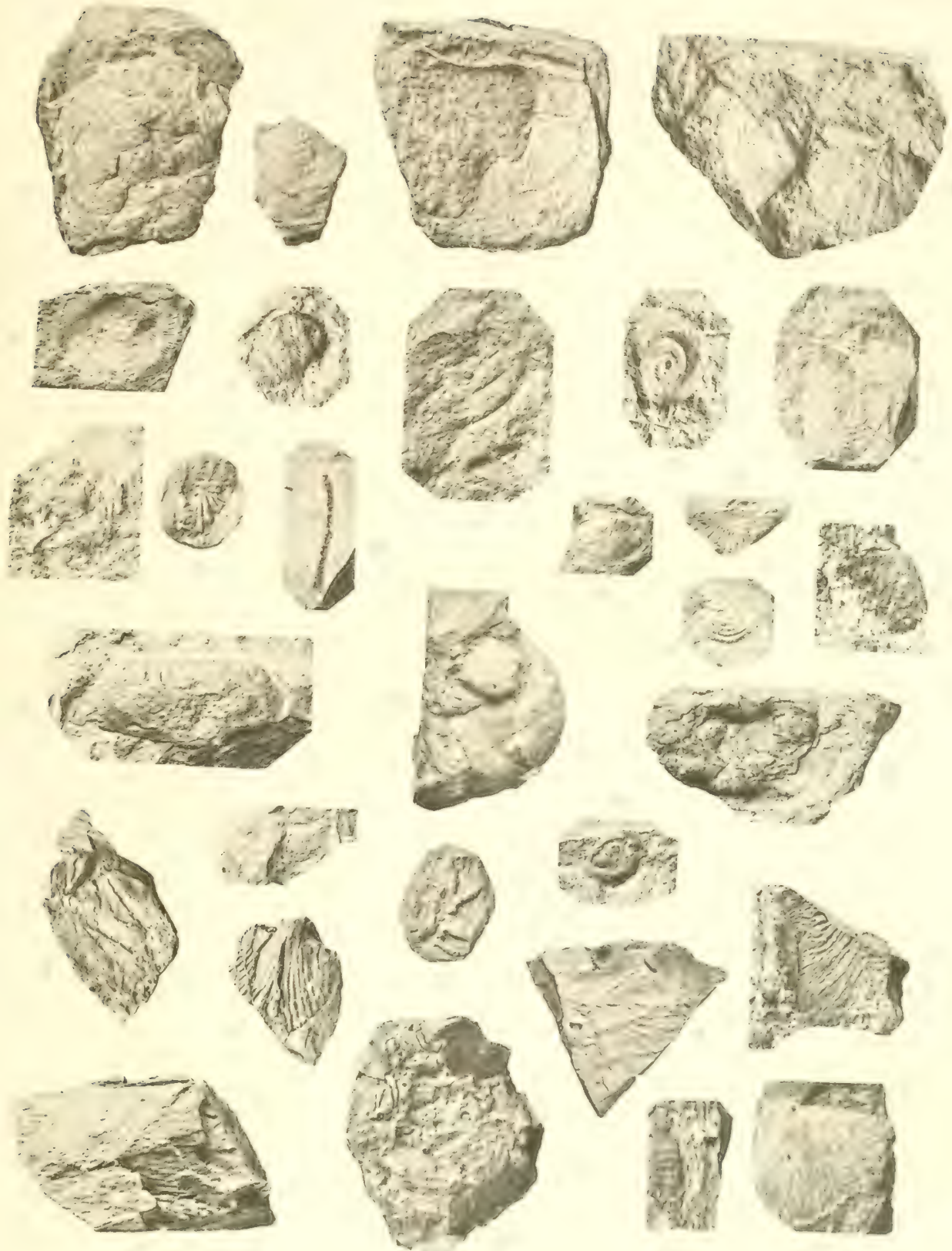



\section{LEGENDE DE LA PLANCHE XII}

Firs. 1. Productus gigunteus, Martin. a Empreinte de la surface externe de la dorsale; $b$ empreinte de la surface interne de la ventrale; $c$ empreinte de la portion inférieure du muscle diducteur; $d$ fragment de la valve ventrale avec ses ornements extérieurs. L'Ardoisière.

Vir. 2. Productus giganteus, Martin. Belle empreinte de la dorsale d'un autre spécimen; a trous des épines. L'Ardoisière.

lis. 3. Productus yigunteus, Martin. Empreinte du bord cardinal d'une dorsale montrant. Ies trous des épines sur les oreillettes. L'Ardoisière.

Firs. 4. Productus gigcunteus, Martin. Autre spécimen; le cỏté frontal de cette empreinte est représenté pl. XIII, fig. 1. L’Ardoisière.

Fro. 5, 6. Productus giguntets, Martin. Moulage d'une eavité interne de Pr. giganteus de l'Ardoisiere.

Firi. 5. Producuts gigunteus, Nartin, Empreinte interne du crochet et des parties aroisinantes, vue du cóté de la face dorsale; $b, c$ empreintes des muscles adducteurs; It septum médian; a retour de la coquille.

liri. b. Prontuctus gigunteus, Martin. La mème, vue du cóté de la face ventrale; $a, b$ empreintes des museles adducteurs; $c$, d empreintes des muscles diducteurs.

Noutu. - Lit figure bi est représentée le crochet en haut, tandis que la figure 5 a ete renversée par mégarde, de sorte que le crochet est en bas et le retour a de la (nequille brisée en haut. L'Ardoisière.

Firs. 7. Prorlucturs pustulesus, Phill. "Portion de valve ventrale; $b$ valve ventrale monfrant nettement le simus; $c$ empreinte de la valve dorsale avee le bourrelet encore indiquè malgré l'écrasenent. L'Ardoisière.

Firi. \&. Protuetus punctutus, Mart. Valve ventrale. L'Ardoisière. 

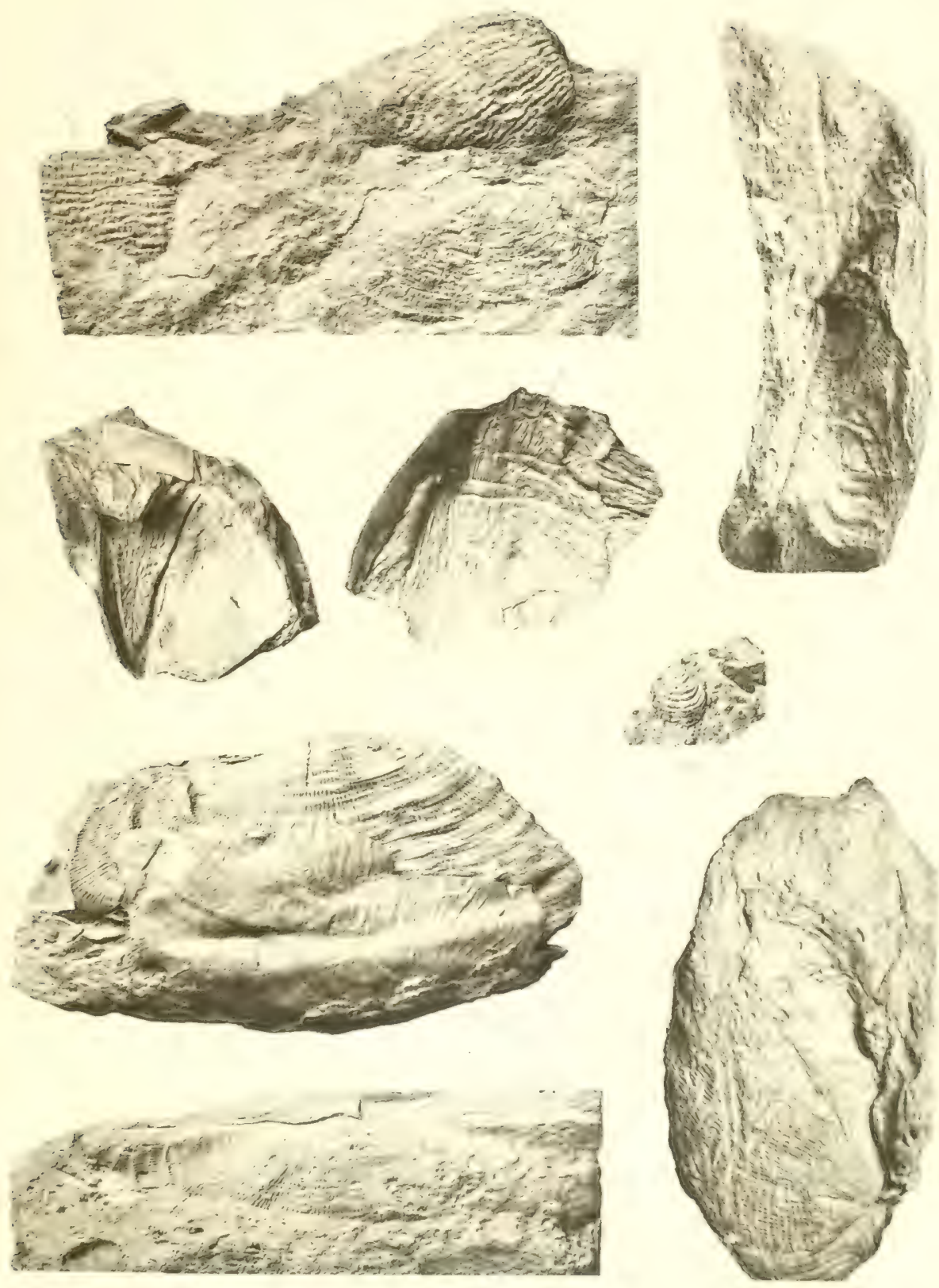




\section{LEGENDE DE LA PLANCHE XIII}

Fin. 1. Productus gigunteus, Mart. Empreinte d'une valve dorsale vue du cóté frontal. Le bord cardinal du mème spécimen est représenté pl. XII, fig. 4. L'Ardoisière.

1 i.. Productus gingunteus, Mart. Autre spécimen. Empreinte d'une dorsale. L'Ardoisière.

Hu.. : Productus fustulosus, Phill. Valve ventrale. L'Ardoisière.

Hi.. 1. Productus pustulosus, Phill. Empreinte de valve dorsale montrant encore le léger bourrelet. L'Ardoisière.

Fu. 5. Productus fimbriatus, J. de C. Sowerby. Empreinte interne d'une valve dorsale. L'Ardoisière.

Ia. i. I'Productus fimbriatus, J. de C. Sowerby. Enpreinte externe de la méme valve.

Fi, i. Productus fimbriatus, J. de C. Sowerby. Empreinte externe d'une valve ventrale. Méme gisement.

1... - Productus semireticulutus, Mart. Var. concinnus. Les deux valves. L'Ardoisière. a Valve dorsale; b valve ventrile. 

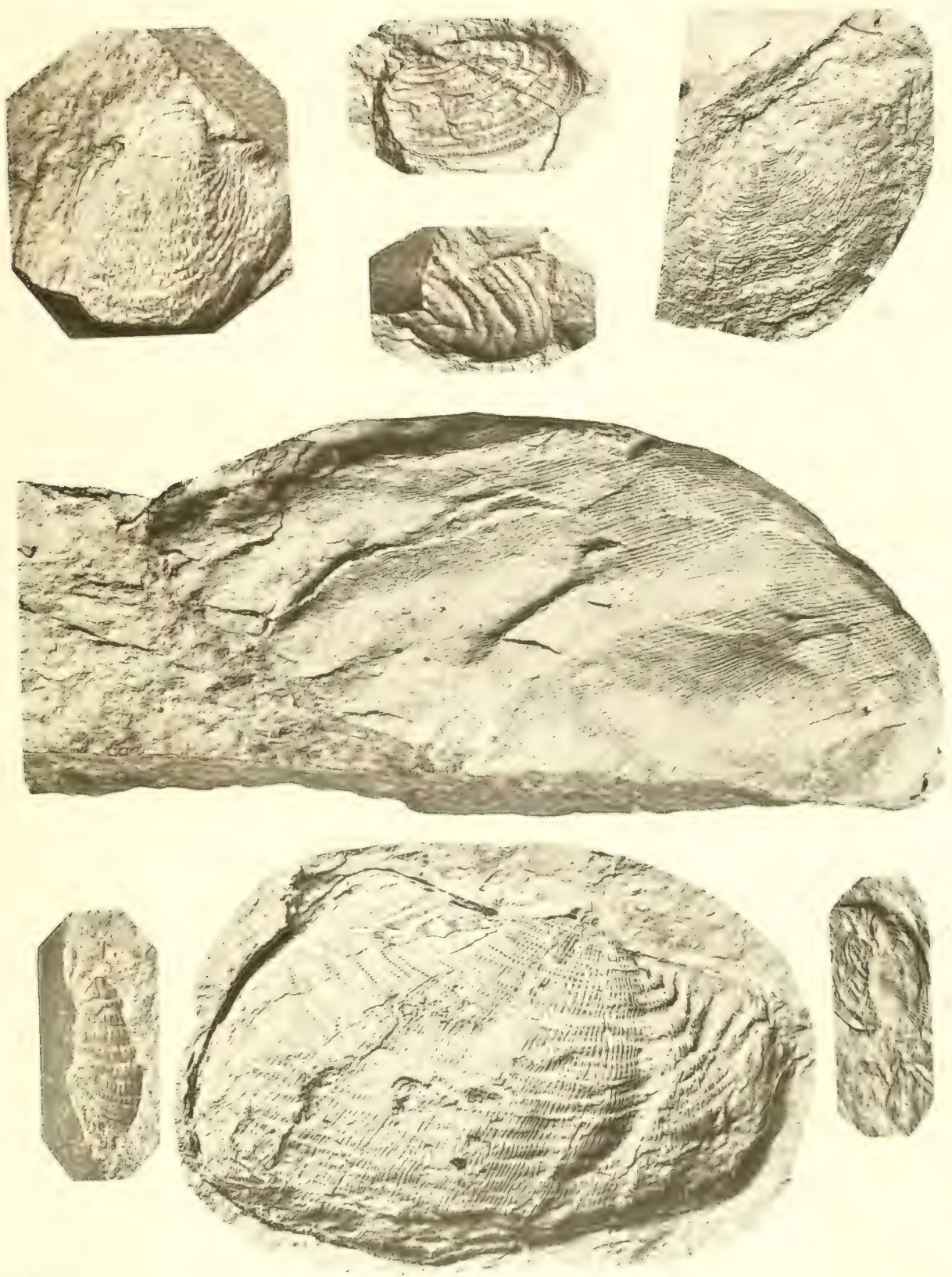



\section{LÉGENDE DE LA PLANCHE XIV}

Fir. 1. Productus cora, d'Orbigny. Magnifique spécimen d'une empreinte de la valve dorsale, vue du cóté de la charnière. L'Ardoisière.

H... 2 Productus cora, d’Orbigny. La même, vue du côté frontal.

Fic, 3. Productus cora, d'Orbigny. Empreinte externe d'une valve ventrale d'un autre spécimen. L'Ardoisière.

Fi:. 1. Productus punctutus, Martin. Empreinte externe d'une valve dorsale. On voit it gauche de la coquille un paquet d'épines aussi fines que des cheveux. L'Ardoisière.

Fin. D. Productus punctutus, Martin. Empreinte externe de valve dorsale. Mème gisement.

Frs. i. Productus giganteus, Martin. Noule interne de la ventrale; a empreinte du muscle diducteur; b empreinte du muscle adducteur. L'Ardoisière.

Fisi. i. Productus gigunteus, Martin. Vu de dos, offrant l'empreinte des aspérités de la face interne. 

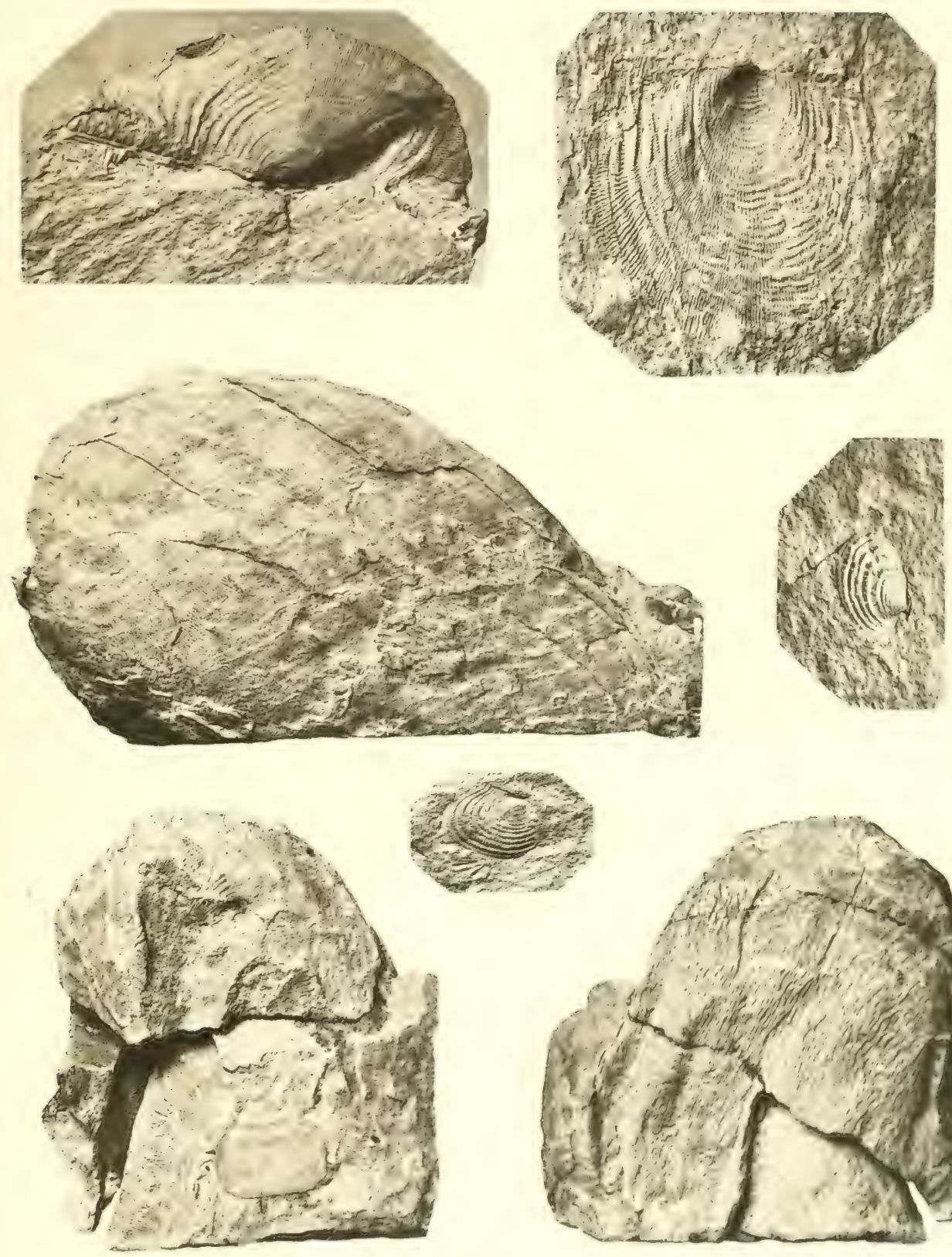



\section{LÉGENDE DE L،A PLANCHE XV}

Fig. 1. Productus gigunteus, Mart. Empreinte du bord cardinal d'une valve dorsale avec empreinte de pygidium de Griffithides Eichtoaldi, F. de Wald. L'Ardoisière.

Fii. ?. Chonetes papilionacea, Phill. Empreinte externe d'une dorsale déformée par pression latérale. L'Ardoisière.

Fis. 3. Chonetes papilionacea, Phill. Empreinte interne d'une valve dorsale. L'Ardoisière.

Fir. 1. Orthis resupinuta, Mart. Enpreinte de la cavité viscérale vue par la face ventrale; $a, b$ fragments du test qui recouvrent encore le moule interne $c$. L'Ardoisière.

Fili, $\therefore$ Orthis resupinatu, Mart. Le mème spécimen, vu par la face dorsale.

Fis. ti. Orthotetes crenistria, Phill. Empreinte d'un grand spécimen. L'Ardoisière.

Fra. i. Murtinice glabru, Mart. Spécimen vu du cóté de la dorsale. L'Ardoisière.

Fin. ‘. Spritifer bisuleatus, Sowerby. Spécimen vu du cóté umbonal. L'Ardoisière.

Frs. 1 Spirifer bisulcatus, Sowerby. Vu du còté de la valve dorsale. L'Ardoisière.

Fo. 10. Nantilus sulcutus, J. Sowerby. Empreinte externe d'un spécimen un pen plus grand que ceux de Belgirjue. L'Ardoisiẻre.

Fig. 11. Nautilus sulcutus, J. Sowerby. Moulage du même.

Fig. 12. P'lychomphulus, sp.? L'Ardoisière.

Fri. 1\%. Straponollus pileopsidens, Phill. L'Ardoisière.

I... 11. P'osidoniclle vetustu, Sow. Moulage d'une valve. L'Ardoisière.

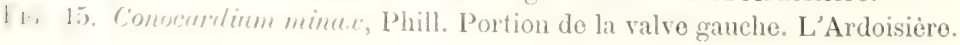




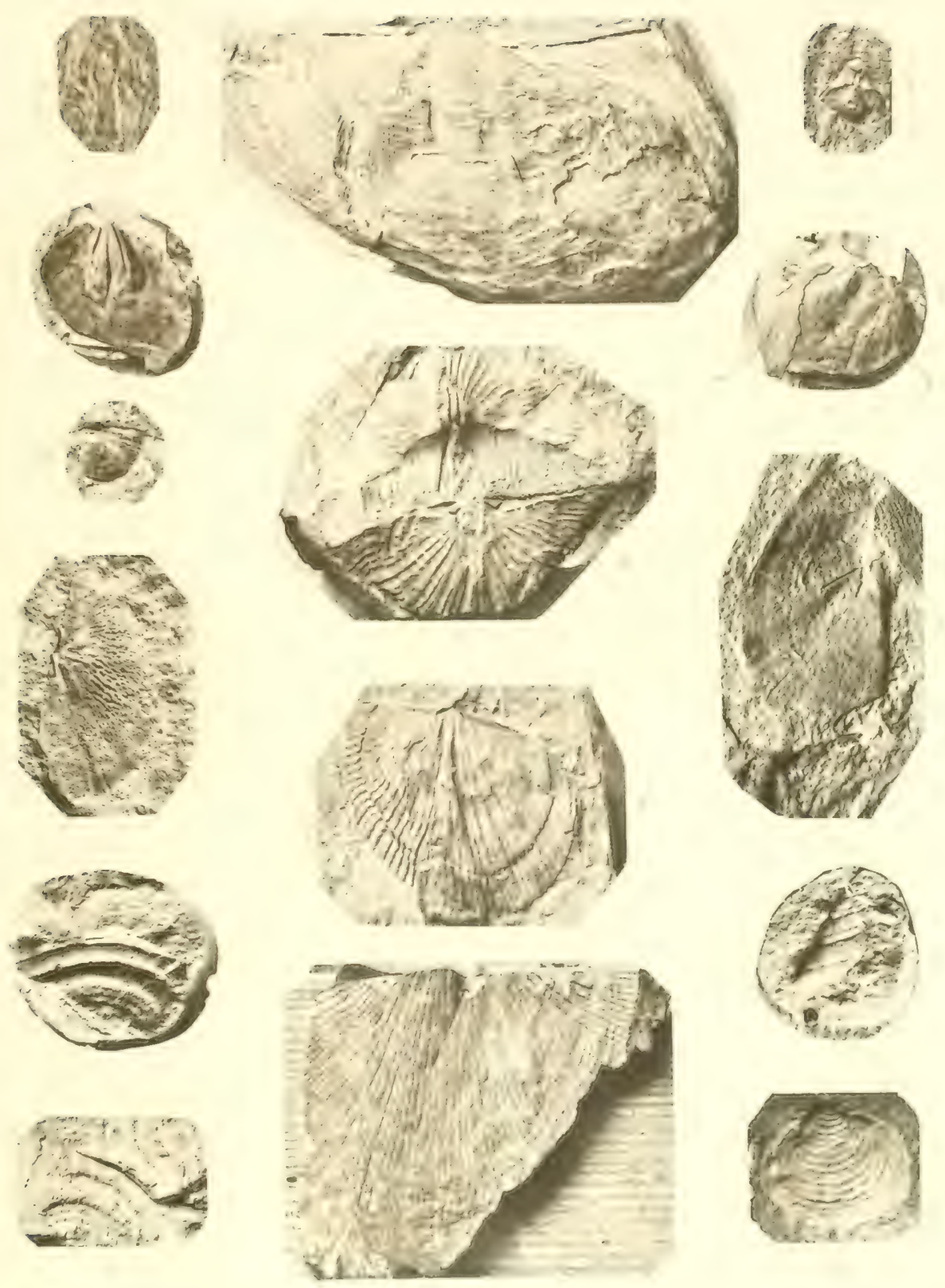





\section{LÉGENDE DE LA PLANCHE XVI}

Fif. 1. Pholidociduris Gundryi, nov. sp. Fragment d'empreinte mal conservée montrant l'aire ambulacraire à 6 rangées de plaques. L'Ardoisière.

Fir. 2. Pholidocidaris Gaudryi, nov, sp. Fragment d'un autre spécimen, montrant une portion de l'aire interambulacraire; $a, c$ plaques offrant l'ornementation; $b$ plaque dont l'ornementation est effacée; $d$ plaque ambulacraire. L'Ardoisière.

I\%, 3. Palechinus Lacasci, nov. sp. Empreinte d'un fragment de test, montrant les aires ambulacraire a et interambulacraire $b$. L'Ardoisière.

W. :. Puluelimus Lacazet, noy. sp. Fragment de test écrasé d'un autre spécimen. L'Ardoisiere.

Virg. ․ Pulcechimus Lacuzci, nov. sp. Une portion d'aire ambulacraire. L'Ardoisière.

Fin. 1i. Pholidocidaris Gundryi? nov. sp. Empreinte d'une grande plaque isolée. L'Arthoisiere.

Fis. T. Pholidociduris Gundryi? nov, sp. Plaques isolées ambulacraires et interambulaeraires, d'ornementation variée et empreintes de piquants. L'Ardoisière.

Fri. - Archeociduris Urii, Flening. Empreinte externe de plagnes interambulacraires. L.Artoisiere.

Firi. :I et 10. Artherecideris Urii, Fleming. Nème gisement.

Firi. 11. Archeucheleris Grïneri, nov. sp. Empreinte d'une portion de test montrant 2 rangres de placyes interambulacraires et l'aire ambulacraire adjacente $\alpha$. L'Ardoisicre.

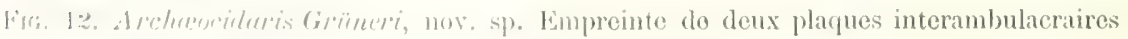
isoleser. I'Ardoisiere.

lik, 13, 15, 16, Rarlioles it fiangres d'épines. L'Ardoisière.

Fin, 11, 17. Rathlinles it 4 rangeres d'épines. L'Ardoisiere. 

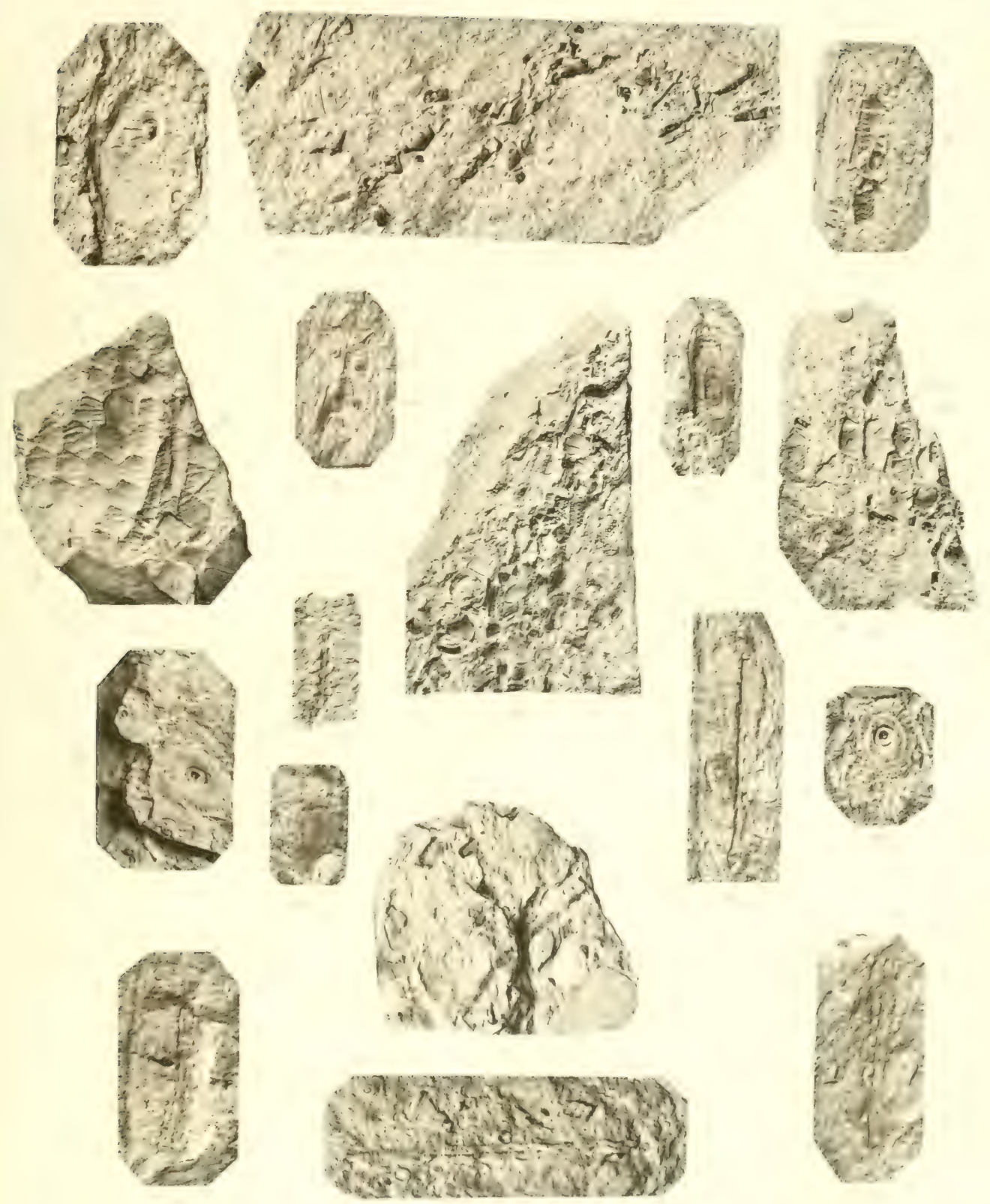




\section{LÉGENDE DE LA PLANCHE XVII}

F... 1. Phillipsia Eichuculdi, Fischer. Groupe otfrant un Phillipsic entier, un Pygidium á cóté, et à droite un autre individu entier, mais incomplètement représenté dans la figure. L'Ardoisière.

I'.. 2, 3. Phillipsia Eichoaldi, Fischer. Autres Pygidiums. L'Ardoisière.

1,.. 4. Palceoflustra Jolieti, nov. sp. L'Ardoisière.

I'i.. 5. Fenestella multiporata, M'Coy. L'Ardoisière.

1. . 6. Potertucrinus crussus, Miller. Moule interne du canal d'une tige. L'Ardoisière.

1... 7. Poteriocrinus crussus, Miller. Moule interne du canal d'une tige. L'Ardoisière.

i': 8, 10. Rarlioles d'Archuecidur'is is 4 rangées d'épines. L'Ardoisière.

1 ' 9. Radioles d'Arehceociduris à 6 rangées d'épines. L'Ardoisière.

i 1.. 11. Sypringopora rumulosu, Goldf. Bloc de roche montrant un grand nombre d'empreintes de Polypiérites. L'Ardoisière.

1.. 12. Monticulipora inflata, de Kon. L'Ardoisière.

1 - 13. Amplexus coralloides, Sowerby. Empreinte externe. L'Ardoisière.

1... 14. Lithostrotion junceun, Fleming. Empreinte d'un fragment de Polypier montrant en a un calice conservé. L’Ardoisière. 


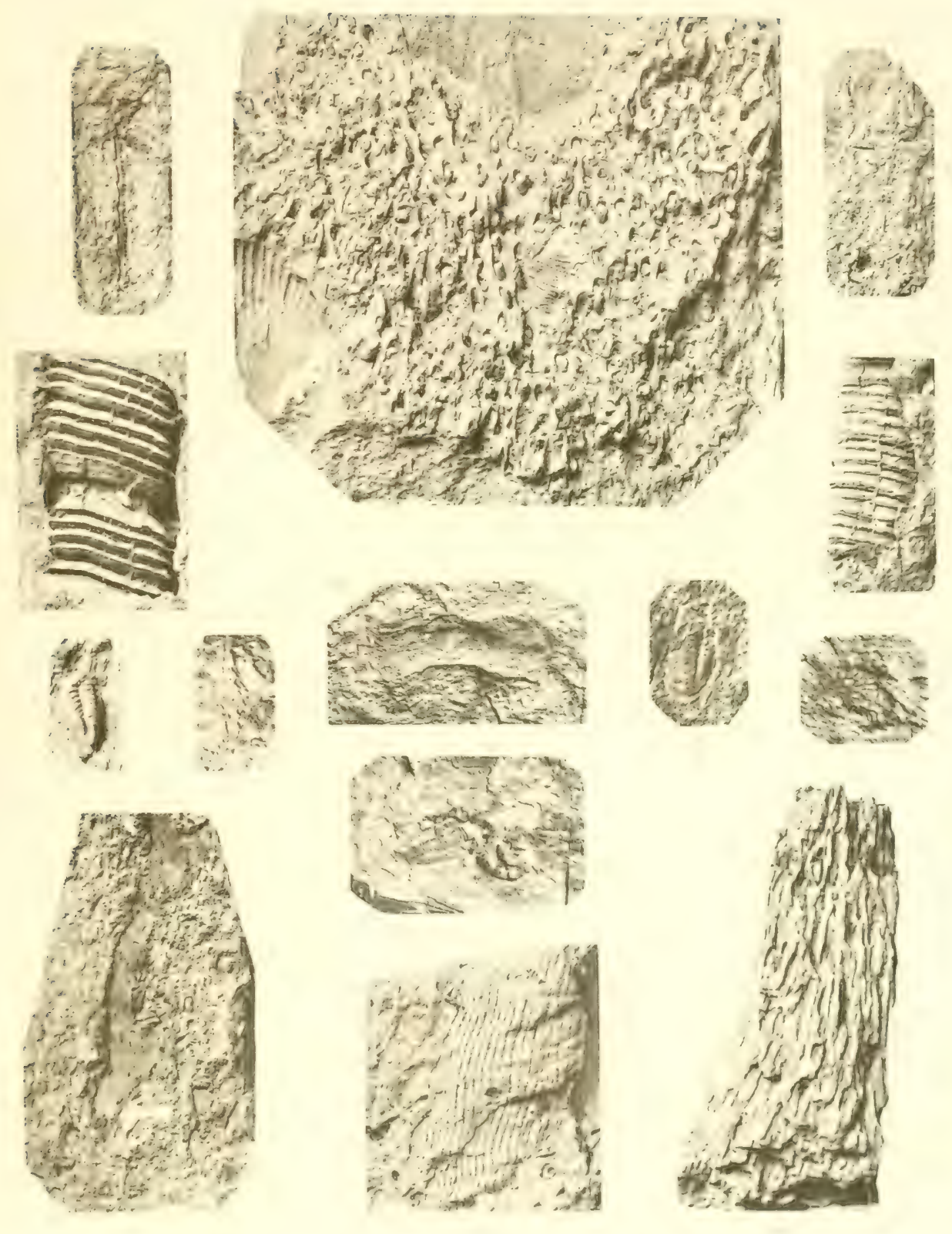






942 
PLEASE DO NOT REAOVE CARDS OR SLIPS FROM THIS POCKET

UNIVERSITY OF TORONTO LIBRARY

2E Julien, Pierre Alphonse

729 Le terrain carbonifère marin

J85 de $\mathrm{la}$ France centrale

1896

Geology 


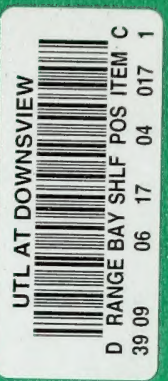

